\title{
IUPAC-NIST Solubility Data Series. 98. Solubility of Polycyclic Aromatic Hydrocarbons in Pure and Organic Solvent Mixtures-Revised and Updated. Part 3. Neat Organic Solvents
}

\author{
William E. Acree, Jr. ${ }^{\text {a) }}$ \\ Department of Chemistry, University of North Texas, Denton, Texas 76203, USA
}

(Received 28 November 2012; accepted 30 November 2012; published online 4 March 2013)

\begin{abstract}
This work updates Vols. 54, 58, and 59 in the IUPAC Solubility Data Series and presents solubility data for polycyclic aromatic hydrocarbon solutes dissolved in neat organic solvents. Published solubility data for acenaphthene, anthracene, biphenyl, carbazole, dibenzofuran, dibenzothiophene, fluoranthene, fluorene, naphthalene, phenanthrene, phenothiazine, pyrene, thianthrene, and xanthene that appeared in the primary literature from 1995 to the end of 2011 are compiled and critically evaluated. Experimental solubility data for more than 550 different solute-organic solvent systems are included. Solubility data published prior to 1995 were contained in three earlier volumes (Vols. 54, 58, and 59) and are not repeated in this volume. (C) 2013 American Institute of Physics. [http://dx.doi.org/ $10.1063 / 1.4775402]$
\end{abstract}

Key words: acenaphthene; alcohols; alkanes; alkanenitriles; alkoxyalcohols; alkyl ethanoates; anthracene; biphenyl; carbazole; chloroalkanes; dialkyl ethers; dibenzofuran; dibeznothiophene; fluoranthene; fluorine; naphthalene; phenanthrene; phenothiazine; polycyclic aromatic hydrocarbons; pyrene; solubility; thainthrene; xanthenes.

\section{CONTENTS}

1.

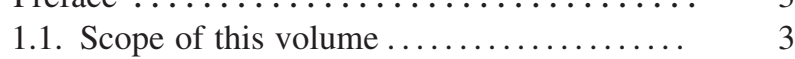

1.2. Procedures used in critical evaluation of published solubility data ............ 3

2. Solubility of Acenaphthene in Organic Solvents 4

2.1. Critical evaluation of experimental solubility data .......................

2.2. Acenaphthene solubility data in saturated hydrocarbons (including cycloalkanes) ....

2.3. Acenaphthene solubility in aromatic hydrocarbons.......................

2.4. Acenaphthene solubility data in esters.....

2.5. Acenaphthene solubility data in ethers ....

2.6. Acenaphthene solubility data in haloalkanes, haloalkenes, and haloaromatic hydrocarbons

2.7. Acenaphthene solubility data in alcohols ..

2.8. Acenaphthene solubility data in alkoxyalcohols

3

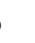

3

4

2.9 . Acenaphthene solubility data in ketones...

2.10. Acenaphthene solubility data in miscellaneous organic solvents ......... 26

3. Solubility of Anthracene in Organic Solvents... 28

3.1. Critical evaluation of experimental solubility data .......................
3.2. Anthracene solubility data in saturated hydrocarbons (including cycloalkanes) ....

3.3. Anthracene solubility data in aromatic hydrocarbons....................... 31

3.4. Anthracene solubility data in esters ...... 32

3.5. Anthracene solubility data in ethers ...... 33

3.6. Anthracene solubility data in haloalkanes, haloalkenes, and haloaromatic hydrocarbons 34

3.7. Anthracene solubility data in alcohols.... 36

3.8. Anthracene solubility data in alkoxyalcohols 42

3.9. Anthracene solubility data in miscellaneous organic solvents .................. 44

4. Solubility of Biphenyl in Organic Solvents.... 50

4.1. Critical evaluation of experimental solubility data .................... 50

4.2. Biphenyl solubility data in saturated hydrocarbons (including cycloalkanes) .... 50

4.3. Biphenyl solubility data in aromatic hydrocarbons....................... 53

4.4. Biphenyl solubility data in ethers ........ 53

4.5. Biphenyl solubility data in alcohols ...... 54

4.6. Biphenyl solubility data in alkoxyalcohols 60

4.7. Biphenyl solubility data in miscellaneous organic solvents ................... 62

5. Solubility of Carbazole in Organic Solvents.... 62

5.1. Critical evaluation of experimental solubility data.................... 62

5.2. Carbazole solubility data in miscellaneous organic solvents ................... 63 
6. Solubility of Dibenzofuran in Organic Solvents .

6.1. Critical evaluation of experimental solubility data

65

6.2. Dibenzofuran solubility data in saturated hydrocarbons (including cycloalkanes) ....

6.3. Dibenzofuran solubility data in aromatic hydrocarbons........................

6.4. Dibenzofuran solubility data in esters ....

6.5. Dibenzofuran solubility data in ethers.....

6.6. Dibenzofuran solubility data in haloalkanes, haloalkenes, and haloaromatic hydrocarbons

6.7. Dibenzofuran solubility data in alcohols...

6.8. Dibenzofuran solubility data in miscellaneous organic solvents...........

7. Solubility of Dibenzothiophene in Organic

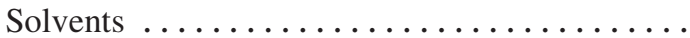

7.1. Critical evaluation of experimental solubility data.

7.2. Dibenzothiophene solubility data in saturated hydrocarbons (including cycloalkanes) ....

7.3. Dibenzothiophene solubility data in esters.

7.4. Dibenzothiophene solubility data in miscellaneous organic solvents .........

8. Solubility of Fluoranthene in Organic Solvents .

8.1. Critical evaluation of experimental



8.2. Fluoranthene solubility data in saturated hydrocarbons (including cycloalkanes)....

8.3. Fluoranthene solubility data in aromatic hydrocarbons......................

8.4. Fluoranthene solubility data in esters......

8.5. Fluoranthene solubility data in ethers .....

8.6. Fluoranthene solubility data in haloalkanes, haloalkenes, and haloaromatic hydrocarbons

8.7. Fluoranthene solubility data in alcohols ...

8.8. Fluoranthene solubility data in miscellaneous organic solvents ......................

9. Solubility of Fluorene in Organic Solvents.....

9.1. Critical evaluation of experimental solubility data ......................

9.2. Fluorene solubility data in saturated hydrocarbons (including cycloalkanes) ....

9.3. Fluorene solubility data in aromatic hydrocarbons......................

9.4. Fluorene solubility data in esters..........

9.5. Fluorene solubility data in ethers..........

9.6. Fluorene solubility data in haloalkanes, haloalkenes, and haloaromatic hydrocarbons

9.7. Fluorene solubility data in alcohols .......

9.8. Fluorene solubility data in ketones........

9.9. Fluorene solubility data in miscellaneous organic solvents .....................

10. Solubility of Naphthalene in Organic Solvents .

10.1. Critical evaluation of experimental solubility data.....................

10.2. Naphthalene solubility data in saturated hydrocarbons (including cycloalkanes)...
10.3. Naphthalene solubility data in aromatic hydrocarbons .................... 118

10.4. Naphthalene solubility data in esters .....

10.5. Naphthalene solubility data in ethers.....

10.6. Naphthalene solubility data in haloalkanes, haloalkenes, and haloaromatic hydrocarbons ..................... 122

10.7. Naphthalene solubility data in alcohols .. 126

10.8. Naphthalene solubility data in ketones ... 129

10.9. Naphthalene solubility data in miscellaneous organic solvents ........ 130

11. Solubility of Phenanthrene in Organic Solvents 131

11.1. Critical evaluation of experimental solubility data......................

11.2. Phenanthrene solubility data in saturated hydrocarbons (including cycloalkanes)...

11.3. Phenanthrene solubility data in aromatic hydrocarbons ..................... 137

11.4. Phenanthrene solubility data in esters ....

11.5. Phenanthrene solubility data in ethers....

11.6. Phenanthrene solubility data in haloalkanes, haloalkenes, and haloaromatic hydrocarbons .................... 140

11.7. Phenanthrene solubility data in alcohols. . 140

11.8. Phenanthrene solubility data in ketones .. 149

11.9. Phenanthrene solubility data in miscellaneous organic solvents ........ 150

12. Solubility of Phenothiazine in Organic Solvents 152

12.1. Critical evaluation of experimental solubility data.....................

12.2. Phenothiazine solubility data in saturated hydrocarbons (including cycloalkanes)...

12.3. Phenothiazine solubility data in ethers ...

12.4. Phenothiazine solubility data in alcohols .

12.5. Phenothiazine solubility data in alkoxyalcohols ......................

12.6. Phenothiazine solubility data in miscellaneous organic solvents ..........

13. Solubility of Pyrene in Organic Solvents .....

13.1. Critical evaluation of experimental solubility data......................

13.2. Pyrene solubility data in saturated hydrocarbons (including cycloalkanes)...

13.3. Pyrene solubility data in aromatic hydrocarbons $\ldots . \ldots \ldots \ldots \ldots \ldots \ldots . . . . . .$.

13.4. Pyrene solubility data in esters..........

13.5. Pyrene solubility data in ethers ..........

13.6. Pyrene solubility data in haloalkanes, haloalkenes, and haloaromatic hydrocarbons ......................

13.7. Pyrene solubility data in alcohols ........

13.8. Pyrene solubility data in alkoxyalcohols .

13.9. Pyrene solubility data in ketones.........

13.10. Pyrene solubility data in miscellaneous organic solvents...................

14. Solubility of Thianthrene in Organic Solvents .

14.1. Critical evaluation of experimental solubility data...................... 
14.2. Thianthrene solubility data in saturated hydrocarbons (including cycloalkanes)...

14.3. Thianthrene solubility data in ethers .....

14.4. Thianthrene solubility data in alcohols...

14.5. Thianthrene solubility data in miscellaneous organic solvents

196

198

200

206

15. Solubility of Xanthene in Organic Solvents ...

15.1. Critical evaluation of experimental solubility data......................

15.2. Xanthene solubility data in saturated hydrocarbons (including cycloalkanes)...

15.3. Xanthene solubility data in ethers........

15.4. Xanthene solubility data in haloalkanes, haloalkenes, and haloaromatic hydrocarbons...$\ldots \ldots \ldots \ldots \ldots \ldots$.

15.5. Xanthene solubility data in alcohols .....

15.6. Xanthene solubility data in alkoxyalcohols

15.7. Xanthene solubility data in miscellaneous organic solvents.....................

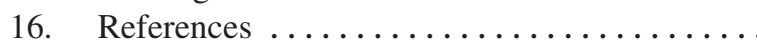

\section{List of Tables}

1. Parameters of the Apelblat equation for describing the solubility of acenaphthene in various organic solvents..............................

2. Parameters of the Apelblat equation for describing the solubility of dibenzofuran in various organic solvents....

3. Parameters of the Apelblat equation for describing the solubility of fluorene in various organic solvents

4. Parameters of the Apelblat equation for describing the solubility of naphthalene in various organic solvents...............................

5. Parameters of the Buchowski $\lambda \mathrm{h}$ equation for describing the solubility of naphthalene in various organic solvents........................

6. Parameters of the Apelblat equation for describing the solubility of pyrene in various organic solvents

\section{Preface}

\subsection{Scope of this volume}

Volumes $54,{ }^{1} 58,{ }^{2}$ and 59 (Ref. 3) in the IUPAC Solubility Data Series dealt with the solubility of polycyclic aromatic hydrocarbons (PAHs) and polycyclic aromatic hetero-atom compounds (PAHaCs) in both neat organic solvents and binary organic solvents. The specific solutes considered included: acenaphthene, acridine, anthracene, benz[a]anthracene, benzo [b]fluorene, benzo[ghi]perylene, benzo[a]pyrene, biphenyl, 2,2'-bipyridine, buckminsterfullerene (C60), carbazole, chrysene, coronene, dibenz[a,h]anthracene, dibenzofuran, dibenzothiophene, 1,2-diphenylbenzene, 1,3-diphenylbenzene, 1,4- diphenylbenzene, fluoranthene, fluorene, indole, naphthacene, naphthalene, perylene, phenanthrene, 1,10-phenanthroline, phenothiazine, phenoxanthiin, phenoxazine, pyrene, thianthrene, thioxanthene, triphenylene, and xanthenes. The three volumes covered the published literature up to 1995, including several articles that were still in press at the time.

This is the last part of the three-part series that updates the three earlier volumes on PAH and PAHaC solubilities. Part 1 (Ref. 4) was devoted to solubilities in binary solvent mixtures, and incorporates compilations based on papers published in the peer-reviewed scientific literature between 1995 to the end of 2011. Part 2 (Ref. 5) dealt with solubilities of PAHs and $\mathrm{PAHaCs}$ dissolved in ternary solvent mixtures. Part 3 (the present paper) will focus on the organic mono-solvents (neat organic solvents). To conserve space, data from the earlier volumes will not be repeated here.

\subsection{Procedures used in critical evaluation of published solubility data}

The different concentration units that are used to express experimental solubility data, and the thermodynamic principles that govern solubility solid-liquid equilibria, were described in Part 1 of this volume. ${ }^{4}$ The only part of the earlier discussion that will be repeated here pertains to the mathematical representation of solubility data for crystalline nonelectrolyte solutes as a function of temperature.

Published solubility data may be found for a given solutesolvent system measured at several different temperatures. The temperature variation can be critically evaluated using standard thermodynamic relationships based on the ideal mole fraction solubility of a solid solute, $x_{1}{ }^{\text {ideal soly }}$ in a liquid solvent $^{6}$

$$
\begin{aligned}
-\ln x_{1} \text { ideal soly }= & \frac{\Delta H_{1} \text { fus }}{R T}\left[1-\frac{T}{T_{\mathrm{mp}}}\right]+\frac{\Delta C_{p, 1}}{R}\left(\frac{T_{\mathrm{mp}}-T}{T}\right) \\
& +\frac{\Delta C_{p, 1}}{R} \ln \left(\frac{T_{\mathrm{mp}}}{T}\right),
\end{aligned}
$$

where $\Delta H_{1}{ }^{\text {fus }}$ is the standard molar enthalpy of fusion of the solute at its normal melting point temperature, $T_{\mathrm{mp}}, \Delta \mathrm{C}_{p, 1}$ is the difference in the molar heat capacities of the liquid and crystalline forms of the solute (i.e., $\Delta \mathrm{C}_{p, 1}=C_{p \text {, liquid }}-C_{p \text {,solid }}$ ) and $R$ is the universal gas constant. Through suitable algebraic manipulations, Eq. (1) can be rearranged to give

$$
\begin{aligned}
\ln x_{1} \text { ideal soly }= & {\left[\frac{\Delta H_{1}{ }^{\text {fus }}}{R T_{\mathrm{mp}}}+\frac{\Delta C_{p, 1}}{R}\left(1+\ln T_{\mathrm{mp}}\right)\right] } \\
& -\left(\frac{\Delta H_{1}{ }^{\text {fus }}}{R}+\frac{\Delta C_{p, 1} T_{\mathrm{mp}}}{R}\right) \frac{1}{T} \\
& +\frac{\Delta C_{p, 1}}{R} \ln T,
\end{aligned}
$$

which has the generalized mathematical form of

$$
\ln x_{1}=A+\frac{B}{T}+C \ln T \text {. }
$$


Though derived for an ideal solution, Eq. (3) has been used successfully to describe solute solubility in many nonideal solutions. The equation is commonly referred to as the Modified Apelblat equation in the literature.

The $\lambda \mathrm{h}$ model, developed by Buchowski et al., ${ }^{7,8}$ is

$$
\ln \left[1+\frac{\lambda\left(1-x_{1}\right)}{x_{1}}\right]=\lambda h\left(\frac{1}{T}-\frac{1}{T_{\mathrm{mp}}}\right),
$$

a second popular mathematical representation for describing how the mole fraction solubility varies with solution temperature. In Eq. (4), $T$ and $T_{\mathrm{mp}}$ refer to the solution temperature and melting point temperature of the solute, respectively. The two model parameters, $\lambda$ and $h$, are determined by least-squares analyses using the measured mole fraction solubilities. Experimental solubility data are considered to be internally consistent if the measured $x_{1}$ values can be accurately described by either Eq. (3) and/or Eq. (4).

\section{Solubility of Acenaphthene in Organic Solvents}

\subsection{Critical evaluation of experimental solubility data}

Volume 58 in the IUPAC Solubility Data Series ${ }^{2}$ contained experimental solubility data for acenaphthene in three saturated hydrocarbons (octadecane, cyclohexane and decahydronaphthene), in four aromatic hydrocarbons (benzene, methylbenzene, 1,2-dimethylbenzene, and 1,2,3,4-tetrahydronaphthalene), in two haloalkanes (trichloromethane and tetrachloromethane), in four alkanols (methanol, ethanol, 1propanol, and 1-octanol) and in two miscellaneous organic solvents (pyridine and thiophene). The majority of systems included measurements at several temperatures covering a 30 to $40 \mathrm{~K}$ range. The compiled solubility data also included phase diagram information for binary 1,2,4,5-tetramethylbenzene + acenaphthene, naphthalene + acenaphthene, 2-methylnaphthalene + acenaphthene, 2,6-dimethylnaphthalene + acenaphthene, 2,7-dimethylnaphthalene + acenaphthene, anthracene + acenaphthene, phenanthrene + acenaphthene, fluorene + acenaphthene, chrysene + acenaphthene, fluoranthene + acenaphthene and 1,2,3,5-tetranitrobenzene + acenaphthene mixtures. Solubility data contained in Vol. 58 will not be republished here. The listing above is provided so that readers will know what solubility data are available in the earlier volume for acenaphthene.

There have been several studies that reported solubility data for acenaphthene dissolved in organic solvents after Vol. 58 was published in 1995. De Fina et al. ${ }^{9}$ determined the solubility of acenaphthene in 36 different organic solvents containing ether-, carbonyl-, hydroxy-, ester-, methyl-, and tert-butyl functional groups. The experimental measurements were performed at the single temperature of $298.15 \mathrm{~K}$ and used to test the limitations and applications of predictive expressions based on Mobile Order theory. Acree and Abraham ${ }^{10}$ measured acenaphthene solubilities at $298.15 \mathrm{~K}$ in 11 additional solvents, namely, squalane, 2-methoxy-2-methylpropane (more commonly called methyl tert-butyl ether), 1,1'-oxybisethane (also called diethyl ether), 2,2'-oxybispropane (also called diisopropyl ether), 3,7-dimethyl-1-octanol, butanone, methyl ethanoate, propanenitrile, butanenitrile, dimethyl sulfoxide, and $\mathrm{N}$-methylformamide. For the majority of the solvents studied by De Fina et al. ${ }^{9}$ and by Acree and Abraham ${ }^{10}$ there is only the single experimental value, and it is not possible to perform a critical evaluation on most of the published data.

There does exist independent solubility data for acenaphthene in cyclohexane in Vol. 58 of the Solubility Data Series $^{2}$ at temperatures slightly greater than 298.15 K. McLauglin and Zainal ${ }^{11}$ conducted acenaphthene solubility measurements from 308.7 to $334.0 \mathrm{~K}$. Choi et al. ${ }^{12}$ subsequently determined the solubility at $303.4-354.5 \mathrm{~K}$. There is approximately a $10 \%-15 \%$ relative deviation between the two independent sets of measurements. Analysis of the two datasets using Eq. (3) with $C=0$ gave the following two mathematical correlations:

$$
\begin{aligned}
& \ln x_{1}=15.534-\frac{5541.3}{T}, \\
& \ln x_{1}=13.202-\frac{4757.9}{T} .
\end{aligned}
$$

The calculated equation coefficients reflect the differences in the two datasets. Equations (5) and (6) provide a reasonably accurate mathematical description of the experimental mole fraction solubilities in the respective dataset. Differences between the experimental data and values back-calculated using Eqs. (5) and (6) were on the order of $3.6 \%$ or less. Equations (5) and (6) give extrapolated values of $x_{1}=0.04726$ and $x_{1}=0.006356$ for the mole fraction solubility of acenaphthene in cyclohexane at $298.15 \mathrm{~K}$, which are both less than the value of $x_{1}=0.07043$ that was reported by De Fina et al. ${ }^{9}$ Differences in chemical purities and experimental methodologies were the likely causes of the deviations noted in the published solubility data for acenaphthene dissolved in cyclohexane.

Two groups have studied the solubility of acenaphthene as a function of temperature. Kotula and Marciniak ${ }^{13}$ published solubility data for acenaphthene in four chlorinated alkanes (trichloromethane, tetrachloromethane, 1,1dichloroethane, and 1,2-dichloroethane) and two chlorinated alkenes (trichloroethene and tetrachloroethene) from 290 to $325 \mathrm{~K}$. He and Liu ${ }^{14}$ employed a synthetic method to measure the solubility of acenaphthene in ethanol, 2-propanol, 1-butanol, and methylbenzene at ten different temperatures covering the range of 278-323 K. The experimental values determined by $\mathrm{He}$ and $\mathrm{Liu}^{14}$ for acenaphthene in ethanol $\left(x_{1}=0.01079\right)$, 2-propanol $\left(x_{1}\right.$ $=0.01197)$, and 1-butanol $\left(x_{1}=0.02367\right)$ differ by an average deviation of less than $5 \%$ from the measured values of De Fina et al. ${ }^{9}$ (ethanol, $x_{1}=0.01068$; 2-propanol, $x_{1}$ $=0.01336$; and 1-butanol, $x_{1}=0.02373$ ). Deviations of this magnitude are often attributed to differences in chemical purities and experimental methodologies. 
TABLE 1. Parameters of the Apelblat equation for describing the solubility of acenaphthene in various organic solvents

\begin{tabular}{|c|c|c|c|c|}
\hline Solvent & $A$ & $B$ & $C$ & MRD (\%) \\
\hline Trichloromethane & 7.354 & -2690.2 & 0 & 0.1 \\
\hline Tetrachloromethane & 8.311 & -3041.8 & 0 & 0.7 \\
\hline 1,1-Dichloroethane & 8.086 & -2959.3 & 0 & 0.4 \\
\hline Trichloroethene & 7.480 & -2743.4 & 0 & 0.6 \\
\hline Tetrachloroethene & 8.454 & -3099.4 & 0 & 0.1 \\
\hline Methylbenzene $\mathrm{a}^{\mathrm{a}}$ & -53.186 & 21.255 & 9.033 & 0.3 \\
\hline 1 -Butanol ${ }^{\mathrm{a}}$ & -93.112 & 844.83 & 15.186 & 0.7 \\
\hline
\end{tabular}

${ }^{\mathrm{a}}$ Numerical values of the coefficients and the percent mean relative deviations were taken from He and Liu. ${ }^{14}$

The internal consistency of the chloroalkane and chloroalkene datasets of measured acenaphthene solubilities, as well as the He and Liu ${ }^{14}$ dataset, were assessed by curve-fitting the measured mole fraction solubility data to Eq. (3). The numerical values of the equation coefficients $(A, B$, and $C)$ are given in Table 1, along with the mean relative deviation (MRD) calculated according to Eq. (7)

$$
\operatorname{MRD}=\frac{1}{N} \sum_{i=1}^{N}\left|\frac{x_{1}{ }^{\exp }-x_{1}^{\text {calc }}}{x_{1} \exp }\right|,
$$

where $N$ is the number of experimental solubility measurements in an individual solute-solvent data set. Examination of the numerical entries in the last column of Table 1 reveals that the largest mean relative percent deviation between the backcalculated values based on Eq. (3) and experimental data is only $0.7 \%$, which is less than the experimental uncertainties in the measured values. Results of the mathematical representation analyses indicate that the experimental data for all ten acenaphthene-organic solvent systems are internally consistent.

The experimental solubility data for acenaphthene dissolved in the different organic solvents are in Secs. 2.2-2.10.

\subsection{Acenaphthene solubility data in saturated hydrocarbons (including cycloalkanes)}

\begin{tabular}{|c|c|}
\hline $\begin{array}{l}\text { Components: } \\
\text { (1) Acenaphthene; } \mathrm{C}_{12} \mathrm{H}_{10} ;[83-32-9] \\
\text { (2) Hexane; } \mathrm{C}_{6} \mathrm{H}_{14} ;[110-54-3]\end{array}$ & $\begin{array}{l}\text { Original Measurements: } \\
{ }^{9} \text { K. M. De Fina, T. L. Sharp, } \\
\text { and W. E. Acree, Jr., Can. J. } \\
\text { Chem. 77, } 1537 \text { (1999). }\end{array}$ \\
\hline Variables: & Prepared by: \\
\hline$T / \mathrm{K}=298.15$ & W. E. Acree, Jr. \\
\hline
\end{tabular}

Experimental Values

\begin{tabular}{|c|c|c|}
\hline$x_{2}{ }^{(\mathrm{s}) \mathrm{a}}$ & $x_{2}{ }^{b}$ & $x_{1}{ }^{\mathrm{c}}$ \\
\hline 1.0000 & 0.9481 & 0.05192 \\
\hline
\end{tabular}

\section{Auxiliary Information}

\section{Method/Apparatus/Procedure:}

Constant-temperature bath, calorimetric thermometer, and an ultraviolet/ visible spectrophotometer.

Excess solute and solvent were placed in amber glass bottles and allowed to equilibrate for several days at constant temperature. Attainment of equilibrium was verified by several repetitive measurements and by approaching equilibrium from supersaturation. Aliquots of saturated solutions were transferred through a coarse filter into tared volumetric flasks, weighed and diluted with methanol. Concentrations were determined by spectrophotometric measurements at $289 \mathrm{~nm}$.

\section{Source and Purity of Chemicals:}

(1) $98 \%$, Aldrich Chemical Company, Milwaukee, WI, USA, was recrystallized several times from methanol.

(2) $99 \%$, Aldrich Chemical Company, stored over molecular sieves before use.

\section{Estimated Error:}

Temperature: $\pm 0.1 \mathrm{~K}$.

$x_{1}: \pm 1.5 \%$ (relative error).

\begin{tabular}{ll}
\hline \hline Components: & Original Measurements: \\
(1) Acenaphthene; $\mathrm{C}_{12} \mathrm{H}_{10} ;[83-32-9]$ & ${ }^{9} \mathrm{~K}$. M. De Fina, T. L. Sharp, \\
(2) Heptane; $\mathrm{C}_{7} \mathrm{H}_{16} ;[142-82-5]$ & and W. E. Acree, Jr., Can. J. \\
& Chem. 77, 1537 (1999). \\
\hline Variables: & Prepared by: \\
$T / \mathrm{K}=298.15$ & W. E. Acree, Jr. \\
\hline
\end{tabular}

Experimental Values

\begin{tabular}{lcc}
\hline \hline$\frac{x_{2}{ }^{(\mathrm{s}) \mathrm{a}}}{x_{2}{ }^{\mathrm{b}}}$ & $x_{1}{ }^{\mathrm{c}}$ \\
\hline 1.0000 & 0.9393 & 0.06075 \\
${ }^{\mathrm{a}} x_{2}{ }^{(\mathrm{s})}:$ initial mole fraction of component 2 in the solution. \\
${ }^{\mathrm{b}} x_{2}:$ mole fraction of component 2 in the saturated solution. \\
${ }^{\mathrm{c}} x_{1}:$ mole fraction solubility of the solute.
\end{tabular}




\section{Auxiliary Information}

\section{Method/Apparatus/Procedure:}

Constant-temperature bath, calorimetric thermometer, and an ultraviolet/ visible spectrophotometer.

Excess solute and solvent were placed in amber glass bottles and allowed to equilibrate for several days at constant temperature. Attainment of equilibrium was verified by several repetitive measurements and by approaching equilibrium from supersaturation. Aliquots of saturated solutions were transferred through a coarse filter into tared volumetric flasks, weighed and diluted with methanol. Concentrations were determined by spectrophotometric measurements at $289 \mathrm{~nm}$.

Source and Purity of Chemicals:

(1) $98 \%$, Aldrich Chemical Company, Milwaukee, WI, USA, was recrystallized several times from methanol.

(2) HPLC Grade, Aldrich Chemical Company, stored over molecular sieves before use.

Estimated Error:

Temperature: $\pm 0.1 \mathrm{~K}$.

$x_{1}: \pm 1.5 \%$ (relative error).

\begin{tabular}{ll}
\hline \hline Components: & Original Measurements: \\
(1) Acenaphthene; $\mathrm{C}_{12} \mathrm{H}_{10} ;[83-32-9]$ & ${ }^{9}$ K. M. De Fina, T. L. Sharp, \\
(2) Octane; $\mathrm{C}_{8} \mathrm{H}_{18} ;[111-65-9]$ & and W. E. Acree, Jr., Can. J. \\
& Chem. 77, 1537 (1999). \\
\hline Variables: & Prepared by: \\
$T / \mathrm{K}=298.15$ & W. E. Acree, Jr. \\
\hline
\end{tabular}

Experimental Values

\begin{tabular}{lcc}
\hline \hline$x_{2}{ }^{(\mathrm{s}) \mathrm{a}}$ & $x_{2}{ }^{\mathrm{b}}$ & $x_{1}{ }^{\mathrm{c}}$ \\
\hline 1.0000 & 0.9317 & 0.06826 \\
\hline
\end{tabular}

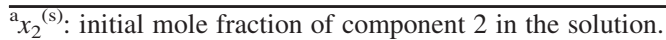

${ }^{b} x_{2}$ : mole fraction of component 2 in the saturated solution.

${ }^{\mathrm{c}} x_{1}$ : mole fraction solubility of the solute.

\section{Auxiliary Information}

\section{Method/Apparatus/Procedure:}

Constant-temperature bath, calorimetric thermometer, and an ultraviolet/ visible spectrophotometer.

Excess solute and solvent were placed in amber glass bottles and allowed to equilibrate for several days at constant temperature. Attainment of equilibrium was verified by several repetitive measurements and by approaching equilibrium from supersaturation. Aliquots of saturated solutions were transferred through a coarse filter into tared volumetric flasks, weighed and diluted with methanol. Concentrations were determined by

spectrophotometric measurements at $289 \mathrm{~nm}$.

\section{Source and Purity of Chemicals:}

(1) 98\%, Aldrich Chemical Company, Milwaukee, WI, USA, was recrystallized several times from methanol.

(2) $99+\%$, anhydrous, Aldrich Chemical Company, stored over molecular sieves before use.

Estimated Error:

Temperature: $\pm 0.1 \mathrm{~K}$

$x_{1}: \pm 1.5 \%$ (relative error).

\begin{tabular}{ll}
\hline \hline Components: & Original Measurements: \\
(1) Acenaphthene; $\mathrm{C}_{12} \mathrm{H}_{10} ;[83-32-9]$ & ${ }^{9}$ K. M. De Fina, T. L. Sharp, \\
(2) Nonane; $\mathrm{C}_{9} \mathrm{H}_{20} ;[111-84-2]$ & and W. E. Acree, Jr., Can. J. \\
& Chem. 77, 1537 (1999). \\
\hline Variables: & Prepared by: \\
$T / \mathrm{K}=298.15$ & W. E. Acree, Jr. \\
\hline
\end{tabular}

Experimental Values

\begin{tabular}{|c|c|c|}
\hline$x_{2}{ }^{(\mathrm{s}) a}$ & $x_{2}{ }^{b}$ & $x_{1}{ }^{c}$ \\
\hline 1.0000 & 0.9279 & 0.07210 \\
\hline
\end{tabular}

\section{Auxiliary Information}

\begin{abstract}
Method/Apparatus/Procedure:
Constant-temperature bath, calorimetric thermometer, and an ultraviolet/ visible spectrophotometer.

Excess solute and solvent were placed in amber glass bottles and allowed to equilibrate for several days at constant temperature. Attainment of equilibrium was verified by several repetitive measurements and by approaching equilibrium from supersaturation. Aliquots of saturated solutions were transferred through a coarse filter into tared volumetric flasks, weighed and diluted with methanol. Concentrations were determined by spectrophotometric measurements at $289 \mathrm{~nm}$.
\end{abstract}

\section{Source and Purity of Chemicals:}

(1) $98 \%$, Aldrich Chemical Company, Milwaukee, WI, USA, was recrystallized several times from methanol.

(2) $99+\%$, TCI America, Portland, OR, USA, stored over molecular sieves before use.

\section{Estimated Error:}

Temperature: $\pm 0.1 \mathrm{~K}$.

$x_{1}: \pm 1.5 \%$ (relative error).

\begin{tabular}{ll}
\hline \hline Components: & Original Measurements: \\
(1) Acenaphthene; $\mathrm{C}_{12} \mathrm{H}_{10} ;[83-32-9]$ & ${ }^{9} \mathrm{~K}$. M. De Fina, T. L. Sharp, \\
(2) Decane; $\mathrm{C}_{10} \mathrm{H}_{22} ;[124-18-5]$ & and W. E. Acree, Jr., Can. J. \\
& Chem. 77, 1537 (1999). \\
\hline Variables: & Prepared by: \\
$T / \mathrm{K}=298.15$ & W. E. Acree, Jr. \\
\hline
\end{tabular}

Experimental Values

\begin{tabular}{lcc}
\hline \hline$x_{2}{ }^{(\mathrm{s}) \mathrm{a}}$ & $x_{2}{ }^{\mathrm{b}}$ & $x_{1}{ }^{\mathrm{c}}$ \\
\hline 1.0000 & 0.9215 & 0.07852 \\
$\frac{{ }^{\mathrm{a}} x_{2}{ }^{(\mathrm{s})}: \text { initial mole fraction of component } 2 \text { in the solution. }}{{ }^{\mathrm{b}} x_{2}: \text { mole fraction of component } 2 \text { in the saturated solution. }}$ \\
${ }^{\mathrm{c}}{ }_{x_{1}}$ : mole fraction solubility of the solute.
\end{tabular}




\section{Auxiliary Information}

\section{Method/Apparatus/Procedure:}

Constant-temperature bath, calorimetric thermometer, and an ultraviolet/ visible spectrophotometer.

Excess solute and solvent were placed in amber glass bottles and allowed to equilibrate for several days at constant temperature. Attainment of equilibrium was verified by several repetitive measurements and by approaching equilibrium from supersaturation. Aliquots of saturated solutions were transferred through a coarse filter into tared volumetric flasks, weighed and diluted with methanol. Concentrations were determined by spectrophotometric measurements at $289 \mathrm{~nm}$.

Source and Purity of Chemicals:

(1) $98 \%$, Aldrich Chemical Company, Milwaukee, WI, USA, was recrystallized several times from methanol.

(2) $99+\%$, TCI America, Portland, OR, USA, stored over molecular sieves before use.

Estimated Error:

Temperature: $\pm 0.1 \mathrm{~K}$.

$x_{1}: \pm 1.5 \%$ (relative error).

\begin{tabular}{ll}
\hline \hline Components: & Original Measurements: \\
(1) Acenaphthene; $\mathrm{C}_{12} \mathrm{H}_{10} ;[83-32-9]$ & ${ }^{17} \mathrm{M} . \mathrm{H}$. Abraham and W. E. \\
(2) Undecane; $\mathrm{C}_{11} \mathrm{H}_{24} ;[1120-21-4]$ & Acree, Jr., New J. Chem. 28, \\
& 1538 (2004). \\
\hline Variables: & Prepared by: \\
$T / \mathrm{K}=298.15$ & W. E. Acree, Jr. \\
\hline
\end{tabular}

Experimental Values

\begin{tabular}{lcc}
\hline \hline$x_{2}{ }^{(\mathrm{s}) \mathrm{a}}$ & $x_{2}{ }^{\mathrm{b}}$ & $x_{1}{ }^{\mathrm{c}}$ \\
\hline 1.0000 & 0.9164 & 0.08355 \\
\hline
\end{tabular}



${ }^{\mathrm{b}} x_{2}$ : mole fraction of component 2 in the saturated solution.

${ }^{\mathrm{c}} x_{1}$ : mole fraction solubility of the solute.

Experimental values were reported as the logarithm of the solute's molar solubility in undecane divided by the molar solubility in water. Mole fraction solubilities were provided by the authors of the paper.

\section{Auxiliary Information}

\section{Method/Apparatus/Procedure:}

Constant-temperature bath, calorimetric thermometer, and an ultraviolet/ visible spectrophotometer.

Excess solute and solvent were placed in amber glass bottles and allowed to equilibrate for several days at constant temperature. Attainment of equilibrium was verified by several repetitive measurements and by approaching equilibrium from supersaturation. Aliquots of saturated solutions were transferred through a coarse filter into tared volumetric flasks, weighed and diluted with methanol. Concentrations were determined by spectrophotometric measurements at $289 \mathrm{~nm}$.

Source and Purity of Chemicals:

(1) $98 \%$, Aldrich Chemical Company, Milwaukee, WI, USA, was

recrystallized several times from methanol.

(2) $99 \%$, Aldrich Chemical Company, stored over molecular sieves before use.

Estimated Error:

Temperature: $\pm 0.1 \mathrm{~K}$.

$x_{1}: \pm 1.5 \%$ (relative error).

\begin{tabular}{ll}
\hline \hline Components: & Original Measurements: \\
(1) Acenaphthene; $\mathrm{C}_{12} \mathrm{H}_{10} ;[83-32-9]$ & ${ }^{17} \mathrm{M}$. H. Abraham and W. E. \\
(2) Dodecane; $\mathrm{C}_{12} \mathrm{H}_{26} ;[112-40-3]$ & Acree, Jr., New J. Chem. 28, \\
& $1538(2004)$. \\
\hline Variables: & Prepared by: \\
$T / \mathrm{K}=298.15$ & W. E. Acree, Jr. \\
\hline
\end{tabular}

Experimental Values

\begin{tabular}{lcc}
\hline \hline$x_{2}{ }^{(\mathrm{s}) \mathrm{a}}$ & $x_{2}{ }^{\mathrm{b}}$ & $x_{1}{ }^{\mathrm{c}}$ \\
\hline 1.0000 & 0.9120 & 0.08799 \\
$\frac{{ }^{\mathrm{a}} x_{2}{ }^{(\mathrm{s})}: \text { initial mole fraction of component } 2 \text { in the solution. }}{{ }^{\mathrm{b}} x_{2}: \text { mole fraction of component } 2 \text { in the saturated solution. }}$ \\
${ }^{\mathrm{c}} x_{1}:$ mole fraction solubility of the solute.
\end{tabular}

Experimental values were reported as the logarithm of the solute's molar solubility in dodecane divided by the molar solubility in water. Mole fraction solubilities were provided by the authors of the paper.

\section{Auxiliary Information}

\begin{tabular}{l}
\hline Method/Apparatus/Procedure: \\
Constant-temperature bath, calorimetric thermometer, and an ultraviolet/ \\
visible spectrophotometer. \\
Excess solute and solvent were placed in amber glass bottles and allowed to \\
equilibrate for several days at constant temperature. Attainment of equilibrium \\
was verified by several repetitive measurements and by approaching \\
equilibrium from supersaturation. Aliquots of saturated solutions were \\
transferred through a coarse filter into tared volumetric flasks, weighed and \\
diluted with methanol. Concentrations were determined by \\
spectrophotometric measurements at $289 \mathrm{~nm}$.
\end{tabular}

Source and Purity of Chemicals:

(1) $98 \%$, Aldrich Chemical Company, Milwaukee, WI, USA, was recrystallized several times from methanol.

(2) $99 \%$, Aldrich Chemical Company, stored over molecular sieves before use.

Estimated Error:

Temperature: $\pm 0.1 \mathrm{~K}$.

$x_{1}: \pm 1.5 \%$ (relative error).

\section{Components:}

(1) Acenaphthene; $\mathrm{C}_{12} \mathrm{H}_{10}$; [83-32-9]

(2) Hexadecane; $\mathrm{C}_{16} \mathrm{H}_{34}$; [544-76-3]

Variables:

$T / \mathrm{K}=298.15$
Original Measurements:

${ }^{9}$ K. M. De Fina, T. L. Sharp, and W. E. Acree, Jr., Can. J. Chem. 77, 1537 (1999).

Prepared by:

W. E. Acree, Jr. 
Experimental Values

\begin{tabular}{lcc}
\hline$x_{2}{ }^{(\mathrm{s}) \mathrm{a}}$ & $x_{2}{ }^{\mathrm{b}}$ & $x_{1}{ }^{\mathrm{c}}$ \\
\hline 1.0000 & 0.8935 & 0.1065 \\
$\frac{{ }^{\mathrm{a}} x_{2}{ }^{(\mathrm{s})}: \text { initial mole fraction of component } 2 \text { in the solution. }}{{ }^{\mathrm{b}}{ }_{x_{2}}: \text { mole fraction of component } 2 \text { in the saturated solution. }}$ \\
${ }^{\mathrm{c}} x_{1}:$ mole fraction solubility of the solute.
\end{tabular}

\section{Auxiliary Information}

\section{Method/Apparatus/Procedure:}

Constant-temperature bath, calorimetric thermometer, and an ultraviolet/ visible spectrophotometer.

Excess solute and solvent were placed in amber glass bottles and allowed to equilibrate for several days at constant temperature. Attainment of equilibrium was verified by several repetitive measurements and by approaching equilibrium from supersaturation. Aliquots of saturated solutions were transferred through a coarse filter into tared volumetric flasks, weighed and diluted with methanol. Concentrations were determined by spectrophotometric measurements at $289 \mathrm{~nm}$.

\section{Source and Purity of Chemicals:}

(1) $98 \%$, Aldrich Chemical Company, Milwaukee, WI, USA, was recrystallized several times from methanol.

(2) $99 \%$, Aldrich Chemical Company, stored over molecular sieves before use.

\section{Estimated Error:}

Temperature: $\pm 0.1 \mathrm{~K}$.

$x_{1}: \pm 1.5 \%$ (relative error).

\section{Components:}

(1) Acenaphthene; $\mathrm{C}_{12} \mathrm{H}_{10} ;$ [83-32-9]

(2) Hexatricontane; $\mathrm{C}_{36} \mathrm{H}_{74} ;[630-06-8]$

Original Measurements:

${ }^{15}$ A. Aoulmi, M. Bouroukba,

R. Solimando, and

M. Rogalski, Fluid Phase Equilib. 110, 283 (1995).

\begin{tabular}{ll}
\hline Variables: & Prepared by: \\
Temperature & W. E. Acree, Jr. \\
\hline
\end{tabular}

Experimental Values

\begin{tabular}{lcc}
\hline \hline$T / \mathrm{K}$ & $x_{2}{ }^{\mathrm{a}}$ & $x_{1}{ }^{\mathrm{b}}$ \\
\hline 350.30 & 1.0000 & 0.0000 \\
348.89 & 0.8878 & 0.1122 \\
348.56 & 0.8482 & 0.1518 \\
347.70 & 0.7926 & 0.2074 \\
346.21 & 0.6886 & 0.3114 \\
345.35 & 0.5931 & 0.4069 \\
344.75 & 0.5537 & 0.4463 \\
343.53 & 0.4629 & 0.5371 \\
348.56 & 0.3953 & 0.6047 \\
350.88 & 0.3005 & 0.6995 \\
353.63 & 0.2517 & 0.7483 \\
356.88 & 0.2029 & 0.7971 \\
359.50 & 0.1510 & 0.8490 \\
362.12 & 0.1006 & 0.8994 \\
365.35 & 0.0000 & 1.0000 \\
\hline
\end{tabular}

${ }^{\mathrm{a}} x_{2}$ : mole fraction of component 2 in the saturated solution.

${ }^{\mathrm{b}} x_{1}$ : mole fraction of the polycyclic aromatic hydrocarbon (component 1 ).
The authors employed a sum of symmetrized functions (SSF) to describe the activity coefficients of acenaphthene and hexatricontane calculated from the solid-liquid equilibrium data. The SSF model gave a calculated eutectic temperature of $T / \mathrm{K}=343.4$ and eutectic mole fraction of acenaphthene of $x_{1}=0.548$.

\section{Auxiliary Information}

Method/Apparatus/Procedure:

Differential scanning calorimeter.

The phase diagram was determined using a differential scanning calorimeter. Measurements were performed at a constant fixed scanning rate of $0.5 \mathrm{~K} / \mathrm{min}$.

\section{Source and Purity of Chemicals:}

(1) Pure Grade, Prolabo, no purification details were given in the paper.

(2) $98+\%$, Fluka Chemical Company, purification details were not given in the paper.

\section{Estimated Error:}

Temperature: Authors state a global accuracy of $\pm 1 \%$ regarding their measurements.

$x_{1}: \pm 0.0002$ (estimated by compiler).

\begin{tabular}{ll}
\hline \hline Components: & Original Measurements: \\
(1) Acenaphthene; $\mathrm{C}_{12} \mathrm{H}_{10} ;[83-32-9]$ & ${ }^{9}$ K. M. De Fina, T. L. Sharp, \\
(2) Cyclohexane; $\mathrm{C}_{6} \mathrm{H}_{12} ;[110-82-7]$ & and W. E. Acree, Jr., Can. J. \\
& Chem. 77, 1537 (1999). \\
\hline Variables: & Prepared by: \\
$T / \mathrm{K}=298.15$ & W. E. Acree, Jr. \\
\hline
\end{tabular}

Experimental Values

\begin{tabular}{|c|c|c|}
\hline$x_{2}{ }^{(\mathrm{s}) \mathrm{a}}$ & $x_{2}{ }^{\mathrm{b}}$ & $x_{1}{ }^{\mathrm{c}}$ \\
\hline 1.0000 & 0.9296 & 0.07043 \\
\hline
\end{tabular}

\section{Auxiliary Information}

\section{Method/Apparatus/Procedure:}

Constant-temperature bath, calorimetric thermometer, and an ultraviolet/ visible spectrophotometer.

Excess solute and solvent were placed in amber glass bottles and allowed to equilibrate for several days at constant temperature. Attainment of equilibrium was verified by several repetitive measurements and by approaching equilibrium from supersaturation. Aliquots of saturated solutions were transferred through a coarse filter into tared volumetric flasks, weighed and diluted with methanol. Concentrations were determined by spectrophotometric measurements at $289 \mathrm{~nm}$.

Source and Purity of Chemicals:

(1) $98 \%$, Aldrich Chemical Company, Milwaukee, WI, USA, was recrystallized several times from methanol.

(2) HPLC Grade, Aldrich Chemical Company, stored over molecular sieves before use.

\section{Estimated Error:}

Temperature: $\pm 0.1 \mathrm{~K}$.

$x_{1}: \pm 1.5 \%$ (relative error). 


\section{Components:}

(1) Acenaphthene; $\mathrm{C}_{12} \mathrm{H}_{10} ;$ [83-32-9]

(2) Methylcyclohexane; $\mathrm{C}_{7} \mathrm{H}_{14} ;$ [108-87-2]

Original Measurements:

${ }^{9}$ K. M. De Fina, T. L. Sharp, and W. E. Acree, Jr., Can. J. Chem. 77, 1537 (1999).

\section{Variables:}

$T / \mathrm{K}=298.15$

Prepared by:

W. E. Acree, Jr.

\section{Experimental Values}

\begin{tabular}{lcc}
\hline \hline$x_{2}{ }^{(\mathrm{s}) \mathrm{a}}$ & $x_{2}{ }^{\mathrm{b}}$ & $x_{1}{ }^{\mathrm{c}}$ \\
\hline 1.0000 & 0.9191 & 0.08093 \\
\hline
\end{tabular}

${ }^{\mathrm{a}} x_{2}{ }^{(\mathrm{s})}$ : initial mole fraction of component 2 in the solution.

${ }^{b} x_{2}$ : mole fraction of component 2 in the saturated solution.

${ }^{c} x_{1}$ : mole fraction solubility of the solute.

\section{Auxiliary Information}

Method/Apparatus/Procedure:
Constant-temperature bath, calorimetric thermometer, and an ultraviolet/
visible spectrophotometer.
Excess solute and solvent were placed in amber glass bottles and allowed to
equilibrate for several days at constant temperature. Attainment of equilibrium
was verified by several repetitive measurements and by approaching
equilibrium from supersaturation. Aliquots of saturated solutions were
transferred through a coarse filter into tared volumetric flasks, weighed and
diluted with methanol. Concentrations were determined by
spectrophotometric measurements at 289 nm.
Source and Purity of Chemicals:
(1) $98 \%$, Aldrich Chemical Company, Milwaukee, WI, USA, was
recrystallized several times from methanol.
(2) $99+\%$, anhydrous, Aldrich Chemical Company, stored over molecular
sieves before use.
Estimated Error:
Temperature: $\pm 0.1 \mathrm{~K}$.
$x_{1}: \pm 1.5 \%$ (relative error).

\begin{tabular}{|c|c|}
\hline $\begin{array}{l}\text { Components: } \\
\text { (1) Acenaphthene; } \mathrm{C}_{12} \mathrm{H}_{10} ;[83-32-9] \\
\text { (2) Cyclooctane; } \mathrm{C}_{8} \mathrm{H}_{16} ;[292-64-8]\end{array}$ & $\begin{array}{l}\text { Original Measurements: } \\
{ }^{9} \text { K. M. De Fina, T. L. Sharp, } \\
\text { and W. E. Acree, Jr., Can. J. } \\
\text { Chem. 77, } 1537 \text { (1999). }\end{array}$ \\
\hline $\begin{array}{l}\text { Variables: } \\
T / \mathrm{K}=298.15\end{array}$ & $\begin{array}{l}\text { Prepared by: } \\
\text { W. E. Acree, Jr. }\end{array}$ \\
\hline
\end{tabular}

Experimental Values

\begin{tabular}{lcc}
\hline \hline$x_{2}{ }^{(\mathrm{s}) \mathrm{a}}$ & $x_{2}{ }^{\mathrm{b}}$ & $x_{1}{ }^{\mathrm{c}}$ \\
\hline 1.0000 & 0.9026 & 0.09739 \\
${ }^{\mathrm{a}} x_{2}{ }^{(\mathrm{s})}:$ initial mole fraction of component 2 in the solution. \\
${ }^{\mathrm{b}}{ }_{x_{2}}:$ mole fraction of component 2 in the saturated solution. \\
${ }^{\mathrm{c}} x_{1}:$ mole fraction solubility of the solute.
\end{tabular}

\section{Auxiliary Information}

\section{Method/Apparatus/Procedure:}

Constant-temperature bath, calorimetric thermometer, and an ultraviolet/ visible spectrophotometer.

Excess solute and solvent were placed in amber glass bottles and allowed to equilibrate for several days at constant temperature. Attainment of equilibrium was verified by several repetitive measurements and by approaching equilibrium from supersaturation. Aliquots of saturated solutions were transferred through a coarse filter into tared volumetric flasks, weighed and diluted with methanol. Concentrations were determined by spectrophotometric measurements at $289 \mathrm{~nm}$.

\section{Source and Purity of Chemicals:}

(1) $98 \%$, Aldrich Chemical Company, Milwaukee, WI, USA, was recrystallized several times from methanol.

(2) $99+\%$, Aldrich Chemical Company, stored over molecular sieves before use.

Estimated Error:

Temperature: $\pm 0.1 \mathrm{~K}$.

$x_{1}: \pm 1.5 \%$ (relative error).

\begin{tabular}{|c|c|}
\hline $\begin{array}{l}\text { Components: } \\
\text { (1) Acenaphthene; } \mathrm{C}_{12} \mathrm{H}_{10} ;[83-32-9] \\
\text { (2) } 2,2,4-\text { Trimethylpentane; } \mathrm{C}_{8} \mathrm{H}_{18} ; \\
\text { [540-84-1] }\end{array}$ & $\begin{array}{l}\text { Original Measurements: } \\
{ }^{9} \text { K. M. De Fina, T. L. Sharp, } \\
\text { and W. E. Acree, Jr., Can. J. } \\
\text { Chem. 77, } 1537 \text { (1999). }\end{array}$ \\
\hline $\begin{array}{l}\text { Variables: } \\
T / \mathrm{K}=298.15\end{array}$ & $\begin{array}{l}\text { Prepared by: } \\
\text { W. E. Acree, Jr. }\end{array}$ \\
\hline
\end{tabular}

Experimental Values

\begin{tabular}{|c|c|c|}
\hline$x_{2}{ }^{(\mathrm{s}) \mathrm{a}}$ & $x_{2}{ }^{\mathrm{b}}$ & $x_{1}{ }^{\mathrm{c}}$ \\
\hline 1.0000 & 0.9533 & 0.04668 \\
\hline
\end{tabular}

\section{Auxiliary Information}

\section{Method/Apparatus/Procedure:}

Constant-temperature bath, calorimetric thermometer, and an ultraviolet/ visible spectrophotometer.

Excess solute and solvent were placed in amber glass bottles and allowed to equilibrate for several days at constant temperature. Attainment of equilibrium was verified by several repetitive measurements and by approaching equilibrium from supersaturation. Aliquots of saturated solutions were transferred through a coarse filter into tared volumetric flasks, weighed and diluted with methanol. Concentrations were determined by spectrophotometric measurements at $289 \mathrm{~nm}$.

\section{Source and Purity of Chemicals:}

(1) $98 \%$, Aldrich Chemical Company, Milwaukee, WI, USA, was recrystallized several times from methanol.

(2) HPLC Grade, Aldrich Chemical Company, stored over molecular sieves before use.

Estimated Error:

Temperature: $\pm 0.1 \mathrm{~K}$.

$x_{1}: \pm 1.5 \%$ (relative error). 


\section{Components:}

(1) Acenaphthene; $\mathrm{C}_{12} \mathrm{H}_{10} ;[83-32-9]$

(2) tert-Butylcyclohexane; $\mathrm{C}_{10} \mathrm{H}_{20}$;

[3178-22-1]

\section{Variables:}

$T / \mathrm{K}=298.15$

Original Measurements:

${ }^{9}$ K. M. De Fina, T. L. Sharp, and W. E. Acree, Jr., Can. J. Chem. 77, 1537 (1999).

Prepared by:

W. E. Acree, Jr.

\section{Experimental Values}

\begin{tabular}{lcc}
\hline \hline$x_{2}{ }^{(\mathrm{s}) \mathrm{a}}$ & $x_{2}{ }^{\mathrm{b}}$ & $x_{1}{ }^{\mathrm{c}}$ \\
\hline 1.0000 & 0.9224 & 0.07763 \\
\hline
\end{tabular}

${ }^{\mathrm{a}} x_{2}{ }^{(\mathrm{s})}$ : initial mole fraction of component 2 in the solution.

${ }^{\mathrm{b}} x_{2}$ : mole fraction of component 2 in the saturated solution.

${ }^{c} x_{1}$ : mole fraction solubility of the solute.

\section{Auxiliary Information}

\section{Method/Apparatus/Procedure:}

Constant-temperature bath, calorimetric thermometer, and an ultraviolet/ visible spectrophotometer.

Excess solute and solvent were placed in amber glass bottles and allowed to equilibrate for several days at constant temperature. Attainment of equilibrium was verified by several repetitive measurements and by approaching equilibrium from supersaturation. Aliquots of saturated solutions were transferred through a coarse filter into tared volumetric flasks, weighed and diluted with methanol. Concentrations were determined by

spectrophotometric measurements at $289 \mathrm{~nm}$.

Source and Purity of Chemicals:

(1) $98 \%$, Aldrich Chemical Company, Milwaukee, WI, USA, was recrystallized several times from methanol.

(2) $99 \%$, Aldrich Chemical Company, stored over molecular sieves before use.

Estimated Error:

Temperature: $\pm 0.1 \mathrm{~K}$.

$x_{1}: \pm 1.5 \%$ (relative error).

\begin{tabular}{ll}
\hline \hline Components: & Original Measurements: \\
(1) Acenaphthene; $\mathrm{C}_{12} \mathrm{H}_{10} ;[83-32-9]$ & ${ }^{10}$ W. E. Acree, Jr. and M. H. \\
(2) Squalane; $\mathrm{C}_{30} \mathrm{H}_{62} ;[111-01-3]$ & Abraham, Fluid Phase \\
& Equilib. 201, 245 (2002). \\
\hline Variables: & Prepared by: \\
$T / \mathrm{K}=298.15$ & W. E. Acree, Jr. \\
\hline
\end{tabular}

Experimental Values

\begin{tabular}{lcc}
\hline \hline$x_{2}{ }^{(\mathrm{s}) \mathrm{a}}$ & $x_{2}{ }^{\mathrm{b}}$ & $x_{1}{ }^{\mathrm{c}}$ \\
\hline 1.0000 & 0.8572 & 0.1428 \\
$\frac{{ }^{a} x_{2}{ }^{(\mathrm{s})}: \text { initial mole fraction of component } 2 \text { in the solution. }}{}$ \\
${ }^{\mathrm{b}} x_{2}:$ mole fraction of component 2 in the saturated solution. \\
${ }^{\mathrm{c}} x_{1}:$ mole fraction solubility of the solute.
\end{tabular}

\section{Auxiliary Information}

Method/Apparatus/Procedure:

Constant-temperature bath, calorimetric thermometer, and an ultraviolet/ visible spectrophotometer.

Excess solute and solvent were placed in amber glass bottles and allowed to equilibrate for several days at constant temperature. Attainment of equilibrium was verified by several repetitive measurements and by approaching equilibrium from supersaturation. Aliquots of saturated solutions were transferred through a coarse filter into tared volumetric flasks, weighed and diluted with 2-propanol. Concentrations were determined by spectrophotometric measurements at $289 \mathrm{~nm}$.

\section{Source and Purity of Chemicals:}

(1) $98 \%$, Aldrich Chemical Company, Milwaukee, WI, USA, was recrystallized several times from methanol.

(2) $99 \%$, Aldrich Chemical Company, stored over molecular sieves before use.

Estimated Error:

Temperature: $\pm 0.1 \mathrm{~K}$.

$x_{1}: \pm 1.5 \%$ (relative error).

\subsection{Acenaphthene solubility in aromatic hydrocarbons}

\begin{tabular}{ll}
\hline \hline Components: & Original Measurements: \\
(1) Acenaphthene; $\mathrm{C}_{12} \mathrm{H}_{10} ;[83-32-9]$ & ${ }^{14} \mathrm{~F}$. He and P. Liu, J. Chem. \\
(2) Methylbenzene; $\mathrm{C}_{7} \mathrm{H}_{8} ;[108-88-8]$ & Eng. Data 52, 2536 (2007). \\
\hline Variables: & Prepared by: \\
Temperature & W. E. Acree, Jr. \\
\hline
\end{tabular}

Experimental Values

\begin{tabular}{lcc}
\hline \hline$T / \mathrm{K}$ & $x_{2}{ }^{\mathrm{a}}$ & $x_{1}{ }^{\mathrm{b}}$ \\
\hline 278.47 & 0.8958 & 0.1042 \\
283.33 & 0.8782 & 0.1218 \\
288.10 & 0.8582 & 0.1418 \\
293.15 & 0.8330 & 0.1670 \\
298.23 & 0.8064 & 0.1936 \\
303.15 & 0.7761 & 0.2239 \\
307.95 & 0.7441 & 0.2559 \\
312.95 & 0.7015 & 0.2985 \\
318.30 & 0.6543 & 0.3457 \\
323.20 & 0.6016 & 0.3984 \\
\hline
\end{tabular}

${ }^{a} x_{2}$ : mole fraction of component 2 in the saturated solution.

${ }^{\mathrm{b}} x_{1}$ : mole fraction solubility of the solute.

The authors refer to the compound has 1,8-dihydroacenaphthalene, which the compiler has taken to be acenaphthene. 


\section{Auxiliary Information}

\section{Method/Apparatus/Procedure:}

Thermostated bath, thermometer.

Very little experimental details were provided. Solubilities were determined by a synthetic method. Known masses of solute and solvent were placed in a dissolving flask. The solute and solvent mixture was stirred with a magnetic stirrer. Small amounts of solute were added at predetermined intervals until no more solid would dissolve. The point at which no more solid would dissolve was taken to be the point of solid-liquid equilibrium. The paper did not specify how the disappearance of the solid was monitored; however, the papers that the authors referenced for the experimental method used a laser monitoring method.

\section{Source and Purity of Chemicals:}

(1) $99.5 \%$, Chemical source and purification method were not given. (2) $99.8 \%$, Analytical Reagent Grade, Tianjin Kewei Company, China, no information given regarding any further purification.

Estimated Error:

Temperature: $\pm 0.2 \mathrm{~K}$.

$x_{1}$ : within 0.0200 (relative uncertainty).

\subsection{Acenaphthene solubility data in esters}

\section{Components:}

(1) Acenaphthene; $\mathrm{C}_{12} \mathrm{H}_{10}$; [83-32-9]

(2) Methyl ethanoate; $\mathrm{C}_{3} \mathrm{H}_{6} \mathrm{O}_{2}$; [79-20-9]

Original Measurements:

${ }^{10}$ W. E. Acree, Jr. and M. H.

Abraham, Fluid Phase

Equilib. 201, 245 (2002).

\section{Variables:}

$T / \mathrm{K}=298.15$

Prepared by:

W. E. Acree, Jr.

Experimental Values

\begin{tabular}{lcc}
\hline \hline$x_{2}{ }^{(\mathrm{s}) \mathrm{a}}$ & $x_{2}{ }^{\mathrm{b}}$ & $x_{1}{ }^{\mathrm{c}}$ \\
\hline 1.0000 & 0.9290 & 0.07100 \\
\hline$x_{2}{ }^{(\mathrm{s})} \mathrm{initat}$
\end{tabular}

${ }^{\mathrm{a}} x_{2}{ }^{(\mathrm{s})}$ : initial mole fraction of component 2 in the solution.

${ }^{\mathrm{b}} x_{2}$ : mole fraction of component 2 in the saturated solution.

${ }^{c} x_{1}$ : mole fraction solubility of the solute.

\section{Auxiliary Information}

\section{Method/Apparatus/Procedure:}

Constant-temperature bath, calorimetric thermometer, and an ultraviolet/ visible spectrophotometer.

Excess solute and solvent were placed in amber glass bottles and allowed to equilibrate for several days at constant temperature. Attainment of equilibrium was verified by several repetitive measurements and by approaching equilibrium from supersaturation. Aliquots of saturated solutions were transferred through a coarse filter into tared volumetric flasks, weighed and diluted with 2-propanol. Concentrations were determined by spectrophotometric measurements at $289 \mathrm{~nm}$.

\section{Source and Purity of Chemicals:}

(1) $98 \%$, Aldrich Chemical Company, Milwaukee, WI, USA, was recrystallized several times from methanol.

(2) $99.5 \%$, anhydrous, Aldrich Chemical Company, stored over molecular sieves before use.
Estimated Error:

Temperature: $\pm 0.1 \mathrm{~K}$.

$x_{1}: \pm 1.5 \%$ (relative error).

\begin{tabular}{ll}
\hline \hline Components: & Original Measurements: \\
(1) Acenaphthene; $\mathrm{C}_{12} \mathrm{H}_{10} ;[83-32-9]$ & ${ }^{9}$ K. M. De Fina, T. L. Sharp, \\
(2) Ethyl ethanoate; $\mathrm{C}_{4} \mathrm{H}_{8} \mathrm{O}_{2} ;[141-78-6]$ & and W. E. Acree, Jr., Can. J. \\
& Chem. 77, 1537 (1999). \\
\hline Variables: & Prepared by: \\
$T / \mathrm{K}=298.15$ & W. E. Acree, Jr. \\
\hline
\end{tabular}

Experimental Values

\begin{tabular}{lcc}
\hline \hline$x_{2}{ }^{(\mathrm{s}) \mathrm{a}}$ & $x_{2}{ }^{\mathrm{b}}$ & $x_{1}{ }^{\mathrm{c}}$ \\
\hline 1.0000 & 0.8914 & 0.1086 \\
\hline${ }^{\mathrm{a}} x_{2}{ }^{(\mathrm{s})}:$ initial mole fraction of component 2 in the solution. \\
${ }^{\mathrm{b}} x_{2}:$ mole fraction of component 2 in the saturated solution. \\
${ }^{\mathrm{c}}{ }_{x_{1}}:$ mole fraction solubility of the solute.
\end{tabular}

\section{Auxiliary Information}

\section{Method/Apparatus/Procedure:}

Constant-temperature bath, calorimetric thermometer, and an ultraviolet/ visible spectrophotometer.

Excess solute and solvent were placed in amber glass bottles and allowed to equilibrate for several days at constant temperature. Attainment of equilibrium was verified by several repetitive measurements and by approaching equilibrium from supersaturation. Aliquots of saturated solutions were transferred through a coarse filter into tared volumetric flasks, weighed and diluted with methanol. Concentrations were determined by spectrophotometric measurements at $289 \mathrm{~nm}$.

\section{Source and Purity of Chemicals:}

(1) $98 \%$, Aldrich Chemical Company, Milwaukee, WI, USA, was recrystallized several times from methanol.

(2) $99.9 \%$, HPLC Grade, Aldrich Chemical Company, stored over molecular sieves before use.

\section{Estimated Error:}

Temperature: $\pm 0.1 \mathrm{~K}$.

$x_{1}: \pm 1.5 \%$ (relative error).

\begin{tabular}{ll}
\hline \hline Components: & Original Measurements: \\
(1) Acenaphthene; $\mathrm{C}_{12} \mathrm{H}_{10} ;[83-32-9]$ & ${ }^{9}$ K. M. De Fina, T. L. Sharp, \\
(2) Butyl ethanoate; $\mathrm{C}_{6} \mathrm{H}_{12} \mathrm{O}_{2} ;[123-86-4]$ & and W. E. Acree, Jr., Can. J. \\
& Chem. 77, 1537 (1999). \\
\hline Variables: & Prepared by: \\
$T / \mathrm{K}=298.15$ & W. E. Acree, Jr. \\
\hline
\end{tabular}


Experimental Values

\begin{tabular}{lcc}
\hline$\frac{x_{2}{ }^{(\mathrm{s}) \mathrm{a}}}{x_{2}{ }^{\mathrm{b}}}$ & $x_{1}{ }^{\mathrm{c}}$ \\
\hline 1.0000 & 0.8630 & 0.1370 \\
$\frac{{ }^{\mathrm{a}} x_{2}{ }^{(\mathrm{s})}: \text { initial mole fraction of component } 2 \text { in the solution. }}{{ }^{\mathrm{b}}{ }_{x_{2}}: \text { mole fraction of component } 2 \text { in the saturated solution. }}$ \\
${ }^{\mathrm{c}} x_{1}:$ mole fraction solubility of the solute.
\end{tabular}

\section{Auxiliary Information}

\section{Method/Apparatus/Procedure:}

Constant-temperature bath, calorimetric thermometer, and an ultraviolet/ visible spectrophotometer.

Excess solute and solvent were placed in amber glass bottles and allowed to equilibrate for several days at constant temperature. Attainment of equilibrium was verified by several repetitive measurements and by approaching equilibrium from supersaturation. Aliquots of saturated solutions were transferred through a coarse filter into tared volumetric flasks, weighed and diluted with methanol. Concentrations were determined by spectrophotometric measurements at $289 \mathrm{~nm}$.

\section{Source and Purity of Chemicals:}

(1) $98 \%$, Aldrich Chemical Company, Milwaukee, WI, USA, was recrystallized several times from methanol.

(2) $99.7 \%$, HPLC Grade, Aldrich Chemical Company, stored over molecular sieves before use.

\section{Estimated Error:}

Temperature: $\pm 0.1 \mathrm{~K}$.

$x_{1}: \pm 1.5 \%$ (relative error)

\subsection{Acenaphthene solubility data in ethers}

\begin{tabular}{|c|c|}
\hline $\begin{array}{l}\text { Components: } \\
\text { (1) Acenaphthene; } \mathrm{C}_{12} \mathrm{H}_{10} ;[83-32-9] \\
\text { (2) } 1,1^{\prime} \text {-Oxybisethane; } \mathrm{C}_{4} \mathrm{H}_{10} \mathrm{O} ;[60-29-7]\end{array}$ & $\begin{array}{l}\text { Original Measurements: } \\
{ }^{10} \text { W. E. Acree, Jr. and M. H. } \\
\text { Abraham, Fluid Phase } \\
\text { Equilib. 201, } 245 \text { (2002). }\end{array}$ \\
\hline $\begin{array}{l}\text { Variables: } \\
T / \mathrm{K}=298.15\end{array}$ & $\begin{array}{l}\text { Prepared by: } \\
\text { W. E. Acree, Jr. }\end{array}$ \\
\hline
\end{tabular}

Experimental Values

\begin{tabular}{|c|c|c|}
\hline$x_{2}^{(\mathrm{s}) \mathrm{a}}$ & $x_{2}{ }^{\mathrm{b}}$ & $x_{1}{ }^{\mathrm{c}}$ \\
\hline 1.0000 & 0.8981 & 0.1019 \\
\hline
\end{tabular}

\section{Auxiliary Information}

\section{Method/Apparatus/Procedure:}

Constant-temperature bath, calorimetric thermometer, and an ultraviolet/ visible spectrophotometer.

Excess solute and solvent were placed in amber glass bottles and allowed to equilibrate for several days at constant temperature. Attainment of equilibrium was verified by several repetitive measurements and by approaching equilibrium from supersaturation. Aliquots of saturated solutions were transferred through a coarse filter into tared volumetric flasks, weighed and diluted with 2-propanol. Concentrations were determined by spectrophotometric measurements at $289 \mathrm{~nm}$.

Source and Purity of Chemicals:

(1) $98 \%$, Aldrich Chemical Company, Milwaukee, WI, USA, was recrystallized several times from methanol.

(2) 99+\%, anhydrous, Aldrich Chemical Company, stored over molecular sieves before use.

Estimated Error:

Temperature: $\pm 0.1 \mathrm{~K}$.

$x_{1}: \pm 1.5 \%$ (relative error)

\begin{tabular}{ll}
\hline \hline Components: & Original Measurements: \\
(1) Acenaphthene; $\mathrm{C}_{12} \mathrm{H}_{10} ;[83-32-9]$ & ${ }^{10}$ W. E. Acree, Jr. and M. H. \\
$(2) 2,2^{\prime}-$ Oxybispropane; $\mathrm{C}_{6} \mathrm{H}_{14} \mathrm{O} ;$ & Abraham, Fluid Phase \\
{$[108-20-3]$} & Equilib. 201, 245 (2002). \\
\hline Variables: & Prepared by: \\
$T / \mathrm{K}=298.15$ & W. E. Acree, Jr. \\
\hline
\end{tabular}

Experimental Values

\begin{tabular}{lcc}
\hline \hline$x_{2}{ }^{(\mathrm{s}) \mathrm{a}}$ & $x_{2}{ }^{\mathrm{b}}$ & $x_{1}{ }^{\mathrm{c}}$ \\
\hline 1.0000 & 0.9149 & 0.08505 \\
\hline
\end{tabular}

${ }^{a} x_{2}{ }^{(\mathrm{s})}$ : initial mole fraction of component 2 in the solution.

${ }^{b} x_{2}$ : mole fraction of component 2 in the saturated solution.

${ }^{c} x_{1}$ : mole fraction solubility of the solute.

\section{Auxiliary Information}

\section{Method/Apparatus/Procedure:}

Constant-temperature bath, calorimetric thermometer, and an ultraviolet/ visible spectrophotometer.

Excess solute and solvent were placed in amber glass bottles and allowed to equilibrate for several days at constant temperature. Attainment of equilibrium was verified by several repetitive measurements and by approaching equilibrium from supersaturation. Aliquots of saturated solutions were transferred through a coarse filter into tared volumetric flasks, weighed and diluted with 2-propanol. Concentrations were determined by spectrophotometric measurements at $289 \mathrm{~nm}$.

Source and Purity of Chemicals:

(1) $98 \%$, Aldrich Chemical Company, Milwaukee, WI, USA, was recrystallized several times from methanol.

(2) $99 \%$, anhydrous, Aldrich Chemical Company, stored over molecular sieves before use.

Estimated Error:

Temperature: $\pm 0.1 \mathrm{~K}$.

$x_{1}: \pm 1.5 \%$ (relative error). 


\section{Components:}

(1) Acenaphthene; $\mathrm{C}_{12} \mathrm{H}_{10} ;$ [83-32-9]

(2) $1,1^{\prime}$-Oxybisbutane; $\mathrm{C}_{8} \mathrm{H}_{18} \mathrm{O}$;

[142-96-1]

Variables:

$T / \mathrm{K}=298.15$

Original Measurements:

${ }^{9}$ K. M. De Fina, T. L. Sharp, and W. E. Acree, Jr., Can. J. Chem. 77, 1537 (1999).

Prepared by:

W. E. Acree, Jr.

Experimental Values

\begin{tabular}{lcc}
\hline \hline$x_{2}{ }^{(\mathrm{s}) \mathrm{a}}$ & $x_{2}{ }^{\mathrm{b}}$ & $x_{1}{ }^{\mathrm{c}}$ \\
\hline 1.0000 & 0.8884 & 0.1116 \\
\hline
\end{tabular}

${ }^{\mathrm{a}} x_{2}{ }^{(\mathrm{s})}$ : initial mole fraction of component 2 in the solution.

${ }^{b} x_{2}$ : mole fraction of component 2 in the saturated solution.

${ }^{c} x_{1}$ : mole fraction solubility of the solute.

\section{Auxiliary Information}

\section{Method/Apparatus/Procedure:}

Constant-temperature bath, calorimetric thermometer, and an ultraviolet/ visible spectrophotometer.

Excess solute and solvent were placed in amber glass bottles and allowed to equilibrate for several days at constant temperature. Attainment of equilibrium was verified by several repetitive measurements and by approaching equilibrium from supersaturation. Aliquots of saturated solutions were transferred through a coarse filter into tared volumetric flasks, weighed and diluted with methanol. Concentrations were determined by

spectrophotometric measurements at $289 \mathrm{~nm}$.

Source and Purity of Chemicals:

(1) $98 \%$, Aldrich Chemical Company, Milwaukee, WI, USA, was recrystallized several times from methanol.

(2) $99 \%$, Aldrich Chemical Company, stored over molecular sieves before use.

Estimated Error:

Temperature: $\pm 0.1 \mathrm{~K}$.

$x_{1}: \pm 1.5 \%$ (relative error).

\begin{tabular}{|c|c|}
\hline $\begin{array}{l}\text { Components: } \\
\text { (1) Acenaphthene; } \mathrm{C}_{12} \mathrm{H}_{10} ;[83-32-9] \\
\text { (2) 2-Methoxy-2-methylpropane; } \mathrm{C}_{5} \mathrm{H}_{12} \mathrm{O} \text {; } \\
\text { [1634-04-4] }\end{array}$ & $\begin{array}{l}\text { Original Measurements: } \\
{ }^{10} \text { W. E. Acree, Jr. and M. H. } \\
\text { Abraham, Fluid Phase } \\
\text { Equilib. 201, } 245 \text { (2002). }\end{array}$ \\
\hline $\begin{array}{l}\text { Variables: } \\
T / \mathrm{K}=298.15\end{array}$ & $\begin{array}{l}\text { Prepared by: } \\
\text { W. E. Acree, Jr. }\end{array}$ \\
\hline
\end{tabular}

Experimental Values

\begin{tabular}{lcc}
\hline \hline$x_{2}{ }^{(\mathrm{s}) \mathrm{a}}$ & $x_{2}{ }^{\mathrm{b}}$ & $x_{1}{ }^{\mathrm{c}}$ \\
\hline 1.0000 & 0.9081 & 0.0919 \\
\hline${ }^{\mathrm{a}} x_{2} \mathrm{(s)}:$ initial mole fraction of component 2 in the solution. \\
${ }^{\mathrm{b}} x_{2}:$ mole fraction of component 2 in the saturated solution. \\
${ }^{\mathrm{c}}{ }_{x_{1}}:$ mole fraction solubility of the solute.
\end{tabular}

\section{Auxiliary Information}

Method/Apparatus/Procedure:

Constant-temperature bath, calorimetric thermometer, and an ultraviolet/ visible spectrophotometer.

Excess solute and solvent were placed in amber glass bottles and allowed to equilibrate for several days at constant temperature. Attainment of equilibrium was verified by several repetitive measurements and by approaching equilibrium from supersaturation. Aliquots of saturated solutions were transferred through a coarse filter into tared volumetric flasks, weighed and diluted with 2-propanol. Concentrations were determined by spectrophotometric measurements at $289 \mathrm{~nm}$.

\section{Source and Purity of Chemicals:}

(1) $98 \%$, Aldrich Chemical Company, Milwaukee, WI, USA, was recrystallized several times from methanol.

(2) $99.8 \%$, anhydrous, Aldrich Chemical Company, stored over molecular sieves before use.

Estimated Error:

Temperature: $\pm 0.1 \mathrm{~K}$.

$x_{1}: \pm 1.5 \%$ (relative error).

\begin{tabular}{|c|c|}
\hline $\begin{array}{l}\text { Components: } \\
\text { (1) Acenaphthene; } \mathrm{C}_{12} \mathrm{H}_{10} ;[83-32-9] \\
\text { (2) Tetrahydrofuran; } \mathrm{C}_{4} \mathrm{H}_{8} \mathrm{O} \text {; } \\
\text { [109-99-9] }\end{array}$ & $\begin{array}{l}\text { Original Measurements: } \\
{ }^{9} \text { K. M. De Fina, T. L. Sharp, } \\
\text { and W. E. Acree, Jr., Can. J. } \\
\text { Chem. 77, } 1537 \text { (1999). }\end{array}$ \\
\hline $\begin{array}{l}\text { Variables: } \\
T / \mathrm{K}=298.15\end{array}$ & $\begin{array}{l}\text { Prepared by: } \\
\text { W. E. Acree, Jr. }\end{array}$ \\
\hline
\end{tabular}

Experimental Values

\begin{tabular}{lcc}
\hline \hline$x_{2}{ }^{(\mathrm{s}) \mathrm{a}}$ & $x_{2}{ }^{\mathrm{b}}$ & $x_{1}{ }^{\mathrm{c}}$ \\
\hline 1.0000 & 0.8027 & 0.1973 \\
\hline${ }^{\mathrm{a}} x_{2}{ }^{(\mathrm{s})}:$ initial mole fraction of component 2 in the solution; \\
${ }^{\mathrm{b}} x_{2}:$ mole fraction of component 2 in the saturated solution. \\
${ }^{\mathrm{c}} x_{1}:$ mole fraction solubility of the solute
\end{tabular}

\section{Auxiliary Information}

\section{Method/Apparatus/Procedure:}

Constant-temperature bath, calorimetric thermometer, and an ultraviolet/ visible spectrophotometer.

Excess solute and solvent were placed in amber glass bottles and allowed to equilibrate for several days at constant temperature. Attainment of equilibrium was verified by several repetitive measurements and by approaching equilibrium from supersaturation. Aliquots of saturated solutions were transferred through a coarse filter into tared volumetric flasks, weighed and diluted with methanol. Concentrations were determined by spectrophotometric measurements at $289 \mathrm{~nm}$.

\section{Source and Purity of Chemicals:}

(1) $98 \%$, Aldrich Chemical Company, Milwaukee, WI, USA, was recrystallized several times from methanol.

(2) $99.9 \%$, anhydrous, Aldrich Chemical Company, stored over molecular sieves before use.

Estimated Error:

Temperature: $\pm 0.1 \mathrm{~K}$.

$x_{1}: \pm 1.5 \%$ (relative error). 


\section{Components:}

(1) Acenaphthene; $\mathrm{C}_{12} \mathrm{H}_{10} ;$; $\left.83-32-9\right]$

(2) 1,4-Dioxane; $\mathrm{C}_{4} \mathrm{H}_{8} \mathrm{O}_{2}$; [123-91-1]

\section{Variables:}

$T / \mathrm{K}=298.15$

Original Measurements:

${ }^{9}$ K. M. De Fina, T. L. Sharp, and W. E. Acree, Jr., Can. J. Chem. 77, 1537 (1999).

Prepared by:

W. E. Acree, Jr.

\section{Experimental Values}

\begin{tabular}{lcc}
\hline$x_{2}{ }^{(\mathrm{s}) \mathrm{a}}$ & $x_{2}{ }^{\mathrm{b}}$ & $x_{1}{ }^{\mathrm{c}}$ \\
\hline 1.0000 & 0.8585 & 0.1415 \\
${ }^{\mathrm{a}} x_{2}{ }^{(\mathrm{s})}:$ initial mole fraction of component 2 in the solution. \\
${ }^{\mathrm{b}} x_{2}:$ mole fraction of component 2 in the saturated solution. \\
${ }^{\mathrm{c}} x_{1}:$ mole fraction solubility of the solute.
\end{tabular}

\section{Auxiliary Information}

\begin{tabular}{l}
\hline Method/Apparatus/Procedure: \\
Constant-temperature bath, calorimetric thermometer, and an ultraviolet/ \\
visible spectrophotometer. \\
Excess solute and solvent were placed in amber glass bottles and allowed to \\
equilibrate for several days at constant temperature. Attainment of equilibrium \\
was verified by several repetitive measurements and by approaching \\
equilibrium from supersaturation. Aliquots of saturated solutions were \\
transferred through a coarse filter into tared volumetric flasks, weighed and \\
diluted with methanol. Concentrations were determined by \\
spectrophotometric measurements at 289 nm. \\
Source and Purity of Chemicals: \\
(1) $98 \%$, Aldrich Chemical Company, Milwaukee, WI, USA, was \\
recrystallized several times from methanol. \\
(2) $99.8 \%$, anhydrous, Aldrich Chemical Company, stored over molecular \\
sieves before use. \\
Estimated Error: \\
Temperature: $\pm 0.1 \mathrm{~K}$. \\
$x_{1}: \pm 1.5 \%$ (relative error).
\end{tabular}

\subsection{Acenaphthene solubility data in haloalkanes, haloalkenes, and haloaromatic hydrocarbons}

\section{Components:}

(1) Acenaphthene; $\mathrm{C}_{12} \mathrm{H}_{10}$; [83-32-9]

(2) Trichloromethane; $\mathrm{CHCl}_{3}$; [67-66-3]
Original Measurements:

${ }^{13}$ I. Kotula and B. Marciniak, J. Chem. Eng. Data 46, 783 (2001).

\begin{tabular}{ll}
\hline Variables: & Prepared by: \\
Temperature & W. E. Acree, Jr. \\
\hline
\end{tabular}

Experimental Values

\begin{tabular}{lcc}
\hline \hline$T / \mathrm{K}$ & $x_{2}{ }^{\mathrm{a}}$ & $x_{1}{ }^{\mathrm{b}}$ \\
\hline 316.05 & 0.6861 & 0.3139 \\
319.45 & 0.6558 & 0.3442 \\
321.45 & 0.6379 & 0.3621 \\
324.05 & 0.6124 & 0.3876 \\
\hline
\end{tabular}

${ }^{a} x_{2}$ : mole fraction of component 2 in the saturated solution.

${ }^{b} x_{1}$ : mole fraction of the polycyclic aromatic hydrocarbon (component 1).

\section{Auxiliary Information}

\section{Method/Apparatus/Procedure:}

Constant-temperature bath, electric glass furnace, heater, mercury thermometer.

Solubilities were determined by a synthetic-dynamic method. Known masses of solute and solvent were placed in a Pyrex test tube and sealed by a flexible rubber cap mounted on a calibrated mercury thermometer. The solute and solvent were heated using an electrical glass furnace equipped with a platinum resistor placed near the heater. Samples were agitated and the temperature at which the solid solute disappeared was observed against a dark screen by means of a stereoscopic microscope.

\section{Source and Purity of Chemicals:}

(1) Analytically pure, International Enzymes Limited, Windsor-Bershire, England, recrystallized twice from distilled benzene and then from anhydrous ethanol.

(2) $99.5+\%$, purchased from either Fluka or Aldrich Chemical Company, was used as received.

Estimated Error:

Temperature: $\pm 0.3 \mathrm{~K}$.

$x_{1}:<1 \%$ (relative error).

\section{Components:}

(2) Tetrachloromethane; $\mathrm{CCl}_{4} ;[56-23-5]$

Variables:

Temperature
(1) Acenaphthene; $\mathrm{C}_{12} \mathrm{H}_{10} ;[83-32-9]$
Original Measurements:

${ }^{13}$ I. Kotula and B. Marciniak, J. Chem. Eng. Data 46, 783 (2001).

Prepared by:

W. E. Acree, Jr.
Experimental Values

\begin{tabular}{lcc}
\hline \hline$T / \mathrm{K}$ & $x_{2}{ }^{\mathrm{a}}$ & $x_{1}{ }^{\mathrm{b}}$ \\
\hline 291.15 & 0.8805 & 0.1195 \\
298.85 & 0.8491 & 0.1509 \\
302.65 & 0.8232 & 0.1768 \\
306.65 & 0.8001 & 0.1999 \\
310.15 & 0.7750 & 0.2250 \\
316.95 & 0.7230 & 0.2770 \\
321.15 & 0.6860 & 0.3140 \\
327.15 & 0.6279 & 0.3721 \\
\hline${ }^{\mathrm{a}} x_{2}:$ mole fraction of component 2 in the saturated solution. \\
${ }^{\mathrm{b}}{ }_{x_{1}}:$ mole fraction of the polycyclic aromatic hydrocarbon (component 1).
\end{tabular}

\begin{tabular}{lcc}
\hline \hline$T / \mathrm{K}$ & $x_{2}{ }^{\mathrm{a}}$ & $x_{1}{ }^{\mathrm{b}}$ \\
\hline 289.25 & 0.8575 & 0.1425 \\
293.55 & 0.8364 & 0.1636 \\
300.45 & 0.7979 & 0.2021 \\
305.25 & 0.7678 & 0.2322 \\
313.65 & 0.7057 & 0.2943
\end{tabular}




\section{Auxiliary Information}

\section{Method/Apparatus/Procedure:}

Constant-temperature bath, electric glass furnace, heater, mercury thermometer.

Solubilities were determined by a synthetic-dynamic method. Known masses of solute and solvent were placed in a Pyrex test tube and sealed by a flexible rubber cap mounted on a calibrated mercury thermometer. The solute and solvent were heated using an electrical glass furnace equipped with a platinum resistor placed near the heater. Samples were agitated and the temperature at which the solid solute disappeared was observed against a dark screen by means of a stereoscopic microscope.

Source and Purity of Chemicals:

(1) Analytically pure, International Enzymes Limited, Windsor-Bershire, England, recrystallized twice from distilled benzene and then from anhydrous ethanol.

(2) Purity not given, Polish Chemical Reagents, Gliwice, Poland, was dried over anhydrous sodium sulfate and then fractionally distilled.

\section{Estimated Error:}

Temperature: $\pm 0.3 \mathrm{~K}$

$x_{1}:<1 \%$ (relative error).

\begin{tabular}{|c|c|}
\hline $\begin{array}{l}\text { Components: } \\
\text { (1) Acenaphthene; } \mathrm{C}_{12} \mathrm{H}_{10} ;[83-32-9] \\
\text { (2) 1,1-Dichloroethane; } \mathrm{C}_{2} \mathrm{H}_{4} \mathrm{Cl}_{2} \text {; } \\
\text { [75-34-3] }\end{array}$ & $\begin{array}{l}\text { Original Measurements: } \\
{ }^{13} \text { I. Kotula and B. Marciniak, } \\
\text { J. Chem. Eng. Data 46, } 783 \\
\text { (2001). }\end{array}$ \\
\hline $\begin{array}{l}\text { Variables: } \\
\text { Temperature }\end{array}$ & $\begin{array}{l}\text { Prepared by: } \\
\text { W. E. Acree, Jr. }\end{array}$ \\
\hline
\end{tabular}

Experimental Values

\begin{tabular}{lcc}
\hline \hline$T / \mathrm{K}$ & $x_{2}{ }^{\mathrm{a}}$ & $x_{1}{ }^{\mathrm{b}}$ \\
\hline 288.65 & 0.8853 & 0.1147 \\
290.15 & 0.8795 & 0.1205 \\
296.85 & 0.8479 & 0.1521 \\
300.35 & 0.8292 & 0.1708 \\
305.35 & 0.7978 & 0.2022 \\
312.95 & 0.7469 & 0.2531 \\
319.15 & 0.6962 & 0.3038 \\
320.45 & 0.6805 & 0.3195 \\
323.75 & 0.6528 & 0.3472
\end{tabular}

${ }^{\mathrm{a}} x_{2}$ : mole fraction of component 2 in the saturated solution.

${ }^{\mathrm{b}} x_{1}$ : mole fraction of the polycyclic aromatic hydrocarbon (component 1 ).

\section{Auxiliary Information}

\section{Method/Apparatus/Procedure:}

Constant-temperature bath, electric glass furnace, heater, mercury thermometer.

Solubilities were determined by a synthetic-dynamic method. Known masses of solute and solvent were placed in a Pyrex test tube and sealed by a flexible rubber cap mounted on a calibrated mercury thermometer. The solute and solvent were heated using an electrical glass furnace equipped with a platinum resistor placed near the heater. Samples were agitated and the temperature at which the solid solute disappeared was observed against a dark screen by means of a stereoscopic microscope.
Source and Purity of Chemicals:

(1) Analytically pure, International Enzymes Limited, Windsor-Bershire, England, recrystallized twice from distilled benzene and then from anhydrous ethanol.

(2) Purity not given, Polish Chemical Reagents, Glowice, Poland, was dried over anhydrous sodium sulfate and then fractionally distilled.

\section{Estimated Error:}

Temperature: $\pm 0.3 \mathrm{~K}$.

$x_{1}:<1 \%$ (relative error).

\begin{tabular}{ll}
\hline \hline Components: & Original Measurements: \\
(1) Acenaphthene; $\mathrm{C}_{12} \mathrm{H}_{10} ;[83-32-9]$ & ${ }^{13}$ I. Kotula and B. Marciniak, \\
(2) 1,2-Dichloroethane; $\mathrm{C}_{2} \mathrm{H}_{4} \mathrm{Cl}_{2} ;$ & J. Chem. Eng. Data 46, 783 \\
{$[107-06-2]$} & $(2001)$. \\
\hline Variables: & Prepared by: \\
Temperature & W. E. Acree, Jr. \\
\hline
\end{tabular}

Experimental Values

\begin{tabular}{lcc}
\hline \hline$T / \mathrm{K}$ & $x_{2}{ }^{\mathrm{a}}$ & $x_{1}{ }^{\mathrm{b}}$ \\
\hline 288.15 & 0.8828 & 0.1172 \\
290.85 & 0.8712 & 0.1288 \\
297.15 & 0.8413 & 0.1587 \\
298.15 & 0.8362 & 0.1638 \\
304.65 & 0.7981 & 0.2019 \\
314.45 & 0.7293 & 0.2707 \\
321.35 & 0.6700 & 0.3300 \\
323.25 & 0.6522 & 0.3478 \\
\hline
\end{tabular}

${ }^{\mathrm{a}} x_{2}$ : mole fraction of component 2 in the saturated solution.

${ }^{\mathrm{b}} x_{1}$ : mole fraction of the polycyclic aromatic hydrocarbon (component 1 ).

\section{Auxiliary Information}

Method/Apparatus/Procedure:

Constant-temperature bath, electric glass furnace, heater, mercury thermometer.

Solubilities were determined by a synthetic-dynamic method. Known masses of solute and solvent were placed in a Pyrex test tube and sealed by a flexible rubber cap mounted on a calibrated mercury thermometer. The solute and solvent were heated using an electrical glass furnace equipped with a platinum resistor placed near the heater. Samples were agitated and the temperature at which the solid solute disappeared was observed against a dark screen by means of a stereoscopic microscope.

Source and Purity of Chemicals:

(1) Analytically pure, International Enzymes Limited, Windsor-Bershire, England, recrystallized twice from distilled benzene and then from anhydrous ethanol.

(2) $99.5+\%$, purchased from either Fluka or Aldrich Chemical Company, was used as received.

\section{Estimated Error:}

Temperature: $\pm 0.3 \mathrm{~K}$.

$x_{1}:<1 \%$ (relative error). 


\section{Components:}

(1) Acenaphthene; $\mathrm{C}_{12} \mathrm{H}_{10} ;$ [83-32-9]

(2) Trichloroethene; $\mathrm{C}_{2} \mathrm{HCl}_{3} ;$ [79-01-6]

Variables:

Temperature

Original Measurements:

${ }^{13}$ I. Kotula and B. Marciniak,

J. Chem. Eng. Data 46, 783

(2001).

Prepared by:

W. E. Acree, Jr.

Experimental Values

\begin{tabular}{lcc}
\hline \hline$T / \mathrm{K}$ & $x_{2}{ }^{\mathrm{a}}$ & $x_{1}{ }^{\mathrm{b}}$ \\
\hline 289.15 & 0.8658 & 0.1342 \\
292.35 & 0.8510 & 0.1490 \\
297.45 & 0.8248 & 0.1752 \\
306.15 & 0.7728 & 0.2272 \\
311.05 & 0.7380 & 0.2620 \\
312.75 & 0.7254 & 0.2746 \\
315.05 & 0.7069 & 0.2931 \\
318.85 & 0.6752 & 0.3248 \\
322.85 & 0.6384 & 0.3616
\end{tabular}

${ }^{\mathrm{a}} x_{2}$ : mole fraction of component 2 in the saturated solution.

${ }^{\mathrm{b}} x_{1}$ : mole fraction of the polycyclic aromatic hydrocarbon (component 1 ).

\section{Auxiliary Information}

\section{Method/Apparatus/Procedure:}

Constant-temperature bath, electric glass furnace, heater, mercury thermometer.

Solubilities were determined by a synthetic-dynamic method. Known masses of solute and solvent were placed in a Pyrex test tube and sealed by a flexible rubber cap mounted on a calibrated mercury thermometer. The solute and solvent were heated using an electrical glass furnace equipped with a platinum resistor placed near the heater. Samples were agitated and the temperature at which the solid solute disappeared was observed against a dark screen by means of a stereoscopic microscope.

\section{Source and Purity of Chemicals:}

(1) Analytically pure, International Enzymes Limited, Windsor-Bershire, England, recrystallized twice from distilled benzene and then from anhydrous ethanol.

(2) $99.5+\%$, purchased from either Fluka or Aldrich Chemical Company, was used as received.

Estimated Error:

Temperature: $\pm 0.3 \mathrm{~K}$.

$x_{1}:<1 \%$ (relative error).

\begin{tabular}{ll}
\hline \hline Components: & Original Measurements: \\
(1) Acenaphthene; $\mathrm{C}_{12} \mathrm{H}_{10} ;[83-32-9]$ & ${ }^{13}$ I. Kotula and B. Marciniak, \\
(2) Tetrachloroethene; $\mathrm{C}_{2} \mathrm{Cl}_{4} ;[127-18-4]$ & J. Chem. Eng. Data 46,783 \\
& $(2001)$. \\
\hline Variables: & Prepared by: \\
Temperature & W. E. Acree, Jr. \\
\hline
\end{tabular}

Experimental Values

\begin{tabular}{lcc}
\hline \hline$T / \mathrm{K}$ & $x_{2}{ }^{\mathrm{a}}$ & $x_{1}{ }^{\mathrm{b}}$ \\
\hline 288.15 & 0.9000 & 0.1000 \\
292.45 & 0.8828 & 0.1172 \\
297.65 & 0.8590 & 0.1410
\end{tabular}

\begin{tabular}{lcc}
\hline \hline$T / \mathrm{K}$ & $x_{2}{ }^{\mathrm{a}}$ & $x_{1}{ }^{\mathrm{b}}$ \\
\hline 301.25 & 0.8401 & 0.1599 \\
306.75 & 0.8082 & 0.1918 \\
311.75 & 0.7741 & 0.2259 \\
314.25 & 0.7556 & 0.2444 \\
319.85 & 0.7905 & 0.2905 \\
322.15 & 0.6887 & 0.3113 \\
\hline
\end{tabular}

${ }^{\mathrm{a}} x_{2}$ : mole fraction of component 2 in the saturated solution.

${ }^{\mathrm{b}} x_{1}$ : mole fraction of the polycyclic aromatic hydrocarbon (component 1 ).

\section{Auxiliary Information}

\section{Method/Apparatus/Procedure:}

Constant-temperature bath, electric glass furnace, heater, mercury thermometer.

Solubilities were determined by a synthetic-dynamic method. Known masses of solute and solvent were placed in a Pyrex test tube and sealed by a flexible rubber cap mounted on a calibrated mercury thermometer. The solute and solvent were heated using an electrical glass furnace equipped with a platinum resistor placed near the heater. Samples were agitated and the temperature at which the solid solute disappeared was observed against a dark screen by means of a stereoscopic microscope.

Source and Purity of Chemicals:

(1) Analytically pure, International Enzymes Limited, Windsor-Bershire, England, recrystallized twice from distilled benzene and then from anhydrous ethanol.

(2) $99.5+\%$, purchased from either Fluka or Aldrich Chemical Company, was used as received.

\section{Estimated Error:}

Temperature: $\pm 0.3 \mathrm{~K}$.

$x_{1}:<1 \%$ (relative error)

\subsection{Acenaphthene solubility data in alcohols}

\section{Components:}

(1) Acenaphthene; $\mathrm{C}_{12} \mathrm{H}_{10}$; [83-32-9]

(2) Methanol; $\mathrm{CH}_{4} \mathrm{O} ;[67-56-1]$

Variables:

$T / \mathrm{K}=298.15$
Original Measurements:

${ }^{9}$ K. M. De Fina, T. L. Sharp, and W. E. Acree, Jr., Can. J. Chem. 77, 1537 (1999).

Prepared by:

W. E. Acree, Jr.
Experimental Values

\begin{tabular}{lcc}
\hline \hline$x_{2}{ }^{(\mathrm{s}) \mathrm{a}}$ & $x_{2}{ }^{\mathrm{b}}$ & $x_{1}{ }^{\mathrm{c}}$ \\
\hline 1.0000 & 0.9946 & 0.00544 \\
$\frac{{ }^{a} x_{2}{ }^{(\mathrm{s})}: \text { initial mole fraction of component } 2 \text { in the solution. }}{}$ \\
${ }^{\mathrm{b}} x_{2}:$ mole fraction of component 2 in the saturated solution. \\
${ }^{c} x_{1}:$ mole fraction solubility of the solute.
\end{tabular}




\section{Auxiliary Information}

\section{Method/Apparatus/Procedure:}

Constant-temperature bath, calorimetric thermometer, and an ultraviolet/ visible spectrophotometer.

Excess solute and solvent were placed in amber glass bottles and allowed to equilibrate for several days at constant temperature. Attainment of equilibrium was verified by several repetitive measurements and by approaching equilibrium from supersaturation. Aliquots of saturated solutions were transferred through a coarse filter into tared volumetric flasks, weighed and diluted with methanol. Concentrations were determined by spectrophotometric measurements at $289 \mathrm{~nm}$.

Source and Purity of Chemicals:

(1) $98 \%$, Aldrich Chemical Company, Milwaukee, WI, USA, was recrystallized several times from methanol.

(2) $99.9+\%$, Aldrich Chemical Company, stored over molecular sieves before use.

Estimated Error:

Temperature: $\pm 0.1 \mathrm{~K}$.

$x_{1}: \pm 1.5 \%$ (relative error).

\begin{tabular}{ll}
\hline \hline Components: & Original Measurements: \\
(1) Acenaphthene; $\mathrm{C}_{12} \mathrm{H}_{10} ;[83-32-9]$ & ${ }^{9}$ K. M. De Fina, T. L. Sharp, \\
(2) Ethanol; $\mathrm{C}_{2} \mathrm{H}_{6} \mathrm{O} ;[64-17-5]$ & and W. E. Acree, Jr., Can. J. \\
& Chem. 77, 1537 (1999). \\
\hline Variables: & Prepared by: \\
$T / \mathrm{K}=298.15$ & W. E. Acree, Jr. \\
\hline
\end{tabular}

Experimental Values

\begin{tabular}{lcc}
\hline \hline$x_{2}{ }^{(\mathrm{s}) \mathrm{a}}$ & $x_{2}{ }^{\mathrm{b}}$ & $x_{1}{ }^{\mathrm{c}}$ \\
\hline 1.0000 & 0.9893 & 0.01068 \\
\hline
\end{tabular}

${ }^{\mathrm{a}} x_{2}{ }^{(\mathrm{s})}$ : initial mole fraction of component 2 in the solution.

${ }^{\mathrm{b}} x_{2}$ : mole fraction of component 2 in the saturated solution.

${ }^{\mathrm{c}} x_{1}$ : mole fraction solubility of the solute.

\section{Auxiliary Information}

\section{Method/Apparatus/Procedure:}

Constant-temperature bath, calorimetric thermometer, and an ultraviolet/ visible spectrophotometer.

Excess solute and solvent were placed in amber glass bottles and allowed to equilibrate for several days at constant temperature. Attainment of equilibrium was verified by several repetitive measurements and by approaching equilibrium from supersaturation. Aliquots of saturated solutions were transferred through a coarse filter into tared volumetric flasks, weighed and diluted with methanol. Concentrations were determined by

spectrophotometric measurements at $289 \mathrm{~nm}$.

\section{Source and Purity of Chemicals:}

(1) $98 \%$, Aldrich Chemical Company, Milwaukee, WI, USA, was recrystallized several times from methanol.

(2) Absolute, Aaper Alcohol and Chemical Company, USA, stored over molecular sieves before use.

Estimated Error:

Temperature: $\pm 0.1 \mathrm{~K}$.

$\underline{x_{1}: \pm 1.5 \% \text { (relative error). }}$

\begin{tabular}{ll}
\hline \hline Components: & Original Measurements: \\
(1) Acenaphthene; $\mathrm{C}_{12} \mathrm{H}_{10} ;[83-32-9]$ & ${ }^{14} \mathrm{~F}$. He and P. Liu, J. Chem. \\
(2) Ethanol; $\mathrm{C}_{2} \mathrm{H}_{6} \mathrm{O} ;[64-17-5]$ & Eng. Data 52, 2536 (2007). \\
\hline Variables: & Prepared by: \\
Temperature & W. E. Acree, Jr. \\
\hline
\end{tabular}

Experimental Values

\begin{tabular}{lcc}
\hline \hline$T / \mathrm{K}$ & $x_{2}{ }^{\mathrm{a}}$ & $x_{1}{ }^{\mathrm{b}}$ \\
\hline 278.10 & 0.9954 & 0.004622 \\
283.53 & 0.9942 & 0.005785 \\
288.25 & 0.9929 & 0.007093 \\
293.25 & 0.9911 & 0.008913 \\
298.20 & 0.9892 & 0.010793 \\
303.20 & 0.9867 & 0.013276 \\
308.20 & 0.9835 & 0.016519 \\
313.05 & 0.9803 & 0.019700 \\
318.25 & 0.9755 & 0.024486 \\
323.15 & 0.9704 & 0.029553 \\
\hline
\end{tabular}

${ }^{a} x_{2}$ : mole fraction of component 2 in the saturated solution.

${ }^{b} x_{1}$ : mole fraction of the polycyclic aromatic hydrocarbon (component 1 ).

The authors refer to the compound as 1,8-dihydroacenaphthalene, which the compiler has taken to be acenaphthene.

\section{Auxiliary Information}

\section{Method/Apparatus/Procedure:}

Thermostated bath, thermometer.

Very little experimental details were provided. Solubilities were determined by a synthetic method. Known masses of solute and solvent were placed in a dissolving flask. The solute and solvent mixture was stirred with a magnetic stirrer. Small amounts of solute were added at predetermined intervals until no more solid would dissolve. The point at which no more solid would dissolve was taken to be the point of solid-liquid equilibrium. The paper did not specify how the disappearance of the solid was monitored; however, the papers that the authors referenced for the experimental method used a laser monitoring method.

\section{Source and Purity of Chemicals:}

(1) $99.5 \%$, Chemical source and purification method were not given.

(2) $99.8 \%$, Analytical Reagent Grade, Tianjin Kewei Company, China, no information given regarding any further purification.

\section{Estimated Error:}

Temperature: $\pm 0.2 \mathrm{~K}$.

$x_{1}$ : within 0.0200 (relative uncertainty).

\begin{tabular}{|c|c|}
\hline $\begin{array}{l}\text { Components: } \\
\text { (1) Acenaphthene; } \mathrm{C}_{12} \mathrm{H}_{10} ;[83-32-9] \\
\text { (2) 1-Propanol; } \mathrm{C}_{3} \mathrm{H}_{8} \mathrm{O} ;[71-23-8]\end{array}$ & $\begin{array}{l}\text { Original Measurements: } \\
{ }^{9} \text { K. M. De Fina, T. L. Sharp, } \\
\text { and W. E. Acree, Jr., Can. J. } \\
\text { Chem. 77, } 1537 \text { (1999). }\end{array}$ \\
\hline $\begin{array}{l}\text { Variables: } \\
T / \mathrm{K}=298.15\end{array}$ & $\begin{array}{l}\text { Prepared by: } \\
\text { W. E. Acree, Jr. }\end{array}$ \\
\hline
\end{tabular}


Experimental Values

\begin{tabular}{lcc}
\hline \hline$x_{2}{ }^{(\mathrm{s}) \mathrm{a}}$ & $x_{2}{ }^{\mathrm{b}}$ & $x_{1}{ }^{\mathrm{c}}$ \\
\hline 1.0000 & 0.9831 & 0.01686 \\
$\frac{{ }^{a} x_{2}{ }^{(\mathrm{s})}: \text { initial mole fraction of component } 2 \text { in the solution. }}{{ }^{\mathrm{b}} x_{2}: \text { mole fraction of component } 2 \text { in the saturated solution. }}$ \\
${ }^{\mathrm{c}} x_{1}:$ mole fraction solubility of the solute.
\end{tabular}

\section{Auxiliary Information}

\section{Method/Apparatus/Procedure:}

Constant-temperature bath, calorimetric thermometer, and an ultraviolet/ visible spectrophotometer.

Excess solute and solvent were placed in amber glass bottles and allowed to equilibrate for several days at constant temperature. Attainment of equilibrium was verified by several repetitive measurements and by approaching equilibrium from supersaturation. Aliquots of saturated solutions were transferred through a coarse filter into tared volumetric flasks, weighed and diluted with methanol. Concentrations were determined by

spectrophotometric measurements at $289 \mathrm{~nm}$

\section{Source and Purity of Chemicals:}

(1) $98 \%$, Aldrich Chemical Company, Milwaukee, WI, USA, was recrystallized several times from methanol.

(2) $99+\%$, anhydrous, Aldrich Chemical Company, stored over molecular sieves before use.

Estimated Error:

Temperature: $\pm 0.1 \mathrm{~K}$.

$x_{1}: \pm 1.5 \%$ (relative error).

\section{Components: \\ (1) Acenaphthene; $\mathrm{C}_{12} \mathrm{H}_{10} ;[83-32-9]$ \\ (2) 2-Propanol; $\mathrm{C}_{3} \mathrm{H}_{8} \mathrm{O}$; [67-63-0]}

\section{Variables:}

$T / \mathrm{K}=298.15$
Original Measurements:

${ }^{9}$ K. M. De Fina, T. L. Sharp, and W. E. Acree, Jr., Can. J. Chem. 77, 1537 (1999).

Prepared by:

W. E. Acree, Jr.

\section{Experimental Values}

\begin{tabular}{lcc}
\hline \hline$x_{2}{ }^{(\mathrm{s}) \mathrm{a}}$ & $x_{2}{ }^{\mathrm{b}}$ & $x_{1}{ }^{\mathrm{c}}$ \\
\hline 1.0000 & 0.9866 & 0.01336
\end{tabular}

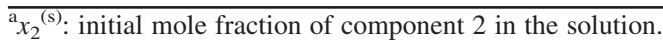

${ }^{b} x_{2}$ : mole fraction of component 2 in the saturated solution.

${ }^{c} x_{1}$ : mole fraction solubility of the solute.

\section{Auxiliary Information}

\section{Method/Apparatus/Procedure:}

Constant-temperature bath, calorimetric thermometer, and an ultraviolet/ visible spectrophotometer.

Excess solute and solvent were placed in amber glass bottles and allowed to equilibrate for several days at constant temperature. Attainment of equilibrium was verified by several repetitive measurements and by approaching equilibrium from supersaturation. Aliquots of saturated solutions were transferred through a coarse filter into tared volumetric flasks, weighed and diluted with methanol. Concentrations were determined by spectrophotometric measurements at $289 \mathrm{~nm}$.
Source and Purity of Chemicals:

(1) $98 \%$, Aldrich Chemical Company, Milwaukee, WI, USA, was recrystallized several times from methanol.

(2) $99+\%$, anhydrous, Aldrich Chemical Company, stored over molecular sieves before use.

\section{Estimated Error:}

Temperature: $\pm 0.1 \mathrm{~K}$.

$x_{1}: \pm 1.5 \%$ (relative error).

\begin{tabular}{ll}
\hline \hline Components: & Original Measurements: \\
(1) Acenaphthene; $\mathrm{C}_{12} \mathrm{H}_{10} ;[83-32-9]$ & ${ }^{14} \mathrm{~F}$. He and P. Liu, J. Chem. \\
(2) 2-Propanol; $\mathrm{C}_{3} \mathrm{H}_{8} \mathrm{O} ;[67-63-0]$ & Eng. Data 52, 2536 (2007). \\
\hline Variables: & Prepared by: \\
Temperature & W. E. Acree, Jr. \\
\hline
\end{tabular}

Experimental Values

\begin{tabular}{lcc}
\hline \hline$T / \mathrm{K}$ & $x_{2}{ }^{\mathrm{a}}$ & $x_{1}{ }^{\mathrm{b}}$ \\
\hline 278.30 & 0.9953 & 0.004687 \\
283.07 & 0.9941 & 0.005923 \\
288.33 & 0.9923 & 0.007701 \\
293.20 & 0.9903 & 0.009671 \\
298.07 & 0.9880 & 0.011972 \\
303.30 & 0.9848 & 0.015233 \\
308.05 & 0.9811 & 0.018871 \\
312.95 & 0.9764 & 0.023581 \\
318.40 & 0.9698 & 0.030246 \\
323.25 & 0.9628 & 0.037196
\end{tabular}

${ }^{a_{1}} x_{2}$ : mole fraction of component 2 in the saturated solution.

${ }^{\mathrm{b}} x_{1}$ : mole fraction of the polycyclic aromatic hydrocarbon (component 1 ).

The authors refer to the compound as 1,8-dihydroacenaphthalene, which the compiler has taken to be acenaphthene.

\section{Auxiliary Information}

\section{Method/Apparatus/Procedure:}

Thermostated bath, thermometer.

Very little experimental details were provided. Solubilities were determined by a synthetic method. Known masses of solute and solvent were placed in a dissolving flask. The solute and solvent mixture was stirred with a magnetic stirrer. Small amounts of solute were added at predetermined intervals until no more solid would dissolve. The point at which no more solid would dissolve was taken to be the point of solid-liquid equilibrium. The paper did not specify how the disappearance of the solid was monitored; however, the papers that the authors referenced for the experimental method used a laser monitoring method.

\section{Source and Purity of Chemicals:}

(1) $99.5 \%$, Chemical source and purification method were not given. (2) $99.8 \%$, Analytical Reagent Grade, Tianjin Kewei Company, China, no information given regarding any further purification.

\section{Estimated Error:}

Temperature: $\pm 0.2 \mathrm{~K}$.

$x_{1}$ : within 0.0200 (relative uncertainty). 


\section{Components: \\ (1) Acenaphthene; $\mathrm{C}_{12} \mathrm{H}_{10} ;$ [83-32-9] \\ (2) 1-Butanol; $\mathrm{C}_{4} \mathrm{H}_{10} \mathrm{O} ;[71-36-3]$}

\section{Variables:}

$T / \mathrm{K}=298.15$
Original Measurements:

${ }^{9}$ K. M. De Fina, T. L. Sharp, and W. E. Acree, Jr., Can. J. Chem. 77, 1537 (1999).

Prepared by:

W. E. Acree, Jr.
Experimental Values

\begin{tabular}{lcc}
\hline \hline$x_{2}{ }^{(\mathrm{s}) \mathrm{a}}$ & $x_{2}{ }^{\mathrm{b}}$ & $x_{1}{ }^{\mathrm{c}}$ \\
\hline 1.0000 & 0.9763 & 0.02373
\end{tabular}

${ }^{\mathrm{a}} x_{2}{ }^{(\mathrm{s})}$ : initial mole fraction of component 2 in the solution.

${ }^{b} x_{2}$ : mole fraction of component 2 in the saturated solution.

${ }^{c} x_{1}$ : mole fraction solubility of the solute.

\section{Auxiliary Information}

Method/Apparatus/Procedure:
Constant-temperature bath, calorimetric thermometer, and an ultraviolet/
visible spectrophotometer.
Excess solute and solvent were placed in amber glass bottles and allowed to
equilibrate for several days at constant temperature. Attainment of equilibrium
was verified by several repetitive measurements and by approaching
equilibrium from supersaturation. Aliquots of saturated solutions were
transferred through a coarse filter into tared volumetric flasks, weighed and
diluted with methanol. Concentrations were determined by
spectrophotometric measurements at 289 nm.
Source and Purity of Chemicals:
(1) $98 \%$, Aldrich Chemical Company, Milwaukee, WI, USA, was
recrystallized several times from methanol.
(2) $99.8+\%$, HPLC Grade, Aldrich Chemical Company, stored over molecular
sieves before use.
Estimated Error:
Temperature: $\pm 0.1 \mathrm{~K}$.
$x_{1}: \pm 1.5 \%$ (relative error).

\begin{tabular}{ll}
\hline \hline Components: & Original Measurements: \\
(1) Acenaphthene; $\mathrm{C}_{12} \mathrm{H}_{10} ;[83-32-9]$ & ${ }^{14} \mathrm{~F}$. He and P. Liu, J. Chem. \\
(2) 1-Butanol; $\mathrm{C}_{4} \mathrm{H}_{10} \mathrm{O} ;[71-36-3]$ & Eng. Data 52, 2536 (2007). \\
\hline Variables: & Prepared by: \\
Temperature & W. E. Acree, Jr. \\
\hline
\end{tabular}

Experimental Values

\begin{tabular}{lcc}
\hline \hline$T / \mathrm{K}$ & $x_{2}{ }^{\mathrm{a}}$ & $x_{1}{ }^{\mathrm{b}}$ \\
\hline 278.25 & 0.9899 & 0.010098 \\
283.05 & 0.9878 & 0.012221 \\
288.05 & 0.9846 & 0.015448 \\
293.25 & 0.9810 & 0.018975 \\
298.25 & 0.9763 & 0.023672 \\
303.15 & 0.9710 & 0.028995 \\
308.10 & 0.9651 & 0.034855
\end{tabular}

\begin{tabular}{lcc}
\hline \hline$T / \mathrm{K}$ & $x_{2}{ }^{\mathrm{a}}$ & $x_{1}{ }^{\mathrm{b}}$ \\
\hline 313.20 & 0.9573 & 0.042705 \\
318.05 & 0.9475 & 0.052472 \\
323.05 & 0.9361 & 0.063929 \\
\hline
\end{tabular}

${ }^{a_{2}}$ : mole fraction of component 2 in the saturated solution.

${ }^{\mathrm{b}} x_{1}$ : mole fraction of the polycyclic aromatic hydrocarbon (component 1 ).

The authors refer to the compound as 1,8-dihydroacenaphthalene, which the compiler has taken to be acenaphthene.

\section{Auxiliary Information}

\section{Method/Apparatus/Procedure:}

Thermostated bath, thermometer.

Very little experimental details were provided. Solubilities were determined by a synthetic method. Known masses of solute and solvent were placed in a dissolving flask. The solute and solvent mixture was stirred with a magnetic stirrer. Small amounts of solute were added at predetermined intervals until no more solid would dissolve. The point at which no more solid would dissolve was taken to be the point of solid-liquid equilibrium. The paper did not specify how the disappearance of the solid was monitored; however, the papers that the authors referenced for the experimental method used a laser monitoring method.

\section{Source and Purity of Chemicals:}

(1) $99.5 \%$, Chemical source and purification method were not given.

(2) $99.8 \%$, Analytical Reagent Grade, Tianjin Kewei Company, China, no information given regarding any further purification.

\section{Estimated Error:}

Temperature: $\pm 0.2 \mathrm{~K}$.

$x_{1}$ : within 0.0200 (relative uncertainty).

\begin{tabular}{ll}
\hline \hline Components: & Original Measurements: \\
(1) Acenaphthene; $\mathrm{C}_{12} \mathrm{H}_{10} ;[83-32-9]$ & ${ }^{9}$ K. M. De Fina, T. L. Sharp, \\
(2) 2-Butanol; $\mathrm{C}_{4} \mathrm{H}_{10} \mathrm{O} ;[78-92-2]$ & and W. E. Acree, Jr., Can. J. \\
& Chem. 77, 1537 (1999). \\
\hline Variables: & Prepared by: \\
$T / \mathrm{K}=298.15$ & W. E. Acree, Jr. \\
\hline
\end{tabular}

Experimental Values

\begin{tabular}{lcc}
\hline \hline$x_{2}{ }^{(\mathrm{s}) \mathrm{a}}$ & $x_{2}{ }^{\mathrm{b}}$ & $x_{1}{ }^{\mathrm{c}}$ \\
\hline 1.0000 & 0.9812 & 0.01877 \\
$\frac{{ }^{\mathrm{a}} x_{2}{ }^{(\mathrm{s})}: \text { initial mole fraction of component } 2 \text { in the solution. }}{{ }^{\mathrm{b}} x_{2}: \text { mole fraction of component } 2 \text { in the saturated solution. }}$ \\
${ }^{\mathrm{c}} x_{1}:$ mole fraction solubility of the solute.
\end{tabular}




\section{Auxiliary Information}

\section{Method/Apparatus/Procedure:}

Constant-temperature bath, calorimetric thermometer, and an ultraviolet/ visible spectrophotometer.

Excess solute and solvent were placed in amber glass bottles and allowed to equilibrate for several days at constant temperature. Attainment of equilibrium was verified by several repetitive measurements and by approaching equilibrium from supersaturation. Aliquots of saturated solutions were transferred through a coarse filter into tared volumetric flasks, weighed and diluted with methanol. Concentrations were determined by spectrophotometric measurements at $289 \mathrm{~nm}$.

Source and Purity of Chemicals:

(1) $98 \%$, Aldrich Chemical Company, Milwaukee, WI, USA, was recrystallized several times from methanol.

(2) $99+\%$, Aldrich Chemical Company, stored over molecular sieves before use.

Estimated Error:

Temperature: $\pm 0.1 \mathrm{~K}$.

$x_{1}: \pm 1.5 \%$ (relative error)

\begin{tabular}{ll}
\hline \hline Components: & Original Measurements: \\
(1) Acenaphthene; $\mathrm{C}_{12} \mathrm{H}_{10} ;[83-32-9]$ & ${ }^{9}$ K. M. De Fina, T. L. Sharp, \\
(2) 2-Methyl-1-propanol; $\mathrm{C}_{4} \mathrm{H}_{10} \mathrm{O} ;$ & and W. E. Acree, Jr., Can. J. \\
[78-83-1] & Chem. 77, 1537 (1999). \\
\hline Variables: & Prepared by: \\
$T / \mathrm{K}=298.15$ & W. E. Acree, Jr. \\
\hline
\end{tabular}

Experimental Values

\begin{tabular}{lcc}
\hline \hline$x_{2}{ }^{(\mathrm{s}) \mathrm{a}}$ & $x_{2}{ }^{\mathrm{b}}$ & $x_{1}{ }^{\mathrm{c}}$ \\
\hline 1.0000 & 0.9831 & 0.01691 \\
$\frac{{ }^{\mathrm{a}} x_{2}{ }^{(\mathrm{s})}: \text { initial mole fraction of component 2 in the solution. }}{{ }^{\mathrm{b}} x_{2}: \text { mole fraction of component } 2 \text { in the saturated solution. }}$ \\
${ }^{\mathrm{c}} x_{1}:$ mole fraction solubility of the solute.
\end{tabular}

\section{Auxiliary Information}

\section{Method/Apparatus/Procedure:}

Constant-temperature bath, calorimetric thermometer, and an ultraviolet/ visible spectrophotometer.

Excess solute and solvent were placed in amber glass bottles and allowed to equilibrate for several days at constant temperature. Attainment of equilibrium was verified by several repetitive measurements and by approaching equilibrium from supersaturation. Aliquots of saturated solutions were transferred through a coarse filter into tared volumetric flasks, weighed and diluted with methanol. Concentrations were determined by

spectrophotometric measurements at $289 \mathrm{~nm}$.

Source and Purity of Chemicals:

(1) $98 \%$, Aldrich Chemical Company, Milwaukee, WI, USA, was recrystallized several times from methanol.

(2) $99+\%$, anhydrous, Aldrich Chemical Company, stored over molecular sieves before use.

\section{Estimated Error:}

Temperature: $\pm 0.1 \mathrm{~K}$

$x_{1}: \pm 1.5 \%$ (relative error).

\begin{tabular}{ll}
\hline \hline Components: & Original Measurements: \\
(1) Acenaphthene; $\mathrm{C}_{12} \mathrm{H}_{10} ;[83-32-9]$ & ${ }^{9}$ K. M. De Fina, T. L. Sharp, \\
(2) 2-Methyl-2-propanol; $\mathrm{C}_{4} \mathrm{H}_{10} \mathrm{O} ;$ & and W. E. Acree, Jr., Can. J. \\
[75-65-0] & Chem. 77, 1537 (1999). \\
\hline Variables: & Prepared by: \\
$T / \mathrm{K}=298.15$ & W. E. Acree, Jr. \\
\hline
\end{tabular}

Experimental Values

\begin{tabular}{|c|c|c|}
\hline$x_{2}{ }^{(\mathrm{s}) \mathrm{a}}$ & $x_{2}{ }^{\mathrm{b}}$ & $x_{1}{ }^{c}$ \\
\hline 1.0000 & 0.9830 & 0.01705 \\
\hline
\end{tabular}

\section{Auxiliary Information}

\begin{abstract}
Method/Apparatus/Procedure:
Constant-temperature bath, calorimetric thermometer, and an ultraviolet/ visible spectrophotometer.

Excess solute and solvent were placed in amber glass bottles and allowed to equilibrate for several days at constant temperature. Attainment of equilibrium was verified by several repetitive measurements and by approaching equilibrium from supersaturation. Aliquots of saturated solutions were transferred through a coarse filter into tared volumetric flasks, weighed and diluted with methanol. Concentrations were determined by spectrophotometric measurements at $289 \mathrm{~nm}$.
\end{abstract}

\section{Source and Purity of Chemicals:}

(1) $98 \%$, Aldrich Chemical Company, Milwaukee, WI, USA, was recrystallized several times from methanol.

(2) $99+\%$, Arco Chemical Company, USA, stored over molecular sieves before use.

\section{Estimated Error:}

Temperature: $\pm 0.1 \mathrm{~K}$.

$x_{1}: \pm 1.5 \%$ (relative error).

\begin{tabular}{ll}
\hline \hline Components: & Original Measurements: \\
(1) Acenaphthene; $\mathrm{C}_{12} \mathrm{H}_{10} ;[83-32-9]$ & ${ }^{9}$ K. M. De Fina, T. L. Sharp, \\
(2) 1-Pentanol; $\mathrm{C}_{5} \mathrm{H}_{12} \mathrm{O} ;[71-41-0]$ & and W. E. Acree, Jr., Can. J. \\
& Chem. 77, 1537 (1999). \\
\hline Variables: & Prepared by: \\
$T / \mathrm{K}=298.15$ & W. E. Acree, Jr. \\
\hline
\end{tabular}

Experimental Values

\begin{tabular}{lcc}
\hline \hline$x_{2}{ }^{(\mathrm{s}) \mathrm{a}}$ & $x_{2}{ }^{\mathrm{b}}$ & $x_{1}{ }^{\mathrm{c}}$ \\
\hline 1.0000 & 0.9682 & 0.03176 \\
${ }^{\mathrm{a}} x_{2}{ }^{(\mathrm{s})}$ : initial mole fraction of component 2 in the solution. \\
${ }^{\mathrm{b}} x_{2}$ : mole fraction of component 2 in the saturated solution. \\
${ }^{\mathrm{c}} x_{1}$ : mole fraction solubility of the solute.
\end{tabular}




\section{Auxiliary Information}

\section{Method/Apparatus/Procedure:}

Constant-temperature bath, calorimetric thermometer, and an ultraviolet/ visible spectrophotometer.

Excess solute and solvent were placed in amber glass bottles and allowed to equilibrate for several days at constant temperature. Attainment of equilibrium was verified by several repetitive measurements and by approaching equilibrium from supersaturation. Aliquots of saturated solutions were transferred through a coarse filter into tared volumetric flasks, weighed and diluted with methanol. Concentrations were determined by spectrophotometric measurements at $289 \mathrm{~nm}$.

Source and Purity of Chemicals:

(1) $98 \%$, Aldrich Chemical Company, Milwaukee, WI, USA, was recrystallized several times from methanol.

(2) $99+\%$, Aldrich Chemical Company, stored over molecular sieves before use.

Estimated Error:

Temperature: $\pm 0.1 \mathrm{~K}$.

$x_{1}: \pm 1.5 \%$ (relative error).

\begin{tabular}{|c|c|}
\hline $\begin{array}{l}\text { Components: } \\
\text { (1) Acenaphthene; } \mathrm{C}_{12} \mathrm{H}_{10} ;[83-32-9] \\
\text { (2) 2-Pentanol; } \mathrm{C}_{5} \mathrm{H}_{12} \mathrm{O} ;[6032-29-7]\end{array}$ & $\begin{array}{l}\text { Original Measurements: } \\
{ }^{9} \text { K. M. De Fina, T. L. Sharp, } \\
\text { and W. E. Acree, Jr., Can. J. } \\
\text { Chem. 77, } 1537 \text { (1999). }\end{array}$ \\
\hline $\begin{array}{l}\text { Variables: } \\
T / \mathrm{K}=298.15\end{array}$ & $\begin{array}{l}\text { Prepared by: } \\
\text { W. E. Acree, Jr. }\end{array}$ \\
\hline
\end{tabular}

Experimental Values

\begin{tabular}{|c|c|c|}
\hline$x_{2}{ }^{(\mathrm{s}) \mathrm{a}}$ & $x_{2}{ }^{b}$ & $x_{1}{ }^{\mathrm{c}}$ \\
\hline 1.0000 & 0.9756 & 0.02443 \\
\hline
\end{tabular}

\section{Auxiliary Information}

\section{Method/Apparatus/Procedure:}

Constant-temperature bath, calorimetric thermometer, and an ultraviolet/ visible spectrophotometer.

Excess solute and solvent were placed in amber glass bottles and allowed to equilibrate for several days at constant temperature. Attainment of equilibrium was verified by several repetitive measurements and by approaching equilibrium from supersaturation. Aliquots of saturated solutions were transferred through a coarse filter into tared volumetric flasks, weighed and diluted with methanol. Concentrations were determined by

spectrophotometric measurements at $289 \mathrm{~nm}$.

\section{Source and Purity of Chemicals:}

(1) 98\%, Aldrich Chemical Company, Milwaukee, WI, USA, was recrystallized several times from methanol.

(2) 99+\%, Acros Organics, USA, stored over molecular sieves before use.

Estimated Error:

Temperature: $\pm 0.1 \mathrm{~K}$.

$x_{1}: \pm 1.5 \%$ (relative error).

\begin{tabular}{ll}
\hline \hline Components: & Original Measurements: \\
(1) Acenaphthene; $\mathrm{C}_{12} \mathrm{H}_{10} ;[83-32-9]$ & ${ }^{9}$ K. M. De Fina, T. L. Sharp, \\
(2) 3-Methyl-1-butanol; $\mathrm{C}_{5} \mathrm{H}_{12} \mathrm{O} ;$ & and W. E. Acree, Jr., Can. J. \\
[123-51-3] & Chem. 77, 1537 (1999). \\
\hline Variables: & Prepared by: \\
$T / \mathrm{K}=298.15$ & W. E. Acree, Jr. \\
\hline
\end{tabular}

Experimental Values

\begin{tabular}{|c|c|c|}
\hline$x_{2}{ }^{(\mathrm{s}) \mathrm{a}}$ & $x_{2}{ }^{\mathrm{b}}$ & $x_{1}{ }^{\mathrm{c}}$ \\
\hline 1.0000 & 0.9765 & 0.02347 \\
\hline
\end{tabular}

\section{Auxiliary Information}

\begin{abstract}
Method/Apparatus/Procedure:
Constant-temperature bath, calorimetric thermometer, and an ultraviolet/ visible spectrophotometer.

Excess solute and solvent were placed in amber glass bottles and allowed to equilibrate for several days at constant temperature. Attainment of equilibrium was verified by several repetitive measurements and by approaching equilibrium from supersaturation. Aliquots of saturated solutions were transferred through a coarse filter into tared volumetric flasks, weighed and diluted with methanol. Concentrations were determined by
\end{abstract} spectrophotometric measurements at $289 \mathrm{~nm}$.

\section{Source and Purity of Chemicals:}

(1) $98 \%$, Aldrich Chemical Company, Milwaukee, WI, USA, was recrystallized several times from methanol.

(2) $99+\%$, anhydrous, Aldrich Chemical Company, stored over molecular sieves before use.

\section{Estimated Error:}

Temperature: $\pm 0.1 \mathrm{~K}$.

$x_{1}: \pm 1.5 \%$ (relative error).

\begin{tabular}{ll}
\hline \hline Components: & Original Measurements: \\
(1) Acenaphthene; $\mathrm{C}_{12} \mathrm{H}_{10} ;[83-32-9]$ & ${ }^{9}$ K. M. De Fina, T. L. Sharp, \\
(2) 2-Methyl-2-butanol; $\mathrm{C}_{5} \mathrm{H}_{12} \mathrm{O} ;$ & and W. E. Acree, Jr., Can. J. \\
[75-85-4] & Chem. 77, 1537 (1999). \\
\hline Variables: & Prepared by: \\
$T / \mathrm{K}=298.15$ & W. E. Acree, Jr. \\
\hline
\end{tabular}

Experimental Values

\begin{tabular}{lcc}
\hline \hline$x_{2}{ }^{(\mathrm{s}) \mathrm{a}}$ & \multicolumn{1}{c}{$x_{2}^{\mathrm{b}}$} & $x_{1}{ }^{\mathrm{c}}$ \\
\hline 1.0000 & 0.9713 & 0.02867 \\
$\frac{{ }^{\mathrm{a}} x_{2}{ }^{(\mathrm{s})}: \text { initial mole fraction of component } 2 \text { in the solution. }}{{ }^{\mathrm{b}} x_{2}: \text { mole fraction of component } 2 \text { in the saturated solution. }}$ \\
${ }^{{ }^{c} x_{1}: \text { mole fraction solubility of the solute. }}$
\end{tabular}




\section{Auxiliary Information}

\section{Method/Apparatus/Procedure:}

Constant-temperature bath, calorimetric thermometer, and an ultraviolet/ visible spectrophotometer.

Excess solute and solvent were placed in amber glass bottles and allowed to equilibrate for several days at constant temperature. Attainment of equilibrium was verified by several repetitive measurements and by approaching equilibrium from supersaturation. Aliquots of saturated solutions were transferred through a coarse filter into tared volumetric flasks, weighed and diluted with methanol. Concentrations were determined by spectrophotometric measurements at $289 \mathrm{~nm}$.

Source and Purity of Chemicals:

(1) $98 \%$, Aldrich Chemical Company, Milwaukee, WI, USA, was recrystallized several times from methanol.

(2) $99+\%$, Acros Organics, USA, stored over molecular sieves before use.

Estimated Error:

Temperature: $\pm 0.1 \mathrm{~K}$.

$x_{1}: \pm 1.5 \%$ (relative error)

\begin{tabular}{ll}
\hline \hline Components: & Original Measurements: \\
(1) Acenaphthene; $\mathrm{C}_{12} \mathrm{H}_{10} ;[83-32-9]$ & ${ }^{9}$ K. M. De Fina, T. L. Sharp, \\
(2) 1-Hexanol; $\mathrm{C}_{6} \mathrm{H}_{14} \mathrm{O} ;[111-27-3]$ & and W. E. Acree, Jr., Can. J. \\
& Chem. 77, 1537 (1999). \\
\hline Variables: & Prepared by: \\
$T / \mathrm{K}=298.15$ & W. E. Acree, Jr. \\
\hline
\end{tabular}

Experimental Values

\begin{tabular}{lcc}
\hline$\frac{x_{2}{ }^{(\mathrm{s}) \mathrm{a}}}{x_{2}{ }^{\mathrm{b}}}$ & $x_{1}{ }^{\mathrm{c}}$ \\
\hline 1.0000 & 0.9608 & 0.03922 \\
$\frac{{ }^{\mathrm{a}} x_{2}{ }^{(\mathrm{s})}: \text { initial mole fraction of component } 2 \text { in the solution. }}{{ }^{\mathrm{b}}{ }_{x_{2}}: \text { mole fraction of component } 2 \text { in the saturated solution. }}$ \\
${ }^{\mathrm{c}} x_{1}:$ mole fraction solubility of the solute.
\end{tabular}

\section{Auxiliary Information}

\section{Method/Apparatus/Procedure:}

Constant-temperature bath, calorimetric thermometer, and an ultraviolet/ visible spectrophotometer.

Excess solute and solvent were placed in amber glass bottles and allowed to equilibrate for several days at constant temperature. Attainment of equilibrium was verified by several repetitive measurements and by approaching equilibrium from supersaturation. Aliquots of saturated solutions were transferred through a coarse filter into tared volumetric flasks, weighed and diluted with methanol. Concentrations were determined by spectrophotometric measurements at $289 \mathrm{~nm}$.

\section{Source and Purity of Chemicals:}

(1) $98 \%$, Aldrich Chemical Company, Milwaukee, WI, USA, was recrystallized several times from methanol.

(2) $99+\%$, Alfa Aesar, USA, stored over molecular sieves before use.

\section{Estimated Error:}

Temperature: $\pm 0.1 \mathrm{~K}$

$x_{1}: \pm 1.5 \%$ (relative error).

\begin{tabular}{ll}
\hline \hline Components: & Original Measurements: \\
(1) Acenaphthene; $\mathrm{C}_{12} \mathrm{H}_{10} ;[83-32-9]$ & ${ }^{9}$ K. M. De Fina, T. L. Sharp, \\
(2) 2-Methyl-1-pentanol; $\mathrm{C}_{6} \mathrm{H}_{14} \mathrm{O} ;$ & and W. E. Acree, Jr., Can. J. \\
{$[105-30-6]$} & Chem. 77, 1537 (1999). \\
\hline Variables: & Prepared by: \\
$T / \mathrm{K}=298.15$ & W. E. Acree, Jr. \\
\hline
\end{tabular}

Experimental Values

\begin{tabular}{|c|c|c|}
\hline$x_{2}{ }^{(s) a}$ & $x_{2}{ }^{b}$ & $x_{1}{ }^{\mathrm{c}}$ \\
\hline 1.0000 & 0.9710 & 0.02904 \\
\hline
\end{tabular}

\section{Auxiliary Information}

\section{Method/Apparatus/Procedure:}

Constant-temperature bath, calorimetric thermometer, and an ultraviolet/ visible spectrophotometer.

Excess solute and solvent were placed in amber glass bottles and allowed to equilibrate for several days at constant temperature. Attainment of equilibrium was verified by several repetitive measurements and by approaching equilibrium from supersaturation. Aliquots of saturated solutions were transferred through a coarse filter into tared volumetric flasks, weighed and diluted with methanol. Concentrations were determined by spectrophotometric measurements at $289 \mathrm{~nm}$.

Source and Purity of Chemicals:

(1) $98 \%$, Aldrich Chemical Company, Milwaukee, WI, USA, was recrystallized several times from methanol.

(2) $99 \%$, Aldrich Chemical Company, stored over molecular sieves before use.

Estimated Error:

Temperature: $\pm 0.1 \mathrm{~K}$.

$x_{1}: \pm 1.5 \%$ (relative error).

\begin{tabular}{ll}
\hline \hline Components: & Original Measurements: \\
(1) Acenaphthene; $\mathrm{C}_{12} \mathrm{H}_{10} ;[83-32-9]$ & ${ }^{9}$ K. M. De Fina, T. L. Sharp, \\
(2) 4-Methyl-2-pentanol; $\mathrm{C}_{6} \mathrm{H}_{14} \mathrm{O} ;$ & and W. E. Acree, Jr., Can. J. \\
{$[108-11-2]$} & Chem. 77, 1537 (1999). \\
\hline Variables: & Prepared by: \\
$T / \mathrm{K}=298.15$ & W. E. Acree, Jr. \\
\hline
\end{tabular}

Experimental Values

\begin{tabular}{lcc}
\hline \hline$x_{2}{ }^{(\mathrm{s}) \mathrm{a}}$ & $x_{2}{ }^{\mathrm{b}}$ & $x_{1}{ }^{\mathrm{c}}$ \\
\hline 1.0000 & 0.9745 & 0.02551 \\
${ }^{{ }^{a} x_{2}{ }^{(\mathrm{s})}: \text { initial mole fraction of component } 2 \text { in the solution. }}$ \\
${ }^{\mathrm{b}} x_{2}:$ mole fraction of component 2 in the saturated solution. \\
${ }^{\mathrm{c}} x_{1}:$ mole fraction solubility of the solute.
\end{tabular}




\section{Auxiliary Information}

\section{Method/Apparatus/Procedure:}

Constant-temperature bath, calorimetric thermometer, and an ultraviolet/ visible spectrophotometer.

Excess solute and solvent were placed in amber glass bottles and allowed to equilibrate for several days at constant temperature. Attainment of equilibrium was verified by several repetitive measurements and by approaching equilibrium from supersaturation. Aliquots of saturated solutions were transferred through a coarse filter into tared volumetric flasks, weighed and diluted with methanol. Concentrations were determined by spectrophotometric measurements at $289 \mathrm{~nm}$.

Source and Purity of Chemicals:

(1) $98 \%$, Aldrich Chemical Company, Milwaukee, WI, USA, was recrystallized several times from methanol.

(2) $99+\%$, Acros Organics, USA, stored over molecular sieves before use.

\section{Estimated Error:}

Temperature: $\pm 0.1 \mathrm{~K}$.

$x_{1}: \pm 1.5 \%$ (relative error).

\begin{tabular}{|c|c|}
\hline $\begin{array}{l}\text { Components: } \\
\text { (1) Acenaphthene; } \mathrm{C}_{12} \mathrm{H}_{10} ;[83-32-9] \\
\text { (2) 1-Heptanol; } \mathrm{C}_{7} \mathrm{H}_{16} \mathrm{O} ;[111-70-6]\end{array}$ & $\begin{array}{l}\text { Original Measurements: } \\
{ }^{9} \text { K. M. De Fina, T. L. Sharp, } \\
\text { and W. E. Acree, Jr., Can. J. } \\
\text { Chem. 77, } 1537 \text { (1999). }\end{array}$ \\
\hline $\begin{array}{l}\text { Variables: } \\
T / \mathrm{K}=298.15\end{array}$ & $\begin{array}{l}\text { Prepared by: } \\
\text { W. E. Acree, Jr. }\end{array}$ \\
\hline
\end{tabular}

Experimental Values

\begin{tabular}{lcc}
\hline \hline$x_{2}{ }^{(\mathrm{s}) \mathrm{a}}$ & $x_{2}{ }^{\mathrm{b}}$ & $x_{1}{ }^{\mathrm{c}}$ \\
\hline 1.0000 & 0.9538 & 0.04617 \\
${ }^{{ }^{\mathrm{a}} x_{2}{ }^{(\mathrm{s})}: \text { initial mole fraction of component } 2 \text { in the solution. }}$ \\
${ }^{\mathrm{b}} x_{2}:$ mole fraction of component 2 in the saturated solution. \\
${ }^{\mathrm{c}} x_{1}:$ mole fraction solubility of the solute.
\end{tabular}

\section{Auxiliary Information}

\section{Method/Apparatus/Procedure:}

Constant-temperature bath, calorimetric thermometer, and an ultraviolet/ visible spectrophotometer.

Excess solute and solvent were placed in amber glass bottles and allowed to equilibrate for several days at constant temperature. Attainment of equilibrium was verified by several repetitive measurements and by approaching equilibrium from supersaturation. Aliquots of saturated solutions were transferred through a coarse filter into tared volumetric flasks, weighed and diluted with methanol. Concentrations were determined by spectrophotometric measurements at $289 \mathrm{~nm}$.

Source and Purity of Chemicals:

(1) $98 \%$, Aldrich Chemical Company, Milwaukee, WI, USA, was recrystallized several times from methanol.

(2) 99+\%, Alfa Aesar, USA, stored over molecular sieves before use.

\section{Estimated Error:}

Temperature: $\pm 0.1 \mathrm{~K}$.

$x_{1}: \pm 1.5 \%$ (relative error).

\begin{tabular}{ll}
\hline \hline Components: & Original Measurements: \\
(1) Acenaphthene; $\mathrm{C}_{12} \mathrm{H}_{10} ;[83-32-9]$ & ${ }^{9}$ K. M. De Fina, T. L. Sharp, \\
(2) 1-Octanol; $\mathrm{C}_{8} \mathrm{H}_{18} \mathrm{O} ;[111-87-5]$ & and W. E. Acree, Jr., Can. J. \\
& Chem. 77, 1537 (1999). \\
\hline Variables: & Prepared by: \\
$T / \mathrm{K}=298.15$ & W. E. Acree, Jr. \\
\hline
\end{tabular}

Experimental Values

\begin{tabular}{lcc}
\hline \hline$x_{2}{ }^{(\mathrm{s}) \mathrm{a}}$ & $x_{2}{ }^{\mathrm{b}}$ & $x_{1}{ }^{\mathrm{c}}$ \\
\hline 1.0000 & 0.9491 & 0.05089 \\
${ }^{\mathrm{a}} x_{2}{ }^{(\mathrm{s})}$ : initial mole fraction of component 2 in the solution. \\
${ }^{\mathrm{b}} x_{2}:$ mole fraction of component 2 in the saturated solution. \\
${ }^{\mathrm{c}} x_{1}$ : mole fraction solubility of the solute.
\end{tabular}

\section{Auxiliary Information}

\begin{abstract}
Method/Apparatus/Procedure:
Constant-temperature bath, calorimetric thermometer, and an ultraviolet/ visible spectrophotometer.

Excess solute and solvent were placed in amber glass bottles and allowed to equilibrate for several days at constant temperature. Attainment of equilibrium was verified by several repetitive measurements and by approaching equilibrium from supersaturation. Aliquots of saturated solutions were transferred through a coarse filter into tared volumetric flasks, weighed and diluted with methanol. Concentrations were determined by spectrophotometric measurements at $289 \mathrm{~nm}$.
\end{abstract}

\section{Source and Purity of Chemicals:}

(1) $98 \%$, Aldrich Chemical Company, Milwaukee, WI, USA, was recrystallized several times from methanol.

(2) $99+\%$, Aldrich Chemical Company, stored over molecular sieves before use.

\section{Estimated Error:}

Temperature: $\pm 0.1 \mathrm{~K}$.

$x_{1}: \pm 1.5 \%$ (relative error).

\begin{tabular}{ll}
\hline \hline Components: & Original Measurements: \\
(1) Acenaphthene; $\mathrm{C}_{12} \mathrm{H}_{10} ;[83-32-9]$ & ${ }^{9}$ K. M. De Fina, T. L. Sharp, \\
(2) 2-Ethyl-1-hexanol; $\mathrm{C}_{8} \mathrm{H}_{18} \mathrm{O} ;$ & and W. E. Acree, Jr., Can. J. \\
{$[104-76-7]$} & Chem. 77, 1537 (1999). \\
\hline Variables: & Prepared by: \\
$T / \mathrm{K}=298.15$ & W. E. Acree, Jr. \\
\hline
\end{tabular}

Experimental Values

\begin{tabular}{lcc}
\hline \hline$\frac{x_{2}{ }^{(\mathrm{s}) \mathrm{a}}}{x_{2}{ }^{\mathrm{b}}}$ & $x_{1}{ }^{\mathrm{c}}$ \\
\hline 1.0000 & 0.9560 & 0.04402 \\
$\frac{{ }^{\mathrm{a}} x_{2}{ }^{(\mathrm{s})}: \text { initial mole fraction of component } 2 \text { in the solution. }}{{ }^{\mathrm{b}} x_{2}: \text { mole fraction of component } 2 \text { in the saturated solution. }}$ \\
${ }^{\mathrm{c}} x_{1}:$ mole fraction solubility of the solute.
\end{tabular}




\section{Auxiliary Information}

\section{Method/Apparatus/Procedure:}

Constant-temperature bath, calorimetric thermometer, and an ultraviolet/ visible spectrophotometer.

Excess solute and solvent were placed in amber glass bottles and allowed to equilibrate for several days at constant temperature. Attainment of equilibrium was verified by several repetitive measurements and by approaching equilibrium from supersaturation. Aliquots of saturated solutions were transferred through a coarse filter into tared volumetric flasks, weighed and diluted with methanol. Concentrations were determined by spectrophotometric measurements at $289 \mathrm{~nm}$.

Source and Purity of Chemicals:

(1) $98 \%$, Aldrich Chemical Company, Milwaukee, WI, USA, was recrystallized several times from methanol.

(2) $99+\%$, Aldrich Chemical Company, stored over molecular sieves before use.

Estimated Error:

Temperature: $\pm 0.1 \mathrm{~K}$.

$x_{1}: \pm 1.5 \%$ (relative error)

\begin{tabular}{|c|c|}
\hline $\begin{array}{l}\text { Components: } \\
\text { (1) Acenaphthene; } \mathrm{C}_{12} \mathrm{H}_{10} ;[83-32-9] \\
\text { (2) 1-Decanol; } \mathrm{C}_{10} \mathrm{H}_{22} \mathrm{O} ;[112-30-1]\end{array}$ & $\begin{array}{l}\text { Original Measurements: } \\
\text { W. E. Acree, Jr., unpublished data }\end{array}$ \\
\hline $\begin{array}{l}\text { Variables: } \\
T / \mathrm{K}=298.15\end{array}$ & $\begin{array}{l}\text { Prepared by: } \\
\text { W. E. Acree, Jr. }\end{array}$ \\
\hline
\end{tabular}

Experimental Values

\begin{tabular}{lcc}
\hline \hline$x_{2}{ }^{(\mathrm{s}) \mathrm{a}}$ & $x_{2}{ }^{\mathrm{b}}$ & $x_{1}^{\mathrm{c}}$ \\
\hline 1.0000 & 0.9332 & 0.06677 \\
${ }^{\mathrm{a}} x_{2}{ }^{(\mathrm{s})}:$ initial mole fraction of component 2 in the solution. \\
${ }^{\mathrm{b}} x_{2}:$ mole fraction of component 2 in the saturated solution. \\
${ }^{\mathrm{c}}{ }_{x_{1}: \text { mole fraction solubility of the solute. }}$
\end{tabular}

\section{Auxiliary Information}

\section{Method/Apparatus/Procedure:}

Constant-temperature bath, calorimetric thermometer, and an ultraviolet/ visible spectrophotometer.

Excess solute and solvent were placed in amber glass bottles and allowed to equilibrate for several days at constant temperature. Attainment of equilibrium was verified by several repetitive measurements and by approaching equilibrium from supersaturation. Aliquots of saturated solutions were transferred through a coarse filter into tared volumetric flasks, weighed and diluted with methanol. Concentrations were determined by spectrophotometric measurements at $289 \mathrm{~nm}$.

\section{Source and Purity of Chemicals:}

(1) $98 \%$, Aldrich Chemical Company, Milwaukee, WI, USA, was recrystallized several times from methanol.

(2) $99+\%$, Alfa Aesar, USA, stored over molecular sieves before use.

\section{Estimated Error:}

Temperature: $\pm 0.1 \mathrm{~K}$

$x_{1}: \pm 1.5 \%$ (relative error).

\begin{tabular}{ll}
\hline \hline Components: & Original Measurements: \\
(1) Acenaphthene; $\mathrm{C}_{12} \mathrm{H}_{10} ;[83-32-9]$ & ${ }^{10}$ W. E. Acree, Jr. and M. H. \\
(2) 3,7-Dimethyl-1-octanol; $\mathrm{C}_{10} \mathrm{H}_{22} \mathrm{O} ;$ & Abraham, Fluid Phase \\
{$[106-21-8]$} & Equilib. 201, 245 (2002). \\
\hline Variables: & Prepared by: \\
$T / \mathrm{K}=298.15$ & W. E. Acree, Jr. \\
\hline
\end{tabular}

Experimental Values

\begin{tabular}{|c|c|c|}
\hline$x_{2}{ }^{(\mathrm{s}) \mathrm{a}}$ & $x_{2}{ }^{\mathrm{b}}$ & $x_{1}{ }^{c}$ \\
\hline 1.0000 & 0.9459 & 0.05410 \\
\hline
\end{tabular}

\section{Auxiliary Information}

\begin{abstract}
Method/Apparatus/Procedure:
Constant-temperature bath, calorimetric thermometer, and an ultraviolet/ visible spectrophotometer.

Excess solute and solvent were placed in amber glass bottles and allowed to equilibrate for several days at constant temperature. Attainment of equilibrium was verified by several repetitive measurements and by approaching equilibrium from supersaturation. Aliquots of saturated solutions were transferred through a coarse filter into tared volumetric flasks, weighed and diluted with 2-propanol. Concentrations were determined by spectrophotometric measurements at $289 \mathrm{~nm}$.
\end{abstract}

\section{Source and Purity of Chemicals:}

(1) $98 \%$, Aldrich Chemical Company, Milwaukee, WI, USA, was recrystallized several times from methanol.

(2) $99 \%$, anhydrous, Aldrich Chemical Company, stored over molecular sieves before use.

Estimated Error:

Temperature: $\pm 0.1 \mathrm{~K}$.

$x_{1}: \pm 1.5 \%$ (relative error).

\begin{tabular}{ll}
\hline \hline Components: & Original Measurements: \\
(1) Acenaphthene; $\mathrm{C}_{12} \mathrm{H}_{10} ;[83-32-9]$ & ${ }^{9} \mathrm{~K}$. M. De Fina, T. L. Sharp, \\
(2) 1,2-Ethanediol; $\mathrm{C}_{2} \mathrm{H}_{6} \mathrm{O}_{2} ;[107-21-1]$ & and W. E. Acree, Jr., Can. J. \\
& Chem. 77, 1537 (1999). \\
\hline Variables: & Prepared by: \\
$T / \mathrm{K}=298.15$ & W. E. Acree, Jr. \\
\hline
\end{tabular}

Experimental Values

\begin{tabular}{lcc}
\hline \hline$x_{2}{ }^{(\mathrm{s}) \mathrm{a}}$ & $x_{2}{ }^{\mathrm{b}}$ & $x_{1}{ }^{\mathrm{c}}$ \\
\hline 1.0000 & 0.9988 & 0.001157 \\
$\frac{{ }^{\mathrm{a}} x_{2}{ }^{(\mathrm{s})}: \text { initial mole fraction of component } 2 \text { in the solution. }}{{ }^{\mathrm{b}} x_{2}: \text { mole fraction of component } 2 \text { in the saturated solution. }}$ \\
${ }^{\mathrm{c}}{ }_{x_{1}}$ : mole fraction solubility of the solute.
\end{tabular}




\section{Auxiliary Information}

\section{Method/Apparatus/Procedure:}

Constant-temperature bath, calorimetric thermometer, and an ultraviolet/ visible spectrophotometer.

Excess solute and solvent were placed in amber glass bottles and allowed to equilibrate for several days at constant temperature. Attainment of equilibrium was verified by several repetitive measurements and by approaching equilibrium from supersaturation. Aliquots of saturated solutions were transferred through a coarse filter into tared volumetric flasks, weighed and diluted with methanol. Concentrations were determined by spectrophotometric measurements at $289 \mathrm{~nm}$.

Source and Purity of Chemicals:

(1) $98 \%$, Aldrich Chemical Company, Milwaukee, WI, USA, was recrystallized several times from methanol.

(2) $99.8 \%$, anhydrous, Aldrich Chemical Company, stored over molecular sieves before use.

Estimated Error:

Temperature: $\pm 0.1 \mathrm{~K}$.

$x_{1}: \pm 1.5 \%$ (relative error).

\subsection{Acenaphthene solubility data in alkoxyalcohols}

\section{Components:}

(1) Acenaphthene; $\mathrm{C}_{12} \mathrm{H}_{10} ;[83-32-9]$

(2) 2-Ethoxyethanol; $\mathrm{C}_{4} \mathrm{H}_{10} \mathrm{O}_{2} ;[110-80-5]$

\begin{tabular}{ll} 
Variables: & Prepared by: \\
$T / \mathrm{K}=298.15$ & W. E. Acree, Jr. \\
\hline
\end{tabular}

Experimental Values

\begin{tabular}{lcc}
\hline \hline$x_{2}{ }^{(\mathrm{s}) \mathrm{a}}$ & $x_{2}^{\mathrm{b}}$ & $x_{1}^{\mathrm{c}}$ \\
\hline 1.0000 & 0.9544 & 0.04561 \\
\hline
\end{tabular}

${ }^{\mathrm{a}} x_{2}{ }^{(\mathrm{s})}$ : initial mole fraction of component 2 in the solution.

${ }^{\mathrm{b}} x_{2}$ : mole fraction of component 2 in the saturated solution.

${ }^{c} x_{1}$ : mole fraction solubility of the solute.

\section{Auxiliary Information}

\section{Method/Apparatus/Procedure:}

Constant-temperature bath, calorimetric thermometer, and an ultraviolet/ visible spectrophotometer.

Excess solute and solvent were placed in amber glass bottles and allowed to equilibrate for several days at constant temperature. Attainment of equilibrium was verified by several repetitive measurements and by approaching equilibrium from supersaturation. Aliquots of saturated solutions were transferred through a coarse filter into tared volumetric flasks, weighed and diluted with methanol. Concentrations were determined by spectrophotometric measurements at $289 \mathrm{~nm}$.

Source and Purity of Chemicals:

(1) $98 \%$, Aldrich Chemical Company, Milwaukee, WI, USA, was recrystallized several times from methanol.

(2) $99 \%$, Aldrich Chemical Company, stored over molecular sieves before use.

\section{Estimated Error:}

Temperature: $\pm 0.1 \mathrm{~K}$.

$x_{1}: \pm 1.5 \%$ (relative error).

\begin{tabular}{ll}
\hline \hline Components: & Original Measurements: \\
(1) Acenaphthene; $\mathrm{C}_{12} \mathrm{H}_{10} ;[83-32-9]$ & W. E. Acree, Jr., \\
(2) 2-Propoxyethanol; $\mathrm{C}_{5} \mathrm{H}_{12} \mathrm{O}_{2} ;[2807-30-9]$ & unpublished data. \\
\hline Variables: & Prepared by: \\
$T / \mathrm{K}=298.15$ & W. E. Acree, Jr. \\
\hline
\end{tabular}

Experimental Values

\begin{tabular}{lcc}
\hline \hline$x_{2}{ }^{(\mathrm{s}) \mathrm{a}}$ & $x_{2}{ }^{\mathrm{b}}$ & $x_{1}{ }^{\mathrm{c}}$ \\
\hline 1.0000 & 0.9392 & 0.06082 \\
$\frac{{ }^{\mathrm{a}} x_{2}{ }^{(\mathrm{s})}: \text { initial mole fraction of component } 2 \text { in the solution. }}{{ }^{\mathrm{b}} x_{2}: \text { mole fraction of component } 2 \text { in the saturated solution. }}$ \\
${ }^{\mathrm{c}} x_{1}:$ mole fraction solubility of the solute.
\end{tabular}

\section{Auxiliary Information}

\section{Method/Apparatus/Procedure:}

Constant-temperature bath, calorimetric thermometer, and an ultraviolet/ visible spectrophotometer.

Excess solute and solvent were placed in amber glass bottles and allowed to equilibrate for several days at constant temperature. Attainment of equilibrium was verified by several repetitive measurements and by approaching equilibrium from supersaturation. Aliquots of saturated solutions were transferred through a coarse filter into tared volumetric flasks, weighed and diluted with methanol. Concentrations were determined by spectrophotometric measurements at $289 \mathrm{~nm}$.

Source and Purity of Chemicals:

(1) $98 \%$, Aldrich Chemical Company, Milwaukee, WI, USA, was recrystallized several times from anhydrous methanol

(2) $99 \%$, Aldrich Chemical Company, stored over molecular sieves before use.

\section{Estimated Error:}

Temperature: $\pm 0.1 \mathrm{~K}$.

$x_{1}: \pm 1.5 \%$ (relative error)

\begin{tabular}{ll}
\hline \hline Components: & Original Measurements: \\
(1) Acenaphthene; $\mathrm{C}_{12} \mathrm{H}_{10} ;[83-32-9]$ & W. E. Acree, Jr., \\
(2) 2-Isopropoxyethanol; $\mathrm{C}_{5} \mathrm{H}_{12} \mathrm{O}_{2} ;[109-59-1]$ & unpublished data. \\
\hline Variables: & Prepared by: \\
$T / \mathrm{K}=298.15$ & W. E. Acree, Jr. \\
\hline
\end{tabular}

\section{Experimental Values}

\begin{tabular}{lcc}
\hline \hline$x_{2}{ }^{(\mathrm{s}) \mathrm{a}}$ & $x_{2}{ }^{\mathrm{b}}$ & $x_{1}{ }^{\mathrm{c}}$ \\
\hline 1.0000 & 0.9400 & 0.05996 \\
$\frac{{ }^{\mathrm{a}} x_{2}{ }^{(\mathrm{s})}: \text { initial mole fraction of component } 2 \text { in the solution. }}{}$ \\
${ }^{\mathrm{b}} x_{2}:$ mole fraction of component 2 in the saturated solution. \\
${ }^{c} x_{1}:$ mole fraction solubility of the solute.
\end{tabular}




\section{Auxiliary Information}

\section{Method/Apparatus/Procedure:}

Constant-temperature bath, calorimetric thermometer, and an ultraviolet/ visible spectrophotometer.

Excess solute and solvent were placed in amber glass bottles and allowed to equilibrate for several days at constant temperature. Attainment of equilibrium was verified by several repetitive measurements and by approaching equilibrium from supersaturation. Aliquots of saturated solutions were transferred through a coarse filter into tared volumetric flasks, weighed and diluted with methanol. Concentrations were determined by spectrophotometric measurements at $280 \mathrm{~nm}$.

Source and Purity of Chemicals:

(1) $98 \%$, Aldrich Chemical Company, Milwaukee, WI, USA, was recrystallized several times from anhydrous methanol.

(2) $99 \%$, Aldrich Chemical Company, stored over molecular sieves before use.

Estimated Error:

Temperature: $\pm 0.1 \mathrm{~K}$.

$x_{1}: \pm 1.5 \%$ (relative error)

\begin{tabular}{ll}
\hline \hline Components: & Original Measurements: \\
(1) Acenaphthene; $\mathrm{C}_{12} \mathrm{H}_{10} ;[83-32-9]$ & W. E. Acree, Jr., \\
(2) 2-Butoxyethanol; $\mathrm{C}_{6} \mathrm{H}_{14} \mathrm{O}_{2} ;[111-76-2]$ & unpublished data. \\
\hline Variables: & Prepared by: \\
$T / \mathrm{K}=298.15$ & W. E. Acree, Jr. \\
\hline
\end{tabular}

Experimental Values

\begin{tabular}{|c|c|c|}
\hline$x_{2}{ }^{(\mathrm{s}) a}$ & $x_{2}{ }^{\mathrm{b}}$ & $x_{1}{ }^{\mathrm{c}}$ \\
\hline 1.0000 & 0.9352 & 0.06484 \\
\hline
\end{tabular}

\section{Auxiliary Information}

\section{Method/Apparatus/Procedure:}

Constant-temperature bath, calorimetric thermometer, and an ultraviolet/ visible spectrophotometer.

Excess solute and solvent were placed in amber glass bottles and allowed to equilibrate for several days at constant temperature. Attainment of equilibrium was verified by several repetitive measurements and by approaching equilibrium from supersaturation. Aliquots of saturated solutions were transferred through a coarse filter into tared volumetric flasks, weighed and diluted with methanol. Concentrations were determined by spectrophotometric measurements at $289 \mathrm{~nm}$.

\section{Source and Purity of Chemicals:}

(1) $98 \%$, Aldrich Chemical Company, Milwaukee, WI, USA, was recrystallized several times from anhydrous methanol.

(2) $99+\%$, Acros Organics, USA, stored over anhydrous sodium sulfate and molecular sieves before use.

\section{Estimated Error:}

Temperature: $\pm 0.1 \mathrm{~K}$

$x_{1}: \pm 1.5 \%$ (relative error).

\subsection{Acenaphthene solubility data in ketones}

\begin{tabular}{ll}
\hline \hline Components: & Original Measurements: \\
(1) Acenaphthene; $\mathrm{C}_{12} \mathrm{H}_{10} ;[83-32-9]$ & ${ }^{9}$ K. M. De Fina, T. L. Sharp, \\
(2) 2-Butanone; $\mathrm{C}_{4} \mathrm{H}_{8} \mathrm{O} ;[78-93-3]$ & and W. E. Acree, Jr., Can. J. \\
& Chem. 77, 1537 (1999). \\
\hline Variables: & Prepared by: \\
$T / \mathrm{K}=298.15$ & W. E. Acree, Jr. \\
\hline
\end{tabular}

Experimental Values

\begin{tabular}{lcc}
\hline \hline$x_{2}{ }^{(\mathrm{s}) \mathrm{a}}$ & $x_{2}{ }^{\mathrm{b}}$ & $x_{1}{ }^{\mathrm{c}}$ \\
\hline 1.0000 & 0.8693 & 0.1307 \\
$\frac{{ }^{\mathrm{a}} x_{2}{ }^{(\mathrm{s})}: \text { initial mole fraction of component } 2 \text { in the solution. }}{{ }^{\mathrm{b}} x_{2}: \text { mole fraction of component } 2 \text { in the saturated solution. }}$ \\
${ }^{\mathrm{c}} x_{1}:$ mole fraction solubility of the solute.
\end{tabular}

\section{Auxiliary Information}

\section{Method/Apparatus/Procedure:}

Constant-temperature bath, calorimetric thermometer, and an ultraviolet/ visible spectrophotometer.

Excess solute and solvent were placed in amber glass bottles and allowed to equilibrate for several days at constant temperature. Attainment of equilibrium was verified by several repetitive measurements and by approaching equilibrium from supersaturation. Aliquots of saturated solutions were transferred through a coarse filter into tared volumetric flasks, weighed and diluted with methanol. Concentrations were determined by spectrophotometric measurements at $289 \mathrm{~nm}$.

\section{Source and Purity of Chemicals:}

(1) $98 \%$, Aldrich Chemical Company, Milwaukee, WI, USA, was recrystallized several times from methanol.

(2) $99.5+\%$, HPLC Grade, Aldrich Chemical Company, stored over molecular sieves before use.

Estimated Error:

Temperature: $\pm 0.1 \mathrm{~K}$.

$x_{1}: \pm 1.5 \%$ (relative error).

\subsection{Acenaphthene solubility data in miscellaneous organic solvents}

\begin{tabular}{ll}
\hline \hline Components: & Original Measurements: \\
(1) Acenaphthene; $\mathrm{C}_{12} \mathrm{H}_{10} ;[83-32-9]$ & ${ }^{10}$ W. E. Acree, Jr. and M. H. \\
(2) Propanenitrile; $\mathrm{C}_{3} \mathrm{H}_{5} \mathrm{~N} ;[107-12-0]$ & Abraham, Fluid Phase \\
& Equilib. 201, 245 (2002). \\
\hline Variables: & Prepared by: \\
$T / \mathrm{K}=298.15$ & W. E. Acree, Jr. \\
\hline
\end{tabular}


Experimental Values

\begin{tabular}{lcc}
\hline \hline$x_{2}{ }^{(\mathrm{s}) \mathrm{a}}$ & $x_{2}{ }^{\mathrm{b}}$ & $x_{1}{ }^{\mathrm{c}}$ \\
\hline 1.0000 & 0.9385 & 0.06154 \\
$\frac{{ }^{\mathrm{a}} x_{2}{ }^{(\mathrm{s})}: \text { initial mole fraction of component } 2 \text { in the solution. }}{{ }^{\mathrm{b}}{ }_{x_{2}}: \text { mole fraction of component } 2 \text { in the saturated solution. }}$ \\
${ }^{\mathrm{c}}{ }_{x_{1}}:$ mole fraction solubility of the solute.
\end{tabular}

\section{Auxiliary Information}

\section{Method/Apparatus/Procedure:}

Constant-temperature bath, calorimetric thermometer, and an ultraviolet/ visible spectrophotometer.

Excess solute and solvent were placed in amber glass bottles and allowed to equilibrate for several days at constant temperature. Attainment of equilibrium was verified by several repetitive measurements and by approaching equilibrium from supersaturation. Aliquots of saturated solutions were transferred through a coarse filter into tared volumetric flasks, weighed and diluted with 2-propanol. Concentrations were determined by spectrophotometric measurements at $289 \mathrm{~nm}$

Source and Purity of Chemicals:

(1) $98 \%$, Aldrich Chemical Company, Milwaukee, WI, USA, was recrystallized several times from methanol.

(2) $99 \%$, Aldrich Chemical Company, stored over molecular sieves before use.

\section{Estimated Error:}

Temperature: $\pm 0.1 \mathrm{~K}$.

$x_{1}: \pm 1.5 \%$ (relative error).

\begin{tabular}{ll}
\hline \hline Components: & Original Measurements: \\
(1) Acenaphthene; $\mathrm{C}_{12} \mathrm{H}_{10} ;[83-32-9]$ & ${ }^{10}$ W. E. Acree, Jr. and M. H. \\
(2) Butanenitrile; $\mathrm{C}_{4} \mathrm{H}_{7} \mathrm{~N} ;[109-74-0]$ & Abraham, Fluid Phase \\
& Equilib. 201, 245 (2002). \\
\hline Variables: & Prepared by: \\
$T / \mathrm{K}=298.15$ & W. E. Acree, Jr. \\
\hline
\end{tabular}

Experimental Values

\begin{tabular}{lcc}
\hline \hline$x_{2}{ }^{(\mathrm{s}) \mathrm{a}}$ & $x_{2}{ }^{\mathrm{b}}$ & $x_{1}{ }^{\mathrm{c}}$ \\
\hline 1.0000 & 0.9026 & 0.09737 \\
\hline
\end{tabular}

${ }^{\mathrm{a}} x_{2}{ }^{(\mathrm{s})}$ : initial mole fraction of component 2 in the solution.

${ }^{b} x_{2}$ : mole fraction of component 2 in the saturated solution.

${ }^{c} x_{1}$ : mole fraction solubility of the solute.

\section{Auxiliary Information}

\footnotetext{
Method/Apparatus/Procedure:

Constant-temperature bath, calorimetric thermometer, and an ultraviolet/ visible spectrophotometer.

Excess solute and solvent were placed in amber glass bottles and allowed to equilibrate for several days at constant temperature. Attainment of equilibrium was verified by several repetitive measurements and by approaching equilibrium from supersaturation. Aliquots of saturated solutions were transferred through a coarse filter into tared volumetric flasks, weighed and diluted with 2-propanol. Concentrations were determined by

spectrophotometric measurements at $289 \mathrm{~nm}$.
}

Source and Purity of Chemicals:

(1) $98 \%$, Aldrich Chemical Company, Milwaukee, WI, USA, was recrystallized several times from methanol.

(2) $99+\%$, Aldrich Chemical Company, stored over molecular sieves before use.

\section{Estimated Error:}

Temperature: $\pm 0.1 \mathrm{~K}$.

$x_{1}: \pm 1.5 \%$ (relative error).

\begin{tabular}{ll}
\hline \hline Components: & Original Measurements: \\
(1) Acenaphthene; $\mathrm{C}_{12} \mathrm{H}_{10} ;[83-32-9]$ & ${ }^{10}$ W. E. Acree, Jr. and M. H. \\
(2) Dimethyl sulfoxide; $\mathrm{C}_{2} \mathrm{H}_{6} \mathrm{OS} ;[67-68-5]$ & Abraham, Fluid Phase \\
& Equilib. 201, 245 (2002). \\
\hline Variables: & Prepared by: \\
$T / \mathrm{K}=298.15$ & W. E. Acree, Jr. \\
\hline
\end{tabular}

Experimental Values

\begin{tabular}{lcc}
\hline \hline$x_{2}{ }^{(\mathrm{s}) \mathrm{a}}$ & $x_{2}{ }^{\mathrm{b}}$ & $x_{1}{ }^{\mathrm{c}}$ \\
\hline 1.0000 & 0.9641 & 0.03588 \\
$\frac{{ }^{\mathrm{a}} x_{2}{ }^{(\mathrm{s})}: \text { initial mole fraction of component } 2 \text { in the solution. }}{{ }^{\mathrm{b}}{ }_{x_{2}}: \text { mole fraction of component } 2 \text { in the saturated solution. }}$ \\
${ }^{\mathrm{c}} x_{1}:$ mole fraction solubility of the solute.
\end{tabular}

\section{Auxiliary Information}

\section{Method/Apparatus/Procedure:}

Constant-temperature bath, calorimetric thermometer, and an ultraviolet/ visible spectrophotometer.

Excess solute and solvent were placed in amber glass bottles and allowed to equilibrate for several days at constant temperature. Attainment of equilibrium was verified by several repetitive measurements and by approaching equilibrium from supersaturation. Aliquots of saturated solutions were transferred through a coarse filter into tared volumetric flasks, weighed and diluted with 2-propanol. Concentrations were determined by spectrophotometric measurements at $289 \mathrm{~nm}$.

\section{Source and Purity of Chemicals:}

(1) $98 \%$, Aldrich Chemical Company, Milwaukee, WI, USA, was recrystallized several times from methanol.

(2) $99.9 \%$, HPLC Grade, Aldrich Chemical Company, stored over molecular sieves before use.

\section{Estimated Error:}

Temperature: $\pm 0.1 \mathrm{~K}$.

$x_{1}: \pm 1.5 \%$ (relative error).

\begin{tabular}{ll}
\hline \hline Components: & Original Measurements: \\
(1) Acenaphthene; $\mathrm{C}_{12} \mathrm{H}_{10} ;[83-32-9]$ & ${ }^{10}$ W. E. Acree, Jr. and M. H. \\
$(2) N$-Methylformamide; $\mathrm{C}_{2} \mathrm{H}_{5} \mathrm{NO} ;$ & Abraham, Fluid Phase \\
{$[123-39-7]$} & Equilib. 201, 245 (2002). \\
\hline Variables: & Prepared by: \\
$T / \mathrm{K}=298.15$ & W. E. Acree, Jr. \\
\hline
\end{tabular}


Experimental Values

\begin{tabular}{lcc}
\hline \hline$x_{2}{ }^{(\mathrm{s}) \mathrm{a}}$ & $x_{2}{ }^{\mathrm{b}}$ & $x_{1}{ }^{\mathrm{c}}$ \\
\hline 1.0000 & 0.9854 & 0.01458 \\
$\frac{{ }^{\mathrm{a}} x_{2}{ }^{(\mathrm{s})}: \text { initial mole fraction of component } 2 \text { in the solution. }}{{ }^{\mathrm{b}}{ }_{x_{2}}: \text { mole fraction of component } 2 \text { in the saturated solution. }}$ \\
${ }^{\mathrm{c}}{ }_{x_{1}}:$ mole fraction solubility of the solute.
\end{tabular}

\section{Auxiliary Information}

Method/Apparatus/Procedure:

Constant-temperature bath, calorimetric thermometer, and an ultraviolet/ visible spectrophotometer.

Excess solute and solvent were placed in amber glass bottles and allowed to equilibrate for several days at constant temperature. Attainment of equilibrium was verified by several repetitive measurements and by approaching equilibrium from supersaturation. Aliquots of saturated solutions were transferred through a coarse filter into tared volumetric flasks, weighed and diluted with 2-propanol. Concentrations were determined by spectrophotometric measurements at $289 \mathrm{~nm}$

Source and Purity of Chemicals:

(1) $98 \%$, Aldrich Chemical Company, Milwaukee, WI, USA, was recrystallized several times from methanol.

(2) $99 \%$, anhydrous, Aldrich Chemical Company, stored over molecular sieves before use.

Estimated Error:

Temperature: $\pm 0.1 \mathrm{~K}$

$x_{1}: \pm 1.5 \%$ (relative error)

\section{Solubility of Anthracene in Organic Solvents}

\subsection{Critical evaluation of experimental solubility data}

Volume 58 in the IUPAC Solubility Data Series ${ }^{2}$ contained experimental solubility data for anthracene dissolved in 12 saturated hydrocarbons (hexane, heptanes, octane, hexadecane, octadecane, cyclohexane, methylcyclohexane, cyclooctane, 2,2,4-trimethylpentane, tert-butylcyclohexane, squalane, and decahydronaphthalene), in five aromatic hydrocarbons (benzene, methylbenzene, 1,4-dimethylbenzene, 4-isopropyl-1-methylbenzene, and 1,2,3,4-tetrahydronaphthalene), in five alkyl alkanoates (ethyl ethanoate, butyl ethanoate, dimethyl hexanedioate, diethyl hexanedioate, and dibutyl oxalate), in two dialkyl ethers (1,1-oxybisethane and 1,1-oxybisbutane) and three cyclic ethers (tetrahydrofuran, tetrahydropyrane and 1,4-dioxane), in five haloalkanes (trichloromethane, tetrachloromethane, 1chlorobutane, 1,4-dichlorobutane, and iodoethane), one haloalkene (trichloroethene) and three haloaromatic hydrocarbons (chlorobenzene, bromobenzene, and iodobenzene), in eight alkanols (methanol, 1-propanol, 2-propanol, 1-butanol, 2-butanol, 2-methyl-1-propanol, 3-methyl-1-butanol, and 1-octanol), in two alkanones (propanone and 2-butanone), and in 15 miscellaneous organic solvents (ethanenitrile, carbon disulfide, acetic anhydride, dimethyl sulfoxide, nitrobenzene, aniline, pyridine, quinoline, thiophene, methoxybenzene, 1-methyl-2pyrrolidinone, $N, N$-dimethylformamide, $N, N$-dimethylacetamide, tetramethylene sulfone, and tributyl phosphate). Except for a few select systems, the majority of the compiled solubility data was measured at either 293.15 or $298.15 \mathrm{~K}$. Anthracene solubility measurements in octadecane covered the temperature range of 466-484 K, measurements in cyclohexane covered the temperature range from 298 to $402 \mathrm{~K}$, measurements in tetrahydronaphthalene covered the range from 324 to $385 \mathrm{~K}$, measurements in benzene covered the range from $293 \mathrm{~K}$ to $364 \mathrm{~K}$, measurements in methylbenzene covered the range from 293 to $333 \mathrm{~K}$, measurements in 1,2,3,4-tetrahydronaphthalene covered the range from 300 to $346 \mathrm{~K}$, measurements in iodobenzene covered the range from 305 to $320 \mathrm{~K}$, measurements in nitrobenzene covered the range from 286 to $313 \mathrm{~K}$, measurements in pyridine covered the range from 293 to $359 \mathrm{~K}$, and measurements in thiophene covered the range from 297 to $358 \mathrm{~K}$. The compiled solubility data also included phase diagram information for binary anthracene + acenaphthene, anthracene + chrysene, anthracene + fluoranthene, and anthracene $+1,2,3,5$-tetranitrobenzene mixtures. Solubility data contained in Vol. 58 will not be republished here. The listing above is provided so that readers will know what solubility data are available in the earlier volume for anthracene.

After Vol. 58 appeared in print, there have been several experimental solubility studies involving anthracene dissolved in organic solvents. Acree and co-workers ${ }^{16-33}$ have measured the solubility of anthracene in four linear alkanes (nonane through dodecane), in two dialkylbenzenes (1,2-dimethylbenzene and 1,3-dimethylbenzene), in several primary (methanol, ethanol, 1-pentanol, 2-methyl-1-butanol, 1-hexanol, 2methyl-1-pentanol, 1-heptanol, 2-ethyl-1-hexanol, 1-decanol, 3,7-dimethyl-1-octanol, 1,2-ethanediol, and 2,2,2-trifluoroethanol), three secondary (2-pentanol, 4-methyl-2-pentanol, and cyclopentanol) and one tertiary (2-methyl-2-propanol) alcohol(s), in four alkyl alkanoates (methyl ethanoate, propyl ethanoate, pentyl ethanoate, and methyl butanoate), in three ethers (2,2'-oxybispropane, 2-methoxy-2-methylpropane, and $1,1^{\prime}$-oxybis[2-methoxyethane]), in three chlorinated alkanes (dichloromethane, trichloromethane, and 1-chlorohexane) and three halogenated benzenes (chlorobenzene, fluorobenzene, and (trifluoromethyl)benzene), in five alkoxyalcohols (2-ethoxyethanol, 2-propoxyethanol, 2-isopropoxyethanol, 2-butoxyethanol, and 3-methoxy-1-butanol) and in several miscellaneous organic solvents (methyl acetoacetate, ethyl acetoacetate, ethanenitrile, propanenitrile, butanenitrile, benzonitrile, $N, N$-dimethylformamide, $N, N$-dimethylacetamide, morpholine, ethanolamine, propylene carbonate, hexanedintitrile (also called adiponitrile) and tributyl phosphate). The authors measured the solubility at only $298.15 \mathrm{~K}$, and for most of the solvents there are no independent experimental measurements to compare the numerical values against.

Shayanfar et al. ${ }^{34}$ measured the solubility of anthracene in binary 2,2,4-trimethylpentane + ethanol solvent mixtures at $298.2,308.2$, and $318.2 \mathrm{~K}$. Since only three temperatures were studied, it is not feasible to use the three-parameter Apelblat equation to assess the internal consistency of the authors' 
experimental data since the equation would yield essentially a perfect fit. The observed mole fraction solubility of anthracene in ethanol reported by Shayanfar et al., ${ }^{34} x_{1}=0.000505$, is approximately $9 \%$ larger than the value given by Roy et al., ${ }^{16}$ $x_{1}=0.000460$. The anthracene samples used both solubility studies were recrystallized from propanone. Shayanfar et al. ${ }^{34}$ started with a sample having an initial purity of $96 \%$. Roy et al. $^{16}$ started with a sample of much higher purity, $99+\%$.

Two research groups have determined phase diagrams for binary mixtures containing anthracene. Auolmi et al. ${ }^{15}$ measured the solid-liquid equilibria data for the anthracene + hexatricontane system by a differential scanning calorimetric method. The authors employed a SSF to describe the activity coefficients of anthracene and hexatricontane calculated from the solid-liquid equilibrium data. The SSF model gave a calculated eutectic temperature of $T / \mathrm{K}=349.9$ and eutectic mole fraction of anthracene of $x_{1}=0.038$. Lisicki and Jamró $z^{35}$ reported solid-liquid equilibria data for binary mixtures containing anthracene with $\mathrm{N}, \mathrm{N}$-dimethylacetamide, 1methyl-2-pyrrolidone, and hexahydro-1-methyl-2H-azepin2-one (also called $N$-methyl- $\varepsilon$-caprolactam). The anthracene $+N, N$-dimethylformamide and anthracene +1 -methyl-2-pyrrolidone systems both exhibited simple eutectic behavior. The published experimental liquidus curves for the latter three anthracene systems did not indicate any outlier data points.

The experimental solubility data for anthracene dissolved in the different organic solvents are given in Secs. 3.2-3.9.

\subsection{Anthracene solubility data in saturated hydrocarbons (including cycloalkanes)}

\begin{tabular}{ll}
\hline \hline Components: & Original Measurements: \\
(1) Anthracene; $\mathrm{C}_{14} \mathrm{H}_{10} ;[120-12-7]$ & ${ }^{16}$ L. E. Roy, C. E. Hernández, \\
(2) Nonane; $\mathrm{C}_{9} \mathrm{H}_{20} ;[111-84-2]$ & and W. E. Acree, Jr., \\
& Polycyclic Aromat. Compd. \\
& $\mathbf{1 3}, 105(1999)$. \\
\hline Variables: & Prepared by: \\
$T / \mathrm{K}=298.15$ & W. E. Acree, Jr. \\
\hline
\end{tabular}

\section{Experimental Values}

\begin{tabular}{lcc}
\hline \hline$\frac{x_{2}{ }^{(\mathrm{s}) \mathrm{a}}}{x_{2}{ }^{\mathrm{b}}}$ & 0.9979 & $x_{1}{ }^{\mathrm{c}}$ \\
\hline 1.0000 & 0.002085 \\
${ }^{\mathrm{a}} x_{2}{ }^{(\mathrm{s})}:$ initial mole fraction of component 2 in the solution. \\
${ }^{\mathrm{b}} x_{2}:$ mole fraction of component 2 in the saturated solution. \\
${ }^{\mathrm{c}} x_{1}:$ mole fraction solubility of the solute.
\end{tabular}

\section{Auxiliary Information}

Method/Apparatus/Procedure:

Constant-temperature bath, calorimetric thermometer, and an ultraviolet/ visible spectrophotometer.

Excess solute and solvent were placed in amber glass bottles and allowed to equilibrate for several days at constant temperature. Attainment of equilibrium was verified by several repetitive measurements and by approaching equilibrium from supersaturation. Aliquots of saturated solutions were transferred through a coarse filter into tared volumetric flasks, weighed and diluted with 2-propanol. Concentrations were determined by spectrophotometric measurements at $356 \mathrm{~nm}$.

Source and Purity of Chemicals:

(1) $99+\%$, Aldrich Chemical Company, Milwaukee, WI, USA, was recrystallized several times from propanone.

(2) $99+\%$, TCI America, Portland, OR, USA, stored over molecular sieves before use.

Estimated Error:

Temperature: $\pm 0.1 \mathrm{~K}$.

$x_{1}: \pm 1.5 \%$ (relative error).

\begin{tabular}{ll}
\hline \hline Components: & Original Measurements: \\
(1) Anthracene; $\mathrm{C}_{14} \mathrm{H}_{10} ;[120-12-7]$ & ${ }^{16}$ L. E. Roy, C. E. Hernández, \\
(2) Decane; $\mathrm{C}_{10} \mathrm{H}_{22} ;[124-18-5]$ & and W. E. Acree, Jr., \\
& Polycyclic Aromat. Compd. \\
& $\mathbf{1 3}, 105$ (1999). \\
\hline Variables: & Prepared by: \\
$T / \mathrm{K}=298.15$ & W. E. Acree, Jr. \\
\hline
\end{tabular}

Experimental Values

\begin{tabular}{lcc}
\hline$\frac{x_{2}{ }^{(\mathrm{s}) \mathrm{a}}}{x_{2}{ }^{\mathrm{b}}}$ & $x_{1}{ }^{\mathrm{c}}$ \\
\hline 1.0000 & 0.9977 & 0.002345 \\
$\frac{{ }^{\mathrm{a}} x_{2}{ }^{(\mathrm{s})}: \text { initial mole fraction of component } 2 \text { in the solution. }}{{ }^{\mathrm{b}}{ }_{x_{2}}: \text { mole fraction of component } 2 \text { in the saturated solution. }}$ \\
${ }^{\mathrm{c}}{ }_{x_{1}}$ : mole fraction solubility of the solute.
\end{tabular}

\section{Auxiliary Information}

\section{Method/Apparatus/Procedure:}

Constant-temperature bath, calorimetric thermometer, and an ultraviolet/ visible spectrophotometer.

Excess solute and solvent were placed in amber glass bottles and allowed to equilibrate for several days at constant temperature. Attainment of equilibrium was verified by several repetitive measurements and by approaching equilibrium from supersaturation. Aliquots of saturated solutions were transferred through a coarse filter into tared volumetric flasks, weighed and diluted with 2-propanol. Concentrations were determined by spectrophotometric measurements at $356 \mathrm{~nm}$.

Source and Purity of Chemicals:

(1) $99+\%$, Aldrich Chemical Company, Milwaukee, WI, USA, was recrystallized several times from propanone.

(2) $99+\%$, TCI America, Portland, OR, USA, stored over molecular sieves before use.

Estimated Error:

Temperature: $\pm 0.1 \mathrm{~K}$.

$x_{1}: \pm 1.5 \%$ (relative error). 


\section{Components:}

(2) Undecane; $\mathrm{C}_{11} \mathrm{H}_{24} ;[1120-21-4]$

\section{Variables:}

$T / \mathrm{K}=298.15$
(1) Anthracene; $\mathrm{C}_{14} \mathrm{H}_{10} ;[120-12-7]$

Original Measurements:

${ }^{17}$ M. H. Abraham and W. E. Acree, Jr., New J. Chem. 28, 1538 (2004).

Prepared by:

W. E. Acree, Jr.

Experimental Values

\begin{tabular}{|c|c|c|}
\hline$x_{2}^{(\mathrm{s}) \mathrm{a}}$ & $x_{2}{ }^{b}$ & $x_{1}{ }^{\mathrm{c}}$ \\
\hline 1.0000 & 0.9974 & 0.002585 \\
\hline
\end{tabular}

Experimental values were reported as the logarithm of the solute's molar solubility in undecane divided by the molar solubility in water. Mole fraction solubilities were provided by the authors of the paper.

\section{Auxiliary Information}

\begin{abstract}
Method/Apparatus/Procedure:
Constant-temperature bath, calorimetric thermometer, and an ultraviolet/ visible spectrophotometer.

Excess solute and solvent were placed in amber glass bottles and allowed to equilibrate for several days at constant temperature. Attainment of equilibrium was verified by several repetitive measurements and by approaching equilibrium from supersaturation. Aliquots of saturated solutions were transferred through a coarse filter into tared volumetric flasks, weighed and diluted with 2-propanol. Concentrations were determined by spectrophotometric measurements at $356 \mathrm{~nm}$.
\end{abstract}

Source and Purity of Chemicals:

(1) $99+\%$, Aldrich Chemical Company, Milwaukee, WI, USA, was recrystallized several times from propanone.

(2) $99 \%$, Aldrich Chemical Company, stored over molecular sieves before use.

\section{Estimated Error:}

Temperature: $\pm 0.1 \mathrm{~K}$

$x_{1}: \pm 1.5 \%$ (relative error).

\begin{tabular}{ll}
\hline \hline Components: & Original Measurements: \\
(1) Anthracene; $\mathrm{C}_{14} \mathrm{H}_{10} ;[120-12-7]$ & ${ }^{17} \mathrm{M} . \mathrm{H}$. Abraham and W. E. \\
(2) Dodecane; $\mathrm{C}_{12} \mathrm{H}_{26} ;[112-40-3]$ & Acree, Jr., New J. Chem. 28, \\
& $1538(2004)$. \\
\hline Variables: & Prepared by: \\
$T / \mathrm{K}=298.15$ & W. E. Acree, Jr. \\
\hline
\end{tabular}

Experimental Values

\begin{tabular}{lcc}
\hline \hline$x_{2}{ }^{(\mathrm{s}) \mathrm{a}}$ & $x_{2}{ }^{\mathrm{b}}$ & $x_{1}{ }^{\mathrm{c}}$ \\
\hline 1.0000 & 0.9972 & 0.002800 \\
$\frac{{ }^{\mathrm{a}} x_{2}{ }^{(\mathrm{s})}: \text { initial mole fraction of component } 2 \text { in the solution. }}{{ }^{\mathrm{b}} x_{2}: \text { mole fraction of component } 2 \text { in the saturated solution. }}$ \\
${ }^{\mathrm{c}}{ }_{x_{1}: \text { mole fraction solubility of the solute. }}$
\end{tabular}

Experimental values were reported as the logarithm of the solute's molar solubility in dodecane divided by the molar solubility in water. Mole fraction solubilities were provided by the authors of the paper.

\section{Auxiliary Information}

\section{Method/Apparatus/Procedure:}

Constant-temperature bath, calorimetric thermometer, and an ultraviolet/ visible spectrophotometer.

Excess solute and solvent were placed in amber glass bottles and allowed to equilibrate for several days at constant temperature. Attainment of equilibrium was verified by several repetitive measurements and by approaching equilibrium from supersaturation. Aliquots of saturated solutions were transferred through a coarse filter into tared volumetric flasks, weighed and diluted with 2-propanol. Concentrations were determined by spectrophotometric measurements at $356 \mathrm{~nm}$.

Source and Purity of Chemicals:

(1) $99+\%$, Aldrich Chemical Company, Milwaukee, WI, USA, was recrystallized several times from propanone.

(2) $99 \%$, Aldrich Chemical Company, stored over molecular sieves before use.

\section{Estimated Error:}

Temperature: $\pm 0.1 \mathrm{~K}$.

$x_{1}: \pm 1.5 \%$ (relative error).

\begin{tabular}{ll}
\hline \hline Components: & Original Measurements: \\
(1) Anthracene; $\mathrm{C}_{14} \mathrm{H}_{10} ;[120-12-7]$ & ${ }^{15}$ A. Aoulmi, M. Bouroukba, \\
(2) Hexatricontane; $\mathrm{C}_{36} \mathrm{H}_{74} ;[630-06-8]$ & R. Solimando, and \\
& M. Rogalski, Fluid Phase \\
& Equilib. 110, 283 (1995). \\
\hline Variables: & Prepared by: \\
Temperature & W. E. Acree, Jr. \\
\hline
\end{tabular}

Experimental Values

\begin{tabular}{lcc}
\hline \hline$T / \mathrm{K}$ & $x_{2}{ }^{\mathrm{a}}$ & $x_{1}{ }^{\mathrm{b}}$ \\
\hline 386.45 & 0.9004 & 0.0996 \\
409.41 & 0.8114 & 0.1886 \\
432.38 & 0.6909 & 0.3091 \\
450.01 & 0.5922 & 0.4078 \\
461.12 & 0.4737 & 0.5263 \\
468.21 & 0.3986 & 0.6014 \\
474.51 & 0.2862 & 0.7138 \\
480.01 & 0.1978 & 0.8022 \\
483.46 & 0.0998 & 0.9002 \\
488.46 & 0.0000 & 1.0000 \\
\hline
\end{tabular}

${ }^{a} x_{2}$ : mole fraction of component 2 in the saturated solution.

${ }^{b} x_{1}$ : mole fraction of the polycyclic aromatic hydrocarbon (component 1 ).

The authors employed a SSF to describe the activity coefficients of anthracene and hexatricontane calculated from the solid-liquid equilibrium data. The SSF model gave a calculated eutectic temperature of $T / \mathrm{K}=349.9$ and eutectic mole fraction of anthracene of $x_{1}=0.038$. 


\section{Auxiliary Information}

Method/Apparatus/Procedure:

Differential scanning calorimeter.

Phase diagram was determined using a differential scanning calorimeter.

Measurements were performed at a constant fixed scanning rate of $0.5 \mathrm{~K} / \mathrm{min}$.

Source and Purity of Chemicals:

(1) $99.9 \%$, Aldrich Chemical Company, Milwaukee, WI, USA, purification details were not given in the paper.

(2) $98+\%$, Fluka Chemical Company, purification details were not given in the paper.

Estimated Error:

Temperature: Authors state a global accuracy of $\pm 1 \%$ regarding their measurements.

$x_{1}: \pm 0.0002$ (estimated by compiler).

\section{Components:}

(1) Anthracene; $\mathrm{C}_{14} \mathrm{H}_{10} ;[120-12-7]$

(2) 2,2,4-Trimethylpentane; $\mathrm{C}_{8} \mathrm{H}_{18}$; [540-84-1]

\section{Variables:}

Temperature
Original Measurements:

${ }^{34}$ A. Shayanfar, S. H. Eghrary, F. Sardari, W. E. Acree, Jr., and A. Jouyban, J. Chem. Eng. Data 56, 2290 (2011).

Prepared by:

W. E. Acree, Jr.
Experimental Values

\begin{tabular}{lcc}
\hline \hline$T / \mathrm{K}$ & $x_{2}{ }^{\mathrm{a}}$ & $x_{1}{ }^{\mathrm{b}}$ \\
\hline 298.2 & 0.9988 & 0.001187 \\
308.2 & 0.9985 & 0.001515 \\
318.2 & 0.9982 & 0.001768
\end{tabular}

${ }^{\mathrm{a}} x_{2}$ : mole fraction of component 2 in the saturated solution.

${ }^{\mathrm{b}} x_{1}$ : mole fraction of the polycyclic aromatic hydrocarbon (component 1 ).

\section{Auxiliary Information}

\section{Method/Apparatus/Procedure:}

Incubator, shaker, and an ultraviolet/visible spectrophotometer.

Solubility was determined by equilibrating an excess amount of solid naphthalene with the organic solvent using a shaker placed in an incubator equipped with a temperature controlling system. After an equilibration period of at least $48 \mathrm{~h}$ the samples were withdrawn and filtered through hydrophobic Durapore filters $(0.45 \mu \mathrm{m})$. The filtered sample was diluted quantitatively with methanol. Concentrations were determined by spectrophotmetric analysis at $356 \mathrm{~nm}$.

Source and Purity of Chemicals:

(1) $96 \%$, Fluka Chemical Company, was recrystallized several from propanone and ethyl ethanoate before use.

(2) $99+\%$, Merck Chemicals, Germany, no information given regarding any further purification.

\section{Estimated Error:}

Temperature: $\pm 0.2 \mathrm{~K}$.

$x_{1}: \pm 2.7 \%$ (relative uncertainty).

\subsection{Anthracene solubility data in aromatic hydrocarbons}

\begin{tabular}{|c|c|}
\hline $\begin{array}{l}\text { Components: } \\
\text { (1) Anthracene; } \mathrm{C}_{14} \mathrm{H}_{10} ;[120-12-7] \\
\text { (2) } 1,2-\text {-Dimethylbenzene; } \mathrm{C}_{8} \mathrm{H}_{10} \text {; } \\
\text { [95-47-6] }\end{array}$ & $\begin{array}{l}\text { Original Measurements: } \\
{ }^{16} \text { L. E. Roy, C. E. Hernández, and } \\
\text { W. E. Acree, Jr., Polycyclic } \\
\text { Aromat. Compd. 13, } 105 \text { (1999). }\end{array}$ \\
\hline $\begin{array}{l}\text { Variables: } \\
T / \mathrm{K}=298.15\end{array}$ & $\begin{array}{l}\text { Prepared by: } \\
\text { W. E. Acree, Jr. }\end{array}$ \\
\hline
\end{tabular}

Experimental Values

\begin{tabular}{|c|c|c|}
\hline$x_{2}{ }^{(\mathrm{s}) \mathrm{a}}$ & $x_{2}{ }^{\mathrm{b}}$ & $x_{1}{ }^{\mathrm{c}}$ \\
\hline 1.0000 & 0.9915 & 0.008458 \\
\hline
\end{tabular}

\section{Auxiliary Information}

\section{Method/Apparatus/Procedure:}

Constant-temperature bath, calorimetric thermometer, and an ultraviolet/ visible spectrophotometer.

Excess solute and solvent were placed in amber glass bottles and allowed to equilibrate for several days at constant temperature. Attainment of equilibrium was verified by several repetitive measurements and by approaching equilibrium from supersaturation. Aliquots of saturated solutions were transferred through a coarse filter into tared volumetric flasks, weighed and diluted with 2-propanol. Concentrations were determined by spectrophotometric measurements at $356 \mathrm{~nm}$.

Source and Purity of Chemicals:

(1) $99+\%$, Aldrich Chemical Company, Milwaukee, WI, USA, was recrystallized several times from propanone.

(2) $98+\%$, HPLC Grade, Aldrich Chemical Company, stored over molecular sieves before use.

Estimated Error:

Temperature: $\pm 0.1 \mathrm{~K}$.

$x_{1}: \pm 1.5 \%$ (relative error).

\section{Components:}

(1) Anthracene; $\mathrm{C}_{14} \mathrm{H}_{10} ;[120-12-7]$

(2) 1,3-Dimethylbenzene; $\mathrm{C}_{8} \mathrm{H}_{10}$; [108-38-3]

Variables:

$T / \mathrm{K}=298.15$
Original Measurements:

${ }^{16}$ L. E. Roy, C. E. Hernández, and W. E. Acree, Jr., Polycyclic Aromat. Compd. 13, 105 (1999).

Prepared by: W. E. Acree, Jr.

\section{Experimental Values}

\begin{tabular}{lcc}
\hline \hline$x_{2}{ }^{(\mathrm{s}) \mathrm{a}}$ & $x_{2}{ }^{\mathrm{b}}$ & $x_{1}{ }^{\mathrm{c}}$ \\
\hline 1.0000 & 0.9920 & 0.007956 \\
$\frac{{ }^{a} x_{2}{ }^{(\mathrm{s})}: \text { initial mole fraction of component } 2 \text { in the solution. }}{{ }^{\mathrm{b}} x_{2}: \text { mole fraction of component } 2 \text { in the saturated solution. }}$ \\
${ }^{\mathrm{c}} x_{1}:$ mole fraction solubility of the solute.
\end{tabular}




\section{Auxiliary Information}

\section{Method/Apparatus/Procedure:}

Constant-temperature bath, calorimetric thermometer, and an ultraviolet/ visible spectrophotometer.

Excess solute and solvent were placed in amber glass bottles and allowed to equilibrate for several days at constant temperature. Attainment of equilibrium was verified by several repetitive measurements and by approaching equilibrium from supersaturation. Aliquots of saturated solutions were transferred through a coarse filter into tared volumetric flasks, weighed and diluted with 2-propanol. Concentrations were determined by spectrophotometric measurements at $356 \mathrm{~nm}$.

Source and Purity of Chemicals:

(1) $99+\%$, Aldrich Chemical Company, Milwaukee, WI, USA, was recrystallized several times from propanone.

(2) $99+\%$, anhydrous, Aldrich Chemical Company, stored over molecular sieves before use.

Estimated Error:

Temperature: $\pm 0.1 \mathrm{~K}$.

$x_{1}: \pm 1.5 \%$ (relative error).

\subsection{Anthracene solubility data in esters}

\section{Components:}

(1) Anthracene; $\mathrm{C}_{14} \mathrm{H}_{10} ;[120-12-7]$

(2) Methyl ethanoate; $\mathrm{C}_{3} \mathrm{H}_{6} \mathrm{O}_{2} ;$;79-20-9]

\begin{tabular}{ll}
\hline Variables: & Prepared by: \\
$T / \mathrm{K}=298.15$ & W. E. Acree, Jr. \\
\hline
\end{tabular}

Experimental Values

\begin{tabular}{lcc}
\hline \hline$x_{2}{ }^{(\mathrm{s}) \mathrm{a}}$ & $x_{2}^{\mathrm{b}}$ & $x_{1}{ }^{\mathrm{c}}$ \\
\hline 1.0000 & 0.9944 & 0.003639 \\
\hline
\end{tabular}

${ }^{\mathrm{a}} x_{2}{ }^{(\mathrm{s})}$ : initial mole fraction of component 2 in the solution.

${ }^{\mathrm{b}} x_{2}$ : mole fraction of component 2 in the saturated solution.

${ }^{c} x_{1}$ : mole fraction solubility of the solute.

\section{Auxiliary Information}

\section{Method/Apparatus/Procedure:}

Constant-temperature bath, calorimetric thermometer, and an ultraviolet/ visible spectrophotometer.

Excess solute and solvent were placed in amber glass bottles and allowed to equilibrate for several days at constant temperature. Attainment of equilibrium was verified by several repetitive measurements and by approaching equilibrium from supersaturation. Aliquots of saturated solutions were transferred through a coarse filter into tared volumetric flasks, weighed and diluted with 2-propanol. Concentrations were determined by spectrophotometric measurements at $356 \mathrm{~nm}$.

Source and Purity of Chemicals:

(1) $99+\%$, Aldrich Chemical Company, Milwaukee, WI, USA, was recrystallized several times from propanone.

(2) $99.5 \%$, anhydrous, Aldrich Chemical Company, stored over molecular sieves before use.
Estimated Error:

Temperature: $\pm 0.1 \mathrm{~K}$.

$x_{1}: \pm 1.5 \%$ (relative error)

\begin{tabular}{ll}
\hline \hline Components: & Original Measurements: \\
(1) Anthracene; $\mathrm{C}_{14} \mathrm{H}_{10} ;[120-12-7]$ & ${ }^{18}$ L. Alcazar, A. Blanco, R. Cano, \\
(2) Propyl ethanoate; $\mathrm{C}_{5} \mathrm{H}_{10} \mathrm{O}_{2} ;$ & L. Fisher, M. Nau, L. Sidransky, \\
[109-60-4] & and W. E. Acree, Jr., J. Chem. \\
& Eng. Data. 53, 201 (2008). \\
\hline Variables: & Prepared by: \\
$T / \mathrm{K}=298.15$ & W. E. Acree, Jr. \\
\hline
\end{tabular}

Experimental Values

\begin{tabular}{lcc}
\hline \hline$x_{2}{ }^{(\mathrm{s}) \mathrm{a}}$ & $x_{2}{ }^{\mathrm{b}}$ & $x_{1}{ }^{\mathrm{c}}$ \\
\hline 1.0000 & 0.9941 & 0.00588
\end{tabular}

${ }^{\mathrm{a}} x_{2}{ }^{(\mathrm{s})}$ : initial mole fraction of component 2 in the solution.

${ }^{b} x_{2}$ : mole fraction of component 2 in the saturated solution.

${ }^{c} x_{1}$ : mole fraction solubility of the solute.

\section{Auxiliary Information}

\section{Method/Apparatus/Procedure:}

Constant-temperature bath, calorimetric thermometer, and an ultraviolet/ visible spectrophotometer.

Excess solute and solvent were placed in amber glass bottles and allowed to equilibrate for several days at constant temperature. Attainment of equilibrium was verified by several repetitive measurements and by approaching equilibrium from supersaturation. Aliquots of saturated solutions were transferred through a coarse filter into tared volumetric flasks, weighed and diluted with methanol. Concentrations were determined by spectrophotometric measurements at $356 \mathrm{~nm}$.

\section{Source and Purity of Chemicals:}

(1) $99+\%$, Aldrich Chemical Company, Milwaukee, WI, USA, was recrystallized several times from propanone.

(2) $99 \%$, anhydrous, Aldrich Chemical Company, stored over molecular sieves before use.

\section{Estimated Error:}

Temperature: $\pm 0.1 \mathrm{~K}$.

$x_{1}: \pm 1.5 \%$ (relative error).

\begin{tabular}{ll}
\hline \hline Components: & Original Measurements: \\
(1) Anthracene; $\mathrm{C}_{14} \mathrm{H}_{10} ;[120-12-7]$ & W. E. Acree, Jr., \\
(2) Pentyl ethanoate; $\mathrm{C}_{7} \mathrm{H}_{14} \mathrm{O}_{2} ;[628-63-7]$ & unpublished data. \\
\hline Variables: & Prepared by: \\
$T / \mathrm{K}=298.15$ & W. E. Acree, Jr. \\
\hline
\end{tabular}


Experimental Values

\begin{tabular}{lcc}
\hline$\frac{x_{2}{ }^{(\mathrm{s}) \mathrm{a}}}{x_{2}{ }^{\mathrm{b}}}$ & $x_{1}{ }^{\mathrm{c}}$ \\
\hline 1.0000 & 0.9924 & 0.007547 \\
$\frac{{ }^{\mathrm{a}} x_{2}{ }^{(\mathrm{s})}: \text { initial mole fraction of component } 2 \text { in the solution. }}{{ }^{\mathrm{b}}{ }_{x_{2}}: \text { mole fraction of component } 2 \text { in the saturated solution. }}$ \\
${ }^{\mathrm{c}} x_{1}:$ mole fraction solubility of the solute.
\end{tabular}

\section{Auxiliary Information}

\section{Method/Apparatus/Procedure:}

Constant-temperature bath, calorimetric thermometer, and an ultraviolet/ visible spectrophotometer.

Excess solute and solvent were placed in amber glass bottles and allowed to equilibrate for several days at constant temperature. Attainment of equilibrium was verified by several repetitive measurements and by approaching equilibrium from supersaturation. Aliquots of saturated solutions were transferred through a coarse filter into tared volumetric flasks, weighed and diluted with 2-propanol. Concentrations were determined by spectrophotometric measurements at $356 \mathrm{~nm}$.

Source and Purity of Chemicals:

(1) $99+\%$, Aldrich Chemical Company, Milwaukee, WI, USA, was recrystallized several times from propanone.

(2) $99 \%$, Aldrich Chemical Company, stored over molecular sieves before use.

Estimated Error:

Temperature: $\pm 0.1 \mathrm{~K}$.

$x_{1}: \pm 1.5 \%$ (relative error).

\begin{tabular}{ll}
\hline \hline Components: & Original Measurements: \\
(1) Anthracene; $\mathrm{C}_{14} \mathrm{H}_{10} ;[120-12-7]$ & W. E. Acree, Jr., \\
(2) Methyl butanoate; $\mathrm{C}_{5} \mathrm{H}_{10} \mathrm{O}_{2} ;[623-42-7]$ & unpublished data. \\
\hline Variables: & Prepared by: \\
$T / \mathrm{K}=298.15$ & W. E. Acree, Jr. \\
\hline
\end{tabular}

Experimental Values

\begin{tabular}{lcc}
\hline \hline$x_{2}{ }^{(\mathrm{s}) \mathrm{a}}$ & $x_{2}{ }^{\mathrm{b}}$ & $x_{1}{ }^{\mathrm{c}}$ \\
\hline 1.0000 & 0.9941 & 0.005893 \\
$\frac{{ }^{\mathrm{a}} x_{2}{ }^{(\mathrm{s})}: \text { initial mole fraction of component } 2 \text { in the solution. }}{{ }^{\mathrm{b}}{ }_{x_{2}}: \text { mole fraction of component } 2 \text { in the saturated solution. }}$ \\
${ }^{\mathrm{c}}{ }_{x_{1}}$ : mole fraction solubility of the solute.
\end{tabular}

\section{Auxiliary Information}

\section{Method/Apparatus/Procedure:}

Constant-temperature bath, calorimetric thermometer, and an ultraviolet/ visible spectrophotometer.

Excess solute and solvent were placed in amber glass bottles and allowed to equilibrate for several days at constant temperature. Attainment of equilibrium was verified by several repetitive measurements and by approaching equilibrium from supersaturation. Aliquots of saturated solutions were transferred through a coarse filter into tared volumetric flasks, weighed and diluted with 2-propanol. Concentrations were determined by spectrophotometric measurements at $356 \mathrm{~nm}$.
Source and Purity of Chemicals:

(1) $99+\%$, Aldrich Chemical Company, Milwaukee, WI, USA, was recrystallized several times from propanone.

(2) $99 \%$, Aldrich Chemical Company, stored over molecular sieves before use.

Estimated Error:

Temperature: $\pm 0.1 \mathrm{~K}$.

$x_{1}: \pm 1.5 \%$ (relative error).

\subsection{Anthracene solubility data in ethers}

\begin{tabular}{ll}
\hline \hline Components: & Original Measurements: \\
(1) Anthracene; $\mathrm{C}_{14} \mathrm{H}_{10} ;[120-12-7]$ & ${ }^{19}$ M. Carrillo, M. Corella, K. Wolcott, \\
$(2) 2,2^{\prime}-$ Oxybispropane; $\mathrm{C}_{6} \mathrm{H}_{14} \mathrm{O} ;$ & K. R. Bowen, and W. E. Acree, Jr., J. \\
{$[108-20-3]$} & Chem. Eng. Data 52, 270 (2007). \\
\hline Variables: & Prepared by: \\
$T / \mathrm{K}=298.15$ & W. E. Acree, Jr. \\
\hline
\end{tabular}

\section{Experimental Values}

\begin{tabular}{lcc}
\hline \hline$\frac{x_{2}{ }^{(\mathrm{s}) \mathrm{a}}}{x_{2}{ }^{\mathrm{b}}}$ & \multicolumn{1}{c}{$x_{1}{ }^{\mathrm{c}}$} \\
\hline 1.0000 & 0.9975 & 0.002515 \\
${ }^{\mathrm{a}}{ }_{x_{2}{ }^{(\mathrm{s})}: \text { initial mole fraction of component } 2 \text { in the solution. }}$ \\
${ }^{\mathrm{b}} x_{2}:$ mole fraction of component 2 in the saturated solution. \\
${ }^{\mathrm{c}}{ }_{x_{1}: \text { mole fraction solubility of the solute. }}$
\end{tabular}

\section{Auxiliary Information}

\section{Method/Apparatus/Procedure:}

Constant-temperature bath, calorimetric thermometer, and an ultraviolet/ visible spectrophotometer.

Excess solute and solvent were placed in amber glass bottles and allowed to equilibrate for several days at constant temperature. Attainment of equilibrium was verified by several repetitive measurements and by approaching equilibrium from supersaturation. Aliquots of saturated solutions were transferred through a coarse filter into tared volumetric flasks, weighed and diluted with methanol. Concentrations were determined by spectrophotometric measurements at $356 \mathrm{~nm}$.

\section{Source and Purity of Chemicals:}

(1) $99+\%$, Aldrich Chemical Company, Milwaukee, WI, USA, was recrystallized several times from propanone.

(2) $99 \%$, anhydrous, Aldrich Chemical Company, stored over molecular sieves before use.

\section{Estimated Error:}

Temperature: $\pm 0.1 \mathrm{~K}$.

$x_{1}: \pm 1.5 \%$ (relative error).

\begin{tabular}{|c|c|}
\hline $\begin{array}{l}\text { Components: } \\
\text { (1) Anthracene; } \mathrm{C}_{14} \mathrm{H}_{10} ;[120-12-7] \\
\text { (2) 2-Methoxy-2-methylpropane; } \mathrm{C}_{5} \mathrm{H}_{12} \mathrm{O} \text {; } \\
{[1634-04-4]}\end{array}$ & $\begin{array}{l}\text { Original Measurements: } \\
{ }^{20} \text { J. R. Powell, M. E. R. } \\
\text { McHale, A.-S. M. Kauppila, } \\
\text { and W. E. Acree, Jr., J. Chem. } \\
\text { Thermodyn. 28, } 1215 \text { (1996). }\end{array}$ \\
\hline $\begin{array}{l}\text { Variables: } \\
T / \mathrm{K}=298.15\end{array}$ & $\begin{array}{l}\text { Prepared by: } \\
\text { W. E. Acree, Jr. }\end{array}$ \\
\hline
\end{tabular}


Experimental Values

\begin{tabular}{lcc}
\hline$\frac{x_{2}{ }^{(\mathrm{s}) \mathrm{a}}}{x_{2}{ }^{\mathrm{b}}}$ & $x_{1}{ }^{\mathrm{c}}$ \\
\hline 1.0000 & 0.9969 & 0.003050 \\
$\frac{{ }^{\mathrm{a}} x_{2}{ }^{(\mathrm{s})}: \text { initial mole fraction of component } 2 \text { in the solution. }}{{ }^{\mathrm{b}}{ }_{x_{2}}: \text { mole fraction of component } 2 \text { in the saturated solution. }}$ \\
${ }^{\mathrm{c}} x_{1}:$ mole fraction solubility of the solute.
\end{tabular}

\section{Auxiliary Information}

\begin{abstract}
Method/Apparatus/Procedure:
Constant-temperature bath, calorimetric thermometer, and an ultraviolet/ visible spectrophotometer.

Excess solute and solvent were placed in amber glass bottles and allowed to equilibrate for several days at constant temperature. Attainment of equilibrium was verified by several repetitive measurements and by approaching equilibrium from supersaturation. Aliquots of saturated solutions were transferred through a coarse filter into tared volumetric flasks, weighed and diluted with methanol. Concentrations were determined by spectrophotometric measurements at $356 \mathrm{~nm}$.
\end{abstract}

Source and Purity of Chemicals:

(1) $99.9+\%$, Gold Label, Aldrich Chemical Company, Milwaukee, WI, USA, was used as received.

(2) $99.9+\%$, Arco Chemical Company, USA, was stored over anhydrous sodium sulfate and molecular sieves before being fractionally distilled.

\section{Estimated Error:}

Temperature: $\pm 0.1 \mathrm{~K}$.

$x_{1}: \pm 1.8 \%$ (relative error).

\begin{tabular}{ll}
\hline \hline Components: & Original Measurements: \\
(1) Anthracene; $\mathrm{C}_{14} \mathrm{H}_{10} ;[120-12-7]$ & ${ }^{21}$ K. S. Coym, L. E. Roy, C. E. \\
$(2) 1,1^{\prime}-$ Oxybis[2-methoxyethane]; & Hernández, and W. E. Acree, Jr., \\
$\mathrm{C}_{6} \mathrm{H}_{14} \mathrm{O}_{3} ;[111-96-6]$ & Chem. Eng. Commun. 162, 215 \\
& (1997). \\
\hline Variables: & Prepared by: \\
$T / \mathrm{K}=298.15$ & W. E. Acree, Jr. \\
\hline
\end{tabular}

Experimental Values

\begin{tabular}{|c|c|c|}
\hline$x_{2}^{(\mathrm{s}) \mathrm{a}}$ & $x_{2}{ }^{\mathrm{b}}$ & $x_{1}{ }^{\mathrm{c}}$ \\
\hline 1.0000 & 0.9886 & 0.01139 \\
\hline
\end{tabular}

\section{Auxiliary Information}

\section{Method/Apparatus/Procedure:}

Constant-temperature bath, calorimetric thermometer, and an ultraviolet/ visible spectrophotometer.

Excess solute and solvent were placed in amber glass bottles and allowed to equilibrate for several days at constant temperature. Attainment of equilibrium was verified by several repetitive measurements and by approaching equilibrium from supersaturation. Aliquots of saturated solutions were transferred through a coarse filter into tared volumetric flasks, weighed and diluted with methanol. Concentrations were determined by spectrophotometric measurements at $356 \mathrm{~nm}$.
Source and Purity of Chemicals:

(1) $99.9+\%$, Acros Organics, USA, was recrystallized several times from propanone.

(2) $99.5 \%$, anhydrous, Aldrich Chemical Company, Milwaukee, WI, USA, was stored over molecular sieves before use.

\section{Estimated Error:}

Temperature: $\pm 0.1 \mathrm{~K}$.

$x_{1}: \pm 1.5 \%$ (relative error).

\subsection{Anthracene solubility data in haloalkanes, haloalkenes, and haloaromatic hydrocarbons}

\begin{tabular}{ll}
\hline \hline Components: & Original Measurements: \\
(1) Anthracene; $\mathrm{C}_{14} \mathrm{H}_{10} ;[120-12-7]$ & ${ }^{16}$ L. E. Roy, C. E. Hernández, and \\
(2) Dichloromethane; $\mathrm{CH}_{2} \mathrm{Cl}_{2} ;$ & W. E. Acree, Jr., Polycyclic \\
[75-09-2] & Aromat. Compd. 13, 105 (1999). \\
\hline Variables: & Prepared by: \\
$T / \mathrm{K}=298.15$ & W. E. Acree, Jr. \\
\hline
\end{tabular}

Experimental Values

\begin{tabular}{lcc}
\hline \hline$x_{2}{ }^{(\mathrm{s}) \mathrm{a}}$ & $x_{2}{ }^{\mathrm{b}}$ & $x_{1}{ }^{\mathrm{c}}$ \\
\hline 1.0000 & 0.9061 & 0.009387
\end{tabular}

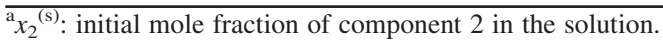

${ }^{\mathrm{b}} \mathrm{x}_{2}$ : mole fraction of component 2 in the saturated solution.

${ }^{c} x_{1}$ : mole fraction solubility of the solute.

\section{Auxiliary Information}

\section{Method/Apparatus/Procedure:}

Constant-temperature bath, calorimetric thermometer, and an ultraviolet/ visible spectrophotometer.

Excess solute and solvent were placed in amber glass bottles and allowed to equilibrate for several days at constant temperature. Attainment of equilibrium was verified by several repetitive measurements and by approaching equilibrium from supersaturation. Aliquots of saturated solutions were transferred through a coarse filter into tared volumetric flasks, weighed and diluted with 2-propanol. Concentrations were determined by spectrophotometric measurements at $356 \mathrm{~nm}$.

\section{Source and Purity of Chemicals:}

(1) $99+\%$, Aldrich Chemical Company, Milwaukee, WI, USA, was recrystallized several times from propanone.

(2) $99.9+\%$, HPLC Grade, Aldrich Chemical Company, stored over molecular sieves before use.

\section{Estimated Error:}

Temperature: $\pm 0.1 \mathrm{~K}$.

$x_{1}: \pm 1.5 \%$ (relative error).

\begin{tabular}{ll}
\hline \hline Components: & Original Measurements: \\
(1) Anthracene; $\mathrm{C}_{14} \mathrm{H}_{10} ;[120-12-7]$ & ${ }^{16}$ L. E. Roy, C. E. Hernández, and \\
(2) Trichloromethane; $\mathrm{CHCl}_{3} ;$ & W. E. Acree, Jr., Polycyclic \\
[67-66-3] & Aromat. Compd. 13, 105 (1999). \\
\hline Variables: & Prepared by: \\
$T / \mathrm{K}=298.15$ & W. E. Acree, Jr. \\
\hline
\end{tabular}


Experimental Values

\begin{tabular}{lcc}
\hline$\frac{x_{2}{ }^{(\mathrm{s}) \mathrm{a}}}{x_{2}{ }^{\mathrm{b}}}$ & $x_{1}{ }^{\mathrm{c}}$ \\
\hline 1.0000 & 0.9892 & 0.01084 \\
$\frac{{ }^{\mathrm{a}} x_{2}{ }^{(\mathrm{s})}: \text { initial mole fraction of component } 2 \text { in the solution. }}{{ }^{\mathrm{b}}{ }_{x_{2}}: \text { mole fraction of component } 2 \text { in the saturated solution. }}$ \\
${ }^{\mathrm{c}}{ }_{x_{1}}:$ mole fraction solubility of the solute.
\end{tabular}

\section{Auxiliary Information}

\section{Method/Apparatus/Procedure:}

Constant-temperature bath, calorimetric thermometer, and an ultraviolet/ visible spectrophotometer.

Excess solute and solvent were placed in amber glass bottles and allowed to equilibrate for several days at constant temperature. Attainment of equilibrium was verified by several repetitive measurements and by approaching equilibrium from supersaturation. Aliquots of saturated solutions were transferred through a coarse filter into tared volumetric flasks, weighed and diluted with 2-propanol. Concentrations were determined by spectrophotometric measurements at $356 \mathrm{~nm}$.

\section{Source and Purity of Chemicals:}

(1) $99+\%$, Aldrich Chemical Company, Milwaukee, WI, USA, was recrystallized several times from propanone.

(2) $99.9+\%$, HPLC Grade, Aldrich Chemical Company, was shaken several times with an aqueous sodium hydroxide solution and then with distilled water to remove the ethanol stabilizer. The resulting solution was dried over both anhydrous calcium chloride and molecular sieves, and then distilled shortly before use.

Estimated Error:

Temperature: $\pm 0.1 \mathrm{~K}$.

$x_{1}: \pm 1.5 \%$ (relative error)

\begin{tabular}{ll}
\hline \hline Components: & Original Measurements: \\
(1) Anthracene; $\mathrm{C}_{14} \mathrm{H}_{10} ;[120-12-7]$ & ${ }^{16}$ L. E. Roy, C. E. Hernández, and \\
$(2) 1-C h l o r o h e x a n e ; \mathrm{C}_{6} \mathrm{H}_{13} \mathrm{Cl} ;$ & W. E. Acree, Jr. Polycyclic \\
[544-10-5] & Aromat. Compd. 13, 105 (1999). \\
\hline Variables: & Prepared by: \\
$T / \mathrm{K}=298.15$ & W. E. Acree, Jr. \\
\hline
\end{tabular}

Experimental Values

\begin{tabular}{|c|c|c|}
\hline$x_{2}{ }^{(\mathrm{s}) \mathrm{a}}$ & $x_{2}{ }^{\mathrm{b}}$ & $x_{1}{ }^{\mathrm{c}}$ \\
\hline 1.0000 & 0.9928 & 0.007177 \\
\hline
\end{tabular}

\section{Auxiliary Information}

\section{Method/Apparatus/Procedure:}

Constant-temperature bath, calorimetric thermometer, and an ultraviolet/ visible spectrophotometer.

Excess solute and solvent were placed in amber glass bottles and allowed to equilibrate for several days at constant temperature. Attainment of equilibrium was verified by several repetitive measurements and by approaching equilibrium from supersaturation. Aliquots of saturated solutions were transferred through a coarse filter into tared volumetric flasks, weighed and diluted with 2-propanol. Concentrations were determined by spectrophotometric measurements at $356 \mathrm{~nm}$.

\section{Source and Purity of Chemicals:}

(1) $99+\%$, Aldrich Chemical Company, Milwaukee, WI, USA, was recrystallized several times from propanone.

(2) $99 \%$, Aldrich Chemical Company, stored over molecular sieves before use.

\section{Estimated Error:}

Temperature: $\pm 0.1 \mathrm{~K}$.

$x_{1}: \pm 1.5 \%$ (relative error).

\begin{tabular}{ll}
\hline \hline Components: & Original Measurements: \\
(1) Anthracene; $\mathrm{C}_{14} \mathrm{H}_{10} ;[120-12-7]$ & ${ }^{16}$ L. E. Roy, C. E. Hernández, and \\
(2) Chlorobenzene; $\mathrm{C}_{6} \mathrm{H}_{5} \mathrm{Cl} ;$ & W. E. Acree, Jr., Polycyclic \\
{$[108-90-7]$} & Aromat. Compd. 13, 105 (1999). \\
\hline Variables: & Prepared by: \\
$T / \mathrm{K}=298.15$ & W. E. Acree, Jr. \\
\hline
\end{tabular}

Experimental Values

\begin{tabular}{lcc}
\hline \hline$x_{2}{ }^{(\mathrm{s}) \mathrm{a}}$ & $x_{2}{ }^{\mathrm{b}}$ & $x_{1}{ }^{\mathrm{c}}$ \\
\hline 1.0000 & 0.9900 & 0.009962 \\
${ }^{\mathrm{a}} x_{2}{ }^{(\mathrm{s})}:$ initial mole fraction of component 2 in the solution. \\
${ }^{\mathrm{b}} x_{2}:$ mole fraction of component 2 in the saturated solution. \\
${ }^{\mathrm{c}} x_{1}:$ mole fraction solubility of the solute.
\end{tabular}

\section{Auxiliary Information}

\section{Method/Apparatus/Procedure:}

Constant-temperature bath, calorimetric thermometer, and an ultraviolet/ visible spectrophotometer.

Excess solute and solvent were placed in amber glass bottles and allowed to equilibrate for several days at constant temperature. Attainment of equilibrium was verified by several repetitive measurements and by approaching equilibrium from supersaturation. Aliquots of saturated solutions were transferred through a coarse filter into tared volumetric flasks, weighed and diluted with 2-propanol. Concentrations were determined by spectrophotometric measurements at $356 \mathrm{~nm}$.

\section{Source and Purity of Chemicals:}

(1) $99+\%$, Aldrich Chemical Company, Milwaukee, WI, USA, was recrystallized several times from propanone.

(2) $99.9 \%$, HPLC Grade, Aldrich Chemical Company, stored over molecular sieves before use.

Estimated Error:

Temperature: $\pm 0.1 \mathrm{~K}$.

$x_{1}: \pm 1.5 \%$ (relative error). 


\section{Components:}

(1) Anthracene; $\mathrm{C}_{14} \mathrm{H}_{10} ;[120-12-7]$

(2) Fluorobenzene; $\mathrm{C}_{6} \mathrm{H}_{5} \mathrm{~F}$; [462-06-6]

Original Measurements:

${ }^{22}$ M. H. Abraham, W. E. Acree,

Jr., A. J. Leo, and D. Hoekman, New J. Chem. 33, 1685 (2009).

\section{Variables:}

$T / \mathrm{K}=298.15$

Prepared by:

W. E. Acree, Jr.

\section{Experimental Values}

\begin{tabular}{|c|c|c|}
\hline$x_{2}{ }^{(\mathrm{s}) \mathrm{a}}$ & $x_{2}{ }^{b}$ & $x_{1}{ }^{\mathrm{c}}$ \\
\hline 1.0000 & 0.9932 & 0.006764 \\
\hline
\end{tabular}

The experimental value was reported as the logarithm of anthracene's molar solubility in fluorobenzene dissolved by the solute's molar solubility in water. Experimental mole fraction solubility was obtained from the authors.

\section{Auxiliary Information}

\section{Method/Apparatus/Procedure:}

Constant-temperature bath, calorimetric thermometer, and an ultraviolet/ visible spectrophotometer.

Excess solute and solvent were placed in amber glass bottles and allowed to equilibrate for several days at constant temperature. Attainment of equilibrium was verified by several repetitive measurements and by approaching equilibrium from supersaturation. Aliquots of saturated solutions were transferred through a coarse filter into tared volumetric flasks, weighed and diluted with 2-propanol. Concentrations were determined by spectrophotometric measurements at $356 \mathrm{~nm}$.

\section{Source and Purity of Chemicals:}

(1) $99+\%$, Aldrich Chemical Company, Milwaukee, WI, USA, was recrystallized several times from propanone.

(2) $99 \%$, Aldrich Chemical Company, stored over molecular sieves before use

\section{Estimated Error:}

Temperature: $\pm 0.1 \mathrm{~K}$

$x_{1}: \pm 1.5 \%$ (relative error).

\section{Components:}

(1) Anthracene; $\mathrm{C}_{14} \mathrm{H}_{10} ;[120-12-7]$

(2) (Trifluoromethyl)benzene; $\mathrm{C}_{7} \mathrm{H}_{5} \mathrm{~F}_{3}$; [98-08-8]

Variables:
$T / \mathrm{K}=298.15$

Original Measurements: W. E. Acree, Jr., unpublished data.
Prepared by:

W. E. Acree, Jr.

\section{Experimental Values}

\begin{tabular}{lcc}
\hline \hline$x_{2}{ }^{(\mathrm{s}) \mathrm{a}}$ & $x_{2}{ }^{\mathrm{b}}$ & $x_{1}{ }^{\mathrm{c}}$ \\
\hline 1.0000 & 0.9961 & 0.003864 \\
$\frac{{ }^{a} x_{2}{ }^{(\mathrm{s})}: \text { initial mole fraction of component } 2 \text { in the solution. }}{{ }^{\mathrm{b}} x_{2}: \text { mole fraction of component } 2 \text { in the saturated solution. }}$ \\
${ }^{c} x_{1}:$ mole fraction solubility of the solute.
\end{tabular}

\section{Auxiliary Information}

\section{Method/Apparatus/Procedure:}

Constant-temperature bath, calorimetric thermometer, and an ultraviolet/ visible spectrophotometer.

Excess solute and solvent were placed in amber glass bottles and allowed to equilibrate for several days at constant temperature. Attainment of equilibrium was verified by several repetitive measurements and by approaching equilibrium from supersaturation. Aliquots of saturated solutions were transferred through a coarse filter into tared volumetric flasks, weighed and diluted with 2-propanol. Concentrations were determined by spectrophotometric measurements at $356 \mathrm{~nm}$.

\section{Source and Purity of Chemicals:}

(1) $99+\%$, Aldrich Chemical Company, Milwaukee, WI, USA, was recrystallized several times from propanone.

(2) $99 \%$, Aldrich Chemical Company, stored over molecular sieves before use.

Estimated Error:

Temperature: $\pm 0.1 \mathrm{~K}$.

$x_{1}: \pm 1.5 \%$ (relative error).

\subsection{Anthracene solubility data in alcohols}

\begin{tabular}{ll}
\hline \hline Components: & Original Measurements: \\
(1) Anthracene; $\mathrm{C}_{14} \mathrm{H}_{10} ;[120-12-7]$ & ${ }^{16}$ L. E. Roy, C. E. Hernández, and \\
(2) Methanol; $\mathrm{CH}_{4} \mathrm{O} ;[67-56-1]$ & W. E. Acree, Jr., Polycyclic \\
& Aromat. Compd. 13, 105 (1999). \\
\hline Variables: & Prepared by: \\
$T / \mathrm{K}=298.15$ & W. E. Acree, Jr. \\
\hline
\end{tabular}

Experimental Values

\begin{tabular}{lcc}
\hline \hline$x_{2}{ }^{(\mathrm{s}) \mathrm{a}}$ & $x_{2}{ }^{\mathrm{b}}$ & $x_{1}{ }^{\mathrm{c}}$ \\
\hline 1.0000 & 0.9998 & 0.000243
\end{tabular}

${ }^{\mathrm{a}} x_{2}{ }^{(\mathrm{s})}$ : initial mole fraction of component 2 in the solution.

${ }^{\mathrm{b}} x_{2}$ : mole fraction of component 2 in the saturated solution.

${ }^{c} x_{1}$ : mole fraction solubility of the solute.

\section{Auxiliary Information}

\section{Method/Apparatus/Procedure:}

Constant-temperature bath, calorimetric thermometer, and an ultraviolet/ visible spectrophotometer.

Excess solute and solvent were placed in amber glass bottles and allowed to equilibrate for several days at constant temperature. Attainment of equilibrium was verified by several repetitive measurements and by approaching equilibrium from supersaturation. Aliquots of saturated solutions were transferred through a coarse filter into tared volumetric flasks, weighed and diluted with 2-propanol. Concentrations were determined by spectrophotometric measurements at $356 \mathrm{~nm}$.

Source and Purity of Chemicals:

(1) $99+\%$, Aldrich Chemical Company, Milwaukee, WI, USA, was recrystallized several times from propanone.

(2) $99.9+\%$, Aldrich Chemical Company, stored over molecular sieves before use. 
Estimated Error:

Temperature: $\pm 0.1 \mathrm{~K}$

$x_{1}: \pm 1.5 \%$ (relative error).

\begin{tabular}{l}
\hline Components: \\
(1) Anthracene; $\mathrm{C}_{14} \mathrm{H}_{10} ;[120-12-7]$ \\
(2) Ethanol; $\mathrm{C}_{2} \mathrm{H}_{6} \mathrm{O} ;[64-17-5]$
\end{tabular}

Original Measurements:

${ }^{16}$ L. E. Roy, C. E. Hernández, and W. E. Acree, Jr., Polycyclic Aromat. Compd. 13, 105 (1999).

Variables:

$T / \mathrm{K}=298.15$

Prepared by:

W. E. Acree, Jr.

Experimental Values

\begin{tabular}{lcc}
\hline \hline$x_{2}{ }^{(\mathrm{s}) \mathrm{a}}$ & $x_{2}{ }^{\mathrm{b}}$ & $x_{1}{ }^{\mathrm{c}}$ \\
\hline 1.0000 & 0.9995 & 0.000460 \\
\hline
\end{tabular}

${ }^{\mathrm{a}} x_{2}{ }^{(\mathrm{s})}$ : initial mole fraction of component 2 in the solution.

${ }^{b} x_{2}$ : mole fraction of component 2 in the saturated solution.

${ }^{c} x_{1}$ : mole fraction solubility of the solute.

\section{Auxiliary Information}

\section{Method/Apparatus/Procedure:}

Constant-temperature bath, calorimetric thermometer, and an ultraviolet/ visible spectrophotometer.

Excess solute and solvent were placed in amber glass bottles and allowed to equilibrate for several days at constant temperature. Attainment of equilibrium was verified by several repetitive measurements and by approaching equilibrium from supersaturation. Aliquots of saturated solutions were transferred through a coarse filter into tared volumetric flasks, weighed and diluted with 2-propanol. Concentrations were determined by spectrophotometric measurements at $356 \mathrm{~nm}$.

\section{Source and Purity of Chemicals:}

(1) $99+\%$, Aldrich Chemical Company, Milwaukee, WI, USA, was recrystallized several times from propanone.

(2) Absolute, Aaper Alcohol and Chemical Company, USA, stored over molecular sieves before use.

\section{Estimated Error:}

Temperature: $\pm 0.1 \mathrm{~K}$.

$x_{1}: \pm 1.5 \%$ (relative error).

\section{Components:}

(1) Anthracene; $\mathrm{C}_{14} \mathrm{H}_{10} ;[120-12-7]$

(2) Ethanol; $\mathrm{C}_{2} \mathrm{H}_{6} \mathrm{O}$; [64-17-5]

\section{Original Measurements:}

${ }^{34}$ A. Shayanfar, S. H. Eghrary, F. Sardari, W. E. Acree, Jr., and A. Jouyban, J. Chem. Eng. Data 56, 2290 (2011).

\begin{tabular}{ll}
\hline Variables: & Prepared by: \\
Temperature & W. E. Acree, Jr. \\
\hline
\end{tabular}

Experimental Values

\begin{tabular}{lcc}
\hline \hline$T / \mathrm{K}$ & $x_{2}{ }^{\mathrm{a}}$ & $x_{1}{ }^{\mathrm{b}}$ \\
\hline 298.2 & 0.9995 & 0.000505 \\
308.2 & 0.9993 & 0.000665 \\
318.2 & 0.9992 & 0.000780 \\
\hline${ }^{\mathrm{a}} x_{2}:$ mole fraction of component 2 in the saturated solution. \\
${ }^{\mathrm{b}} x_{1}:$ mole fraction of the polycyclic aromatic hydrocarbon (component 1$)$.
\end{tabular}

\section{Auxiliary Information}

\section{Method/Apparatus/Procedure:}

Incubator, shaker, and an ultraviolet/visible spectrophotometer.

Solubility was determined by equilibrating an excess amount of solid naphthalene with the organic solvent using a shaker placed in an incubator equipped with a temperature controlling system. After an equilibration period of at least $48 \mathrm{~h}$ the samples were withdrawn and filtered through hydrophobic Durapore filters $(0.45 \mu \mathrm{m})$. The filtered sample was diluted quantitatively with methanol. Concentrations were determined by spectrophotmetric analysis at $356 \mathrm{~nm}$.

\section{Source and Purity of Chemicals:}

(1) $96 \%$, Fluka Chemical Company, was recrystallized several from propanone and ethyl ethanoate before use.

(2) $99+\%$, Absolute, Merck Chemicals, Germany, no information given regarding any further purification.

\section{Estimated Error:}

Temperature: $\pm 0.2 \mathrm{~K}$.

$x_{1}: \pm 2.7 \%$ (relative uncertainty)

\begin{tabular}{ll}
\hline \hline Components: & Original Measurements: \\
(1) Anthracene; $\mathrm{C}_{14} \mathrm{H}_{10} ;[120-12-7]$ & ${ }^{23}$ K. M. De Fina, T. T. Van, A. \\
(2) 2-Methyl-2-propanol; $\mathrm{C}_{4} \mathrm{H}_{10} \mathrm{O} ;$ & Ibarra, E. Hamilton, J. Martinez, \\
[75-65-0] & A. Valdez, and W. E. Acree, Jr., \\
& Phys. Chem. Liq. 39, 249 (2001). \\
\hline Variables: & Prepared by: \\
$T / \mathrm{K}=298.15$ & W. E. Acree, Jr. \\
\hline
\end{tabular}

\section{Experimental Values}

\begin{tabular}{|c|c|c|}
\hline$x_{2}{ }^{(\mathrm{s}) \mathrm{a}}$ & $x_{2}{ }^{b}$ & $x_{1}{ }^{\mathrm{c}}$ \\
\hline 1.0000 & 0.9996 & 0.000430 \\
\hline
\end{tabular}




\section{Auxiliary Information}

\section{Method/Apparatus/Procedure:}

Constant-temperature bath, calorimetric thermometer, and an ultraviolet/ visible spectrophotometer.

Excess solute and solvent were placed in amber glass bottles and allowed to equilibrate for several days at constant temperature. Attainment of equilibrium was verified by several repetitive measurements and by approaching equilibrium from supersaturation. Aliquots of saturated solutions were transferred through a coarse filter into tared volumetric flasks, weighed and diluted with methanol. Concentrations were determined by

spectrophotometric measurements at $356 \mathrm{~nm}$.

Source and Purity of Chemicals:

(1) $99.9+\%$, Aldrich Chemical Company, Milwaukee, WI, USA, was used as received.

(2) $99+\%$, Arco Chemical Company, USA, stored over molecular sieves and distilled shortly before use.

Estimated Error:

Temperature: $\pm 0.1 \mathrm{~K}$.

$x_{1}: \pm 1.5 \%$ (relative error)

\section{Components:}

(1) Anthracene; $\mathrm{C}_{14} \mathrm{H}_{10} ;[120-12-7]$

(2) 1-Pentanol; $\mathrm{C}_{5} \mathrm{H}_{12} \mathrm{O}$; [71-41-0]

\begin{tabular}{ll}
\hline Variables: & Prepared by: \\
$T / \mathrm{K}=298.15$ & W. E. Acree, Jr. \\
\hline
\end{tabular}

Experimental Values

\begin{tabular}{lcc}
\hline \hline$\frac{x_{2}{ }^{(\mathrm{s}) \mathrm{a}}}{x_{2}{ }^{\mathrm{b}}}$ & $x_{1}{ }^{\mathrm{c}}$ \\
\hline 1.0000 & 0.9989 & 0.001097 \\
$\frac{{ }^{\mathrm{a}} x_{2}{ }^{(\mathrm{s})}: \text { initial mole fraction of component } 2 \text { in the solution. }}{{ }^{\mathrm{b}} x_{2}: \text { mole fraction of component } 2 \text { in the saturated solution. }}$ \\
${ }^{\mathrm{c}} x_{1}:$ mole fraction solubility of the solute.
\end{tabular}

\section{Auxiliary Information}

\section{Method/Apparatus/Procedure:}

Constant-temperature bath, calorimetric thermometer, and an ultraviolet/ visible spectrophotometer.

Excess solute and solvent were placed in amber glass bottles and allowed to equilibrate for several days at constant temperature. Attainment of equilibrium was verified by several repetitive measurements and by approaching equilibrium from supersaturation. Aliquots of saturated solutions were transferred through a coarse filter into tared volumetric flasks, weighed and diluted with methanol. Concentrations were determined by

spectrophotometric measurements at $356 \mathrm{~nm}$.

\section{Source and Purity of Chemicals:}

(1) $99+\%$, Aldrich Chemical Company, Milwaukee, WI, USA, was used as received.

(2) $99 \%$, Aldrich Chemical Company, stored over anhydrous sodium sulfate and molecular sieves before being fractionally distilled.

\section{Estimated Error:}

Temperature: $\pm 0.1 \mathrm{~K}$.

$x_{1}: \pm 1.3 \%$ (relative error).

\begin{tabular}{ll}
\hline \hline Components: & Original Measurements: \\
(1) Anthracene; $\mathrm{C}_{14} \mathrm{H}_{10} ;[120-12-7]$ & ${ }^{24}$ J. R. Powell, M. E. R. McHale, \\
(2) 2-Pentanol; $\mathrm{C}_{5} \mathrm{H}_{12} \mathrm{O} ;[6032-29-7]$ & A.-S. M. Kauppila, and W. E. \\
& Acree, Jr., J. Chem. Eng. Data 41, \\
& 728 (1996). \\
\hline Variables: & Prepared by: \\
$T / \mathrm{K}=298.15$ & W. E. Acree, Jr. \\
\hline
\end{tabular}

Experimental Values

\begin{tabular}{lcc}
\hline \hline$x_{2}{ }^{(\mathrm{s}) \mathrm{a}}$ & $x_{2}{ }^{\mathrm{b}}$ & $x_{1}{ }^{\mathrm{c}}$ \\
\hline 1.0000 & 0.9992 & 0.000800 \\
\hline
\end{tabular}

${ }^{\mathrm{a}} x_{2}{ }^{(\mathrm{s})}$ : initial mole fraction of component 2 in the solution.

${ }^{b} x_{2}$ : mole fraction of component 2 in the saturated solution.

${ }^{c} x_{1}$ : mole fraction solubility of the solute.

\section{Auxiliary Information}

\section{Method/Apparatus/Procedure:}

Constant-temperature bath, calorimetric thermometer, and an ultraviolet/ visible spectrophotometer.

Excess solute and solvent were placed in amber glass bottles and allowed to equilibrate for several days at constant temperature. Attainment of equilibrium was verified by several repetitive measurements and by approaching equilibrium from supersaturation. Aliquots of saturated solutions were transferred through a coarse filter into tared volumetric flasks, weighed and diluted with methanol. Concentrations were determined by spectrophotometric measurements at $356 \mathrm{~nm}$.

\section{Source and Purity of Chemicals:}

(1) $99+\%$, Aldrich Chemical Company, Milwaukee, WI, USA, was used as received.

(2) $99+\%$, Acros Organics, USA, stored over anhydrous sodium sulfate and molecular sieves before being fractionally distilled.

\section{Estimated Error:}

Temperature: $\pm 0.1 \mathrm{~K}$.

$x_{1}: \pm 1.3 \%$ (relative error)

\begin{tabular}{ll}
\hline \hline Components: & Original Measurements: \\
(1) Anthracene; $\mathrm{C}_{14} \mathrm{H}_{10} ;[120-12-7]$ & ${ }^{25} \mathrm{C}$. I. Monárrez, P. G. Taylor, \\
(2) 2-Methyl-1-butanol; $\mathrm{C}_{5} \mathrm{H}_{12} \mathrm{O} ;$ & A. M. Tran, and W. E. Acree, Jr., J. \\
[137-32-6] & Chem. Eng. Data 48, 1341 (2003). \\
\hline Variables: & Prepared by: \\
$T / \mathrm{K}=298.15$ & W. E. Acree, Jr. \\
\hline
\end{tabular}

Experimental Values

\begin{tabular}{lcc}
\hline \hline$x_{2}{ }^{(\mathrm{s}) \mathrm{a}}$ & $x_{2}{ }^{\mathrm{b}}$ & $x_{1}{ }^{\mathrm{c}}$ \\
\hline 1.0000 & 0.9992 & 0.000786 \\
$\frac{{ }^{\mathrm{a}} x_{2}{ }^{(\mathrm{s})}: \text { initial mole fraction of component } 2 \text { in the solution. }}{{ }^{\mathrm{b}} x_{2}: \text { mole fraction of component } 2 \text { in the saturated solution. }}$ \\
${ }^{{ }^{c} x_{1}: \text { mole fraction solubility of the solute. }}$
\end{tabular}




\section{Auxiliary Information}

\section{Method/Apparatus/Procedure:}

Constant-temperature bath, calorimetric thermometer, and an ultraviolet/ visible spectrophotometer.

Excess solute and solvent were placed in amber glass bottles and allowed to equilibrate for several days at constant temperature. Attainment of equilibrium was verified by several repetitive measurements and by approaching equilibrium from supersaturation. Aliquots of saturated solutions were transferred through a coarse filter into tared volumetric flasks, weighed and diluted with methanol. Concentrations were determined by spectrophotometric measurements at $356 \mathrm{~nm}$.

Source and Purity of Chemicals:

(1) $99 \%$, Aldrich Chemical Company, Milwaukee, WI, USA, was recrystallized several times from propanone.

(2) $99+\%$, Aldrich Chemical Company, stored over molecular sieves and distilled shortly before use.

Estimated Error:

Temperature: $\pm 0.1 \mathrm{~K}$.

$x_{1}: \pm 1.0 \%$ (relative error).

\begin{tabular}{ll}
\hline \hline Components: & Original Measurements: \\
(1) Anthracene; $\mathrm{C}_{14} \mathrm{H}_{10} ;[120-12-7]$ & ${ }^{16}$ L. E. Roy, C. E. Hernández, and \\
(2) 1-Hexanol; $\mathrm{C}_{6} \mathrm{H}_{14} \mathrm{O} ;[111-27-3]$ & W. E. Acree, Jr., Polycyclic \\
& Aromat. Compd. 13, 105 (1999). \\
\hline Variables: & Prepared by: \\
$T / \mathrm{K}=298.15$ & W. E. Acree, Jr. \\
\hline
\end{tabular}

Experimental Values

\begin{tabular}{|c|c|c|}
\hline$x_{2}{ }^{(\mathrm{s}) \mathrm{a}}$ & $x_{2}{ }^{b}$ & $x_{1}{ }^{\mathrm{c}}$ \\
\hline 1.0000 & 0.9985 & 0.001483 \\
\hline
\end{tabular}

\section{Auxiliary Information}

\section{Method/Apparatus/Procedure:}

Constant-temperature bath, calorimetric thermometer, and an ultraviolet/ visible spectrophotometer.

Excess solute and solvent were placed in amber glass bottles and allowed to equilibrate for several days at constant temperature. Attainment of equilibrium was verified by several repetitive measurements and by approaching equilibrium from supersaturation. Aliquots of saturated solutions were transferred through a coarse filter into tared volumetric flasks, weighed and diluted with 2-propanol. Concentrations were determined by spectrophotometric measurements at $356 \mathrm{~nm}$.

Source and Purity of Chemicals:

(1) $99+\%$, Aldrich Chemical Company, Milwaukee, WI, USA, was recrystallized several times from propanone.

(2) $99+\%$, Alfa Aesar, USA, stored over molecular sieves before use.

\section{Estimated Error:}

Temperature: $\pm 0.1 \mathrm{~K}$.

$x_{1}: \pm 1.5 \%$ (relative error).

\begin{tabular}{ll}
\hline \hline Components: & Original Measurements: \\
(1) Anthracene; $\mathrm{C}_{14} \mathrm{H}_{10} ;[120-12-7]$ & ${ }^{26}$ J. R. Powell, K. A. Fletcher, K. \\
(2) 2-Methyl-1-pentanol; $\mathrm{C}_{6} \mathrm{H}_{14} \mathrm{O} ;$ & S. Coym, W. E. Acree, Jr., V. G. \\
[105-30-6] & Varanasi, and S. W. Campbell, Int. \\
& J. Thermophys. 18, 1495 (1997). \\
\hline Variables: & Prepared by: \\
$T / \mathrm{K}=298.15$ & W. E. Acree, Jr. \\
\hline
\end{tabular}

Experimental Values

\begin{tabular}{lcc}
\hline \hline$x_{2}{ }^{(\mathrm{s}) \mathrm{a}}$ & $x_{2}{ }^{\mathrm{b}}$ & $x_{1}{ }^{\mathrm{c}}$ \\
\hline 1.0000 & 0.9990 & 0.000996
\end{tabular}

${ }^{\mathrm{a}} x_{2}{ }^{(\mathrm{s})}$ : initial mole fraction of component 2 in the solution.

${ }^{b} x_{2}$ : mole fraction of component 2 in the saturated solution.

${ }^{c} x_{1}$ : mole fraction solubility of the solute.

\section{Auxiliary Information}

\section{Method/Apparatus/Procedure:}

Constant-temperature bath, calorimetric thermometer, and an ultraviolet/ visible spectrophotometer.

Excess solute and solvent were placed in amber glass bottles and allowed to equilibrate for several days at constant temperature. Attainment of equilibrium was verified by several repetitive measurements and by approaching equilibrium from supersaturation. Aliquots of saturated solutions were transferred through a coarse filter into tared volumetric flasks, weighed and diluted with methanol. Concentrations were determined by spectrophotometric measurements at $356 \mathrm{~nm}$.

\section{Source and Purity of Chemicals:}

(1) $99+\%$, Acros Organics, USA, was recrystallized several times from propanone before use.

(2) 99\%, Aldrich Chemical Company, Milwaukee, WI, USA, stored over anhydrous sodium sulfate and molecular sieves before being fractionally distilled.

\section{Estimated Error:}

Temperature: $\pm 0.1 \mathrm{~K}$.

$x_{1}: \pm 1.5 \%$ (relative error).

\section{Components:}

\begin{tabular}{ll} 
& $728(1996)$. \\
\hline Variables: & Prepared by: \\
$T / \mathrm{K}=298.15$ & W. E. Acree, Jr. \\
\hline
\end{tabular}

Experimental Values

\begin{tabular}{lcc}
\hline \hline$x_{2}{ }^{(\mathrm{s}) \mathrm{a}}$ & $x_{2}{ }^{\mathrm{b}}$ & $x_{1}{ }^{\mathrm{c}}$ \\
\hline 1.0000 & 0.9992 & 0.000779 \\
$\frac{{ }^{a} x_{2}{ }^{(\mathrm{s})}: \text { initial mole fraction of component } 2 \text { in the solution. }}{{ }^{\mathrm{b}} x_{2}: \text { mole fraction of component } 2 \text { in the saturated solution. }}$ \\
${ }^{\mathrm{c}}{ }_{x_{1}: \text { mole fraction solubility of the solute. }}$
\end{tabular}

(1) Anthracene; $\mathrm{C}_{14} \mathrm{H}_{10} ;[120-12-7]$

(2) 4-Methyl-2-pentanol; $\mathrm{C}_{6} \mathrm{H}_{14} \mathrm{O}$;

[108-11-2]

Original Measurements:

${ }^{24}$ J. R. Powell, M. E. R. McHale, A.-S. M. Kauppila, and W. E. Acree, Jr., J. Chem. Eng. Data 41, Prepared by: 


\section{Auxiliary Information}

\section{Method/Apparatus/Procedure:}

Constant-temperature bath, calorimetric thermometer, and an ultraviolet/ visible spectrophotometer.

Excess solute and solvent were placed in amber glass bottles and allowed to equilibrate for several days at constant temperature. Attainment of equilibrium was verified by several repetitive measurements and by approaching equilibrium from supersaturation. Aliquots of saturated solutions were transferred through a coarse filter into tared volumetric flasks, weighed and diluted with methanol. Concentrations were determined by

spectrophotometric measurements at $356 \mathrm{~nm}$.

Source and Purity of Chemicals:

(1) $99+\%$, Aldrich Chemical Company, Milwaukee, WI, USA, was used as received.

(2) $99+\%$, Acros Organics, USA, stored over anhydrous sodium sulfate and molecular sieves before being fractionally distilled.

Estimated Error:

Temperature: $\pm 0.1 \mathrm{~K}$.

$x_{1}: \pm 1.3 \%$ (relative error)

\section{Components:}

(1) Anthracene; $\mathrm{C}_{14} \mathrm{H}_{10} ;[120-12-7]$

(2) 1-Heptanol; $\mathrm{C}_{7} \mathrm{H}_{16} \mathrm{O}$; [111-27-3]

Original Measurements:

${ }^{16}$ L. E. Roy, C. E. Hernández, and

W. E. Acree, Jr., Polycyclic

Aromat. Compd. 13, 105 (1999).

\section{Variables:}

$T / \mathrm{K}=298.15$

\section{Prepared by:}

W. E. Acree, Jr.

Experimental Values

\begin{tabular}{lcc}
\hline \hline$x_{2}{ }^{(\mathrm{s}) \mathrm{a}}$ & $x_{2}{ }^{\mathrm{b}}$ & $x_{1}{ }^{\mathrm{c}}$ \\
\hline 1.0000 & 0.9981 & 0.001869 \\
\hline
\end{tabular}

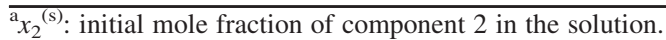

${ }^{b} x_{2}$ : mole fraction of component 2 in the saturated solution.

${ }^{\mathrm{c}} x_{1}$ : mole fraction solubility of the solute.

\section{Auxiliary Information}

\section{Method/Apparatus/Procedure:}

Constant-temperature bath, calorimetric thermometer, and an ultraviolet/ visible spectrophotometer.

Excess solute and solvent were placed in amber glass bottles and allowed to equilibrate for several days at constant temperature. Attainment of equilibrium was verified by several repetitive measurements and by approaching equilibrium from supersaturation. Aliquots of saturated solutions were transferred through a coarse filter into tared volumetric flasks, weighed and diluted with 2-propanol. Concentrations were determined by spectrophotometric measurements at $356 \mathrm{~nm}$

\section{Source and Purity of Chemicals:}

(1) $99+\%$, Aldrich Chemical Company, Milwaukee, WI, USA, was recrystallized several times from propanone.

(2) $99+\%$, Alfa Aesar, USA, stored over molecular sieves before use.

\section{Estimated Error:}

Temperature: $\pm 0.1 \mathrm{~K}$

$x_{1}: \pm 1.5 \%$ (relative error).

\begin{tabular}{ll}
\hline \hline Components: & Original Measurements: \\
(1) Anthracene; $\mathrm{C}_{14} \mathrm{H}_{10} ;[120-12-7]$ & ${ }^{27}$ J. R. Powell, M. E. R. McHale, \\
(2) 2-Ethyl-1-hexanol; $\mathrm{C}_{8} \mathrm{H}_{18} \mathrm{O} ;$ & A.-S. M. Kauppila, P. Otero, M. \\
[104-76-7] & Jayasekera, and W. E. Acree, Jr., J. \\
& Chem. Eng. Data 40, 1270 (1995). \\
\hline Variables: & Prepared by: \\
$T / \mathrm{K}=298.15$ & W. E. Acree, Jr. \\
\hline
\end{tabular}

Experimental Values

\begin{tabular}{lcc}
\hline \hline$x_{2}{ }^{(\mathrm{s}) \mathrm{a}}$ & $x_{2}{ }^{\mathrm{b}}$ & $x_{1}{ }^{\mathrm{c}}$ \\
\hline 1.0000 & 0.9986 & 0.001397 \\
\hline
\end{tabular}

${ }^{\mathrm{a}} x_{2}{ }^{(\mathrm{s})}$ : initial mole fraction of component 2 in the solution.

${ }^{b} x_{2}$ : mole fraction of component 2 in the saturated solution.

${ }^{c} x_{1}$ : mole fraction solubility of the solute.

\section{Auxiliary Information}

\section{Method/Apparatus/Procedure:}

Constant-temperature bath, calorimetric thermometer, and an ultraviolet/ visible spectrophotometer.

Excess solute and solvent were placed in amber glass bottles and allowed to equilibrate for several days at constant temperature. Attainment of equilibrium was verified by several repetitive measurements and by approaching equilibrium from supersaturation. Aliquots of saturated solutions were transferred through a coarse filter into tared volumetric flasks, weighed and diluted with methanol. Concentrations were determined by spectrophotometric measurements at $356 \mathrm{~nm}$.

\section{Source and Purity of Chemicals:}

(1) $99.9+\%$, Aldrich Chemical Company, Milwaukee, WI, USA, was used as received.

(2) $99+\%$, Aldrich Chemical Company, stored over anhydrous sodium sulfate and molecular sieves before being fractionally distilled.

\section{Estimated Error:}

Temperature: $\pm 0.1 \mathrm{~K}$.

$x_{1}: \pm 1.3 \%$ (relative error)

\begin{tabular}{ll}
\hline \hline Components: & Original Measurements: \\
(1) Anthracene; $\mathrm{C}_{14} \mathrm{H}_{10} ;[120-12-7]$ & ${ }^{28}$ W. E. Acree, Jr. and M. H. \\
(2) 1-Decanol; $\mathrm{C}_{10} \mathrm{H}_{22} \mathrm{O} ;[112-30-1]$ & Abraham, Can. J. Chem. 79, \\
& 1466 (2001). \\
\hline Variables: & Prepared by: \\
$T / \mathrm{K}=298.15$ & W. E. Acree, Jr. \\
\hline
\end{tabular}

Experimental Values

\begin{tabular}{|c|c|c|}
\hline$x_{2}{ }^{(\mathrm{s}) \mathrm{a}}$ & $x_{2}{ }^{b}$ & $x_{1}{ }^{\mathrm{c}}$ \\
\hline 1.0000 & 0.9966 & 0.003433 \\
\hline
\end{tabular}


The experimental value was reported as the molar solubility of anthracene in 1-decanol. Mole fraction solubility was provided by the authors of the paper.

\section{Auxiliary Information}

\section{Method/Apparatus/Procedure:}

Constant-temperature bath, calorimetric thermometer, and an ultraviolet/ visible spectrophotometer.

Excess solute and solvent were placed in amber glass bottles and allowed to equilibrate for several days at constant temperature. Attainment of equilibrium was verified by several repetitive measurements and by approaching equilibrium from supersaturation. Aliquots of saturated solutions were transferred through a coarse filter into tared volumetric flasks, weighed and diluted with 2-propanol. Concentrations were determined by spectrophotometric measurements at $356 \mathrm{~nm}$.

Source and Purity of Chemicals:

(1) $99+\%$, Aldrich Chemical Company, Milwaukee, WI, USA, was recrystallized several times from propanone.

(2) $99+\%$, Alfa Aesar, USA, stored over molecular sieves before use.

\section{Estimated Error:}

Temperature: $\pm 0.1 \mathrm{~K}$.

$x_{1}: \pm 1.5 \%$ (relative error).

\begin{tabular}{|c|c|}
\hline $\begin{array}{l}\text { Components: } \\
\text { (1) Anthracene; } \mathrm{C}_{14} \mathrm{H}_{10} ;[120-12-7] \\
\text { (2) 3,7-Dimethyl-1-octanol; } \mathrm{C}_{10} \mathrm{H}_{22} \mathrm{O} \text {; } \\
\text { [106-21-8] }\end{array}$ & $\begin{array}{l}\text { Original Measurements: } \\
\text { W. E. Acree, Jr., } \\
\text { unpublished data. }\end{array}$ \\
\hline $\begin{array}{l}\text { Variables: } \\
T / \mathrm{K}=298.15 \\
\end{array}$ & $\begin{array}{l}\text { Prepared by: } \\
\text { W. E. Acree, Jr. }\end{array}$ \\
\hline
\end{tabular}

Experimental Values

\begin{tabular}{lcc}
\hline \hline$\frac{x_{2}{ }^{(\mathrm{s}) \mathrm{a}}}{x_{2}{ }^{\mathrm{b}}}$ & \multicolumn{1}{c}{$x_{1}{ }^{\mathrm{c}}$} \\
\hline 1.0000 & 0.9980 & 0.002001 \\
${ }^{\mathrm{a}}{ }_{x_{2}}{ }^{(\mathrm{s})}$ : initial mole fraction of component 2 in the solution. \\
${ }^{\mathrm{b}} x_{2}:$ mole fraction of component 2 in the saturated solution. \\
${ }^{\mathrm{c}} x_{1}$ : mole fraction solubility of the solute.
\end{tabular}

\section{Auxiliary Information}

\footnotetext{
Method/Apparatus/Procedure:

Constant-temperature bath, calorimetric thermometer, and an ultraviolet/ visible spectrophotometer.

Excess solute and solvent were placed in amber glass bottles and allowed to equilibrate for several days at constant temperature. Attainment of equilibrium was verified by several repetitive measurements and by approaching equilibrium from supersaturation. Aliquots of saturated solutions were transferred through a coarse filter into tared volumetric flasks, weighed and diluted with 2-propanol. Concentrations were determined by spectrophotometric measurements at $356 \mathrm{~nm}$.

Source and Purity of Chemicals:

(1) $99+\%$, Aldrich Chemical Company, Milwaukee, WI, USA, was recrystallized several times from propanone.

(2) $99 \%$, anhydrous, Aldrich Chemical Company, stored over molecular sieves before use.
}

Estimated Error:

Temperature: $\pm 0.1 \mathrm{~K}$.

$x_{1}: \pm 1.5 \%$ (relative error).

\section{Components:}

(1) Anthracene; $\mathrm{C}_{14} \mathrm{H}_{10} ;[120-12-7]$

(2) Cyclopentanol; $\mathrm{C}_{5} \mathrm{H}_{10} \mathrm{O}$; [96-41-3]

Variables:

$T / \mathrm{K}=298.15$
Original Measurements:

${ }^{16}$ L. E. Roy, C. E. Hernández, and

W. E. Acree, Jr., Polycyclic

Aromat. Compd. 13, 105 (1999).

Prepared by:

W. E. Acree, Jr.

\begin{tabular}{|c|c|c|}
\hline$x_{2}{ }^{(\mathrm{s}) \mathrm{a}}$ & $x_{2}{ }^{\mathrm{b}}$ & $x_{1}{ }^{\mathrm{c}}$ \\
\hline 1.0000 & 0.9987 & 0.001330 \\
\hline
\end{tabular}

\section{Auxiliary Information}

\section{Method/Apparatus/Procedure:}

Constant-temperature bath, calorimetric thermometer, and an ultraviolet/ visible spectrophotometer.

Excess solute and solvent were placed in amber glass bottles and allowed to equilibrate for several days at constant temperature. Attainment of equilibrium was verified by several repetitive measurements and by approaching equilibrium from supersaturation. Aliquots of saturated solutions were transferred through a coarse filter into tared volumetric flasks, weighed and diluted with 2-propanol. Concentrations were determined by spectrophotometric measurements at $356 \mathrm{~nm}$.

\section{Source and Purity of Chemicals:}

(1) $99+\%$, Aldrich Chemical Company, Milwaukee, WI, USA, was recrystallized several times from propanone.

(2) $99 \%$, Aldrich Chemical Company, stored over molecular sieves before use.

\section{Estimated Error:}

Temperature: $\pm 0.1 \mathrm{~K}$.

$x_{1}: \pm 1.5 \%$ (relative error).

\begin{tabular}{|c|c|}
\hline $\begin{array}{l}\text { Components: } \\
\text { (1) Anthracene; } \mathrm{C}_{14} \mathrm{H}_{10} ;[120-12-7] \\
\text { (2) 1,2-Ethanediol; } \mathrm{C}_{2} \mathrm{H}_{6} \mathrm{O}_{2} ;[107-21-1]\end{array}$ & $\begin{array}{l}\text { Original Measurements: } \\
{ }^{16} \text { L. E. Roy, C. E. Hernández, and } \\
\text { W. E. Acree, Jr., Polycyclic } \\
\text { Aromat. Compd. 13, } 105 \text { (1999). }\end{array}$ \\
\hline $\begin{array}{l}\text { Variables: } \\
T / \mathrm{K}=298.15\end{array}$ & $\begin{array}{l}\text { Prepared by: } \\
\text { W. E. Acree, Jr. }\end{array}$ \\
\hline
\end{tabular}

Experimental Values

\begin{tabular}{lcc}
\hline \hline$x_{2}{ }^{(\mathrm{s}) \mathrm{a}}$ & $x_{2}^{\mathrm{b}}$ & ${ }_{x_{1}}{ }^{\mathrm{c}}$ \\
\hline 1.0000 & 0.9999 & 0.0000715 \\
$\frac{{ }^{\mathrm{a}} x_{2}{ }^{(\mathrm{s})}: \text { initial mole fraction of component 2 in the solution. }}{{ }^{\mathrm{b}} x_{2}: \text { mole fraction of component } 2 \text { in the saturated solution. }}$ \\
${ }^{\mathrm{c}} x_{1}:$ mole fraction solubility of the solute.
\end{tabular}




\section{Auxiliary Information}

\section{Method/Apparatus/Procedure:}

Constant-temperature bath, calorimetric thermometer, and an ultraviolet/ visible spectrophotometer.

Excess solute and solvent were placed in amber glass bottles and allowed to equilibrate for several days at constant temperature. Attainment of equilibrium was verified by several repetitive measurements and by approaching equilibrium from supersaturation. Aliquots of saturated solutions were transferred through a coarse filter into tared volumetric flasks, weighed and diluted with 2-propanol. Concentrations were determined by spectrophotometric measurements at $356 \mathrm{~nm}$.

Source and Purity of Chemicals:

(1) $99+\%$, Aldrich Chemical Company, Milwaukee, WI, USA, was recrystallized several times from propanone.

(2) $99+\%$, Aldrich Chemical Company, stored over molecular sieves before use.

Estimated Error:

Temperature: $\pm 0.1 \mathrm{~K}$

$x_{1}: \pm 1.5 \%$ (relative error).

\begin{tabular}{ll}
\hline \hline Components: & Original Measurements: \\
(1) Anthracene; $\mathrm{C}_{14} \mathrm{H}_{10} ;[120-12-7]$ & ${ }^{16} \mathrm{~L}$. E. Roy, C. E. Hernández, and \\
$(2) 2,2,2-$ Trifluoroethanol; $\mathrm{C}_{2} \mathrm{H}_{3} \mathrm{~F}_{3} \mathrm{O} ;$ & W. E. Acree, Jr., Polycyclic \\
{$[75-89-8]$} & Aromat. Compd. 13, 105 (1999). \\
\hline Variables: & Prepared by: \\
$T / \mathrm{K}=298.15$ & W. E. Acree, Jr. \\
\hline
\end{tabular}

Experimental Values

\begin{tabular}{|c|c|c|}
\hline$x_{2}{ }^{(s) a}$ & $x_{2}{ }^{\mathrm{b}}$ & $x_{1}{ }^{\mathrm{c}}$ \\
\hline 1.0000 & 0.9999 & 0.0000865 \\
\hline
\end{tabular}

\section{Auxiliary Information}

\section{Method/Apparatus/Procedure:}

Constant-temperature bath, calorimetric thermometer, and an ultraviolet/ visible spectrophotometer.

Excess solute and solvent were placed in amber glass bottles and allowed to equilibrate for several days at constant temperature. Attainment of equilibrium was verified by several repetitive measurements and by approaching equilibrium from supersaturation. Aliquots of saturated solutions were transferred through a coarse filter into tared volumetric flasks, weighed and diluted with 2-propanol. Concentrations were determined by spectrophotometric measurements at $356 \mathrm{~nm}$.

Source and Purity of Chemicals:

(1) $99+\%$, Aldrich Chemical Company, Milwaukee, WI, USA, was recrystallized several times from propanone.

(2) $99+\%$, Aldrich Chemical Company, stored over molecular sieves before use.

Estimated Error:

Temperature: $\pm 0.1 \mathrm{~K}$.

$x_{1}: \pm 1.5 \%$ (relative error)

\subsection{Anthracene solubility data in alkoxyalcohols}

\begin{tabular}{ll}
\hline \hline Components: & Original Measurements: \\
(1) Anthracene; $\mathrm{C}_{14} \mathrm{H}_{10} ;[120-12-7]$ & ${ }^{29}$ C. E. Hernández, L. E. Roy, T. \\
(2) 2-Ethoxyethanol; $\mathrm{C}_{4} \mathrm{H}_{10} \mathrm{O}_{2} ;$ & Deng, M. B. Tuggle, and W. E. Acree, \\
[110-80-5] & Jr., Phys. Chem. Liq. 37, 677 (1999). \\
\hline Variables: & Prepared by: \\
$T / \mathrm{K}=298.15$ & W. E. Acree, Jr. \\
\hline
\end{tabular}

Experimental Values

\begin{tabular}{|c|c|c|}
\hline$x_{2}{ }^{(\mathrm{s}) \mathrm{a}}$ & $x_{2}{ }^{b}$ & $x_{1}{ }^{\mathrm{c}}$ \\
\hline 1.0000 & 0.9971 & 0.002921 \\
\hline
\end{tabular}

\section{Auxiliary Information}

\section{Method/Apparatus/Procedure:}

Constant-temperature bath, calorimetric thermometer, and an ultraviolet/ visible spectrophotometer.

Excess solute and solvent were placed in amber glass bottles and allowed to equilibrate for several days at constant temperature. Attainment of equilibrium was verified by several repetitive measurements and by approaching equilibrium from supersaturation. Aliquots of saturated solutions were transferred through a coarse filter into tared volumetric flasks, weighed and diluted with methanol. Concentrations were determined by spectrophotometric measurements at $356 \mathrm{~nm}$.

\section{Source and Purity of Chemicals:}

(1) $99+\%$, Acros Organics, USA, was recrystallized several times from propanone.

(2) $99 \%$, Aldrich Chemical Company, Milwaukee, WI, USA, stored over molecular sieves before use.

Estimated Error:

Temperature: $\pm 0.1 \mathrm{~K}$.

$x_{1}: \pm 1.5 \%$ (relative error)

\begin{tabular}{ll}
\hline \hline Components: & Original Measurements: \\
(1) Anthracene; $\mathrm{C}_{14} \mathrm{H}_{10} ;[120-12-7]$ & ${ }^{30}$ C. E. Hernández, L. E. Roy, G. D. \\
(2) 2-Propoxyethanol; $\mathrm{C}_{5} \mathrm{H}_{12} \mathrm{O}_{2} ;$ & Reddy, G. L. Martinez, A. Jackson, G. \\
[2807-30-9] & Brown, and W. E. Acree, Jr., Chem. \\
& Eng. Commun. 169, 137 (1998). \\
\hline Variables: & Prepared by: \\
$T / \mathrm{K}=298.15$ & W. E. Acree, Jr. \\
\hline
\end{tabular}

Experimental Values

\begin{tabular}{|c|c|c|}
\hline$x_{2}{ }^{(\mathrm{s}) \mathrm{a}}$ & $x_{2}{ }^{b}$ & $x_{1}{ }^{\mathrm{c}}$ \\
\hline 1.0000 & 0.9967 & 0.003343 \\
\hline
\end{tabular}




\section{Auxiliary Information}

\section{Method/Apparatus/Procedure:}

Constant-temperature bath, calorimetric thermometer, and an ultraviolet/ visible spectrophotometer.

Excess solute and solvent were placed in amber glass bottles and allowed to equilibrate for several days at constant temperature. Attainment of equilibrium was verified by several repetitive measurements and by approaching equilibrium from supersaturation. Aliquots of saturated solutions were transferred through a coarse filter into tared volumetric flasks, weighed and diluted with methanol. Concentrations were determined by spectrophotometric measurements at $356 \mathrm{~nm}$.

Source and Purity of Chemicals:

(1) $99+\%$, Acros Organics, USA, was recrystallized several times from propanone.

(2) $99 \%$, Aldrich Chemical Company, Milwaukee, WI, USA, stored over molecular sieves before use.

Estimated Error:

Temperature: $\pm 0.1 \mathrm{~K}$.

$x_{1}: \pm 1.5 \%$ (relative error).

\begin{tabular}{|c|c|}
\hline $\begin{array}{l}\text { Components: } \\
\text { (1) Anthracene; } \mathrm{C}_{14} \mathrm{H}_{10} ;[120-12-7] \\
\text { (2) 2-Isopropoxyethanol; } \mathrm{C}_{5} \mathrm{H}_{12} \mathrm{O}_{2} \text {; } \\
\text { [109-59-1] }\end{array}$ & $\begin{array}{l}\text { Original Measurements: } \\
{ }^{31} \text { C. E. Hernández, L. E. Roy, G. D. } \\
\text { Reddy, G. L. Martinez, A. Jackson, G. } \\
\text { Brown, T. L. Borders, J. T. Sanders, } \\
\text { and W. E. Acree, Jr., Phys. Chem. Liq } \\
\text { 36, } 257 \text { (1998). }\end{array}$ \\
\hline $\begin{array}{l}\text { Variables: } \\
T / \mathrm{K}=298.15\end{array}$ & $\begin{array}{l}\text { Prepared by: } \\
\text { W. E. Acree, Jr. }\end{array}$ \\
\hline
\end{tabular}

Experimental Values

\begin{tabular}{lcc}
\hline \hline$x_{2}{ }^{(\mathrm{s}) \mathrm{a}}$ & $x_{2}{ }^{\mathrm{b}}$ & $x_{1}{ }^{\mathrm{c}}$ \\
\hline 1.0000 & 0.9969 & 0.003093 \\
\hline
\end{tabular}

${ }^{\mathrm{a}} x_{2}{ }^{(\mathrm{s})}$ : initial mole fraction of component 2 in the solution.

${ }^{b} x_{2}$ : mole fraction of component 2 in the saturated solution.

${ }^{c} x_{1}$ : mole fraction solubility of the solute.

\section{Auxiliary Information}

\footnotetext{
Method/Apparatus/Procedure:

Constant-temperature bath, calorimetric thermometer, and an ultraviolet/ visible spectrophotometer.

Excess solute and solvent were placed in amber glass bottles and allowed to equilibrate for several days at constant temperature. Attainment of equilibrium was verified by several repetitive measurements and by approaching equilibrium from supersaturation. Aliquots of saturated solutions were transferred through a coarse filter into tared volumetric flasks, weighed and diluted with methanol. Concentrations were determined by spectrophotometric measurements at $356 \mathrm{~nm}$.
}

Source and Purity of Chemicals:

(1) $99+\%$, Acros Organics, USA, was recrystallized several times from propanone.

(2) $99 \%$, Aldrich Chemical Company, Milwaukee, WI, USA, stored over molecular sieves before use.
Estimated Error:

Temperature: $\pm 0.1 \mathrm{~K}$.

$x_{1}: \pm 1.5 \%$ (relative error).

\begin{tabular}{ll}
\hline \hline Components: & Original Measurements: \\
(1) Anthracene; $\mathrm{C}_{14} \mathrm{H}_{10} ;[120-12-7]$ & ${ }^{32}$ M. E. R. McHale, A.-S. M. \\
(2) 2-Butoxyethanol; $\mathrm{C}_{6} \mathrm{H}_{14} \mathrm{O}_{2} ;$ & Kauppila, J. R. Powell, and W. E. \\
[111-76-2] & Acree, Jr., J. Chem. Thermodyn. 28, \\
& 209 (1996). \\
\hline Variables: & Prepared by: \\
$T / \mathrm{K}=298.15$ & W. E. Acree, Jr. \\
\hline
\end{tabular}

Experimental Values

\begin{tabular}{lcc}
\hline \hline$x_{2}{ }^{(\mathrm{s}) \mathrm{a}}$ & $x_{2}{ }^{\mathrm{b}}$ & $x_{1}{ }^{\mathrm{c}}$ \\
\hline 1.0000 & 0.9962 & 0.003785
\end{tabular}

${ }^{\mathrm{a}} x_{2}{ }^{(\mathrm{s})}$ : initial mole fraction of component 2 in the solution.

${ }^{\mathrm{b}} x_{2}$ : mole fraction of component 2 in the saturated solution.

${ }^{c} x_{1}$ : mole fraction solubility of the solute.

\section{Auxiliary Information}

\section{Method/Apparatus/Procedure:}

Constant-temperature bath, calorimetric thermometer, and an ultraviolet/ visible spectrophotometer.

Excess solute and solvent were placed in amber glass bottles and allowed to equilibrate for several days at constant temperature. Attainment of equilibrium was verified by several repetitive measurements and by approaching equilibrium from supersaturation. Aliquots of saturated solutions were transferred through a coarse filter into tared volumetric flasks, weighed and diluted with methanol. Concentrations were determined by spectrophotometric measurements at $356 \mathrm{~nm}$.

\section{Source and Purity of Chemicals:}

(1) $99.9+\%$, Gold Label, Aldrich Chemical Company, Milwaukee, WI, USA, was used as received.

(2) $99+\%$, Acros Organics, USA, stored over anhydrous sodium sulfate and molecular sieves before use.

\section{Estimated Error:}

Temperature: $\pm 0.1 \mathrm{~K}$.

$x_{1}: \pm 1.5 \%$ (relative error).

\begin{tabular}{ll}
\hline \hline Components: & Original Measurements: \\
(1) Anthracene; $\mathrm{C}_{14} \mathrm{H}_{10} ;[120-12-7]$ & ${ }^{33}$ C. E. Hernández, L. E. Roy, G. \\
(2) 3-Methoxy-1-butanol; $\mathrm{C}_{5} \mathrm{H}_{12} \mathrm{O}_{2} ;$ & D. Reddy, T. L. Borders, J. T. \\
[2517-43-3] & Sanders, and W. E. Acree, Jr., \\
& Phys. Chem. Liq. 37, 31 (1998). \\
\hline Variables: & Prepared by: \\
$T / \mathrm{K}=298.15$ & W. E. Acree, Jr. \\
\hline
\end{tabular}


Experimental Values

\begin{tabular}{lcc}
\hline$\frac{x_{2}{ }^{(\mathrm{s}) \mathrm{a}}}{x_{2}{ }^{\mathrm{b}}}$ & $x_{1}{ }^{\mathrm{c}}$ \\
\hline 1.0000 & 0.9973 & 0.002702 \\
$\frac{{ }^{\mathrm{a}} x_{2}{ }^{(\mathrm{s})}: \text { initial mole fraction of component } 2 \text { in the solution. }}{{ }^{\mathrm{b}}{ }_{x_{2}}: \text { mole fraction of component } 2 \text { in the saturated solution. }}$ \\
${ }^{\mathrm{c}} x_{1}:$ mole fraction solubility of the solute.
\end{tabular}

\section{Auxiliary Information}

\section{Method/Apparatus/Procedure:}

Constant-temperature bath, calorimetric thermometer, and an ultraviolet/ visible spectrophotometer.

Excess solute and solvent were placed in amber glass bottles and allowed to equilibrate for several days at constant temperature. Attainment of equilibrium was verified by several repetitive measurements and by approaching equilibrium from supersaturation. Aliquots of saturated solutions were transferred through a coarse filter into tared volumetric flasks, weighed and diluted with methanol. Concentrations were determined by spectrophotometric measurements at $356 \mathrm{~nm}$.

\section{Source and Purity of Chemicals:}

(1) $99+\%$, Acros Organics, USA, was recrystallized several times from propanone.

(2) $99 \%$, Aldrich Chemical Company, Milwaukee, WI, USA, stored over molecular sieves before use.

\section{Estimated Error:}

Temperature: $\pm 0.1 \mathrm{~K}$

$x_{1}: \pm 1.5 \%$ (relative error).

\subsection{Anthracene solubility data in miscellaneous organic solvents}

\begin{tabular}{ll}
\hline \hline Components: & Original Measurements: \\
(1) Anthracene; $\mathrm{C}_{14} \mathrm{H}_{10} ;[120-12-7]$ & ${ }^{16}$ L. E. Roy, C. E. Hernández, and \\
(2) Methyl acetoacetate; $\mathrm{C}_{5} \mathrm{H}_{8} \mathrm{O}_{3} ;$ & W. E. Acree, Jr., Polycyclic \\
{$[105-45-3]$} & Aromat. Compd. 13, 105 (1999). \\
\hline Variables: & Prepared by: \\
$T / \mathrm{K}=298.15$ & W. E. Acree, Jr. \\
\hline
\end{tabular}

Experimental Values

\begin{tabular}{lcc}
\hline \hline$x_{2}{ }^{(\mathrm{s}) \mathrm{a}}$ & $x_{2}^{\mathrm{b}}$ & $x_{1}{ }^{\mathrm{c}}$ \\
\hline 1.0000 & 0.9968 & 0.003191 \\
$\frac{{ }^{a} x_{2}{ }^{(\mathrm{s})}: \text { initial mole fraction of component } 2 \text { in the solution. }}{{ }^{\mathrm{b}} x_{2}: \text { mole fraction of component } 2 \text { in the saturated solution. }}$ \\
${ }^{\mathrm{c}} x_{1}:$ mole fraction solubility of the solute.
\end{tabular}

\section{Auxiliary Information}

\section{Method/Apparatus/Procedure:}

Constant-temperature bath, calorimetric thermometer, and an ultraviolet/ visible spectrophotometer.

Excess solute and solvent were placed in amber glass bottles and allowed to equilibrate for several days at constant temperature. Attainment of equilibrium was verified by several repetitive measurements and by approaching equilibrium from supersaturation. Aliquots of saturated solutions were transferred through a coarse filter into tared volumetric flasks, weighed and diluted with 2-propanol. Concentrations were determined by spectrophotometric measurements at $356 \mathrm{~nm}$.

Source and Purity of Chemicals:

(1) $99+\%$, Aldrich Chemical Company, Milwaukee, WI, USA, was recrystallized several times from propanone.

(2) $99 \%$, Aldrich Chemical Company, stored over molecular sieves before use.

Estimated Error:

Temperature: $\pm 0.1 \mathrm{~K}$.

$x_{1}: \pm 1.5 \%$ (relative error)

\begin{tabular}{ll}
\hline \hline Components: & Original Measurements:. \\
(1) Anthracene; $\mathrm{C}_{14} \mathrm{H}_{10} ;[120-12-7]$ & ${ }^{16}$ L. E. Roy, C. E. Hernández, and \\
(2) Ethyl acetoacetate; $\mathrm{C}_{6} \mathrm{H}_{10} \mathrm{O}_{3} ;$ & W. E. Acree, Jr., Polycyclic \\
{$[141-97-9]$} & Aromat. Compd. 13, 105 (1999) \\
\hline Variables: & Prepared by: \\
$T / \mathrm{K}=298.15$ & W. E. Acree, Jr. \\
\hline
\end{tabular}

Experimental Values

\begin{tabular}{lcc}
\hline \hline$x_{2}{ }^{(\mathrm{s}) \mathrm{a}}$ & $x_{2}{ }^{\mathrm{b}}$ & $x_{1}{ }^{\mathrm{c}}$ \\
\hline 1.0000 & 0.9955 & 0.004533 \\
$\frac{{ }^{\mathrm{a}} x_{2}{ }^{(\mathrm{s})}: \text { initial mole fraction of component } 2 \text { in the solution. }}{{ }^{\mathrm{b}} x_{2}: \text { mole fraction of component } 2 \text { in the saturated solution. }}$ \\
${ }^{\mathrm{c}} x_{1}:$ mole fraction solubility of the solute.
\end{tabular}

Auxiliary Information

\section{Method/Apparatus/Procedure:}

Constant-temperature bath, calorimetric thermometer, and an ultraviolet/ visible spectrophotometer.

Excess solute and solvent were placed in amber glass bottles and allowed to equilibrate for several days at constant temperature. Attainment of equilibrium was verified by several repetitive measurements and by approaching equilibrium from supersaturation. Aliquots of saturated solutions were transferred through a coarse filter into tared volumetric flasks, weighed and diluted with 2-propanol. Concentrations were determined by spectrophotometric measurements at $356 \mathrm{~nm}$.

\section{Source and Purity of Chemicals:}

(1) $99+\%$, Aldrich Chemical Company, Milwaukee, WI, USA, was recrystallized several times from propanone.

(2) $99 \%$, Aldrich Chemical Company, stored over molecular sieves before use.

\section{Estimated Error:}

Temperature: $\pm 0.1 \mathrm{~K}$.

$x_{1}: \pm 1.5 \%$ (relative error). 


\section{Components:}

(1) Anthracene; $\mathrm{C}_{14} \mathrm{H}_{10} ;[120-12-7]$

(2) Ethanenitrile; $\mathrm{C}_{2} \mathrm{H}_{3} \mathrm{~N}$; [75-05-8]

Original Measurements:

${ }^{16}$ L. E. Roy, C. E. Hernández, and

W. E. Acree, Jr., Polycyclic

Aromat. Compd. 13, 105 (1999).

Variables:

$T / \mathrm{K}=298.15$

Prepared by:

W. E. Acree, Jr.

\section{Experimental Values}

\begin{tabular}{lcc}
\hline \hline$x_{2}{ }^{(\mathrm{s}) \mathrm{a}}$ & $x_{2}{ }^{\mathrm{b}}$ & $x_{1}{ }^{\mathrm{c}}$ \\
\hline 1.0000 & 0.9992 & 0.000830 \\
\hline
\end{tabular}

${ }^{\mathrm{a}} x_{2}{ }^{(\mathrm{s})}$ : initial mole fraction of component 2 in the solution.

${ }^{b} x_{2}$ : mole fraction of component 2 in the saturated solution.

${ }^{c} x_{1}$ : mole fraction solubility of the solute.

\section{Auxiliary Information}

Method/Apparatus/Procedure:
Constant-temperature bath, calorimetric thermometer, and an ultraviolet/
visible spectrophotometer.
Excess solute and solvent were placed in amber glass bottles and allowed to
equilibrate for several days at constant temperature. Attainment of equilibrium
was verified by several repetitive measurements and by approaching
equilibrium from supersaturation. Aliquots of saturated solutions were
transferred through a coarse filter into tared volumetric flasks, weighed and
diluted with 2-propanol. Concentrations were determined by
spectrophotometric measurements at 356 nm.
Source and Purity of Chemicals:
(1) $99+\%$, Aldrich Chemical Company, Milwaukee, WI, USA, was
recrystallized several times from propanone.
(2) $99.9+\%$, HPLC Grade, Aldrich Chemical Company, USA, stored over
molecular sieves before use.
Estimated Error:
Temperature: \pm 0.1 K.
$x_{1}: \pm 1.5 \%$ (relative error).

\begin{tabular}{ll}
\hline \hline Components: & Original Measurements: \\
(1) Anthracene; $\mathrm{C}_{14} \mathrm{H}_{10} ;[120-12-7]$ & W. E. Acree, Jr., \\
(2) Propanenitrile; $\mathrm{C}_{3} \mathrm{H}_{5} \mathrm{~N} ;[107-12-0]$ & unpublished data. \\
\hline Variables: & Prepared by: \\
$T / \mathrm{K}=298.15$ & W. E. Acree, Jr. \\
\hline
\end{tabular}

Experimental Values

\begin{tabular}{|c|c|c|}
\hline$x_{2}^{(\mathrm{s}) \mathrm{a}}$ & $x_{2}{ }^{\mathrm{b}}$ & $x_{1}{ }^{\mathrm{c}}$ \\
\hline 1.0000 & 0.9971 & 0.002879 \\
\hline
\end{tabular}

\section{Auxiliary Information}

\section{Method/Apparatus/Procedure:}

Constant-temperature bath, calorimetric thermometer, and an ultraviolet/ visible spectrophotometer.

Excess solute and solvent were placed in amber glass bottles and allowed to equilibrate for several days at constant temperature. Attainment of equilibrium was verified by several repetitive measurements and by approaching equilibrium from supersaturation. Aliquots of saturated solutions were transferred through a coarse filter into tared volumetric flasks, weighed and diluted with 2-propanol. Concentrations were determined by spectrophotometric measurements at $356 \mathrm{~nm}$.

\section{Source and Purity of Chemicals:}

(1) $99+\%$, Aldrich Chemical Company, Milwaukee, WI, USA, was recrystallized several times from propanone.

(2) $99 \%$, Aldrich Chemical Company, stored over molecular sieves before use.

\section{Estimated Error:}

Temperature: $\pm 0.1 \mathrm{~K}$.

$x_{1}: \pm 1.5 \%$ (relative error).

\begin{tabular}{ll}
\hline \hline Components: & Original Measurements: \\
(1) Anthracene; $\mathrm{C}_{14} \mathrm{H}_{10} ;[120-12-7]$ & W. E. Acree, Jr., \\
(2) Butanenitrile; $\mathrm{C}_{4} \mathrm{H}_{7} \mathrm{~N} ;[109-74-0]$ & unpublished data. \\
\hline Variables: & Prepared by: \\
$T / \mathrm{K}=298.15$ & W. E. Acree, Jr. \\
\hline
\end{tabular}

Experimental Values

\begin{tabular}{|c|c|c|}
\hline$x_{2}{ }^{(s) a}$ & $x_{2}{ }^{b}$ & $x_{1}{ }^{\mathrm{c}}$ \\
\hline 1.0000 & 0.9955 & 0.004527 \\
\hline
\end{tabular}

\section{Auxiliary Information}

\section{Method/Apparatus/Procedure:}

Constant-temperature bath, calorimetric thermometer, and an ultraviolet/ visible spectrophotometer.

Excess solute and solvent were placed in amber glass bottles and allowed to equilibrate for several days at constant temperature. Attainment of equilibrium was verified by several repetitive measurements and by approaching equilibrium from supersaturation. Aliquots of saturated solutions were transferred through a coarse filter into tared volumetric flasks, weighed and diluted with 2-propanol. Concentrations were determined by spectrophotometric measurements at $356 \mathrm{~nm}$.

\section{Source and Purity of Chemicals:}

(1) $99+\%$, Aldrich Chemical Company, Milwaukee, WI, USA, was recrystallized several times from propanone.

(2) $99 \%$, Aldrich Chemical Company, stored over molecular sieves before use.

Estimated Error:

Temperature: $\pm 0.1 \mathrm{~K}$.

$x_{1}: \pm 1.5 \%$ (relative error) 


\section{Components:}

(1) Anthracene; $\mathrm{C}_{14} \mathrm{H}_{10} ;[120-12-7]$

(2) Benzonitrile; $\mathrm{C}_{7} \mathrm{H}_{5} \mathrm{~N}$; [100-47-0]

Variables:

$T / \mathrm{K}=298.15$

Original Measurements:

${ }^{16}$ L. E. Roy, C. E. Hernández, and

W. E. Acree, Jr., Polycyclic

Aromat. Compd. 13, 105 (1999).

Prepared by:

W. E. Acree, Jr.

Experimental Values

\begin{tabular}{lcc}
\hline \hline$x_{2}{ }^{(\mathrm{s}) \mathrm{a}}$ & $x_{2}{ }^{\mathrm{b}}$ & $x_{1}{ }^{\mathrm{c}}$ \\
\hline 1.0000 & 0.9916 & 0.008426 \\
\hline
\end{tabular}

${ }^{\mathrm{a}} x_{2}{ }^{(\mathrm{s})}$ : initial mole fraction of component 2 in the solution.

${ }^{b} x_{2}$ : mole fraction of component 2 in the saturated solution.

${ }^{c} x_{1}$ : mole fraction solubility of the solute.

\section{Auxiliary Information}

\begin{abstract}
Method/Apparatus/Procedure:
Constant-temperature bath, calorimetric thermometer, and an ultraviolet/ visible spectrophotometer.

Excess solute and solvent were placed in amber glass bottles and allowed to equilibrate for several days at constant temperature. Attainment of equilibrium was verified by several repetitive measurements and by approaching equilibrium from supersaturation. Aliquots of saturated solutions were transferred through a coarse filter into tared volumetric flasks, weighed and diluted with 2-propanol. Concentrations were determined by

spectrophotometric measurements at $356 \mathrm{~nm}$.
\end{abstract}

Source and Purity of Chemicals:

(1) $99+\%$, Aldrich Chemical Company, Milwaukee, WI, USA, was

recrystallized several times from propanone.

(2) $99 \%$, Aldrich Chemical Company, stored over molecular sieves before use.

Estimated Error:

Temperature: $\pm 0.1 \mathrm{~K}$

$x_{1}: \pm 1.5 \%$ (relative error).

\begin{tabular}{ll}
\hline \hline Components: & Original Measurements: \\
(1) Anthracene; $\mathrm{C}_{14} \mathrm{H}_{10} ;[120-12-7]$ & ${ }^{16}$ L. E. Roy, C. E. Hernández, and \\
$(2) N, N$-Dimethylformamide; $\mathrm{C}_{3} \mathrm{H}_{7} \mathrm{NO} ;$ & W. E. Acree, Jr., Polycyclic \\
{$[111-84-2]$} & Aromat. Compd. 13, 105 (1999). \\
\hline Variables: & Prepared by: \\
$T / \mathrm{K}=298.15$ & W. E. Acree, Jr. \\
\hline
\end{tabular}

Experimental Values

\begin{tabular}{|c|c|c|}
\hline$x_{2}^{(\mathrm{s}) \mathrm{a}}$ & $x_{2}{ }^{\mathrm{b}}$ & $x_{1}{ }^{\mathrm{c}}$ \\
\hline 1.0000 & 0.9922 & 0.007839 \\
\hline
\end{tabular}

\section{Auxiliary Information}

Method/Apparatus/Procedure:

Constant-temperature bath, calorimetric thermometer, and an ultraviolet/ visible spectrophotometer.

Excess solute and solvent were placed in amber glass bottles and allowed to equilibrate for several days at constant temperature. Attainment of equilibrium was verified by several repetitive measurements and by approaching equilibrium from supersaturation. Aliquots of saturated solutions were transferred through a coarse filter into tared volumetric flasks, weighed and diluted with 2-propanol. Concentrations were determined by spectrophotometric measurements at $356 \mathrm{~nm}$.

\section{Source and Purity of Chemicals:}

(1) $99+\%$, Aldrich Chemical Company, Milwaukee, WI, USA, was recrystallized several times from propanone.

(2) $99.8 \%$, anhydrous, Aldrich Chemical Company, stored over molecular sieves before use.

Estimated Error:

Temperature: $\pm 0.1 \mathrm{~K}$.

$x_{1}: \pm 1.5 \%$ (relative error).

\begin{tabular}{|c|c|}
\hline $\begin{array}{l}\text { Components: } \\
\text { (1) Anthracene; } \mathrm{C}_{14} \mathrm{H}_{10} ;[120-12-7] \\
\text { (2) } N, N \text {-Dimethylacetamide; } \mathrm{C}_{4} \mathrm{H}_{9} \mathrm{NO} \text {; } \\
{[127-19-5]}\end{array}$ & $\begin{array}{l}\text { Original Measurements: } \\
{ }^{16} \text { L. E. Roy, C. E. Hernández, and } \\
\text { W. E. Acree, Jr., Polycyclic } \\
\text { Aromat. Compd. 13, } 105 \text { (1999). }\end{array}$ \\
\hline $\begin{array}{l}\text { Variables: } \\
T / \mathrm{K}=298.15\end{array}$ & $\begin{array}{l}\text { Prepared by: } \\
\text { W. E. Acree, Jr. }\end{array}$ \\
\hline
\end{tabular}

Experimental Values

\begin{tabular}{|c|c|c|}
\hline$x_{2}{ }^{(\mathrm{s}) \mathrm{a}}$ & $x_{2}{ }^{b}$ & $x_{1}{ }^{\mathrm{c}}$ \\
\hline 1.0000 & 0.9873 & 0.01267 \\
\hline
\end{tabular}

\section{Auxiliary Information}

\section{Method/Apparatus/Procedure:}

Constant-temperature bath, calorimetric thermometer, and an ultraviolet/ visible spectrophotometer.

Excess solute and solvent were placed in amber glass bottles and allowed to equilibrate for several days at constant temperature. Attainment of equilibrium was verified by several repetitive measurements and by approaching equilibrium from supersaturation. Aliquots of saturated solutions were transferred through a coarse filter into tared volumetric flasks, weighed and diluted with 2-propanol. Concentrations were determined by spectrophotometric measurements at $356 \mathrm{~nm}$.

\section{Source and Purity of Chemicals:}

(1) $99+\%$, Aldrich Chemical Company, Milwaukee, WI, USA, was recrystallized several times from propanone.

(2) $99.8 \%$, anhydrous, Aldrich Chemical Company, stored over molecular sieves before use.

\section{Estimated Error:}

Temperature: $\pm 0.1 \mathrm{~K}$.

$x_{1}: \pm 1.5 \%$ (relative error). 


\section{Components:}

(1) Anthracene; $\mathrm{C}_{14} \mathrm{H}_{10} ;[120-12-7]$

(2) N,N-Dimethylacetamide; $\mathrm{C}_{4} \mathrm{H}_{9} \mathrm{NO}$;

[127-19-5]

Variables:

Temperature

re

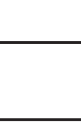

\begin{tabular}{ll}
$1335(2000)$. \\
\hline Prepared by: \\
W. E. Acree, Jr.
\end{tabular}

Experimental Values

\begin{tabular}{lcc}
\hline \hline$T / \mathrm{K}$ & $x_{2}{ }^{\mathrm{a}}$ & $x_{1}{ }^{\mathrm{b}}$ \\
\hline 299.5 & 0.9900 & 0.0100 \\
318.3 & 0.9802 & 0.0198 \\
332.2 & 0.9699 & 0.0301 \\
350.3 & 0.9498 & 0.0502 \\
363.1 & 0.9301 & 0.0699 \\
378.9 & 0.8979 & 0.1021 \\
398.6 & 0.8504 & 0.1496 \\
408.7 & 0.8144 & 0.1856 \\
418.0 & 0.7760 & 0.2240 \\
422.9 & 0.7506 & 0.2494 \\
431.5 & 0.6991 & 0.3009 \\
446.0 & 0.5989 & 0.4011 \\
\hline
\end{tabular}

${ }^{\mathrm{a}} x_{2}$ : mole fraction of component 2 in the saturated solution.

${ }^{\mathrm{b}} \mathrm{x}_{1}$ : mole fraction of the polycyclic aromatic hydrocarbon (component 1 ).

The authors reported that the binary system exhibits simple eutectic behavior, with a eutectic temperature of $T / \mathrm{K}=250.3$ as determined by calorimetric measurements. The eutectic composition was not determined.

\section{Auxiliary Information}

\section{Method/Apparatus/Procedure:}

Temperature-controlled heating/cooling system and a shaking-mixing system. Synthetic mixtures of known compositions were weighed in small glass ampoules. The mixture composition was known to within \pm 0.0002 mole fraction. Ampoules were cooled to very low temperatures of about $220 \mathrm{~K}$, pumped down, and sealed. The sealed mixtures were then melted, cooled rapidly, and reheated. The rate of reheating was at an approximate rate of $8 \times 10^{-5} \mathrm{~K} / \mathrm{s}$ just before complete dissolution. The temperature at which the solid completely dissolved was recorded. The average of two or three independent measurements was taken as the solid-liquid equilibrium.

\section{Source and Purity of Chemicals:}

(1) $99.9 \%$, Heybreck, Switzerland, was used as received.

(2) Purest grade, Merck-Schuchardt, Federal Republic of Germany, was distilled under reduced pressure and dried over molecular sieves to produce a sample of $99.9 \%$ purity (mass percent).

\section{Estimated Error:}

Temperature: $\pm 0.2 \mathrm{~K}$ (estimated by compiler). $x_{1}: \pm 0.0002$.

\begin{tabular}{|c|c|}
\hline $\begin{array}{l}\text { Components: } \\
\text { (1) Anthracene; } \mathrm{C}_{14} \mathrm{H}_{10} ;[120-12-7] \\
\text { (2) 1-Methyl-2-pyrrolidone; } \mathrm{C}_{5} \mathrm{H}_{9} \mathrm{NO} \text {; } \\
\text { [872-50-4] }\end{array}$ & $\begin{array}{l}\text { Original Measurements: } \\
{ }^{35} \text { Z. Lisicki and M. E. Jamróz, } \\
\text { J. Chem. Thermodyn. 32, } \\
1335 \text { (2000). }\end{array}$ \\
\hline $\begin{array}{l}\text { Variables: } \\
\text { Temperature }\end{array}$ & $\begin{array}{l}\text { Prepared by: } \\
\text { W. E. Acree, Jr. }\end{array}$ \\
\hline
\end{tabular}

Experimental Values

\begin{tabular}{lcc}
\hline \hline$T / \mathrm{K}$ & $x_{2}{ }^{\mathrm{a}}$ & $x_{1}{ }^{\mathrm{b}}$ \\
\hline 272.9 & 0.9892 & 0.0108 \\
296.3 & 0.9799 & 0.0201 \\
318.1 & 0.9700 & 0.0300 \\
333.4 & 0.9497 & 0.0503 \\
344.7 & 0.9395 & 0.0605 \\
361.2 & 0.9090 & 0.0910 \\
381.3 & 0.8698 & 0.1302 \\
393.7 & 0.8299 & 0.1701 \\
403.7 & 0.7994 & 0.2006 \\
418.0 & 0.9497 & 0.2503 \\
438.7 & 0.6698 & 0.3302 \\
451.1 & 0.5888 & 0.4112 \\
461.4 & 0.4995 & 0.5005 \\
471.0 & 0.3998 & 0.6002 \\
477.5 & 0.2990 & 0.7010 \\
\hline
\end{tabular}

${ }^{\mathrm{a}} x_{2}$ : mole fraction of component 2 in the saturated solution.

${ }^{\mathrm{b}} x_{1}$ : mole fraction of the polycyclic aromatic hydrocarbon (component 1 ).

The authors reported that the binary system exhibits simple eutectic behavior, with a eutectic temperature of $T / \mathrm{K}=245.1$ as determined by calorimetric measurements. The eutectic composition was not determined.

\section{Auxiliary Information}

\section{Method/Apparatus/Procedure:}

Temperature-controlled heating/cooling system and a shaking-mixing system. Synthetic mixtures of known compositions were weighed in small glass ampoules. The mixture composition was known to within \pm 0.0002 mole fraction. Ampoules were cooled to very low temperatures of about $220 \mathrm{~K}$, pumped down, and sealed. The sealed mixtures were then melted, cooled rapidly, and reheated. The rate of reheating was at an approximate rate of $8 \times 10^{-5} \mathrm{~K} / \mathrm{s}$ just before complete dissolution. The temperature at which the solid completely dissolved was recorded. The average of two or three independent measurements was taken as the solid-liquid equilibrium.

\section{Source and Purity of Chemicals:}

(1) $99.9 \%$, Heybreck, Switzerland, was used as received.

(2) Technical Grade, BASF, Federal Republic of Germany, was purified by treatment with a $2 \%$ solution of potassium permanganate, and then distilled under reduced pressure to collect the distillate having $99.8 \%$ purity (mass percent). The purified sample was dried over molecular sieves.

Estimated Error:

Temperature: $\pm 0.2 \mathrm{~K}$ (estimated by compiler). $x_{1}: \pm 0.0002$.

\begin{tabular}{ll}
\hline \hline Components: & Original Measurements: \\
(1) Anthracene; $\mathrm{C}_{14} \mathrm{H}_{10} ;[120-12-7]$ & ${ }^{35} \mathrm{Z}$. Lisicki and M. E. \\
(2) Hexahydro-1-methyl-2H-azepin-2-one $(\mathrm{N}-$ & Jamróz, J. Chem. \\
Methyl-e-caprolactam); $\mathrm{C}_{7} \mathrm{H}_{13} \mathrm{NO} ;$ [2556-73-2] & Thermodyn. 32, 1335 \\
& $(2000)$. \\
\hline Variables: & Prepared by: \\
Temperature & W. E. Acree, Jr. \\
\hline
\end{tabular}


Experimental Values

\begin{tabular}{lcc}
\hline \hline$T / \mathrm{K}$ & $x_{2}{ }^{\mathrm{a}}$ & $x_{1}{ }^{\mathrm{b}}$ \\
\hline 279.6 & 1.0000 & 0.0000 \\
287.4 & 0.9905 & 0.0095 \\
309.0 & 0.9696 & 0.0304 \\
322.9 & 0.9601 & 0.0399 \\
332.4 & 0.9491 & 0.0509 \\
349.1 & 0.9281 & 0.0719 \\
356.1 & 0.9195 & 0.0805 \\
364.3 & 0.9016 & 0.0984 \\
377.8 & 0.8777 & 0.1223 \\
389.4 & 0.8469 & 0.1531 \\
405.5 & 0.7948 & 0.2052 \\
424.9 & 0.7004 & 0.2996 \\
439.9 & 0.5993 & 0.4007 \\
453.2 & 0.4999 & 0.5001 \\
460.2 & 0.3990 & 0.6010 \\
466.6 & 0.3003 & 0.6997 \\
472.9 & 0.2012 & 0.7988 \\
480.1 & 0.0994 & 0.9006 \\
489.4 & 0.0000 & 1.0000 \\
\hline
\end{tabular}

${ }^{\mathrm{a}} x_{2}$ : mole fraction of component 2 in the saturated solution.

${ }^{b} x_{1}$ : mole fraction of the polycyclic aromatic hydrocarbon (component 1 ).

\section{Auxiliary Information}

\section{Method/Apparatus/Procedure:}

Temperature-controlled heating/cooling system and a shaking-mixing system. Synthetic mixtures of known compositions were weighed in small glass ampoules. The mixture composition was known to within \pm 0.0002 mole fraction. Ampoules were cooled to very low temperatures of about $220 \mathrm{~K}$, pumped down, and sealed. The sealed mixtures were then melted, cooled rapidly, and reheated. The rate of reheating was at an approximate rate of $8 \times 10^{-5} \mathrm{~K} / \mathrm{s}$ just before complete dissolution. The temperature at which the solid completely dissolved was recorded. The average of two or three independent measurements was taken as the solid-liquid equilibrium.

\section{Source and Purity of Chemicals:}

(1) $99.9 \%$, Heybreck, Switzerland, was used as received.

(2) Technical Grade, Leuna Werke, Federal Republic of Germany, was purified by super-rectification and then dried over molecular sieves to give a sample having a purity of $99.9 \%$ by mass.

\section{Estimated Error:}

Temperature: $\pm 0.2 \mathrm{~K}$ (estimated by compiler). $x_{1}: \pm 0.0002$.

\begin{tabular}{ll}
\hline \hline Components: & Original Measurements: \\
(1) Anthracene; $\mathrm{C}_{14} \mathrm{H}_{10} ;[120-12-7]$ & W. E. Acree, Jr., \\
(2) Morpholine; $\mathrm{C}_{4} \mathrm{H}_{9} \mathrm{NO} ;[110-91-8]$ & unpublished data. \\
\hline Variables: & Prepared by: \\
$T / \mathrm{K}=298.15$ & W. E. Acree, Jr. \\
\hline
\end{tabular}

\section{Experimental Values}

\begin{tabular}{lcc}
\hline \hline$x_{2}{ }^{(\mathrm{s}) \mathrm{a}}$ & $x_{2}{ }^{\mathrm{b}}$ & $x_{1}{ }^{\mathrm{c}}$ \\
\hline 1.0000 & 0.9918 & 0.008193 \\
\hline
\end{tabular}

${ }^{\mathrm{a}} x_{2}{ }^{(\mathrm{s})}$ : initial mole fraction of component 2 in the solution

${ }^{\mathrm{b}} x_{1}$ : mole fraction solubility of the solute

${ }^{c} x_{2}$ : mole fraction of component 2 in the saturated solution.

\section{Auxiliary Information}

\section{Method/Apparatus/Procedure:}

Constant-temperature bath, calorimetric thermometer, and an ultraviolet/ visible spectrophotometer.

Excess solute and solvent were placed in amber glass bottles and allowed to equilibrate for several days at constant temperature. Attainment of equilibrium was verified by several repetitive measurements and by approaching equilibrium from supersaturation. Aliquots of saturated solutions were transferred through a coarse filter into tared volumetric flasks, weighed and diluted with 2-propanol. Concentrations were determined by spectrophotometric measurements at $356 \mathrm{~nm}$.

\section{Source and Purity of Chemicals:}

(1) $99+\%$, Aldrich Chemical Company, Milwaukee, WI, USA, was recrystallized several times from propanone.

(2) $99 \%$, Aldrich Chemical Company, stored over molecular sieves before use.

Estimated Error:

Temperature: $\pm 0.1 \mathrm{~K}$.

$x_{1}: \pm 1.5 \%$ (relative error).

\begin{tabular}{ll}
\hline \hline Components: & Original Measurements: \\
(1) Anthracene; $\mathrm{C}_{14} \mathrm{H}_{10} ;[120-12-7]$ & W. E. Acree, Jr., \\
(2) Ethanolamine; $\mathrm{C}_{2} \mathrm{H}_{7} \mathrm{NO} ;[141-43-5]$ & unpublished data. \\
\hline Variables: & Prepared by: \\
$T / \mathrm{K}=298.15$ & W. E. Acree, Jr. \\
\hline
\end{tabular}

Experimental Values

\begin{tabular}{|c|c|c|}
\hline$x_{2}{ }^{(\mathrm{s}) \mathrm{a}}$ & $x_{2}^{b}$ & $x_{1}{ }^{c}$ \\
\hline 1.0000 & 0.9997 & 0.0002587 \\
\hline
\end{tabular}

\section{Auxiliary Information}

\section{Method/Apparatus/Procedure:}

Constant-temperature bath, calorimetric thermometer, and an ultraviolet/ visible spectrophotometer.

Excess solute and solvent were placed in amber glass bottles and allowed to equilibrate for several days at constant temperature. Attainment of equilibrium was verified by several repetitive measurements and by approaching equilibrium from supersaturation. Aliquots of saturated solutions were transferred through a coarse filter into tared volumetric flasks, weighed and diluted with 2-propanol. Concentrations were determined by spectrophotometric measurements at $356 \mathrm{~nm}$.

\section{Source and Purity of Chemicals:}

(1) $99+\%$, Aldrich Chemical Company, Milwaukee, WI, USA, was recrystallized several times from propanone.

(2) $99 \%$, Aldrich Chemical Company, stored over molecular sieves before use.

Estimated Error:

Temperature: $\pm 0.1 \mathrm{~K}$.

$x_{1}: \pm 1.5 \%$ (relative error) 


\section{Components:}

(1) Anthracene; $\mathrm{C}_{14} \mathrm{H}_{10} ;[120-12-7]$

(2) Propylene carbonate; $\mathrm{C}_{4} \mathrm{H}_{6} \mathrm{O}_{3} ;$; $\left.108-32-7\right]$

Variables:

$T / \mathrm{K}=298.15$

Original Measurements:

W. E. Acree, Jr.,

unpublished data.

Prepared by:

W. E. Acree, Jr.

Experimental Values

\begin{tabular}{lcc}
\hline \hline$x_{2}{ }^{(\mathrm{s}) \mathrm{a}}$ & $x_{2}{ }^{\mathrm{b}}$ & $x_{1}{ }^{\mathrm{c}}$ \\
\hline 1.0000 & 0.9979 & 0.002102 \\
\hline
\end{tabular}

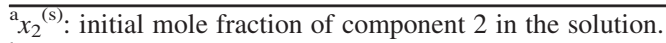

${ }^{\mathrm{b}} x_{2}$ : mole fraction of component 2 in the saturated solution.

${ }^{c} x_{1}$ : mole fraction solubility of the solute.

\section{Auxiliary Information}

\section{Method/Apparatus/Procedure:}

Constant-temperature bath, calorimetric thermometer, and an ultraviolet/ visible spectrophotometer.

Excess solute and solvent were placed in amber glass bottles and allowed to equilibrate for several days at constant temperature. Attainment of equilibrium was verified by several repetitive measurements and by approaching equilibrium from supersaturation. Aliquots of saturated solutions were transferred through a coarse filter into tared volumetric flasks, weighed and diluted with 2-propanol. Concentrations were determined by

spectrophotometric measurements at $356 \mathrm{~nm}$.

\section{Source and Purity of Chemicals:}

(1) $99+\%$, Aldrich Chemical Company, Milwaukee, WI, USA, was recrystallized several times from propanone.

(2) $99.7 \%$, anhydrous, Aldrich Chemical Company, stored over molecular sieves before use.

Estimated Error:

Temperature: $\pm 0.1 \mathrm{~K}$

$x_{1}: \pm 1.5 \%$ (relative error).

\section{Components:}

(1) Anthracene; $\mathrm{C}_{14} \mathrm{H}_{10} ;[120-12-7]$

(2) Hexanedinitrile; $\mathrm{C}_{6} \mathrm{H}_{8} \mathrm{~N}_{2}$; [111-69-3]

\begin{tabular}{ll}
\hline Variables: & Prepared by: \\
$T / \mathrm{K}=298.15$ & W. E. Acree, Jr. \\
\hline
\end{tabular}

Experimental Values

\begin{tabular}{lcc}
\hline \hline$x_{2}{ }^{(\mathrm{s}) \mathrm{a}}$ & $x_{2}{ }^{\mathrm{b}}$ & $x_{1}{ }^{\mathrm{c}}$ \\
\hline 1.0000 & 0.9972 & 0.002801 \\
\hline
\end{tabular}

${ }^{{ }} x_{2}{ }^{(\mathrm{s})}$ : initial mole fraction of component 2 in the solution.

${ }^{\mathrm{b}} x_{2}$ : mole fraction of component 2 in the saturated solution.

${ }^{c} x_{1}$ : mole fraction solubility of the solute.

\section{Auxiliary Information}

Method/Apparatus/Procedure:

Constant-temperature bath, calorimetric thermometer, and an ultraviolet/ visible spectrophotometer.

Excess solute and solvent were placed in amber glass bottles and allowed to equilibrate for several days at constant temperature. Attainment of equilibrium was verified by several repetitive measurements and by approaching equilibrium from supersaturation. Aliquots of saturated solutions were transferred through a coarse filter into tared volumetric flasks, weighed and diluted with 2-propanol. Concentrations were determined by spectrophotometric measurements at $356 \mathrm{~nm}$.

\section{Source and Purity of Chemicals:}

(1) $99+\%$, Aldrich Chemical Company, Milwaukee, WI, USA, was recrystallized several times from propanone.

(2) $99 \%$, Aldrich Chemical Company, stored over molecular sieves before use.

\section{Estimated Error:}

Temperature: $\pm 0.1 \mathrm{~K}$.

$x_{1}: \pm 1.5 \%$ (relative error).

\begin{tabular}{ll}
\hline \hline Components: & Original Measurements: \\
(1) Anthracene; $\mathrm{C}_{14} \mathrm{H}_{10} ;[120-12-7]$ & W. E. Acree, Jr., \\
(2) Tributyl phosphate; $\mathrm{C}_{12} \mathrm{H}_{27} \mathrm{O}_{4} \mathrm{P} ;[126-73-8]$ & unpublished data. \\
\hline Variables: & Prepared by: \\
$T / \mathrm{K}=298.15$ & W. E. Acree, Jr. \\
\hline
\end{tabular}

Experimental Values

\begin{tabular}{|c|c|c|}
\hline$x_{2}{ }^{(s) a}$ & $x_{2}{ }^{\mathrm{b}}$ & $x_{1}{ }^{\mathrm{c}}$ \\
\hline 1.0000 & 0.9850 & 0.01498 \\
\hline
\end{tabular}

\section{Auxiliary Information}

\section{Method/Apparatus/Procedure:}

Constant-temperature bath, calorimetric thermometer, and an ultraviolet/ visible spectrophotometer.

Excess solute and solvent were placed in amber glass bottles and allowed to equilibrate for several days at constant temperature. Attainment of equilibrium was verified by several repetitive measurements and by approaching equilibrium from supersaturation. Aliquots of saturated solutions were transferred through a coarse filter into tared volumetric flasks, weighed and diluted with 2-propanol. Concentrations were determined by spectrophotometric measurements at $356 \mathrm{~nm}$.

\section{Source and Purity of Chemicals:}

(1) $99+\%$, Aldrich Chemical Company, Milwaukee, WI, USA, was recrystallized several times from propanone.

(2) $99+\%$, Aldrich Chemical Company, stored over molecular sieves before use.

\section{Estimated Error:}

Temperature: $\pm 0.1 \mathrm{~K}$.

$x_{1}: \pm 1.5 \%$ (relative error). 


\section{Solubility of Biphenyl in Organic Solvents}

\subsection{Critical evaluation of experimental solubility data}

Volume 58 in the IUPAC Solubility Data Series ${ }^{2}$ contained experimental solubility data for biphenyl in three linear alkanes (hexane, heptane and octane) and eight cycloalkanes (cyclohexane, methylcyclohexane, cis-1,2dimethylcyclohexane, trans-1,2-dimethylcyclohexane, cis1,3-dimethylcyclohexane, cis-1,4-dimethylcyclohexane, trans-1,2-dimethylcyclohexane, and decahydronaphthalene), in four aromatic hydrocarbons (benzene, methylbenzene, ethylbenzene and 1,2,3,4-tetrahydronaphthalene), in one cyclic ether (1,4-dioxane), in six halogenated alkanes (dichloromethane, trichloromethane, tetrachloromethane, 1,2dibromethane, 1,1-dichloroethane, and 1,2-dichloroethane) and two halogenated benzenes (chlorobenzene and hexachlorobenzene), in one alkanol (1-octanol) and four miscellaneous organic solvents (carbon disulfide, pyridine, thiophene and nitrobenzene). For several of the solvents, the reporting authors made measurements at more than one temperature. Biphenyl solubility data in both hexane and octane covered the temperature range from 298 to $313 \mathrm{~K}$, data in heptane, cyclohexane, benzene, carbon tetrachloride, carbon disulfide, pyridine, and thiophene covered the range from about 298 to $335 \mathrm{~K}$, data in decahydronaphthalene and 1,2,3,4-tetrahydronaphthalene covered the range from about 309 to $328 \mathrm{~K}$, data in 1,4-dioxane covered the range from 300 to $341 \mathrm{~K}$, and data for hexafluorobenzene covered the range from 307 to $337 \mathrm{~K}$. The compiled solubility data also included phase diagram information for binary biphenyl + tetracosane, biphenyl + octacosane, biphenyl + naphthalene, biphenyl + 2-methylnaphthalene, biphenyl $+2,6$-dimethylnaphtalene, biphenyl + 1,2-diphenylethane, diphenyl + diphenyl ether, biphenyl + 1octadecanol, biphenyl + indole, biphenyl + diphenylamine, and biphenyl + octadecanoic acid mixtures. Solubility data contained in Vol. 58 will not be republished here. The listing above is provided so that readers will know what solubility data are available in the earlier volume for biphenyl.

A search of the published chemical and engineering literature covering the period of 1995 to 2011 found only two solubility studies involving biphenyl and one study reporting the phase diagrams for two binary systems containing biphenyl. De Fina et al. ${ }^{36}$ determined the solubility of biphenyl at $298.15 \mathrm{~K}$ in 26 different alkane, dialkyl ether and alcohol solvents based on a spectrophotometric method. Grubbs et $a l .{ }^{37}$ later measured the solubility of biphenyl in five alkoxyalcohols (2-ethoxyethanol, 2-propoxyethanol, 2-isopropoxyethanol, 2-butoxyethanol, and 3-methoxy-1-butanol) at $298.15 \mathrm{~K}$. It is not possible to conduct a critical evaluation of these two sets of published biphenyl solubility data because independent values are not available, and because the measurements were performed at only a single temperature. Phase diagrams of the binary biphenyl + octadecane and biphenyl + naphthalene systems were reported by Mengarelli et $a .^{38}$ Freezing-point temperatures for the biphenyl + naphthalene system were in good agreement with calculated values assuming an ideal solution. In the case of the binary biphenyl + octadecane system, the authors noted that activity coefficients of the polycyclic aromatic hydrocarbon solute, calculated from the freezing point temperatures, differed by $2 \%$ from calculated values using Flory's model with regular solution residual contribution and a $2 / 3$ exponent in the definition of the volume fraction.

The experimental solubility data for biphenyl dissolved in the different organic solvents are given in Secs. 4.2-4.7.

\subsection{Biphenyl solubility data in saturated hydrocarbons (including cycloalkanes)}

\begin{tabular}{ll}
\hline \hline Components: & Original Measurements: \\
(1) Biphenyl; $\mathrm{C}_{12} \mathrm{H}_{10} ;[92-52-4]$ & ${ }^{36} \mathrm{~K}$. M. De Fina, T. L. Sharp, and W. E. \\
(2) Nonane; $\mathrm{C}_{9} \mathrm{H}_{20} ;[111-84-2]$ & Acree, Jr., Can. J. Chem. 77, 1589 (1999). \\
\hline Variables: & Prepared by: \\
$T / \mathrm{K}=298.15$ & W. E. Acree, Jr. \\
\hline
\end{tabular}

Experimental Values

\begin{tabular}{lcc}
\hline \hline$x_{2}{ }^{(\mathrm{s}) \mathrm{a}}$ & $x_{2}{ }^{\mathrm{b}}$ & $x_{1}{ }^{\mathrm{c}}$ \\
\hline 1.0000 & 0.8449 & 0.1551 \\
$\frac{{ }^{\mathrm{a}} x_{2}{ }^{(\mathrm{s})}: \text { initial mole fraction of component } 2 \text { in the solution. }}{{ }^{\mathrm{b}} x_{2}: \text { mole fraction of component } 2 \text { in the saturated solution. }}$ \\
${ }^{\mathrm{c}} x_{1}:$ mole fraction solubility of the solute.
\end{tabular}

Auxiliary Information

\section{Method/Apparatus/Procedure:}

Constant-temperature bath, calorimetric thermometer, and an ultraviolet/ visible spectrophotometer.

Excess solute and solvent were placed in amber glass bottles and allowed to equilibrate for several days at constant temperature. Attainment of equilibrium was verified by several repetitive measurements and by approaching equilibrium from supersaturation. Aliquots of saturated solutions were transferred through a coarse filter into tared volumetric flasks, weighed and diluted with 2-propanol. Concentrations were determined by spectrophotometric measurements at $250 \mathrm{~nm}$.

Source and Purity of Chemicals:

(1) $99 \%$, Aldrich Chemical Company, Milwaukee, WI, USA, was recrystallized several times from methanol.

(2) 99+\%, TCI America, Portland, OR, USA, stored over molecular sieves before use.

Estimated Error:

Temperature: $\pm 0.1 \mathrm{~K}$.

$x_{1}: \pm 1.5 \%$ (relative error)

\begin{tabular}{ll}
\hline \hline Components: & Original Measurements: \\
(1) Biphenyl; $\mathrm{C}_{12} \mathrm{H}_{10} ;[92-52-4]$ & ${ }^{36}$ K. M. De Fina, T. L. Sharp, and W. E. \\
(2) Decane; $\mathrm{C}_{10} \mathrm{H}_{22} ;[124-18-5]$ & Acree, Jr., Can. J. Chem. 77, 1589(1999). \\
\hline Variables: & Prepared by: \\
$T / \mathrm{K}=298.15$ & W. E. Acree, Jr. \\
\hline
\end{tabular}


Experimental Values

\begin{tabular}{lcc}
\hline \hline$x_{2}{ }^{(\mathrm{s})}$ & $x_{2}$ & $x_{1}$ \\
\hline 1.0000 & 0.8364 & 0.1636 \\
$\frac{{ }^{\mathrm{a}} x_{2}{ }^{(\mathrm{s})}: \text { initial mole fraction of component } 2 \text { in the solution. }}{{ }^{\mathrm{b}}{ }_{x_{2}}: \text { mole fraction of component } 2 \text { in the saturated solution. }}$ \\
${ }^{\mathrm{c}}{ }_{x_{1}}:$ mole fraction solubility of the solute.
\end{tabular}

\section{Auxiliary Information}

\section{Method/Apparatus/Procedure:}

Constant-temperature bath, calorimetric thermometer, and an ultraviolet/ visible spectrophotometer.

Excess solute and solvent were placed in amber glass bottles and allowed to equilibrate for several days at constant temperature. Attainment of equilibrium was verified by several repetitive measurements and by approaching equilibrium from supersaturation. Aliquots of saturated solutions were transferred through a coarse filter into tared volumetric flasks, weighed and diluted with 2-propanol. Concentrations were determined by

spectrophotometric measurements at $250 \mathrm{~nm}$.

Source and Purity of Chemicals:

(1) $99 \%$, Aldrich Chemical Company, Milwaukee, WI, USA, was recrystallized several times from methanol.

(2) $99+\%$, TCI America, Portland, OR, USA, stored over molecular sieves before use.

\section{Estimated Error:}

Temperature: $\pm 0.1 \mathrm{~K}$.

$x_{1}: \pm 1.5 \%$ (relative error).

\begin{tabular}{ll}
\hline \hline Components: & Original Measurements: \\
(1) Biphenyl; $\mathrm{C}_{12} \mathrm{H}_{10} ;[92-52-4]$ & ${ }^{36} \mathrm{~K}$. M. De Fina, T. L. Sharp, and \\
(2) Hexadecane; $\mathrm{C}_{16} \mathrm{H}_{34} ;[544-76-3]$ & W. E. Acree, Jr., Can. J. Chem. 77, \\
& $1589(1999)$. \\
\hline Variables: & Prepared by: \\
$T / \mathrm{K}=298.15$ & W. E. Acree, Jr. \\
\hline
\end{tabular}

Experimental Values

\begin{tabular}{lcc}
\hline \hline$x_{2}{ }^{(\mathrm{s}) \mathrm{a}}$ & $x_{2}{ }^{\mathrm{b}}$ & $x_{1}{ }^{\mathrm{c}}$ \\
\hline 1.0000 & 0.7849 & 0.2151 \\
${ }^{\mathrm{a}} x_{2}{ }^{\left({ }^{(}\right)}:$initial mole fraction of component 2 in the solution. \\
${ }^{\mathrm{b}} x_{2}:$ mole fraction of component 2 in the saturated solution. \\
${ }^{\mathrm{c}} x_{1}:$ mole fraction solubility of the solute.
\end{tabular}

\section{Auxiliary Information}

\section{Method/Apparatus/Procedure:}

Constant-temperature bath, calorimetric thermometer, and an ultraviolet/ visible spectrophotometer.

Excess solute and solvent were placed in amber glass bottles and allowed to equilibrate for several days at constant temperature. Attainment of equilibrium was verified by several repetitive measurements and by approaching equilibrium from supersaturation. Aliquots of saturated solutions were transferred through a coarse filter into tared volumetric flasks, weighed and diluted with 2-propanol. Concentrations were determined by spectrophotometric measurements at $250 \mathrm{~nm}$.
Source and Purity of Chemicals:

(1) $99 \%$, Aldrich Chemical Company, Milwaukee, WI, USA, was recrystallized several times from methanol.

(2) $99 \%$, Aldrich Chemical Company, stored over molecular sieves before use.

Estimated Error:

Temperature: $\pm 0.1 \mathrm{~K}$.

$x_{1}: \pm 1.5 \%$ (relative error).

\begin{tabular}{|c|c|}
\hline $\begin{array}{l}\text { Components: } \\
\text { (1) Biphenyl; } \mathrm{C}_{12} \mathrm{H}_{10} ;[92-52-4] \\
\text { (2) Octadecane; } \mathrm{C}_{18} \mathrm{H}_{38} ;[593-45-3]\end{array}$ & $\begin{array}{l}\text { Original Measurements: } \\
{ }^{38} \text { A. C. Mengarelli, S. B. Bottini, } \\
\text { and E. A. Brignole, J. Chem. Eng. } \\
\text { Data 40, } 746 \text { (1995). }\end{array}$ \\
\hline Variables: & Prepared by: \\
\hline Temperature & W. E. Acree, Jr. \\
\hline
\end{tabular}

Experimental Values

\begin{tabular}{lcc}
\hline \hline$T / \mathrm{K}$ & $x_{2}{ }^{\mathrm{a}}$ & $x_{1}{ }^{\mathrm{b}}$ \\
\hline 301.25 & 1.000 & 0.000 \\
299.60 & 0.845 & 0.155 \\
299.12 & 0.775 & 0.225 \\
298.81 & 0.754 & 0.246 \\
298.77 & 0.748 & 0.252 \\
298.57 & 0.734 & 0.266 \\
303.86 & 0.708 & 0.292 \\
312.25 & 0.645 & 0.355 \\
317.60 & 0.586 & 0.414 \\
324.95 & 0.476 & 0.524 \\
328.75 & 0.378 & 0.622 \\
332.22 & 0.288 & 0.712 \\
334.21 & 0.206 & 0.794 \\
336.57 & 0.132 & 0.868 \\
338.77 & 0.063 & 0.937 \\
341.62 & 0.000 & 1.000 \\
\hline
\end{tabular}

${ }^{a} x_{2}$ : mole fraction of component 2 in the saturated solution.

${ }^{\mathrm{b}} x_{1}$ : mole fraction of the polycyclic aromatic hydrocarbon (component 1).

\section{Auxiliary Information}

\begin{abstract}
Method/Apparatus/Procedure:
Thermostatted liquid bath, platinum resistance thermometer, and a coiled wire driven by a small electrical motor to provide stirring.

A liquid mixture of known composition was prepared by mass and placed in a glass tube. The solution was slowly cooled until a solid phase appeared. The temperature of the solution was recorded continuously with a platinum resistance thermometer. A break point in the temperature versus time curve indicated formation of a solid phase. The temperature at break point was taken to be the solid-liquid equilibrium temperature, and the composition of the solution gives the solubility of the crystalline solute in the liquid solvent.
\end{abstract}

Source and Purity of Chemicals:

(1) Chemical purity and source not given in the paper.

(2) $97 \%$, Aldrich Chemical Company, Milwaukee, WI, USA, purification details were not given in the paper.

\section{Estimated Error:}

Temperature: $\pm 0.5 \mathrm{~K}$.

$x_{1}: \pm 0.002$ (estimated by compiler). 


\section{Components:}

(1) Biphenyl; $\mathrm{C}_{12} \mathrm{H}_{10} ;[92-52-4]$

(2) Cyclooctane; $\mathrm{C}_{8} \mathrm{H}_{16} ;[292-64-8]$

Variables:

$T / \mathrm{K}=298.15$

Original Measurements:

${ }^{36}$ K. M. De Fina, T. L. Sharp, and

W. E. Acree, Jr., Can. J. Chem. 77, 1589 (1999).

Prepared by:

W. E. Acree, Jr.

\section{Experimental Values}

\begin{tabular}{lcc}
\hline \hline$x_{2}{ }^{(\mathrm{s}) \mathrm{a}}$ & $x_{2}{ }^{\mathrm{b}}$ & $x_{1}{ }^{\mathrm{c}}$ \\
\hline 1.0000 & 0.7806 & 0.2194 \\
\hline
\end{tabular}

${ }^{\mathrm{a}} x_{2}{ }^{(\mathrm{s})}$ : initial mole fraction of component 2 in the solution.

${ }^{b} x_{2}$ : mole fraction of component 2 in the saturated solution.

${ }^{c} x_{1}$ : mole fraction solubility of the solute.

\section{Auxiliary Information}

\begin{abstract}
Method/Apparatus/Procedure:
Constant-temperature bath, calorimetric thermometer, and an ultraviolet/ visible spectrophotometer.

Excess solute and solvent were placed in amber glass bottles and allowed to equilibrate for several days at constant temperature. Attainment of equilibrium was verified by several repetitive measurements and by approaching equilibrium from supersaturation. Aliquots of saturated solutions were transferred through a coarse filter into tared volumetric flasks, weighed and diluted with 2-propanol. Concentrations were determined by

spectrophotometric measurements at $250 \mathrm{~nm}$.
\end{abstract}

Source and Purity of Chemicals:

(1) $99 \%$, Aldrich Chemical Company, Milwaukee, WI, USA, was recrystallized several times from methanol.

(2) $99+\%$, Aldrich Chemical Company, stored over molecular sieves before use.

\section{Estimated Error:}

Temperature: $\pm 0.1 \mathrm{~K}$.

$x_{1}: \pm 1.5 \%$ (relative error).

\begin{tabular}{|c|c|}
\hline $\begin{array}{l}\text { Components: } \\
\text { (1) Biphenyl; } \mathrm{C}_{12} \mathrm{H}_{10} ;[92-52-4] \\
\text { (2) } 2,2,4-\text { Trimethylpentane; } \mathrm{C}_{8} \mathrm{H}_{18} \text {; } \\
\text { [540-84-1] }\end{array}$ & $\begin{array}{l}\text { Original Measurements: } \\
{ }^{36} \text { K. M. De Fina, T. L. Sharp, and } \\
\text { W. E. Acree, Jr., Can. J. Chem. 77, } \\
1589 \text { (1999). }\end{array}$ \\
\hline $\begin{array}{l}\text { Variables: } \\
T / \mathrm{K}=298.15\end{array}$ & $\begin{array}{l}\text { Prepared by: } \\
\text { W. E. Acree, Jr. }\end{array}$ \\
\hline
\end{tabular}

Experimental Values

\begin{tabular}{lcc}
\hline \hline$x_{2}{ }^{(\mathrm{s}) \mathrm{a}}$ & $x_{2}{ }^{\mathrm{b}}$ & $x_{1}{ }^{\mathrm{c}}$ \\
\hline 1.0000 & 0.8906 & 0.1094 \\
${ }^{\mathrm{a}}{ }_{x_{2}}{ }^{(\mathrm{s})}:$ initial mole fraction of component 2 in the solution. \\
${ }^{\mathrm{b}} x_{2}:$ mole fraction of component 2 in the saturated solution. \\
${ }^{\mathrm{c}} x_{1}:$ mole fraction solubility of the solute.
\end{tabular}

\section{Auxiliary Information}

Method/Apparatus/Procedure:

Constant-temperature bath, calorimetric thermometer, and an ultraviolet/ visible spectrophotometer.

Excess solute and solvent were placed in amber glass bottles and allowed to equilibrate for several days at constant temperature. Attainment of equilibrium was verified by several repetitive measurements and by approaching equilibrium from supersaturation. Aliquots of saturated solutions were transferred through a coarse filter into tared volumetric flasks, weighed and diluted with 2-propanol. Concentrations were determined by spectrophotometric measurements at $250 \mathrm{~nm}$.

\section{Source and Purity of Chemicals:}

(1) $99 \%$, Aldrich Chemical Company, Milwaukee, WI, USA, was recrystallized several times from methanol.

(2) HPLC Grade, Aldrich Chemical Company, stored over molecular sieves before use.

Estimated Error:

Temperature: $\pm 0.1 \mathrm{~K}$.

$x_{1}: \pm 1.5 \%$ (relative error).

\begin{tabular}{|c|c|}
\hline $\begin{array}{l}\text { Components: } \\
\text { (1) Biphenyl; } \mathrm{C}_{12} \mathrm{H}_{10} ;[92-52-4] \\
\text { (2) tert-Butylcyclohexane; } \mathrm{C}_{10} \mathrm{H}_{20} \text {; } \\
\text { [3178-22-1] }\end{array}$ & $\begin{array}{l}\text { Original Measurements: } \\
{ }^{36} \text { K. M. De Fina, T. L. Sharp, and } \\
\text { W. E. Acree, Jr., Can. J. Chem. 77, } \\
\text { 1589 (1999). }\end{array}$ \\
\hline $\begin{array}{l}\text { Variables: } \\
T / \mathrm{K}=298.15\end{array}$ & $\begin{array}{l}\text { Prepared by: } \\
\text { W. E. Acree, Jr. }\end{array}$ \\
\hline
\end{tabular}

Experimental Values

\begin{tabular}{lcc}
\hline \hline$x_{2}{ }^{(\mathrm{s}) \mathrm{a}}$ & $x_{2}{ }^{\mathrm{b}}$ & $x_{1}{ }^{\mathrm{c}}$ \\
\hline 1.0000 & 0.8260 & 0.1740 \\
$\frac{{ }^{\mathrm{a}} x_{2}{ }^{(\mathrm{s})}: \text { initial mole fraction of component } 2 \text { in the solution. }}{{ }^{\mathrm{b}} x_{2}: \text { mole fraction of component } 2 \text { in the saturated solution. }}$ \\
${ }^{\mathrm{c}} x_{1}:$ mole fraction solubility of the solute.
\end{tabular}

\section{Auxiliary Information}

\section{Method/Apparatus/Procedure:}

Constant-temperature bath, calorimetric thermometer, and an ultraviolet/ visible spectrophotometer.

Excess solute and solvent were placed in amber glass bottles and allowed to equilibrate for several days at constant temperature. Attainment of equilibrium was verified by several repetitive measurements and by approaching equilibrium from supersaturation. Aliquots of saturated solutions were transferred through a coarse filter into tared volumetric flasks, weighed and diluted with 2-propanol. Concentrations were determined by spectrophotometric measurements at $250 \mathrm{~nm}$.

\section{Source and Purity of Chemicals:}

(1) $99 \%$, Aldrich Chemical Company, Milwaukee, WI, USA, was recrystallized several times from methanol.

(2) $99+\%$, Aldrich Chemical Company, stored over molecular sieves before use.

Estimated Error:

Temperature: $\pm 0.1 \mathrm{~K}$.

$x_{1}: \pm 1.5 \%$ (relative error) 


\subsection{Biphenyl solubility data in aromatic hydrocarbons}

\begin{tabular}{ll}
\hline \hline Components: & Original Measurements: \\
(1) Biphenyl; $\mathrm{C}_{12} \mathrm{H}_{10} ;[92-52-4]$ & ${ }^{38}$ A. C. Mengarelli, S. B. Bottini, \\
(2) Naphthalene; $\mathrm{C}_{10} \mathrm{H}_{8} ;[91-20-3]$ & and E. A. Brignole, J. Chem. Eng. \\
& Data 40, 746 (1995). \\
\hline Variables: & Prepared by: \\
Temperature & W. E. Acree, Jr. \\
\hline
\end{tabular}

Experimental Values

\begin{tabular}{lcc}
\hline \hline$T / \mathrm{K}$ & $x_{2}{ }^{\mathrm{a}}$ & $x_{1}{ }^{\mathrm{b}}$ \\
\hline 351.88 & 1.00 & 0.00 \\
347.49 & 0.92 & 0.08 \\
342.42 & 0.83 & 0.17 \\
336.26 & 0.74 & 0.26 \\
329.50 & 0.64 & 0.36 \\
321.50 & 0.55 & 0.45 \\
317.69 & 0.50 & 0.50 \\
312.20 & 0.44 & 0.56 \\
316.15 & 0.39 & 0.61 \\
320.99 & 0.34 & 0.66 \\
328.76 & 0.23 & 0.77 \\
335.20 & 0.12 & 0.88 \\
341.62 & 0.00 & 1.00 \\
\hline
\end{tabular}

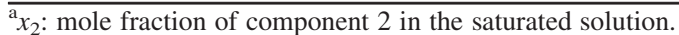

${ }^{b} x_{1}$ : mole fraction of the polycyclic aromatic hydrocarbon (component 1 ).

\section{Auxiliary Information}

\section{Method/Apparatus/Procedure:}

Thermostatted liquid bath, platinum resistance thermometer, and a coiled wire driven by a small electrical motor to provide stirring.

A liquid mixture of known composition was prepared by mass and placed in a glass tube. The solution was slowly cooled until a solid phase appeared. The temperature of the solution was recorded continuously with a platinum resistance thermometer. A break point in the temperature versus time curve indicated formation of a solid phase. The temperature at break point was taken to be the solid-liquid equilibrium temperature, and the composition of the solution gives the solubility of the crystalline solute in the liquid solvent.

Source and Purity of Chemicals:

(1) Chemical purity and source not given in the paper.

(2) Chemical purity and source not given in the paper.

Estimated Error:

Temperature: $\pm 0.5 \mathrm{~K}$

$x_{1}: \pm 0.01$ (estimated by compiler).
Experimental Values

\begin{tabular}{lcc}
\hline \hline$x_{2}{ }^{(\mathrm{s}) \mathrm{a}}$ & $x_{2}{ }^{\mathrm{b}}$ & $x_{1}{ }^{\mathrm{c}}$ \\
\hline 1.0000 & 0.7340 & 0.2660 \\
$\frac{{ }^{a} x_{2}{ }^{(\mathrm{s})}: \text { initial mole fraction of component } 2 \text { in the solution. }}{{ }^{\mathrm{b}} x_{2}: \text { mole fraction of component } 2 \text { in the saturated solution. }}$ \\
${ }^{\mathrm{c}} x_{1}:$ mole fraction solubility of the solute.
\end{tabular}

\section{Auxiliary Information}

\section{Method/Apparatus/Procedure:}

Constant-temperature bath, calorimetric thermometer, and an ultraviolet/ visible spectrophotometer.

Excess solute and solvent were placed in amber glass bottles and allowed to equilibrate for several days at constant temperature. Attainment of equilibrium was verified by several repetitive measurements and by approaching equilibrium from supersaturation. Aliquots of saturated solutions were transferred through a coarse filter into tared volumetric flasks, weighed and diluted with 2-propanol. Concentrations were determined by spectrophotometric measurements at $250 \mathrm{~nm}$.

\section{Source and Purity of Chemicals:}

(1) $99 \%$, Aldrich Chemical Company, Milwaukee, WI, USA, was recrystallized several times from methanol.

(2) $99 \%$, Aldrich Chemical Company, stored over molecular sieves before use.

\section{Estimated Error:}

Temperature: $\pm 0.1 \mathrm{~K}$.

$x_{1}: \pm 1.5 \%$ (relative error).

\section{Components:}

(1) Biphenyl; $\mathrm{C}_{12} \mathrm{H}_{10} ;[92-52-4]$

(2) 2-Methoxy-2-methylpropane; $\mathrm{C}_{5} \mathrm{H}_{12} \mathrm{O}$; [1634-04-4]

\section{Variables:}

$T / \mathrm{K}=298.15$

Original Measurements: W. E. Acree, Jr., unpublished data.

Prepared by:

W. E. Acree, Jr.

\section{Experimental Values}

\begin{tabular}{lcc}
\hline \hline$x_{2}{ }^{(\mathrm{s}) \mathrm{a}}$ & $x_{2}{ }^{\mathrm{b}}$ & $x_{1}{ }^{\mathrm{c}}$ \\
\hline 1.0000 & 0.7150 & 0.2850 \\
${ }^{\mathrm{a}} x_{2}{ }^{(\mathrm{s})}$ : initial mole fraction of component 2 in the solution. \\
${ }^{\mathrm{b}} x_{2}:$ mole fraction of component 2 in the saturated solution. \\
${ }^{\mathrm{c}} x_{1}$ : mole fraction solubility of the solute.
\end{tabular}

\subsection{Biphenyl solubility data in ethers}

\begin{tabular}{|c|c|}
\hline $\begin{array}{l}\text { Components: } \\
\text { (1) Biphenyl; } \mathrm{C}_{12} \mathrm{H}_{10} ;[92-52-4] \\
\text { (2) } 1,1^{\prime}-\mathrm{O} y \mathrm{~b} \text { bisbutane; } \mathrm{C}_{8} \mathrm{H}_{18} \mathrm{O} \text {; } \\
\text { [142-96-1] }\end{array}$ & $\begin{array}{l}\text { Original Measurements: } \\
{ }^{36} \text { K. M. De Fina, T. L. Sharp, and W. } \\
\text { E. Acree, Jr., Can. J. Chem. 77, } 1589 \\
\text { (1999). }\end{array}$ \\
\hline $\begin{array}{l}\text { Variables: } \\
T / \mathrm{K}=298.15\end{array}$ & $\begin{array}{l}\text { Prepared by: } \\
\text { W. E. Acree, Jr. }\end{array}$ \\
\hline
\end{tabular}




\section{Auxiliary Information}

\section{Method/Apparatus/Procedure:}

Constant-temperature bath, calorimetric thermometer, and an ultraviolet/ visible spectrophotometer.

Excess solute and solvent were placed in amber glass bottles and allowed to equilibrate for several days at constant temperature. Attainment of equilibrium was verified by several repetitive measurements and by approaching equilibrium from supersaturation. Aliquots of saturated solutions were transferred through a coarse filter into tared volumetric flasks, weighed and diluted with 2-propanol. Concentrations were determined by spectrophotometric measurements at $250 \mathrm{~nm}$.

Source and Purity of Chemicals:

(1) $99 \%$, Aldrich Chemical Company, Milwaukee, WI, USA, was recrystallized several times from methanol.

(2) $99.9+\%$, Arco Chemical Company, USA, stored over molecular sieves before use.

Estimated Error:

Temperature: $\pm 0.1 \mathrm{~K}$

$x_{1}: \pm 1.5 \%$ (relative error).
Estimated Error:

Temperature: $\pm 0.1 \mathrm{~K}$.

$x_{1}: \pm 1.5 \%$ (relative error).

\begin{tabular}{ll}
\hline \hline Components: & Original Measurements: \\
(1) Biphenyl; $\mathrm{C}_{12} \mathrm{H}_{10} ;[92-52-4]$ & ${ }^{36} \mathrm{~K}$. M. De Fina, T. L. Sharp, and W. E. \\
(2) Ethanol; $\mathrm{C}_{2} \mathrm{H}_{6} \mathrm{O} ;[64-17-5]$ & Acree, Jr., Can. J. Chem. 77, 1589 (1999). \\
\hline Variables: & Prepared by: \\
$T / \mathrm{K}=298.15$ & W. E. Acree, Jr. \\
\hline
\end{tabular}

Experimental Values

\begin{tabular}{lcc}
\hline \hline$x_{2}{ }^{(\mathrm{s}) \mathrm{a}}$ & $x_{2}{ }^{\mathrm{b}}$ & $x_{1}{ }^{\mathrm{c}}$ \\
\hline 1.0000 & 0.9654 & 0.03456
\end{tabular}

${ }^{\mathrm{a}} x_{2}{ }^{(\mathrm{s})}$ : initial mole fraction of component 2 in the solution.

${ }^{b} x_{2}$ : mole fraction of component 2 in the saturated solution.

${ }^{c} x_{1}$ : mole fraction solubility of the solute.

\section{Auxiliary Information}

\section{Method/Apparatus/Procedure:}

Constant-temperature bath, calorimetric thermometer, and an ultraviolet/ visible spectrophotometer.

Excess solute and solvent were placed in amber glass bottles and allowed to equilibrate for several days at constant temperature. Attainment of equilibrium was verified by several repetitive measurements and by approaching equilibrium from supersaturation. Aliquots of saturated solutions were transferred through a coarse filter into tared volumetric flasks, weighed and diluted with 2-propanol. Concentrations were determined by spectrophotometric measurements at $250 \mathrm{~nm}$.

\section{Experimental Values}

\begin{tabular}{lcc}
\hline \hline$x_{2}{ }^{(\mathrm{s}) \mathrm{a}}$ & $x_{2}{ }^{\mathrm{b}}$ & $x_{1}{ }^{\mathrm{c}}$ \\
\hline 1.0000 & 0.9815 & 0.01851 \\
\hline
\end{tabular}

${ }^{\mathrm{a}} x_{2}{ }^{(\mathrm{s})}$ : initial mole fraction of component 2 in the solution.

${ }^{b} x_{2}$ : mole fraction of component 2 in the saturated solution.

${ }^{c} x_{1}$ : mole fraction solubility of the solute.

\section{Auxiliary Information}

\section{Method/Apparatus/Procedure:}

Constant-temperature bath, calorimetric thermometer, and an ultraviolet/ visible spectrophotometer.

Excess solute and solvent were placed in amber glass bottles and allowed to equilibrate for several days at constant temperature. Attainment of equilibrium was verified by several repetitive measurements and by approaching equilibrium from supersaturation. Aliquots of saturated solutions were transferred through a coarse filter into tared volumetric flasks, weighed and diluted with 2-propanol. Concentrations were determined by spectrophotometric measurements at $250 \mathrm{~nm}$.

\section{Source and Purity of Chemicals:}

(1) $99 \%$, Aldrich Chemical Company, Milwaukee, WI, USA, was recrystallized several times from methanol.

(2) $99.9+\%$, Aldrich Chemical Company, stored over molecular sieves before use.

\section{Source and Purity of Chemicals:}

(1) $99 \%$, Aldrich Chemical Company, Milwaukee, WI, USA, was recrystallized several times from methanol.

(2) Absolute, Aaper Alcohol and Chemical Company, USA, stored over molecular sieves before use.

\section{Estimated Error:}

Temperature: $\pm 0.1 \mathrm{~K}$.

$x_{1}: \pm 1.5 \%$ (relative error).

\begin{tabular}{ll}
\hline \hline Components: & Original Measurements: \\
(1) Biphenyl; $\mathrm{C}_{12} \mathrm{H}_{10} ;[92-52-4]$ & ${ }^{36} \mathrm{~K}$. M. De Fina, T. L. Sharp, and \\
(2) 1-Propanol; $\mathrm{C}_{3} \mathrm{H}_{8} \mathrm{O} ;[71-23-8]$ & W. E. Acree, Jr., Can. J. Chem. 77, \\
& $1589(1999)$. \\
\hline Variables: & Prepared by: \\
$T / \mathrm{K}=298.15$ & W. E. Acree, Jr. \\
\hline
\end{tabular}


Experimental Values

\begin{tabular}{lcc}
\hline$\frac{x_{2}{ }^{(\mathrm{s}) \mathrm{a}}}{x_{2}{ }^{\mathrm{b}}}$ & $x_{1}{ }^{\mathrm{c}}$ \\
\hline 1.0000 & 0.9538 & 0.04620 \\
$\frac{{ }^{\mathrm{a}} x_{2}{ }^{(\mathrm{s})}: \text { initial mole fraction of component } 2 \text { in the solution. }}{{ }^{\mathrm{b}}{ }_{x_{2}}: \text { mole fraction of component } 2 \text { in the saturated solution. }}$ \\
${ }^{\mathrm{c}} x_{1}:$ mole fraction solubility of the solute.
\end{tabular}

\section{Auxiliary Information}

\section{Method/Apparatus/Procedure:}

Constant-temperature bath, calorimetric thermometer, and an ultraviolet/ visible spectrophotometer.

Excess solute and solvent were placed in amber glass bottles and allowed to equilibrate for several days at constant temperature. Attainment of equilibrium was verified by several repetitive measurements and by approaching equilibrium from supersaturation. Aliquots of saturated solutions were transferred through a coarse filter into tared volumetric flasks, weighed and diluted with 2-propanol. Concentrations were determined by spectrophotometric measurements at $250 \mathrm{~nm}$

Source and Purity of Chemicals:

(1) $99 \%$, Aldrich Chemical Company, Milwaukee, WI, USA, was recrystallized several times from methanol.

(2) $99+\%$, anhydrous, Aldrich Chemical Company, stored over molecular sieves before use.

Estimated Error:

Temperature: $\pm 0.1 \mathrm{~K}$

$x_{1}: \pm 1.5 \%$ (relative error).

\section{Components:}

(1) Biphenyl; $\mathrm{C}_{12} \mathrm{H}_{10} ;[92-52-4]$

(2) 2-Propanol; $\mathrm{C}_{3} \mathrm{H}_{8} \mathrm{O}$; [67-63-0]

\section{Variables:}

$T / \mathrm{K}=298.15$

\section{Original Measurements:}

${ }^{36}$ K. M. De Fina, T. L. Sharp, and W. E. Acree, Jr., Can. J. Chem. 77, 1589 (1999).

Prepared by:

W. E. Acree, Jr.

\section{Experimental Values}

\begin{tabular}{lcc}
\hline \hline$x_{2}{ }^{(\mathrm{s}) \mathrm{a}}$ & $x_{2}^{\mathrm{b}}$ & $x_{1}{ }^{\mathrm{c}}$ \\
\hline 1.0000 & 0.9647 & 0.03533 \\
${ }^{{ }^{\mathrm{a}} x_{2}{ }^{(\mathrm{s})}: \text { initial mole fraction of component } 2 \text { in the solution. }}$ \\
${ }^{\mathrm{b}} x_{2}:$ mole fraction of component 2 in the saturated solution. \\
${ }^{\mathrm{c}} x_{1}:$ mole fraction solubility of the solute.
\end{tabular}

\section{Auxiliary Information}

\section{Method/Apparatus/Procedure:}

Constant-temperature bath, calorimetric thermometer, and an ultraviolet/ visible spectrophotometer.

Excess solute and solvent were placed in amber glass bottles and allowed to equilibrate for several days at constant temperature. Attainment of equilibrium was verified by several repetitive measurements and by approaching equilibrium from supersaturation. Aliquots of saturated solutions were transferred through a coarse filter into tared volumetric flasks, weighed and diluted with 2-propanol. Concentrations were determined by spectrophotometric measurements at $250 \mathrm{~nm}$.
Source and Purity of Chemicals:

(1) $99 \%$, Aldrich Chemical Company, Milwaukee, WI, USA, was recrystallized several times from methanol.

(2) $99+\%$, anhydrous, Aldrich Chemical Company, stored over molecular sieves before use.

\section{Estimated Error:}

Temperature: $\pm 0.1 \mathrm{~K}$.

$x_{1}: \pm 1.5 \%$ (relative error).

\begin{tabular}{ll}
\hline \hline Components: & Original Measurements: \\
(1) Biphenyl; $\mathrm{C}_{12} \mathrm{H}_{10} ;[92-52-4]$ & ${ }^{36}$ K. M. De Fina, T. L. Sharp, and \\
(2) 1-Butanol; $\mathrm{C}_{4} \mathrm{H}_{10} \mathrm{O} ;[71-36-3]$ & W. E. Acree, Jr., Can. J. Chem. 77, \\
& $1589(1999)$. \\
\hline Variables: & Prepared by: \\
$T / \mathrm{K}=298.15$ & W. E. Acree, Jr. \\
\hline
\end{tabular}

\section{Experimental Values}

\begin{tabular}{lcc}
\hline \hline$x_{2}{ }^{(\mathrm{s}) \mathrm{a}}$ & $x_{2}{ }^{\mathrm{b}}$ & $x_{1}{ }^{\mathrm{c}}$ \\
\hline 1.0000 & 0.9421 & 0.05788 \\
${ }^{\mathrm{a}} x_{2}{ }^{(\mathrm{s})}$ : initial mole fraction of component 2 in the solution. \\
${ }^{\mathrm{b}} x_{2}$ : mole fraction of component 2 in the saturated solution. \\
${ }^{\mathrm{c}} x_{1}$ : mole fraction solubility of the solute.
\end{tabular}

\section{Auxiliary Information}

\section{Method/Apparatus/Procedure:}

Constant-temperature bath, calorimetric thermometer, and an ultraviolet/ visible spectrophotometer.

Excess solute and solvent were placed in amber glass bottles and allowed to equilibrate for several days at constant temperature. Attainment of equilibrium was verified by several repetitive measurements and by approaching equilibrium from supersaturation. Aliquots of saturated solutions were transferred through a coarse filter into tared volumetric flasks, weighed and diluted with 2-propanol. Concentrations were determined by spectrophotometric measurements at $250 \mathrm{~nm}$.

\section{Source and Purity of Chemicals:}

(1) $99 \%$, Aldrich Chemical Company, Milwaukee, WI, USA, was recrystallized several times from methanol.

(2) $99.8+\%$, HPLC Grade, Aldrich Chemical Company, stored over molecular sieves before use.

\section{Estimated Error:}

Temperature: $\pm 0.1 \mathrm{~K}$.

$x_{1}: \pm 1.5 \%$ (relative error).

\begin{tabular}{ll}
\hline \hline Components: & Original Measurements: \\
(1) Biphenyl; $\mathrm{C}_{12} \mathrm{H}_{10} ;[92-52-4]$ & ${ }^{36}$ K. M. De Fina, T. L. Sharp, and \\
(2) 2-Butanol; $\mathrm{C}_{4} \mathrm{H}_{10} \mathrm{O} ;[78-92-2]$ & W. E. Acree, Jr., Can. J. Chem. 77, \\
& $1589(1999)$. \\
\hline Variables: & Prepared by: \\
$T / \mathrm{K}=298.15$ & W. E. Acree, Jr. \\
\hline
\end{tabular}


Experimental Values

\begin{tabular}{lcc}
\hline$x_{2}{ }^{(\mathrm{s}) \mathrm{a}}$ & $x_{2}{ }^{\mathrm{b}}$ & $x_{1}{ }^{\mathrm{c}}$ \\
\hline 1.0000 & 0.9499 & 0.05005 \\
$\frac{{ }^{\mathrm{a}} x_{2}{ }^{(\mathrm{s})}: \text { initial mole fraction of component } 2 \text { in the solution. }}{{ }^{\mathrm{b}}{ }_{x_{2}}: \text { mole fraction of component } 2 \text { in the saturated solution. }}$ \\
${ }^{\mathrm{c}} x_{1}:$ mole fraction solubility of the solute.
\end{tabular}

\section{Auxiliary Information}

\section{Method/Apparatus/Procedure:}

Constant-temperature bath, calorimetric thermometer, and an ultraviolet/ visible spectrophotometer.

Excess solute and solvent were placed in amber glass bottles and allowed to equilibrate for several days at constant temperature. Attainment of equilibrium was verified by several repetitive measurements and by approaching equilibrium from supersaturation. Aliquots of saturated solutions were transferred through a coarse filter into tared volumetric flasks, weighed and diluted with 2-propanol. Concentrations were determined by spectrophotometric measurements at $250 \mathrm{~nm}$

\section{Source and Purity of Chemicals:}

(1) $99 \%$, Aldrich Chemical Company, Milwaukee, WI, USA, was recrystallized several times from methanol.

(2) $99+\%$, anhydrous, Aldrich Chemical Company, stored over molecular sieves before use.

Estimated Error:

Temperature: $\pm 0.1 \mathrm{~K}$

$x_{1}: \pm 1.5 \%$ (relative error).

\begin{tabular}{ll}
\hline \hline Components: & Original Measurements: \\
(1) Biphenyl; $\mathrm{C}_{12} \mathrm{H}_{10} ;[92-52-4]$ & ${ }^{36}$ K. M. De Fina, T. L. Sharp, and \\
(2) 2-Methyl-1-propanol; $\mathrm{C}_{4} \mathrm{H}_{10} \mathrm{O} ;$ & W. E. Acree, Jr., Can. J. Chem. 77, \\
[78-83-1] & $1589(1999)$. \\
\hline Variables: & Prepared by: \\
$T / \mathrm{K}=298.15$ & W. E. Acree, Jr. \\
\hline
\end{tabular}

Experimental Values

\begin{tabular}{|c|c|c|}
\hline$x_{2}^{(s) a}$ & $x_{2}{ }^{b}$ & $x_{1}{ }^{\mathrm{c}}$ \\
\hline 1.0000 & 0.9609 & 0.03906 \\
\hline
\end{tabular}

\section{Auxiliary Information}

\section{Method/Apparatus/Procedure:}

Constant-temperature bath, calorimetric thermometer, and an ultraviolet/ visible spectrophotometer.

Excess solute and solvent were placed in amber glass bottles and allowed to equilibrate for several days at constant temperature. Attainment of equilibrium was verified by several repetitive measurements and by approaching equilibrium from supersaturation. Aliquots of saturated solutions were transferred through a coarse filter into tared volumetric flasks, weighed and diluted with 2-propanol. Concentrations were determined by spectrophotometric measurements at $250 \mathrm{~nm}$.
Source and Purity of Chemicals:

(1) $99 \%$, Aldrich Chemical Company, Milwaukee, WI, USA, was recrystallized several times from methanol.

(2) $99+\%$, anhydrous, Aldrich Chemical Company, stored over molecular sieves before use.

\section{Estimated Error:}

Temperature: $\pm 0.1 \mathrm{~K}$.

$x_{1}: \pm 1.5 \%$ (relative error).

\begin{tabular}{ll}
\hline \hline Components: & Original Measurements: \\
(1) Biphenyl; $\mathrm{C}_{12} \mathrm{H}_{10} ;[92-52-4]$ & ${ }^{36}$ K. M. De Fina, T. L. Sharp, and \\
(2) 2-Methyl-2-propanol; $\mathrm{C}_{4} \mathrm{H}_{10} \mathrm{O} ;$ & W. E. Acree, Jr., Can. J. Chem. 77, \\
[75-65-0] & $1589(1999)$. \\
\hline Variables: & Prepared by: \\
$T / \mathrm{K}=298.15$ & W. E. Acree, Jr. \\
\hline
\end{tabular}

Experimental Values

\begin{tabular}{|c|c|c|}
\hline$x_{2}{ }^{(\mathrm{s}) \mathrm{a}}$ & $x_{2}{ }^{b}$ & $x_{1}{ }^{\mathrm{c}}$ \\
\hline 1.0000 & 0.9588 & 0.04118 \\
\hline
\end{tabular}

\section{Auxiliary Information}

\section{Method/Apparatus/Procedure:}

Constant-temperature bath, calorimetric thermometer, and an ultraviolet/ visible spectrophotometer.

Excess solute and solvent were placed in amber glass bottles and allowed to equilibrate for several days at constant temperature. Attainment of equilibrium was verified by several repetitive measurements and by approaching equilibrium from supersaturation. Aliquots of saturated solutions were transferred through a coarse filter into tared volumetric flasks, weighed and diluted with 2-propanol. Concentrations were determined by spectrophotometric measurements at $250 \mathrm{~nm}$.

\section{Source and Purity of Chemicals:}

(1) $99 \%$, Aldrich Chemical Company, Milwaukee, WI, USA, was recrystallized several times from methanol.

(2) $99+\%$, Arco Chemical Company, stored over molecular sieves before use.

\section{Estimated Error:}

Temperature: $\pm 0.1 \mathrm{~K}$.

$x_{1}: \pm 1.5 \%$ (relative error).

\begin{tabular}{ll}
\hline \hline Components: & Original Measurements: \\
(1) Biphenyl; $\mathrm{C}_{12} \mathrm{H}_{10} ;[92-52-4]$ & ${ }^{36} \mathrm{~K}$. M. De Fina, T. L. Sharp, and \\
(2) 1-Pentanol; $\mathrm{C}_{5} \mathrm{H}_{12} \mathrm{O} ;[71-41-0]$ & W. E. Acree, Jr., Can. J. Chem. 77, \\
& $1589(1999)$. \\
\hline Variables: & Prepared by: \\
$T / \mathrm{K}=298.15$ & W. E. Acree, Jr.
\end{tabular}


Experimental Values

\begin{tabular}{lcc}
\hline$\frac{x_{2}{ }^{(\mathrm{s}) \mathrm{a}}}{x_{2}{ }^{\mathrm{b}}}$ & $x_{1}{ }^{\mathrm{c}}$ \\
\hline 1.0000 & 0.9243 & 0.07573 \\
$\frac{{ }^{\mathrm{a}} x_{2}{ }^{(\mathrm{s})}: \text { initial mole fraction of component } 2 \text { in the solution. }}{{ }^{\mathrm{b}}{ }_{x_{2}}: \text { mole fraction of component } 2 \text { in the saturated solution. }}$ \\
${ }^{\mathrm{c}}{ }_{x_{1}}:$ mole fraction solubility of the solute.
\end{tabular}

\section{Auxiliary Information}

\section{Method/Apparatus/Procedure:}

Constant-temperature bath, calorimetric thermometer, and an ultraviolet/ visible spectrophotometer.

Excess solute and solvent were placed in amber glass bottles and allowed to equilibrate for several days at constant temperature. Attainment of equilibrium was verified by several repetitive measurements and by approaching equilibrium from supersaturation. Aliquots of saturated solutions were transferred through a coarse filter into tared volumetric flasks, weighed and diluted with 2-propanol. Concentrations were determined by spectrophotometric measurements at $250 \mathrm{~nm}$.

\section{Source and Purity of Chemicals:}

(1) $99 \%$, Aldrich Chemical Company, Milwaukee, WI, USA, was recrystallized several times from methanol.

(2) $99+\%$, Aldrich Chemical Company, stored over molecular sieves before use.

\section{Estimated Error:}

Temperature: $\pm 0.1 \mathrm{~K}$

$x_{1}: \pm 1.5 \%$ (relative error).

\begin{tabular}{ll}
\hline \hline Components: & Original Measurements: \\
(1) Biphenyl; $\mathrm{C}_{12} \mathrm{H}_{10} ;[92-52-4]$ & ${ }^{36} \mathrm{~K}$. M. De Fina, T. L. Sharp, and \\
(2) 2-Pentanol; $\mathrm{C}_{5} \mathrm{H}_{12} \mathrm{O} ;[6032-29-7]$ & W. E. Acree, Jr., Can. J. Chem. 77, \\
& $1589(1999)$. \\
\hline Variables: & Prepared by: \\
$T / \mathrm{K}=298.15$ & W. E. Acree, Jr. \\
\hline
\end{tabular}

Experimental Values

\begin{tabular}{|c|c|c|}
\hline$x_{2}{ }^{(\mathrm{s}) \mathrm{a}}$ & $x_{2}{ }^{b}$ & $x_{1}{ }^{c}$ \\
\hline 1.0000 & 0.9347 & 0.06525 \\
\hline
\end{tabular}

\section{Auxiliary Information}

\section{Method/Apparatus/Procedure:}

Constant-temperature bath, calorimetric thermometer, and an ultraviolet/ visible spectrophotometer.

Excess solute and solvent were placed in amber glass bottles and allowed to equilibrate for several days at constant temperature. Attainment of equilibrium was verified by several repetitive measurements and by approaching equilibrium from supersaturation. Aliquots of saturated solutions were transferred through a coarse filter into tared volumetric flasks, weighed and diluted with 2-propanol. Concentrations were determined by spectrophotometric measurements at $250 \mathrm{~nm}$.

Source and Purity of Chemicals:

(1) $99 \%$, Aldrich Chemical Company, Milwaukee, WI, USA, was recrystallized several times from methanol.

(2) $99+\%$, Acros Organics, USA, stored over molecular sieves before use.

Estimated Error:

Temperature: $\pm 0.1 \mathrm{~K}$.

$x_{1}: \pm 1.5 \%$ (relative error)

\begin{tabular}{ll}
\hline \hline Components: & Original Measurements: \\
(1) Biphenyl; $\mathrm{C}_{12} \mathrm{H}_{10} ;[92-52-4]$ & ${ }^{36} \mathrm{~K}$. M. De Fina, T. L. Sharp, and \\
(2) 3-Methyl-1-butanol; $\mathrm{C}_{5} \mathrm{H}_{12} \mathrm{O} ;$ & W. E. Acree, Jr., Can. J. Chem. 77, \\
[123-51-3] & 1589 (1999). \\
\hline Variables: & Prepared by: \\
$T / \mathrm{K}=298.15$ & W. E. Acree, Jr. \\
\hline
\end{tabular}

Experimental Values

\begin{tabular}{lcc}
\hline \hline$x_{2}{ }^{(\mathrm{s}) \mathrm{a}}$ & $x_{2}{ }^{\mathrm{b}}$ & $x_{1}{ }^{\mathrm{c}}$ \\
\hline 1.0000 & 0.9434 & 0.05664 \\
$\frac{{ }^{\mathrm{a}} x_{2}{ }^{(\mathrm{s})}: \text { initial mole fraction of component } 2 \text { in the solution. }}{{ }^{\mathrm{b}} x_{2}: \text { mole fraction of component } 2 \text { in the saturated solution. }}$ \\
${ }^{{ }^{x_{1}} \text { : mole fraction solubility of the solute. }}$
\end{tabular}

${ }^{b} x_{2}$ : mole fraction of component 2 in the saturated solution.

$x_{1}$ : mole fraction solubility of the solute.

\section{Auxiliary Information}

\section{Method/Apparatus/Procedure:}

Constant-temperature bath, calorimetric thermometer, and an ultraviolet/ visible spectrophotometer.

Excess solute and solvent were placed in amber glass bottles and allowed to equilibrate for several days at constant temperature. Attainment of equilibrium was verified by several repetitive measurements and by approaching equilibrium from supersaturation. Aliquots of saturated solutions were transferred through a coarse filter into tared volumetric flasks, weighed and diluted with 2-propanol. Concentrations were determined by spectrophotometric measurements at $250 \mathrm{~nm}$.

Source and Purity of Chemicals:

(1) $99 \%$, Aldrich Chemical Company, Milwaukee, WI, USA, was recrystallized several times from methanol.

(2) 99+\%, anhydrous, Aldrich Chemical Company, stored over molecular sieves before use.

Estimated Error:

Temperature: $\pm 0.1 \mathrm{~K}$.

$x_{1}: \pm 1.5 \%$ (relative error).

Components:

(1) Biphenyl; $\mathrm{C}_{12} \mathrm{H}_{10} ;[92-52-4]$

(2) 2-Methyl-2-butanol; $\mathrm{C}_{5} \mathrm{H}_{12} \mathrm{O}$ [75-85-4]

Variables:

$T / \mathrm{K}=298.15$
Original Measurements:

${ }^{36}$ K. M. De Fina, T. L. Sharp, and W. E. Acree, Jr., Can. J. Chem. 77, 1589 (1999)

Prepared by: W. E. Acree, Jr. 
Experimental Values

\begin{tabular}{lcc}
\hline \hline$x_{2}{ }^{(\mathrm{s}) \mathrm{a}}$ & $x_{2}{ }^{\mathrm{b}}$ & $x_{1}{ }^{\mathrm{c}}$ \\
\hline 1.0000 & 0.9288 & 0.07120 \\
\hline${ }^{\mathrm{a}}{ }^{(\mathrm{s})}$. initial mole fraction of ${ }^{2}$ &
\end{tabular}

${ }_{x_{2}}(\mathrm{~s})$ initial mole fraction of component 2 in the solution.

${ }^{\mathrm{b}} x_{2}$ : mole fraction of component 2 in the saturated solution.

${ }^{c} x_{1}$ : mole fraction solubility of the solute.

\section{Auxiliary Information}

\section{Method/Apparatus/Procedure:}

Constant-temperature bath, calorimetric thermometer, and an ultraviolet/ visible spectrophotometer.

Excess solute and solvent were placed in amber glass bottles and allowed to equilibrate for several days at constant temperature. Attainment of equilibrium was verified by several repetitive measurements and by approaching equilibrium from supersaturation. Aliquots of saturated solutions were transferred through a coarse filter into tared volumetric flasks, weighed and diluted with 2-propanol. Concentrations were determined by spectrophotometric measurements at $250 \mathrm{~nm}$.

Source and Purity of Chemicals:

(1) $99 \%$, Aldrich Chemical Company, Milwaukee, WI, USA, was recrystallized several times from methanol.

(2) $99+\%$, Acros Organics, USA, stored over molecular sieves before use.

\section{Estimated Error:}

Temperature: $\pm 0.1 \mathrm{~K}$.

$x_{1}: \pm 1.5 \%$ (relative error).

\begin{tabular}{ll}
\hline \hline Components: & Original Measurements: \\
(1) Biphenyl; $\mathrm{C}_{12} \mathrm{H}_{10} ;[92-52-4]$ & ${ }^{36} \mathrm{~K}$. M. De Fina, T. L. Sharp, and \\
(2) 1-Hexanol; $\mathrm{C}_{6} \mathrm{H}_{14} \mathrm{O} ;[111-27-3]$ & W. E. Acree, Jr., Can. J. Chem. 77, \\
& 1589 (1999). \\
\hline Variables: & Prepared by: \\
$T / \mathrm{K}=298.15$ & W. E. Acree, Jr. \\
\hline
\end{tabular}

Experimental Values

\begin{tabular}{lcc}
\hline \hline$x_{2}{ }^{(\mathrm{s}) \mathrm{a}}$ & $x_{2}{ }^{\mathrm{b}}$ & $x_{1}{ }^{\mathrm{c}}$ \\
\hline 1.0000 & 0.9141 & 0.08592 \\
$\frac{{ }^{\mathrm{a}} x_{2}{ }^{(\mathrm{s})}: \text { initial mole fraction of component } 2 \text { in the solution. }}{}$ \\
${ }^{\mathrm{b}}{ }_{x_{2}}:$ mole fraction of component 2 in the saturated solution. \\
${ }^{\mathrm{c}} x_{1}:$ mole fraction solubility of the solute.
\end{tabular}

\section{Auxiliary Information}

\footnotetext{
Method/Apparatus/Procedure:

Constant-temperature bath, calorimetric thermometer, and an ultraviolet/ visible spectrophotometer.

Excess solute and solvent were placed in amber glass bottles and allowed to equilibrate for several days at constant temperature. Attainment of equilibrium was verified by several repetitive measurements and by approaching equilibrium from supersaturation. Aliquots of saturated solutions were transferred through a coarse filter into tared volumetric flasks, weighed and diluted with 2-propanol. Concentrations were determined by spectrophotometric measurements at $250 \mathrm{~nm}$.
}

Source and Purity of Chemicals:

(1) $99 \%$, Aldrich Chemical Company, Milwaukee, WI, USA, was recrystallized several times from methanol.

(2) $99+\%$, Alfa Aesar, USA, stored over molecular sieves before use.

Estimated Error:

Temperature: $\pm 0.1 \mathrm{~K}$.

$x_{1}: \pm 1.5 \%$ (relative error).

\begin{tabular}{ll}
\hline \hline Components: & Original Measurements: \\
(1) Biphenyl; $\mathrm{C}_{12} \mathrm{H}_{10} ;[92-52-4]$ & ${ }^{36} \mathrm{~K}$. M. De Fina, T. L. Sharp, and \\
(2) 2-Methyl-1-pentanol; $\mathrm{C}_{6} \mathrm{H}_{14} \mathrm{O} ;$ & W. E. Acree, Jr., Can. J. Chem. 77, \\
[105-30-6] & 1589 (1999). \\
\hline Variables: & Prepared by: \\
$T / \mathrm{K}=298.15$ & W. E. Acree, Jr. \\
\hline
\end{tabular}

Experimental Values

\begin{tabular}{lcc}
\hline \hline$x_{2}{ }^{(\mathrm{s}) \mathrm{a}}$ & $x_{2}{ }^{\mathrm{b}}$ & $x_{1}{ }^{\mathrm{c}}$ \\
\hline 1.0000 & 0.9278 & 0.07216 \\
$\frac{{ }^{\mathrm{a}} x_{2}{ }^{(\mathrm{s})}: \text { initial mole fraction of component } 2 \text { in the solution. }}{{ }^{\mathrm{b}} x_{2}: \text { mole fraction of component } 2 \text { in the saturated solution. }}$ \\
${ }^{\mathrm{c}} x_{1}$ : mole fraction solubility of the solute.
\end{tabular}

\section{Auxiliary Information}

\section{Method/Apparatus/Procedure:}

Constant-temperature bath, calorimetric thermometer, and an ultraviolet/ visible spectrophotometer.

Excess solute and solvent were placed in amber glass bottles and allowed to equilibrate for several days at constant temperature. Attainment of equilibrium was verified by several repetitive measurements and by approaching equilibrium from supersaturation. Aliquots of saturated solutions were transferred through a coarse filter into tared volumetric flasks, weighed and diluted with 2-propanol. Concentrations were determined by spectrophotometric measurements at $250 \mathrm{~nm}$.

Source and Purity of Chemicals:

(1) $99 \%$, Aldrich Chemical Company, Milwaukee, WI, USA, was recrystallized several times from methanol.

(2) $99 \%$, Aldrich Chemical Company, stored over molecular sieves before use.

\section{Estimated Error:}

Temperature: $\pm 0.1 \mathrm{~K}$.

$x_{1}: \pm 1.5 \%$ (relative error).

\begin{tabular}{ll}
\hline \hline Components: & Original Measurements: \\
(1) Biphenyl; $\mathrm{C}_{12} \mathrm{H}_{10} ;[92-52-4]$ & ${ }^{36}$ K. M. De Fina, T. L. Sharp, and \\
(2) 4-Methyl-2-pentanol; $\mathrm{C}_{6} \mathrm{H}_{14} \mathrm{O} ;$ & W. E. Acree, Jr., Can. J. Chem. 77, \\
[108-11-2] & 1589 (1999). \\
\hline Variables: & Prepared by: \\
$T / \mathrm{K}=298.15$ & W. E. Acree, Jr. \\
\hline
\end{tabular}


Experimental Values

\begin{tabular}{lcc}
\hline$\frac{x_{2}{ }^{(\mathrm{s}) \mathrm{a}}}{x_{2}{ }^{\mathrm{b}}}$ & $x_{1}{ }^{\mathrm{c}}$ \\
\hline 1.0000 & 0.9388 & 0.06115 \\
$\frac{{ }^{\mathrm{a}} x_{2}{ }^{(\mathrm{s})}: \text { initial mole fraction of component } 2 \text { in the solution. }}{{ }^{\mathrm{b}}{ }_{x_{2}}: \text { mole fraction of component } 2 \text { in the saturated solution. }}$ \\
${ }^{\mathrm{c}} x_{1}:$ mole fraction solubility of the solute.
\end{tabular}

\section{Auxiliary Information}

\section{Method/Apparatus/Procedure:}

Constant-temperature bath, calorimetric thermometer, and an ultraviolet/ visible spectrophotometer.

Excess solute and solvent were placed in amber glass bottles and allowed to equilibrate for several days at constant temperature. Attainment of equilibrium was verified by several repetitive measurements and by approaching equilibrium from supersaturation. Aliquots of saturated solutions were transferred through a coarse filter into tared volumetric flasks, weighed and diluted with 2-propanol. Concentrations were determined by spectrophotometric measurements at $250 \mathrm{~nm}$.

Source and Purity of Chemicals:

(1) $99 \%$, Aldrich Chemical Company, Milwaukee, WI, USA, was recrystallized several times from methanol.

(2) $99+\%$, Acros Organics, USA, stored over molecular sieves before use.

Estimated Error:

Temperature: $\pm 0.1 \mathrm{~K}$.

$x_{1}: \pm 1.5 \%$ (relative error).

\begin{tabular}{ll}
\hline \hline Components: & Original Measurements: \\
(1) Biphenyl; $\mathrm{C}_{12} \mathrm{H}_{10} ;[92-52-4]$ & ${ }^{36} \mathrm{~K}$. M. De Fina, T. L. Sharp, and \\
(2) 1-Heptanol; $\mathrm{C}_{7} \mathrm{H}_{16} \mathrm{O} ;[111-70-6]$ & W. E. Acree, Jr., Can. J. Chem. 77, \\
& $1589(1999)$. \\
\hline Variables: & Prepared by: \\
$T / \mathrm{K}=298.15$ & W. E. Acree, Jr. \\
\hline
\end{tabular}

Experimental Values

\begin{tabular}{lcc}
\hline \hline$x_{2}{ }^{(\mathrm{s}) \mathrm{a}}$ & $x_{2}{ }^{\mathrm{b}}$ & $x_{1}{ }^{\mathrm{c}}$ \\
\hline 1.0000 & 0.8999 & 0.1001 \\
\hline
\end{tabular}

${ }^{\mathrm{a}} x_{2}{ }^{(\mathrm{s})}$ : initial mole fraction of component 2 in the solution.



${ }^{c} x_{1}$ : mole fraction solubility of the solute.

\section{Auxiliary Information}

\footnotetext{
Method/Apparatus/Procedure:

Constant-temperature bath, calorimetric thermometer, and an ultraviolet/ visible spectrophotometer.

Excess solute and solvent were placed in amber glass bottles and allowed to equilibrate for several days at constant temperature. Attainment of equilibrium was verified by several repetitive measurements and by approaching equilibrium from supersaturation. Aliquots of saturated solutions were transferred through a coarse filter into tared volumetric flasks, weighed and diluted with 2-propanol. Concentrations were determined by

spectrophotometric measurements at $250 \mathrm{~nm}$.
}

Source and Purity of Chemicals:

(1) $99 \%$, Aldrich Chemical Company, Milwaukee, WI, USA, was recrystallized several times from methanol.

(2) $99+\%$, Alfa Aesar, USA, stored over molecular sieves before use.

Estimated Error:

Temperature: $\pm 0.1 \mathrm{~K}$.

$x_{1}: \pm 1.5 \%$ (relative error).

\begin{tabular}{ll}
\hline \hline Components: & Original Measurements: \\
(1) Biphenyl; $\mathrm{C}_{12} \mathrm{H}_{10} ;[92-52-4]$ & ${ }^{36} \mathrm{~K}$. M. De Fina, T. L. Sharp, and \\
(2) 1-Octanol; $\mathrm{C}_{8} \mathrm{H}_{18} \mathrm{O} ;[111-87-5]$ & W. E. Acree, Jr., Can. J. Chem. 77, \\
& 1589 (1999). \\
\hline Variables: & Prepared by: \\
$T / \mathrm{K}=298.15$ & W. E. Acree, Jr. \\
\hline
\end{tabular}

Experimental Values

\begin{tabular}{lcc}
\hline \hline$x_{2}{ }^{(\mathrm{s}) \mathrm{a}}$ & $x_{2}{ }^{\mathrm{b}}$ & $x_{1}{ }^{\mathrm{c}}$ \\
\hline 1.0000 & 0.8903 & 0.1097 \\
\hline${ }^{\mathrm{a}}{ }_{x_{2}}{ }^{(\mathrm{s})}:$ initial mole fraction of component 2 in the solution. \\
${ }^{\mathrm{b}} x_{2}:$ mole fraction of component 2 in the saturated solution. \\
${ }^{\mathrm{c}}{ }_{x_{1}}$ : mole fraction solubility of the solute.
\end{tabular}

${ }^{b} x_{2}$ : mole fraction of component 2 in the saturated solution.

$x_{1}$ : mole fraction solubility of the solute.

\section{Auxiliary Information}

\section{Method/Apparatus/Procedure:}

Constant-temperature bath, calorimetric thermometer, and an ultraviolet/ visible spectrophotometer.

Excess solute and solvent were placed in amber glass bottles and allowed to equilibrate for several days at constant temperature. Attainment of equilibrium was verified by several repetitive measurements and by approaching equilibrium from supersaturation. Aliquots of saturated solutions were transferred through a coarse filter into tared volumetric flasks, weighed and diluted with 2-propanol. Concentrations were determined by spectrophotometric measurements at $250 \mathrm{~nm}$.

Source and Purity of Chemicals:

(1) $99 \%$, Aldrich Chemical Company, Milwaukee, WI, USA, was recrystallized several times from methanol.

(2) $99+\%$, anhydrous, Aldrich Chemical Company, stored over molecular sieves before use.

Estimated Error:

Temperature: $\pm 0.1 \mathrm{~K}$.

$x_{1}: \pm 1.5 \%$ (relative error).

\begin{tabular}{ll}
\hline \hline Components: & Original Measurements: \\
(1) Biphenyl; $\mathrm{C}_{12} \mathrm{H}_{10} ;[92-52-4]$ & ${ }^{36} \mathrm{~K}$. M. De Fina, T. L. Sharp, and \\
(2) 2-Ethyl-1-hexanol; $\mathrm{C}_{8} \mathrm{H}_{18} \mathrm{O} ;$ & W. E. Acree, Jr., Can. J. Chem. 77, \\
{$[104-76-7]$} & $1589(1999)$. \\
\hline Variables: & Prepared by: \\
$T / \mathrm{K}=298.15$ & W. E. Acree, Jr. \\
\hline
\end{tabular}


Experimental Values

\begin{tabular}{lcc}
\hline$\frac{x_{2}{ }^{(\mathrm{s}) \mathrm{a}}}{x_{2}{ }^{\mathrm{b}}}$ & $x_{1}{ }^{\mathrm{c}}$ \\
\hline 1.0000 & 0.9052 & 0.09481 \\
$\frac{{ }^{\mathrm{a}} x_{2}{ }^{(\mathrm{s})}: \text { initial mole fraction of component } 2 \text { in the solution. }}{{ }^{\mathrm{b}}{ }_{x_{2}}: \text { mole fraction of component } 2 \text { in the saturated solution. }}$ \\
${ }^{\mathrm{c}} x_{1}:$ mole fraction solubility of the solute.
\end{tabular}

\section{Auxiliary Information}

\section{Method/Apparatus/Procedure:}

Constant-temperature bath, calorimetric thermometer, and an ultraviolet/ visible spectrophotometer.

Excess solute and solvent were placed in amber glass bottles and allowed to equilibrate for several days at constant temperature. Attainment of equilibrium was verified by several repetitive measurements and by approaching equilibrium from supersaturation. Aliquots of saturated solutions were transferred through a coarse filter into tared volumetric flasks, weighed and diluted with 2-propanol. Concentrations were determined by spectrophotometric measurements at $250 \mathrm{~nm}$

\section{Source and Purity of Chemicals:}

(1) $99 \%$, Aldrich Chemical Company, Milwaukee, WI, USA, was recrystallized several times from methanol.

(2) $99+\%$, Aldrich Chemical Company, stored over molecular sieves before use.

\section{Estimated Error:}

Temperature: $\pm 0.1 \mathrm{~K}$

$x_{1}: \pm 1.5 \%$ (relative error).

\begin{tabular}{|c|c|}
\hline $\begin{array}{l}\text { Components: } \\
\text { (1) Biphenyl; } \mathrm{C}_{12} \mathrm{H}_{10} ;[92-52-4] \\
\text { (2) } 1,2 \text {-Ethanediol; } \mathrm{C}_{2} \mathrm{H}_{6} \mathrm{O}_{2} \text {; } \\
\text { [107-21-1] }\end{array}$ & $\begin{array}{l}\text { Original Measurements: } \\
{ }^{36} \text { K. M. De Fina, T. L. Sharp, and } \\
\text { W. E. Acree, Jr., Can. J. Chem. 77, } \\
1589 \text { (1999). }\end{array}$ \\
\hline $\begin{array}{l}\text { Variables: } \\
T / \mathrm{K}=298.15\end{array}$ & $\begin{array}{l}\text { Prepared by: } \\
\text { W. E. Acree, Jr. }\end{array}$ \\
\hline
\end{tabular}

Experimental Values

\begin{tabular}{|c|c|c|}
\hline$x_{2}^{(s) a}$ & $x_{2}{ }^{b}$ & $x_{1}{ }^{\mathrm{c}}$ \\
\hline 1.0000 & 0.9973 & 0.00269 \\
\hline
\end{tabular}

\section{Auxiliary Information}

\section{Method/Apparatus/Procedure:}

Constant-temperature bath, calorimetric thermometer, and an ultraviolet/ visible spectrophotometer.

Excess solute and solvent were placed in amber glass bottles and allowed to equilibrate for several days at constant temperature. Attainment of equilibrium was verified by several repetitive measurements and by approaching equilibrium from supersaturation. Aliquots of saturated solutions were transferred through a coarse filter into tared volumetric flasks, weighed and diluted with 2-propanol. Concentrations were determined by spectrophotometric measurements at $250 \mathrm{~nm}$.
Source and Purity of Chemicals:

(1) $99 \%$, Aldrich Chemical Company, Milwaukee, WI, USA, was recrystallized several times from methanol.

(2) $99.8 \%$, anhydrous, Aldrich Chemical Company, stored over molecular sieves before use.

\section{Estimated Error:}

Temperature: $\pm 0.1 \mathrm{~K}$.

$x_{1}: \pm 1.5 \%$ (relative error).

\subsection{Biphenyl solubility data in alkoxyalcohols}

\begin{tabular}{ll}
\hline \hline Components: & Original Measurements: \\
(1) Biphenyl; $\mathrm{C}_{12} \mathrm{H}_{10} ;[92-52-4]$ & ${ }^{37}$ L. M. Grubbs, M. Saifullah, N. E. De \\
(2) 2-Ethoxyethanol; $\mathrm{C}_{4} \mathrm{H}_{10} \mathrm{O}_{2} ;$ & La Rosa, S. Ye, S. S. Achi, W. E. \\
[111-27-3] & Acree, Jr., and M. H. Abraham, Fluid \\
& Phase Equilib. 298, 48 (2010). \\
\hline Variables: & Prepared by: \\
$T / \mathrm{K}=298.15$ & W. E. Acree, Jr. \\
\hline
\end{tabular}

Experimental Values

\begin{tabular}{lcc}
\hline \hline$x_{2}{ }^{(\mathrm{s}) \mathrm{a}}$ & $x_{2}{ }^{\mathrm{b}}$ & $x_{1}{ }^{\mathrm{c}}$ \\
\hline 1.0000 & 0.8589 & 0.1411 \\
\hline${ }^{\mathrm{a}} x_{2}{ }^{(\mathrm{s})}:$ initial mole fraction of component 2 in the solution. \\
${ }^{\mathrm{b}} x_{2}:$ mole fraction of component 2 in the saturated solution. \\
${ }^{\mathrm{c}}{ }_{x_{1}: \text { mole fraction solubility of the solute. }}$
\end{tabular}

\section{Auxiliary Information}

\section{Method/Apparatus/Procedure:}

Constant-temperature bath, calorimetric thermometer, and an ultraviolet visible spectrophotometer.

Excess solute and solvent were placed in amber glass bottles and allowed to equilibrate for several days at constant temperature. Attainment of equilibrium was verified by several repetitive measurements and by approaching equilibrium from supersaturation. Aliquots of saturated solutions were transferred through a coarse filter into tared volumetric flasks, weighed and diluted with methanol or 2-propanol. Concentrations were determined by spectrophotometric measurements at $250 \mathrm{~nm}$.

\section{Source and Purity of Chemicals:}

(1) $99 \%$, Aldrich Chemical Company, Milwaukee, WI, USA, was recrystallized several times from methanol.

(2) $99 \%$, Aldrich Chemical Company, stored over molecular sieves and distilled shortly before use.

Estimated Error:

Temperature: $\pm 0.1 \mathrm{~K}$.

$x_{1}: \pm 1.5 \%$ (relative error). 


\section{Components:}

(1) Biphenyl; $\mathrm{C}_{12} \mathrm{H}_{10} ;[92-52-4]$

(2) 2-Propoxyethanol; $\mathrm{C}_{5} \mathrm{H}_{12} \mathrm{O}_{2}$;

[2807-30-9]

\begin{tabular}{ll}
\hline Variables: & Prepared by: \\
$T / \mathrm{K}=298.15$ & W. E. Acree, Jr. \\
\hline
\end{tabular}

Experimental Values

\begin{tabular}{lcc}
\hline \hline$x_{2}{ }^{(\mathrm{s}) \mathrm{a}}$ & $x_{2}{ }^{\mathrm{b}}$ & $x_{1}{ }^{\mathrm{c}}$ \\
\hline 1.0000 & 0.8317 & 0.1683
\end{tabular}

${ }^{{ }} x_{2}{ }^{(\mathrm{s})}$ : initial mole fraction of component 2 in the solution.

${ }^{b} x_{2}$ : mole fraction of component 2 in the saturated solution.

${ }^{c} x_{1}$ : mole fraction solubility of the solute.

\section{Auxiliary Information}

\begin{abstract}
Method/Apparatus/Procedure:
Constant-temperature bath, calorimetric thermometer, and an ultraviolet/ visible spectrophotometer.

Excess solute and solvent were placed in amber glass bottles and allowed to equilibrate for several days at constant temperature. Attainment of equilibrium was verified by several repetitive measurements and by approaching equilibrium from supersaturation. Aliquots of saturated solutions were transferred through a coarse filter into tared volumetric flasks, weighed and diluted with methanol or 2-propanol. Concentrations were determined by spectrophotometric measurements at $250 \mathrm{~nm}$.
\end{abstract}

\section{Source and Purity of Chemicals:}

(1) $99 \%$, Aldrich Chemical Company, Milwaukee, WI, USA, was recrystallized several times from methanol.

(2) $99+\%$, Aldrich Chemical Company, stored over molecular sieves and distilled shortly before use.

\section{Estimated Error:}

Temperature: $\pm 0.1 \mathrm{~K}$.

$x_{1}: \pm 1.5 \%$ (relative error).

\section{Auxiliary Information}

\section{Method/Apparatus/Procedure:}

Constant-temperature bath, calorimetric thermometer, and an ultraviolet/ visible spectrophotometer.

Excess solute and solvent were placed in amber glass bottles and allowed to equilibrate for several days at constant temperature. Attainment of equilibrium was verified by several repetitive measurements and by approaching equilibrium from supersaturation. Aliquots of saturated solutions were transferred through a coarse filter into tared volumetric flasks, weighed and diluted with methanol or 2-propanol. Concentrations were determined by spectrophotometric measurements at $250 \mathrm{~nm}$.

\section{Source and Purity of Chemicals:}

(1) $99 \%$, Aldrich Chemical Company, Milwaukee, WI, USA, was recrystallized several times from methanol.

(2) $99 \%$, Aldrich Chemical Company, stored over molecular sieves and distilled shortly before use.

Estimated Error:

Temperature: $\pm 0.1 \mathrm{~K}$.

$x_{1}: \pm 1.5 \%$ (relative error)

\begin{tabular}{ll}
\hline \hline Components: & Original Measurements: \\
(1) Biphenyl; $\mathrm{C}_{12} \mathrm{H}_{10} ;[92-52-4]$ & ${ }^{37}$ L. M. Grubbs, M. Saifullah, N. E. De \\
(2) 2-Butoxyethanol; $\mathrm{C}_{6} \mathrm{H}_{14} \mathrm{O}_{2} ;$ & La Rosa, S. Ye, S. S. Achi, W. E. \\
[111-76-2] & Acree, Jr., and M. H. Abraham, Fluid \\
& Phase Equilib. 298, 48 (2010). \\
\hline Variables: & Prepared by: \\
$T / \mathrm{K}=298.15$ & W. E. Acree, Jr. \\
\hline
\end{tabular}

Experimental Values

\begin{tabular}{lcc}
\hline \hline$x_{2}{ }^{(\mathrm{s}) \mathrm{a}}$ & $x_{2}{ }^{\mathrm{b}}$ & $x_{1}{ }^{\mathrm{c}}$ \\
\hline 1.0000 & 0.8449 & 0.1551 \\
${ }^{\mathrm{a}} x_{2}{ }^{(\mathrm{s})}:$ initial mole fraction of component 2 in the solution. \\
${ }^{\mathrm{b}} x_{2}:$ mole fraction of component 2 in the saturated solution. \\
${ }^{\mathrm{c}} x_{1}$ : mole fraction solubility of the solute.
\end{tabular}

\section{Auxiliary Information}

\begin{tabular}{ll}
\hline \hline Components: & Original Measurements: \\
(1) Biphenyl; $\mathrm{C}_{12} \mathrm{H}_{10} ;[92-52-4]$ & ${ }^{37}$ L. M. Grubbs, M. Saifullah, N. E. De \\
(2) 2-Isopropoxyethanol; $\mathrm{C}_{5} \mathrm{H}_{12} \mathrm{O}_{2} ;$ & La Rosa, S. Ye, S. S. Achi, W. E. \\
{$[109-59-1]$} & Acree, Jr., and M. H. Abraham, Fluid \\
& Phase Equilib. 298, 48 (2010). \\
\hline Variables: & Prepared by: \\
$T / \mathrm{K}=298.15$ & W. E. Acree, Jr. \\
\hline
\end{tabular}

Experimental Values

\begin{tabular}{lcc}
\hline \hline$x_{2}{ }^{(\mathrm{s}) \mathrm{a}}$ & $x_{2}{ }^{\mathrm{b}}$ & $x_{1}{ }^{\mathrm{c}}$ \\
\hline 1.0000 & 0.8387 & 0.1613 \\
$\frac{{ }^{\mathrm{a}} x_{2}{ }^{(\mathrm{s})}: \text { initial mole fraction of component } 2 \text { in the solution. }}{{ }^{\mathrm{b}} x_{2}: \text { mole fraction of component } 2 \text { in the saturated solution. }}$ \\
${ }^{\mathrm{c}} x_{1}:$ mole fraction solubility of the solute.
\end{tabular}

\section{Method/Apparatus/Procedure:}

Constant-temperature bath, calorimetric thermometer, and an ultraviolet/ visible spectrophotometer.

Excess solute and solvent were placed in amber glass bottles and allowed to equilibrate for several days at constant temperature. Attainment of equilibrium was verified by several repetitive measurements and by approaching equilibrium from supersaturation. Aliquots of saturated solutions were transferred through a coarse filter into tared volumetric flasks, weighed and diluted with methanol or 2-propanol. Concentrations were determined by spectrophotometric measurements at $250 \mathrm{~nm}$.

\section{Source and Purity of Chemicals:}

(1) $99 \%$, Aldrich Chemical Company, Milwaukee, WI, USA, was recrystallized several times from methanol.

(2) $99+\%$, Acros Organics, USA, stored over molecular sieves and distilled shortly before use.

Estimated Error:

Temperature: $\pm 0.1 \mathrm{~K}$.

$x_{1}: \pm 1.5 \%$ (relative error) 


\begin{tabular}{ll}
\hline \hline Components: & Original Measurements: \\
(1) Biphenyl; $\mathrm{C}_{12} \mathrm{H}_{10} ;[92-52-4]$ & ${ }^{37}$ L. M. Grubbs, M. Saifullah, N. E. De \\
(2) 3-Methoxy-1-butanol; $\mathrm{C}_{5} \mathrm{H}_{12} \mathrm{O}_{2} ;$ & La Rosa, S. Ye, S. S. Achi, W. E. \\
[2517-43-3] & Acree, Jr., and M. H. Abraham, Fluid \\
& Phase Equilib. 298, 48 (2010). \\
\hline Variables: & Prepared by: \\
$T / \mathrm{K}=298.15$ & W. E. Acree, Jr. \\
\hline
\end{tabular}

Experimental Values

\begin{tabular}{lcc}
\hline \hline$x_{2}{ }^{(\mathrm{s}) \mathrm{a}}$ & $x_{2}{ }^{\mathrm{b}}$ & $x_{1}{ }^{\mathrm{c}}$ \\
\hline 1.0000 & 0.8536 & 0.1464 \\
\hline
\end{tabular}



${ }^{b} x_{2}$ : mole fraction of component 2 in the saturated solution.

${ }^{c} x_{1}$ : mole fraction solubility of the solute.

\section{Auxiliary Information}

\section{Method/Apparatus/Procedure:}

Constant-temperature bath, calorimetric thermometer, and an ultraviolet/ visible spectrophotometer.

Excess solute and solvent were placed in amber glass bottles and allowed to equilibrate for several days at constant temperature. Attainment of equilibrium was verified by several repetitive measurements and by approaching equilibrium from supersaturation. Aliquots of saturated solutions were transferred through a coarse filter into tared volumetric flasks, weighed and diluted with methanol or 2-propanol. Concentrations were determined by spectrophotometric measurements at $250 \mathrm{~nm}$.

Source and Purity of Chemicals:

(1) $99 \%$, Aldrich Chemical Company, Milwaukee, WI, USA, was recrystallized several times from methanol.

(2) $99 \%$, Aldrich Chemical Company, stored over molecular sieves and distilled shortly before use.

Estimated Error:

Temperature: $\pm 0.1 \mathrm{~K}$.

$x_{1}: \pm 1.5 \%$ (relative error).

\subsection{Biphenyl solubility data in miscellaneous organic solvents}

\section{Components:}

(1) Biphenyl; $\mathrm{C}_{12} \mathrm{H}_{10} ;[92-52-4]$

(2) Propylene carbonate; $\mathrm{C}_{4} \mathrm{H}_{6} \mathrm{O}_{3} ;$; $\left.108-32-7\right]$

Variables:

$T / \mathrm{K}=298.15$
Original Measurements:

W. E. Acree, Jr., unpublished data.

Prepared by:

W. E. Acree, Jr.
Experimental Values

\begin{tabular}{lcc}
\hline \hline$x_{2}{ }^{(\mathrm{s}) \mathrm{a}}$ & $x_{2}{ }^{\mathrm{b}}$ & $x_{1}{ }^{\mathrm{c}}$ \\
\hline 1.0000 & 0.8700 & 0.1300 \\
$\frac{{ }^{\mathrm{a}} x_{2}{ }^{(\mathrm{s})}: \text { initial mole fraction of component } 2 \text { in the solution. }}{{ }^{\mathrm{b}} x_{2}: \text { mole fraction of component } 2 \text { in the saturated solution. }}$ \\
${ }^{\mathrm{c}} x_{1}$ : mole fraction solubility of the solute.
\end{tabular}

\section{Auxiliary Information}

\section{Method/Apparatus/Procedure:}

Constant-temperature bath, calorimetric thermometer, and an ultraviolet/ visible spectrophotometer.

Excess solute and solvent were placed in amber glass bottles and allowed to equilibrate for several days at constant temperature. Attainment of equilibrium was verified by several repetitive measurements and by approaching equilibrium from supersaturation. Aliquots of saturated solutions were transferred through a coarse filter into tared volumetric flasks, weighed and diluted with 2-propanol. Concentrations were determined by spectrophotometric measurements at $250 \mathrm{~nm}$.

Source and Purity of Chemicals:

(1) $99 \%$, Aldrich Chemical Company, Milwaukee, WI, USA, was recrystallized several times from methanol.

(2) $99.7 \%$, anhydrous, Aldrich Chemical Company, stored over molecular sieves before use.

Estimated Error:

Temperature: $\pm 0.1 \mathrm{~K}$.

$x_{1}: \pm 1.5 \%$ (relative error).

\section{Solubility of Carbazole in Organic Solvents}

\subsection{Critical evaluation of experimental solubility data}

Volume 58 in the IUPAC Solubility Data Series ${ }^{2}$ contained experimental solubility data for carbazole dissolved in 13 saturated hydrocarbons (hexane, heptane, octane, decane, dodecane, hexadecane, cyclohexane, methylcyclohexane, cyclooctane, 2,2,4-trimethylpentane, tert-butylcyclohexane, squalane, and decahydronapthalene), in three aromatic hydrocarbons (benzene, methylcyclohexane, and 1,4-dimethylbenzene), in two alkyl alkanoates (ethyl ethanoate and butyl butanoate), in seven ethers (1,1'-oxybisethane, 1,1'-oxybisbutane, 1,1'-oxybispentane, tetrahydropyran, tetrahydrofuran, 1,4-dioxane, and methoxybenzene), in six chloroalkanes (trichloromethane, tetrachloromethane, 1-chlorohexane, 1-chlorooctane, 1-chlorotetradecane, and chlorocyclohexane), in seven alkanols (methanol, ethanol, 1-propanol, 2-propnaol, 
1-butanol, 1-octanol, and 1,2-ethanediol), in three alkanones (propanone, butanone, and cyclohexanone) and in acetophenone, and in 11 miscellaneous organic solvents (carbon disulfide, pyridine, quinoline, thiophene, dimethyl sulfoxide, nitromethane, acetic anhydride, 1-methyl-2-pyrrolidinone, $\mathrm{N}, \mathrm{N}$-dimethylacetamide, $\mathrm{N}, \mathrm{N}$-dimethylformamide, tetramethylene sulfone, and tributyl phosphate). Except for cyclohexane, decahydronaphthalene, benzene, methylbenzene 1,2,3,4-tetrahydronaphthalene, tetrachloromethane, 2-propanol, pyridine, and thiophene, solubility measurements were made at only a single temperature of $298 \mathrm{~K}$, or at a single ambient temperature estimated at between 298 and $303 \mathrm{~K}$. Solubility measurements in methylbenzene, tetrachloromethane, and 2-propanol covered $40 \mathrm{~K}$ in temperature from 293 to $333 \mathrm{~K}$. Solubility data for carbazole in cyclohexane (from 293 to $357 \mathrm{~K}$ ), decahydronaphthalene (from 333 to $502 \mathrm{~K}$ ), 1,2,3,4-tetrahydronaphthalene (from 345 to $495 \mathrm{~K}$ ), and pyridine (from 302 to $439 \mathrm{~K}$ ) covered a much larger temperature interval, albeit measurements were made at only a few temperatures within the given interval. Solubility data contained in Vol. 58 will not be republished here. The listing above is provided so that readers will know what solubility data are available in the earlier volume for carbazole.

There has been very little carbazole solubility data published during the last 17 years. The only data that could be found pertained to phase diagrams determined by Lisicki and Jamró $^{35}$ for carbazole $+N, N$-dimethylacetamide, carbazole + 1-methyl-2-pyrrolidone, and carbazole + hexahydro-1methyl- $2 \mathrm{H}$-azepin-2-one (also called $\mathrm{N}$-methyl- $\varepsilon$-caprolactam). The published experimental liquidus curves for the latter three carbazole systems did not indicate any outlier data points. The experimental data are listed in Sec. 5.2.

\subsection{Carbazole solubility data in miscellaneous organic solvents}

\begin{tabular}{ll}
\hline \hline Components: & Original Measurements: \\
(1) Carbazole; $\mathrm{C}_{12} \mathrm{H}_{9} \mathrm{~N} ;[86-74-8]$ & ${ }^{35} \mathrm{Z}$. Lisicki and M. E. Jamróz, \\
$(2) \mathrm{N}, \mathrm{N}$-Dimethylacetamide; $\mathrm{C}_{4} \mathrm{H}_{9} \mathrm{NO} ;$ & J. Chem. Thermodyn. 32, \\
{$[127-19-5]$} & $1335(2000)$. \\
\hline Variables: & Prepared by: \\
Temperature & W. E. Acree, Jr. \\
\hline
\end{tabular}

Experimental Values

\begin{tabular}{lcc}
\hline \hline$T / \mathrm{K}$ & $x_{2}{ }^{\mathrm{a}}$ & $x_{1}{ }^{\mathrm{b}}$ \\
\hline 253.2 & 1.0000 & 0.0000 \\
253.0 & 0.9898 & 0.0102 \\
252.1 & 0.9779 & 0.0221 \\
251.6 & 0.9690 & 0.0310 \\
251.3 & 0.9597 & 0.0403 \\
256.2 & 0.9472 & 0.0528 \\
269.1 & 0.9252 & 0.0748 \\
285.3 & 0.8825 & 0.1175 \\
291.7 & 0.8584 & 0.1416 \\
302.8 & 0.8217 & 0.1783 \\
311.3 & 0.7801 & 0.2199
\end{tabular}

\begin{tabular}{lcc}
\hline \hline$T / \mathrm{K}$ & $x_{2}{ }^{\mathrm{a}}$ & $x_{1}{ }^{\mathrm{b}}$ \\
\hline 311.4 & 0.7786 & 0.2214 \\
317.5 & 0.7491 & 0.2509 \\
318.7 & 0.7416 & 0.2584 \\
321.2 & 0.7284 & 0.2716 \\
331.5 & 0.7185 & 0.2815 \\
343.8 & 0.7033 & 0.2967 \\
359.3 & 0.6790 & 0.3210 \\
363.3 & 0.6737 & 0.3263 \\
377.4 & 0.6490 & 0.3510 \\
398.1 & 0.6101 & 0.3899 \\
411.9 & 0.5773 & 0.4227 \\
420.9 & 0.5567 & 0.4433 \\
444.3 & 0.4842 & 0.5158 \\
518.7 & 0.0000 & 1.0000 \\
\hline
\end{tabular}

${ }^{a} x_{2}$ : mole fraction of component 2 in the saturated solution.

${ }^{\mathrm{b}} x_{1}$ : mole fraction of the polycyclic aromatic hydrocarbon (component 1).

The authors report that the data reveal the presence of a eutectic point at a carbazole mole fraction of $x_{1}=0.0475$ and eutectic temperature of $T / \mathrm{K}=250.8$. An incongruent melting temperature of $T / \mathrm{K}=322.8$ and $x_{1}=0.27$ suggested formation of a 1:1 carbazole- $N, N$-dimethylacetamide molecular complex.

\section{Auxiliary Information}

\section{Method/Apparatus/Procedure:}

Temperature-controlled heating/cooling system and a shaking-mixing system. Synthetic mixtures of known compositions were weighed in small glass ampoules. The mixture composition was known to within \pm 0.0002 mole fraction. Ampoules were cooled to very low temperatures of about $220 \mathrm{~K}$, pumped down and sealed. The sealed mixtures were then melted, cooled rapidly, and reheated. The rate of reheating was at an approximate rate of $8 \times 10^{-5} \mathrm{~K} / \mathrm{s}$ just before complete dissolution. The temperatures at which the solid completely dissolved were recorded. The average of two or three independent measurements was taken as the solid-liquid equilibrium.

Source and Purity of Chemicals:

(1) $98 \%$, Merck-Schuchardt, Federal Republic of Germany, was purified by melting with potassium hydroxide, followed by repeated recrystallization from dimethylbenzene to yield a sample having a purity of $99.9 \%$ (by mass). (2) Purest grade, Merck-Schuchardt, was distilled under reduced pressure and dried over molecular sieves to produce a sample of $99.9 \%$ purity (mass percent).

\section{Estimated Error:}

Temperature: $\pm 0.2 \mathrm{~K}$ (estimated by compiler). $x_{1}: \pm 0.0002$.

\begin{tabular}{|c|c|}
\hline $\begin{array}{l}\text { Components: } \\
\text { (1) Carbazole; } \mathrm{C}_{12} \mathrm{H}_{9} \mathrm{~N} \text {; [86-74-8] } \\
\text { (2) 1-Methyl-2-pyrrolidone; } \mathrm{C}_{5} \mathrm{H}_{9} \mathrm{NO} \text {; } \\
\text { [872-50-4] }\end{array}$ & $\begin{array}{l}\text { Original Measurements: } \\
{ }^{35} \text { Z. Lisicki and M. E. Jamróz, } \\
\text { J. Chem. Thermodyn. 32, } \\
1335 \text { (2000). }\end{array}$ \\
\hline $\begin{array}{l}\text { Variables: } \\
\text { Temperature }\end{array}$ & $\begin{array}{l}\text { Prepared by: } \\
\text { W. E. Acree, Jr. }\end{array}$ \\
\hline
\end{tabular}


Experimental Values

\begin{tabular}{lcc}
\hline \hline$T / \mathrm{K}$ & $x_{2}{ }^{\mathrm{a}}$ & $x_{1}{ }^{\mathrm{b}}$ \\
\hline 248.5 & 1.0000 & 0.0000 \\
252.7 & 0.9941 & 0.0059 \\
260.2 & 0.9902 & 0.0098 \\
268.7 & 0.9804 & 0.0196 \\
284.1 & 0.9520 & 0.0480 \\
296.4 & 0.9090 & 0.0910 \\
299.6 & 0.8935 & 0.1065 \\
302.2 & 0.8750 & 0.1250 \\
306.9 & 0.8371 & 0.1629 \\
309.8 & 0.8097 & 0.1903 \\
312.7 & 0.8000 & 0.2000 \\
319.2 & 0.7778 & 0.2222 \\
324.5 & 0.7499 & 0.2501 \\
330.5 & 0.7140 & 0.2860 \\
334.2 & 0.6875 & 0.3125 \\
353.7 & 0.6667 & 0.3333 \\
373.4 & 0.6360 & 0.3640 \\
393.3 & 0.6002 & 0.3998 \\
418.1 & 0.5499 & 0.4501 \\
437.2 & 0.5003 & 0.4997 \\
456.3 & 0.4398 & 0.5602 \\
518.7 & 0.0000 & 1.0000 \\
\hline$x_{2}:$ & &
\end{tabular}

${ }^{a} x_{2}$ : mole fraction of component 2 in the saturated solution.

${ }^{b} x_{1}$ : mole fraction of the polycyclic aromatic hydrocarbon (component 1).

The authors report that the binary system has two incongruent melting temperatures: the first at $T / \mathrm{K}=309.2$ and a carbazole mole fraction of $x_{1}=0.189$, and the second at $T / \mathrm{K}$ $=334.5$ and $x_{1}=0.313$. The composition of the complexes could not be established explicitly. An eutectic temperature of $T / \mathrm{K}=241.3$ was determined based on calorimetric measurements.

\section{Auxiliary Information}

\section{Method/Apparatus/Procedure:}

Temperature-controlled heating/cooling system and a shaking-mixing system. Synthetic mixtures of known compositions were weighed in small glass ampoules. The mixture composition was known to within \pm 0.0002 mole fraction. Ampoules were cooled to very low temperatures of about $220 \mathrm{~K}$, pumped down and sealed. The sealed mixtures were then melted, cooled rapidly, and reheated. The rate of reheating was at an approximate rate of $8 \times 10^{-5} \mathrm{~K} / \mathrm{s}$ just before complete dissolution. The temperatures at which the solid completely dissolved were recorded. The average of two or three independent measurements was taken as the solid-liquid equilibrium.

\section{Source and Purity of Chemicals:}

(1) $98 \%$, Merck-Schuchardt, Federal Republic of Germany, was purified by melting with potassium hydroxide, followed by repeated recrystallization from dimethylbenzene to yield a sample having a purity of $99.9 \%$ (by mass). (2) Technical Grade, BASF, Federal Republic of Germany, was purified by treatment with a $2 \%$ solution of potassium permanganate, and then distilled under reduced pressure to collect the distillate having $99.8 \%$ purity (mass percent). The purified sample was dried over molecular sieves.

\begin{tabular}{|c|c|}
\hline $\begin{array}{l}\text { Components: } \\
\text { (1) Carbazole; } \mathrm{C}_{12} \mathrm{H}_{9} \mathrm{~N} \text {; [86-74-8] } \\
\text { (2) Hexahydro-1-methyl- } 2 \mathrm{H} \text {-azepin-2-one }(\mathrm{N} \text { - } \\
\text { Methyl-e-caprolactam); } \mathrm{C}_{7} \mathrm{H}_{13} \mathrm{NO} ;[2556-73-2]\end{array}$ & $\begin{array}{l}\text { Original Measurements: } \\
{ }^{35} \text { Z. Lisicki and M. E. } \\
\text { Jamróz, J. Chem. } \\
\text { Thermodyn. 32, } 1335 \\
\text { (2000). }\end{array}$ \\
\hline $\begin{array}{l}\text { Variables: } \\
\text { Temperature }\end{array}$ & $\begin{array}{l}\text { Prepared by: } \\
\text { W. E. Acree, Jr. }\end{array}$ \\
\hline
\end{tabular}

Experimental Values

\begin{tabular}{lcc}
\hline \hline$T / \mathrm{K}$ & $x_{2}{ }^{\mathrm{a}}$ & $x_{1}{ }^{\mathrm{b}}$ \\
\hline 279.6 & 1.0000 & 0.0000 \\
282.7 & 0.7489 & 0.2511 \\
306.4 & 0.7284 & 0.2716 \\
322.1 & 0.7111 & 0.2889 \\
332.0 & 0.6930 & 0.3070 \\
341.9 & 0.6793 & 0.3207 \\
363.0 & 0.6459 & 0.3541 \\
394.3 & 0.5998 & 0.4002 \\
398.4 & 0.5910 & 0.4090 \\
422.1 & 0.5417 & 0.4583 \\
439.3 & 0.4909 & 0.5091 \\
462.4 & 0.4012 & 0.5988 \\
463.2 & 0.3975 & 0.6025 \\
481.7 & 0.3009 & 0.6991 \\
496.8 & 0.1996 & 0.8004 \\
508.8 & 0.0988 & 0.9012 \\
518.7 & 0.0000 & 1.0000 \\
\hline
\end{tabular}

${ }^{a} x_{2}$ : mole fraction of component 2 in the saturated solution.

${ }^{\mathrm{b}} x_{1}$ : mole fraction of the polycyclic aromatic hydrocarbon (component 1 ).

\section{Auxiliary Information}

\section{Method/Apparatus/Procedure:}

Temperature-controlled heating/cooling system and a shaking-mixing system. Synthetic mixtures of known compositions were weighed in small glass ampoules. The mixture composition was known to within \pm 0.0002 mole fraction. Ampoules were cooled to very low temperatures of about $220 \mathrm{~K}$, pumped down, and sealed. The sealed mixtures were then melted, cooled rapidly, and reheated. The rate of reheating was at an approximate rate of $8 \times 10^{-5} \mathrm{~K} / \mathrm{s}$ just before complete dissolution. The temperature at which the solid completely dissolved was recorded. The average of two or three independent measurements was taken as the solid-liquid equilibrium.

\section{Source and Purity of Chemicals:}

(1) $98 \%$, Merck-Schuchardt, Federal Republic of Germany, was purified by melting with potassium hydroxide, followed by repeated recrystallization from dimethylbenzene to yield a sample having a purity of $99.9 \%$ (by mass). (2) Technical Grade, Leuna Werke, Federal Republic of Germany, was purified by super-rectification and then dried over molecular sieves to give a sample having a purity of $99.9 \%$ by mass.

\section{Estimated Error:}

Temperature: $\pm 0.2 \mathrm{~K}$ (estimated by compiler). $x_{1}: \pm 0.0002$.

\section{Estimated Error:}

Temperature: $\pm 0.2 \mathrm{~K}$ (estimated by compiler).

$x_{1}: \pm 0.0002$. 
TABLE 2. Parameters of the Apelblat equation for describing the solubility of dibenzofuran in various organic solvents

\begin{tabular}{|c|c|c|c|c|}
\hline Solvent & $A$ & $B$ & $C$ & MRD (\%) \\
\hline Methylbenzene & 40.193 & -4126.3 & -4.863 & 1.3 \\
\hline Ethyl ethanoate & 36.051 & -4126.4 & -4.151 & 1.9 \\
\hline 2,2'-Oxybispropane & 20.096 & -4126.8 & -1.421 & 1.8 \\
\hline Tetrachloromethane & 33.557 & -4126.5 & -3.725 & 1.7 \\
\hline Ethanol & -13.514 & -4127.5 & 4.258 & 10.9 \\
\hline 1-Butanol & -14.149 & -4127.5 & 4.410 & 10.1 \\
\hline
\end{tabular}

\section{Solubility of Dibenzofuran in Organic Solvents}

\subsection{Critical evaluation of experimental solubility data}

The dibenzofuran solubility data that was contained in Vol. 58 of the IUPAC Solubility Data Series ${ }^{2}$ was very limited. The solubility data included mole fraction solubilities of dibenzofuran in cyclohexane (from 328 to $345 \mathrm{~K}$ ), decahydronaphthalene (from 326 to $337 \mathrm{~K}$ ), benzene (from 322 to $339 \mathrm{~K}$ ), 1,2,3,4-tetrahydronaphthalene (from 319 to $340 \mathrm{~K}$ ), pyridine (from 324 to $341 \mathrm{~K}$ ), and thiophene (from 314 to $341 \mathrm{~K}$ ) measured by Coon et al. ${ }^{39}$ along with the published solidliquid equilibrium data for the binary dibenzofuran + benzene phase diagram from a paper by Domanska et al. ${ }^{40}$ Solubility data contained in Vol. 58 will not be republished here. The listing above is provided so that readers will know what solubility data are available in the earlier volume for dibenzofuran.

There have been three studies that have reported solubility data for dibenzofuran in organic solvents after Vol. 58 was published in 1995. First, Wei et al. ${ }^{41}$ determined the solubility of dibenzofuran in methylbenzene, ethyl ethanoate, tetrachloromethane, 2,2'-oxybispropane, ethanol, and 1-butanol from 283.15 to $333.15 \mathrm{~K}$. The internal consistency of the six datasets of measured dibenzofuran solubilities were assessed by curvefitting the measured the mole fraction solubility to Eq. (3). The numerical values of the equation coefficients $(A, B$, and $C)$ are given in Table 2, along with the respective MRDs. Examination of the numerical entries in the last column of Table 2 reveals that the largest mean relative percent deviation between the back-calculated values based on Eq. (3) and experimental data is less than $2 \%$ for the four nonalkanol solvents, which is less than the experimental uncertainties in the measured values. Much larger mean relative percent deviations of $10.9 \%$ and $10.1 \%$ were noted for ethanol and 1-butanol, respectively. The experimental uncertainties associated with solubility data for the two alcohol solvents were on the order of $\pm 6 \%$. Results of the mathematical representation analyses indicate that the experimental data for the first four dibenzofuran-organic solvent systems are internally consistent. Deviations for ethanol and 1-butanol are larger than desired; however, given the experimental uncertainty in the measured values they are still considered to fall within an "acceptable" level.
The final two studies involved determination of phase diagrams. Hafsaoui and Mahmound ${ }^{42}$ used a differential scanning calorimetric method in determining solid-liquid equilibrium data for the dibenzofuran + pentacosane system. Lisicki and Jamróz ${ }^{35}$ reported solid-liquid equilibria data for binary mixtures containing dibenzofuran with $\mathrm{N}, \mathrm{N}$-dimethylacetamide, 1-methyl-2-pyrrolidone, and hexahydro-1-methyl- $2 \mathrm{H}$ azepin-2-one (also called $N$-methyl- $\varepsilon$-caprolactam). The dibenzofuran $+N, N$-dimethylformamide and dibenzofuran + 1-methyl-2-pyrrolidone systems both exhibited simple eutectic behavior. The published experimental liquidus curves for the latter three dibenzofuran systems did not indicate any outlier data points.

The experimental solubility data for dibenzofuran dissolved in the different organic solvents are given in Secs. 6.2-6.8.

\subsection{Dibenzofuran solubility data in saturated hydrocarbons (including cycloalkanes)}

\begin{tabular}{|c|c|}
\hline $\begin{array}{l}\text { Components: } \\
\text { (1) Dibenzofuran; } \mathrm{C}_{12} \mathrm{H}_{8} \mathrm{O} ;[132-64-9] \\
\text { (2) Pentacosane; } \mathrm{C}_{25} \mathrm{H}_{52} ;[629-99-2]\end{array}$ & $\begin{array}{l}\text { Original Measurements: } \\
{ }^{42} \text { S. L. Hafsaoui and R. } \\
\text { Mahmoud, J. Therm. Anal. } \\
\text { Calorim. 88, } 565 \text { (2007). }\end{array}$ \\
\hline Variables: & Prepared by: \\
\hline Temperature & W. E. Acree, Jr. \\
\hline
\end{tabular}

Experimental Values

\begin{tabular}{lcc}
\hline \hline$T / \mathrm{K}$ & $x_{2}{ }^{\mathrm{a}}$ & $x_{1}{ }^{\mathrm{b}}$ \\
\hline 324.10 & 1.000 & 0.000 \\
323.65 & 0.901 & 0.099 \\
322.35 & 0.798 & 0.202 \\
319.34 & 0.702 & 0.298 \\
326.43 & 0.601 & 0.399 \\
332.21 & 0.501 & 0.499 \\
337.75 & 0.399 & 0.601 \\
344.04 & 0.299 & 0.701 \\
348.94 & 0.199 & 0.801 \\
352.84 & 0.101 & 0.899 \\
355.10 & 0.000 & 1.000 \\
\hline
\end{tabular}

${ }^{a} x_{2}$ : mole fraction of component 2 in the saturated solution.

${ }^{\mathrm{b}} x_{1}$ : mole fraction of the polycyclic aromatic hydrocarbon (component 1 ). 


\section{Auxiliary Information}

\section{Method/Apparatus/Procedure:}

Differential scanning calorimeter.

Mixtures of dibenzofuran and pentacosane were prepared by mass. The resulting mixtures were heated slowly with agitation until the entire sample melted. After melting the sample was immersed in liquid nitrogen to solidify the mixture completely. A small amount of solid was removed and sealed in a pan, the capsule was crimped, and placed in the calorimetric block of the differential scanning calorimeter for analysis.

Source and Purity of Chemicals:

(1) $98 \%$, Fluka Chemical Company, was used as received.

(2) $99 \%$, Fluka Chemical Company, was used as received.

\section{Estimated Error:}

Temperature: $\pm 0.7 \mathrm{~K}$.

$x_{1}: \pm 0.002$ (estimated by compiler).

\subsection{Dibenzofuran solubility data in aromatic hydrocarbons}

\begin{tabular}{ll}
\hline \hline $\begin{array}{l}\text { Components: } \\
\text { (1) Dibenzofuran; } \mathrm{C}_{12} \mathrm{H}_{8} \mathrm{O} ;[132-64-9]\end{array}$ & $\begin{array}{l}\text { Original Measurements: } \\
{ }^{41} \text { Y. Wei, L. Dang, Z. Zhang, } \\
\text { (2) Methylbenzene; } \mathrm{C}_{7} \mathrm{H}_{8} ;[108-88-3]\end{array}$ \\
& W. Cui, and H. Wei, J. Chem. \\
& Eng. Data 57, 1279 (2012). \\
\hline Variables: & Prepared by: \\
Temperature & W. E. Acree, Jr. \\
\hline
\end{tabular}

Experimental Values

\begin{tabular}{lcc}
\hline \hline$T / \mathrm{K}$ & $x_{2}{ }^{\mathrm{a}}$ & $x_{1}{ }^{\mathrm{b}}$ \\
\hline 283.15 & 0.8423 & 0.1577 \\
288.15 & 0.8145 & 0.1855 \\
293.15 & 0.7769 & 0.2231 \\
298.15 & 0.7344 & 0.2656 \\
303.15 & 0.6987 & 0.3013 \\
308.15 & 0.6563 & 0.3437 \\
313.15 & 0.6009 & 0.3991 \\
318.15 & 0.5453 & 0.4547 \\
323.15 & 0.4855 & 0.5145 \\
328.15 & 0.4183 & 0.5817 \\
333.15 & 0.3762 & 0.6238
\end{tabular}

${ }^{\mathrm{a}} x_{2}$ : mole fraction of component 2 in the saturated solution.

${ }^{\mathrm{b}} \mathrm{x}_{1}$ : mole fraction of the polycyclic aromatic hydrocarbon (component 1 ).

\section{Auxiliary Information}

\section{Method/Apparatus/Procedure:}

Jacketed vessel, thermostatted bath and gas chromatograph equipped with a flame ionization detector.

The authors' description states that the method is based on adding excess solute to a stirred solution kept at fixed temperature for at least $24 \mathrm{~h}$. After stirring was stopped, the solid was allowed to settle to the bottom of the container. Three samples of about $10 \mathrm{~mL}$ each were carefully withdrawn and filtered through a $0.045 \mu \mathrm{m}$ PTFE filter. The filtered samples were then analyzed using a gas chromatograph equipped with a flame ionization detector.
Source and Purity of Chemicals:

(1) $99.5 \%$ (by gas chromatography), Tianjin Kewei Chemical Reagents, Company, Ltd., China, recrystallized twice. Recrystallization solvent was not specified.

(2) $99.5 \%$, Analytical Reagent Grade, Tianjin Damao Chemical Reagent Company, China, was used without further purification.

\section{Estimated Error:}

Temperature: $\pm 0.1 \mathrm{~K}$.

$x_{1}: \pm 3 \%$ (relative error).

\subsection{Dibenzofuran solubility data in esters}

\begin{tabular}{ll}
\hline \hline Components: & Original Measurements: \\
(1) Dibenzofuran; $\mathrm{C}_{12} \mathrm{H}_{8} \mathrm{O} ;[132-64-9]$ & ${ }^{41}$ Y. Wei, L. Dang, Z. Zhang, \\
(2) Ethyl ethanoate; $\mathrm{C}_{4} \mathrm{H}_{8} \mathrm{O}_{2} ;[141-78-6]$ & W. Cui, and H. Wei, J. Chem. \\
& Eng. Data 57, 1279 (2012). \\
\hline Variables: & Prepared by: \\
Temperature & W. E. Acree, Jr. \\
\hline
\end{tabular}

Experimental Values

\begin{tabular}{lcc}
\hline \hline$T / \mathrm{K}$ & $x_{2}{ }^{\mathrm{a}}$ & $x_{1}{ }^{\mathrm{b}}$ \\
\hline 283.15 & 0.8545 & 0.1455 \\
288.15 & 0.8321 & 0.1679 \\
293.15 & 0.8011 & 0.1989 \\
298.15 & 0.7685 & 0.2315 \\
303.15 & 0.7315 & 0.2685 \\
308.15 & 0.6723 & 0.3277 \\
313.15 & 0.6128 & 0.3872 \\
318.15 & 0.5589 & 0.4411 \\
323.15 & 0.4983 & 0.5017 \\
328.15 & 0.4349 & 0.5651 \\
333.15 & 0.3751 & 0.6249 \\
\hline
\end{tabular}

${ }^{\mathrm{a}} x_{2}$ : mole fraction of component 2 in the saturated solution.

${ }^{b} x_{1}$ : mole fraction of the polycyclic aromatic hydrocarbon (component 1 ).

\section{Auxiliary Information}

\section{Method/Apparatus/Procedure:}

Jacketed vessel, thermostatted bath and gas chromatograph equipped with a flame ionization detector.

The authors' description states that the method is based on adding excess solute to a stirred solution kept at fixed temperature for at least $24 \mathrm{~h}$. After stirring was stopped, the solid was allowed to settle to the bottom of the container. Three samples of about $10 \mathrm{~mL}$ each were carefully withdrawn and filtered through a $0.045 \mu \mathrm{m}$ PTFE filter. The filtered samples were then analyzed using a gas chromatograph equipped with a flame ionization detector.

Source and Purity of Chemicals:

(1) $99.5 \%$ (by gas chromatography), Tianjin Kewei Chemical Reagents, Company, Ltd., China, recrystallized twice. Recrystallization solvent was not specified.

(2) $99.5 \%$, Analytical Reagent Grade, Tianjin Damao Chemical Reagent Company, China, was used without further purification.

\section{Estimated Error:}

Temperature: $\pm 0.1 \mathrm{~K}$.

$x_{1}: \pm 3 \%$ (relative error). 


\subsection{Dibenzofuran solubility data in ethers}

\begin{tabular}{|c|c|}
\hline $\begin{array}{l}\text { Components: } \\
\text { (1) Dibenzofuran; } \mathrm{C}_{12} \mathrm{H}_{8} \mathrm{O} ;[132-64-9] \\
\text { (2) } 2,2^{\prime}-\mathrm{O} \text { - } \\
\text { [108-20-3] }\end{array}$ & $\begin{array}{l}\text { Original Measurements: } \\
{ }^{41} \text { Y. Wei, L. Dang, Z. Zhang, } \\
\text { W. Cui, and H. Wei, J. Chem. } \\
\text { Eng. Data 57, } 1279 \text { (2012). }\end{array}$ \\
\hline $\begin{array}{l}\text { Variables: } \\
\text { Temperature }\end{array}$ & $\begin{array}{l}\text { Prepared by: } \\
\text { W. E. Acree, Jr. }\end{array}$ \\
\hline
\end{tabular}

Experimental Values

\begin{tabular}{lcc}
\hline \hline$T / \mathrm{K}$ & $x_{2}{ }^{\mathrm{a}}$ & $x_{1}{ }^{\mathrm{b}}$ \\
\hline 283.15 & 0.9162 & 0.0838 \\
288.15 & 0.8958 & 0.1042 \\
293.15 & 0.8698 & 0.1302 \\
298.15 & 0.8452 & 0.1548 \\
303.15 & 0.8168 & 0.1832 \\
308.15 & 0.7634 & 0.2366 \\
313.15 & 0.7155 & 0.2845 \\
318.15 & 0.6589 & 0.3411 \\
323.15 & 0.5881 & 0.4119 \\
328.15 & 0.4919 & 0.5081 \\
333.15 & 0.4202 & 0.5798 \\
\hline
\end{tabular}

${ }^{\mathrm{a}} x_{2}$ : mole fraction of component 2 in the saturated solution.

${ }^{\mathrm{b}} x_{1}$ : mole fraction of the polycyclic aromatic hydrocarbon (component 1).

\section{Auxiliary Information}

\section{Method/Apparatus/Procedure:}

Jacketed vessel, thermostatted bath and gas chromatograph equipped with a flame ionization detector.

The authors' description states that the method is based on adding excess solute to a stirred solution kept at fixed temperature for at least $24 \mathrm{~h}$. After stirring was stopped, the solid was allowed to settle to the bottom of the container. Three samples of about $10 \mathrm{~mL}$ each were carefully withdrawn and filtered through a $0.045 \mu \mathrm{m}$ PTFE filter. The filtered samples were then analyzed using a gas chromatograph equipped with a flame ionization detector.

Source and Purity of Chemicals:

(1) $99.5 \%$ (by gas chromatography), Tianjin Kewei Chemical Reagents, Company, Ltd., China, recrystallized twice. Recrystallization solvent was not specified.

(2) $99.5 \%$, Analytical Reagent Grade, Tianjin Damao Chemical Reagent Company, China, was used without further purification.

Estimated Error:

Temperature: $\pm 0.1 \mathrm{~K}$.

$x_{1}: \pm 3 \%$ (relative error).

\subsection{Dibenzofuran solubility data in haloalkanes, haloalkenes, and haloaromatic hydrocarbons}

\section{Components:}

(1) Dibenzofuran; $\mathrm{C}_{12} \mathrm{H}_{8} \mathrm{O}$; [132-64-9]

(2) Tetrachloromethane; $\mathrm{CCl}_{4} ;[56-23-5]$
Variables:

Temperature
Original Measurements:

${ }^{41}$ Y. Wei, L. Dang, Z. Zhang, W. Cui, and H. Wei, J. Chem. Eng. Data 57, 1279 (2012).
Prepared by:

W. E. Acree, Jr.
Experimental Values

\begin{tabular}{lcc}
\hline \hline$T / \mathrm{K}$ & $x_{2}{ }^{\mathrm{a}}$ & $x_{1}{ }^{\mathrm{b}}$ \\
\hline 283.15 & 0.8661 & 0.1339 \\
288.15 & 0.8386 & 0.1614 \\
293.15 & 0.8087 & 0.1913 \\
298.15 & 0.7842 & 0.2158 \\
303.15 & 0.7361 & 0.2639 \\
308.15 & 0.6934 & 0.3066 \\
313.15 & 0.6458 & 0.3542 \\
318.15 & 0.5881 & 0.4119 \\
323.15 & 0.5086 & 0.4914 \\
328.15 & 0.4521 & 0.5479 \\
333.15 & 0.3652 & 0.6348 \\
\hline
\end{tabular}

${ }^{a} x_{2}$ : mole fraction of component 2 in the saturated solution.

${ }^{\mathrm{b}} x_{1}$ : mole fraction of the polycyclic aromatic hydrocarbon (component 1 ).

\section{Auxiliary Information}

Method/Apparatus/Procedure:

Jacketed vessel, thermostatted bath and gas chromatograph equipped with a flame ionization detector.

The authors' description states that the method is based on adding excess solute to a stirred solution kept at fixed temperature for at least $24 \mathrm{~h}$. After stirring was stopped, the solid was allowed to settle to the bottom of the container. Three samples of about $10 \mathrm{~mL}$ each were carefully withdrawn and filtered through a $0.045 \mu \mathrm{m}$ PTFE filter. The filtered samples were then analyzed using a gas chromatograph equipped with a flame ionization detector.

Source and Purity of Chemicals:

(1) $99.5 \%$ (by gas chromatography), Tianjin Kewei Chemical Reagents, Company, Ltd., China, recrystallized twice. Recrystallization solvent was not specified.

(2) 99.5\%, Analytical Reagent Grade, Tianjin Damao Chemical Reagent Company, China, was used without further purification.

\section{Estimated Error:}

Temperature: $\pm 0.1 \mathrm{~K}$.

$x_{1}: \pm 3 \%$ (relative error).

\subsection{Dibenzofuran solubility data in alcohols}

\section{Components:}

(1) Dibenzofuran; $\mathrm{C}_{12} \mathrm{H}_{8} \mathrm{O}$; [132-64-9]

(2) Ethanol; $\mathrm{C}_{2} \mathrm{H}_{6} \mathrm{O} ;[64-17-5]$
Original Measurements:

${ }^{41}$ Y. Wei, L. Dang, Z. Zhang, W. Cui, and H. Wei, J. Chem. Eng. Data 57, 1279 (2012).

Variables: $\quad$ Prepared by:


Experimental Values

\begin{tabular}{lcc}
\hline \hline$T / \mathrm{K}$ & $x_{2}{ }^{\mathrm{a}}$ & $x_{1}{ }^{\mathrm{b}}$ \\
\hline 283.15 & 0.9789 & 0.0211 \\
288.15 & 0.9729 & 0.0271 \\
293.15 & 0.9678 & 0.0322 \\
298.15 & 0.9586 & 0.0414 \\
303.15 & 0.9511 & 0.0489 \\
308.15 & 0.9293 & 0.0707 \\
313.15 & 0.8977 & 0.1023 \\
318.15 & 0.8659 & 0.1341 \\
323.15 & 0.8029 & 0.1971 \\
328.15 & 0.7169 & 0.2831 \\
333.15 & 0.6659 & 0.3341
\end{tabular}

${ }^{\mathrm{a}} x_{2}$ : mole fraction of component 2 in the saturated solution.

${ }^{b} x_{1}$ : mole fraction of the polycyclic aromatic hydrocarbon (component 1 ).

\section{Auxiliary Information}

Method/Apparatus/Procedure:

Jacketed vessel, thermostatted bath and gas chromatograph equipped with a flame ionization detector.

The authors' description states that the method is based on adding excess solute to a stirred solution kept at fixed temperature for at least $24 \mathrm{~h}$. After stirring was stopped, the solid was allowed to settle to the bottom of the container. Three samples of about $10 \mathrm{~mL}$ each were carefully withdrawn and filtered through a $0.045 \mu \mathrm{m}$ PTFE filter. The filtered samples were then analyzed using a gas chromatograph equipped with a flame ionization detector.

Source and Purity of Chemicals:

(1) $99.5 \%$ (by gas chromatography), Tianjin Kewei Chemical Reagents, Company, Ltd., China, recrystallized twice. Recrystallization solvent was not specified.

(2) $99.5 \%$, Analytical Reagent Grade, Tianjin Damao Chemical Reagent Company, China, was used without further purification.

Estimated Error:

Temperature: $\pm 0.1 \mathrm{~K}$.

$x_{1}: \pm 6 \%$ (relative error).

\section{Components:}

(1) Dibenzofuran; $\mathrm{C}_{12} \mathrm{H}_{8} \mathrm{O}$; [132-64-9]

(2) 1-Butanol; $\mathrm{C}_{4} \mathrm{H}_{10} \mathrm{O}$; [71-36-3]

Original Measurements:

${ }^{41}$ Y. Wei, L. Dang, Z. Zhang, W. Cui, and H. Wei, J. Chem Eng. Data 57, 1279 (2012).

\section{Variables:}

Prepared by:

Temperature W. E. Acree, Jr.

\begin{tabular}{lcc}
\hline \hline$T / \mathrm{K}$ & $x_{2}{ }^{\mathrm{a}}$ & $x_{1}{ }^{\mathrm{b}}$ \\
\hline 313.15 & 0.8932 & 0.1068 \\
318.15 & 0.8336 & 0.1664 \\
323.15 & 0.7699 & 0.2301 \\
328.15 & 0.6362 & 0.3638 \\
333.15 & 0.4859 & 0.5141
\end{tabular}

${ }^{\mathrm{a}} x_{2}$ : mole fraction of component 2 in the saturated solution.

${ }^{b} x_{1}$ : mole fraction of the polycyclic aromatic hydrocarbon (component 1 ).

\section{Auxiliary Information}

\section{Method/Apparatus/Procedure:}

Jacketed vessel, thermostatted bath and gas chromatograph equipped with a flame ionization detector

The authors' description states that the method is based on adding excess solute to a stirred solution kept at fixed temperature for at least $24 \mathrm{~h}$. After stirring was stopped, the solid was allowed to settle to the bottom of the container. Three samples of about $10 \mathrm{~mL}$ each were carefully withdrawn and filtered through a $0.045 \mu \mathrm{m}$ PTFE filter. The filtered samples were then analyzed using a gas chromatograph equipped with a flame ionization detector.

\section{Source and Purity of Chemicals:}

(1) $99.5 \%$ (by gas chromatography), Tianjin Kewei Chemical Reagents, Company, Ltd., China, recrystallized twice. Recrystallization solvent was not specified.

(2) $99.5 \%$, Analytical Reagent Grade, Tianjin Damao Chemical Reagent Company, China, was used without further purification.

\section{Estimated Error:}

Temperature: $\pm 0.1 \mathrm{~K}$.

$x_{1}: \pm 6 \%$ (relative error).

\subsection{Dibenzofuran solubility data in miscellaneous organic solvents}

\begin{tabular}{ll}
\hline \hline Components: & Original Measurements: \\
(1) Dibenzofuran; $\mathrm{C}_{12} \mathrm{H}_{8} \mathrm{O} ;[132-64-9]$ & ${ }^{35} \mathrm{Z}$. Lisicki and M. E. Jamróz, \\
$(2) \mathrm{N}, \mathrm{N}$-Dimethylacetamide; $\mathrm{C}_{4} \mathrm{H}_{9} \mathrm{NO} ;$ & J. Chem. Thermodyn. 32, \\
{$[127-19-5]$} & $1335(2000)$. \\
\hline Variables: & Prepared by: \\
Temperature & W. E. Acree, Jr. \\
\hline
\end{tabular}

Experimental Values

\begin{tabular}{lcc}
\hline \hline$T / \mathrm{K}$ & $x_{2}{ }^{\mathrm{a}}$ & $x_{1}{ }^{\mathrm{b}}$ \\
\hline 283.15 & 0.9719 & 0.0281 \\
288.15 & 0.9674 & 0.0326 \\
293.15 & 0.9579 & 0.0421 \\
298.15 & 0.9469 & 0.0531 \\
303.15 & 0.9329 & 0.0671 \\
308.15 & 0.9123 & 0.0877
\end{tabular}


Experimental Values

\begin{tabular}{lcc}
\hline \hline$T / \mathrm{K}$ & $x_{2}{ }^{\mathrm{a}}$ & $x_{1}{ }^{\mathrm{b}}$ \\
\hline 253.2 & 1.0000 & 0.0000 \\
252.8 & 0.9894 & 0.0106 \\
252.4 & 0.9779 & 0.0221 \\
251.7 & 0.9654 & 0.0346 \\
249.5 & 0.9462 & 0.0538 \\
247.9 & 0.9305 & 0.0695 \\
249.6 & 0.9137 & 0.0863 \\
256.7 & 0.8915 & 0.1085 \\
272.7 & 0.8227 & 0.1773 \\
283.4 & 0.7723 & 0.2277 \\
298.9 & 0.6764 & 0.3236 \\
313.4 & 0.5765 & 0.4235 \\
323.2 & 0.4745 & 0.5255 \\
332.7 & 0.3584 & 0.6416 \\
337.7 & 0.3003 & 0.6997 \\
344.3 & 0.2084 & 0.7916 \\
351.0 & 0.1021 & 0.8979 \\
354.1 & 0.0546 & 0.9454 \\
355.8 & 0.0000 & 1.0000
\end{tabular}

${ }^{\mathrm{a}} x_{2}$ : mole fraction of component 2 in the saturated solution.

${ }^{b} x_{1}$ : mole fraction of the polycyclic aromatic hydrocarbon (component 1 ).

The authors reported that the binary system exhibits simple eutectic behavior, with a eutectic temperature of $T / \mathrm{K}=247.3$ and eutectic composition of $x_{1}=0.079$.

\section{Auxiliary Information}

\section{Method/Apparatus/Procedure:}

Temperature-controlled heating/cooling system and a shaking-mixing system. Synthetic mixtures of known compositions were weighed in small glass ampoules. The mixture composition was known to within \pm 0.0002 mole fraction. Ampoules were cooled to very low temperatures of about $220 \mathrm{~K}$, pumped down and sealed. The sealed mixtures were then melted, cooled rapidly, and reheated. The rate of reheating was at an approximate rate of $8 \times 10^{-5} \mathrm{~K} / \mathrm{s}$ just before complete dissolution. The temperatures at which the solid completely dissolved were recorded. The average of two or three independent measurements was taken as the solid-liquid equilibrium.

Source and Purity of Chemicals:

(1) $98 \%$, Merck-Schuchardt, Federal Republic of Germany, was recrystallized twice from ethanol in the presence of activated carbon, and then recrystallized from methylbenzene.

(2) Purest grade, Merck-Schuchardt, was distilled under reduced pressure and dried over molecular sieves to produce a sample of $99.9 \%$ purity (mass percent).

\section{Estimated Error:}

Temperature: $\pm 0.2 \mathrm{~K}$ (estimated by compiler). $x_{1}: \pm 0.0002$.

\begin{tabular}{ll}
\hline \hline Components: & Original Measurements: \\
(1) Dibenzofuran; $\mathrm{C}_{12} \mathrm{H}_{8} \mathrm{O} ;[132-64-9]$ & ${ }^{35} \mathrm{Z}$. Lisicki and M. E. Jamróz, \\
(2) 1-Methyl-2-pyrrolidone; $\mathrm{C}_{5} \mathrm{H}_{9} \mathrm{NO} ;$ & J. Chem. Thermodyn. 32, \\
[872-50-4] & $1335(2000)$. \\
\hline Variables: & Prepared by: \\
Temperature & W. E. Acree, Jr. \\
\hline
\end{tabular}

Experimental Values

\begin{tabular}{lcc}
\hline \hline$T / \mathrm{K}$ & $x_{2}{ }^{\mathrm{a}}$ & $x_{1}{ }^{\mathrm{b}}$ \\
\hline 248.5 & 1.0000 & 0.0000 \\
247.9 & 0.9709 & 0.0291 \\
247.6 & 0.9648 & 0.0352 \\
244.8 & 0.9047 & 0.0953 \\
244.3 & 0.8791 & 0.1209 \\
243.0 & 0.8580 & 0.1420 \\
247.3 & 0.8434 & 0.1566 \\
249.0 & 0.8399 & 0.1601 \\
252.7 & 0.8275 & 0.1725 \\
258.3 & 0.8115 & 0.1885 \\
270.1 & 0.7716 & 0.2284 \\
286.3 & 0.7009 & 0.2991 \\
301.4 & 0.6200 & 0.3800 \\
317.1 & 0.5125 & 0.4875 \\
327.3 & 0.4094 & 0.5906 \\
335.8 & 0.3210 & 0.6790 \\
342.9 & 0.2336 & 0.7664 \\
352.1 & 0.1074 & 0.8926 \\
353.4 & 0.0675 & 0.9325 \\
355.8 & 0.0000 & 1.0000 \\
\hline
\end{tabular}

${ }^{\mathrm{a}} x_{2}$ : mole fraction of component 2 in the saturated solution.

${ }^{\mathrm{b}} x_{1}$ : mole fraction of the polycyclic aromatic hydrocarbon (component 1).

The authors reported that the binary system exhibits simple eutectic behavior, with a eutectic temperature of $T / \mathrm{K}=242.1$ and eutectic composition of $x_{1}=0.141$.

\section{Auxiliary Information}

\section{Method/Apparatus/Procedure:}

Temperature-controlled heating/cooling system and a shaking-mixing system. Synthetic mixtures of known compositions were weighed in small glass ampoules. The mixture composition was known to within \pm 0.0002 mole fraction. Ampoules were cooled to very low temperatures of about $220 \mathrm{~K}$, pumped down, and sealed. The sealed mixtures were then melted, cooled rapidly, and reheated. The rate of reheating was at an approximate rate of $8 \times 10^{-5} \mathrm{~K} / \mathrm{s}$ just before complete dissolution. The temperature at which the solid completely dissolved was recorded. The average of two or three independent measurements was taken as the solid-liquid equilibrium.

\section{Source and Purity of Chemicals:}

(1) $98 \%$, Merck-Schuchardt, Federal Republic of Germany, was recrystallized twice from ethanol in the presence of activated carbon, and then recrystallized from methylbenzene.

(2) Technical Grade, BASF, Federal Republic of Germany, was purified by treatment with a $2 \%$ solution of potassium permanganate, and then distilled under reduced pressure to collect the distillate having $99.8 \%$ purity (mass percent). The purified sample was dried over molecular sieves.

Estimated Error:

Temperature: $\pm 0.2 \mathrm{~K}$ (estimated by compiler).

$x_{1}: \pm 0.0002$.

\section{Components:}

(1) Dibenzofuran; $\mathrm{C}_{12} \mathrm{H}_{8} \mathrm{O}$; [132-64-9]

(2) Hexahydro-1-methyl- $2 \mathrm{H}$-azepin-2-one $(\mathrm{N}$ Methyl-e-caprolactam); $\mathrm{C}_{7} \mathrm{H}_{13} \mathrm{NO}$; [2556-73-2]
Original Measurements:

${ }^{35}$ Z. Lisicki and M. E. Jamróz, J. Chem. Thermodyn. 32, 1335 (2000).

Variables:

Temperature

\section{Prepared by:}

W. E. Acree, Jr. 
Experimental Values

\begin{tabular}{lcc}
\hline \hline$T / \mathrm{K}$ & $x_{2}{ }^{\mathrm{a}}$ & $x_{1}{ }^{\mathrm{b}}$ \\
\hline 279.6 & 1.0000 & 0.0000 \\
286.3 & 0.7030 & 0.2970 \\
296.6 & 0.6479 & 0.3521 \\
305.7 & 0.6049 & 0.3951 \\
306.3 & 0.5977 & 0.4023 \\
314.1 & 0.5495 & 0.4505 \\
320.2 & 0.5056 & 0.4944 \\
329.9 & 0.4110 & 0.5890 \\
330.9 & 0.3998 & 0.6002 \\
337.9 & 0.3141 & 0.6859 \\
345.5 & 0.2093 & 0.7907 \\
351.8 & 0.0888 & 0.9112 \\
355.8 & 0.0000 & 1.0000 \\
\hline
\end{tabular}

${ }^{\mathrm{a}} x_{2}$ : mole fraction of component 2 in the saturated solution.

${ }^{b} x_{1}$ : mole fraction of the polycyclic aromatic hydrocarbon (component 1 ).

Auxiliary Information

\section{Method/Apparatus/Procedure:}

Temperature controlled heating/cooling system and a shaking-mixing system. Synthetic mixtures of known compositions were weighed in small glass ampoules. The mixture composition was known to within \pm 0.0002 mole fraction. Ampoules were cooled to very low temperatures of about $220 \mathrm{~K}$, pumped down and sealed. The sealed mixtures were then melted, cooled rapidly, and reheated. The rate of reheating was at an approximate rate of $8 \times 10^{-5} \mathrm{~K} / \mathrm{s}$ just before complete dissolution. The temperatures at which the solid completely dissolved were recorded. The average of two or three independent measurements was taken as the solid-liquid equilibrium.

\section{Source and Purity of Chemicals:}

(1) $98 \%$, Merck-Schuchardt, Federal Republic of Germany, was recrystallized twice from ethanol in the presence of activated carbon, and then recrystallized from methylbenzene.

(2) Technical Grade, Leuna Werke, Federal Republic of Germany, was purified by super-rectification and then dried over molecular sieves to give a sample having a purity of $99.9 \%$ by mass.

Estimated Error:

Temperature: $\pm 0.2 \mathrm{~K}$ (estimated by compiler). $x_{1}: \pm 0.0002$.

\section{Solubility of Dibenzothiophene in Organic Solvents}

\subsection{Critical evaluation of experimental solubility data}

The dibenzothiophene solubility data that was contained in Vol. 58 of the IUPAC Solubility Data Series ${ }^{2}$ was very limited. The solubility data included mole fraction solubilities of dibenzothiophene in cyclohexane (from 321 to $343 \mathrm{~K}$ ), decahydronaphthalene (from 311 to $334 \mathrm{~K}$ ), benzene (from 310 to $342 \mathrm{~K}$ ), 1,2,3,4-tetrahydronaphthalene (from 309 to $338 \mathrm{~K}$ ), pyridine (from 307 to $335 \mathrm{~K}$ ), and thiophene (from 309 to $337 \mathrm{~K}$ ) measured by Coon et al. ${ }^{39}$ along with the published solid-liquid equilibrium data for the binary dibenzothiophene + benzene phase diagram from a paper by Domanska et al. ${ }^{40}$ Solubility data contained in Vol. 58 will not be republished here. The listing above is provided so that readers will know what solubility data are available in the earlier volume for dibenzothiophene.

A search of the published chemical and engineering literature from 1995 to 2011 found two papers reporting experimental dibenzothiophene solubility data. Ramirez-Verduzco et $a .^{43}$ determined the solubility of dibenzothiophene in cyclohexane, tetradecane, hexadecane, dihydro- $2(3 H)$-furanone (commonly referred to as $\gamma$-butyrolactone), and acetonitrile as a function of temperature. Each of the five systems covered a temperature range of more than $40 \mathrm{~K}$. The authors noted that their experimental results for the dibenzothiophene + cyclohexane system were in good agreement with corresponding values previously reported by Coon et al. ${ }^{39}$ The highest deviation noted in the solid-liquidus temperature was $1.2 \mathrm{~K}$. The experimental data were correlated with both the two-parameter Wilson and UNIQUAC models. Interaction coefficients calculated from the experimental solid-liquid equilibrium data provided a reasonably accurate mathematical description of the measured values. Absolute average relative deviations between calculated and observed values were less than $3 \%$.

Lisicki and Jamróz ${ }^{35}$ reported solid-liquid equilibria data for binary mixtures containing dibenzothiophene with $\mathrm{N}, \mathrm{N}$ dimethylacetamide, 1-methyl-2-pyrrolidone and hexahydro1-methyl- $2 H$-azepin-2-one (also called $N$-methyl- $\varepsilon$-caprolactam). The dibenzothiophene $+N, N$-dimethylformamide and dibenzothiophene + 1-methyl-2-pyrrolidone systems both exhibited simple eutectic behavior. The published experimental liquidus curves for the latter three dibenzothiophene systems did not indicate any outlier data points.

The experimental solubility data for dibenzothiophene dissolved in the different organic solvents are given in Secs. 7.2-7.4.

\subsection{Dibenzothiophene solubility data in saturated hydrocarbons (including cycloalkanes)}

\begin{tabular}{|c|c|}
\hline $\begin{array}{l}\text { Components: } \\
\text { (1) Dibenzothiophene; } \mathrm{C}_{12} \mathrm{H}_{8} \mathrm{~S} \text {; } \\
{[132-65-0]} \\
\text { (2) Tetradecane; } \mathrm{C}_{14} \mathrm{H}_{30} \text {; } \\
{[629-59-4]}\end{array}$ & $\begin{array}{l}\text { Original Measurements: } \\
{ }^{43} \text { L. F. Ramirez-Verduzco, A. Rojas- } \\
\text { Aguilar, J. A. De los Reyes, J. A. Munoz- } \\
\text { Arroyo, and F. Murrieta-Guevara, J. } \\
\text { Chem. Eng. Data 52, } 2212 \text { (2007). }\end{array}$ \\
\hline $\begin{array}{l}\text { Variables: } \\
\text { Temperature }\end{array}$ & $\begin{array}{l}\text { Prepared by: } \\
\text { W. E. Acree, Jr. }\end{array}$ \\
\hline
\end{tabular}


Experimental Values

\begin{tabular}{lcc}
\hline \hline$T / \mathrm{K}$ & $x_{2}{ }^{\mathrm{a}}$ & $x_{1}{ }^{\mathrm{b}}$ \\
\hline 278.2 & 0.9770 & 0.0230 \\
283.9 & 0.9700 & 0.0300 \\
291.0 & 0.9600 & 0.0400 \\
298.3 & 0.9475 & 0.0525 \\
316.1 & 0.9041 & 0.0959 \\
337.2 & 0.7965 & 0.2035 \\
346.7 & 0.7018 & 0.2982 \\
352.6 & 0.5993 & 0.4007 \\
356.2 & 0.5035 & 0.4965
\end{tabular}

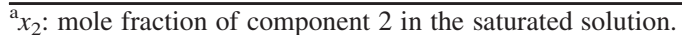

${ }^{\mathrm{b}} x_{1}$ : mole fraction of the polycyclic aromatic hydrocarbon (component 1 ).

The authors report that the eutectic point occurred at $x_{1}=0.0230$ and $T / \mathrm{K}=278.2$.

\section{Auxiliary Information}

\section{Method/Apparatus/Procedure:}

Heating circulating bath and platinum resistance temperature probe.

Synthetic mixtures of known compositions were prepared by mass. Samples were slowly heated in a temperature bath, and the temperature at which the las amount of solid solute dissolved was measured. The temperature obtained was taken to represent the saturation point for the mixture being studied.

Source and Purity of Chemicals:

(1) $98.0 \%$, Aldrich Chemical Company, Milwaukee, WI, USA, was used as received.

(2) $99.0 \%$, Aldrich Chemical Company, was used as received.

\section{Estimated Error:}

Temperature: $\pm 0.5 \mathrm{~K}$.

$x_{1}: \pm 0.0005$

\begin{tabular}{ll}
\hline \hline Components: & Original Measurements: \\
(1) Dibenzothiophene; $\mathrm{C}_{12} \mathrm{H}_{8} \mathrm{~S} ;$ & ${ }^{43}$ L. F. Ramirez-Verduzco, A. Rojas- \\
[132-65-0] & Aguilar, J. A. De los Reyes, J. A. Munoz- \\
(2) Hexadecane; $\mathrm{C}_{16} \mathrm{H}_{34} ;$ & Arroyo, and F. Murrieta-Guevara, J. \\
[544-76-3] & Chem. Eng. Data 52, 2212 (2007). \\
\hline Variables: & Prepared by: \\
Temperature & W. E. Acree, Jr. \\
\hline
\end{tabular}

Experimental Values

\begin{tabular}{lcc}
\hline \hline$T / \mathrm{K}$ & $x_{2}{ }^{\mathrm{a}}$ & $x_{1}{ }^{\mathrm{b}}$ \\
\hline 291.1 & 0.9939 & 0.0061 \\
290.9 & 0.9816 & 0.0184 \\
290.8 & 0.9695 & 0.0305 \\
290.6 & 0.9573 & 0.0427 \\
290.6 & 0.9561 & 0.0439 \\
290.7 & 0.9549 & 0.0451 \\
291.0 & 0.9537 & 0.0463 \\
292.2 & 0.9525 & 0.0475 \\
293.0 & 0.9503 & 0.0497 \\
293.7 & 0.9496 & 0.0504 \\
304.4 & 0.9274 & 0.0726 \\
315.0 & 0.8992 & 0.1008 \\
319.9 & 0.8799 & 0.1201 \\
331.5 & 0.8217 & 0.1783
\end{tabular}

\begin{tabular}{lcc}
\hline \hline$T / \mathrm{K}$ & $x_{2}{ }^{\mathrm{a}}$ & $x_{1}{ }^{\mathrm{b}}$ \\
\hline 334.7 & 0.8000 & 0.2000 \\
341.5 & 0.7435 & 0.2565 \\
350.9 & 0.6263 & 0.3737 \\
356.9 & 0.4979 & 0.5021 \\
357.0 & 0.4906 & 0.5094 \\
360.1 & 0.3953 & 0.6047 \\
362.7 & 0.3046 & 0.6954 \\
\hline
\end{tabular}

${ }^{\mathrm{a}} x_{2}$ : mole fraction of component 2 in the saturated solution.

${ }^{b} x_{1}$ : mole fraction of the polycyclic aromatic hydrocarbon (component 1).

The authors report that the eutectic point occurred at $x_{1}=0.0439$ and $T / \mathrm{K}=290.6$.

\section{Auxiliary Information}

\section{Method/Apparatus/Procedure:}

Heating circulating bath and platinum resistance temperature probe.

Synthetic mixtures of known compositions were prepared by mass. Samples were slowly heated in a temperature bath, and the temperature at which the last amount of solid solute dissolved was measured. The temperature obtained was taken to represent the saturation point for the mixture being studied.

Source and Purity of Chemicals:

(1) $98.0 \%$, Aldrich Chemical Company, Milwaukee, WI, USA, was used as received.

(2) $99.0 \%$, Aldrich Chemical Company, was used as received.

Estimated Error:

Temperature: $\pm 0.5 \mathrm{~K}$.

$x_{1}: \pm 0.0005$.

\begin{tabular}{|c|c|}
\hline $\begin{array}{l}\text { Components: } \\
\text { (1) Dibenzothiophene; } \mathrm{C}_{12} \mathrm{H}_{8} \mathrm{~S} \text {; } \\
\text { [132-65-0] } \\
\text { (2) Cyclohexane; } \mathrm{C}_{6} \mathrm{H}_{12} ; \\
{[110-82-7]}\end{array}$ & $\begin{array}{l}\text { Original Measurements: } \\
{ }^{43} \text { L. F. Ramirez-Verduzco, A. Rojas- } \\
\text { Aguilar, J. A. De los Reyes, J. A. Munoz- } \\
\text { Arroyo, and F. Murrieta-Guevara, J. } \\
\text { Chem. Eng. Data 52, } 2212 \text { (2007). }\end{array}$ \\
\hline $\begin{array}{l}\text { Variables: } \\
\text { Temperature }\end{array}$ & $\begin{array}{l}\text { Prepared by: } \\
\text { W. E. Acree, Jr. }\end{array}$ \\
\hline
\end{tabular}

Experimental Values

\begin{tabular}{lcc}
\hline \hline$T / \mathrm{K}$ & $x_{2}{ }^{\mathrm{a}}$ & $x_{1}{ }^{\mathrm{b}}$ \\
\hline 309.3 & 0.9504 & 0.0496 \\
321.1 & 0.9129 & 0.0871 \\
328.8 & 0.8709 & 0.1291 \\
335.6 & 0.8011 & 0.1989 \\
349.6 & 0.5076 & 0.4924 \\
353.5 & 0.4070 & 0.5930
\end{tabular}

${ }^{a} x_{2}$ : mole fraction of component 2 in the saturated solution.

${ }^{b} x_{1}$ : mole fraction of the polycyclic aromatic hydrocarbon (component 1 ). 


\section{Auxiliary Information}

\section{Method/Apparatus/Procedure:}

Heating circulating bath and platinum resistance temperature probe. Synthetic mixtures of known compositions were prepared by mass. Samples were slowly heated in a temperature bath, and the temperature at which the last amount of solid solute dissolved was measured. The temperature obtained was taken to represent the saturation point for the mixture being studied.

\section{Source and Purity of Chemicals:}

(1) $98.0 \%$, Aldrich Chemical Company, Milwaukee, WI, USA, was used as received.

(2) $99.9 \%$, Aldrich Chemical Company, was used as received.

Estimated Error:

Temperature: $\pm 0.5 \mathrm{~K}$.

$x_{1}: \pm 0.0005$

\subsection{Dibenzothiophene solubility data in esters}

\begin{tabular}{ll}
\hline \hline Components: & Original Measurements: \\
(1) Dibenzothiophene; $\mathrm{C}_{12} \mathrm{H}_{8} \mathrm{~S} ;$ & ${ }^{43}$ L. F. Ramirez-Verduzco, A. \\
{$[132-65-0]$} & Rojas-Aguilar, J. A. De los Reyes, \\
(2) Dihydro-2( $3 \mathrm{H})$-furanone & J. A. Munoz-Arroyo, and F. \\
$\left(\gamma\right.$-butyrolactone); $\mathrm{C}_{4} \mathrm{H}_{6} \mathrm{O}_{2} ;[$ [96-48-0] & Murrieta-Guevara, J. Chem. Eng. \\
& Data 52, 2212 (2007). \\
\hline Variables: & Prepared by: \\
Temperature & W. E. Acree, Jr. \\
\hline
\end{tabular}

Experimental Values

\begin{tabular}{lcc}
\hline \hline$T / \mathrm{K}$ & $x_{2}{ }^{\mathrm{a}}$ & $x_{1}{ }^{\mathrm{b}}$ \\
\hline 281.3 & 0.9401 & 0.0599 \\
289.7 & 0.9238 & 0.0762 \\
300.1 & 0.8953 & 0.1047 \\
307.9 & 0.8652 & 0.1348 \\
314.6 & 0.8332 & 0.1668 \\
320.2 & 0.7989 & 0.2011 \\
325.2 & 0.7624 & 0.2376 \\
329.4 & 0.7257 & 0.2743 \\
337.6 & 0.6370 & 0.3630 \\
344.6 & 0.5365 & 0.4635 \\
351.5 & 0.4167 & 0.5833 \\
354.8 & 0.3497 & 0.6503 \\
362.4 & 0.1924 & 0.8076 \\
\hline${ }^{\mathrm{a}} x_{2}:$ mole fraction of component 2 in the saturated solution. \\
${ }^{\mathrm{b}}{ }_{1}:$ mole fraction of the polycyclic aromatic hydrocarbon (component 1$)$.
\end{tabular}

\section{Auxiliary Information}

\begin{abstract}
Method/Apparatus/Procedure:
Heating circulating bath and platinum resistance temperature probe. Synthetic mixtures of known compositions were prepared by mass. Samples were slowly heated in a temperature bath, and the temperature at which the last amount of solid solute dissolved was measured. The temperature obtained was taken to represent the saturation point for the mixture being studied.
\end{abstract}

Source and Purity of Chemicals:

(1) $98.0 \%$, Aldrich Chemical Company, Milwaukee, WI, USA, was used as received.

(2) $99.0 \%$, Aldrich Chemical Company, was used as received.

Estimated Error:

Temperature: $\pm 0.5 \mathrm{~K}$.

$x_{1}: \pm 0.0005$.

\subsection{Dibenzothiophene solubility data in miscellaneous organic solvents}

\begin{tabular}{|c|c|}
\hline $\begin{array}{l}\text { Components: } \\
\text { (1) Dibenzothiophene; } \mathrm{C}_{12} \mathrm{H}_{8} \mathrm{~S} \text {; } \\
\text { [132-65-0] } \\
\text { (2) Ethanenitrile; } \mathrm{C}_{2} \mathrm{H}_{3} \mathrm{~N} \text {; } \\
\text { [75-05-8] }\end{array}$ & $\begin{array}{l}\text { Original Measurements: } \\
{ }^{43} \text { L. F. Ramirez-Verduzco, A. Rojas- } \\
\text { Aguilar, J. A. De los Reyes, J. A. Munoz- } \\
\text { Arroyo, and F. Murrieta-Guevara, J. } \\
\text { Chem. Eng. Data 52, } 2212 \text { (2007). }\end{array}$ \\
\hline $\begin{array}{l}\text { Variables: } \\
\text { Temperature }\end{array}$ & $\begin{array}{l}\text { Prepared by: } \\
\text { W. E. Acree, Jr. }\end{array}$ \\
\hline
\end{tabular}

Experimental Values

\begin{tabular}{lcc}
\hline \hline$T / \mathrm{K}$ & $x_{2}{ }^{\mathrm{a}}$ & $x_{1}{ }^{\mathrm{b}}$ \\
\hline 303.7 & 0.9801 & 0.0199 \\
323.1 & 0.9478 & 0.0522 \\
332.0 & 0.8979 & 0.1021 \\
338.1 & 0.8014 & 0.1986 \\
341.8 & 0.6908 & 0.3092 \\
344.8 & 0.6044 & 0.3956 \\
347.1 & 0.5396 & 0.4604 \\
\hline
\end{tabular}

${ }^{\mathrm{a}} x_{2}$ : mole fraction of component 2 in the saturated solution.

${ }^{b} x_{1}$ : mole fraction of the polycyclic aromatic hydrocarbon (component 1 ).

\section{Auxiliary Information}

\section{Method/Apparatus/Procedure:}

Heating circulating bath and platinum resistance temperature probe.

Synthetic mixtures of known compositions were prepared by mass. Samples were slowly heated in a temperature bath, and the temperature at which the last amount of solid solute dissolved was measured. The temperature obtained was taken to represent the saturation point for the mixture being studied.

Source and Purity of Chemicals:

(1) $98.0 \%$, Aldrich Chemical Company, Milwaukee, WI, USA, was used as received.

(2) $99.9 \%$, Fermont Company, was used as received.

Estimated Error:

Temperature: $\pm 0.5 \mathrm{~K}$.

$x_{1}: \pm 0.0005$.

\section{Components:}

(1) Dibenzothiophene; $\mathrm{C}_{12} \mathrm{H}_{8} \mathrm{~S}$; [132-65-0]

(2) $\mathrm{N}, \mathrm{N}$-Dimethylacetamide; $\mathrm{C}_{4} \mathrm{H}_{9} \mathrm{NO}$; [127-19-5]

Variables:

Temperature
Original Measurements:

${ }^{35}$ Z. Lisicki and M. E. Jamróz, J. Chem. Thermodyn. 32, 1335 (2000).

Prepared by:

W. E. Acree, Jr. 
Experimental Values

\begin{tabular}{lcc}
\hline \hline$T / \mathrm{K}$ & $x_{2}{ }^{\mathrm{a}}$ & $x_{1}{ }^{\mathrm{b}}$ \\
\hline 253.2 & 1.0000 & 0.0000 \\
252.9 & 0.9892 & 0.0108 \\
252.1 & 0.9790 & 0.0210 \\
251.3 & 0.9673 & 0.0327 \\
250.6 & 0.9463 & 0.0537 \\
250.9 & 0.9250 & 0.0750 \\
256.5 & 0.9140 & 0.0860 \\
269.0 & 0.8912 & 0.1088 \\
289.7 & 0.8299 & 0.1701 \\
300.7 & 0.7848 & 0.2152 \\
318.0 & 0.6875 & 0.3175 \\
331.9 & 0.5729 & 0.4271 \\
341.4 & 0.4727 & 0.5273 \\
347.8 & 0.3979 & 0.6021 \\
355.3 & 0.2926 & 0.7074 \\
361.4 & 0.1906 & 0.8094 \\
366.9 & 0.0990 & 0.9010 \\
369.0 & 0.0498 & 0.9502 \\
371.8 & 0.0000 & 1.0000 \\
\hline
\end{tabular}

${ }^{\mathrm{a}} x_{2}$ : mole fraction of component 2 in the saturated solution.

${ }^{b} x_{1}$ : mole fraction of the polycyclic aromatic hydrocarbon (component 1 ).

The authors reported that the binary system exhibits simple eutectic behavior, with a eutectic temperature of $T / \mathrm{K}=249.6$ and eutectic composition of $x_{1}=0.073$.

\section{Auxiliary Information}

Method/Apparatus/Procedure:
Temperature-controlled heating/cooling system and a shaking-mixing system.
Synthetic mixtures of known compositions were weighed in small glass
ampoules. The mixture composition was known to within \pm 0.0002 . mole
fraction. Ampoules were cooled to very low temperatures of about $220 \mathrm{~K}$,
pumped down and sealed. The sealed mixtures were then melted, cooled
rapidly, and reheated. The rate of reheating was at an approximate rate of
$8 \times 10^{-5} \mathrm{~K} / \mathrm{s}$ just before complete dissolution. The temperatures at which the
solid completely dissolved were recorded. The average of two or three
independent measurements was taken as the solid-liquid equilibrium.
Source and Purity of Chemicals:
(1) $98 \%$, Merck-Schuchardt, Federal Republic of Germany, was recrystallized
twice from ethanol in the presence of activated carbon, and then recrystallized
from methylbenzene.
(2) Purest grade, Merck-Schuchardt, was distilled under reduced pressure and
dried over molecular sieves to produce a sample of $99.9 \%$ purity (mass
percent).
Estimated Error:
Temperature: $\pm 0.2 \mathrm{~K}$ (estimated by compiler).
$x_{1}: \pm 0.0002$.

\begin{tabular}{ll}
\hline \hline Components: & Original Measurements: \\
(1) Dibenzothiophene; $\mathrm{C}_{12} \mathrm{H}_{8} \mathrm{~S} ;[132-65-0]$ & ${ }^{35} \mathrm{Z}$. Lisicki and M. E. Jamróz, \\
(2) 1-Methyl-2-pyrrolidone; $\mathrm{C}_{5} \mathrm{H}_{9} \mathrm{NO} ;$ & J. Chem. Thermodyn. 32, \\
[872-50-4] & $1335(2000)$. \\
\hline Variables: & Prepared by: \\
Temperature & W. E. Acree, Jr. \\
\hline
\end{tabular}

Experimental Values

\begin{tabular}{lcc}
\hline \hline$T / \mathrm{K}$ & $x_{2}{ }^{\mathrm{a}}$ & $x_{1}{ }^{\mathrm{b}}$ \\
\hline 248.5 & 1.0000 & 0.0000 \\
247.3 & 0.9798 & 0.0202 \\
246.3 & 0.9648 & 0.0352 \\
245.7 & 0.9463 & 0.0537 \\
244.7 & 0.9195 & 0.0805 \\
248.1 & 0.8797 & 0.1203 \\
276.6 & 0.8390 & 0.1610 \\
284.8 & 0.7940 & 0.2060 \\
298.2 & 0.7393 & 0.2607 \\
309.2 & 0.6871 & 0.3129 \\
318.0 & 0.6389 & 0.3611 \\
325.9 & 0.5859 & 0.4141 \\
330.5 & 0.5525 & 0.4475 \\
336.6 & 0.4936 & 0.5064 \\
342.1 & 0.4444 & 0.5556 \\
347.3 & 0.3851 & 0.6149 \\
353.5 & 0.2968 & 0.7032 \\
359.3 & 0.2133 & 0.7867 \\
366.3 & 0.0977 & 0.9023 \\
371.8 & 0.0000 & 1.0000 \\
\hline
\end{tabular}

${ }^{\mathrm{a}} x_{2}$ : mole fraction of component 2 in the saturated solution.

${ }^{\mathrm{b}} x_{1}$ : mole fraction of the polycyclic aromatic hydrocarbon (component 1).

The authors reported that the binary system exhibits simple eutectic behavior, with a eutectic temperature of $T / \mathrm{K}=242.7$ and eutectic composition of $x_{1}=0.111$.

\section{Auxiliary Information}

\section{Method/Apparatus/Procedure:}

Temperature-controlled heating/cooling system and a shaking-mixing system. Synthetic mixtures of known compositions were weighed in small glass ampoules. The mixture composition was known to within \pm 0.0002 mole fraction. Ampoules were cooled to very low temperatures of about $220 \mathrm{~K}$, pumped down and sealed. The sealed mixtures were then melted, cooled rapidly, and reheated. The rate of reheating was at an approximate rate of $8 \times 10^{-5} \mathrm{~K} / \mathrm{s}$ just before complete dissolution. The temperatures at which the solid completely dissolved were recorded. The average of two or three independent measurements was taken as the solid-liquid equilibrium.

\section{Source and Purity of Chemicals:}

(1) $98 \%$, Merck-Schuchardt, Federal Republic of Germany, was recrystallized twice from ethanol in the presence of activated carbon, and then recrystallized from methylbenzene.

(2) Technical Grade, BASF, Federal Republic of Germany, was purified by treatment with a $2 \%$ solution of potassium permanganate, and then distilled under reduced pressure to collect the distillate having $99.8 \%$ purity (mass percent). The purified sample was dried over molecular sieves.

Estimated Error:

Temperature: $\pm 0.2 \mathrm{~K}$ (estimated by compiler).

$x_{1}: \pm 0.0002$.

\section{Components:}

(1) Dibenzothiophene; $\mathrm{C}_{12} \mathrm{H}_{8} \mathrm{~S}$; [132-65-0]

(2) Hexahydro-1-methyl- $2 \mathrm{H}$-azepin-2-one $(\mathrm{N}$ Methyl-e-caprolactam); $\mathrm{C}_{7} \mathrm{H}_{13} \mathrm{NO}$; [2556-73-2]
Original Measurements:

${ }^{35}$ Z. Lisicki and M. E. Jamróz, J. Chem. Thermodyn. 32, 1335 (2000).

\section{Prepared by:}

W. E. Acree, Jr. 
Experimental Values

\begin{tabular}{lcc}
\hline \hline$T / \mathrm{K}$ & $x_{2}{ }^{\mathrm{a}}$ & $x_{1}{ }^{\mathrm{b}}$ \\
\hline 279.6 & 1.0000 & 0.0000 \\
282.3 & 0.7978 & 0.2022 \\
296.7 & 0.7494 & 0.2506 \\
305.4 & 0.7023 & 0.2977 \\
313.8 & 0.6489 & 0.3511 \\
321.6 & 0.5982 & 0.4018 \\
328.4 & 0.5493 & 0.4507 \\
335.5 & 0.4948 & 0.5052 \\
345.9 & 0.3917 & 0.6083 \\
354.3 & 0.2859 & 0.7141 \\
360.4 & 0.1977 & 0.8023 \\
365.6 & 0.1091 & 0.8909 \\
371.8 & 0.0000 & 1.0000 \\
\hline
\end{tabular}

${ }^{a} x_{2}$ : mole fraction of component 2 in the saturated solution.

${ }^{b} x_{1}$ : mole fraction of the polycyclic aromatic hydrocarbon (component 1 ).

\section{Auxiliary Information}

\section{Method/Apparatus/Procedure:}

Temperature-controlled heating/cooling system and a shaking-mixing system. Synthetic mixtures of known compositions were weighed in small glass ampoules. The mixture composition was known to within \pm 0.0002 mole fraction. Ampoules were cooled to very low temperatures of about $220 \mathrm{~K}$, pumped down and sealed. The sealed mixtures were then melted, cooled rapidly, and reheated. The rate of reheating was at an approximate rate of $8 \times 10^{-5} \mathrm{~K} / \mathrm{s}$ just before complete dissolution. The temperatures at which the solid completely dissolved were recorded. The average of two or three independent measurements was taken as the solid-liquid equilibrium.

\section{Source and Purity of Chemicals:}

(1) $98 \%$, Merck-Schuchardt, Federal Republic of Germany, was recrystallized twice from ethanol in the presence of activated carbon, and then recrystallized from methylbenzene.

(2) Technical Grade, Leuna Werke, Federal Republic of Germany, was purified by super-rectification and then dried over molecular sieves to give a sample having a purity of $99.9 \%$ by mass.

\section{Estimated Error:}

Temperature: $\pm 0.2 \mathrm{~K}$ (estimated by compiler). $x_{1}: \pm 0.0002$.

\section{Solubility of Fluoranthene in Organic Solvents}

\subsection{Critical evaluation of experimental solubility data}

Vol. 59 in the IUPAC Solubility Data Series ${ }^{3}$ contained experimental solubility data for fluoranthene in one cycloalkane (cyclohexane), in one aromatic hydrocarbon (methylbenzene), in one cyclic ether (1,4-dioxane), in one chlorinated alkane (tetrachloromethane) and chlorinated aromatic hydrocarbon (chlorobenzene), in three alkanols (methanol, ethanol and 1-octanol) and one substituted phenol (1-hydroxy-2-methylbenzene), in one alkanone (propanone), and in three miscellaneous organic solvents (pyridine, nitrobenzene and dimethylsulfoxide). For each of the aforemen- tioned systems there was only a single measurement made near $298.15 \mathrm{~K}$. The volume also included phase diagram information for binary fluoranthene + benzene, fluoranthene $+1,2,4,5$ tetramethylbenzene, fluoranthene + naphthalene, fluoranthene + 2-methylnaphthalene, fluoranthene +2,7-dimethylnaphthalene, fluoranthene + fluorene, fluoranthene + anthracene, fluoranthene + phenanthrene, fluoranthene + acenaphthene, fluoranthene + chrysene, fluoranthene +1 ,4-dinitrobenzene, fluoranthene +1 ,4-dinitrobenzene, fluoranthene $+1,3,5$-trinitrobenzene, fluoranthene $+1,2,3,5$-tetranitrobenzene, fluoranthene + 2,3-nitromethylbenzene, fluoranthene + 2,4dinitrophenol, fluoranthene + 2,4,6-trinitromethylbenzene, fluoranthene $+2,4,6$-trinitromethoxybenzene, fluoranthene + 2,4,6-trinitroaniline, fluoranthene +2 -chloro-1,3,5-trinitrobenzene, and fluoranthene +3 -methyl-2,4,6-trinitrophenol mixtures. Solubility data contained in Vol. 59 will not be republished here. The listing above is provided so that readers will know what solubility data are available in the earlier volume for fluoranthene.

There have been three studies that reported solubility data for fluoranthene dissolved in organic solvents after Vol. 59 was published in 1995. Hernández and Acree ${ }^{44}$ determined the solubility of fluoranthene in 36 different organic solvents containing ether-, hydroxy-, ester-, chloro-, methyl-, and tert-butyl functional groups. Roy et $a l^{45}$ later measured fluoranthene solubilities in benzene, methylbenzene, trichloromethane, tetrachloromethane, 1,2-dichloroethane, 1-chlorohexane, 1,2-ethanediol, 2,2,2-trifluoroethanol, ethanenitrile, $\mathrm{N}, \mathrm{N}$-dimethylformamide, and $\mathrm{N}, \mathrm{N}$-dimethylformamide. The experimental measurements were performed at the single temperature of $298.15 \mathrm{~K}$ and used to test the limitations and applications of predictive expressions based on Mobile Order theory. For the majority of the solvents studied by Hernández and Acree $^{44}$ and by Roy et al. ${ }^{45}$ there is only the single experimental value, and it is not possible to perform a critical evaluation on most of the published data.

There exist independent experimental values for the solubility of fluoranthene in both methanol $\left[c_{1}=0.0704 \mathrm{~mol} \mathrm{dm}^{-3}\right.$ at $296.15 \mathrm{~K}$ (Ref. 46)] and 1-octanol $\left[c_{1}=0.1726 \mathrm{~mol} \mathrm{dm}^{-3}\right.$ at 298.15 K (Ref. 47) and $c_{1}=0.198 \mathrm{~mol} \mathrm{dm}^{-3}$ at $296.15 \mathrm{~K}$ (Ref. 46)] measured at or near $298.15 \mathrm{~K}$ in Vol. 59 of the Solubility Data Series. ${ }^{3}$ The experimental solubility data determined by Hernández and Acree, ${ }^{44}$ when converted to molar solubilities $\left(c_{1}=0.0557 \mathrm{~mol} \mathrm{dm}^{-3}\right.$ for methanol and $c_{1}$ $=0.198 \mathrm{~mol} \mathrm{dm}^{-3}$ for 1-octanol), are in reasonably good agreement with the earlier published values. McLauglin and Zainal $^{11}$ conducted fluoranthene solubility measurements in cyclohexane from 301.8 to $344.2 \mathrm{~K}$. The dataset is in Vol. 59. ${ }^{3}$ Analysis of the measured fluoranthene mole fraction solubilities using Eq. (3) gave the following mathematical correlation:

$$
\ln x_{1}=10.549-\frac{4127.4}{T}+3.544 \ln T .
$$

Equation (8) provides a reasonably accurate mathematical description of the solubility behavior of fluoranthene in cyclohexane. Differences between the experimental data and values 
back-calculated using Eq. (8) were on the order of $11 \%$ or less. Equation (8) gives an extrapolated value of $x_{1}=0.01502$ for the mole fraction solubility of fluoranthene in cyclohexane, which is less than the value of $x_{1}=0.01807$ that was reported by Hernández and Acree. ${ }^{44}$ Differences in chemical purities and experimental methodologies were the likely causes of the deviations noted in the published solubility data for fluoranthene dissolved in methanol, 1-octanol and cyclohexane noted above.

The experimental solubility data for fluoranthene in the different organic solvents are given in Secs. 8.2-8.8.

\subsection{Fluoranthene solubility data in saturated hydrocarbons (including cycloalkanes)}

\begin{tabular}{ll}
\hline \hline Components: & Original Measurements: \\
(1) Fluoranthene; $\mathrm{C}_{16} \mathrm{H}_{10} ;[206-44-0]$ & ${ }^{44} \mathrm{C}$. E. Hernández and W. E. \\
(2) Hexane; $\mathrm{C}_{6} \mathrm{H}_{14} ;[110-54-3]$ & Acree, Jr., Can. J. Chem. 76, \\
& $1312(1998)$. \\
\hline Variables: & Prepared by: \\
$T / \mathrm{K}=298.15$ & W. E. Acree, Jr. \\
\hline
\end{tabular}

Experimental Values

\begin{tabular}{lcc}
\hline \hline$x_{2}{ }^{(\mathrm{s}) \mathrm{a}}$ & $x_{2}{ }^{\mathrm{b}}$ & $x_{1}{ }^{\mathrm{c}}$ \\
\hline 1.0000 & 0.9852 & 0.01476 \\
\hline
\end{tabular}

${ }^{\mathrm{a}} x_{2}{ }^{(\mathrm{s})}$ : initial mole fraction of component 2 in the solution.

${ }^{\mathrm{b}} x_{2}$ : mole fraction of component 2 in the saturated solution.

${ }^{c} x_{1}$ : mole fraction solubility of the solute.

\section{Auxiliary Information}

\section{Method/Apparatus/Procedure:}

Constant-temperature bath, calorimetric thermometer, and an ultraviolet/ visible spectrophotometer.

Excess solute and solvent were placed in amber glass bottles and allowed to equilibrate for several days at constant temperature. Attainment of equilibrium was verified by several repetitive measurements and by approaching equilibrium from supersaturation. Aliquots of saturated solutions were transferred through a coarse filter into tared volumetric flasks, weighed and diluted with methanol. Concentrations were determined by

spectrophotometric measurements at $357 \mathrm{~nm}$.

Source and Purity of Chemicals:

(1) $98 \%$, Aldrich Chemical Company, Milwaukee, WI, USA, was recrystallized several times from methanol.

(2) $99 \%$, Aldrich Chemical Company, stored over molecular sieves before use.

Estimated Error:

Temperature: $\pm 0.1 \mathrm{~K}$.

$x_{1}: \pm 1.5 \%$ (relative error).

\begin{tabular}{ll}
\hline \hline Components: & Original Measurements: \\
(1) Fluoranthene; $\mathrm{C}_{16} \mathrm{H}_{10} ;[206-44-0]$ & ${ }^{44} \mathrm{C}$. E. Hernández and W. E. \\
(2) Heptane; $\mathrm{C}_{7} \mathrm{H}_{16} ;[142-82-5]$ & Acree, Jr., Can. J. Chem. 76, \\
& $1312(1998)$. \\
\hline Variables: & Prepared by: \\
$T / \mathrm{K}=298.15$ & W. E. Acree, Jr. \\
\hline
\end{tabular}

Experimental Values

\begin{tabular}{lcc}
\hline \hline$\frac{x_{2}{ }^{(\mathrm{s}) \mathrm{a}}}{x_{2}{ }^{\mathrm{b}}}$ & $x_{1}{ }^{\mathrm{c}}$ \\
\hline $\begin{array}{l}1.0000 \\
{ }^{\mathrm{a}} x_{2}{ }^{(\mathrm{s})}: \text { initial mole fraction of component } 2 \text { in the solution. }\end{array}$ \\
${ }^{\mathrm{b}} x_{2}:$ mole fraction of component 2 in the saturated solution. \\
${ }^{\mathrm{c}} x_{1}$ : mole fraction solubility of the solute.
\end{tabular}

Auxiliary Information

Method/Apparatus/Procedure:

Constant-temperature bath, calorimetric thermometer, and an ultraviolet/ visible spectrophotometer.

Excess solute and solvent were placed in amber glass bottles and allowed to equilibrate for several days at constant temperature. Attainment of equilibrium was verified by several repetitive measurements and by approaching equilibrium from supersaturation. Aliquots of saturated solutions were transferred through a coarse filter into tared volumetric flasks, weighed and diluted with methanol. Concentrations were determined by spectrophotometric measurements at $357 \mathrm{~nm}$.

Source and Purity of Chemicals:

(1) $98 \%$, Aldrich Chemical Company, Milwaukee, WI, USA, was recrystallized several times from methanol.

(2) HPLC Grade, Aldrich Chemical Company, stored over molecular sieves before use.

Estimated Error:

Temperature: $\pm 0.1 \mathrm{~K}$.

$x_{1}: \pm 1.5 \%$ (relative error).

\begin{tabular}{ll}
\hline \hline Components: & Original Measurements: \\
(1) Fluoranthene; $\mathrm{C}_{16} \mathrm{H}_{10} ;[206-44-0]$ & ${ }^{44} \mathrm{C}$. E. Hernández and W. E. \\
(2) Octane; $\mathrm{C}_{8} \mathrm{H}_{18} ;[111-65-9]$ & Acree, Jr., Can. J. Chem. 76, \\
& $1312(1998)$. \\
\hline Variables: & Prepared by: \\
$T / \mathrm{K}=298.15$ & W. E. Acree, Jr. \\
\hline
\end{tabular}

Experimental Values

\begin{tabular}{lcc}
\hline \hline$x_{2}{ }^{(\mathrm{s}) \mathrm{a}}$ & $x_{2}{ }^{\mathrm{b}}$ & $x_{1}{ }^{\mathrm{c}}$ \\
\hline 1.0000 & 0.9774 & 0.02260 \\
$\frac{{ }^{\mathrm{a}} x_{2}{ }^{(\mathrm{s})}: \text { initial mole fraction of component } 2 \text { in the solution. }}{{ }^{\mathrm{b}} x_{x_{2}}: \text { mole fraction of component } 2 \text { in the saturated solution. }}$ \\
${ }^{\mathrm{c}} x_{1}:$ mole fraction solubility of the solute.
\end{tabular}




\section{Auxiliary Information}

\section{Method/Apparatus/Procedure:}

Constant-temperature bath, calorimetric thermometer, and an ultraviolet/ visible spectrophotometer.

Excess solute and solvent were placed in amber glass bottles and allowed to equilibrate for several days at constant temperature. Attainment of equilibrium was verified by several repetitive measurements and by approaching equilibrium from supersaturation. Aliquots of saturated solutions were transferred through a coarse filter into tared volumetric flasks, weighed and diluted with methanol. Concentrations were determined by spectrophotometric measurements at $357 \mathrm{~nm}$.

Source and Purity of Chemicals:

(1) $98 \%$, Aldrich Chemical Company, Milwaukee, WI, USA, was recrystallized several times from methanol.

(2) $99+\%$, anhydrous, Aldrich Chemical Company, stored over molecular sieves before use.

Estimated Error:

Temperature: $\pm 0.1 \mathrm{~K}$

$x_{1}: \pm 1.5 \%$ (relative error).

\begin{tabular}{|c|c|}
\hline $\begin{array}{l}\text { Components: } \\
\text { (1) Fluoranthene; } \mathrm{C}_{16} \mathrm{H}_{10} ;[206-44-0] \\
\text { (2) Nonane; } \mathrm{C}_{9} \mathrm{H}_{20} ;[111-84-2]\end{array}$ & $\begin{array}{l}\text { Original Measurements: } \\
{ }^{44} \text { C. E. Hernández and W. E. } \\
\text { Acree, Jr., Can. J. Chem. 76, } \\
1312 \text { (1998). }\end{array}$ \\
\hline $\begin{array}{l}\text { Variables: } \\
T / \mathrm{K}=298.15\end{array}$ & $\begin{array}{l}\text { Prepared by: } \\
\text { W. E. Acree, Jr. }\end{array}$ \\
\hline
\end{tabular}

Experimental Values

\begin{tabular}{lcc}
\hline \hline$x_{2}{ }^{(\mathrm{s}) \mathrm{a}}$ & $x_{2}{ }^{\mathrm{b}}$ & $x_{1}{ }^{\mathrm{c}}$ \\
\hline 1.0000 & 0.9736 & 0.02642 \\
\hline
\end{tabular}

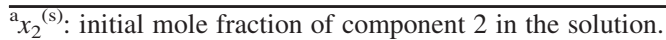

${ }^{b} x_{2}$ : mole fraction of component 2 in the saturated solution.

${ }^{c} x_{1}$ : mole fraction solubility of the solute.

\section{Auxiliary Information}

\section{Method/Apparatus/Procedure:}

Constant-temperature bath, calorimetric thermometer, and an ultraviolet/ visible spectrophotometer.

Excess solute and solvent were placed in amber glass bottles and allowed to equilibrate for several days at constant temperature. Attainment of equilibrium was verified by several repetitive measurements and by approaching equilibrium from supersaturation. Aliquots of saturated solutions were transferred through a coarse filter into tared volumetric flasks, weighed and diluted with methanol. Concentrations were determined by

spectrophotometric measurements at $357 \mathrm{~nm}$.

Source and Purity of Chemicals:

(1) $98 \%$, Aldrich Chemical Company, Milwaukee, WI, USA, was recrystallized several times from methanol.

(2) $99+\%$, TCI America, Portland, OR, USA, stored over molecular sieves before use.

Estimated Error:

Temperature: $\pm 0.1 \mathrm{~K}$.

$x_{1}: \pm 1.5 \%$ (relative error).

\begin{tabular}{ll}
\hline \hline Components: & Original Measurements: \\
(1) Fluoranthene; $\mathrm{C}_{16} \mathrm{H}_{10} ;[206-44-0]$ & ${ }^{44} \mathrm{C}$. E. Hernández and W. E. \\
(2) Decane; $\mathrm{C}_{10} \mathrm{H}_{22} ;[124-18-5]$ & Acree, Jr., Can. J. Chem. 76, \\
& $1312(1998)$. \\
\hline Variables: & Prepared by: \\
$T / \mathrm{K}=298.15$ & W. E. Acree, Jr. \\
\hline
\end{tabular}

Experimental Values

\begin{tabular}{|c|c|c|}
\hline$x_{2}{ }^{(\mathrm{s}) a}$ & $x_{2}{ }^{b}$ & $x_{1}{ }^{c}$ \\
\hline 1.0000 & 0.9698 & 0.03015 \\
\hline
\end{tabular}

\section{Auxiliary Information}

\section{Method/Apparatus/Procedure:}

Constant-temperature bath, calorimetric thermometer, and an ultraviolet/ visible spectrophotometer.

Excess solute and solvent were placed in amber glass bottles and allowed to equilibrate for several days at constant temperature. Attainment of equilibrium was verified by several repetitive measurements and by approaching equilibrium from supersaturation. Aliquots of saturated solutions were transferred through a coarse filter into tared volumetric flasks, weighed and diluted with methanol. Concentrations were determined by spectrophotometric measurements at $357 \mathrm{~nm}$.

Source and Purity of Chemicals:

(1) $98 \%$, Aldrich Chemical Company, Milwaukee, WI, USA, was recrystallized several times from methanol.

(2) $99+\%$, TCI America, Portland, OR, USA, stored over molecular sieves before use.

\section{Estimated Error:}

Temperature: $\pm 0.1 \mathrm{~K}$.

$x_{1}: \pm 1.5 \%$ (relative error)

\begin{tabular}{ll}
\hline \hline Components: & Original Measurements: \\
(1) Fluoranthene; $\mathrm{C}_{16} \mathrm{H}_{10} ;[206-44-0]$ & ${ }^{44} \mathrm{C}$. E. Hernández and W. E. \\
(2) Hexadecane; $\mathrm{C}_{16} \mathrm{H}_{34} ;[544-76-3]$ & Acree, Jr., Can. J. Chem. 76, \\
& 1312 (1998). \\
\hline Variables: & Prepared by: \\
$T / \mathrm{K}=298.15$ & W. E. Acree, Jr. \\
\hline
\end{tabular}

Experimental Values

\begin{tabular}{lcc}
\hline \hline$x_{2}{ }^{(\mathrm{s}) \mathrm{a}}$ & $x_{2}{ }^{\mathrm{b}}$ & $x_{1}{ }^{\mathrm{c}}$ \\
\hline 1.0000 & 0.9495 & 0.05046 \\
$\frac{{ }^{\mathrm{a}} x_{2}{ }^{(\mathrm{s})}: \text { initial mole fraction of component } 2 \text { in the solution. }}{{ }^{\mathrm{b}} x_{2}: \text { mole fraction of component } 2 \text { in the saturated solution. }}$ \\
${ }^{\mathrm{c}} x_{1}$ : mole fraction solubility of the solute.
\end{tabular}




\section{Auxiliary Information}

\section{Method/Apparatus/Procedure:}

Constant-temperature bath, calorimetric thermometer, and an ultraviolet/ visible spectrophotometer.

Excess solute and solvent were placed in amber glass bottles and allowed to equilibrate for several days at constant temperature. Attainment of equilibrium was verified by several repetitive measurements and by approaching equilibrium from supersaturation. Aliquots of saturated solutions were transferred through a coarse filter into tared volumetric flasks, weighed and diluted with methanol. Concentrations were determined by

spectrophotometric measurements at $357 \mathrm{~nm}$.

Source and Purity of Chemicals:

(1) $98 \%$, Aldrich Chemical Company, Milwaukee, WI, USA, was recrystallized several times from methanol.

(2) $99 \%$, Aldrich Chemical Company, stored over molecular sieves before use.

Estimated Error:

Temperature: $\pm 0.1 \mathrm{~K}$.

$x_{1}: \pm 1.5 \%$ (relative error)

\begin{tabular}{ll}
\hline \hline Components: & Original Measurements: \\
(1) Fluoranthene; $\mathrm{C}_{16} \mathrm{H}_{10} ;[206-44-0]$ & ${ }^{15}$ A. Aoulmi, M. Bouroukba, \\
(2) Octacosane; $\mathrm{C}_{28} \mathrm{H}_{58} ;[630-02-4]$ & R. Solimando, and M. Rogalski, \\
& Fluid Phase Equilib. 110, 283 \\
& $(1995)$. \\
\hline Variables: & Prepared by: \\
Temperature & W. E. Acree, Jr. \\
\hline
\end{tabular}

Experimental Values

\begin{tabular}{lcc}
\hline \hline$T / \mathrm{K}$ & $x_{2}{ }^{\mathrm{a}}$ & $x_{1}{ }^{\mathrm{b}}$ \\
\hline 333.91 & 1.0000 & 0.0000 \\
333.75 & 0.8993 & 0.1007 \\
333.65 & 0.7978 & 0.2022 \\
333.21 & 0.7001 & 0.2999 \\
349.40 & 0.5979 & 0.4021 \\
362.55 & 0.5001 & 0.4999 \\
370.34 & 0.3976 & 0.6024 \\
372.26 & 0.3350 & 0.6650 \\
373.09 & 0.3001 & 0.6999 \\
377.28 & 0.2004 & 0.7996 \\
379.29 & 0.1002 & 0.8998 \\
382.35 & 0.0000 & 1.0000 \\
\hline
\end{tabular}

${ }^{\mathrm{a}} x_{2}$ : mole fraction of component 2 in the saturated solution.

${ }^{\mathrm{b}} x_{1}$ : mole fraction of the polycyclic aromatic hydrocarbon (component 1 ).

The authors employed a SSF to describe the activity coefficients of fluoranthene and octacosane calculated from the solid-liquid equilibrium data. The SSF model gave a calculated eutectic temperature of $T / \mathrm{K}=329.7$ and eutectic mole fraction of fluoranthene of $x_{1}=0.270$.

\section{Auxiliary Information}

\section{Method/Apparatus/Procedure:}

Differential scanning calorimeter.

The phase diagram was determined using a differential scanning calorimeter Measurements were performed at a constant fixed scanning rate of $0.5 \mathrm{~K} / \mathrm{min}$.

Source and Purity of Chemicals:

(1) $98 \%$, Aldrich Chemical Company, Milwaukee, WI, USA, no purification details were given in the paper.

(2) $99 \%$, Aldrich Chemical Company, purification details were not given in the paper.

\section{Estimated Error:}

Temperature: Authors state a global accuracy of $\pm 1 \%$ regarding their measurements.

$x_{1}: \pm 0.0002$ (estimated by compiler).

\begin{tabular}{ll}
\hline \hline Components: & Original Measurements: \\
(1) Fluoranthene; $\mathrm{C}_{16} \mathrm{H}_{10} ;[206-44-0]$ & ${ }^{44} \mathrm{C}$. E. Hernández and W. E. \\
(2) Cyclohexane; $\mathrm{C}_{6} \mathrm{H}_{12} ;[110-82-7]$ & Acree, Jr., Can. J. Chem. 76, \\
& $1312(1998)$. \\
\hline Variables: & Prepared by: \\
$T / \mathrm{K}=298.15$ & W. E. Acree, Jr. \\
\hline
\end{tabular}

Experimental Values

\begin{tabular}{lcc}
\hline \hline$x_{2}^{(\mathrm{s}) \mathrm{a}}$ & $x_{2}{ }^{\mathrm{b}}$ & $x_{1}{ }^{\mathrm{c}}$ \\
\hline 1.0000 & 0.9819 & 0.01807 \\
\hline
\end{tabular}

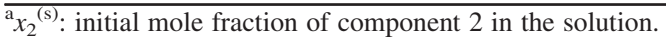

${ }^{\mathrm{b}} x_{2}$ : mole fraction of component 2 in the saturated solution.

${ }^{\mathrm{c}} x_{1}$ : mole fraction solubility of the solute.

\section{Auxiliary Information}

\section{Method/Apparatus/Procedure:}

Constant-temperature bath, calorimetric thermometer, and an ultraviolet/ visible spectrophotometer.

Excess solute and solvent were placed in amber glass bottles and allowed to equilibrate for several days at constant temperature. Attainment of equilibrium was verified by several repetitive measurements and by approaching equilibrium from supersaturation. Aliquots of saturated solutions were transferred through a coarse filter into tared volumetric flasks, weighed and diluted with methanol. Concentrations were determined by spectrophotometric measurements at $357 \mathrm{~nm}$.

Source and Purity of Chemicals:

(1) $98 \%$, Aldrich Chemical Company, Milwaukee, WI, USA, was recrystallized several times from methanol.

(2) HPLC Grade, Aldrich Chemical Company, stored over molecular sieves before use.

Estimated Error:

Temperature: $\pm 0.1 \mathrm{~K}$.

$x_{1}: \pm 1.5 \%$ (relative error)

\section{Components:}

(1) Fluoranthene; $\mathrm{C}_{16} \mathrm{H}_{10}$; [206-44-0]

(2) Methylcyclohexane; $\mathrm{C}_{7} \mathrm{H}_{14} ;$; $\left.108-87-2\right]$

Variables:

$T / \mathrm{K}=298.15$
Original Measurements:

${ }^{44}$ C. E. Hernández and W. E. Acree, Jr., Can. J. Chem. 76, 1312 (1998).

Prepared by:

W. E. Acree, Jr. 
Experimental Values

\begin{tabular}{lcc}
\hline$x_{2}{ }^{(\mathrm{s}) \mathrm{a}}$ & $x_{2}{ }^{\mathrm{b}}$ & $x_{1}{ }^{\mathrm{c}}$ \\
\hline 1.0000 & 0.9782 & 0.02179 \\
$\frac{{ }^{\mathrm{a}} x_{2}{ }^{(\mathrm{s})}: \text { initial mole fraction of component } 2 \text { in the solution. }}{{ }^{\mathrm{b}}{ }_{x_{2}}: \text { mole fraction of component } 2 \text { in the saturated solution. }}$ \\
${ }^{\mathrm{c}} x_{1}:$ mole fraction solubility of the solute.
\end{tabular}

\section{Auxiliary Information}

\section{Method/Apparatus/Procedure:}

Constant-temperature bath, calorimetric thermometer, and an ultraviolet/ visible spectrophotometer.

Excess solute and solvent were placed in amber glass bottles and allowed to equilibrate for several days at constant temperature. Attainment of equilibrium was verified by several repetitive measurements and by approaching equilibrium from supersaturation. Aliquots of saturated solutions were transferred through a coarse filter into tared volumetric flasks, weighed and diluted with methanol. Concentrations were determined by spectrophotometric measurements at $357 \mathrm{~nm}$

\section{Source and Purity of Chemicals:}

(1) $98 \%$, Aldrich Chemical Company, Milwaukee, WI, USA, was recrystallized several times from methanol.

(2) $99+\%$, anhydrous, Aldrich Chemical Company, stored over molecular sieves before use.

Estimated Error:

Temperature: $\pm 0.1 \mathrm{~K}$

$x_{1}: \pm 1.5 \%$ (relative error).

\section{Components: \\ (1) Fluoranthene; $\mathrm{C}_{16} \mathrm{H}_{10} ;[206-44-0]$ \\ (2) Cyclooctane; $\mathrm{C}_{8} \mathrm{H}_{16} ;[292-64-8]$}

\section{Variables:}

$T / \mathrm{K}=298.15$
Original Measurements:

${ }^{44}$ C. E. Hernández and W. E. Acree, Jr., Can. J. Chem. 76, 1312 (1998).

Prepared by: W. E. Acree, Jr.

\section{Experimental Values}

\begin{tabular}{lcc}
\hline \hline$x_{2}{ }^{(\mathrm{s}) \mathrm{a}}$ & $x_{2}{ }^{\mathrm{b}}$ & $x_{1}{ }^{\mathrm{c}}$ \\
\hline 1.0000 & 0.9699 & 0.03011
\end{tabular}

${ }^{\mathrm{a}} x_{2}{ }^{(\mathrm{s})}$ : initial mole fraction of component 2 in the solution.

${ }^{\mathrm{b}} x_{2}$ : mole fraction of component 2 in the saturated solution.

${ }^{c} x_{1}$ : mole fraction solubility of the solute.

\section{Auxiliary Information}

\section{Method/Apparatus/Procedure:}

Constant-temperature bath, calorimetric thermometer, and an ultraviolet/ visible spectrophotometer.

Excess solute and solvent were placed in amber glass bottles and allowed to equilibrate for several days at constant temperature. Attainment of equilibrium was verified by several repetitive measurements and by approaching equilibrium from supersaturation. Aliquots of saturated solutions were transferred through a coarse filter into tared volumetric flasks, weighed and diluted with methanol. Concentrations were determined by spectrophotometric measurements at $357 \mathrm{~nm}$.
Source and Purity of Chemicals:

(1) $98 \%$, Aldrich Chemical Company, Milwaukee, WI, USA, was recrystallized several times from methanol.

(2) $99+\%$, Aldrich Chemical Company, stored over molecular sieves before use.

\section{Estimated Error:}

Temperature: $\pm 0.1 \mathrm{~K}$.

$x_{1}: \pm 1.5 \%$ (relative error).

\begin{tabular}{ll}
\hline \hline Components: & Original Measurements: \\
(1) Fluoranthene; $\mathrm{C}_{16} \mathrm{H}_{10} ;[206-44-0]$ & ${ }^{44} \mathrm{C}$. E. Hernández and W. E. \\
$(2)$ 2,2,4-Trimethylpentane; $\mathrm{C}_{8} \mathrm{H}_{18} ;$ & Acree, Jr., Can. J. Chem. 76, \\
{$[540-84-1]$} & $1312(1998)$. \\
\hline Variables: & Prepared by: \\
$T / \mathrm{K}=298.15$ & W. E. Acree, Jr. \\
\hline
\end{tabular}

\section{Experimental Values}

\begin{tabular}{lcc}
\hline \hline$x_{2}{ }^{(\mathrm{s}) \mathrm{a}}$ & $x_{2}{ }^{\mathrm{b}}$ & $x_{1}{ }^{\mathrm{c}}$ \\
\hline 1.0000 & 0.9884 & 0.01162 \\
\hline${ }^{\mathrm{a}} x_{2}{ }^{(\mathrm{s})}:$ initial mole fraction of component 2 in the solution. \\
${ }^{\mathrm{b}} x_{2}:$ mole fraction of component 2 in the saturated solution. \\
${ }^{\mathrm{c}}{ }_{x_{1}}:$ mole fraction solubility of the solute.
\end{tabular}

\section{Auxiliary Information}

\section{Method/Apparatus/Procedure:}

Constant-temperature bath, calorimetric thermometer, and an ultraviolet/ visible spectrophotometer.

Excess solute and solvent were placed in amber glass bottles and allowed to equilibrate for several days at constant temperature. Attainment of equilibrium was verified by several repetitive measurements and by approaching equilibrium from supersaturation. Aliquots of saturated solutions were transferred through a coarse filter into tared volumetric flasks, weighed and diluted with methanol. Concentrations were determined by spectrophotometric measurements at $357 \mathrm{~nm}$.

\section{Source and Purity of Chemicals:}

(1) $98 \%$, Aldrich Chemical Company, Milwaukee, WI, USA, was recrystallized several times from methanol.

(2) HPLC Grade, Aldrich Chemical Company, stored over molecular sieves before use.

\section{Estimated Error:}

Temperature: $\pm 0.1 \mathrm{~K}$.

$x_{1}: \pm 1.5 \%$ (relative error).

\begin{tabular}{ll}
\hline \hline Components: & Original Measurements: \\
(1) Fluoranthene; $\mathrm{C}_{16} \mathrm{H}_{10} ;[206-44-0]$ & ${ }^{44} \mathrm{C}$. E. Hernández and W. E. \\
(2) tert-Butylcyclohexane; $\mathrm{C}_{10} \mathrm{H}_{20} ;$ & Acree, Jr., Can. J. Chem. 76, \\
{$[3178-22-1]$} & $1312(1998)$. \\
\hline Variables: & Prepared by: \\
$T / \mathrm{K}=298.15$ & W. E. Acree, Jr. \\
\hline
\end{tabular}


Experimental Values

\begin{tabular}{lcc}
\hline$x_{2}{ }^{(\mathrm{s}) \mathrm{a}}$ & $x_{2}{ }^{\mathrm{b}}$ & $x_{1}{ }^{\mathrm{c}}$ \\
\hline 1.0000 & 0.9752 & 0.02482 \\
$\frac{{ }^{\mathrm{a}} x_{2}{ }^{(\mathrm{s})}: \text { initial mole fraction of component } 2 \text { in the solution. }}{{ }^{\mathrm{b}}{ }_{x_{2}}: \text { mole fraction of component } 2 \text { in the saturated solution. }}$ \\
${ }^{{ }^{c} x_{1}: \text { mole fraction solubility of the solute. }}$
\end{tabular}

\section{Auxiliary Information}

\section{Method/Apparatus/Procedure:}

Constant-temperature bath, calorimetric thermometer, and an ultraviolet/ visible spectrophotometer.

Excess solute and solvent were placed in amber glass bottles and allowed to equilibrate for several days at constant temperature. Attainment of equilibrium was verified by several repetitive measurements and by approaching equilibrium from supersaturation. Aliquots of saturated solutions were transferred through a coarse filter into tared volumetric flasks, weighed and diluted with methanol. Concentrations were determined by spectrophotometric measurements at $357 \mathrm{~nm}$.

Source and Purity of Chemicals:

(1) $98 \%$, Aldrich Chemical Company, Milwaukee, WI, USA, was recrystallized several times from methanol.

(2) $99+\%$, Aldrich Chemical Company, stored over molecular sieves before use.

Estimated Error:

Temperature: $\pm 0.1 \mathrm{~K}$.

$x_{1}: \pm 1.5 \%$ (relative error).

\begin{tabular}{ll}
\hline \hline Components: & Original Measurements: \\
(1) Fluoranthene; $\mathrm{C}_{16} \mathrm{H}_{10} ;[206-44-0]$ & ${ }^{15}$ A. Aoulmi, M. Bouroukba, \\
(2) Squalane; $\mathrm{C}_{30} \mathrm{H}_{62} ;[111-01-3]$ & R. Solimando, and M. Rogalski, \\
& $\begin{array}{l}\text { Fluid Phase Equilib. 110, 283 } \\
\text { (1995). }\end{array}$ \\
\hline Variables: & Prepared by: \\
Temperature & W. E. Acree, Jr. \\
\hline
\end{tabular}

Experimental Values

\begin{tabular}{lcc}
\hline \hline$T / \mathrm{K}$ & $x_{2}{ }^{\mathrm{a}}$ & $x_{1}{ }^{\mathrm{b}}$ \\
\hline 339.75 & 0.7944 & 0.2056 \\
354.45 & 0.6928 & 0.3072 \\
362.85 & 0.5987 & 0.4013 \\
369.05 & 0.4970 & 0.5030 \\
372.95 & 0.4000 & 0.6000 \\
374.65 & 0.3505 & 0.6495 \\
375.75 & 0.2998 & 0.7002 \\
377.60 & 0.2008 & 0.7992 \\
378.35 & 0.1502 & 0.8498 \\
378.95 & 0.1019 & 0.8981 \\
380.05 & 0.0510 & 0.9490 \\
\hline
\end{tabular}

${ }^{\mathrm{a}} x_{2}$ : mole fraction of component 2 in the saturated solution.

${ }^{b} x_{1}$ : mole fraction of the polycyclic aromatic hydrocarbon (component 1).
The authors employed a SSF to describe the activity coefficients of fluoranthene and squalane calculated from the solidliquid equilibrium data. The SSF model gave a calculated eutectic temperature of $T / \mathrm{K}=234.1$ and eutectic mole fraction of fluoranthene of $x_{1}=0.006$.

\section{Auxiliary Information}

\section{Method/Apparatus/Procedure:}

Simple thermal analysis device constructed in the authors' laboratory, and a high precision platinum-resistance thermometer.

The phase diagram was determined using a simple thermal device. A sample of known composition was placed in a thermostatted glass tube, melted and then slowly cooled at a rate of about $1 \mathrm{~K} / \mathrm{min}$. The temperature of the sample was measured with a high precision platinum-resistance thermometer. The crystallization temperature was obtained from a plot of the cooling curve versus time. Each measurement was repeated three times.

Source and Purity of Chemicals:

(1) $98 \%$, Aldrich Chemical Company, Milwaukee, WI, USA, no purification details were given in the paper.

(2) $99 \%$, Aldrich Chemical Company, purification details were not given in the paper.

\section{Estimated Error:}

Temperature: Authors state a global accuracy of $\pm 1 \%$ regarding their measurements.

$x_{1}: \pm 0.0002$ (estimated by compiler).

\subsection{Fluoranthene solubility data in aromatic hydrocarbons}

\begin{tabular}{ll}
\hline \hline Components: & Original Measurements: \\
(1) Fluoranthene; $\mathrm{C}_{16} \mathrm{H}_{10} ;[206-44-0]$ & ${ }^{45}$ L. E. Roy, C. E. Hernández, and \\
(2) Benzene; $\mathrm{C}_{6} \mathrm{H}_{6} ;[71-43-2]$ & W. E. Acree, Jr., Polycyclic \\
& Aromat. Compd. 13, 205 (1999). \\
\hline Variables: & Prepared by: \\
$T / \mathrm{K}=298.15$ & W. E. Acree, Jr. \\
\hline
\end{tabular}

\section{Experimental Values}

\begin{tabular}{lcc}
\hline \hline$x_{2}{ }^{(\mathrm{s}) \mathrm{a}}$ & $x_{2}{ }^{\mathrm{b}}$ & $x_{1}{ }^{\mathrm{c}}$ \\
\hline 1.0000 & 0.8789 & 0.1211 \\
$\frac{{ }^{\mathrm{a}} x_{2}{ }^{(\mathrm{s})}: \text { initial mole fraction of component } 2 \text { in the solution. }}{{ }^{\mathrm{b}} x_{2}: \text { mole fraction of component } 2 \text { in the saturated solution. }}$ \\
${ }^{\mathrm{c}} x_{1}:$ mole fraction solubility of the solute.
\end{tabular}




\section{Auxiliary Information}

\section{Method/Apparatus/Procedure:}

Constant-temperature bath, calorimetric thermometer, and an ultraviolet/ visible spectrophotometer.

Excess solute and solvent were placed in amber glass bottles and allowed to equilibrate for several days at constant temperature. Attainment of equilibrium was verified by several repetitive measurements and by approaching equilibrium from supersaturation. Aliquots of saturated solutions were transferred through a coarse filter into tared volumetric flasks, weighed and diluted with methanol. Concentrations were determined by spectrophotometric measurements at $357 \mathrm{~nm}$.

Source and Purity of Chemicals:

(1) $98 \%$, Aldrich Chemical Company, Milwaukee, WI, USA, was recrystallized several times from methanol.

(2) $99.9+\%$, HPLC Grade, Aldrich Chemical Company, stored over molecular sieves and distilled shortly before use.

Estimated Error:

Temperature: $\pm 0.1 \mathrm{~K}$.

$x_{1}: \pm 1.5 \%$ (relative error).

\begin{tabular}{ll}
\hline \hline Components: & Original Measurements: \\
(1) Fluoranthene; $\mathrm{C}_{16} \mathrm{H}_{10} ;[206-44-0]$ & ${ }^{45}$ L. E. Roy, C. E. Hernández, and \\
(2) Methylbenzene; $\mathrm{C}_{7} \mathrm{H}_{8} ;[108-88-8]$ & W. E. Acree, Jr., Polycyclic \\
& Aromat. Compd. 13, 205 (1999). \\
\hline Variables: & Prepared by: \\
$T / \mathrm{K}=298.15$ & W. E. Acree, Jr. \\
\hline
\end{tabular}

Experimental Values

\begin{tabular}{lcc}
\hline \hline$x_{2}{ }^{(\mathrm{s}) \mathrm{a}}$ & $x_{2}{ }^{\mathrm{b}}$ & $x_{1}{ }^{\mathrm{c}}$ \\
\hline 1.0000 & 0.8840 & 0.1160 \\
${ }^{\mathrm{a}} x_{2}{ }^{(\mathrm{s})}:$ initial mole fraction of component 2 in the solution. \\
${ }^{\mathrm{b}} x_{2}:$ mole fraction of component 2 in the saturated solution. \\
${ }^{\mathrm{c}} x_{1}:$ mole fraction solubility of the solute.
\end{tabular}

\section{Auxiliary Information}

\section{Method/Apparatus/Procedure:}

Constant-temperature bath, calorimetric thermometer, and an ultraviolet/ visible spectrophotometer.

Excess solute and solvent were placed in amber glass bottles and allowed to equilibrate for several days at constant temperature. Attainment of equilibrium was verified by several repetitive measurements and by approaching equilibrium from supersaturation. Aliquots of saturated solutions were transferred through a coarse filter into tared volumetric flasks, weighed and diluted with methanol. Concentrations were determined by

spectrophotometric measurements at $357 \mathrm{~nm}$.

Source and Purity of Chemicals:

(1) $98 \%$, Aldrich Chemical Company, Milwaukee, WI, USA, was recrystallized several times from methanol.

(2) $99.8 \%$, Aldrich Chemical Company, stored over molecular sieves and distilled shortly before use.

Estimated Error:

Temperature: $\pm 0.1 \mathrm{~K}$.

$x_{1}: \pm 1.5 \%$ (relative error)

\subsection{Fluoranthene solubility data in esters}

\begin{tabular}{ll}
\hline \hline Components: & Original Measurements: \\
(1) Fluoranthene; $\mathrm{C}_{16} \mathrm{H}_{10} ;[206-44-0]$ & ${ }^{44} \mathrm{C}$. E. Hernández and W. E. \\
(2) Ethyl ethanoate; $\mathrm{C}_{4} \mathrm{H}_{8} \mathrm{O}_{2} ;[141-78-6]$ & Acree, Jr., Can. J. Chem. 76, \\
& 1312 (1998). \\
\hline Variables: & Prepared by: \\
$T / \mathrm{K}=298.15$ & W. E. Acree, Jr. \\
\hline
\end{tabular}

Experimental Values

\begin{tabular}{|c|c|c|}
\hline$x_{2}{ }^{(\mathrm{s}) \mathrm{a}}$ & $x_{2}{ }^{b}$ & $x_{1}{ }^{\mathrm{c}}$ \\
\hline 1.0000 & 0.9141 & 0.08589 \\
\hline
\end{tabular}

\section{Auxiliary Information}

\section{Method/Apparatus/Procedure:}

Constant-temperature bath, calorimetric thermometer, and an ultraviolet/ visible spectrophotometer.

Excess solute and solvent were placed in amber glass bottles and allowed to equilibrate for several days at constant temperature. Attainment of equilibrium was verified by several repetitive measurements and by approaching equilibrium from supersaturation. Aliquots of saturated solutions were transferred through a coarse filter into tared volumetric flasks, weighed and diluted with methanol. Concentrations were determined by spectrophotometric measurements at $357 \mathrm{~nm}$.

\section{Source and Purity of Chemicals:}

(1) $98 \%$, Aldrich Chemical Company, Milwaukee, WI, USA, was recrystallized several times from methanol.

(2) $99.9 \%$, HPLC Grade, Aldrich Chemical Company, stored over molecular sieves before use.

\section{Estimated Error:}

Temperature: $\pm 0.1 \mathrm{~K}$.

$x_{1}: \pm 1.5 \%$ (relative error).

\begin{tabular}{ll}
\hline \hline Components: & Original Measurements: \\
(1) Fluoranthene; $\mathrm{C}_{16} \mathrm{H}_{10} ;[206-44-0]$ & ${ }^{44} \mathrm{C}$. E. Hernández and W. E. \\
(2) Butyl ethanoate; $\mathrm{C}_{6} \mathrm{H}_{12} \mathrm{O}_{2} ;[123-86-4]$ & Acree, Jr., Can. J. Chem. 76, \\
& $1312(1998)$. \\
\hline Variables: & Prepared by: \\
$T / \mathrm{K}=298.15$ & W. E. Acree, Jr. \\
\hline
\end{tabular}

Experimental Values

\begin{tabular}{|c|c|c|}
\hline$x_{2}{ }^{(\mathrm{s}) \mathrm{a}}$ & $x_{2}{ }^{b}$ & $x_{1}{ }^{c}$ \\
\hline 1.0000 & 0.8894 & 0.1106 \\
\hline
\end{tabular}




\section{Auxiliary Information}

Method/Apparatus/Procedure:

Constant-temperature bath, calorimetric thermometer, and an ultraviolet/ visible spectrophotometer.

Excess solute and solvent were placed in amber glass bottles and allowed to equilibrate for several days at constant temperature. Attainment of equilibrium was verified by several repetitive measurements and by approaching equilibrium from supersaturation. Aliquots of saturated solutions were transferred through a coarse filter into tared volumetric flasks, weighed and diluted with methanol. Concentrations were determined by spectrophotometric measurements at $357 \mathrm{~nm}$.

Source and Purity of Chemicals:

(1) $98 \%$, Aldrich Chemical Company, Milwaukee, WI, USA, was recrystallized several times from methanol.

(2) $99 \%$, Aldrich Chemical Company, stored over molecular sieves before use.

Estimated Error:

Temperature: $\pm 0.1 \mathrm{~K}$.

$x_{1}: \pm 1.5 \%$ (relative error).

\subsection{Fluoranthene solubility data in ethers}

\section{Components:}

(1) Fluoranthene; $\mathrm{C}_{16} \mathrm{H}_{10} ;[206-44-0]$

(2) 1,1'-Oxybisbutane; $\mathrm{C}_{8} \mathrm{H}_{18} \mathrm{O}$; [142-96-1]

\begin{tabular}{ll}
\hline Variables: & Prepared by: \\
$T / \mathrm{K}=298.15$ & W. E. Acree, Jr. \\
\hline
\end{tabular}

Experimental Values

\begin{tabular}{lcc}
\hline \hline$x_{2}{ }^{(\mathrm{s}) \mathrm{a}}$ & $x_{2}^{\mathrm{b}}$ & $x_{1}{ }^{\mathrm{c}}$ \\
\hline 1.0000 & 0.9482 & 0.05177 \\
\hline
\end{tabular}

${ }^{\mathrm{a}} x_{2}{ }^{(\mathrm{s})}$ : initial mole fraction of component 2 in the solution.

${ }^{\mathrm{b}} x_{2}$ : mole fraction of component 2 in the saturated solution.

${ }^{c} x_{1}$ : mole fraction solubility of the solute.

\section{Auxiliary Information}

\section{Method/Apparatus/Procedure:}

Constant-temperature bath, calorimetric thermometer, and an ultraviolet/ visible spectrophotometer.

Excess solute and solvent were placed in amber glass bottles and allowed to equilibrate for several days at constant temperature. Attainment of equilibrium was verified by several repetitive measurements and by approaching equilibrium from supersaturation. Aliquots of saturated solutions were transferred through a coarse filter into tared volumetric flasks, weighed and diluted with methanol. Concentrations were determined by spectrophotometric measurements at $357 \mathrm{~nm}$.

Source and Purity of Chemicals:

(1) $98 \%$, Aldrich Chemical Company, Milwaukee, WI, USA, was recrystallized several times from methanol.

(2) $99 \%$, Aldrich Chemical Company, stored over molecular sieves before use.

Estimated Error:

Temperature: $\pm 0.1 \mathrm{~K}$.

$x_{1}: \pm 1.5 \%$ (relative error).

\begin{tabular}{ll}
\hline \hline Components: & Original Measurements: \\
(1) Fluoranthene; $\mathrm{C}_{16} \mathrm{H}_{10} ;[206-44-0]$ & ${ }^{44} \mathrm{C}$. E. Hernández and W. E. \\
(2) 2-Methoxy-2-methylpropane; $\mathrm{C}_{5} \mathrm{H}_{12} \mathrm{O} ;$ & Acree, Jr., Can. J. Chem. 76, \\
{$[1634-04-4]$} & $1312(1998)$. \\
\hline Variables: & Prepared by: \\
$T / \mathrm{K}=298.15$ & W. E. Acree, Jr. \\
\hline
\end{tabular}

Experimental Values

\begin{tabular}{lcc}
\hline \hline$x_{2}{ }^{(\mathrm{s}) \mathrm{a}}$ & $x_{2}{ }^{\mathrm{b}}$ & $x_{1}{ }^{\mathrm{c}}$ \\
\hline 1.0000 & 0.9524 & 0.04755 \\
$\frac{{ }^{\mathrm{a}} x_{2}{ }^{(\mathrm{s})}: \text { initial mole fraction of component 2 in the solution. }}{{ }^{\mathrm{b}} x_{2}: \text { mole fraction of component } 2 \text { in the saturated solution. }}$ \\
${ }^{\mathrm{c}}{ }_{x_{1}}:$ mole fraction solubility of the solute.
\end{tabular}

\section{Auxiliary Information}

\section{Method/Apparatus/Procedure:}

Constant-temperature bath, calorimetric thermometer, and an ultraviolet/ visible spectrophotometer.

Excess solute and solvent were placed in amber glass bottles and allowed to equilibrate for several days at constant temperature. Attainment of equilibrium was verified by several repetitive measurements and by approaching equilibrium from supersaturation. Aliquots of saturated solutions were transferred through a coarse filter into tared volumetric flasks, weighed and diluted with methanol. Concentrations were determined by spectrophotometric measurements at $357 \mathrm{~nm}$.

Source and Purity of Chemicals:

(1) $98 \%$, Aldrich Chemical Company, Milwaukee, WI, USA, was recrystallized several times from methanol.

(2) $99.9+\%$, Arco Chemical Company, USA, stored over molecular sieves before use.

Estimated Error:

Temperature: $\pm 0.1 \mathrm{~K}$.

$x_{1}: \pm 1.5 \%$ (relative error).

\subsection{Fluoranthene solubility data in haloalkanes, haloalkenes, and haloaromatic hydrocarbons}

Components:
(1) Fluoranthene; $\mathrm{C}_{16} \mathrm{H}_{10} ;[206-44-0]$
(2) Trichloromethane; $\mathrm{CHCl}_{3} ;[67-66-3]$

Variables:

$T / \mathrm{K}=298.15$
Original Measurements:

${ }^{45}$ L. E. Roy, C. E. Hernández, and W. E. Acree, Jr.,

Polycyclic Aromat. Compd.

13, 205 (1999).

Prepared by:

W. E. Acree, Jr. 
Experimental Values

\begin{tabular}{lcc}
\hline$\frac{x_{2}{ }^{(\mathrm{s}) \mathrm{a}}}{x_{2}{ }^{\mathrm{b}}}$ & $x_{1}{ }^{\mathrm{c}}$ \\
\hline 1.0000 & 0.8574 & 0.1426 \\
$\frac{{ }^{\mathrm{a}} x_{2}{ }^{(\mathrm{s})}: \text { initial mole fraction of component } 2 \text { in the solution. }}{{ }^{\mathrm{b}}{ }_{x_{2}}: \text { mole fraction of component } 2 \text { in the saturated solution. }}$ \\
${ }^{\mathrm{c}} x_{1}:$ mole fraction solubility of the solute.
\end{tabular}

\section{Auxiliary Information}

\section{Method/Apparatus/Procedure:}

Constant-temperature bath, calorimetric thermometer, and an ultraviolet/ visible spectrophotometer.

Excess solute and solvent were placed in amber glass bottles and allowed to equilibrate for several days at constant temperature. Attainment of equilibrium was verified by several repetitive measurements and by approaching equilibrium from supersaturation. Aliquots of saturated solutions were transferred through a coarse filter into tared volumetric flasks, weighed and diluted with methanol. Concentrations were determined by spectrophotometric measurements at $357 \mathrm{~nm}$.

\section{Source and Purity of Chemicals:}

(1) $98 \%$, Aldrich Chemical Company, Milwaukee, WI, USA, was recrystallized several times from methanol.

(2) $99.9+\%$, HPLC Grade, Aldrich Chemical Company, was shaken several times with an aqueous sodium hydroxide solution and then with distilled water to remove the ethanol stabilizer. The resulting solution was dried over both anhydrous calcium chloride and molecular sieves, and then distilled shortly before use.

\section{Estimated Error:}

Temperature: $\pm 0.1 \mathrm{~K}$

$x_{1}: \pm 1.5 \%$ (relative error)

\section{Components:}

(1) Fluoranthene; $\mathrm{C}_{16} \mathrm{H}_{10} ;[206-44-0]$

(2) Tetrachloromethane; $\mathrm{CCl}_{4} ;[56-23-5]$

\begin{tabular}{ll} 
& 13, $205(1999)$. \\
\hline Variables: & Prepared by: \\
$T / \mathrm{K}=298.15$ & W. E. Acree, Jr. \\
\hline
\end{tabular}

Experimental Values

\begin{tabular}{lcc}
\hline \hline$x_{2}{ }^{(\mathrm{s}) \mathrm{a}}$ & $x_{2}{ }^{\mathrm{b}}$ & $x_{1}{ }^{\mathrm{c}}$ \\
\hline 1.0000 & 0.9184 & 0.08157 \\
$\frac{{ }^{\mathrm{a}} x_{2}{ }^{(\mathrm{s})}: \text { initial mole fraction of component } 2 \text { in the solution. }}{{ }^{\mathrm{b}} x_{2}: \text { mole fraction of component } 2 \text { in the saturated solution. }}$ \\
${ }^{\mathrm{c}} x_{1}:$ mole fraction solubility of the solute.
\end{tabular}

Original Measurements:

${ }^{45}$ L. E. Roy, C. E. Hernández, and W. E. Acree, Jr., Polycyclic Aromat. Compd. Prepared by:

$T / \mathrm{K}=298.15$

\section{Method/Apparatus/Procedure:}

Auxiliary Information

Constant-temperature bath, calorimetric thermometer, and an ultraviolet/ visible spectrophotometer.

Excess solute and solvent were placed in amber glass bottles and allowed to equilibrate for several days at constant temperature. Attainment of equilibrium was verified by several repetitive measurements and by approaching equilibrium from supersaturation. Aliquots of saturated solutions were transferred through a coarse filter into tared volumetric flasks, weighed and diluted with methanol. Concentrations were determined by

spectrophotometric measurements at $357 \mathrm{~nm}$.

\section{Source and Purity of Chemicals:}

(1) $98 \%$, Aldrich Chemical Company, Milwaukee, WI, USA, was recrystallized several times from methanol.

(2) $99.8 \%$, HPLC Grade, Aldrich Chemical Company, stored over molecular sieves and distilled shortly before use.

Estimated Error:

Temperature: $\pm 0.1 \mathrm{~K}$.

$x_{1}: \pm 1.5 \%$ (relative error) 


\section{Components:}

(1) Fluoranthene; $\mathrm{C}_{16} \mathrm{H}_{10} ;$; 206-44-0]

(2) 1-Chlorobutane; $\mathrm{C}_{4} \mathrm{H}_{9} \mathrm{Cl}$; [109-69-3]

Original Measurements:

${ }^{44}$ C. E. Hernández and W. E. Acree, Jr., Can. J. Chem. 76, 1312 (1998).

\section{Variables:}

$T / \mathrm{K}=298.15$

Prepared by:

W. E. Acree, Jr.

\section{Experimental Values}

\begin{tabular}{lcc}
\hline \hline$x_{2}{ }^{(\mathrm{s}) \mathrm{a}}$ & $x_{2}{ }^{\mathrm{b}}$ & $x_{1}{ }^{\mathrm{c}}$ \\
\hline 1.0000 & 0.8867 & 0.1133 \\
\hline
\end{tabular}

${ }^{\mathrm{a}} x_{2}{ }^{(\mathrm{s})}$ : initial mole fraction of component 2 in the solution.

${ }^{b} x_{2}$ : mole fraction of component 2 in the saturated solution.

${ }^{c} x_{1}$ : mole fraction solubility of the solute.

\section{Auxiliary Information}

\begin{abstract}
Method/Apparatus/Procedure:
Constant-temperature bath, calorimetric thermometer, and an ultraviolet/ visible spectrophotometer.

Excess solute and solvent were placed in amber glass bottles and allowed to equilibrate for several days at constant temperature. Attainment of equilibrium was verified by several repetitive measurements and by approaching equilibrium from supersaturation. Aliquots of saturated solutions were transferred through a coarse filter into tared volumetric flasks, weighed and diluted with methanol. Concentrations were determined by

spectrophotometric measurements at $357 \mathrm{~nm}$.
\end{abstract}

Source and Purity of Chemicals:

(1) $98 \%$, Aldrich Chemical Company, Milwaukee, WI, USA, was recrystallized several times from methanol.

(2) $99.5+\%$, HPLC Grade, Sigma-Aldrich Chemical Company, stored over molecular sieves before use.

\section{Estimated Error:}

Temperature: $\pm 0.1 \mathrm{~K}$.

$x_{1}: \pm 1.5 \%$ (relative error).

\begin{tabular}{ll}
\hline \hline Components: & Original Measurements: \\
(1) Fluoranthene; $\mathrm{C}_{16} \mathrm{H}_{10} ;$ [206-44-0] & ${ }^{45}$ L. E. Roy, C. E. Hernández, and \\
(2) 1-Chlorohexane; $\mathrm{C}_{6} \mathrm{H}_{13} \mathrm{Cl} ;$ & W. E. Acree, Jr., Polycyclic \\
[544-10-5] & Aromat. Compd. 13, 205 (1999). \\
\hline Variables: & Prepared by: \\
$T / \mathrm{K}=298.15$ & W. E. Acree, Jr. \\
\hline
\end{tabular}

Experimental Values

\begin{tabular}{lcc}
\hline \hline$x_{2}{ }^{(\mathrm{s}) \mathrm{a}}$ & \multicolumn{1}{c}{$x_{2}^{\mathrm{b}}$} & $x_{1}{ }^{\mathrm{c}}$ \\
\hline 1.0000 & 0.8685 & 0.1315 \\
$\frac{{ }^{\mathrm{a}} x_{2}{ }^{(\mathrm{s})}: \text { initial mole fraction of component } 2 \text { in the solution. }}{{ }^{\mathrm{b}} x_{2}: \text { mole fraction of component } 2 \text { in the saturated solution. }}$ \\
${ }^{\mathrm{c}} x_{1}:$ mole fraction solubility of the solute.
\end{tabular}

\section{Auxiliary Information}

\section{Method/Apparatus/Procedure:}

Constant-temperature bath, calorimetric thermometer, and an ultraviolet/ visible spectrophotometer.

Excess solute and solvent were placed in amber glass bottles and allowed to equilibrate for several days at constant temperature. Attainment of equilibrium was verified by several repetitive measurements and by approaching equilibrium from supersaturation. Aliquots of saturated solutions were transferred through a coarse filter into tared volumetric flasks, weighed and diluted with methanol. Concentrations were determined by spectrophotometric measurements at $357 \mathrm{~nm}$.

\section{Source and Purity of Chemicals:}

(1) $98 \%$, Aldrich Chemical Company, Milwaukee, WI, USA, was recrystallized several times from methanol.

(2) $99 \%$, Aldrich Chemical Company, stored over molecular sieves and distilled shortly before use.

Estimated Error:

Temperature: $\pm 0.1 \mathrm{~K}$.

$x_{1}: \pm 1.5 \%$ (relative error).

\begin{tabular}{ll}
\hline \hline Components: & Original Measurements: \\
(1) Fluoranthene; $\mathrm{C}_{16} \mathrm{H}_{10} ;[206-44-0]$ & ${ }^{44} \mathrm{C}$. E. Hernández and W. E. \\
(2) 1-Chlorooctane; $\mathrm{C}_{8} \mathrm{H}_{17} \mathrm{Cl} ;[111-85-3]$ & Acree, Jr., Can. J. Chem. 76, \\
& 1312 (1998). \\
\hline Variables: & Prepared by: \\
$T / \mathrm{K}=298.15$ & W. E. Acree, Jr. \\
\hline
\end{tabular}

Experimental Values

\begin{tabular}{lcc}
\hline \hline$x_{2}{ }^{(\mathrm{s}) \mathrm{a}}$ & $x_{2}{ }^{\mathrm{b}}$ & $x_{1}{ }^{\mathrm{c}}$ \\
\hline 1.0000 & 0.8633 & 0.1367 \\
$\frac{{ }^{\mathrm{a}} x_{2}{ }^{(\mathrm{s})}: \text { initial mole fraction of component } 2 \text { in the solution. }}{}$ \\
${ }^{\mathrm{b}} x_{2}:$ mole fraction of component 2 in the saturated solution. \\
${ }^{\mathrm{c}} x_{1}:$ mole fraction solubility of the solute.
\end{tabular}

\section{Auxiliary Information}

\section{Method/Apparatus/Procedure:}

Constant-temperature bath, calorimetric thermometer, and an ultraviolet/ visible spectrophotometer.

Excess solute and solvent were placed in amber glass bottles and allowed to equilibrate for several days at constant temperature. Attainment of equilibrium was verified by several repetitive measurements and by approaching equilibrium from supersaturation. Aliquots of saturated solutions were transferred through a coarse filter into tared volumetric flasks, weighed and diluted with methanol. Concentrations were determined by spectrophotometric measurements at $357 \mathrm{~nm}$.

\section{Source and Purity of Chemicals:}

(1) $98 \%$, Aldrich Chemical Company, Milwaukee, WI, USA, was recrystallized several times from methanol.

(2) $99 \%$, Aldrich Chemical Company, stored over molecular sieves before use.

\section{Estimated Error:}

Temperature: $\pm 0.1 \mathrm{~K}$.

$x_{1}: \pm 1.5 \%$ (relative error). 


\section{Components:}

(1) Fluoranthene; $\mathrm{C}_{16} \mathrm{H}_{10}$; [206-44-0]

(2) Chlorocyclohexane; $\mathrm{C}_{6} \mathrm{H}_{11} \mathrm{Cl}$;

[542-18-7]

Variables:

$T / \mathrm{K}=298.15$

Original Measurements:

${ }^{44}$ C. E. Hernández and W. E.

Acree, Jr., Can. J. Chem. 76, 1312 (1998).

Prepared by:

W. E. Acree, Jr.

Experimental Values

\begin{tabular}{lcc}
\hline \hline$x_{2}{ }^{(\mathrm{s}) \mathrm{a}}$ & $x_{2}{ }^{\mathrm{b}}$ & $x_{1}{ }^{\mathrm{c}}$ \\
\hline 1.0000 & 0.8841 & 0.1159 \\
\hline
\end{tabular}

${ }^{\mathrm{a}} x_{2}{ }^{(\mathrm{s})}$ : initial mole fraction of component 2 in the solution.

${ }^{b} x_{2}$ : mole fraction of component 2 in the saturated solution.

${ }^{c} x_{1}$ : mole fraction solubility of the solute.

\title{
Auxiliary Information
}

\begin{abstract}
Method/Apparatus/Procedure:
Constant-temperature bath, calorimetric thermometer, and an ultraviolet/ visible spectrophotometer.

Excess solute and solvent were placed in amber glass bottles and allowed to equilibrate for several days at constant temperature. Attainment of equilibrium was verified by several repetitive measurements and by approaching equilibrium from supersaturation. Aliquots of saturated solutions were transferred through a coarse filter into tared volumetric flasks, weighed and diluted with methanol. Concentrations were determined by

spectrophotometric measurements at $357 \mathrm{~nm}$.
\end{abstract}

Source and Purity of Chemicals:

(1) $98 \%$, Aldrich Chemical Company, Milwaukee, WI, USA, was recrystallized several times from methanol.

(2) $99 \%$, Aldrich Chemical Company, stored over molecular sieves before use.

Estimated Error:

Temperature: $\pm 0.1 \mathrm{~K}$

$x_{1}: \pm 1.5 \%$ (relative error).

\subsection{Fluoranthene solubility data in alcohols}

\section{Components:}

(1) Fluoranthene; $\mathrm{C}_{16} \mathrm{H}_{10} ;$ [206-44-0]

(2) Methanol; $\mathrm{CH}_{4} \mathrm{O} ;[67-56-1]$

\section{Variables:}

$T / \mathrm{K}=298.15$
Original Measurements:

${ }^{44}$ C. E. Hernández and W. E. Acree, Jr., Can. J. Chem. 76, 1312 (1998).

Prepared by:

W. E. Acree, Jr.
Experimental Values

\begin{tabular}{|c|c|c|}
\hline$x_{2}{ }^{(\mathrm{s}) \mathrm{a}}$ & $x_{2}{ }^{b}$ & $x_{1}{ }^{\mathrm{c}}$ \\
\hline 1.0000 & 0.9977 & 0.002267 \\
\hline
\end{tabular}

\section{Auxiliary Information}

Method/Apparatus/Procedure:

Constant-temperature bath, calorimetric thermometer, and an ultraviolet/ visible spectrophotometer.

Excess solute and solvent were placed in amber glass bottles and allowed to equilibrate for several days at constant temperature. Attainment of equilibrium was verified by several repetitive measurements and by approaching equilibrium from supersaturation. Aliquots of saturated solutions were transferred through a coarse filter into tared volumetric flasks, weighed and diluted with methanol. Concentrations were determined by spectrophotometric measurements at $357 \mathrm{~nm}$.

\section{Source and Purity of Chemicals:}

(1) $98 \%$, Aldrich Chemical Company, Milwaukee, WI, USA, was recrystallized several times from methanol.

(2) $99.9+\%$, Aldrich Chemical Company, stored over molecular sieves before use.

Estimated Error:

Temperature: $\pm 0.1 \mathrm{~K}$.

$x_{1}: \pm 1.5 \%$ (relative error).

\begin{tabular}{ll}
\hline \hline Components: & Original Measurements: \\
(1) Fluoranthene; $\mathrm{C}_{16} \mathrm{H}_{10} ;[206-44-0]$ & ${ }^{44} \mathrm{C}$. E. Hernández and W. E. \\
(2) Ethanol; $\mathrm{C}_{2} \mathrm{H}_{6} \mathrm{O} ;[64-17-5]$ & Acree, Jr., Can. J. Chem. 76, \\
& 1312 (1998). \\
\hline Variables: & Prepared by: \\
$T / \mathrm{K}=298.15$ & W. E. Acree, Jr. \\
\hline
\end{tabular}

Experimental Values

\begin{tabular}{|c|c|c|}
\hline$x_{2}{ }^{(\mathrm{s}) \mathrm{a}}$ & $x_{2}{ }^{b}$ & $x_{1}{ }^{\mathrm{c}}$ \\
\hline 1.0000 & 0.9946 & 0.00544 \\
\hline
\end{tabular}

\section{Auxiliary Information}

\section{Method/Apparatus/Procedure:}

Constant-temperature bath, calorimetric thermometer, and an ultraviolet/ visible spectrophotometer.

Excess solute and solvent were placed in amber glass bottles and allowed to equilibrate for several days at constant temperature. Attainment of equilibrium was verified by several repetitive measurements and by approaching equilibrium from supersaturation. Aliquots of saturated solutions were transferred through a coarse filter into tared volumetric flasks, weighed and diluted with methanol. Concentrations were determined by spectrophotometric measurements at $357 \mathrm{~nm}$.

\section{Source and Purity of Chemicals:}

(1) $98 \%$, Aldrich Chemical Company, Milwaukee, WI, USA, was recrystallized several times from methanol.

(2) Absolute, Aaper Alcohol and Chemical Company, USA, stored over molecular sieves before use.

\section{Estimated Error:}

Temperature: $\pm 0.1 \mathrm{~K}$.

$x_{1}: \pm 1.5 \%$ (relative error). 


\section{Components:}

(1) Fluoranthene; $\mathrm{C}_{16} \mathrm{H}_{10}$; [206-44-0]

(2) 1-Propanol; $\mathrm{C}_{3} \mathrm{H}_{8} \mathrm{O}$; [71-23-8]

\section{Variables:}

$T / \mathrm{K}=298.15$

Original Measurements:

${ }^{44}$ C. E. Hernández and W. E. Acree, Jr., Can. J. Chem. 76, 1312 (1998).

Prepared by:

W. E. Acree, Jr.

Experimental Values

\begin{tabular}{lcc}
\hline \hline$x_{2}{ }^{(\mathrm{s}) \mathrm{a}}$ & $x_{2}{ }^{\mathrm{b}}$ & $x_{1}{ }^{\mathrm{c}}$ \\
\hline 1.0000 & 0.9933 & 0.00670 \\
\hline
\end{tabular}

${ }^{\mathrm{a}} x_{2}{ }^{(\mathrm{s})}$ : initial mole fraction of component 2 in the solution.

${ }^{b} x_{2}$ : mole fraction of component 2 in the saturated solution.

${ }^{c} x_{1}$ : mole fraction solubility of the solute.

\section{Auxiliary Information}

Method/Apparatus/Procedure:
Constant-temperature bath, calorimetric thermometer, and an ultraviolet/
visible spectrophotometer.
Excess solute and solvent were placed in amber glass bottles and allowed to
equilibrate for several days at constant temperature. Attainment of equilibrium
was verified by several repetitive measurements and by approaching
equilibrium from supersaturation. Aliquots of saturated solutions were
transferred through a coarse filter into tared volumetric flasks, weighed and
diluted with methanol. Concentrations were determined by
spectrophotometric measurements at $357 \mathrm{~nm}$.
Source and Purity of Chemicals:
(1) $98 \%$, Aldrich Chemical Company, Milwaukee, WI, USA, was
recrystallized several times from methanol.
(2) $99+\%$, anhydrous, Aldrich Chemical Company, stored over molecular
sieves before use.
Estimated Error:
Temperature: $\pm 0.1 \mathrm{~K}$.
$x_{1}: \pm 1.5 \%$ (relative error).

\begin{tabular}{ll}
\hline \hline Components: & Original Measurements: \\
(1) Fluoranthene; $\mathrm{C}_{16} \mathrm{H}_{10} ;[206-44-0]$ & ${ }^{44} \mathrm{C}$. E. Hernández and W. E. \\
(2) 2-Propanol; $\mathrm{C}_{3} \mathrm{H}_{8} \mathrm{O} ;[67-63-0]$ & Acree, Jr., Can. J. Chem. 76, \\
& $1312(1998)$. \\
\hline Variables: & Prepared by: \\
$T / \mathrm{K}=298.15$ & W. E. Acree, Jr. \\
\hline
\end{tabular}

Experimental Values

\begin{tabular}{lcc}
\hline \hline$x_{2}{ }^{(\mathrm{s}) \mathrm{a}}$ & $x_{2}{ }^{\mathrm{b}}$ & $x_{1}{ }^{\mathrm{c}}$ \\
\hline 1.0000 & 0.9952 & 0.00475 \\
$\frac{{ }^{\mathrm{a}}{ }^{(\mathrm{s})}: \text { initial mole fraction of component } 2 \text { in the solution. }}{{ }^{\mathrm{b}}{ }{ }_{2}: \text { mole fraction of component } 2 \text { in the saturated solution. }}$ \\
${ }^{\mathrm{c}} x_{1}:$ mole fraction solubility of the solute.
\end{tabular}

\section{Auxiliary Information}

\section{Method/Apparatus/Procedure:}

Constant-temperature bath, calorimetric thermometer, and an ultraviolet/ visible spectrophotometer.

Excess solute and solvent were placed in amber glass bottles and allowed to equilibrate for several days at constant temperature. Attainment of equilibrium was verified by several repetitive measurements and by approaching equilibrium from supersaturation. Aliquots of saturated solutions were transferred through a coarse filter into tared volumetric flasks, weighed and diluted with methanol. Concentrations were determined by spectrophotometric measurements at $357 \mathrm{~nm}$.

\section{Source and Purity of Chemicals:}

(1) $98 \%$, Aldrich Chemical Company, Milwaukee, WI, USA, was recrystallized several times from methanol.

(2) $99+\%$, anhydrous, Aldrich Chemical Company, stored over molecular sieves before use.

Estimated Error:

Temperature: $\pm 0.1 \mathrm{~K}$.

$x_{1}: \pm 1.5 \%$ (relative error)

\begin{tabular}{ll}
\hline \hline Components: & Original Measurements: \\
(1) Fluoranthene; $\mathrm{C}_{16} \mathrm{H}_{10} ;[206-44-0]$ & ${ }^{44} \mathrm{C}$. E. Hernández and W. E. \\
(2) 1-Butanol; $\mathrm{C}_{4} \mathrm{H}_{10} \mathrm{O} ;[71-36-3]$ & Acree, Jr., Can. J. Chem. 76, \\
& 1312 (1998). \\
\hline Variables: & Prepared by: \\
$T / \mathrm{K}=298.15$ & W. E. Acree, Jr. \\
\hline
\end{tabular}

Experimental Values

\begin{tabular}{|c|c|c|}
\hline$x_{2}{ }^{(\mathrm{s}) \mathrm{a}}$ & $x_{2}{ }^{b}$ & $x_{1}{ }^{\mathrm{c}}$ \\
\hline 1.0000 & 0.9900 & 0.00996 \\
\hline
\end{tabular}

\section{Auxiliary Information}

\section{Method/Apparatus/Procedure:}

Constant-temperature bath, calorimetric thermometer, and an ultraviolet/ visible spectrophotometer.

Excess solute and solvent were placed in amber glass bottles and allowed to equilibrate for several days at constant temperature. Attainment of equilibrium was verified by several repetitive measurements and by approaching equilibrium from supersaturation. Aliquots of saturated solutions were transferred through a coarse filter into tared volumetric flasks, weighed and diluted with methanol. Concentrations were determined by spectrophotometric measurements at $357 \mathrm{~nm}$.

\section{Source and Purity of Chemicals:}

(1) $98 \%$, Aldrich Chemical Company, Milwaukee, WI, USA, was recrystallized several times from methanol.

(2) $99.8+\%$, HPLC Grade, Aldrich Chemical Company, stored over molecular sieves before use.

\section{Estimated Error:}

Temperature: $\pm 0.1 \mathrm{~K}$.

$x_{1}: \pm 1.5 \%$ (relative error). 


\section{Components:}

(1) Fluoranthene; $\mathrm{C}_{16} \mathrm{H}_{10}$; [206-44-0]

(2) 2-Butanol; $\mathrm{C}_{4} \mathrm{H}_{10} \mathrm{O}$; [78-92-2]

\section{Variables:}

$T / \mathrm{K}=298.15$

Original Measurements:

${ }^{44}$ C. E. Hernández and W. E. Acree, Jr., Can. J. Chem. 76, 1312 (1998).

Prepared by:

W. E. Acree, Jr.

Experimental Values

\begin{tabular}{lcc}
\hline \hline$x_{2}{ }^{(\mathrm{s}) \mathrm{a}}$ & $x_{2}{ }^{\mathrm{b}}$ & $x_{1}{ }^{\mathrm{c}}$ \\
\hline 1.0000 & 0.9930 & 0.00702 \\
\hline
\end{tabular}

${ }^{\mathrm{a}} x_{2}{ }^{(\mathrm{s})}$ : initial mole fraction of component 2 in the solution.

${ }^{b} x_{2}$ : mole fraction of component 2 in the saturated solution.

${ }^{c} x_{1}$ : mole fraction solubility of the solute.

\section{Auxiliary Information}

Method/Apparatus/Procedure:
Constant-temperature bath, calorimetric thermometer, and an ultraviolet/
visible spectrophotometer.
Excess solute and solvent were placed in amber glass bottles and allowed to
equilibrate for several days at constant temperature. Attainment of equilibrium
was verified by several repetitive measurements and by approaching
equilibrium from supersaturation. Aliquots of saturated solutions were
transferred through a coarse filter into tared volumetric flasks, weighed and
diluted with methanol. Concentrations were determined by
spectrophotometric measurements at 357 nm.
Source and Purity of Chemicals:
(1) $98 \%$, Aldrich Chemical Company, Milwaukee, WI, USA, was
recrystallized several times from methanol.
(2) $99+\%$, anhydrous, Aldrich Chemical Company, stored over molecular
sieves before use.
Estimated Error:
Temperature: $\pm 0.1 \mathrm{~K}$.
$x_{1}: \pm 1.5 \%$ (relative error).

\begin{tabular}{ll}
\hline \hline Components: & Original Measurements: \\
(1) Fluoranthene; $\mathrm{C}_{16} \mathrm{H}_{10} ;[206-44-0]$ & ${ }^{44} \mathrm{C}$. E. Hernández and W. E. \\
(2) 2-Methyl-1-propanol; $\mathrm{C}_{4} \mathrm{H}_{10} \mathrm{O} ;$ & Acree, Jr., Can. J. Chem. 76, \\
[78-83-1] & $1312(1998)$. \\
\hline Variables: & Prepared by: \\
$T / \mathrm{K}=298.15$ & W. E. Acree, Jr. \\
\hline
\end{tabular}

Experimental Values

\begin{tabular}{lcc}
\hline \hline$x_{2}{ }^{(\mathrm{s}) \mathrm{a}}$ & $x_{2}{ }^{\mathrm{b}}$ & $x_{1}{ }^{\mathrm{c}}$ \\
\hline 1.0000 & 0.9950 & 0.00495 \\
${ }^{\mathrm{a}} x_{2}{ }^{(\mathrm{s})}:$ initial mole fraction of component 2 in the solution. \\
${ }^{\mathrm{b}}{ }_{x_{2}: \text { mole fraction of component } 2 \text { in the saturated solution. }}$ \\
${ }^{\mathrm{c}}{ }_{x_{1}: \text { mole fraction solubility of the solute. }}$
\end{tabular}

\section{Auxiliary Information}

\section{Method/Apparatus/Procedure:}

Constant-temperature bath, calorimetric thermometer, and an ultraviolet/ visible spectrophotometer.

Excess solute and solvent were placed in amber glass bottles and allowed to equilibrate for several days at constant temperature. Attainment of equilibrium was verified by several repetitive measurements and by approaching equilibrium from supersaturation. Aliquots of saturated solutions were transferred through a coarse filter into tared volumetric flasks, weighed and diluted with methanol. Concentrations were determined by spectrophotometric measurements at $357 \mathrm{~nm}$.

\section{Source and Purity of Chemicals:}

(1) $98 \%$, Aldrich Chemical Company, Milwaukee, WI, USA, was recrystallized several times from methanol.

(2) $99+\%$, anhydrous, Aldrich Chemical Company, stored over molecular sieves before use.

Estimated Error:

Temperature: $\pm 0.1 \mathrm{~K}$.

$x_{1}: \pm 1.5 \%$ (relative error).

\begin{tabular}{ll}
\hline \hline Components: & Original Measurements: \\
(1) Fluoranthene; $\mathrm{C}_{16} \mathrm{H}_{10} ;[206-44-0]$ & ${ }^{44} \mathrm{C}$. E. Hernández and W. E. \\
(2) 1-Pentanol; $\mathrm{C}_{5} \mathrm{H}_{12} \mathrm{O} ;[71-41-0]$ & Acree, Jr., Can. J. Chem. 76, \\
& $1312(1998)$. \\
\hline Variables: & Prepared by: \\
$T / \mathrm{K}=298.15$ & W. E. Acree, Jr. \\
\hline
\end{tabular}

Experimental Values

\begin{tabular}{|c|c|c|}
\hline$x_{2}{ }^{(\mathrm{s}) \mathrm{a}}$ & $x_{2}{ }^{b}$ & $x_{1}{ }^{\mathrm{c}}$ \\
\hline 1.0000 & 0.9855 & 0.01446 \\
\hline
\end{tabular}

\section{Auxiliary Information}

\section{Method/Apparatus/Procedure:}

Constant-temperature bath, calorimetric thermometer, and an ultraviolet/ visible spectrophotometer.

Excess solute and solvent were placed in amber glass bottles and allowed to equilibrate for several days at constant temperature. Attainment of equilibrium was verified by several repetitive measurements and by approaching equilibrium from supersaturation. Aliquots of saturated solutions were transferred through a coarse filter into tared volumetric flasks, weighed and diluted with methanol. Concentrations were determined by spectrophotometric measurements at $357 \mathrm{~nm}$.

\section{Source and Purity of Chemicals:}

(1) $98 \%$, Aldrich Chemical Company, Milwaukee, WI, USA, was recrystallized several times from methanol.

(2) $99+\%$, Aldrich Chemical Company, stored over molecular sieves before use.

Estimated Error:

Temperature: $\pm 0.1 \mathrm{~K}$.

$x_{1}: \pm 1.5 \%$ (relative error). 


\section{Components:}

(1) Fluoranthene; $\mathrm{C}_{16} \mathrm{H}_{10} ;$; $\left.206-44-0\right]$

(2) 2-Pentanol; $\mathrm{C}_{5} \mathrm{H}_{12} \mathrm{O}$; [6032-29-7]

\section{Variables:}

$T / \mathrm{K}=298.15$

Original Measurements:

${ }^{44}$ C. E. Hernández and W. E. Acree, Jr., Can. J. Chem. 76, 1312 (1998).

Prepared by:

W. E. Acree, Jr.

\section{Experimental Values}

\begin{tabular}{lcc}
\hline \hline$x_{2}{ }^{(\mathrm{s}) \mathrm{a}}$ & $x_{2}{ }^{\mathrm{b}}$ & $x_{1}{ }^{\mathrm{c}}$ \\
\hline 1.0000 & 0.9898 & 0.01021 \\
\hline
\end{tabular}

${ }^{\mathrm{a}} x_{2}{ }^{(\mathrm{s})}$ : initial mole fraction of component 2 in the solution.

${ }^{b} x_{2}$ : mole fraction of component 2 in the saturated solution.

${ }^{c} x_{1}$ : mole fraction solubility of the solute.

\section{Auxiliary Information}

\begin{abstract}
Method/Apparatus/Procedure:
Constant-temperature bath, calorimetric thermometer, and an ultraviolet/ visible spectrophotometer.

Excess solute and solvent were placed in amber glass bottles and allowed to equilibrate for several days at constant temperature. Attainment of equilibrium was verified by several repetitive measurements and by approaching equilibrium from supersaturation. Aliquots of saturated solutions were transferred through a coarse filter into tared volumetric flasks, weighed and diluted with methanol. Concentrations were determined by

spectrophotometric measurements at $357 \mathrm{~nm}$.
\end{abstract}

Source and Purity of Chemicals:

(1) $98 \%$, Aldrich Chemical Company, Milwaukee, WI, USA, was recrystallized several times from methanol.

(2) $99+\%$, Acros Organics, USA, stored over molecular sieves before use.

Estimated Error:

Temperature: $\pm 0.1 \mathrm{~K}$.

$x_{1}: \pm 1.5 \%$ (relative error).

\begin{tabular}{ll}
\hline \hline Components: & Original Measurements: \\
(1) Fluoranthene; $\mathrm{C}_{16} \mathrm{H}_{10} ;[206-44-0]$ & ${ }^{44} \mathrm{C}$. E. Hernández and W. E. \\
(2) 3-Methyl-1-butanol; $\mathrm{C}_{5} \mathrm{H}_{12} \mathrm{O} ;$ & Acree, Jr., Can. J. Chem. 76, 1312 \\
{$[123-51-3]$} & $(1998)$. \\
\hline Variables: & Prepared by: \\
$T / \mathrm{K}=298.15$ & W. E. Acree, Jr. \\
\hline
\end{tabular}

Experimental Values

\begin{tabular}{lcc}
\hline \hline$x_{2}{ }^{(\mathrm{s}) \mathrm{a}}$ & $x_{2}{ }^{\mathrm{b}}$ & $x_{1}{ }^{\mathrm{c}}$ \\
\hline 1.0000 & 0.9914 & 0.00862 \\
$\frac{{ }^{\mathrm{a}} x_{2}{ }^{(\mathrm{s})}: \text { initial mole fraction of component 2 in the solution. }}{{ }^{\mathrm{b}} x_{2}: \text { mole fraction of component } 2 \text { in the saturated solution. }}$ \\
${ }^{\mathrm{c}}{ }_{x_{1}}:$ mole fraction solubility of the solute.
\end{tabular}

\section{Auxiliary Information}

\section{Method/Apparatus/Procedure:}

Constant-temperature bath, calorimetric thermometer, and an ultraviolet/ visible spectrophotometer.

Excess solute and solvent were placed in amber glass bottles and allowed to equilibrate for several days at constant temperature. Attainment of equilibrium was verified by several repetitive measurements and by approaching equilibrium from supersaturation. Aliquots of saturated solutions were transferred through a coarse filter into tared volumetric flasks, weighed and diluted with methanol. Concentrations were determined by spectrophotometric measurements at $357 \mathrm{~nm}$.

\section{Source and Purity of Chemicals:}

(1) $98 \%$, Aldrich Chemical Company, Milwaukee, WI, USA, was recrystallized several times from methanol.

(2) $99+\%$, anhydrous, Aldrich Chemical Company, stored over molecular sieves before use.

Estimated Error:

Temperature: $\pm 0.1 \mathrm{~K}$.

$x_{1}: \pm 1.5 \%$ (relative error).

\begin{tabular}{|c|c|}
\hline $\begin{array}{l}\text { Components: } \\
\text { (1) Fluoranthene; } \mathrm{C}_{16} \mathrm{H}_{10} ;[206-44-0] \\
\text { (2) 2-Methyl-2-butanol; } \mathrm{C}_{5} \mathrm{H}_{12} \mathrm{O} \text {; } \\
\text { [75-85-4] }\end{array}$ & $\begin{array}{l}\text { Original Measurements: } \\
{ }^{44} \text { C. E. Hernández and W. E. } \\
\text { Acree, Jr., Can. J. Chem. 76, } 1312 \\
\text { (1998). }\end{array}$ \\
\hline $\begin{array}{l}\text { Variables: } \\
T / \mathrm{K}=298.15\end{array}$ & $\begin{array}{l}\text { Prepared by: } \\
\text { W. E. Acree, Jr. }\end{array}$ \\
\hline
\end{tabular}

Experimental Values

\begin{tabular}{|c|c|c|}
\hline$x_{2}{ }^{(\mathrm{s}) \mathrm{a}}$ & $x_{2}{ }^{b}$ & $x_{1}{ }^{\mathrm{c}}$ \\
\hline 1.0000 & 0.9903 & 0.00970 \\
\hline
\end{tabular}

\section{Auxiliary Information}

\section{Method/Apparatus/Procedure:}

Constant-temperature bath, calorimetric thermometer, and an ultraviolet/ visible spectrophotometer.

Excess solute and solvent were placed in amber glass bottles and allowed to equilibrate for several days at constant temperature. Attainment of equilibrium was verified by several repetitive measurements and by approaching equilibrium from supersaturation. Aliquots of saturated solutions were transferred through a coarse filter into tared volumetric flasks, weighed and diluted with methanol. Concentrations were determined by spectrophotometric measurements at $357 \mathrm{~nm}$.

\section{Source and Purity of Chemicals:}

(1) $98 \%$, Aldrich Chemical Company, Milwaukee, WI, USA, was recrystallized several times from methanol.

(2) $99+\%$, Acros Organics, USA, stored over molecular sieves before use.

\section{Estimated Error:}

Temperature: $\pm 0.1 \mathrm{~K}$.

$x_{1}: \pm 1.5 \%$ (relative error). 


\section{Components:}

(1) Fluoranthene; $\mathrm{C}_{16} \mathrm{H}_{10} ;[206-44-0]$

(2) 1-Hexanol; $\mathrm{C}_{6} \mathrm{H}_{14} \mathrm{O}$; [111-27-3]

Original Measurements:

${ }^{44}$ C. E. Hernández and W. E.

Acree, Jr., Can. J. Chem. 76, 1312 (1998).

\section{Variables:}

$T / \mathrm{K}=298.15$

Prepared by:

W. E. Acree, Jr

\section{Experimental Values}

\begin{tabular}{lcc}
\hline \hline$x_{2}{ }^{(\mathrm{s}) \mathrm{a}}$ & $x_{2}{ }^{\mathrm{b}}$ & $x_{1}{ }^{\mathrm{c}}$ \\
\hline 1.0000 & 0.9801 & 0.01986 \\
\hline
\end{tabular}

${ }^{\mathrm{a}} x_{2}{ }^{(\mathrm{s})}$ : initial mole fraction of component 2 in the solution.

${ }^{\mathrm{b}} x_{2}$ : mole fraction of component 2 in the saturated solution.

${ }^{c} x_{1}$ : mole fraction solubility of the solute.

\section{Auxiliary Information}

\begin{abstract}
Method/Apparatus/Procedure:
Constant-temperature bath, calorimetric thermometer, and an ultraviolet/ visible spectrophotometer.

Excess solute and solvent were placed in amber glass bottles and allowed to equilibrate for several days at constant temperature. Attainment of equilibrium was verified by several repetitive measurements and by approaching equilibrium from supersaturation. Aliquots of saturated solutions were transferred through a coarse filter into tared volumetric flasks, weighed and diluted with methanol. Concentrations were determined by

spectrophotometric measurements at $357 \mathrm{~nm}$.
\end{abstract}

Source and Purity of Chemicals:

(1) $98 \%$, Aldrich Chemical Company, Milwaukee, WI, USA, was recrystallized several times from methanol.

(2) $99+\%$, Alfa Aesar, USA, stored over molecular sieves before use.

\section{Estimated Error:}

Temperature: $\pm 0.1 \mathrm{~K}$.

$x_{1}: \pm 1.5 \%$ (relative error).

\begin{tabular}{|c|c|}
\hline $\begin{array}{l}\text { Components: } \\
\text { (1) Fluoranthene; } \mathrm{C}_{16} \mathrm{H}_{10} ; \text { [206-44-0] } \\
\text { (2) 2-Methyl-1-pentanol; } \mathrm{C}_{6} \mathrm{H}_{14} \mathrm{O} \text {; } \\
\text { [105-30-6] }\end{array}$ & $\begin{array}{l}\text { Original Measurements: } \\
{ }^{44} \text { C. E. Hernández and W. E. } \\
\text { Acree, Jr., Can. J. Chem. 76, } 1312 \\
(1998) .\end{array}$ \\
\hline $\begin{array}{l}\text { Variables: } \\
T / \mathrm{K}=298.15\end{array}$ & $\begin{array}{l}\text { Prepared by: } \\
\text { W. E. Acree, Jr. }\end{array}$ \\
\hline
\end{tabular}

Experimental Values

\begin{tabular}{lcc}
\hline \hline$x_{2}{ }^{(\mathrm{s}) \mathrm{a}}$ & $x_{2}{ }^{\mathrm{b}}$ & $x_{1}{ }^{\mathrm{c}}$ \\
\hline 1.0000 & 0.9883 & 0.01172 \\
\hline${ }^{\mathrm{a}} x_{2}{ }^{(\mathrm{s})}:$ initial mole fraction of component 2 in the solution. \\
${ }^{\mathrm{b}} x_{2}:$ mole fraction of component 2 in the saturated solution. \\
${ }^{\mathrm{c}}{ }_{x_{1}}$ : mole fraction solubility of the solute.
\end{tabular}

\section{Auxiliary Information}

Method/Apparatus/Procedure:

Constant-temperature bath, calorimetric thermometer, and an ultraviolet/ visible spectrophotometer.

Excess solute and solvent were placed in amber glass bottles and allowed to equilibrate for several days at constant temperature. Attainment of equilibrium was verified by several repetitive measurements and by approaching equilibrium from supersaturation. Aliquots of saturated solutions were transferred through a coarse filter into tared volumetric flasks, weighed and diluted with methanol. Concentrations were determined by spectrophotometric measurements at $357 \mathrm{~nm}$.

\section{Source and Purity of Chemicals:}

(1) $98 \%$, Aldrich Chemical Company, Milwaukee, WI, USA, was recrystallized several times from methanol.

(2) $99 \%$, Aldrich Chemical Company, stored over molecular sieves before use.

\section{Estimated Error:}

Temperature: $\pm 0.1 \mathrm{~K}$.

$x_{1}: \pm 1.5 \%$ (relative error).

\begin{tabular}{ll}
\hline \hline Components: & $\begin{array}{l}\text { Original Measurements: } \\
\text { (1) Fluoranthene; } \mathrm{C}_{16} \mathrm{H}_{10} ;[206-44-0]\end{array}$ \\
$\begin{array}{l}{ }^{44} \mathrm{C} . \text { E. Hernández and W. E. } \\
\text { (2) 4-Methyl-2-pentanol; } \mathrm{C}_{6} \mathrm{H}_{14} \mathrm{O} ;\end{array}$ & $\begin{array}{l}\text { Acree, Jr., Can. J. Chem. 76, 1312 } \\
\text { [108-11-2] }\end{array}$ \\
\hline Variables: & Prepared by: \\
$T / \mathrm{K}=298.15$ & W. E. Acree, Jr. \\
\hline
\end{tabular}

Experimental Values

\begin{tabular}{|c|c|c|}
\hline$x_{2}{ }^{(\mathrm{s}) \mathrm{a}}$ & $x_{2}{ }^{b}$ & $x_{1}{ }^{\mathrm{c}}$ \\
\hline 1.0000 & 0.9905 & $0.00948^{\mathrm{d}}$ \\
\hline \multicolumn{3}{|c|}{$\begin{array}{l}{ }^{\mathrm{a}} x_{2}{ }^{(\mathrm{s})} \text { : initial mole fraction of component } 2 \text { in the solution. } \\
{ }^{\mathrm{b}} x_{2} \text { : mole fraction of component } 2 \text { in the saturated solution. } \\
{ }^{{ }_{2}} x_{1} \text { : mole fraction solubility of the solute. } \\
{ }^{\mathrm{d}} \text { There is a typographical error in the published mole fraction solubility. The } \\
\text { correct value is } 0.00948 \text {. }\end{array}$} \\
\hline
\end{tabular}

\section{Auxiliary Information}

\section{Method/Apparatus/Procedure:}

Constant-temperature bath, calorimetric thermometer, and an ultraviolet/ visible spectrophotometer.

Excess solute and solvent were placed in amber glass bottles and allowed to equilibrate for several days at constant temperature. Attainment of equilibrium was verified by several repetitive measurements and by approaching equilibrium from supersaturation. Aliquots of saturated solutions were transferred through a coarse filter into tared volumetric flasks, weighed and diluted with methanol. Concentrations were determined by spectrophotometric measurements at $357 \mathrm{~nm}$.

\section{Source and Purity of Chemicals:}

(1) $98 \%$, Aldrich Chemical Company, Milwaukee, WI, USA, was recrystallized several times from methanol.

(2) $99+\%$, Acros Organics, USA, stored over molecular sieves before use. 
Estimated Error:

Temperature: $\pm 0.1 \mathrm{~K}$

$x_{1}: \pm 1.5 \%$ (relative error).
Original Measurements:

${ }^{44}$ C. E. Hernández and W. E.

Acree, Jr., Can. J. Chem. 76, 1312 (1998).

\section{Variables:}

$T / \mathrm{K}=298.15$

\section{Prepared by:}

W. E. Acree, Jr.

Experimental Values

\begin{tabular}{lcc}
\hline \hline$x_{2}{ }^{(\mathrm{s}) \mathrm{a}}$ & $x_{2}{ }^{\mathrm{b}}$ & $x_{1}{ }^{\mathrm{c}}$ \\
\hline 1.0000 & 0.9748 & 0.02524 \\
\hline
\end{tabular}

${ }^{\mathrm{a}} x_{2}{ }^{(\mathrm{s})}$ : initial mole fraction of component 2 in the solution.

${ }^{b} x_{2}$ : mole fraction of component 2 in the saturated solution.

${ }^{\mathrm{c}} x_{1}$ : mole fraction solubility of the solute.

\section{Auxiliary Information}

\section{Method/Apparatus/Procedure:}

Constant-temperature bath, calorimetric thermometer, and an ultraviolet/ visible spectrophotometer.

Excess solute and solvent were placed in amber glass bottles and allowed to equilibrate for several days at constant temperature. Attainment of equilibrium was verified by several repetitive measurements and by approaching equilibrium from supersaturation. Aliquots of saturated solutions were transferred through a coarse filter into tared volumetric flasks, weighed and diluted with methanol. Concentrations were determined by

spectrophotometric measurements at $357 \mathrm{~nm}$.

\section{Source and Purity of Chemicals:}

(1) $98 \%$, Aldrich Chemical Company, Milwaukee, WI, USA, was recrystallized several times from methanol.

(2) $99+\%$, Alfa Aesar, USA, stored over molecular sieves before use.

Estimated Error:

Temperature: $\pm 0.1 \mathrm{~K}$.

$x_{1}: \pm 1.5 \%$ (relative error)

\begin{tabular}{ll}
\hline \hline Components: & $\begin{array}{l}\text { Original Measurements: } \\
\text { (1) Fluoranthene; } \mathrm{C}_{16} \mathrm{H}_{10} ;[206-44-0]\end{array}$ \\
$\begin{array}{l}{ }^{44} \mathrm{C} . \text { E. Hernández and W. E. } \\
\text { (2) 1-Octanol; } \mathrm{C}_{8} \mathrm{H}_{18} \mathrm{O} ;[111-87-5]\end{array}$ & $\begin{array}{l}\text { Acree, Jr., Can. J. Chem. 76, 1312 } \\
(1998) .\end{array}$ \\
\hline Variables: & Prepared by: \\
$T / \mathrm{K}=298.15$ & W. E. Acree, Jr. \\
\hline
\end{tabular}

Experimental Values

\begin{tabular}{lcc}
\hline \hline$x_{2}{ }^{(\mathrm{s}) \mathrm{a}}$ & $x_{2}{ }^{\mathrm{b}}$ & $x_{1}{ }^{\mathrm{c}}$ \\
\hline 1.0000 & 0.9687 & 0.03125 \\
\hline
\end{tabular}

${ }^{\mathrm{a}} x_{2}{ }^{(\mathrm{s})}$ : initial mole fraction of component 2 in the solution.

${ }^{b_{x_{2}}}$ : mole fraction of component 2 in the saturated solution.

${ }^{c} x_{1}$ : mole fraction solubility of the solute.

\section{Auxiliary Information}

Method/Apparatus/Procedure:

Constant-temperature bath, calorimetric thermometer, and an ultraviolet/ visible spectrophotometer.

Excess solute and solvent were placed in amber glass bottles and allowed to equilibrate for several days at constant temperature. Attainment of equilibrium was verified by several repetitive measurements and by approaching equilibrium from supersaturation. Aliquots of saturated solutions were transferred through a coarse filter into tared volumetric flasks, weighed and diluted with methanol. Concentrations were determined by spectrophotometric measurements at $357 \mathrm{~nm}$.

\section{Source and Purity of Chemicals:}

(1) $98 \%$, Aldrich Chemical Company, Milwaukee, WI, USA, was recrystallized several times from methanol.

(2) $99+\%$, anhydrous, Aldrich Chemical Company, stored over molecular sieves before use.

\section{Estimated Error:}

Temperature: $\pm 0.1 \mathrm{~K}$.

$x_{1}: \pm 1.5 \%$ (relative error)

\begin{tabular}{ll}
\hline \hline Components: & Original Measurements: \\
(1) Fluoranthene; $\mathrm{C}_{16} \mathrm{H}_{10} ;[206-44-0]$ & ${ }^{44} \mathrm{C}$. E. Hernández and W. E. \\
(2) 2-Ethyl-1-hexanol; $\mathrm{C}_{8} \mathrm{H}_{18} \mathrm{O} ;$ & Acree, Jr., Can. J. Chem. 76, 1312 \\
[104-76-7] & $(1998)$. \\
\hline Variables: & Prepared by: \\
$T / \mathrm{K}=298.15$ & W. E. Acree, Jr. \\
\hline
\end{tabular}

Experimental Values

\begin{tabular}{lcc}
\hline \hline$x_{2}{ }^{(\mathrm{s}) \mathrm{a}}$ & $x_{2}{ }^{\mathrm{b}}$ & $x_{1}{ }^{\mathrm{c}}$ \\
\hline 1.0000 & 0.9822 & 0.01782 \\
$\frac{{ }^{\mathrm{a}} x_{2}{ }^{(\mathrm{s})}: \text { initial mole fraction of component } 2 \text { in the solution. }}{{ }^{\mathrm{b}} x_{2}: \text { mole fraction of component } 2 \text { in the saturated solution. }}$ \\
${ }^{\mathrm{c}} x_{1}:$ mole fraction solubility of the solute.
\end{tabular}

\section{Auxiliary Information}

\section{Method/Apparatus/Procedure:}

Constant-temperature bath, calorimetric thermometer, and an ultraviolet/ visible spectrophotometer.

Excess solute and solvent were placed in amber glass bottles and allowed to equilibrate for several days at constant temperature. Attainment of equilibrium was verified by several repetitive measurements and by approaching equilibrium from supersaturation. Aliquots of saturated solutions were transferred through a coarse filter into tared volumetric flasks, weighed and diluted with methanol. Concentrations were determined by

spectrophotometric measurements at $357 \mathrm{~nm}$.

\section{Source and Purity of Chemicals:}

(1) $98 \%$, Aldrich Chemical Company, Milwaukee, WI, USA, was recrystallized several times from methanol.

(2) $99+\%$, Aldrich Chemical Company, stored over molecular sieves before use.

\section{Estimated Error:}

Temperature: $\pm 0.1 \mathrm{~K}$.

$x_{1}: \pm 1.5 \%$ (relative error). 


\section{Components:}

(1) Fluoranthene; $\mathrm{C}_{16} \mathrm{H}_{10} ;$; $\left.206-44-0\right]$

(2) Cyclopentanol; $\mathrm{C}_{5} \mathrm{H}_{10} \mathrm{O}$; [96-41-3]

\section{Variables:}

$T / \mathrm{K}=298.15$

Original Measurements:

${ }^{44}$ C. E. Hernández and W. E.

Acree, Jr., Can. J. Chem. 76, 1312 (1998).

\section{Prepared by:}

W. E. Acree, Jr

\section{Experimental Values}

\begin{tabular}{lcc}
\hline \hline$x_{2}{ }^{(\mathrm{s}) \mathrm{a}}$ & $x_{2}{ }^{\mathrm{b}}$ & $x_{1}{ }^{\mathrm{c}}$ \\
\hline 1.0000 & 0.9823 & 0.01772 \\
\hline
\end{tabular}

${ }^{\mathrm{a}} x_{2}{ }^{(\mathrm{s})}$ : initial mole fraction of component 2 in the solution.

${ }^{\mathrm{b}} x_{2}$ : mole fraction of component 2 in the saturated solution.

${ }^{c} x_{1}$ : mole fraction solubility of the solute.

\section{Auxiliary Information}

\begin{abstract}
Method/Apparatus/Procedure:
Constant-temperature bath, calorimetric thermometer, and an ultraviolet/ visible spectrophotometer.

Excess solute and solvent were placed in amber glass bottles and allowed to equilibrate for several days at constant temperature. Attainment of equilibrium was verified by several repetitive measurements and by approaching equilibrium from supersaturation. Aliquots of saturated solutions were transferred through a coarse filter into tared volumetric flasks, weighed and diluted with methanol. Concentrations were determined by

spectrophotometric measurements at $357 \mathrm{~nm}$.
\end{abstract}

Source and Purity of Chemicals:

(1) $98 \%$, Aldrich Chemical Company, Milwaukee, WI, USA, was recrystallized several times from methanol.

(2) $99 \%$, Aldrich Chemical Company, stored over molecular sieves before use.

Estimated Error:

Temperature: $\pm 0.1 \mathrm{~K}$.

$x_{1}: \pm 1.5 \%$ (relative error).

\section{Components:}

(1) Fluoranthene; $\mathrm{C}_{16} \mathrm{H}_{10} ;$; 206-44-0]

(2) 1,2-Ethanediol; $\mathrm{C}_{2} \mathrm{H}_{6} \mathrm{O}_{2} ;[107-21-1]$

Original Measurements:

${ }^{45}$ L. E. Roy, C. E. Hernández and

W. E. Acree, Jr., Polycyclic

Aromat. Compd. 13, 205 (1999).

\section{Variables:}

$T / \mathrm{K}=298.15$

\section{Prepared by:}

W. E. Acree, Jr.

\begin{tabular}{|c|c|c|}
\hline$x_{2}^{(\mathrm{s}) \mathrm{a}}$ & $x_{2}{ }^{\mathrm{b}}$ & $x_{1}{ }^{\mathrm{c}}$ \\
\hline 1.0000 & 0.9992 & 0.000750 \\
\hline
\end{tabular}

\section{Auxiliary Information}

Method/Apparatus/Procedure:

Constant-temperature bath, calorimetric thermometer, and an ultraviolet/ visible spectrophotometer.

Excess solute and solvent were placed in amber glass bottles and allowed to equilibrate for several days at constant temperature. Attainment of equilibrium was verified by several repetitive measurements and by approaching equilibrium from supersaturation. Aliquots of saturated solutions were transferred through a coarse filter into tared volumetric flasks, weighed and diluted with methanol. Concentrations were determined by spectrophotometric measurements at $357 \mathrm{~nm}$.

\section{Source and Purity of Chemicals:}

(1) $98 \%$, Aldrich Chemical Company, Milwaukee, WI, USA, was recrystallized several times from methanol.

(2) $99+\%$, Aldrich Chemical Company, stored over molecular sieves and distilled shortly before use.

Estimated Error:

Temperature: $\pm 0.1 \mathrm{~K}$.

$x_{1}: \pm 1.5 \%$ (relative error).

\begin{tabular}{|c|c|}
\hline $\begin{array}{l}\text { Components: } \\
\text { (1) Fluoranthene; } \mathrm{C}_{16} \mathrm{H}_{10} ;[206-44-0] \\
\text { (2) } 2,2,2 \text {-Trifluoroethanol; } \mathrm{C}_{2} \mathrm{H}_{3} \mathrm{~F}_{3} \mathrm{O} \text {; } \\
\text { [75-89-8] }\end{array}$ & $\begin{array}{l}\text { Original Measurements: } \\
{ }^{45} \text { L. E. Roy, C. E. Hernández, and } \\
\text { W. E. Acree, Jr., Polycyclic } \\
\text { Aromat. Compd. 13, } 205 \text { (1999). }\end{array}$ \\
\hline $\begin{array}{l}\text { Variables: } \\
T / \mathrm{K}=298.15\end{array}$ & $\begin{array}{l}\text { Prepared by: } \\
\text { W. E. Acree, Jr. }\end{array}$ \\
\hline
\end{tabular}

Experimental Values

\begin{tabular}{|c|c|c|}
\hline$x_{2}{ }^{(\mathrm{s}) \mathrm{a}}$ & $x_{2}{ }^{b}$ & $x_{1}{ }^{\mathrm{c}}$ \\
\hline 1.0000 & 0.9992 & 0.000772 \\
\hline
\end{tabular}

\section{Auxiliary Information}

\section{Method/Apparatus/Procedure:}

Constant-temperature bath, calorimetric thermometer, and an ultraviolet/ visible spectrophotometer.

Excess solute and solvent were placed in amber glass bottles and allowed to equilibrate for several days at constant temperature. Attainment of equilibrium was verified by several repetitive measurements and by approaching equilibrium from supersaturation. Aliquots of saturated solutions were transferred through a coarse filter into tared volumetric flasks, weighed and diluted with methanol. Concentrations were determined by spectrophotometric measurements at $357 \mathrm{~nm}$.

\section{Source and Purity of Chemicals:}

(1) $98 \%$, Aldrich Chemical Company, Milwaukee, WI, USA, was recrystallized several times from methanol.

(2) $99+\%$, Aldrich Chemical Company, stored over molecular sieves and distilled shortly before use.

\section{Estimated Error:}

Temperature: $\pm 0.1 \mathrm{~K}$.

$x_{1}: \pm 1.5 \%$ (relative error). 


\subsection{Fluoranthene solubility data in miscellaneous organic solvents}

Components:
(1) Fluoranthene; $\mathrm{C}_{16} \mathrm{H}_{10} ;[206-44-0]$
(2) Ethanenitrile; $\mathrm{C}_{2} \mathrm{H}_{3} \mathrm{~N} ;[75-05-8]$

\section{Components}

(2) Ethanenitrile; $\mathrm{C}_{2} \mathrm{H}_{3} \mathrm{~N}$; [75-05-8]

\section{Variables:}

$T / \mathrm{K}=298.15$

Original Measurements:

${ }^{45}$ L. E. Roy, C. E. Hernández, and

W. E. Acree, Jr., Polycyclic

Aromat. Compd. 13, 205 (1999).

Prepared by:

W. E. Acree, Jr.

Experimental Values

\begin{tabular}{lcc}
\hline \hline$x_{2}{ }^{(\mathrm{s}) \mathrm{a}}$ & $x_{2}{ }^{\mathrm{b}}$ & $x_{1}{ }^{\mathrm{c}}$ \\
\hline 1.0000 & 0.9868 & 0.01315 \\
\hline
\end{tabular}

${ }^{\mathrm{a}} x_{2}{ }^{(\mathrm{s})}$ : initial mole fraction of component 2 in the solution.

${ }^{b} x_{2}$ : mole fraction of component 2 in the saturated solution.

${ }^{c} x_{1}$ : mole fraction solubility of the solute.

\section{Auxiliary Information}

\section{Method/Apparatus/Procedure:}

Constant-temperature bath, calorimetric thermometer, and an ultraviolet/ visible spectrophotometer.

Excess solute and solvent were placed in amber glass bottles and allowed to equilibrate for several days at constant temperature. Attainment of equilibrium was verified by several repetitive measurements and by approaching equilibrium from supersaturation. Aliquots of saturated solutions were transferred through a coarse filter into tared volumetric flasks, weighed and diluted with methanol. Concentrations were determined by spectrophotometric measurements at $357 \mathrm{~nm}$.

Source and Purity of Chemicals:

(1) $98 \%$, Aldrich Chemical Company, Milwaukee, WI, USA, was recrystallized several times from methanol.

(2) $99.9+\%$, HPLC Grade, Aldrich Chemical Company, stored over molecular sieves and distilled shortly before use.

\section{Estimated Error:}

Temperature: $\pm 0.1 \mathrm{~K}$.

$x_{1}: \pm 1.5 \%$ (relative error).

\begin{tabular}{ll}
\hline \hline Components: & Original Measurements: \\
(1) Fluoranthene; $\mathrm{C}_{16} \mathrm{H}_{10} ;[206-44-0]$ & ${ }^{45}$ L. E. Roy, C. E. Hernández, and \\
$(2) N, N$-Dimethylformamide; $\mathrm{C}_{3} \mathrm{H}_{7} \mathrm{NO} ;$ & W. E. Acree, Jr. Polycyclic \\
{$[68-12-2]$} & Aromat. Compd. 13, 205 (1999). \\
\hline Variables: & Prepared by: \\
$T / \mathrm{K}=298.15$ & W. E. Acree, Jr. \\
\hline
\end{tabular}

Experimental Values

\begin{tabular}{lcc}
\hline \hline$x_{2}{ }^{(\mathrm{s}) \mathrm{a}}$ & $x_{2}{ }^{\mathrm{b}}$ & $x_{1}{ }^{\mathrm{c}}$ \\
\hline 1.0000 & 0.8203 & 0.1797 \\
$\frac{{ }^{\mathrm{a}} x_{2}{ }^{(\mathrm{s})}: \text { initial mole fraction of component } 2 \text { in the solution. }}{{ }^{\mathrm{b}} x_{2}: \text { mole fraction of component } 2 \text { in the saturated solution. }}$ \\
${ }^{\mathrm{c}} x_{1}:$ mole fraction solubility of the solute.
\end{tabular}

\section{Auxiliary Information}

Method/Apparatus/Procedure:

Constant-temperature bath, calorimetric thermometer, and an ultraviolet/ visible spectrophotometer.

Excess solute and solvent were placed in amber glass bottles and allowed to equilibrate for several days at constant temperature. Attainment of equilibrium was verified by several repetitive measurements and by approaching equilibrium from supersaturation. Aliquots of saturated solutions were transferred through a coarse filter into tared volumetric flasks, weighed and diluted with methanol. Concentrations were determined by spectrophotometric measurements at $357 \mathrm{~nm}$.

\section{Source and Purity of Chemicals:}

(1) $98 \%$, Aldrich Chemical Company, Milwaukee, WI, USA, was recrystallized several times from methanol.

(2) $99.8 \%$, anhydrous, Aldrich Chemical Company, stored over molecular sieves and distilled shortly before use.

\section{Estimated Error:}

Temperature: $\pm 0.1 \mathrm{~K}$.

$x_{1}: \pm 1.5 \%$ (relative error)

\begin{tabular}{ll}
\hline \hline Components: & Original Measurements: \\
(1) Fluoranthene; $\mathrm{C}_{16} \mathrm{H}_{10} ;[206-44-0]$ & ${ }^{45}$ L. E. Roy, C. E. Hernández, and \\
$(2) N, N$-Dimethylacetamide; $\mathrm{C}_{4} \mathrm{H}_{9} \mathrm{NO} ;$ & W. E. Acree, Jr., Polycyclic \\
{$[127-19-5]$} & Aromat. Compd. 13, 205 (1999). \\
\hline Variables: & Prepared by: \\
$T / \mathrm{K}=298.15$ & W. E. Acree, Jr. \\
\hline
\end{tabular}

Experimental Values

\begin{tabular}{lcc}
\hline \hline$x_{2}{ }^{(\mathrm{s}) \mathrm{a}}$ & $x_{2}{ }^{\mathrm{b}}$ & $x_{1}{ }^{\mathrm{c}}$ \\
\hline 1.0000 & 0.7630 & 0.2370 \\
$\frac{{ }^{\mathrm{a}} x_{2}{ }^{(\mathrm{s})}: \text { initial mole fraction of component } 2 \text { in the solution. }}{{ }^{\mathrm{b}} x_{2}: \text { mole fraction of component } 2 \text { in the saturated solution. }}$ \\
${ }^{\mathrm{c}} x_{1}:$ mole fraction solubility of the solute.
\end{tabular}

\section{Auxiliary Information}

\section{Method/Apparatus/Procedure:}

Constant-temperature bath, calorimetric thermometer, and an ultraviolet/ visible spectrophotometer.

Excess solute and solvent were placed in amber glass bottles and allowed to equilibrate for several days at constant temperature. Attainment of equilibrium was verified by several repetitive measurements and by approaching equilibrium from supersaturation. Aliquots of saturated solutions were transferred through a coarse filter into tared volumetric flasks, weighed and diluted with methanol. Concentrations were determined by

spectrophotometric measurements at $357 \mathrm{~nm}$.

\section{Source and Purity of Chemicals:}

(1) $98 \%$, Aldrich Chemical Company, Milwaukee, WI, USA, was recrystallized several times from methanol.

(2) $99.8 \%$, anhydrous, Aldrich Chemical Company, stored over molecular sieves and distilled shortly before use.

\section{Estimated Error:}

Temperature: $\pm 0.1 \mathrm{~K}$.

$x_{1}: \pm 1.5 \%$ (relative error). 


\section{Solubility of Fluorene in Organic Solvents}

\subsection{Critical evaluation of experimental solubility data}

Volume 59 in the IUPAC Solubility Data Series ${ }^{3}$ contained experimental solubility data for fluorene dissolved in two cycloalkanes (cyclohexane and decahydronaphthalene), in four aromatic hydrocarbons (benzene, methylbenzene, 1,4dimethylbenzene, and 1,2,3,4-tetrahydronaphalene), in one chlorinated alkane (tetrachloromethane) and one chlorinated benzene (chlorobenzene), in three alkanols (methanol, ethanol, and 1-octanol), and in four miscellaneous organic solvents (nitrobenzene, pyridine, aniline, and thiophene). Except for the fluorene + methanol system, each data set contained mole fraction solubilities measured at several temperatures. The volume also included phase diagram information for binary fluorene + benzene, fluorene + ethylbenzene, fluorene $+1,3$ dimethylbenzene, fluorene + 1,2,4,5-tetramethylbenzene, fluorene + naphthalene, fluorene +2 -methylnaphthalene, fluorene + 2,7-dimethylnaphthalene, fluorene + fluoranthene, fluorene + acenaphthene, fluorene + chrysene, fluorene $+1,2$ dinitrobenzene, fluorene $+1,3$-dinitrobenzene, fluorene $+1,4$ dinitrobenzene, fluorene + 1,3,5-trinitrobenzene, fluorene $+1,2,3,5$-tetranitrobenzene, fluorene $+2,4$-dinitromethylbenzene, fluorene $+2,4,6$-trinitromethylbenzene, fluorene $+2,4$ dinitrophenol, fluorene $+2,4,6$-trinitrophenol, and fluorene + 6-methyl-2,3,4-trinitrophenol mixtures. Solubility data contained in Vol. 59 will not be republished here. The listing above is provided so that readers will know what solubility data are available in the earlier volume for fluorene.

There have been several studies that reported solubility data for fluorene in organic solvents after Vol. 59 was published in 1995. Monárrez et al. ${ }^{48}$ investigated the solubility of fluorene in 13 primary, four secondary, and two tertiary alcohols. In a followup study, Stovall et al. ${ }^{49}$ measured the solubility of fluorene in several alkane, dialkyl ether and alkanenitrile solvents. Experimental results from both studies were used to test the applications and limitations of the Abraham solvation parameter model in regards to solubility predictions. The authors measured the solubility at only $298.15 \mathrm{~K}$, and except for five alkanol solvents there are no independent experimental measurements to compare the numerical values against.

Two research groups studied the solubility behavior of fluorene as a function of temperature. Zou et al. ${ }^{50}$ examined the solubility of fluorene in methylbenzene, ethanol, 2propanol, and 1-butanol at ten different temperatures using a synthetic method with laser monitoring. Han and Wang ${ }^{51}$ later measured the solubility of fluorene in benzene, trichloromethane, propanone, 1-propanol, 2-methyl-1-propanol, and methylbenzene from 283 to $323 \mathrm{~K}$ also using a laser monitoring method. The solubility measurements in the alkanol solvents from these two studies can be compared to the values reported by Monárrez et al. ${ }^{48}$ Reasonably good agreement is found in the case of ethanol $\left(x_{1}=0.00743\right.$ in Ref. 48 versus $x_{1}=0.00802$ in Ref. 50), 1-propanol $\left(x_{1}\right.$ $=0.01232$ in Ref. 48 versus $x_{1}=0.01339$ in Ref. 51), and 2methyl-1-propanol $\left(x_{1}=0.01006\right.$ in Ref. 48 versus $x_{1}$ $=0.00902$ in Ref. 50). Much larger deviations were observed in the case of 2-propanol $\left(x_{1}=0.00949\right.$ in Ref. 48 versus $x_{1}=0.00423$ in Ref. 50) and 1-butanol $\left(x_{1}=0.01689\right.$ in Ref. 48 versus $x_{1}=0.01030$ in Ref. 50 ). No explanation is given for the larger deviations noted for the latter two alkanol solvents.

The internal consistency of the Zou et al. ${ }^{50}$ and Han and Wang $^{51}$ datasets were assessed by curve-fitting the measured mole fraction solubility to Eq. (3). The numerical values of the equation coefficients $(A, B$, and $C$ ) are given in Table 3 , along with the MRD calculated according to Eq. (7). Examination of the numerical entries in the last column of Table 3 reveals that the largest mean relative percent deviation between the backcalculated values based on Eq. (3) and experimental data is only $7.1 \%$. Deviations for the other systems are much less and are often even less than the experimental uncertainties in the measured values. Results of the mathematical representation analyses indicate that the experimental data for all ten fluorene-organic solvent systems are internally consistent. It is further noted that the two sets of solubility data for fluorene in methylbenzene are in good agreement with each other, with the calculated average absolute relative deviation being less than $2 \%$.

TABLE 3. Parameters of the Apelblat equation for describing the solubility of fluorene in various organic solvents

\begin{tabular}{|c|c|c|c|c|}
\hline Solvent & $A$ & $B$ & $C$ & MRD (\%) \\
\hline Methylbenzene $^{\mathrm{a}}$ & -103.83 & 1180.4 & 17.162 & 1.2 \\
\hline Ethanol $^{\mathrm{a}}$ & -102.96 & 1448.2 & 16.369 & $<0.1$ \\
\hline 2-Propanol ${ }^{\mathrm{a}}$ & -68.045 & -1896.3 & 12.096 & $<0.1$ \\
\hline 1-Butanol ${ }^{\mathrm{a}}$ & -88.212 & 508.8 & 14.390 & 0.3 \\
\hline Benzene $^{b}$ & -97.752 & 2365.2 & 15.401 & 1.2 \\
\hline Trichloromethane $^{\mathrm{b}}$ & -64.383 & 801.3 & 10.474 & 0.7 \\
\hline Propanone $^{\mathrm{b}}$ & -64.406 & 487.2 & 10.545 & 2.2 \\
\hline 1-Butanol ${ }^{\mathrm{b}}$ & -65.649 & 305.9 & 10.586 & 2.8 \\
\hline 2-Methyl-1-propanol ${ }^{\mathrm{b}}$ & -96.548 & 710.9 & 10.709 & 7.1 \\
\hline Methylbenzene ${ }^{\mathrm{b}}$ & -101.009 & 1132.4 & 16.702 & 3.5 \\
\hline
\end{tabular}

${ }^{\mathrm{a}}$ Numerical values of the coefficients and the percent mean relative deviations were taken from Zou et al. ${ }^{50}$

${ }^{\mathrm{b}}$ Numerical values of the coefficients and the percent mean relative deviations were taken from Han and Wang. ${ }^{51}$ 
Lisicki and Jamró $z^{35}$ reported solid-liquid equilibria data for binary mixtures containing fluorene with $N, N$-dimethylacetamide, 1-methyl-2-pyrrolidone and hexahydro-1-methyl-

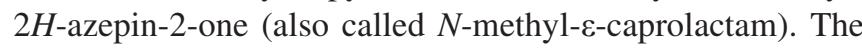
fluorene $+N, N$-dimethylformamide and anthracene +1 methyl-2-pyrrolidone systems both exhibited simple eutectic behavior. The published experimental liquidus curves for the latter three fluorene systems did not indicate any outlier data points.

The experimental solubility data for fluorene dissolved in the different organic solvents are given in Secs. 9.2-9.9.

\subsection{Fluorene solubility data in saturated hydrocarbons (including cycloalkanes)}

\begin{tabular}{ll}
\hline \hline Components: & Original Measurements: \\
(1) Fluorene; $\mathrm{C}_{13} \mathrm{H}_{10} ;[92-52-4]$ & ${ }^{49}$ D. M. Stovall, K. R. Hoover, \\
(2) Hexane; $\mathrm{C}_{6} \mathrm{H}_{14} ;[110-54-3]$ & W. E. Acree, Jr., and M. H. \\
& Abraham, Polycyclic Aromat. \\
& Compd. 25, 313 (2005). \\
\hline Variables: & Prepared by: \\
$T / \mathrm{K}=298.15$ & W. E. Acree, Jr. \\
\hline
\end{tabular}

Experimental Values

\begin{tabular}{lcc}
\hline \hline$x_{2}{ }^{(\mathrm{s}) \mathrm{a}}$ & $x_{2}{ }^{\mathrm{b}}$ & $x_{1}{ }^{\mathrm{c}}$ \\
\hline 1.0000 & 0.9696 & 0.03041 \\
${ }^{\mathrm{a}} x_{2}{ }^{(\mathrm{s})}:$ initial mole fraction of component 2 in the solution. \\
${ }^{\mathrm{b}}{ }_{x_{2}}$ : mole fraction of component 2 in the saturated solution. \\
${ }^{\mathrm{c}}{ }_{x_{1}}$ : mole fraction solubility of the solute.
\end{tabular}

\section{Auxiliary Information}

Method/Apparatus/Procedure:

Constant-temperature bath, calorimetric thermometer, and an ultraviolet/ visible spectrophotometer.

Excess solute and solvent were placed in amber glass bottles and allowed to equilibrate for several days at constant temperature. Attainment of equilibrium was verified by several repetitive measurements and by approaching equilibrium from supersaturation. Aliquots of saturated solutions were transferred through a coarse filter into tared volumetric flasks, weighed and diluted with 2-propanol. Concentrations were determined by

spectrophotometric measurements at $280 \mathrm{~nm}$.

Source and Purity of Chemicals:

(1) $98 \%$, Aldrich Chemical Company, Milwaukee, WI, USA, was recrystallized several times from methanol.

(2) $99 \%$, Aldrich Chemical Company, stored over molecular sieves before use.

\section{Estimated Error:}

Temperature: $\pm 0.1 \mathrm{~K}$.

$x_{1}: \pm 1.5 \%$ (relative error).

\begin{tabular}{ll}
\hline \hline Components: & Original Measurements: \\
(1) Fluorene; $\mathrm{C}_{13} \mathrm{H}_{10} ;[92-52-4]$ & ${ }^{49}$ D. M. Stovall, K. R. Hoover, \\
(2) Heptane; $\mathrm{C}_{7} \mathrm{H}_{16} ;[142-82-5]$ & W. E. Acree, Jr., and M. H. \\
& Abraham, Polycyclic Aromat. \\
& Compd. 25, 313 (2005). \\
\hline Variables: & Prepared by: \\
$T / \mathrm{K}=298.15$ & W. E. Acree, Jr. \\
\hline
\end{tabular}

Experimental Values

\begin{tabular}{lcc}
\hline \hline$x_{2}{ }^{(\mathrm{s}) \mathrm{a}}$ & $x_{2}{ }^{\mathrm{b}}$ & $x_{1}{ }^{\mathrm{c}}$ \\
\hline 1.0000 & 0.9659 & 0.03406 \\
$\frac{{ }^{\mathrm{a}} x_{2}{ }^{(\mathrm{s})}: \text { initial mole fraction of component } 2 \text { in the solution. }}{{ }^{\mathrm{b}} x_{2}: \text { mole fraction of component } 2 \text { in the saturated solution. }}$ \\
${ }^{\mathrm{c}} x_{1}:$ mole fraction solubility of the solute.
\end{tabular}

Auxiliary Information

Method/Apparatus/Procedure:

Constant-temperature bath, calorimetric thermometer, and an ultraviolet/ visible spectrophotometer.

Excess solute and solvent were placed in amber glass bottles and allowed to equilibrate for several days at constant temperature. Attainment of equilibrium was verified by several repetitive measurements and by approaching equilibrium from supersaturation. Aliquots of saturated solutions were transferred through a coarse filter into tared volumetric flasks, weighed and diluted with 2-propanol. Concentrations were determined by spectrophotometric measurements at $280 \mathrm{~nm}$.

\section{Source and Purity of Chemicals:}

(1) $98 \%$, Aldrich Chemical Company, Milwaukee, WI, USA, was recrystallized several times from methanol.

(2) $99 \%$, anhydrous, Aldrich Chemical Company, stored over molecular sieves before use.

Estimated Error:

Temperature: $\pm 0.1 \mathrm{~K}$.

$x_{1}: \pm 1.5 \%$ (relative error).

\begin{tabular}{ll}
\hline \hline Components: & Original Measurements: \\
(1) Fluorene; $\mathrm{C}_{13} \mathrm{H}_{10} ;[92-52-4]$ & ${ }^{49}$ D. M. Stovall, K. R. Hoover, \\
(2) Octane; $\mathrm{C}_{8} \mathrm{H}_{18} ;[111-65-9]$ & W. E. Acree, Jr., and M. H. \\
& Abraham, Polycyclic Aromat. \\
& Compd. 25, 313 (2005). \\
\hline Variables: & Prepared by: \\
$T / \mathrm{K}=298.15$ & W. E. Acree, Jr. \\
\hline
\end{tabular}

Experimental Values

\begin{tabular}{lcc}
\hline \hline$x_{2}{ }^{(\mathrm{s}) \mathrm{a}}$ & $x_{2}{ }^{\mathrm{b}}$ & $x_{1}{ }^{\mathrm{c}}$ \\
\hline 1.0000 & 0.9625 & 0.03750 \\
$\frac{{ }^{a} x_{2}{ }^{(\mathrm{s})}: \text { initial mole fraction of component } 2 \text { in the solution. }}{{ }^{\mathrm{b}} x_{2}: \text { mole fraction of component } 2 \text { in the saturated solution. }}$ \\
${ }^{c} x_{1}:$ mole fraction solubility of the solute.
\end{tabular}




\section{Auxiliary Information}

\section{Method/Apparatus/Procedure:}

Constant-temperature bath, calorimetric thermometer, and an ultraviolet/ visible spectrophotometer.

Excess solute and solvent were placed in amber glass bottles and allowed to equilibrate for several days at constant temperature. Attainment of equilibrium was verified by several repetitive measurements and by approaching equilibrium from supersaturation. Aliquots of saturated solutions were transferred through a coarse filter into tared volumetric flasks, weighed and diluted with 2-propanol. Concentrations were determined by spectrophotometric measurements at $280 \mathrm{~nm}$.

Source and Purity of Chemicals:

(1) $98 \%$, Aldrich Chemical Company, Milwaukee, WI, USA, was recrystallized several times from methanol.

(2) $99+\%$, anhydrous, Aldrich Chemical Company, stored over molecular sieves before use.

Estimated Error:

Temperature: $\pm 0.1 \mathrm{~K}$

$x_{1}: \pm 1.5 \%$ (relative error).

\begin{tabular}{ll}
\hline \hline Components: & Original Measurements: \\
(1) Fluorene; $\mathrm{C}_{13} \mathrm{H}_{10} ;[92-52-4]$ & ${ }^{49}$ D. M. Stovall, K. R. Hoover, \\
(2) Nonane; $\mathrm{C}_{9} \mathrm{H}_{20} ;[111-84-2]$ & W. E. Acree, Jr., and M. H. \\
& Abraham, Polycyclic Aromat. \\
& Compd. 25, 313 (2005). \\
\hline Variables: & Prepared by: \\
$T / \mathrm{K}=298.15$ & W. E. Acree, Jr. \\
\hline
\end{tabular}

Experimental Values

\begin{tabular}{lcc}
\hline \hline$x_{2}{ }^{(\mathrm{s}) \mathrm{a}}$ & $x_{2}{ }^{\mathrm{b}}$ & $x_{1}{ }^{\mathrm{c}}$ \\
\hline 1.0000 & 0.9573 & 0.04269
\end{tabular}

${ }^{\mathrm{a}} x_{2}{ }^{(\mathrm{s})}$ : initial mole fraction of component 2 in the solution.

${ }^{\mathrm{b}} x_{2}$ : mole fraction of component 2 in the saturated solution.

${ }^{c} x_{1}$ : mole fraction solubility of the solute.

\section{Auxiliary Information}

Method/Apparatus/Procedure:
Constant-temperature bath, calorimetric thermometer, and an ultraviolet/
visible spectrophotometer.
Excess solute and solvent were placed in amber glass bottles and allowed to
equilibrate for several days at constant temperature. Attainment of equilibrium
was verified by several repetitive measurements and by approaching
equilibrium from supersaturation. Aliquots of saturated solutions were
transferred through a coarse filter into tared volumetric flasks, weighed and
diluted with 2-propanol. Concentrations were determined by
spectrophotometric measurements at $280 \mathrm{~nm}$.
Source and Purity of Chemicals:
(1) $98 \%$, Aldrich Chemical Company, Milwaukee, WI, USA, was
recrystallized several times from methanol.
(2) $99+\%$, TCI America, Portland, OR, USA, stored over molecular sieves
before use.
Estimated Error:
Temperature: $\pm 0.1 \mathrm{~K}$.
$x_{1}: \pm 1.5 \%$ (relative error).

\begin{tabular}{ll}
\hline \hline Components: & Original Measurements: \\
(1) Fluorene; $\mathrm{C}_{13} \mathrm{H}_{10} ;[92-52-4]$ & ${ }^{49} \mathrm{D}$. M. Stovall, K. R. Hoover, \\
(2) Decane; $\mathrm{C}_{10} \mathrm{H}_{22} ;[124-18-5]$ & W. E. Acree, Jr., and M. H. \\
& Abraham, Polycyclic Aromat. \\
& Compd. 25, 313 (2005). \\
\hline Variables: & Prepared by: \\
$T / \mathrm{K}=298.15$ & W. E. Acree, Jr. \\
\hline
\end{tabular}

Experimental Values

\begin{tabular}{lcc}
\hline \hline$x_{2}{ }^{(\mathrm{s}) \mathrm{a}}$ & $x_{2}{ }^{\mathrm{b}}$ & $x_{1}{ }^{\mathrm{c}}$ \\
\hline 1.0000 & 0.9533 & 0.04669 \\
$\frac{{ }^{\mathrm{a}} x_{2}{ }^{(\mathrm{s})}: \text { initial mole fraction of component } 2 \text { in the solution. }}{{ }^{\mathrm{b}} x_{2}: \text { mole fraction of component } 2 \text { in the saturated solution. }}$ \\
${ }^{\mathrm{c}} x_{1}:$ mole fraction solubility of the solute.
\end{tabular}

\section{Auxiliary Information}

\section{Method/Apparatus/Procedure:}

Constant-temperature bath, calorimetric thermometer, and an ultraviolet/ visible spectrophotometer.

Excess solute and solvent were placed in amber glass bottles and allowed to equilibrate for several days at constant temperature. Attainment of equilibrium was verified by several repetitive measurements and by approaching equilibrium from supersaturation. Aliquots of saturated solutions were transferred through a coarse filter into tared volumetric flasks, weighed and diluted with 2-propanol. Concentrations were determined by spectrophotometric measurements at $280 \mathrm{~nm}$.

\section{Source and Purity of Chemicals:}

(1) $98 \%$, Aldrich Chemical Company, Milwaukee, WI, USA, was recrystallized several times from methanol.

(2) 99+\%, TCI America, Portland, OR, USA, stored over molecular sieves before use.

\section{Estimated Error:}

Temperature: $\pm 0.1 \mathrm{~K}$.

$x_{1}: \pm 1.5 \%$ (relative error)

\begin{tabular}{ll}
\hline \hline Components: & Original Measurements: \\
(1) Fluorene; $\mathrm{C}_{13} \mathrm{H}_{10} ;[92-52-4]$ & ${ }^{49} \mathrm{D}$. M. Stovall, K. R. Hoover, \\
(2) Undecane; $\mathrm{C}_{11} \mathrm{H}_{24} ;[1120-21-4]$ & W. E. Acree, Jr., and M. H. \\
& Abraham, Polycyclic Aromat. \\
& Compd. 25, 313 (2005). \\
\hline Variables: & Prepared by: \\
$T / \mathrm{K}=298.15$ & W. E. Acree, Jr. \\
\hline
\end{tabular}

Experimental Values

\begin{tabular}{lcc}
\hline \hline$x_{2}{ }^{(\mathrm{s}) \mathrm{a}}$ & $x_{2}{ }^{\mathrm{b}}$ & $x_{1}{ }^{\mathrm{c}}$ \\
\hline 1.0000 & 0.9495 & 0.05048 \\
\hline${ }^{{ }^{a} x_{2}{ }^{(\mathrm{s})}: \text { initial mole fraction of component } 2 \text { in the solution. }}$ \\
${ }^{\mathrm{b}} x_{2}:$ mole fraction of component 2 in the saturated solution. \\
${ }^{{ }^{c} x_{1}: \text { mole fraction solubility of the solute. }}$
\end{tabular}




\section{Auxiliary Information}

\section{Method/Apparatus/Procedure:}

Constant-temperature bath, calorimetric thermometer, and an ultraviolet/ visible spectrophotometer.

Excess solute and solvent were placed in amber glass bottles and allowed to equilibrate for several days at constant temperature. Attainment of equilibrium was verified by several repetitive measurements and by approaching equilibrium from supersaturation. Aliquots of saturated solutions were transferred through a coarse filter into tared volumetric flasks, weighed and diluted with 2-propanol. Concentrations were determined by spectrophotometric measurements at $280 \mathrm{~nm}$.

Source and Purity of Chemicals:

(1) $98 \%$, Aldrich Chemical Company, Milwaukee, WI, USA, was recrystallized several times from methanol.

(2) $99 \%$, Acros Organics, USA, stored over molecular sieves before use.

Estimated Error:

Temperature: $\pm 0.1 \mathrm{~K}$.

$x_{1}: \pm 1.5 \%$ (relative error)

\begin{tabular}{ll}
\hline \hline Components: & Original Measurements: \\
(1) Fluorene; $\mathrm{C}_{13} \mathrm{H}_{10} ;[92-52-4]$ & ${ }^{49} \mathrm{D}$. M. Stovall, K. R. Hoover, \\
(2) Dodecane; $\mathrm{C}_{12} \mathrm{H}_{26} ;[112-40-3]$ & W. E. Acree, Jr., and M. H. \\
& Abraham, Polycyclic Aromat. \\
& Compd. 25, 313 (2005). \\
\hline Variables: & Prepared by: \\
$T / \mathrm{K}=298.15$ & W. E. Acree, Jr. \\
\hline
\end{tabular}

Experimental Values

\begin{tabular}{lcc}
\hline \hline$x_{2}{ }^{(\mathrm{s}) \mathrm{a}}$ & $x_{2}{ }^{\mathrm{b}}$ & $x_{1}{ }^{\mathrm{c}}$ \\
\hline 1.0000 & 0.9470 & 0.05302 \\
\hline
\end{tabular}

${ }^{\mathrm{a}} x_{2}{ }^{(\mathrm{s})}$ : initial mole fraction of component 2 in the solution.

${ }^{\mathrm{b}} x_{2}$ : mole fraction of component 2 in the saturated solution.

${ }^{c} x_{1}$ : mole fraction solubility of the solute.

\section{Auxiliary Information}

\section{Method/Apparatus/Procedure:}

Constant-temperature bath, calorimetric thermometer, and an ultraviolet/ visible spectrophotometer.

Excess solute and solvent were placed in amber glass bottles and allowed to equilibrate for several days at constant temperature. Attainment of equilibrium was verified by several repetitive measurements and by approaching equilibrium from supersaturation. Aliquots of saturated solutions were transferred through a coarse filter into tared volumetric flasks, weighed and diluted with 2-propanol. Concentrations were determined by

spectrophotometric measurements at $280 \mathrm{~nm}$.

Source and Purity of Chemicals:

(1) $98 \%$, Aldrich Chemical Company, Milwaukee, WI, USA, was recrystallized several times from methanol.

(2) $99+\%$, anhydrous, Aldrich Chemical Company, stored over molecular sieves before use.

Estimated Error:

Temperature: $\pm 0.1 \mathrm{~K}$.

$x_{1}: \pm 1.5 \%$ (relative error).

\begin{tabular}{ll}
\hline \hline Components: & Original Measurements: \\
(1) Fluorene; $\mathrm{C}_{13} \mathrm{H}_{10} ;[92-52-4]$ & ${ }^{49}$ D. M. Stovall, K. R. Hoover, \\
(2) Hexadecane; $\mathrm{C}_{16} \mathrm{H}_{34} ;[544-76-3]$ & W. E. Acree, Jr., and M. H. \\
& Abraham, Polycyclic Aromat. \\
& Compd. 25, 313 (2005). \\
\hline Variables: & Prepared by: \\
$T / \mathrm{K}=298.15$ & W. E. Acree, Jr. \\
\hline
\end{tabular}

Experimental Values

\begin{tabular}{lcc}
\hline \hline$x_{2}{ }^{(\mathrm{s}) \mathrm{a}}$ & $x_{2}{ }^{\mathrm{b}}$ & $x_{1}{ }^{\mathrm{c}}$ \\
\hline 1.0000 & 0.9322 & 0.06781 \\
$\frac{{ }^{\mathrm{a}} x_{2}{ }^{(\mathrm{s})}: \text { initial mole fraction of component } 2 \text { in the solution. }}{{ }^{\mathrm{b}} x_{2}: \text { mole fraction of component } 2 \text { in the saturated solution. }}$ \\
${ }^{\mathrm{c}} x_{1}:$ mole fraction solubility of the solute.
\end{tabular}

\section{Auxiliary Information}

\section{Method/Apparatus/Procedure:}

Constant-temperature bath, calorimetric thermometer, and an ultraviolet/ visible spectrophotometer.

Excess solute and solvent were placed in amber glass bottles and allowed to equilibrate for several days at constant temperature. Attainment of equilibrium was verified by several repetitive measurements and by approaching equilibrium from supersaturation. Aliquots of saturated solutions were transferred through a coarse filter into tared volumetric flasks, weighed and diluted with 2-propanol. Concentrations were determined by spectrophotometric measurements at $280 \mathrm{~nm}$.

\section{Source and Purity of Chemicals:}

(1) $98 \%$, Aldrich Chemical Company, Milwaukee, WI, USA, was recrystallized several times from methanol.

(2) $99 \%$, Aldrich Chemical Company, stored over molecular sieves before use.

\section{Estimated Error:}

Temperature: $\pm 0.1 \mathrm{~K}$.

$x_{1}: \pm 1.5 \%$ (relative error).

\begin{tabular}{ll}
\hline \hline Components: & Original Measurements: \\
(1) Fluorene; $\mathrm{C}_{13} \mathrm{H}_{10} ;[92-52-4]$ & ${ }^{15}$ A. Aoulmi, M. Bouroukba, \\
(2) Octacosane; $\mathrm{C}_{28} \mathrm{H}_{58} ;[630-02-4]$ & R. Solimando, and \\
& M. Rogalski, Fluid Phase \\
& Equilib. 110, 283 (1995). \\
\hline Variables: & Prepared by: \\
Temperature & W. E. Acree, Jr. \\
\hline
\end{tabular}


Experimental Values

\begin{tabular}{lcc}
\hline \hline$T / \mathrm{K}$ & $x_{2}{ }^{\mathrm{a}}$ & $x_{1}{ }^{\mathrm{b}}$ \\
\hline 333.91 & 1.0000 & 0.0000 \\
332.95 & 0.8981 & 0.1019 \\
332.69 & 0.7881 & 0.2119 \\
332.19 & 0.7540 & 0.2460 \\
340.80 & 0.6916 & 0.3084 \\
352.41 & 0.6011 & 0.3989 \\
359.00 & 0.4935 & 0.5065 \\
365.60 & 0.4002 & 0.5998 \\
370.67 & 0.2997 & 0.7003 \\
377.23 & 0.2002 & 0.7998 \\
382.34 & 0.1001 & 0.8999 \\
387.25 & 0.0000 & 1.0000 \\
\hline
\end{tabular}

${ }^{\mathrm{a}} x_{2}$ : mole fraction of component 2 in the saturated solution.

${ }^{b} x_{1}$ : mole fraction of the polycyclic aromatic hydrocarbon (component 1 ).

The authors employed a SSF to describe the activity coefficients of fluorene and octacosane calculated from the solidliquid equilibrium data. The SSF model gave a calculated eutectic temperature of $T / \mathrm{K}=332.3$ and eutectic mole fraction of fluorene of $x_{1}=0.226$.

\section{Auxiliary Information}

\section{Method/Apparatus/Procedure:}

Differential scanning calorimeter.

The phase diagram was determined using a differential scanning calorimeter Measurements were performed at a constant fixed scanning rate of $0.5 \mathrm{~K} / \mathrm{min}$.

\section{Source and Purity of Chemicals:}

(1) $98 \%$, Aldrich Chemical Company, Milwaukee, WI, USA, no purification details were given in the paper.

(2) $99 \%$, Aldrich Chemical Company, purification details were not given in the paper.

\section{Estimated Error:}

Temperature: Authors state a global accuracy of $\pm 1 \%$ regarding their measurements.

$x_{1}: \pm 0.0002$ (estimated by compiler).

\begin{tabular}{ll}
\hline \hline Components: & Original Measurements: \\
(1) Fluorene; $\mathrm{C}_{13} \mathrm{H}_{10} ;[92-52-4]$ & ${ }^{49} \mathrm{D}$. M. Stovall, K. R. Hoover, \\
(2) Cyclohexane; $\mathrm{C}_{6} \mathrm{H}_{12} ;[110-82-7]$ & W. E. Acree, Jr., and M. H. \\
& Abraham, Polycyclic Aromat. \\
& Compd. 25, 313 (2005). \\
\hline Variables: & Prepared by: \\
$T / \mathrm{K}=298.15$ & W. E. Acree, Jr. \\
\hline
\end{tabular}

Experimental Values

\begin{tabular}{|c|c|c|}
\hline$x_{2}{ }^{(\mathrm{s}) \mathrm{a}}$ & $x_{2}{ }^{b}$ & $x_{1}{ }^{c}$ \\
\hline 1.0000 & 0.9623 & 0.03768 \\
\hline
\end{tabular}

\section{Auxiliary Information}

Method/Apparatus/Procedure:

Constant-temperature bath, calorimetric thermometer, and an ultraviolet/ visible spectrophotometer.

Excess solute and solvent were placed in amber glass bottles and allowed to equilibrate for several days at constant temperature. Attainment of equilibrium was verified by several repetitive measurements and by approaching equilibrium from supersaturation. Aliquots of saturated solutions were transferred through a coarse filter into tared volumetric flasks, weighed and diluted with 2-propanol. Concentrations were determined by spectrophotometric measurements at $280 \mathrm{~nm}$.

\section{Source and Purity of Chemicals:}

(1) $98 \%$, Aldrich Chemical Company, Milwaukee, WI, USA, was recrystallized several times from methanol.

(2) $99.9+\%$, HPLC Grade, Aldrich Chemical Company, stored over molecular sieves before use.

Estimated Error:

Temperature: $\pm 0.1 \mathrm{~K}$.

$x_{1}: \pm 1.5 \%$ (relative error)

\begin{tabular}{ll}
\hline \hline Components: & Original Measurements: \\
(1) Fluorene; $\mathrm{C}_{13} \mathrm{H}_{10} ;[92-52-4]$ & ${ }^{49}$ D. M. Stovall, K. R. Hoover, W. E. \\
(2) Methylcyclohexane; $\mathrm{C}_{7} \mathrm{H}_{14} ;$ & Acree, Jr., and M. H. Abraham, \\
{$[108-87-2]$} & $\begin{array}{l}\text { Polycyclic Aromat. Compd. 25, 313 } \\
\text { (2005). }\end{array}$ \\
\hline Variables: & Prepared by: \\
$T / \mathrm{K}=298.15$ & W. E. Acree, Jr. \\
\hline
\end{tabular}

\section{Experimental Values}

\begin{tabular}{lcc}
\hline \hline$x_{2}{ }^{(\mathrm{s}) \mathrm{a}}$ & $x_{2}{ }^{\mathrm{b}}$ & $x_{1}{ }^{\mathrm{c}}$ \\
\hline 1.0000 & 0.9602 & 0.03975 \\
$\frac{{ }^{a} x_{2}{ }^{(\mathrm{s})}: \text { initial mole fraction of component } 2 \text { in the solution. }}{{ }^{\mathrm{b}} x_{2}: \text { mole fraction of component } 2 \text { in the saturated solution. }}$ \\
${ }^{c} x_{1}:$ mole fraction solubility of the solute.
\end{tabular}

\section{Auxiliary Information}

\section{Method/Apparatus/Procedure:}

Constant-temperature bath, calorimetric thermometer, and an ultraviolet/ visible spectrophotometer.

Excess solute and solvent were placed in amber glass bottles and allowed to equilibrate for several days at constant temperature. Attainment of equilibrium was verified by several repetitive measurements and by approaching equilibrium from supersaturation. Aliquots of saturated solutions were transferred through a coarse filter into tared volumetric flasks, weighed and diluted with 2-propanol. Concentrations were determined by spectrophotometric measurements at $280 \mathrm{~nm}$.

\section{Source and Purity of Chemicals:}

(1) $98 \%$, Aldrich Chemical Company, Milwaukee, WI, USA, was recrystallized several times from methanol.

(2) $99+\%$, anhydrous, Aldrich Chemical Company, stored over molecular sieves before use.

\section{Estimated Error:}

Temperature: $\pm 0.1 \mathrm{~K}$.

$x_{1}: \pm 1.5 \%$ (relative error). 


\section{Components:}

(1) Fluorene; $\mathrm{C}_{13} \mathrm{H}_{10} ;[92-52-4]$

(2) Cyclooctane; $\mathrm{C}_{8} \mathrm{H}_{16}$; [292-64-8]

\section{Original Measurements:}

${ }^{49}$ D. M. Stovall, K. R. Hoover, W. E. Acree, Jr., and M. H. Abraham,

Polycyclic Aromat. Compd. 25, 313 (2005).

\begin{tabular}{ll}
\hline Variables: & Prepared by: \\
$T / \mathrm{K}=298.15$ & W. E. Acree, Jr. \\
\hline
\end{tabular}

Experimental Values

\begin{tabular}{lcc}
\hline \hline$x_{2}{ }^{(\mathrm{s}) \mathrm{a}}$ & $x_{2}{ }^{\mathrm{b}}$ & $x_{1}{ }^{\mathrm{c}}$ \\
\hline 1.0000 & 0.9502 & 0.04977 \\
\hline
\end{tabular}

${ }^{\mathrm{a}_{2}}{ }^{(\mathrm{s})}$ : initial mole fraction of component 2 in the solution.

${ }^{b} x_{2}$ : mole fraction of component 2 in the saturated solution.

${ }^{c} x_{1}$ : mole fraction solubility of the solute.

\section{Auxiliary Information}

\section{Method/Apparatus/Procedure:}

Constant-temperature bath, calorimetric thermometer, and an ultraviolet/ visible spectrophotometer.

Excess solute and solvent were placed in amber glass bottles and allowed to equilibrate for several days at constant temperature. Attainment of equilibrium was verified by several repetitive measurements and by approaching equilibrium from supersaturation. Aliquots of saturated solutions were transferred through a coarse filter into tared volumetric flasks, weighed and diluted with 2-propanol. Concentrations were determined by

spectrophotometric measurements at $280 \mathrm{~nm}$.

\section{Source and Purity of Chemicals:}

(1) $98 \%$, Aldrich Chemical Company, Milwaukee, WI, USA, was recrystallized several times from methanol.

(2) 99+\%, Lancaster Synthesis, USA, stored over molecular sieves before use.

\section{Estimated Error:}

Temperature: $\pm 0.1 \mathrm{~K}$.

$x_{1}: \pm 1.5 \%$ (relative error).

\begin{tabular}{|c|c|}
\hline $\begin{array}{l}\text { Components: } \\
\text { (1) Fluorene; } \mathrm{C}_{13} \mathrm{H}_{10} ;[92-52-4] \\
\text { (2) 2,2,4-Trimethylpentane; } \mathrm{C}_{8} \mathrm{H}_{18} \text {; } \\
\text { [540-84-1] }\end{array}$ & $\begin{array}{l}\text { Original Measurements: } \\
{ }^{49} \text { D. M. Stovall, K. R. Hoover, W. E. } \\
\text { Acree, Jr., and M. H. Abraham, } \\
\text { Polycyclic Aromat. Compd. 25, } 313 \\
(2005) \text {. }\end{array}$ \\
\hline $\begin{array}{l}\text { Variables: } \\
T / \mathrm{K}=298.15\end{array}$ & $\begin{array}{l}\text { Prepared by: } \\
\text { W. E. Acree, Jr. }\end{array}$ \\
\hline
\end{tabular}

Experimental Values

\begin{tabular}{lcc}
\hline \hline$x_{2}{ }^{(\mathrm{s}) \mathrm{a}}$ & $x_{2}{ }^{\mathrm{b}}$ & $x_{1}{ }^{\mathrm{c}}$ \\
\hline 1.0000 & 0.9746 & 0.02537 \\
\hline
\end{tabular}

${ }^{\mathrm{a}} x_{2}{ }^{(\mathrm{s})}$ : initial mole fraction of component 2 in the solution.

${ }^{\mathrm{b}} x_{2}$ : mole fraction of component 2 in the saturated solution.

${ }^{c} x_{1}$ : mole fraction solubility of the solute.

\section{Auxiliary Information}

\section{Method/Apparatus/Procedure:}

Constant-temperature bath, calorimetric thermometer, and an ultraviolet/ visible spectrophotometer.

Excess solute and solvent were placed in amber glass bottles and allowed to equilibrate for several days at constant temperature. Attainment of equilibrium was verified by several repetitive measurements and by approaching equilibrium from supersaturation. Aliquots of saturated solutions were transferred through a coarse filter into tared volumetric flasks, weighed and diluted with 2-propanol. Concentrations were determined by spectrophotometric measurements at $280 \mathrm{~nm}$.

Source and Purity of Chemicals:

(1) $98 \%$, Aldrich Chemical Company, Milwaukee, WI, USA, was recrystallized several times from methanol.

(2) $99.7 \%$, HPLC Grade, Aldrich Chemical Company, stored over molecular sieves before use.

Estimated Error:

Temperature: $\pm 0.1 \mathrm{~K}$.

$x_{1}: \pm 1.5 \%$ (relative error).

\begin{tabular}{ll}
\hline \hline Components: & Original Measurements: \\
(1) Fluorene; $\mathrm{C}_{13} \mathrm{H}_{10} ;[92-52-4]$ & ${ }^{49} \mathrm{D}$. M. Stovall, K. R. Hoover, W. \\
(2) tert-Butylcyclohexane; $\mathrm{C}_{10} \mathrm{H}_{20} ;$ & E. Acree, Jr., and M. H. Abraham, \\
{$[3178-22-1]$} & Polycyclic Aromat. Compd. 25, \\
& $313(2005)$. \\
\hline Variables: & Prepared by: \\
$T / \mathrm{K}=298.15$ & W. E. Acree, Jr. \\
\hline
\end{tabular}

Experimental Values

\begin{tabular}{lcc}
\hline \hline$x_{2}{ }^{(\mathrm{s}) \mathrm{a}}$ & $x_{2}{ }^{\mathrm{b}}$ & $x_{1}{ }^{\mathrm{c}}$ \\
\hline 1.0000 & 0.9561 & 0.04385 \\
$\frac{{ }^{a} x_{2}{ }^{(\mathrm{s})}: \text { initial mole fraction of component } 2 \text { in the solution. }}{{ }^{\mathrm{b}} x_{2}: \text { mole fraction of component } 2 \text { in the saturated solution. }}$ \\
${ }^{{ }^{2} x_{1}: \text { mole fraction solubility of the solute. }}$
\end{tabular}

\section{Auxiliary Information}

\section{Method/Apparatus/Procedure:}

Constant-temperature bath, calorimetric thermometer, and an ultraviolet/ visible spectrophotometer.

Excess solute and solvent were placed in amber glass bottles and allowed to equilibrate for several days at constant temperature. Attainment of equilibrium was verified by several repetitive measurements and by approaching equilibrium from supersaturation. Aliquots of saturated solutions were transferred through a coarse filter into tared volumetric flasks, weighed and diluted with 2-propanol. Concentrations were determined by spectrophotometric measurements at $280 \mathrm{~nm}$.

\section{Source and Purity of Chemicals:}

(1) $98 \%$, Aldrich Chemical Company, Milwaukee, WI, USA, was recrystallized several times from methanol.

(2) $99+\%$, Aldrich Chemical Company, stored over molecular sieves before use.

Estimated Error:

Temperature: $\pm 0.1 \mathrm{~K}$.

$x_{1}: \pm 1.5 \%$ (relative error). 


\section{Components:}

(1) Fluorene; $\mathrm{C}_{13} \mathrm{H}_{10} ;[92-52-4]$

(2) Squalane; $\mathrm{C}_{30} \mathrm{H}_{62} ;[111-01-3]$

\begin{tabular}{ll}
\hline Variables: & Prepared by: \\
Temperature & W. E. Acree, Jr. \\
\hline
\end{tabular}

Experimental Values

\begin{tabular}{lcc}
\hline \hline$T / \mathrm{K}$ & $x_{2}{ }^{\mathrm{a}}$ & $x_{1}{ }^{\mathrm{b}}$ \\
\hline 324.35 & 0.7999 & 0.2001 \\
340.45 & 0.6983 & 0.3017 \\
351.85 & 0.5992 & 0.4008 \\
360.95 & 0.5014 & 0.4986 \\
365.15 & 0.4504 & 0.5496 \\
368.75 & 0.3899 & 0.6101 \\
371.25 & 0.3502 & 0.6498 \\
373.85 & 0.2968 & 0.7032 \\
376.35 & 0.2506 & 0.7494 \\
377.95 & 0.2024 & 0.7976 \\
380.05 & 0.1514 & 0.8486 \\
381.65 & 0.1000 & 0.9000 \\
383.85 & 0.0540 & 0.9460
\end{tabular}

${ }^{\mathrm{a}} x_{2}$ : mole fraction of component 2 in the saturated solution.

${ }^{b} x_{1}$ : mole fraction of the polycyclic aromatic hydrocarbon (component 1).

The authors employed a SSF to describe the activity coefficients of fluorene and squalane calculated from the solid-liquid equilibrium data. The SSF model gave a calculated eutectic temperature of $T / \mathrm{K}=234.0$ and eutectic mole fraction of fluorene of $x_{1}=0.01$.

\section{Auxiliary Information}

\section{Method/Apparatus/Procedure:}

Simple thermal analysis device constructed in the authors' laboratory, and a high precision platinum-resistance thermometer.

Phase diagram was determined using a simple thermal device. A sample of known composition was placed in a thermostatted glass tube, melted and then slowly cooled at a rate of about $1 \mathrm{~K} / \mathrm{min}$. The temperature of the sample was measured with a high precision platinum-resistance thermometer. The crystallization temperature was obtained from a plot of the cooling curve versus time. Each measurement was repeated three times.

\section{Source and Purity of Chemicals:}

(1) $98 \%$, Aldrich Chemical Company, Milwaukee, WI, USA, no purification details were given in the paper.

(2) $99 \%$, Aldrich Chemical Company, purification details were not given in the paper.

\section{Estimated Error:}

Temperature: Authors state a global accuracy of $\pm 1 \%$ regarding their measurements.

$x_{1}: \pm 0.0002$ (estimated by compiler).

\subsection{Fluorene solubility data in aromatic hydrocarbons}

\begin{tabular}{ll}
\hline \hline Components: & Original Measurements: \\
(1) Fluorene; $\mathrm{C}_{13} \mathrm{H}_{10} ;[92-52-4]$ & ${ }^{51}$ Y. Han and Z. Wang, J. Chem. \\
(2) Benzene; $\mathrm{C}_{6} \mathrm{H}_{6} ;[71-43-2]$ & Eng. Data 54, 148 (2009). \\
\hline Variables: & Prepared by: \\
Temperature & W. E. Acree, Jr. \\
\hline
\end{tabular}

Experimental Values

\begin{tabular}{lcl}
\hline \hline$T / \mathrm{K}$ & $x_{2}{ }^{\mathrm{a}}$ & \multicolumn{1}{c}{$x_{1}{ }^{\mathrm{b}}$} \\
\hline 283.00 & 0.9117 & 0.08832 \\
288.00 & 0.9018 & 0.09824 \\
293.00 & 0.8909 & 0.1091 \\
298.00 & 0.8743 & 0.1257 \\
303.20 & 0.8568 & 0.1432 \\
308.00 & 0.8416 & 0.1584 \\
313.00 & 0.8165 & 0.1835 \\
318.00 & 0.7909 & 0.2091 \\
323.00 & 0.7654 & 0.2346 \\
\hline
\end{tabular}

${ }^{a} x_{2}$ : mole fraction of component 2 in the saturated solution.

${ }^{\mathrm{b}} x_{1}$ : mole fraction of the polycyclic aromatic hydrocarbon (component 1).

\section{Auxiliary Information}

\section{Method/Apparatus/Procedure:}

Double-jacketed glass vessel, laser generator, water bath, photoelectric transformer, digital display.

Solubilities were determined by a synthetic-dynamic method. Known masses of solute and solvent were placed in a dissolving flask. The solid in the solution dissolved as the temperature was increased. The temperature at which the last amount of solid dissolved was recorded as the solid-liquid equilibrium temperature. The disappearance of the solid solute was determined by laser monitoring.

\section{Source and Purity of Chemicals:}

(1) $99+\%$, Chemical source and purification method were not given. (2) $99.8+\%$, Analytical Reagent Grade, Tianjin Kewei Company, China, no information given regarding any further purification.

\section{Estimated Error:}

Temperature: $\pm 0.2 \mathrm{~K}$.

$x_{1}$ : within 0.0200 (relative uncertainty).

\begin{tabular}{|c|c|}
\hline $\begin{array}{l}\text { Components: } \\
\text { (1) Fluorene; } \mathrm{C}_{13} \mathrm{H}_{10} ;[92-52-4] \\
\text { (2) Methylbenzene; } \mathrm{C}_{7} \mathrm{H}_{8} ;[108-88-8]\end{array}$ & $\begin{array}{l}\text { Original Measurements: } \\
{ }^{51} \text { Y. Han and Z. Wang, J. Chem. } \\
\text { Eng. Data 54, } 148 \text { (2009). }\end{array}$ \\
\hline $\begin{array}{l}\text { Variables: } \\
\text { Temperature }\end{array}$ & $\begin{array}{l}\text { Prepared by: } \\
\text { W. E. Acree, Jr. }\end{array}$ \\
\hline
\end{tabular}


Experimental Values

\begin{tabular}{lcc}
\hline \hline$T / \mathrm{K}$ & $x_{2}{ }^{\mathrm{a}}$ & $x_{1}{ }^{\mathrm{b}}$ \\
\hline 283.00 & 0.9370 & 0.06295 \\
288.00 & 0.9191 & 0.08088 \\
293.00 & 0.9033 & 0.09674 \\
298.00 & 0.8761 & 0.1239 \\
303.20 & 0.8475 & 0.1525 \\
308.00 & 0.8048 & 0.1952 \\
313.00 & 0.7503 & 0.2497 \\
318.00 & 0.7001 & 0.2999 \\
323.00 & 0.6304 & 0.3696
\end{tabular}

${ }^{a_{x_{2}}}$ : mole fraction of component 2 in the saturated solution.

${ }^{\mathrm{b}} x_{1}$ : mole fraction of the polycyclic aromatic hydrocarbon (component 1 ).

\section{Auxiliary Information}

\section{Method/Apparatus/Procedure:}

Double-jacketed glass vessel, laser generator, water bath, photoelectric transformer, digital display.

Solubilities were determined by a synthetic-dynamic method. Known masses of solute and solvent were placed in a dissolving flask. The solid in the solution dissolved as the temperature was increased. The temperature at which the last amount of solid dissolved was recorded as the solid-liquid equilibrium temperature. The disappearance of the solid solute was determined by laser monitoring.

\section{Source and Purity of Chemicals:}

(1) $99+\%$, Chemical source and purification method were not given.

(2) $99.8+\%$, Analytical Reagent Grade, Tianjin Kewei Company, China, no information given regarding any further purification.

\section{Estimated Error:}

Temperature: $\pm 0.2 \mathrm{~K}$.

$x_{1}$ : within 0.0200 (relative uncertainty).

\begin{tabular}{|c|c|}
\hline $\begin{array}{l}\text { Components: } \\
\text { (1) Fluorene; } \mathrm{C}_{13} \mathrm{H}_{10} ;[92-52-4] \\
\text { (2) Methylbenzene; } \mathrm{C}_{7} \mathrm{H}_{8} ;[108-88-8]\end{array}$ & $\begin{array}{l}\text { Original Measurements: } \\
{ }^{50} \text { Z. Zou, L. Dang, P. Liu, and } \\
\text { H. Wei, J. Chem. Eng. Data } \\
\text { 52, } 1501 \text { (2007). }\end{array}$ \\
\hline $\begin{array}{l}\text { Variables: } \\
\text { Temperature }\end{array}$ & $\begin{array}{l}\text { Prepared by: } \\
\text { W. E. Acree, Jr. }\end{array}$ \\
\hline
\end{tabular}

Experimental Values

\begin{tabular}{lcl}
\hline \hline$T / \mathrm{K}$ & $x_{2}{ }^{\mathrm{a}}$ & $x_{1}{ }^{\mathrm{b}}$ \\
\hline 283.27 & 0.9366 & 0.06341 \\
288.77 & 0.9181 & 0.08185 \\
293.72 & 0.8981 & 0.1019 \\
298.89 & 0.8705 & 0.1295 \\
303.17 & 0.8455 & 0.1545 \\
308.81 & 0.7995 & 0.2005 \\
313.17 & 0.7471 & 0.2521 \\
319.27 & 0.6923 & 0.3077 \\
324.95 & 0.6329 & 0.3671 \\
\hline
\end{tabular}

${ }^{\mathrm{a}} x_{2}$ : mole fraction of component 2 in the saturated solution.

${ }^{b} x_{1}$ : mole fraction of the polycyclic aromatic hydrocarbon (component 1 ).

\section{Auxiliary Information}

Method/Apparatus/Procedure:

Thermostated bath, thermometer

Very little experimental details were provided. Solubilities were determined by a synthetic method. Known masses of solute and solvent were placed in a dissolving flask. The solute and solvent mixture was stirred with a magnetic stirrer. Small amounts of solute were added at predetermined intervals until no more solid would dissolve. The point at which no more solid would dissolve was taken to be the point of solid-liquid equilibrium. The paper did not specify how the disappearance of the solid was monitored; however, the papers that the authors referenced for the experimental method used a laser monitoring method.

\section{Source and Purity of Chemicals:}

(1) $99.5 \%$, Chemical source and purification method were not given. (2) $99.8 \%$, Analytical Reagent Grade, Tianjin Kewei Company, China, no information given regarding any further purification.

Estimated Error:

Temperature: $\pm 0.2 \mathrm{~K}$.

$x_{1}$ : within 0.0200 (relative uncertainty).

\subsection{Fluorene solubility data in esters}

\begin{tabular}{|c|c|}
\hline $\begin{array}{l}\text { Components: } \\
\text { (1) Fluorene; } \mathrm{C}_{13} \mathrm{H}_{10} ;[92-52-4] \\
\text { (2) } 1,2,3-\text { Triacetoxypropane (Triacetin); } \\
\mathrm{C}_{9} \mathrm{H}_{14} \mathrm{O}_{6} ;[102-76-1]\end{array}$ & $\begin{array}{l}\text { Original Measurements: } \\
{ }^{49} \text { D. M. Stovall, K. R. Hoover, W } \\
\text { E. Acree, Jr., and M. H. Abraham } \\
\text { Polycyclic Aromat. Compd. 25, } \\
313 \text { (2005). }\end{array}$ \\
\hline $\begin{array}{l}\text { Variables: } \\
T / \mathrm{K}=298.15\end{array}$ & $\begin{array}{l}\text { Prepared by: } \\
\text { W. E. Acree, Jr. }\end{array}$ \\
\hline
\end{tabular}

Experimental Values

\begin{tabular}{lcc}
\hline \hline$\frac{x_{2}{ }^{(\mathrm{s}) \mathrm{a}}}{x_{2}{ }^{\mathrm{b}}}$ & $x_{1}{ }^{\mathrm{c}}$ \\
\hline 1.0000 & 0.9333 & 0.06667 \\
${ }^{\mathrm{a}}{ }_{x_{2}{ }^{(\mathrm{s})}: \text { initial mole fraction of component } 2 \text { in the solution. }}$ \\
${ }^{\mathrm{b}} x_{2}:$ mole fraction of component 2 in the saturated solution. \\
${ }^{\mathrm{c}}{ }_{x_{1}: \text { mole fraction solubility of the solute. }}$
\end{tabular}

Auxiliary Information

\section{Method/Apparatus/Procedure:}

Constant-temperature bath, calorimetric thermometer, and an ultraviolet/ visible spectrophotometer.

Excess solute and solvent were placed in amber glass bottles and allowed to equilibrate for several days at constant temperature. Attainment of equilibrium was verified by several repetitive measurements and by approaching equilibrium from supersaturation. Aliquots of saturated solutions were transferred through a coarse filter into tared volumetric flasks, weighed and diluted with 2-propanol. Concentrations were determined by spectrophotometric measurements at $280 \mathrm{~nm}$.

Source and Purity of Chemicals:

(1) $98 \%$, Aldrich Chemical Company, Milwaukee, WI, USA, was recrystallized several times from methanol.

(2) $99 \%$, Acros Organics, USA, stored over molecular sieves before use. 
Estimated Error:

Temperature: $\pm 0.1 \mathrm{~K}$.

$x_{1}: \pm 1.5 \%$ (relative error)

\subsection{Fluorene solubility data in ethers}

\begin{tabular}{ll}
\hline \hline Components: & Original Measurements: \\
(1) Fluorene; $\mathrm{C}_{13} \mathrm{H}_{10} ;[92-52-4]$ & ${ }^{49} \mathrm{D}$. M. Stovall, K. R. Hoover, W. \\
$(2) 2,2^{\prime}-\mathrm{O} x y b i s p r o p a n e ; \mathrm{C}_{6} \mathrm{H}_{14} \mathrm{O} ;$ & E. Acree, Jr., and M. H. Abraham, \\
{$[108-20-3]$} & Polycyclic Aromat. Compd. 25, \\
& 313 (2005). \\
\hline Variables: & Prepared by: \\
$T / \mathrm{K}=298.15$ & W. E. Acree, Jr. \\
\hline
\end{tabular}

Experimental Values

\begin{tabular}{lcc}
\hline \hline$x_{2}{ }^{(\mathrm{s}) \mathrm{a}}$ & $x_{2}{ }^{\mathrm{b}}$ & $x_{1}{ }^{\mathrm{c}}$ \\
\hline 1.0000 & 0.9468 & 0.05324 \\
${ }^{{ }^{a} x_{2}{ }^{(\mathrm{s})}: \text { initial mole fraction of component } 2 \text { in the solution. }}$ \\
${ }^{\mathrm{b}} x_{2}:$ mole fraction of component 2 in the saturated solution. \\
${ }^{{ }^{c} x_{1}: \text { mole fraction solubility of the solute. }}$
\end{tabular}

\section{Auxiliary Information}

\section{Method/Apparatus/Procedure:}

Constant-temperature bath, calorimetric thermometer, and an ultraviolet/ visible spectrophotometer.

Excess solute and solvent were placed in amber glass bottles and allowed to equilibrate for several days at constant temperature. Attainment of equilibrium was verified by several repetitive measurements and by approaching equilibrium from supersaturation. Aliquots of saturated solutions were transferred through a coarse filter into tared volumetric flasks, weighed and diluted with 2-propanol. Concentrations were determined by spectrophotometric measurements at $280 \mathrm{~nm}$.

Source and Purity of Chemicals:

(1) $98 \%$, Aldrich Chemical Company, Milwaukee, WI, USA, was recrystallized several times from methanol.

(2) $99 \%$, anhydrous, Aldrich Chemical Company, stored over molecular sieves before use.

Estimated Error:

Temperature: $\pm 0.1 \mathrm{~K}$.

$x_{1}: \pm 1.5 \%$ (relative error).

\begin{tabular}{ll}
\hline \hline Components: & Original Measurements: \\
(1) Fluorene; $\mathrm{C}_{13} \mathrm{H}_{10} ;[92-52-4]$ & ${ }^{49}$ D. M. Stovall, K. R. Hoover, W. \\
(2) $1,1^{\prime}-$-Oxybisbutane; $\mathrm{C}_{8} \mathrm{H}_{18} \mathrm{O} ;$ & E. Acree, Jr., and M. H. Abraham, \\
{$[142-96-1]$} & Polycyclic Aromat. Compd. 25, \\
& 313 (2005). \\
\hline Variables: & Prepared by: \\
$T / \mathrm{K}=298.15$ & W. E. Acree, Jr. \\
\hline
\end{tabular}

Experimental Values

\begin{tabular}{|c|c|c|}
\hline$x_{2}{ }^{(\mathrm{s}) \mathrm{a}}$ & $x_{2}{ }^{b}$ & $x_{1}{ }^{\mathrm{c}}$ \\
\hline 1.0000 & 0.9277 & 0.07226 \\
\hline
\end{tabular}

Auxiliary Information

\section{Method/Apparatus/Procedure:}

Constant-temperature bath, calorimetric thermometer, and an ultraviolet/ visible spectrophotometer.

Excess solute and solvent were placed in amber glass bottles and allowed to equilibrate for several days at constant temperature. Attainment of equilibrium was verified by several repetitive measurements and by approaching equilibrium from supersaturation. Aliquots of saturated solutions were transferred through a coarse filter into tared volumetric flasks, weighed and diluted with 2-propanol. Concentrations were determined by spectrophotometric measurements at $280 \mathrm{~nm}$.

\section{Source and Purity of Chemicals:}

(1) $98 \%$, Aldrich Chemical Company, Milwaukee, WI, USA, was recrystallized several times from methanol.

(2) $99.3 \%$, anhydrous, Aldrich Chemical Company, stored over molecular sieves before use.

\section{Estimated Error:}

Temperature: $\pm 0.1 \mathrm{~K}$.

$x_{1}: \pm 1.5 \%$ (relative error)

\subsection{Fluorene solubility data in haloalkanes, haloalkenes, and haloaromatic hydrocarbons}

\begin{tabular}{|c|c|}
\hline $\begin{array}{l}\text { Components: } \\
\text { (1) Fluorene; } \mathrm{C}_{13} \mathrm{H}_{10} ;[92-52-4] \\
\text { (2) Trichloromethane; } \mathrm{CHCl}_{3} ;[67-66-3]\end{array}$ & $\begin{array}{l}\text { Original Measurements: } \\
{ }^{51} \text { Y. Han and Z. Wang, J. Chem. } \\
\text { Eng. Data 54, } 148 \text { (2009). }\end{array}$ \\
\hline $\begin{array}{l}\text { Variables: } \\
\text { Temperature }\end{array}$ & $\begin{array}{l}\text { Prepared by: } \\
\text { W. E. Acree, Jr. }\end{array}$ \\
\hline
\end{tabular}

Experimental Values

\begin{tabular}{lcl}
\hline \hline$T / \mathrm{K}$ & $x_{2}{ }^{\mathrm{a}}$ & \multicolumn{1}{c}{$x_{1}{ }^{\mathrm{b}}$} \\
\hline 283.00 & 0.9107 & 0.08928 \\
288.00 & 0.8984 & 0.1016 \\
293.00 & 0.8856 & 0.1144 \\
298.00 & 0.8682 & 0.1318 \\
303.20 & 0.8474 & 0.1526 \\
308.00 & 0.8269 & 0.1731 \\
313.00 & 0.8048 & 0.1952 \\
318.00 & 0.7801 & 0.2199 \\
323.00 & 0.7503 & 0.2497
\end{tabular}

${ }^{a} x_{2}$ : mole fraction of component 2 in the saturated solution.

${ }^{b} x_{1}$ : mole fraction of the polycyclic aromatic hydrocarbon (component 1). 


\section{Auxiliary Information}

\section{Method/Apparatus/Procedure:}

Double-jacketed glass vessel, laser generator, water bath, photoelectric transformer, digital display.

Solubilities were determined by a synthetic-dynamic method. Known masses of solute and solvent were placed in a dissolving flask. The solid in the solution dissolved as the temperature was increased. The temperature at which last amount of solid dissolved was recorded as the solid-liquid equilibrium temperature. The disappearance of the solid solute was determined by laser monitoring.

Source and Purity of Chemicals:

(1) $99+\%$, Chemical source and purification method were not given.

(2) $99.8+\%$, Analytical Reagent Grade, Tianjin Kewei Company, China, no information given regarding any further purification.

Estimated Error:

Temperature: $\pm 0.2 \mathrm{~K}$.

$x_{1}$ : within 0.0200 (relative uncertainty).

\subsection{Fluorene solubility data in alcohols}

\section{Components:}

(1) Fluorene; $\mathrm{C}_{13} \mathrm{H}_{10} ;[92-52-4]$

(2) Methanol; $\mathrm{CH}_{4} \mathrm{O} ;[67-56-1]$

Original Measurements:

${ }^{48}$ C. I. Monárrez, W. E. Acree,

Jr., and M. H. Abraham, Phys. Chem. Liq. 40, 581 (2002).

\section{Variables:}

$T / \mathrm{K}=298.15$

Prepared by:

W. E. Acree, Jr.

\section{Experimental Values}

\begin{tabular}{lcc}
\hline \hline$x_{2}{ }^{(\mathrm{s})}$ & $x_{2}$ & $x_{1}$ \\
\hline 1.0000 & 0.9955 & 0.00449 \\
\hline
\end{tabular}

${ }^{\mathrm{a}} x_{2}{ }^{(\mathrm{s})}$ : initial mole fraction of component 2 in the solution.

${ }^{b} x_{2}$ : mole fraction of component 2 in the saturated solution.

${ }^{c} x_{1}$ : mole fraction solubility of the solute.

\section{Auxiliary Information}

\section{Method/Apparatus/Procedure:}

Constant-temperature bath, calorimetric thermometer, and an ultraviolet/ visible spectrophotometer.

Excess solute and solvent were placed in amber glass bottles and allowed to equilibrate for several days at constant temperature. Attainment of equilibrium was verified by several repetitive measurements and by approaching equilibrium from supersaturation. Aliquots of saturated solutions were transferred through a coarse filter into tared volumetric flasks, weighed and diluted with 2-propanol. Concentrations were determined by

spectrophotometric measurements at $280 \mathrm{~nm}$.

Source and Purity of Chemicals:

(1) $98 \%$, Aldrich Chemical Company, Milwaukee, WI, USA, was recrystallized several times from methanol.

(2) $99.8 \%$, anhydrous, Aldrich Chemical Company, stored over molecular sieves before use.

Estimated Error:

Temperature: $\pm 0.1 \mathrm{~K}$.

$x_{1}: \pm 1.5 \%$ (relative error).

\begin{tabular}{ll}
\hline \hline Components: & Original Measurements: \\
(1) Fluorene; $\mathrm{C}_{13} \mathrm{H}_{10} ;[92-52-4]$ & ${ }^{48}$ C. I. Monárrez, W. E. Acree, Jr., and \\
(2) Ethanol; $\mathrm{C}_{2} \mathrm{H}_{6} \mathrm{O} ;[64-17-5]$ & M. H. Abraham, Phys. Chem. Liq. 40, \\
& $581(2002)$. \\
\hline Variables: & Prepared by: \\
$T / \mathrm{K}=298.15$ & W. E. Acree, Jr. \\
\hline
\end{tabular}

Experimental Values

\begin{tabular}{lcc}
\hline \hline$\frac{x_{2}{ }^{(\mathrm{s})}}{x_{2}}$ & $x_{1}$ \\
\hline 1.0000 & 0.9926 & 0.00743 \\
${ }^{\mathrm{a}} x_{2}{ }^{(\mathrm{s})}:$ initial mole fraction of component 2 in the solution. \\
${ }^{\mathrm{b}} x_{2}:$ mole fraction of component 2 in the saturated solution. \\
${ }^{\mathrm{c}} x_{1}$ : mole fraction solubility of the solute.
\end{tabular}

\section{Auxiliary Information}

\section{Method/Apparatus/Procedure:}

Constant-temperature bath, calorimetric thermometer, and an ultraviolet/ visible spectrophotometer.

Excess solute and solvent were placed in amber glass bottles and allowed to equilibrate for several days at constant temperature. Attainment of equilibrium was verified by several repetitive measurements and by approaching equilibrium from supersaturation. Aliquots of saturated solutions were transferred through a coarse filter into tared volumetric flasks, weighed and diluted with 2-propanol. Concentrations were determined by spectrophotometric measurements at $280 \mathrm{~nm}$.

Source and Purity of Chemicals:

(1) $98 \%$, Aldrich Chemical Company, Milwaukee, WI, USA, was recrystallized several times from methanol.

(2) Absolute, Aaper Alcohol and Chemical Company, USA, stored over molecular sieves before use.

Estimated Error:

Temperature: $\pm 0.1 \mathrm{~K}$.

$x_{1}: \pm 1.5 \%$ (relative error).

\begin{tabular}{ll}
\hline \hline Components: & Original Measurements: \\
(1) Fluorene; $\mathrm{C}_{13} \mathrm{H}_{10} ;[92-52-4]$ & ${ }^{50}$ Z. Zou, L. Dang, P. Liu, and H. Wei, \\
(2) Ethanol; $\mathrm{C}_{2} \mathrm{H}_{6} \mathrm{O} ;[64-17-5]$ & J. Chem. Eng. Data 52, 1501 (2007). \\
\hline Variables: & Prepared by: \\
Temperature & W. E. Acree, Jr. \\
\hline
\end{tabular}


Experimental Values

\begin{tabular}{lcc}
\hline \hline$T / \mathrm{K}$ & $x_{2}{ }^{\mathrm{a}}$ & $x_{1}{ }^{\mathrm{b}}$ \\
\hline 278.98 & 0.9963 & 0.00372 \\
283.99 & 0.9954 & 0.00455 \\
289.01 & 0.9945 & 0.00554 \\
294.08 & 0.9933 & 0.00668 \\
298.35 & 0.9920 & 0.00802 \\
303.36 & 0.9903 & 0.00973 \\
308.77 & 0.9881 & 0.01185 \\
313.17 & 0.9861 & 0.01387 \\
319.27 & 0.9823 & 0.01766 \\
324.95 & 0.9784 & 0.02161 \\
\hline
\end{tabular}

${ }^{\mathrm{a}} x_{2}$ : mole fraction of component 2 in the saturated solution.

${ }^{b} x_{1}$ : mole fraction of the polycyclic aromatic hydrocarbon (component 1 ).

\section{Auxiliary Information}

\begin{abstract}
Method/Apparatus/Procedure:
Thermostated bath, thermometer.

Very little experimental details were provided. Solubilities were determined by a synthetic method. Known masses of solute and solvent were placed in a dissolving flask. The solute and solvent mixture was stirred with a magnetic stirrer. Small amounts of solute were added at predetermined intervals until no more solid would dissolve. The point at which no more solid would dissolve was taken to be the point of solid-liquid equilibrium. The paper did not specify how the disappearance of the solid was monitored; however, the papers that the authors referenced for the experimental method used a laser monitoring method.
\end{abstract}

Source and Purity of Chemicals:

(1) $99.5 \%$, Chemical source and purification method were not given.

(2) $99.8 \%$, Analytical Reagent Grade, Tianjin Kewei Company, China, no information given regarding any further purification.

Estimated Error:

Temperature: $\pm 0.2 \mathrm{~K}$.

$x_{1}$ : within 0.0200 (relative uncertainty).

\begin{tabular}{ll}
\hline \hline Components: & Original Measurements: \\
(1) Fluorene; $\mathrm{C}_{13} \mathrm{H}_{10} ;[92-52-4]$ & ${ }^{48}$ C. I. Monárrez, W. E. Acree, Jr., and \\
(2) 1-Propanol; $\mathrm{C}_{3} \mathrm{H}_{8} \mathrm{O} ;[71-23-8]$ & M. H. Abraham, Phys. Chem. Liq. 40, \\
& 581 (2002). \\
\hline Variables: & Prepared by: \\
$T / \mathrm{K}=298.15$ & W. E. Acree, Jr. \\
\hline
\end{tabular}

Experimental Values

\begin{tabular}{lcc}
\hline \hline$x_{2}{ }^{(\mathrm{s}) \mathrm{a}}$ & $x_{2}{ }^{\mathrm{b}}$ & $x_{1}{ }^{\mathrm{c}}$ \\
\hline 1.0000 & 0.9877 & 0.01232 \\
\hline
\end{tabular}

${ }^{\mathrm{a}} x_{2}{ }^{(\mathrm{s})}$ : initial mole fraction of component 2 in the solution.

${ }^{\mathrm{b}} x_{2}$ : mole fraction of component 2 in the saturated solution.

${ }^{c} x_{1}$ : mole fraction solubility of the solute.

\section{Auxiliary Information}

\section{Method/Apparatus/Procedure:}

Constant-temperature bath, calorimetric thermometer, and an ultraviolet/ visible spectrophotometer.

Excess solute and solvent were placed in amber glass bottles and allowed to equilibrate for several days at constant temperature. Attainment of equilibrium was verified by several repetitive measurements and by approaching equilibrium from supersaturation. Aliquots of saturated solutions were transferred through a coarse filter into tared volumetric flasks, weighed and diluted with 2-propanol. Concentrations were determined by spectrophotometric measurements at $280 \mathrm{~nm}$.

\section{Source and Purity of Chemicals:}

(1) $98 \%$, Aldrich Chemical Company, Milwaukee, WI, USA, was recrystallized several times from methanol.

(2) $99+\%$, anhydrous, Aldrich Chemical Company, stored over molecular sieves before use.

Estimated Error:

Temperature: $\pm 0.1 \mathrm{~K}$.

$x_{1}: \pm 1.5 \%$ (relative error).

\begin{tabular}{|c|c|}
\hline $\begin{array}{l}\text { Components: } \\
\text { (1) Fluorene; } \mathrm{C}_{13} \mathrm{H}_{10} ;[92-52-4] \\
\text { (2) 1-Propanol; } \mathrm{C}_{3} \mathrm{H}_{8} \mathrm{O} ;[71-23-8]\end{array}$ & $\begin{array}{l}\text { Original Measurements: } \\
\text { 51 Y. Han and Z. Wang, J. Chem. Eng. } \\
\text { Data 54, } 148 \text { (2009). }\end{array}$ \\
\hline $\begin{array}{l}\text { Variables: } \\
\text { Temperature }\end{array}$ & Prepared by: \\
\hline
\end{tabular}

Experimental Values

\begin{tabular}{lcc}
\hline \hline$T / \mathrm{K}$ & $x_{2}{ }^{\mathrm{a}}$ & $x_{1}{ }^{\mathrm{b}}$ \\
\hline 283.00 & 0.9918 & 0.00817 \\
288.00 & 0.9910 & 0.00903 \\
293.00 & 0.9985 & 0.01154 \\
298.40 & 0.9866 & 0.01339 \\
302.20 & 0.9837 & 0.01631 \\
308.00 & 0.9812 & 0.01882 \\
313.00 & 0.9785 & 0.02151 \\
318.00 & 0.9752 & 0.02483 \\
323.00 & 0.9711 & 0.02890
\end{tabular}

${ }^{\mathrm{a}} x_{2}$ : mole fraction of component 2 in the saturated solution.

${ }^{b} x_{1}$ : mole fraction of the polycyclic aromatic hydrocarbon (component 1 ).

\section{Auxiliary Information}

\section{Method/Apparatus/Procedure:}

Double-jacketed glass vessel, laser generator, water bath, photoelectric transformer, and digital display.

Solubilities were determined by a synthetic-dynamic method. Known masses of solute and solvent were placed in a dissolving flask. The solid in the solution dissolved as the temperature was increased. The temperature at which last amount of solid dissolved was recorded as the solid-liquid equilibrium temperature. The disappearance of the solid solute was determined by laser monitoring.

Source and Purity of Chemicals:

(1) $99+\%$, Chemical source and purification method were not given. (2) $99.8+\%$, Analytical Reagent Grade, Tianjin Kewei Company, China, no information given regarding any further purification. 
Estimated Error:

Temperature: $\pm 0.2 \mathrm{~K}$

$x_{1}$ : within 0.0200 (relative uncertainty).

\section{Components:}

(1) Fluorene; $\mathrm{C}_{13} \mathrm{H}_{10} ;[$ [92-52-4]

(2) 2-Propanol; $\mathrm{C}_{3} \mathrm{H}_{8} \mathrm{O}$; [67-63-0]

Original Measurements:

${ }^{48}$ C. I. Monárrez, W. E. Acree, Jr., and M. H. Abraham, Phys. Chem. Liq. 40 581 (2002).

\section{Variables:}

$T / \mathrm{K}=298.15$

\section{Prepared by:}

W. E. Acree, Jr.

Experimental Values

\begin{tabular}{lcc}
\hline \hline$x_{2}{ }^{(\mathrm{s}) \mathrm{a}}$ & $x_{2}{ }^{\mathrm{b}}$ & $x_{1}{ }^{\mathrm{c}}$ \\
\hline 1.0000 & 0.9905 & 0.00949 \\
\hline
\end{tabular}



${ }^{b} x_{2}$ : mole fraction of component 2 in the saturated solution.

${ }^{c} x_{1}$ : mole fraction solubility of the solute.

\section{Auxiliary Information}

\section{Method/Apparatus/Procedure:}

Constant-temperature bath, calorimetric thermometer, and an ultraviolet/ visible spectrophotometer.

Excess solute and solvent were placed in amber glass bottles and allowed to equilibrate for several days at constant temperature. Attainment of equilibrium was verified by several repetitive measurements and by approaching equilibrium from supersaturation. Aliquots of saturated solutions were transferred through a coarse filter into tared volumetric flasks, weighed and diluted with 2-propanol. Concentrations were determined by

spectrophotometric measurements at $280 \mathrm{~nm}$.

\section{Source and Purity of Chemicals:}

(1) $98 \%$, Aldrich Chemical Company, Milwaukee, WI, USA, was recrystallized several times from methanol.

(2) $99+\%$, anhydrous, Aldrich Chemical Company, stored over molecular sieves before use.

\section{Estimated Error:}

Temperature: $\pm 0.1 \mathrm{~K}$.

$x_{1}: \pm 1.5 \%$ (relative error).

\section{Components:}

(1) Fluorene; $\mathrm{C}_{13} \mathrm{H}_{10} ;[92-52-4]$

Original Measurements:

(2) 2-Propanol; $\mathrm{C}_{3} \mathrm{H}_{8} \mathrm{O}$; [67-63-0]

${ }^{50}$ Z. Zou, L. Dang, P. Liu, and H. Wei, J. Chem. Eng. Data 52, 1501 (2007).

\begin{tabular}{ll}
\hline Variables: & Prepared by: \\
Temperature & W. E. Acree, Jr. \\
\hline
\end{tabular}

\begin{tabular}{lcc}
\hline \hline$T / \mathrm{K}$ & $x_{2}{ }^{\mathrm{a}}$ & $x_{1}{ }^{\mathrm{b}}$ \\
\hline 313.78 & 0.9895 & 0.01047 \\
318.24 & 0.9864 & 0.01355 \\
323.17 & 0.9821 & 0.01787 \\
328.25 & 0.9763 & 0.02368 \\
333.45 & 0.9686 & 0.03136
\end{tabular}

${ }^{\mathrm{a}} x_{2}$ : mole fraction of component 2 in the saturated solution.

${ }^{b} x_{1}$ : mole fraction of the polycyclic aromatic hydrocarbon (component 1 ).

\section{Auxiliary Information}

Method/Apparatus/Procedure:

Thermostated bath, thermometer.

Very little experimental details were provided. Solubilities were determined by a synthetic method. Known masses of solute and solvent were placed in a dissolving flask. The solute and solvent mixture was stirred with a magnetic stirrer. Small amounts of solute were added at predetermined intervals until no more solid would dissolve. The point at which no more solid would dissolve was taken to be the point of solid-liquid equilibrium. The paper did not specify how the disappearance of the solid was monitored; however, the papers that the authors referenced for the experimental method used a laser monitoring method.

Source and Purity of Chemicals:

(1) $99.5 \%$, Chemical source and purification method were not given. (2) $99.8 \%$, Analytical Reagent Grade, Tianjin Kewei Company, China, no information given regarding any further purification.

Estimated Error:

Temperature: $\pm 0.2 \mathrm{~K}$.

$x_{1}$ : within 0.0200 .

\begin{tabular}{ll}
\hline \hline Components: & Original Measurements: \\
(1) Fluorene; $\mathrm{C}_{13} \mathrm{H}_{10} ;[92-52-4]$ & ${ }^{48}$ C. I. Monárrez, W. E. Acree, Jr., and \\
(2) 1-Butanol; $\mathrm{C}_{4} \mathrm{H}_{10} \mathrm{O} ;[71-36-3]$ & M. H. Abraham, Phys. Chem. Liq. 40, \\
& 581 (2002). \\
\hline Variables: & Prepared by: \\
$T / \mathrm{K}=298.15$ & W. E. Acree, Jr. \\
\hline
\end{tabular}

Experimental Values

\begin{tabular}{|c|c|c|}
\hline$x_{2}{ }^{(\mathrm{s}) \mathrm{a}}$ & $x_{2}{ }^{b}$ & $x_{1}{ }^{\mathrm{c}}$ \\
\hline 1.0000 & 0.9831 & 0.01686 \\
\hline
\end{tabular}

Experimental Values

\begin{tabular}{lcc}
\hline \hline$T / \mathrm{K}$ & $x_{2}{ }^{\mathrm{a}}$ & $x_{1}{ }^{\mathrm{b}}$ \\
\hline 288.28 & 0.9978 & 0.00221 \\
293.69 & 0.9969 & 0.00313 \\
299.50 & 0.9955 & 0.00453 \\
303.17 & 0.9943 & 0.00565 \\
309.06 & 0.9918 & 0.00820
\end{tabular}




\section{Auxiliary Information}

\section{Method/Apparatus/Procedure:}

Constant-temperature bath, calorimetric thermometer, and an ultraviolet/ visible spectrophotometer.

Excess solute and solvent were placed in amber glass bottles and allowed to equilibrate for several days at constant temperature. Attainment of equilibrium was verified by several repetitive measurements and by approaching equilibrium from supersaturation. Aliquots of saturated solutions were transferred through a coarse filter into tared volumetric flasks, weighed and diluted with 2-propanol. Concentrations were determined by spectrophotometric measurements at $280 \mathrm{~nm}$.

Source and Purity of Chemicals:

(1) $98 \%$, Aldrich Chemical Company, Milwaukee, WI, USA, was recrystallized several times from methanol.

(2) $99.8 \%$, anhydrous, Aldrich Chemical Company, stored over molecular sieves before use.

Estimated Error:

Temperature: $\pm 0.1 \mathrm{~K}$.

$x_{1}: \pm 1.5 \%$ (relative error).

\begin{tabular}{|c|c|}
\hline $\begin{array}{l}\text { Components: } \\
\text { (1) Fluorene; } \mathrm{C}_{13} \mathrm{H}_{10} ;[92-52-4] \\
\text { (2) 1-Butanol; } \mathrm{C}_{4} \mathrm{H}_{10} \mathrm{O} ;[71-36-3]\end{array}$ & $\begin{array}{l}\text { Original Measurements: } \\
{ }^{50} \text { Z. Zou, L. Dang, P. Liu, and H. Wei, } \\
\text { J. Chem. Eng. Data 52, } 1501 \text { (2007). }\end{array}$ \\
\hline $\begin{array}{l}\text { Variables: } \\
\text { Temperature }\end{array}$ & $\begin{array}{l}\text { Prepared by: } \\
\text { W. E. Acree, J }\end{array}$ \\
\hline
\end{tabular}

Experimental Values

\begin{tabular}{lcc}
\hline \hline$T / \mathrm{K}$ & $x_{2}{ }^{\mathrm{a}}$ & $x_{1}{ }^{\mathrm{b}}$ \\
\hline 284.27 & 0.9941 & 0.00590 \\
288.82 & 0.9928 & 0.00724 \\
293.76 & 0.9909 & 0.00906 \\
298.88 & 0.9887 & 0.01130 \\
304.06 & 0.9861 & 0.01390 \\
308.28 & 0.9832 & 0.01679 \\
313.93 & 0.9788 & 0.02122 \\
318.16 & 0.9749 & 0.02510 \\
323.16 & 0.9696 & 0.03038 \\
328.25 & 0.9630 & 0.03703 \\
333.35 & 0.9542 & 0.04578 \\
338.35 & 0.9442 & 0.05584 \\
\hline
\end{tabular}

${ }^{a_{x_{2}}}$ : mole fraction of component 2 in the saturated solution.

${ }^{b} x_{1}$ : mole fraction of the polycyclic aromatic hydrocarbon (component 1 ).

\section{Auxiliary Information}

\footnotetext{
Method/Apparatus/Procedure:

Thermostated bath, thermometer.

Very little experimental details were provided. Solubilities were determined by a synthetic method. Known masses of solute and solvent were placed in a dissolving flask. The solute and solvent mixture was stirred with a magnetic stirrer. Small amounts of solute were added at predetermined intervals until no more solid would dissolve. The point at which no more solid would dissolve was taken to be the point of solid-liquid equilibrium. The paper did not specify how the disappearance of the solid was monitored; however, the papers that the authors referenced for the experimental method used a laser monitoring method.
}

Source and Purity of Chemicals:

(1) $99.5 \%$, Chemical source and purification method were not given.

(2) $99.8 \%$, Analytical Reagent Grade, Tianjin Kewei Company, China, no information given regarding any further purification.

Estimated Error:

Temperature: $\pm 0.2 \mathrm{~K}$.

$x_{1}$ : within 0.0200 .

\begin{tabular}{ll}
\hline \hline Components: & Original Measurements: \\
(1) Fluorene; $\mathrm{C}_{13} \mathrm{H}_{10} ;[92-52-4]$ & ${ }^{48} \mathrm{C}$. I. Monárrez, W. E. Acree, Jr., and \\
(2) 2-Butanol; $\mathrm{C}_{4} \mathrm{H}_{10} \mathrm{O} ;[78-92-2]$ & M. H. Abraham, Phys. Chem. Liq. 40, \\
& 581 (2002). \\
\hline Variables: & Prepared by: \\
$T / \mathrm{K}=298.15$ & W. E. Acree, Jr. \\
\hline
\end{tabular}

Experimental Values

\begin{tabular}{lcc}
\hline \hline$x_{2}{ }^{(\mathrm{s}) \mathrm{a}}$ & $x_{2}{ }^{\mathrm{b}}$ & $x_{1}{ }^{\mathrm{c}}$ \\
\hline 1.0000 & 0.9879 & 0.01206 \\
\hline${ }^{\mathrm{a}}{ }_{x_{2}}{ }^{(\mathrm{s})}:$ initial mole fraction of component 2 in the solution. \\
${ }^{\mathrm{b}} x_{2}:$ mole fraction of component 2 in the saturated solution. \\
${ }^{\mathrm{c}}{ }_{x_{1}}$ : mole fraction solubility of the solute.
\end{tabular}

$x_{2}$ : mole fraction of component 2 in the
${ }^{x_{1}}$ : mole fraction solubility of the solute.

\section{Auxiliary Information}

\section{Method/Apparatus/Procedure:}

Constant-temperature bath, calorimetric thermometer, and an ultraviolet/ visible spectrophotometer.

Excess solute and solvent were placed in amber glass bottles and allowed to equilibrate for several days at constant temperature. Attainment of equilibrium was verified by several repetitive measurements and by approaching equilibrium from supersaturation. Aliquots of saturated solutions were transferred through a coarse filter into tared volumetric flasks, weighed and diluted with 2-propanol. Concentrations were determined by spectrophotometric measurements at $280 \mathrm{~nm}$.

\section{Source and Purity of Chemicals:}

(1) $98 \%$, Aldrich Chemical Company, Milwaukee, WI, USA, was recrystallized several times from methanol.

(2) 99+\%, anhydrous, Aldrich Chemical Company, stored over molecular sieves before use.

\section{Estimated Error:}

Temperature: $\pm 0.1 \mathrm{~K}$.

$x_{1}: \pm 1.5 \%$ (relative error).

\begin{tabular}{ll}
\hline \hline Components: & Original Measurements: \\
(1) Fluorene; $\mathrm{C}_{13} \mathrm{H}_{10} ;[92-52-4]$ & ${ }^{48} \mathrm{C}$. I. Monárrez, W. E. Acree, Jr., and \\
(2) 2-Methyl-1-propanol; $\mathrm{C}_{4} \mathrm{H}_{10} \mathrm{O} ;$ & M. H. Abraham, Phys. Chem. Liq. 40, \\
[78-83-1] & $581(2002)$. \\
\hline Variables: & Prepared by: \\
$T / \mathrm{K}=298.15$ & W. E. Acree, Jr. \\
\hline
\end{tabular}


Experimental Values

\begin{tabular}{lcc}
\hline$\frac{x_{2}{ }^{(\mathrm{s}) \mathrm{a}}}{x_{2}{ }^{\mathrm{b}}}$ & $x_{1}{ }^{\mathrm{c}}$ \\
\hline 1.0000 & 0.9899 & 0.01006 \\
$\frac{{ }^{\mathrm{a}} x_{2}{ }^{(\mathrm{s})}: \text { initial mole fraction of component } 2 \text { in the solution. }}{{ }^{\mathrm{b}}{ }_{x_{2}}: \text { mole fraction of component } 2 \text { in the saturated solution. }}$ \\
${ }^{\mathrm{c}} x_{1}:$ mole fraction solubility of the solute.
\end{tabular}

\section{Auxiliary Information}

\section{Method/Apparatus/Procedure:}

Constant-temperature bath, calorimetric thermometer, and an ultraviolet/ visible spectrophotometer.

Excess solute and solvent were placed in amber glass bottles and allowed to equilibrate for several days at constant temperature. Attainment of equilibrium was verified by several repetitive measurements and by approaching equilibrium from supersaturation. Aliquots of saturated solutions were transferred through a coarse filter into tared volumetric flasks, weighed and diluted with 2-propanol. Concentrations were determined by spectrophotometric measurements at $280 \mathrm{~nm}$.

\section{Source and Purity of Chemicals:}

(1) $98 \%$, Aldrich Chemical Company, Milwaukee, WI, USA, was recrystallized several times from methanol.

(2) $99+\%$, anhydrous, Aldrich Chemical Company, stored over molecular sieves before use.

\section{Estimated Error:}

Temperature: $\pm 0.1 \mathrm{~K}$.

$x_{1}: \pm 1.5 \%$ (relative error).

\begin{tabular}{|c|c|}
\hline $\begin{array}{l}\text { Components: } \\
\text { (1) Fluorene; } \mathrm{C}_{13} \mathrm{H}_{10} ;[92-52-4] \\
\text { (2) 2-Methyl-1-propanol; } \mathrm{C}_{4} \mathrm{H}_{10} \mathrm{O} \text {; } \\
\text { [78-83-1] }\end{array}$ & $\begin{array}{l}\text { Original Measurements: } \\
{ }^{51} \text { Y. Han and Z. Wang, J. Chem. } \\
\text { Eng. Data 54, } 148 \text { (2009). }\end{array}$ \\
\hline $\begin{array}{l}\text { Variables: } \\
\text { Temperature }\end{array}$ & $\begin{array}{l}\text { Prepared by: } \\
\text { W. E. Acree, Jr. }\end{array}$ \\
\hline
\end{tabular}

Experimental Values

\begin{tabular}{lcc}
\hline \hline$T / \mathrm{K}$ & $x_{2}{ }^{\mathrm{a}}$ & $x_{1}{ }^{\mathrm{b}}$ \\
\hline 283.00 & 0.9957 & 0.00425 \\
288.00 & 0.9946 & 0.00544 \\
293.00 & 0.9931 & 0.00691 \\
298.40 & 0.9910 & 0.00902 \\
303.20 & 0.9879 & 0.01212 \\
308.00 & 0.9844 & 0.01563 \\
313.00 & 0.9808 & 0.01924 \\
318.00 & 0.9770 & 0.02296 \\
323.00 & 0.9733 & 0.02668 \\
\hline
\end{tabular}

${ }^{a} x_{2}$ : mole fraction of component 2 in the saturated solution.

${ }^{b} x_{1}$ : mole fraction of the polycyclic aromatic hydrocarbon (component 1 ).

\section{Auxiliary Information}

\section{Method/Apparatus/Procedure:}

Double-jacketed glass vessel, laser generator, water bath, photoelectric transformer, digital display.

Solubilities were determined by a synthetic-dynamic method. Known masses of solute and solvent were placed in a dissolving flask. The solid in the solution dissolved as the temperature was increased. The temperature at which last amount of solid dissolved was recorded as the solid-liquid equilibrium temperature. The disappearance of the solid solute was determined by laser monitoring.

\section{Source and Purity of Chemicals:}

(1) $99+\%$, Chemical source and purification method were not given. (2) $99.8+\%$, Analytical Reagent Grade, Tianjin Kewei Company, China, no information given regarding any further purification.

Estimated Error:

Temperature: $\pm 0.2 \mathrm{~K}$.

$x_{1}$ : within 0.0200 (relative uncertainty).

\begin{tabular}{ll}
\hline \hline Components: & Original Measurements: \\
(1) Fluorene; $\mathrm{C}_{13} \mathrm{H}_{10} ;[92-52-4]$ & ${ }^{48} \mathrm{C}$. I. Monárrez, W. E. Acree, Jr., \\
(2) 2-Methyl-2-propanol; $\mathrm{C}_{4} \mathrm{H}_{10} \mathrm{O} ;$ & and M. H. Abraham, Phys. Chem. \\
[75-65-0] & Liq. 40, 581 (2002). \\
\hline Variables: & Prepared by: \\
$T / \mathrm{K}=298.15$ & W. E. Acree, Jr. \\
\hline
\end{tabular}

\section{Experimental Values}

\begin{tabular}{|c|c|c|}
\hline$\underline{x}_{2}{ }^{(\mathrm{s}) \mathrm{a}}$ & $x_{2}{ }^{\mathrm{b}}$ & $x_{1}{ }^{\mathrm{c}}$ \\
\hline 1.0000 & 0.9898 & 0.01016 \\
\hline
\end{tabular}

\section{Auxiliary Information}

\section{Method/Apparatus/Procedure:}

Constant-temperature bath, calorimetric thermometer, and an ultraviolet/ visible spectrophotometer.

Excess solute and solvent were placed in amber glass bottles and allowed to equilibrate for several days at constant temperature. Attainment of equilibrium was verified by several repetitive measurements and by approaching equilibrium from supersaturation. Aliquots of saturated solutions were transferred through a coarse filter into tared volumetric flasks, weighed and diluted with 2-propanol. Concentrations were determined by spectrophotometric measurements at $280 \mathrm{~nm}$.

Source and Purity of Chemicals:

(1) $98 \%$, Aldrich Chemical Company, Milwaukee, WI, USA, was recrystallized several times from methanol.

(2) $99.5+\%$, anhydrous, Aldrich Chemical Company, stored over molecular sieves before use.

\section{Estimated Error:}

Temperature: $\pm 0.1 \mathrm{~K}$.

$x_{1}: \pm 1.5 \%$ (relative error). 


\section{Components:}

(1) Fluorene; $\mathrm{C}_{13} \mathrm{H}_{10} ;[92-52-4]$

(2) 1-Pentanol; $\mathrm{C}_{5} \mathrm{H}_{12} \mathrm{O} ;[$ [71-41-0]

Variables:

$T / \mathrm{K}=298.15$

Original Measurements:

${ }^{48}$ C. I. Monárrez, W. E. Acree,

Jr., and M. H. Abraham, Phys. Chem. Liq. 40, 581 (2002).

Prepared by:

W. E. Acree, Jr.

Experimental Values

\begin{tabular}{lcc}
\hline \hline$x_{2}{ }^{(\mathrm{s}) \mathrm{a}}$ & $x_{2}{ }^{\mathrm{b}}$ & $x_{1}{ }^{\mathrm{c}}$ \\
\hline 1.0000 & 0.9771 & 0.02290 \\
\hline
\end{tabular}

${ }^{\mathrm{a}} x_{2}{ }^{(\mathrm{s})}$ : initial mole fraction of component 2 in the solution.

${ }^{b} x_{2}$ : mole fraction of component 2 in the saturated solution.

${ }^{c} x_{1}$ : mole fraction solubility of the solute.

\section{Auxiliary Information}

\begin{abstract}
Method/Apparatus/Procedure:
Constant-temperature bath, calorimetric thermometer, and an ultraviolet/ visible spectrophotometer.

Excess solute and solvent were placed in amber glass bottles and allowed to equilibrate for several days at constant temperature. Attainment of equilibrium was verified by several repetitive measurements and by approaching equilibrium from supersaturation. Aliquots of saturated solutions were transferred through a coarse filter into tared volumetric flasks, weighed and diluted with 2-propanol. Concentrations were determined by

spectrophotometric measurements at $280 \mathrm{~nm}$.
\end{abstract}

Source and Purity of Chemicals:

(1) $98 \%$, Aldrich Chemical Company, Milwaukee, WI, USA, was recrystallized several times from methanol.

(2) $99+\%$, Aldrich Chemical Company, stored over molecular sieves before use.

\section{Estimated Error:}

Temperature: $\pm 0.1 \mathrm{~K}$.

$x_{1}: \pm 1.5 \%$ (relative error)

\begin{tabular}{ll}
\hline \hline Components: & Original Measurements: \\
(1) Fluorene; $\mathrm{C}_{13} \mathrm{H}_{10} ;[92-52-4]$ & ${ }^{48} \mathrm{C}$. I. Monárrez, W. E. Acree, \\
(2) 2-Pentanol; $\mathrm{C}_{5} \mathrm{H}_{12} \mathrm{O} ;[6032-29-7]$ & Jr., and M. H. Abraham, Phys. \\
& Chem. Liq. 40, 581 (2002). \\
\hline Variables: & Prepared by: \\
$T / \mathrm{K}=298.15$ & W. E. Acree, Jr. \\
\hline
\end{tabular}

Experimental Values

\begin{tabular}{lcc}
\hline \hline$x_{2}{ }^{(\mathrm{s}) \mathrm{a}}$ & $x_{2}{ }^{\mathrm{b}}$ & $x_{1}{ }^{\mathrm{c}}$ \\
\hline 1.0000 & 0.9837 & 0.01634 \\
${ }^{\mathrm{a}}{ }_{x_{2}}{ }^{(\mathrm{s})}:$ initial mole fraction of component 2 in the solution. \\
${ }^{\mathrm{b}} x_{2}:$ mole fraction of component 2 in the saturated solution. \\
${ }^{\mathrm{c}} x_{1}:$ mole fraction solubility of the solute.
\end{tabular}

\section{Auxiliary Information}

\section{Method/Apparatus/Procedure:}

Constant-temperature bath, calorimetric thermometer, and an ultraviolet/ visible spectrophotometer.

Excess solute and solvent were placed in amber glass bottles and allowed to equilibrate for several days at constant temperature. Attainment of equilibrium was verified by several repetitive measurements and by approaching equilibrium from supersaturation. Aliquots of saturated solutions were transferred through a coarse filter into tared volumetric flasks, weighed and diluted with 2-propanol. Concentrations were determined by spectrophotometric measurements at $280 \mathrm{~nm}$.

\section{Source and Purity of Chemicals:}

(1) $98 \%$, Aldrich Chemical Company, Milwaukee, WI, USA, was recrystallized several times from methanol.

(2) $99+\%$, Acros Organics, USA, stored over molecular sieves before use.

\section{Estimated Error:}

Temperature: $\pm 0.1 \mathrm{~K}$.

$x_{1}: \pm 1.5 \%$ (relative error).

\begin{tabular}{ll}
\hline \hline Components: & Original Measurements: \\
(1) Fluorene; $\mathrm{C}_{13} \mathrm{H}_{10} ;[92-52-4]$ & ${ }^{49}$ D. M. Stovall, K. R. Hoover, W. \\
(2) 2-Methyl-1-butanol; $\mathrm{C}_{5} \mathrm{H}_{12} \mathrm{O} ;$ & E. Acree, Jr., and M. H. Abraham, \\
[137-32-6] & Polycyclic Aromat. Compd. 25, \\
& 313 (2005). \\
\hline Variables: & Prepared by: \\
$T / \mathrm{K}=298.15$ & W. E. Acree, Jr. \\
\hline
\end{tabular}

Experimental Values

\begin{tabular}{|c|c|c|}
\hline$x_{2}{ }^{(\mathrm{s}) \mathrm{a}}$ & $x_{2}{ }^{b}$ & $x_{1}{ }^{\mathrm{c}}$ \\
\hline 1.0000 & 0.9816 & 0.01842 \\
\hline
\end{tabular}

\section{Auxiliary Information}

\section{Method/Apparatus/Procedure:}

Constant-temperature bath, calorimetric thermometer, and an ultraviolet/ visible spectrophotometer.

Excess solute and solvent were placed in amber glass bottles and allowed to equilibrate for several days at constant temperature. Attainment of equilibrium was verified by several repetitive measurements and by approaching equilibrium from supersaturation. Aliquots of saturated solutions were transferred through a coarse filter into tared volumetric flasks, weighed and diluted with 2-propanol. Concentrations were determined by spectrophotometric measurements at $280 \mathrm{~nm}$.

\section{Source and Purity of Chemicals:}

(1) $98 \%$, Aldrich Chemical Company, Milwaukee, WI, USA, was recrystallized several times from methanol.

(2) $99+\%$, Aldrich Chemical Company, stored over molecular sieves before use.

\section{Estimated Error:}

Temperature: $\pm 0.1 \mathrm{~K}$.

$x_{1}: \pm 1.5 \%$ (relative error). 


\section{Components:}

(1) Fluorene; $\mathrm{C}_{13} \mathrm{H}_{10} ;[92-52-4]$

(2) 3-Methyl-1-butanol; $\mathrm{C}_{5} \mathrm{H}_{12} \mathrm{O}$;

[123-51-3]

Variables:

$T / \mathrm{K}=298.15$

Original Measurements:

${ }^{48}$ C. I. Monárrez, W. E. Acree, Jr., and

M. H. Abraham, Phys. Chem. Liq. 40, 581 (2002).

Prepared by:

W. E. Acree, Jr.

\section{Experimental Values}

\begin{tabular}{lcc}
\hline \hline$x_{2}{ }^{(\mathrm{s}) \mathrm{a}}$ & $x_{2}{ }^{\mathrm{b}}$ & $x_{1}{ }^{\mathrm{c}}$ \\
\hline 1.0000 & 0.9854 & 0.01461 \\
\hline
\end{tabular}

${ }^{\mathrm{a}} x_{2}{ }^{(\mathrm{s})}$ : initial mole fraction of component 2 in the solution.

${ }^{b} x_{2}$ : mole fraction of component 2 in the saturated solution.

${ }^{c} x_{1}$ : mole fraction solubility of the solute.

\section{Auxiliary Information}

Method/Apparatus/Procedure:
Constant-temperature bath, calorimetric thermometer, and an ultraviolet/
visible spectrophotometer.
Excess solute and solvent were placed in amber glass bottles and allowed to
equilibrate for several days at constant temperature. Attainment of equilibrium
was verified by several repetitive measurements and by approaching
equilibrium from supersaturation. Aliquots of saturated solutions were
transferred through a coarse filter into tared volumetric flasks, weighed and
diluted with 2-propanol. Concentrations were determined by
spectrophotometric measurements at $280 \mathrm{~nm}$.
Source and Purity of Chemicals:
(1) $98 \%$, Aldrich Chemical Company, Milwaukee, WI, USA, was
recrystallized several times from methanol.
(2) $99+\%$, anhydrous, Aldrich Chemical Company, stored over molecular
sieves before use.
Estimated Error:
Temperature: $\pm 0.1 \mathrm{~K}$.
$x_{1}: \pm 1.5 \%$ (relative error).

\begin{tabular}{ll}
\hline \hline Components: & Original Measurements: \\
(1) Fluorene; $\mathrm{C}_{13} \mathrm{H}_{10} ;[92-52-4]$ & ${ }^{48} \mathrm{C}$. I. Monárrez, W. E. Acree, Jr., and \\
(2) 2-Methyl-2-butanol; $\mathrm{C}_{5} \mathrm{H}_{12} \mathrm{O} ;$ & M. H. Abraham, Phys. Chem. Liq. 40, \\
[75-85-4] & 581 (2002). \\
\hline Variables: & Prepared by: \\
$T / \mathrm{K}=298.15$ & W. E. Acree, Jr. \\
\hline
\end{tabular}

Experimental Values

\begin{tabular}{lcc}
\hline \hline$x_{2}{ }^{(\mathrm{s}) \mathrm{a}}$ & $x_{2}{ }^{\mathrm{b}}$ & $x_{1}{ }^{\mathrm{c}}$ \\
\hline 1.0000 & 0.9815 & 0.01854 \\
${ }^{\mathrm{a}}{ }_{x_{2}}{ }^{(\mathrm{s})}:$ initial mole fraction of component 2 in the solution. \\
${ }^{\mathrm{b}} x_{2}:$ mole fraction of component 2 in the saturated solution. \\
${ }^{\mathrm{c}} x_{1}:$ mole fraction solubility of the solute.
\end{tabular}

\section{Auxiliary Information}

\section{Method/Apparatus/Procedure:}

Constant-temperature bath, calorimetric thermometer, and an ultraviolet/ visible spectrophotometer.

Excess solute and solvent were placed in amber glass bottles and allowed to equilibrate for several days at constant temperature. Attainment of equilibrium was verified by several repetitive measurements and by approaching equilibrium from supersaturation. Aliquots of saturated solutions were transferred through a coarse filter into tared volumetric flasks, weighed and diluted with 2-propanol. Concentrations were determined by spectrophotometric measurements at $280 \mathrm{~nm}$.

\section{Source and Purity of Chemicals:}

(1) $98 \%$, Aldrich Chemical Company, Milwaukee, WI, USA, was recrystallized several times from methanol.

(2) $99+\%$, Acros Organics, USA, stored over molecular sieves before use.

\section{Estimated Error:}

Temperature: $\pm 0.1 \mathrm{~K}$.

$x_{1}: \pm 1.5 \%$ (relative error).

\begin{tabular}{ll}
\hline \hline Components: & Original Measurements: \\
(1) Fluorene; $\mathrm{C}_{13} \mathrm{H}_{10} ;[92-52-4]$ & ${ }^{48}$ C. I. Monárrez, W. E. Acree, \\
(2) 1-Hexanol; $\mathrm{C}_{6} \mathrm{H}_{14} \mathrm{O} ;[111-27-3]$ & Jr., and M. H. Abraham, Phys. \\
& Chem. Liq. 40, 581 (2002). \\
\hline Variables: & Prepared by: \\
$T / \mathrm{K}=298.15$ & W. E. Acree, Jr. \\
\hline
\end{tabular}

Experimental Values

\begin{tabular}{lcc}
\hline \hline$x_{2}{ }^{(\mathrm{s}) \mathrm{a}}$ & $x_{2}{ }^{\mathrm{b}}$ & $x_{1}{ }^{\mathrm{c}}$ \\
\hline 1.0000 & 0.9724 & 0.02757 \\
$\frac{{ }^{a} x_{2}{ }^{(\mathrm{s})}: \text { initial mole fraction of component } 2 \text { in the solution. }}{{ }^{\mathrm{b}} x_{2}: \text { mole fraction of component } 2 \text { in the saturated solution. }}$ \\
${ }^{c} x_{1}:$ mole fraction solubility of the solute.
\end{tabular}

\section{Auxiliary Information}

\section{Method/Apparatus/Procedure:}

Constant-temperature bath, calorimetric thermometer, and an ultraviolet/ visible spectrophotometer.

Excess solute and solvent were placed in amber glass bottles and allowed to equilibrate for several days at constant temperature. Attainment of equilibrium was verified by several repetitive measurements and by approaching equilibrium from supersaturation. Aliquots of saturated solutions were transferred through a coarse filter into tared volumetric flasks, weighed and diluted with 2-propanol. Concentrations were determined by spectrophotometric measurements at $280 \mathrm{~nm}$.

\section{Source and Purity of Chemicals:}

(1) $98 \%$, Aldrich Chemical Company, Milwaukee, WI, USA, was recrystallized several times from methanol.

(2) $99+\%$, Alfa Aesar, USA, stored over molecular sieves before use.

\section{Estimated Error:}

Temperature: $\pm 0.1 \mathrm{~K}$.

$x_{1}: \pm 1.5 \%$ (relative error). 


\section{Components:}

(1) Fluorene; $\mathrm{C}_{13} \mathrm{H}_{10} ;[92-52-4]$

(2) 2-Methyl-1-pentanol; $\mathrm{C}_{6} \mathrm{H}_{14} \mathrm{O}$; [105-30-6]

\section{Variables:}

$T / \mathrm{K}=298.15$
Original Measurements:

${ }^{48}$ C. I. Monárrez, W. E. Acree, Jr., and

M. H. Abraham, Phys. Chem. Liq. 40, 581 (2002).

Prepared by:

W. E. Acree, Jr.

\section{Experimental Values}

\begin{tabular}{lcc}
\hline \hline$x_{2}{ }^{(\mathrm{s}) \mathrm{a}}$ & $x_{2}{ }^{\mathrm{b}}$ & $x_{1}{ }^{\mathrm{c}}$ \\
\hline 1.0000 & 0.9803 & 0.01973 \\
\hline
\end{tabular}

${ }^{\mathrm{a}} x_{2}{ }^{(\mathrm{s})}$ : initial mole fraction of component 2 in the solution.

${ }^{b} x_{2}$ : mole fraction of component 2 in the saturated solution.

${ }^{c} x_{1}$ : mole fraction solubility of the solute.

\section{Auxiliary Information}

Method/Apparatus/Procedure:
Constant-temperature bath, calorimetric thermometer, and an ultraviolet/
visible spectrophotometer.
Excess solute and solvent were placed in amber glass bottles and allowed to
equilibrate for several days at constant temperature. Attainment of equilibrium
was verified by several repetitive measurements and by approaching
equilibrium from supersaturation. Aliquots of saturated solutions were
transferred through a coarse filter into tared volumetric flasks, weighed and
diluted with 2-propanol. Concentrations were determined by
spectrophotometric measurements at 280 nm.
Source and Purity of Chemicals:
(1) $98 \%$, Aldrich Chemical Company, Milwaukee, WI, USA, was
recrystallized several times from methanol.
(2) $99 \%$, Aldrich Chemical Company, stored over molecular sieves before use.
Estimated Error:
Temperature: $\pm 0.1 \mathrm{~K}$.
$x_{1}: \pm 1.5 \%$ (relative error).

\begin{tabular}{ll}
\hline \hline Components: & Original Measurements: \\
(1) Fluorene; $\mathrm{C}_{13} \mathrm{H}_{10} ;[92-52-4]$ & ${ }^{48} \mathrm{C}$. I. Monárrez, W. E. Acree, Jr., and \\
(2) 4-Methyl-2-pentanol; $\mathrm{C}_{6} \mathrm{H}_{14} \mathrm{O} ;$ & M. H. Abraham, Phys. Chem. Liq. 40, \\
{$[108-11-2]$} & 581 (2002). \\
\hline Variables: & Prepared by: \\
$T / \mathrm{K}=298.15$ & W. E. Acree, Jr. \\
\hline
\end{tabular}

Experimental Values

\begin{tabular}{|c|c|c|}
\hline$x_{2}^{(\mathrm{s}) \mathrm{a}}$ & $x_{2}{ }^{b}$ & $x_{1}{ }^{\mathrm{c}}$ \\
\hline 1.0000 & 0.9832 & 0.01676 \\
\hline
\end{tabular}

\section{Auxiliary Information}

Method/Apparatus/Procedure:

Constant-temperature bath, calorimetric thermometer, and an ultraviolet/ visible spectrophotometer.

Excess solute and solvent were placed in amber glass bottles and allowed to equilibrate for several days at constant temperature. Attainment of equilibrium was verified by several repetitive measurements and by approaching equilibrium from supersaturation. Aliquots of saturated solutions were transferred through a coarse filter into tared volumetric flasks, weighed and diluted with 2-propanol. Concentrations were determined by spectrophotometric measurements at $280 \mathrm{~nm}$.

\section{Source and Purity of Chemicals:}

(1) $98 \%$, Aldrich Chemical Company, Milwaukee, WI, USA, was recrystallized several times from methanol.

(2) $99+\%$, Acros Organics, USA, stored over molecular sieves before use.

\section{Estimated Error:}

Temperature: $\pm 0.1 \mathrm{~K}$.

$x_{1}: \pm 1.5 \%$ (relative error).

\begin{tabular}{ll}
\hline \hline Components: & Original Measurements: \\
(1) Fluorene; $\mathrm{C}_{13} \mathrm{H}_{10} ;[92-52-4]$ & ${ }^{48} \mathrm{C}$. I. Monárrez, W. E. Acree, Jr., \\
(2) 1-Heptanol; $\mathrm{C}_{7} \mathrm{H}_{16} \mathrm{O} ;[111-70-6]$ & and M. H. Abraham, Phys. Chem. \\
& Liq. 40, 581 (2002). \\
\hline Variables: & Prepared by: \\
$T / \mathrm{K}=298.15$ & W. E. Acree, Jr. \\
\hline
\end{tabular}

Experimental Values

\begin{tabular}{lcc}
\hline \hline$x_{2}{ }^{(\mathrm{s}) \mathrm{a}}$ & $x_{2}{ }^{\mathrm{b}}$ & $x_{1}{ }^{\mathrm{c}}$ \\
\hline 1.0000 & 0.9650 & 0.03503 \\
$\frac{{ }^{\mathrm{a}} x_{2}{ }^{(\mathrm{s})}: \text { initial mole fraction of component } 2 \text { in the solution. }}{{ }^{\mathrm{b}}{ }_{x_{2}}: \text { mole fraction of component } 2 \text { in the saturated solution. }}$ \\
${ }^{\mathrm{c}}{ }_{x_{1}}:$ mole fraction solubility of the solute.
\end{tabular}

\section{Auxiliary Information}

\section{Method/Apparatus/Procedure:}

Constant-temperature bath, calorimetric thermometer, and an ultraviolet/ visible spectrophotometer.

Excess solute and solvent were placed in amber glass bottles and allowed to equilibrate for several days at constant temperature. Attainment of equilibrium was verified by several repetitive measurements and by approaching equilibrium from supersaturation. Aliquots of saturated solutions were transferred through a coarse filter into tared volumetric flasks, weighed and diluted with 2-propanol. Concentrations were determined by spectrophotometric measurements at $280 \mathrm{~nm}$.

\section{Source and Purity of Chemicals:}

(1) $98 \%$, Aldrich Chemical Company, Milwaukee, WI, USA, was recrystallized several times from methanol.

(2) $99+\%$, Alfa Aesar, USA, stored over molecular sieves before use.

\section{Estimated Error:}

Temperature: $\pm 0.1 \mathrm{~K}$.

$x_{1}: \pm 1.5 \%$ (relative error). 


\section{Components:}

(1) Fluorene; $\mathrm{C}_{13} \mathrm{H}_{10} ;[92-52-4]$

(2) 1-Octanol; $\mathrm{C}_{8} \mathrm{H}_{18} \mathrm{O} ;[111-87-5]$

Original Measurements:

${ }^{48}$ C. I. Monárrez, W. E. Acree, Jr., and M. H. Abraham, Phys. Chem. Liq. 40, 581 (2002).

\section{Variables:}

$T / \mathrm{K}=298.15$

Prepared by:

W. E. Acree, Jr.

\section{Experimental Values}

\begin{tabular}{lcc}
\hline \hline$x_{2}{ }^{(\mathrm{s}) \mathrm{a}}$ & $x_{2}{ }^{\mathrm{b}}$ & $x_{1}{ }^{\mathrm{c}}$ \\
\hline 1.0000 & 0.9612 & 0.03884 \\
\hline
\end{tabular}

${ }^{\mathrm{a}} x_{2}{ }^{(\mathrm{s})}$ : initial mole fraction of component 2 in the solution.

${ }^{b} x_{2}$ : mole fraction of component 2 in the saturated solution.

${ }^{c} x_{1}$ : mole fraction solubility of the solute.

\section{Auxiliary Information}

Method/Apparatus/Procedure:
Constant-temperature bath, calorimetric thermometer, and an ultraviolet/
visible spectrophotometer.
Excess solute and solvent were placed in amber glass bottles and allowed to
equilibrate for several days at constant temperature. Attainment of equilibrium
was verified by several repetitive measurements and by approaching
equilibrium from supersaturation. Aliquots of saturated solutions were
transferred through a coarse filter into tared volumetric flasks, weighed and
diluted with 2-propanol. Concentrations were determined by
spectrophotometric measurements at $280 \mathrm{~nm}$.
Source and Purity of Chemicals:
(1) $98 \%$, Aldrich Chemical Company, Milwaukee, WI, USA, was
recrystallized several times from methanol.
(2) $99+\%$, anhydrous, Aldrich Chemical Company, stored over molecular
sieves before use.
Estimated Error:
Temperature: $\pm 0.1 \mathrm{~K}$.
$x_{1}: \pm 1.5 \%$ (relative error).

\begin{tabular}{ll}
\hline \hline Components: & Original Measurements: \\
(1) Fluorene; $\mathrm{C}_{13} \mathrm{H}_{10} ;[92-52-4]$ & ${ }^{48} \mathrm{C}$. I. Monárrez, W. E. Acree, Jr., \\
(2) 2-Ethyl-1-hexanol; $\mathrm{C}_{8} \mathrm{H}_{18} \mathrm{O} ;$ & and M. H. Abraham, Phys. Chem. \\
{$[104-76-7]$} & Liq. 40, 581 (2002). \\
\hline Variables: & Prepared by: \\
$T / \mathrm{K}=298.15$ & W. E. Acree, Jr. \\
\hline
\end{tabular}

Experimental Values

\begin{tabular}{lcc}
\hline \hline$x_{2}{ }^{(\mathrm{s}) \mathrm{a}}$ & $x_{2}{ }^{\mathrm{b}}$ & $x_{1}{ }^{\mathrm{c}}$ \\
\hline 1.0000 & 0.9721 & 0.02794 \\
${ }^{\mathrm{a}} x_{2}{ }^{(\mathrm{s})}:$ initial mole fraction of component 2 in the solution. \\
${ }^{\mathrm{b}}{ }_{x_{2}}:$ mole fraction of component 2 in the saturated solution. \\
${ }^{\mathrm{c}} x_{1}:$ mole fraction solubility of the solute.
\end{tabular}

\section{Auxiliary Information}

Method/Apparatus/Procedure:

Constant-temperature bath, calorimetric thermometer, and an ultraviolet/ visible spectrophotometer.

Excess solute and solvent were placed in amber glass bottles and allowed to equilibrate for several days at constant temperature. Attainment of equilibrium was verified by several repetitive measurements and by approaching equilibrium from supersaturation. Aliquots of saturated solutions were transferred through a coarse filter into tared volumetric flasks, weighed and diluted with 2-propanol. Concentrations were determined by spectrophotometric measurements at $280 \mathrm{~nm}$.

\section{Source and Purity of Chemicals:}

(1) $98 \%$, Aldrich Chemical Company, Milwaukee, WI, USA, was recrystallized several times from methanol.

(2) $99+\%$, Aldrich Chemical Company, stored over molecular sieves before use.

Estimated Error:

Temperature: $\pm 0.1 \mathrm{~K}$.

$x_{1}: \pm 1.5 \%$ (relative error).

\begin{tabular}{|c|c|}
\hline $\begin{array}{l}\text { Components: } \\
\text { (1) Fluorene; } \mathrm{C}_{13} \mathrm{H}_{10} ;[92-52-4] \\
\text { (2) 1-Decanol; } \mathrm{C}_{10} \mathrm{H}_{22} \mathrm{O} ;[112-30-1]\end{array}$ & $\begin{array}{l}\text { Original Measurements: } \\
{ }^{48} \text { C. I. Monárrez, W. E. Acree, Jr., } \\
\text { and M. H. Abraham, Phys. Chem. } \\
\text { Liq. 40, } 581 \text { (2002). }\end{array}$ \\
\hline $\begin{array}{l}\text { Variables: } \\
T / \mathrm{K}=298.15\end{array}$ & $\begin{array}{l}\text { Prepared by: } \\
\text { W. E. Acree, Jr. }\end{array}$ \\
\hline
\end{tabular}

Experimental Values

\begin{tabular}{|c|c|c|}
\hline$x_{2}{ }^{(\mathrm{s}) \mathrm{a}}$ & $x_{2}{ }^{b}$ & $x_{1}{ }^{\mathrm{c}}$ \\
\hline 1.0000 & 0.9533 & 0.04669 \\
\hline
\end{tabular}

\section{Auxiliary Information}

\section{Method/Apparatus/Procedure:}

Constant-temperature bath, calorimetric thermometer, and an ultraviolet/ visible spectrophotometer.

Excess solute and solvent were placed in amber glass bottles and allowed to equilibrate for several days at constant temperature. Attainment of equilibrium was verified by several repetitive measurements and by approaching equilibrium from supersaturation. Aliquots of saturated solutions were transferred through a coarse filter into tared volumetric flasks, weighed and diluted with 2-propanol. Concentrations were determined by spectrophotometric measurements at $280 \mathrm{~nm}$.

\section{Source and Purity of Chemicals:}

(1) $98 \%$, Aldrich Chemical Company, Milwaukee, WI, USA, was recrystallized several times from methanol.

(2) $99+\%$, Alfa Aesar, USA, stored over molecular sieves before use.

\section{Estimated Error:}

Temperature: $\pm 0.1 \mathrm{~K}$.

$x_{1}: \pm 1.5 \%$ (relative error). 


\subsection{Fluorene solubility data in ketones}

\begin{tabular}{ll}
\hline \hline Components: & Original Measurements: \\
(1) Fluorene; $\mathrm{C}_{13} \mathrm{H}_{10} ;[92-52-4]$ & ${ }^{51} \mathrm{Y}$. Han and Z. Wang, J. Chem. \\
(2) Propanone; $\mathrm{C}_{3} \mathrm{H}_{6} \mathrm{O} ;[67-64-1]$ & Eng. Data 54, 148 (2009). \\
\hline Variables: & Prepared by: \\
Temperature & W. E. Acree, Jr. \\
\hline
\end{tabular}

Experimental Values

\begin{tabular}{lcc}
\hline \hline$T / \mathrm{K}$ & $x_{2}{ }^{\mathrm{a}}$ & $x_{1}{ }^{\mathrm{b}}$ \\
\hline 283.00 & 0.9555 & 0.04448 \\
288.00 & 0.9510 & 0.04896 \\
293.00 & 0.9442 & 0.05577 \\
298.00 & 0.9313 & 0.06872 \\
303.10 & 0.9207 & 0.07931 \\
308.20 & 0.9093 & 0.09074 \\
313.00 & 0.8962 & 0.1038 \\
318.00 & 0.8802 & 0.1198 \\
323.00 & 0.8602 & 0.1398
\end{tabular}

${ }^{\mathrm{a}} x_{2}$ : mole fraction of component 2 in the saturated solution.

${ }^{b} x_{1}$ : mole fraction of the polycyclic aromatic hydrocarbon (component 1).

\section{Auxiliary Information}

Method/Apparatus/Procedure:

Double-jacketed glass vessel, laser generator, water bath, photoelectric transformer, and digital display.

Solubilities were determined by a synthetic-dynamic method. Known masses of solute and solvent were placed in a dissolving flask. The solid in the solution dissolved as the temperature was increased. The temperature at which last amount of solid dissolved was recorded as the solid-liquid equilibrium temperature. The disappearance of the solid solute was determined by laser monitoring.

\section{Source and Purity of Chemicals:}

(1) $99+\%$, Chemical source and purification method were not given.

(2) $99.8+\%$, Analytical Reagent Grade, Tianjin Kewei Company, China, no information given regarding any further purification.

\section{Estimated Error:}

Temperature: $\pm 0.2 \mathrm{~K}$.

$x_{1}$ : within 0.0200 (relative uncertainty).

\subsection{Fluorene solubility data in miscellaneous organic solvents}

\begin{tabular}{ll}
\hline \hline Components: & Original Measurements: \\
(1) Fluorene; $\mathrm{C}_{13} \mathrm{H}_{10} ;[92-52-4]$ & ${ }^{49}$ D. M. Stovall, K. R. Hoover, W. \\
(2) Propanenitrile; $\mathrm{C}_{3} \mathrm{H}_{5} \mathrm{~N} ;[107-12-0]$ & E. Acree, Jr., and M. H. Abraham, \\
& Polycyclic Aromat. Compd. 25, \\
& 313 (2005). \\
\hline Variables: & Prepared by: \\
$T / \mathrm{K}=298.15$ & W. E. Acree, Jr. \\
\hline
\end{tabular}

Experimental Values

\begin{tabular}{lcc}
\hline \hline$x_{2}{ }^{(\mathrm{s}) \mathrm{a}}$ & $x_{2}{ }^{\mathrm{b}}$ & $x_{1}{ }^{\mathrm{c}}$ \\
\hline 1.0000 & 0.9418 & 0.05815 \\
$\frac{{ }^{\mathrm{a}} x_{2}{ }^{(\mathrm{s})}: \text { initial mole fraction of component } 2 \text { in the solution. }}{{ }^{\mathrm{b}} x_{2}: \text { mole fraction of component } 2 \text { in the saturated solution. }}$ \\
${ }^{\mathrm{c}} x_{1}:$ mole fraction solubility of the solute.
\end{tabular}

\section{Auxiliary Information}

\begin{abstract}
Method/Apparatus/Procedure:
Constant-temperature bath, calorimetric thermometer, and an ultraviolet/ visible spectrophotometer.

Excess solute and solvent were placed in amber glass bottles and allowed to equilibrate for several days at constant temperature. Attainment of equilibrium was verified by several repetitive measurements and by approaching equilibrium from supersaturation. Aliquots of saturated solutions were transferred through a coarse filter into tared volumetric flasks, weighed and diluted with 2-propanol. Concentrations were determined by spectrophotometric measurements at $280 \mathrm{~nm}$.
\end{abstract}

\section{Source and Purity of Chemicals:}

(1) $98 \%$, Aldrich Chemical Company, Milwaukee, WI, USA, was recrystallized several times from methanol.

(2) $99 \%$, Aldrich Chemical Company, stored over molecular sieves before use.

\section{Estimated Error:}

Temperature: $\pm 0.1 \mathrm{~K}$.

$x_{1}: \pm 1.5 \%$ (relative error)

\begin{tabular}{ll}
\hline \hline Components: & Original Measurements: \\
(1) Fluorene; $\mathrm{C}_{13} \mathrm{H}_{10} ;[92-52-4]$ & ${ }^{49} \mathrm{D}$. M. Stovall, K. R. Hoover, W. \\
(2) Butanenitrile; $\mathrm{C}_{4} \mathrm{H}_{7} \mathrm{~N} ;[109-74-0]$ & E. Acree, Jr., and M. H. Abraham, \\
& Polycyclic Aromat. Compd. 25, \\
& $313(2005)$. \\
\hline Variables: & Prepared by: \\
$T / \mathrm{K}=298.15$ & W. E. Acree, Jr. \\
\hline
\end{tabular}

Experimental Values

\begin{tabular}{lcc}
\hline \hline$x_{2}{ }^{(\mathrm{s}) \mathrm{a}}$ & $x_{2}{ }^{\mathrm{b}}$ & $x_{1}{ }^{\mathrm{c}}$ \\
\hline 1.0000 & 0.9145 & 0.08548 \\
\hline
\end{tabular}

${ }^{\mathrm{a}} x_{2}{ }^{(\mathrm{s})}$ : initial mole fraction of component 2 in the solution.

${ }^{\mathrm{b}} x_{2}$ : mole fraction of component 2 in the saturated solution.

${ }^{c} x_{1}$ : mole fraction solubility of the solute. 


\section{Auxiliary Information}

\section{Method/Apparatus/Procedure:}

Constant-temperature bath, calorimetric thermometer, and an ultraviolet/ visible spectrophotometer.

Excess solute and solvent were placed in amber glass bottles and allowed to equilibrate for several days at constant temperature. Attainment of equilibrium was verified by several repetitive measurements and by approaching equilibrium from supersaturation. Aliquots of saturated solutions were transferred through a coarse filter into tared volumetric flasks, weighed and diluted with 2-propanol. Concentrations were determined by spectrophotometric measurements at $280 \mathrm{~nm}$.

Source and Purity of Chemicals:

(1) $98 \%$, Aldrich Chemical Company, Milwaukee, WI, USA, was recrystallized several times from methanol.

(2) $99+\%$, Aldrich Chemical Company, stored over molecular sieves before use.

Estimated Error:

Temperature: $\pm 0.1 \mathrm{~K}$.

$x_{1}: \pm 1.5 \%$ (relative error).

\begin{tabular}{ll}
\hline \hline Components: & Original Measurements: \\
(1) Fluorene; $\mathrm{C}_{13} \mathrm{H}_{10} ;[92-52-4]$ & ${ }^{49} \mathrm{D}$. M. Stovall, K. R. Hoover, W. \\
(2) Hexanedinitrile; $\mathrm{C}_{6} \mathrm{H}_{8} \mathrm{~N}_{2} ;$ & E. Acree, Jr., and M. H. Abraham, \\
{$[111-69-3]$} & Polycyclic Aromat. Compd. 25, \\
& 313 (2005). \\
\hline Variables: & Prepared by: \\
$T / \mathrm{K}=298.15$ & W. E. Acree, Jr. \\
\hline
\end{tabular}

Experimental Values

\begin{tabular}{|c|c|c|}
\hline$x_{2}{ }^{(\mathrm{s}) \mathrm{a}}$ & $x_{2}{ }^{\mathrm{b}}$ & $x_{1}{ }^{\mathrm{c}}$ \\
\hline 1.0000 & 0.9541 & 0.04589 \\
\hline
\end{tabular}

\section{Auxiliary Information}

\section{Method/Apparatus/Procedure:}

Constant-temperature bath, calorimetric thermometer, and an ultraviolet/ visible spectrophotometer.

Excess solute and solvent were placed in amber glass bottles and allowed to equilibrate for several days at constant temperature. Attainment of equilibrium was verified by several repetitive measurements and by approaching equilibrium from supersaturation. Aliquots of saturated solutions were transferred through a coarse filter into tared volumetric flasks, weighed and diluted with 2-propanol. Concentrations were determined by spectrophotometric measurements at $280 \mathrm{~nm}$.

Source and Purity of Chemicals:

(1) $98 \%$, Aldrich Chemical Company, Milwaukee, WI, USA, was recrystallized several times from methanol.

(2) $99 \%$, Aldrich Chemical Company, stored over molecular sieves before use.

\section{Estimated Error:}

Temperature: $\pm 0.1 \mathrm{~K}$.

$x_{1}: \pm 1.5 \%$ (relative error).

\begin{tabular}{ll}
\hline \hline Components: & Original Measurements: \\
(1) Fluorene; $\mathrm{C}_{13} \mathrm{H}_{10} ;[92-52-4]$ & ${ }^{35} \mathrm{Z}$. Lisicki and M. E. Jamróz, \\
$(2) \mathrm{N}, \mathrm{N}$-Dimethylacetamide; $\mathrm{C}_{4} \mathrm{H}_{9} \mathrm{NO} ;$ & J. Chem. Thermodyn. 32, \\
{$[127-19-5]$} & $1335(2000)$. \\
\hline Variables: & Prepared by: \\
Temperature & W. E. Acree, Jr. \\
\hline
\end{tabular}

Experimental Values

\begin{tabular}{lcc}
\hline \hline$T / \mathrm{K}$ & $x_{2}{ }^{\mathrm{a}}$ & $x_{1}{ }^{\mathrm{b}}$ \\
\hline 253.2 & 1.0000 & 0.0000 \\
252.9 & 0.9898 & 0.0102 \\
252.3 & 0.9792 & 0.0208 \\
251.6 & 0.9698 & 0.0302 \\
250.7 & 0.9599 & 0.0401 \\
254.0 & 0.9500 & 0.0500 \\
272.7 & 0.9180 & 0.0820 \\
285.7 & 0.8913 & 0.1087 \\
304.8 & 0.8298 & 0.1702 \\
314.7 & 0.7839 & 0.2161 \\
332.7 & 0.6739 & 0.3261 \\
342.8 & 0.5938 & 0.4062 \\
353.8 & 0.4822 & 0.5178 \\
360.1 & 0.4158 & 0.5842 \\
371.6 & 0.2972 & 0.7028 \\
375.5 & 0.2133 & 0.7867 \\
379.9 & 0.1459 & 0.8541 \\
385.1 & 0.0481 & 0.9519 \\
387.7 & 0.0000 & 1.0000 \\
\hline
\end{tabular}

${ }^{a} x_{2}$ : mole fraction of component 2 in the saturated solution.

${ }^{\mathrm{b}} x_{1}$ : mole fraction of the polycyclic aromatic hydrocarbon (component 1 ).

The authors reported that the binary system exhibits simple eutectic behavior, with a eutectic temperature of $T / \mathrm{K}=250.1$ and eutectic composition of $x_{1}=0.046$.

\section{Auxiliary Information}

\section{Method/Apparatus/Procedure:}

Temperature-controlled heating/cooling system and a shaking-mixing system. Synthetic mixtures of known compositions were weighed in small glass ampoules. The mixture composition was known to within \pm 0.0002 mole fraction. Ampoules were cooled to very low temperatures of about $220 \mathrm{~K}$, pumped down, and sealed. The sealed mixtures were then melted, cooled rapidly, and reheated. The rate of reheating was at an approximate rate of $8 \times$ $10^{-5} \mathrm{~K} / \mathrm{s}$ just before complete dissolution. The temperature at which the solid completely dissolved was recorded. The average of two or three independent measurements was taken as the solid-liquid equilibrium.

\section{Source and Purity of Chemicals:}

(1) $98 \%$, Merck-Schuchardt, Federal Republic of Germany, was recrystallized twice from ethanol in the presence of activated carbon, and then recrystallized from methylbenzene.

(2) Purest grade, Merck-Schuchardt, was distilled under reduced pressure and dried over molecular sieves to produce a sample of $99.9 \%$ purity (mass percent).

\section{Estimated Error:}

Temperature: $\pm 0.2 \mathrm{~K}$ (estimated by compiler).

$x_{1}: \pm 0.0002$. 


\begin{tabular}{ll}
\hline \hline Components: & Original Measurements: \\
(1) Fluorene; $\mathrm{C}_{13} \mathrm{H}_{10} ;[92-52-4]$ & ${ }^{35} \mathrm{Z}$. Lisicki and M. E. Jamróz, \\
(2) 1-Methyl-2-pyrrolidone; $\mathrm{C}_{5} \mathrm{H}_{9} \mathrm{NO} ;$ & J. Chem. Thermodyn. 32, \\
{$[872-50-4]$} & $1335(2000)$. \\
\hline Variables: & Prepared by: \\
Temperature & W. E. Acree, Jr. \\
\hline
\end{tabular}

Experimental Values

\begin{tabular}{lcc}
\hline \hline$T / \mathrm{K}$ & $x_{2}{ }^{\mathrm{a}}$ & $x_{1}{ }^{\mathrm{b}}$ \\
\hline 248.5 & 1.0000 & 0.0000 \\
247.7 & 0.9781 & 0.0219 \\
246.8 & 0.9503 & 0.0497 \\
246.0 & 0.9338 & 0.0662 \\
245.6 & 0.9241 & 0.0759 \\
249.1 & 0.9139 & 0.0861 \\
262.0 & 0.8933 & 0.1067 \\
274.4 & 0.8724 & 0.1276 \\
283.0 & 0.8509 & 0.1491 \\
293.7 & 0.8201 & 0.1799 \\
295.4 & 0.8139 & 0.1861 \\
303.7 & 0.7845 & 0.2155 \\
322.6 & 0.6936 & 0.3064 \\
339.0 & 0.5885 & 0.4115 \\
351.7 & 0.4840 & 0.5160 \\
356.3 & 0.4398 & 0.5602 \\
358.8 & 0.4130 & 0.5870 \\
363.3 & 0.3602 & 0.6398 \\
366.2 & 0.3263 & 0.6737 \\
374.1 & 0.2295 & 0.7705 \\
374.7 & 0.2216 & 0.7784 \\
383.8 & 0.0793 & 0.9207 \\
384.9 & 0.0624 & 0.9376 \\
387.7 & 0.0000 & 1.0000 \\
${ }^{a} x_{2}:$ mole fraction of component 2 in the saturated solution. \\
${ }^{{ }} x_{1}:$ mole fraction of the polycyclic aromatic hydrocarbon (component 1$)$. \\
\end{tabular}

The authors reported that the binary system exhibits simple eutectic behavior, with a eutectic temperature of $T / \mathrm{K}=245.3$ and eutectic composition of $x_{1}=0.082$.

\section{Auxiliary Information}

\section{Method/Apparatus/Procedure:}

Temperature-controlled heating/cooling system and a shaking-mixing system. Synthetic mixtures of known compositions were weighed in small glass ampoules. The mixture composition was known to within \pm 0.0002 mole fraction. Ampoules were cooled to very low temperatures of about $220 \mathrm{~K}$, pumped down, and sealed. The sealed mixtures were then melted, cooled rapidly, and reheated. The rate of reheating was at an approximate rate of $8 \times 10^{-5} \mathrm{~K} / \mathrm{s}$ just before complete dissolution. The temperature at which the solid completely dissolved was recorded. The average of two or three independent measurements was taken as the solid-liquid equilibrium.

\section{Source and Purity of Chemicals:}

(1) $98 \%$, Merck-Schuchardt, Federal Republic of Germany, was recrystallized twice from ethanol in the presence of activated carbon, and then recrystallized from methylbenzene.

(2) Technical Grade, BASF, Federal Republic of Germany, was purified by treatment with a $2 \%$ solution of potassium permanganate, and then distilled under reduced pressure to collect the distillate having $99.8 \%$ purity (mass percent). The purified sample was dried over molecular sieves.
Estimated Error:

Temperature: $\pm 0.2 \mathrm{~K}$ (estimated by compiler). $x_{1}: \pm 0.0002$.

\begin{tabular}{|c|c|}
\hline $\begin{array}{l}\text { Components: } \\
\text { (1) Fluorene; } \mathrm{C}_{13} \mathrm{H}_{10} ;[92-52-4] \\
\text { (2) Hexahydro-1-methyl-2H-azepin-2-one } \\
\text { ( } N \text {-Methyl- } \varepsilon \text {-caprolactam); } \mathrm{C}_{7} \mathrm{H}_{13} \mathrm{NO} ; \\
\text { [2556-73-2] }\end{array}$ & $\begin{array}{l}\text { Original Measurements: } \\
{ }^{35} \text { Z. Lisicki and M. E. Jamróz, } \\
\text { J. Chem. Thermodyn. 32, } \\
1335 \text { (2000). }\end{array}$ \\
\hline $\begin{array}{l}\text { ables: } \\
\text { perature }\end{array}$ & $\begin{array}{l}\text { Prepared by: } \\
\text { W. E. Acree, Jr. }\end{array}$ \\
\hline
\end{tabular}

Experimental Values

\begin{tabular}{lcc}
\hline \hline$T / \mathrm{K}$ & $x_{2}{ }^{\mathrm{a}}$ & $x_{1}{ }^{\mathrm{b}}$ \\
\hline 279.6 & 1.0000 & 0.0000 \\
304.3 & 0.8049 & 0.1951 \\
315.7 & 0.7463 & 0.2537 \\
324.3 & 0.6988 & 0.3012 \\
332.3 & 0.6499 & 0.3501 \\
339.2 & 0.5970 & 0.4030 \\
347.5 & 0.5290 & 0.4710 \\
352.0 & 0.4911 & 0.5089 \\
356.3 & 0.4508 & 0.5492 \\
361.3 & 0.4004 & 0.5996 \\
365.9 & 0.3480 & 0.6520 \\
370.1 & 0.2964 & 0.7036 \\
377.7 & 0.1978 & 0.8022 \\
383.5 & 0.0999 & 0.9001 \\
387.7 & 0.0000 & 1.0000 \\
\hline
\end{tabular}

${ }^{a} x_{2}$ : mole fraction of component 2 in the saturated solution.

${ }^{\mathrm{b}} x_{1}$ : mole fraction of the polycyclic aromatic hydrocarbon (component 1 ).

\section{Auxiliary Information}

\section{Method/Apparatus/Procedure:}

Temperature-controlled heating/cooling system and a shaking-mixing system. Synthetic mixtures of known compositions were weighed in small glass ampoules. The mixture composition was known to within \pm 0.0002 mole fraction. Ampoules were cooled to very low temperatures of about $220 \mathrm{~K}$, pumped down, and sealed. The sealed mixtures were then melted, cooled rapidly, and reheated. The rate of reheating was at an approximate rate of $8 \times 10^{-5} \mathrm{~K} / \mathrm{s}$ just before complete dissolution. The temperature at which the solid completely dissolved was recorded. The average of two or three independent measurements was taken as the solid-liquid equilibrium.

\section{Source and Purity of Chemicals:}

(1) $98 \%$, Merck-Schuchardt, Federal Republic of Germany, was recrystallized twice from ethanol in the presence of activated carbon, and then recrystallized from methylbenzene.

(2) Technical Grade, Leuna Werke, Federal Republic of Germany, was purified by super-rectification and then dried over molecular sieves to give a sample having a purity of $99.9 \%$ by mass.

\section{Estimated Error:}

Temperature: $\pm 0.2 \mathrm{~K}$ (estimated by compiler). $x_{1}: \pm 0.0002$. 


\section{Solubility of Naphthalene in Organic Solvents}

\subsection{Critical evaluation of experimental solubility data}

Volume 59 in the IUPAC Solubility Data Series ${ }^{3}$ contained experimental solubility data for naphthalene dissolved in five linear alkanes (hexane, heptane, octane, hexadecane, and octadecane) and eight cycloalkanes (cyclohexane, methylcyclohexane, cis-1,2-dimethyl-cyclohexane, trans-1,2dimethylcyclohexane, cis-1,3-dimethylcyclohexane, cis-1,4dimethylcylcohexane, trans-1,4-dimethylcyclohexane, and decahydronaphthalene), in five aromatic hydrocarbons (benzene, methylbenzene, ethylbenzene, 1,2,3,4-tetrahydronaphthalene, and 4-isopropyl-1-methylbenzene), in five esters (furfuryl acetate, methyl 2-furoate, ethyl 2-furoate, propyl 2-furoate, and butyl 2-furoate), in four dialkyl ethers (1,1'-oxybisethane, 1,1'-oxybisbutane, 1-ethoxypropane, and 2-ethoxypropane), and three cyclic ethers (2,2-dimethyloxetane, 3,3-dimethyloxetane, and tetrahydrofuran), in eight halogenated alkanes (dichloromethane, trichloromethane, tetrachloromethane, diiodomethane, 1,1-dibromoethane, 1,2-dibromoethane, 1,1-dichloroethane, 1,2-dichloroethane), one chloroalkene (trichloroethene) and two halogenated benzenes (chlorobenzene and hexafluorobenzene), in 14 alkanols (methanol, ethanol, 1-propanol, 2-propanol, 1-butanol, 2butanol, 2-methyl-1-propanol, 2-methyl-2-propanol, 1-pentanol, 1-hexanol, 1-octanol, cyclohexanol, methylcyclohexanol, and furfuryl alcohol), in one alkanone (propanone) and in seven miscellaneous organic solvents (carbon disulfide, nitrobenzene, aniline, thiophene, pyridine, perfluorodibutyl ether, and perfluorotripropylamine). The volume also included phase diagram information for binary naphthalene + benzene, naphthalene + methylbenzene, naphthalene + ethylbenzene, naphthalene + 1,2-dimethylbenzene, naphthalene $+1,3$ dimethylbenzene, naphthalene + 1,4-dimethylbenzene, naphthalene + biphenyl, naphthalene +1 ,2-diphenylethane, naphthalene + indene, naphthalene + fluoranthene, naphthalene + fluorene, naphthalene + chrysene, naphthalene + pyrene and naphthalene + acenaphthene, naphthalene +1 ,4-dichlorobenzene, naphthalene + octafluoronaphthalene, naphthalene $+1,2$-benzenediol, naphthalene $+1,3$-benzenediol, naphthalene +1 ,4-benzenediol, naphthalene +2 -nitrobenzaldehyde, naphthalene +3 -nitrobenzaldehyde, naphthalene +4 -nitrobenzaldehyde, naphthalene + 1-chloro-3-nitrobenzene, naphthalene +1 -chloro-4-nitrobenzene, naphthalene $+2,4-$ dinitroaniline, naphthalene + 3,5-dinitrobenzoic acid, naphthalene $+1,3,5$-trimethyl-2,4,6-trinitrobenzene, naphthalene +4 -nitro-1-methylbenzene, naphthalene +1 ,3-dinitrobenzene, naphthalene $+1,3,5$-trinitrobenzene, naphthalene + 2-nitrophenol, naphthalene + 4-nitrophenol, naphthalene + 2,4-dinitro-1-methylbenzene, naphthalene + 2,4-dinitrophenol, naphthalene $+2,4,6$-trinitrophenol, and naphthalene + isoquinoline. Solubility data contained in Vol. 59 will not be republished here. The listing above is provided so that readers will know what solubility data are available in the earlier volume for naphthalene.
There have been several studies that reported solubility data for naphthalene in organic solvents after Vol. 59 was published in 1995. Numerous research groups have studied the solubility behavior of naphthalene as a function of temperature. Cui et $a .^{52}$ examined the solubility of naphthalene in heptanes, methylbenzene, ethanol, 1-butanol, propanone, and in a mixture of dimethylbenzene isomers (13.3\% 1,2-dimethylbenzene, $\quad 66.4 \%$ 1,3-dimethylbenzene, and $20.3 \% \quad 1,4-$ dimethylbenzene) using a dynamic method with laser detection system. Plots of naphthalene mole fraction solubilities versus temperature given in the paper fell on smooth curves. As part of the study the authors determined curve-fit equation coefficients for mathematical describing the measured mole fraction solubilities with the Apelblat (see Eq. (3)) and Buchowski $\lambda \mathrm{h}$ (see Eq. (4)) models. The calculated equation coefficients are given in Tables 4 and 5, respectively. Average deviations between the experimental data and calculated values based on the Apelblat and Buchowski $\lambda \mathrm{h}$ models were on the order of $4.6 \%$ and $8.1 \%$, respectively. Li et al. ${ }^{53}$ studied the solubility of naphthalene in ethyl ethanoate, 2-methylpropyl ethanoate, tetrahydrofuran, butanoic acid, $N, N$-dimethylformamide, and 2-methyl-2-pyrrolidone between 278 and $328 \mathrm{~K}$. The calculated coefficients for the Apelblat mathematical representation are given in Table 4. The six datasets are judged to be internally consistent, with mean relative deviations between the observed data and values calculated based on Apelblat model being less than $7.5 \%$. Both research groups studied the solubility of naphthalene in propanone. There is less than a 5\% difference between the two datasets.

Kotula and Marciniak ${ }^{13}$ published solubility data for naphthalene in four chlorinated alkanes (trichloromethane, tetrachloromethane, 1,1-dichloroethane, and 1,2-dichloroethane) and two chlorinated alkenes (trichloroethene and tetrachloroethene) from 290 to $325 \mathrm{~K}$. The internal consistency of the chloroalkane and chloroalkene datasets of measured acenaphthene solubilities were assessed by curve-fitting the measured the mole fraction solubility data to Eq. (3). The numerical values of the equation coefficients $(A, B$, and $C)$ are given in Table 4 along with the respective mean relative deviations. Examination of the numerical entries in the last column of the table reveals that the Apelblat equation provides a very good description of the solubility behavior in the four chloroalkane and two chloroalkene solvents with the average mean relative deviations being on the order of $0.4 \%$ or less.

Published studies ${ }^{15,39,42,54-58}$ have also included phase diagrams for binary naphthalene mixtures containing a variety of different organic compounds, including saturated hydrocarbons (eicosane, pentacosane, octacosane, trans-decahydronaphthalene, and cis-decahydronaphthalene), polycyclic aromatic hydrocarbons (biphenyl and phenanthrene) and chlorinated benzenes (1,2-dichlorobenzene, 1,3-dichlorobenzene, and 1,4-dichlorobenzene). Phase diagrams and discussions given in the papers did not suggest any inconsistencies in the individual datasets.

The experimental solubility data for naphthalene dissolved in the different organic solvents are given in Secs. 10.2-10.9. 
TABLE 4. Parameters of the Apelblat equation for describing the solubility of naphthalene in various organic solvents

\begin{tabular}{|c|c|c|c|c|}
\hline Solvent & $A$ & $B$ & $C$ & MRD (\%) \\
\hline Heptane $^{\mathrm{a}}$ & -127.81 & 195.1 & 20.824 & \\
\hline Methylbenzene $^{a}$ & -69.291 & 1194.7 & 11.240 & \\
\hline Dimethylbenzene mixture ${ }^{a}$ & -11.758 & -1499.4 & 2.7243 & \\
\hline 1-Butanol ${ }^{\mathrm{a}}$ & -87.689 & -2104.5 & 15.999 & \\
\hline Propanone $^{a}$ & -55.641 & 19.068 & 9.488 & \\
\hline Ethyl ethanoate $^{\mathrm{b}}$ & -188.03 & 6434.2 & 28.986 & 4.4 \\
\hline Tetrahydrofuran ${ }^{\mathrm{b}}$ & 2.900 & -1600.7 & 0.252 & 6.9 \\
\hline Butanoic acid ${ }^{\mathrm{b}}$ & -150.15 & 4199.0 & 23.552 & 2.3 \\
\hline$N, N$-Dimethylformamide ${ }^{\mathrm{b}}$ & -52.982 & 341.7 & 8.873 & 4.4 \\
\hline 1-Methyl-2-pyrrolidone ${ }^{\mathrm{b}}$ & -97.615 & 2991.6 & 15.198 & 4.9 \\
\hline Trichloromethane & 48.913 & -4126.1 & -6.357 & 0.2 \\
\hline Tetrachloromethane & 41.257 & -4126.2 & -5.049 & 0.2 \\
\hline
\end{tabular}

${ }^{\mathrm{a}}$ Numerical values of the coefficients were taken from Cui et al. ${ }^{52}$

${ }^{\mathrm{b}}$ Numerical values of the coefficients were taken from Li et al. ${ }^{53}$

\subsection{Naphthalene solubility data in saturated hydrocarbons (including cycloalkanes)}

\begin{tabular}{ll}
\hline \hline Components: & Original Measurements: \\
(1) Naphthalene; $\mathrm{C}_{10} \mathrm{H}_{8} ;[91-20-3]$ & ${ }^{52}$ T.-B. Cui, T.-L. Luo, C. Zhang, \\
(2) Heptane; $\mathrm{C}_{7} \mathrm{H}_{16} ;[142-82-5]$ & Z.-B. Mao, and G.-J. Liu, J. Chem. \\
& Eng. Data 54, 1065 (2009). \\
\hline Variables: & Prepared by: \\
Temperature & W. E. Acree, Jr. \\
\hline
\end{tabular}

Experimental Values

\begin{tabular}{lcc}
\hline \hline$T / \mathrm{K}$ & $x_{2}{ }^{\mathrm{a}}$ & $x_{1}{ }^{\mathrm{b}}$ \\
\hline 290.25 & 0.9405 & 0.05948 \\
294.30 & 0.9295 & 0.07054 \\
297.85 & 0.9202 & 0.07982 \\
301.37 & 0.9111 & 0.08893 \\
303.04 & 0.9022 & 0.09778 \\
305.30 & 0.8934 & 0.1066 \\
307.53 & 0.8850 & 0.1150 \\
309.63 & 0.8766 & 0.1234 \\
311.20 & 0.8674 & 0.1326 \\
312.60 & 0.8588 & 0.1412
\end{tabular}

TABLE 5. Parameters of the Buchowski $\lambda$ h equation for describing the solubility of naphthalene in various organic solvents ${ }^{\mathrm{a}}$

\begin{tabular}{llr}
\hline \hline Solvent & \multicolumn{1}{c}{$\lambda$} & \multicolumn{1}{c}{$h$} \\
\hline Heptane & 0.62352 & 9451.2 \\
Methylbenzene & 1.4722 & 2962.2 \\
Dimethylbenzene mixture of & 1.6190 & 2906.0 \\
isomers & & \\
Ethanol & 0.62042 & 15339.0 \\
1-Butanol & 1.8991 & 6048.3 \\
Propanone & 1.0642 & 4097.0 \\
\hline
\end{tabular}

${ }^{\mathrm{a}}$ Numerical values of the coefficients were taken from Cui et al. ${ }^{52}$

\begin{tabular}{lcc}
\hline \hline$T / \mathrm{K}$ & $x_{2}{ }^{\mathrm{a}}$ & $x_{1}{ }^{\mathrm{b}}$ \\
\hline 314.13 & 0.8502 & 0.1498 \\
315.15 & 0.8417 & 0.1583 \\
316.63 & 0.8318 & 0.1682 \\
317.65 & 0.8230 & 0.1770 \\
321.33 & 0.7910 & 0.2090 \\
324.25 & 0.7698 & 0.2302 \\
326.98 & 0.7354 & 0.2646 \\
329.45 & 0.6956 & 0.3044 \\
330.26 & 0.6706 & 0.3294 \\
331.50 & 0.6495 & 0.3505 \\
333.75 & 0.6110 & 0.3890 \\
\hline
\end{tabular}

${ }^{\mathrm{a}} x_{2}$ : mole fraction of component 2 in the saturated solution.

${ }^{b} x_{1}$ : mole fraction of the polycyclic aromatic hydrocarbon (component 1 ).

\section{Auxiliary Information}

Method/Apparatus/Procedure:

Double-jacketed glass vessel, laser generator, water bath, photoelectric transformer, and digital display.

Solubilities were determined by a synthetic-dynamic method. Known masses of solute and solvent were placed in a dissolving flask. The solid in the solution dissolved as the temperature was increased. The temperature at which the last amount of solid dissolved was recorded as the solid-liquid equilibrium temperature. The disappearance of the solid solute was determined by laser monitoring.

Source and Purity of Chemicals:

(1) Initial purity not given, Tianjin Kermel Chemical Reagent Purchase and Supply Station, China, was dissolved in acetone, filtered and the acetone evaporated. The sample was then recrystallized several times from methanol. The purity of the recrystallized sample was $99.8 \%$ as determined by UV spectrometry.

(2) 99.7\%, Tianjin Kermel Chemical Reagent Company, Ltd, China, no information given regarding any further purification.

\section{Estimated Error:}

Temperature: $\pm 0.2 \mathrm{~K}$.

$x_{1}: 5 \%$ (relative error) 


\section{Components:}

(1) Naphthalene; $\mathrm{C}_{10} \mathrm{H}_{8} ;$ [91-20-3]

(2) Eicosane; $\mathrm{C}_{20} \mathrm{H}_{42}$; [112-95-8]

\begin{tabular}{ll} 
& Phase Equilib. 110, 283 (1995). \\
\hline Variables: & Prepared by: \\
Temperature & W. E. Acree, Jr. \\
\hline
\end{tabular}

Experimental Values

\begin{tabular}{lcc}
\hline \hline$T / \mathrm{K}$ & $x_{2}{ }^{\mathrm{a}}$ & $x_{1}{ }^{\mathrm{b}}$ \\
\hline 309.79 & 1.0000 & 0.0000 \\
309.42 & 0.8997 & 0.1003 \\
308.89 & 0.7912 & 0.2088 \\
307.30 & 0.6966 & 0.3034 \\
317.40 & 0.5895 & 0.4105 \\
323.20 & 0.4975 & 0.5025 \\
330.90 & 0.4012 & 0.5988 \\
337.37 & 0.3013 & 0.6987 \\
343.46 & 0.2004 & 0.7996 \\
348.36 & 0.0996 & 0.9004 \\
352.55 & 0.0000 & 1.0000 \\
\hline
\end{tabular}

${ }^{\mathrm{a}} x_{2}$ : mole fraction of component 2 in the saturated solution.

${ }^{\mathrm{b}} x_{1}$ : mole fraction of the polycyclic aromatic hydrocarbon (component 1 ).

The authors employed a SSF to describe the activity coefficients of naphthalene and eicosane calculated from the solidliquid equilibrium data. The SSF model gave a calculated eutectic temperature of $T / \mathrm{K}=307.6$ and eutectic mole fraction of naphthalene of $x_{1}=0.312$.

\section{Auxiliary Information}

\section{Method/Apparatus/Procedure:}

Differential scanning calorimeter.

The phase diagram was determined using a differential scanning calorimeter Measurements were performed at a constant fixed scanning rate of $0.5 \mathrm{~K} / \mathrm{min}$.

\section{Source and Purity of Chemicals:}

(1) $99 \%$, Prolabo, no purification details were given in the paper. (2) $99 \%$, Aldrich Chemical Company, Milwaukee, WI, USA, purification details were not given in the paper.

\section{Estimated Error:}

Temperature: Authors state a global accuracy of $\pm 1 \%$ regarding their measurements.

$x_{1}: \pm 0.0002$ (estimated by compiler).

\begin{tabular}{ll}
\hline \hline Components: & Original Measurements: \\
(1) Naphthalene; $\mathrm{C}_{10} \mathrm{H}_{8} ;[91-20-3]$ & ${ }^{42}$ S. L. Hafsaoui and R. Mahmoud, \\
(2) Pentacosane; $\mathrm{C}_{25} \mathrm{H}_{52} ;[629-99-2]$ & J. Therm. Anal. Calorim. 88, 565 \\
& $(2007)$. \\
\hline Variables: & Prepared by: \\
Temperature & W. E. Acree, Jr. \\
\hline
\end{tabular}

Experimental Values

\begin{tabular}{|c|c|c|}
\hline$T / \mathrm{K}$ & $x_{2}{ }^{\mathrm{a}}$ & $x_{1}{ }^{\mathrm{b}}$ \\
\hline 324.10 & 1.000 & 0.000 \\
\hline 323.72 & 0.899 & 0.101 \\
\hline 321.48 & 0.800 & 0.200 \\
\hline 318.13 & 0.702 & 0.298 \\
\hline 317.38 & 0.599 & 0.401 \\
\hline 324.23 & 0.501 & 0.499 \\
\hline 330.20 & 0.401 & 0.599 \\
\hline 336.70 & 0.302 & 0.698 \\
\hline 344.48 & 0.199 & 0.801 \\
\hline 348.14 & 0.102 & 0.898 \\
\hline 353.42 & 0.000 & 1.000 \\
\hline
\end{tabular}

\section{Auxiliary Information}

Method/Apparatus/Procedure:

Differential scanning calorimeter.

Mixtures of naphthalene and pentacosane were prepared by mass. The resulting mixtures were heated slowly with agitation until the entire sample melted. After melting the sample was immersed in liquid nitrogen to solidify the mixture completely. A small amount of solid was removed and sealed in a pan, the capsule was crimped, and placed in the calorimetric block of the differential scanning calorimeter for analysis.

Source and Purity of Chemicals:

(1) $99 \%$, Fluka Chemical Company, was used as received.

(2) $99 \%$, Fluka Chemical Company, was used as received.

Estimated Error:

Temperature: $\pm 0.7 \mathrm{~K}$.

$x_{1}: \pm 0.002$ (estimated by compiler).

\begin{tabular}{|c|c|}
\hline $\begin{array}{l}\text { Components: } \\
\text { (1) Naphthalene; } \mathrm{C}_{10} \mathrm{H}_{8} ;[91-20-3] \\
\text { (2) Pentacosane; } \mathrm{C}_{25} \mathrm{H}_{52} ;[629-99-2]\end{array}$ & $\begin{array}{l}\text { Original Measurements: } \\
{ }^{54} \text { P. M. Ghogomu, R. Rakolosaona, } \\
\text { M. Bouroukba, D. Petitjean, N. } \\
\text { Hubert, and M. Dirand, Mol. Cryst. } \\
\text { Liq. Cryst. 408, } 103 \text { (2004). }\end{array}$ \\
\hline $\begin{array}{l}\text { Variables: } \\
\text { Temperature }\end{array}$ & $\begin{array}{l}\text { Prepared by: } \\
\text { W. E. Acree, Jr. }\end{array}$ \\
\hline
\end{tabular}


Experimental Values

\begin{tabular}{lcc}
\hline \hline$T / \mathrm{K}$ & $x_{2}{ }^{\mathrm{a}}$ & $x_{1}{ }^{\mathrm{b}}$ \\
\hline 327.05 & 1.000 & 0.000 \\
326.65 & 0.874 & 0.126 \\
325.45 & 0.768 & 0.232 \\
324.85 & 0.675 & 0.325 \\
323.35 & 0.593 & 0.407 \\
322.15 & 0.524 & 0.476 \\
320.85 & 0.426 & 0.574 \\
319.05 & 0.356 & 0.644 \\
321.45 & 0.308 & 0.698 \\
336.75 & 0.268 & 0.732 \\
345.15 & 0.144 & 0.856 \\
348.65 & 0.085 & 0.915 \\
350.65 & 0.036 & 0.964 \\
354.05 & 0.000 & 1.000 \\
\hline
\end{tabular}

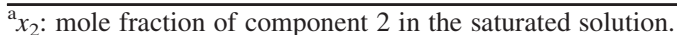

${ }^{\mathrm{b}} x_{1}$ : mole fraction of the polycyclic aromatic hydrocarbon (component 1 ).

\section{Auxiliary Information}

\section{Method/Apparatus/Procedure:}

Coupled simple thermal analysis-differential thermal analysis device constructed in the authors' laboratory.

Mixtures of naphthalene and pentacosane were prepared by mass, melted and mixed carefully, and then quenched in a crystallizing dish maintained at very low temperature. The solidified mixtures were ground to a fine powder and stored at ambient room temperature. The solidus-liquidus equilibrium temperature was determined by calorimetric methods using a coupled simple thermal analysis-differential thermal analysis device constructed in the authors' laboratory.

\section{Source and Purity of Chemicals:}

(1) $99 \%$, Fluka Chemical Company, was used as received.

(2) $98 \%$, Fluka Chemical Company, was used as received.

\section{Estimated Error:}

Temperature: $\pm 0.5 \mathrm{~K}$.

$x_{1}: \pm 0.002$ (estimated by compiler).

\begin{tabular}{ll}
\hline \hline Components: & Original Measurements: \\
(1) Naphthalene; $\mathrm{C}_{10} \mathrm{H}_{8} ;[91-20-3]$ & ${ }^{15}$ A. Aoulmi, M. Bouroukba, R. \\
(2) Octacosane; $\mathrm{C}_{28} \mathrm{H}_{58} ;[630-02-4]$ & Solimando, and M. Rogalski, Fluid \\
& Phase Equilib. 110, 283 (1995). \\
\hline Variables: & Prepared by: \\
Temperature & W. E. Acree, Jr. \\
\hline
\end{tabular}

Experimental Values

\begin{tabular}{lcc}
\hline \hline$T / \mathrm{K}$ & $x_{2}{ }^{\mathrm{a}}$ & $x_{1}{ }^{\mathrm{b}}$ \\
\hline 333.91 & 1.0000 & 0.0000 \\
333.65 & 0.8970 & 0.1030 \\
333.38 & 0.7990 & 0.2010 \\
331.79 & 0.6997 & 0.3003 \\
330.63 & 0.6003 & 0.3997 \\
329.57 & 0.4976 & 0.5024 \\
336.47 & 0.3505 & 0.6495 \\
338.32 & 0.3004 & 0.6996 \\
341.17 & 0.2505 & 0.7495 \\
344.59 & 0.1993 & 0.8007 \\
348.73 & 0.1000 & 0.9000 \\
352.55 & 0.0000 & 1.0000 \\
\hline
\end{tabular}

${ }^{a} x_{2}$ : mole fraction of component 2 in the saturated solution.

${ }^{b} x_{1}$ : mole fraction of the polycyclic aromatic hydrocarbon (component 1).

The authors employed a SSF to describe the activity coefficients of naphthalene and octacosane calculated from the solid-liquid equilibrium data. The SSF model gave a calculated eutectic temperature of $T / \mathrm{K}=329.2$ and eutectic mole fraction of naphthalene of $x_{1}=0.524$.

\section{Auxiliary Information}

Method/Apparatus/Procedure:

Differential scanning calorimeter.

The phase diagram was determined using a differential scanning calorimeter. Measurements were performed at a constant fixed scanning rate of $0.5 \mathrm{~K} / \mathrm{min}$.

\section{Source and Purity of Chemicals:}

(1) $99 \%$, Prolabo, no purification details were given in the paper. (2) $99 \%$, Aldrich Chemical Company, Milwaukee, WI, USA, purification details were not given in the paper.

\section{Estimated Error:}

Temperature: Authors state a global accuracy of $\pm 1 \%$ regarding their measurements.

$x_{1}: \pm 0.0002$ (estimated by compiler).

\begin{tabular}{ll}
\hline \hline Components: & Original Measurements: \\
(1) Naphthalene; $\mathrm{C}_{10} \mathrm{H}_{8} ;[91-20-3]$ & ${ }^{59}$ A. Shayanfar, S. Soltani, F. \\
(2) 2,2,4-Trimethylpentane; $\mathrm{C}_{8} \mathrm{H}_{18} ;$ & Jabbaribar, A. A. Hamidi, W. E. \\
[540-84-1] & Acree, Jr., and A. Jouyban, J. \\
& Chem. Eng. Data 53, 574 (2008). \\
\hline Variables: & Prepared by: \\
$T / \mathrm{K}=298.15$ & W. E. Acree, Jr. \\
\hline
\end{tabular}

Experimental Values

\begin{tabular}{lcc}
\hline \hline$c_{1}{ }^{\mathrm{a}}$ & $x_{2}{ }^{\mathrm{b}}$ & $x_{1}{ }^{\mathrm{c}}$ \\
\hline 0.692 & 0.8871 & 0.1129 \\
\hline
\end{tabular}

${ }^{\mathrm{a}} c_{1}$ : molar solubility of the solute.

${ }^{\mathrm{b}} x_{2}$ : mole fraction of component 2 in the saturated solution.

${ }^{\mathrm{c}} x_{1}$ : mole fraction solubility of the solute. 
The mole fraction solubility of the solute was calculated by the compiler using the experimental density data given in the paper.

\section{Auxiliary Information}

\section{Method/Apparatus/Procedure:}

Incubator, shaker, and an ultraviolet/visible spectrophotometer.

Solubility was determined by equilibrating an excess amount of solid naphthalene with the organic solvent using a shaker placed in an incubator equipped with a temperature controlling system. After an equilibration period of at least $48 \mathrm{~h}$, the samples were withdrawn and filtered through hydrophobic Durapore filters $(0.45 \mu \mathrm{m})$. The filtered sample was diluted quantitatively with methanol. Concentrations were determined by spectrophotmetric analysis at $274 \mathrm{~nm}$.

\section{Source and Purity of Chemicals:}

(1) $99 \%$, Fluka Chemical Company, was used as received.

(2) $99.5 \%$, Merck Chemical Company, Germany. No purification details were provided in the paper.

Estimated Error:

Temperature: $\pm 0.2 \mathrm{~K}$.

$x_{1}: \pm 2 \%$ (relative error).

\begin{tabular}{ll}
\hline \hline Components: & Original Measurements: \\
(1) Naphthalene; $\mathrm{C}_{10} \mathrm{H}_{8} ;[91-20-3]$ & ${ }^{55}$ T. Tsuji, K. Sue, T. Hiaki, and N. \\
(2) cis-Decahydronaphthalene; $\mathrm{C}_{10} \mathrm{H}_{18} ;$ & Itoh, Fluid Phase Equilib. 257, 183 \\
[493-01-6] & $(2007)$. \\
\hline Variables: & Prepared by: \\
Temperature & W. E. Acree, Jr. \\
\hline
\end{tabular}

Experimental Values

\begin{tabular}{lcc}
\hline \hline$T / \mathrm{K}$ & $x_{2}{ }^{\mathrm{a}}$ & $x_{1}{ }^{\mathrm{b}}$ \\
\hline 353.14 & 0.0000 & 1.0000 \\
347.35 & 0.1073 & 0.8927 \\
342.01 & 0.2024 & 0.7976 \\
336.54 & 0.2985 & 0.7015 \\
330.14 & 0.4178 & 0.5822 \\
325.40 & 0.4971 & 0.5029 \\
321.47 & 0.5488 & 0.4512 \\
308.48 & 0.7041 & 0.2959 \\
302.29 & 0.7631 & 0.2369 \\
295.66 & 0.8045 & 0.1955 \\
272.67 & 0.9087 & 0.0913 \\
244.47 & 0.9642 & 0.0358 \\
227.99 & 0.9800 & 0.0200 \\
226.69 & 0.9895 & 0.0105 \\
228.07 & 1.0000 & 0.0000
\end{tabular}

${ }^{a} x_{2}$ : mole fraction of component 2 in the saturated solution.

${ }^{\mathrm{b}} \mathrm{x}_{1}$ : mole fraction of the polycyclic aromatic hydrocarbon (component 1 ).

\section{Auxiliary Information}

\section{Method/Apparatus/Procedure:}

Pyrex glass sample cell, resistance thermometer, view type Dewar flask and a magnetic stirrer.

Solubilities were determined by a synthetic-dynamic method. Known masses of solute and solvent were placed in the sample. The solid in the solution dissolved as the temperature was increased. The temperature at which last amount of solid dissolved was recorded as the solid-liquid equilibrium temperature.

Source and Purity of Chemicals:

(1) $99.0 \%$, Wako Pure Chemical Industries Ltd., Osaka, Japan, used as received.

(2) $97.0 \%$, Wako Pure Chemical Industries Ltd. Gas chromatographic analysis showed the purity to be $97.8 \%$. Sample was further purified by distillation by a rotary evaporator.

\section{Estimated Error:}

Temperature: $\pm 0.2 \mathrm{~K}$ (estimated by compiler). $x_{1}:<2 \%$ (relative error, estimated by compiler).

\begin{tabular}{ll}
\hline \hline Components: & Original Measurements: \\
(1) Naphthalene; $\mathrm{C}_{10} \mathrm{H}_{8} ;[91-20-3]$ & ${ }^{55} \mathrm{~T}$. Tsuji, K. Sue, T. Hiaki, and N. \\
(2) trans-Decahydronaphthalene; & Itoh, Fluid Phase Equilib. 257, 183 \\
$\mathrm{C}_{10} \mathrm{H}_{18} ;[493-02-7]$ & $(2007)$. \\
\hline Variables: & Prepared by: \\
Temperature & W. E. Acree, Jr. \\
\hline
\end{tabular}

Experimental Values

\begin{tabular}{lcc}
\hline \hline$T / \mathrm{K}$ & $x_{2}{ }^{\mathrm{a}}$ & $x_{1}{ }^{\mathrm{b}}$ \\
\hline 353.14 & 0.0000 & 1.0000 \\
349.21 & 0.0687 & 0.9313 \\
341.67 & 0.2164 & 0.7836 \\
335.97 & 0.3245 & 0.6755 \\
328.04 & 0.4634 & 0.5366 \\
320.76 & 0.5739 & 0.4261 \\
313.52 & 0.6539 & 0.3461 \\
308.20 & 0.7117 & 0.2883 \\
290.96 & 0.8272 & 0.1728 \\
269.03 & 0.9244 & 0.0756 \\
264.10 & 0.9384 & 0.0616 \\
251.96 & 0.9610 & 0.0390 \\
242.00 & 0.9798 & 0.0202 \\
241.98 & 0.9864 & 0.0136 \\
242.31 & 0.9899 & 0.0101 \\
242.71 & 1.0000 & 0.0000 \\
\hline
\end{tabular}

${ }^{a} x_{2}$ : mole fraction of component 2 in the saturated solution.

${ }^{b} x_{1}$ : mole fraction of the polycyclic aromatic hydrocarbon (component 1 ).

\section{Auxiliary Information}

\section{Method/Apparatus/Procedure:}

Pyrex glass sample cell, resistance thermometer, view type Dewar flask, and a magnetic stirrer.

Solubilities were determined by a synthetic-dynamic method. Known masses of solute and solvent were placed in the sample. The solid in the solution dissolved as the temperature was increased. The temperature at which last amount of solid dissolved was recorded as the solid-liquid equilibrium temperature. 
Source and Purity of Chemicals:

(1) $99.0 \%$, Wako Pure Chemical Industries Ltd., Osaka, Japan, used as received.

(2) $97.0 \%$, Wako Pure Chemical Industries Ltd. Gas chromatographic analysis showed the purity to be $99.9 \%$, and the chemical was used as received.

\section{Estimated Error:}

Temperature: $\pm 0.2 \mathrm{~K}$ (estimated by compiler).

$x_{1}:<2 \%$ (relative error, estimated by compiler)

\begin{tabular}{ll}
\hline \hline Components: & Original Measurements: \\
(1) Naphthalene; $\mathrm{C}_{10} \mathrm{H}_{8} ;[91-20-3]$ & ${ }^{15}$ A. Aoulmi, M. Bouroukba, R. \\
(2) Squalane; $\mathrm{C}_{30} \mathrm{H}_{62} ;[111-01-3]$ & Solimando, and M. Rogalski, Fluid \\
& Phase Equilib. 110, 283 (1995). \\
\hline Variables: & Prepared by: \\
Temperature & W. E. Acree, Jr. \\
\hline
\end{tabular}

Experimental Values

\begin{tabular}{lcc}
\hline \hline$T / \mathrm{K}$ & $x_{2}{ }^{\mathrm{a}}$ & $x_{1}{ }^{\mathrm{b}}$ \\
\hline 327.75 & 0.4472 & 0.5528 \\
332.05 & 0.3975 & 0.6025 \\
335.55 & 0.3486 & 0.6514 \\
338.55 & 0.2971 & 0.7029 \\
341.05 & 0.2447 & 0.7553 \\
344.05 & 0.1984 & 0.8016 \\
346.35 & 0.1487 & 0.8513 \\
348.65 & 0.0983 & 0.9017 \\
350.55 & 0.0492 & 0.9508
\end{tabular}

${ }^{\mathrm{a}} x_{2}$ : mole fraction of component 2 in the saturated solution.

${ }^{\mathrm{b}} \mathrm{x}_{1}$ : mole fraction of the polycyclic aromatic hydrocarbon (component 1 ).

The authors employed a SSF to describe the activity coefficients of naphthalene and squalane calculated from the solidliquid equilibrium data. The SSF model gave a calculated eutectic temperature of $T / \mathrm{K}=233.6$ and eutectic mole fraction of naphthalene of $x_{1}=0.04$.

\section{Auxiliary Information}

\begin{abstract}
Method/Apparatus/Procedure:
Simple thermal analysis device constructed in the authors' laboratory, and a high precision platinum-resistance thermometer.

Phase diagram was determined using a simple thermal device. A sample of known composition was placed in a thermostatted glass tube, melted, and then slowly cooled at a rate of about $1 \mathrm{~K} / \mathrm{min}$. The temperature of the sample was measured with a high precision platinum-resistance thermometer. The crystallization temperature was obtained from a plot of the cooling curve versus time. Each measurement was repeated three times.
\end{abstract}

Source and Purity of Chemicals:

(1) $99 \%$, Prolaboo, no purification details were given in the paper.

(2) $99 \%$, Aldrich Chemical Company, Milwaukee, WI, USA, purification details were not given in the paper.

\section{Estimated Error:}

Temperature: Authors state a global accuracy of $\pm 1 \%$ regarding their measurements.

$x_{1}: \pm 0.0002$ (estimated by compiler).

\subsection{Naphthalene solubility data in aromatic hydrocarbons}

\begin{tabular}{ll}
\hline \hline Components: & Original Measurements: \\
(1) Naphthalene; $\mathrm{C}_{10} \mathrm{H}_{8} ;[91-20-3]$ & ${ }^{52}$ T.-B. Cui, T.-L. Luo, C. Zhang, \\
(2) Methylbenzene; $\mathrm{C}_{7} \mathrm{H}_{8} ;[108-88-3]$ & Z.-B. Mao, and G.-J. Liu, J. Chem. \\
& Eng. Data 54, 1065 (2009). \\
\hline Variables: & Prepared by: \\
Temperature & W. E. Acree, Jr. \\
\hline
\end{tabular}

Temperature

Experimental Values

\begin{tabular}{lcc}
\hline \hline$T / \mathrm{K}$ & $x_{2}{ }^{\mathrm{a}}$ & $x_{1}{ }^{\mathrm{b}}$ \\
\hline 291.35 & 0.7605 & 0.2395 \\
301.45 & 0.6897 & 0.3103 \\
304.54 & 0.6604 & 0.3396 \\
308.53 & 0.6261 & 0.3739 \\
311.93 & 0.5945 & 0.4055 \\
315.25 & 0.5613 & 0.4387 \\
318.55 & 0.5283 & 0.4717 \\
322.23 & 0.4973 & 0.5027 \\
324.37 & 0.4629 & 0.5371 \\
327.55 & 0.4111 & 0.5889 \\
331.35 & 0.3626 & 0.6374 \\
334.65 & 0.3227 & 0.6773 \\
\hline
\end{tabular}

${ }^{a} x_{2}$ : mole fraction of component 2 in the saturated solution.

${ }^{{ }} x_{1}$ : mole fraction of the polycyclic aromatic hydrocarbon (component 1 ).

\section{Auxiliary Information}

\section{Method/Apparatus/Procedure:}

Double-jacketed glass vessel, laser generator, water bath, photoelectric transformer, and digital display.

Solubilities were determined by a synthetic-dynamic method. Known masses of solute and solvent were placed in a dissolving flask. The solid in the solution dissolved as the temperature was increased. The temperature at which last amount of solid dissolved was recorded as the solid-liquid equilibrium temperature. The disappearance of the solid solute was determined by laser monitoring.

\section{Source and Purity of Chemicals:}

(1) Initial purity not given, Tianjin Kermel Chemical Reagent Purchase and Supply Station, China, was dissolved in acetone, filtered and the acetone evaporated. The sample was then recrystallized several times from methanol. The purity of the recrystallized sample was $99.8 \%$ as determined by UV spectrometry.

(2) $99.5 \%$, Tianjin Kermel Chemical Reagent Company, Ltd, China, no information given regarding any further purification.

\section{Estimated Error:}

Temperature: $\pm 0.16 \mathrm{~K}$

$x_{1}: 2 \%$ (relative error) 


\section{Components:}

(1) Naphthalene; $\mathrm{C}_{10} \mathrm{H}_{8} ;$ [91-20-3]

(2) Dimethylbenzene (mixture of isomers);

$\mathrm{C}_{8} \mathrm{H}_{10}$

13.3\% 1,2-Dimethylbenzene; [95-47-6]

66.4\% 1,3-Dimethylbenzene; [108-38-3]

20.3\% 1,4-Dimethylbenzene; [106-42-3]

\begin{tabular}{ll}
\hline Variables: & Prepared by: \\
Temperature & W. E. Acree, Jr. \\
\hline
\end{tabular}

Experimental Values

\begin{tabular}{lcc}
\hline \hline$T / \mathrm{K}$ & $x_{2}{ }^{\mathrm{a}}$ & $x_{1}{ }^{\mathrm{b}}$ \\
\hline 299.42 & 0.7082 & 0.2918 \\
302.70 & 0.6821 & 0.3179 \\
306.14 & 0.6566 & 0.3434 \\
309.84 & 0.6285 & 0.3715 \\
311.96 & 0.6019 & 0.3981 \\
315.52 & 0.5605 & 0.4395 \\
319.25 & 0.5209 & 0.4791 \\
322.13 & 0.4917 & 0.5083 \\
325.05 & 0.4554 & 0.5446 \\
328.66 & 0.4198 & 0.5802 \\
330.46 & 0.3914 & 0.6086 \\
333.53 & 0.3533 & 0.6467 \\
335.56 & 0.3150 & 0.6850 \\
337.97 & 0.2804 & 0.7196
\end{tabular}

${ }^{\mathrm{a}} x_{2}$ : mole fraction of component 2 in the saturated solution.

${ }^{b} x_{1}$ : mole fraction of the polycyclic aromatic hydrocarbon (component 1 ).

\section{Auxiliary Information}

\section{Method/Apparatus/Procedure:}

Double-jacketed glass vessel, laser generator, water bath, photoelectric transformer, and digital display.

Solubilities were determined by a synthetic-dynamic method. Known masses of solute and solvent were placed in a dissolving flask. The solid in the solution dissolved as the temperature was increased. The temperature at which last amount of solid dissolved was recorded as the solid-liquid equilibrium temperature. The disappearance of the solid solute was determined by laser monitoring.

\section{Source and Purity of Chemicals:}

(1) Initial purity not given, Tianjin Kermel Chemical Reagent Purchase and Supply Station, China, was dissolved in acetone, filtered and the acetone evaporated. The sample was then recrystallized several times from methanol. The purity of the recrystallized sample was $99.8 \%$ as determined by UV spectrometry.

(2) Mixture of isomers, Tianjin Kermel Chemical Reagent Company, Ltd, China, no information given regarding any further purification.

\section{Estimated Error:}

Temperature: $\pm 0.1 \mathrm{~K}$.

$x_{1}: 1 \%$ (relative error).

\begin{tabular}{ll}
\hline \hline Components: & Original Measurements: \\
(1) Naphthalene; $\mathrm{C}_{10} \mathrm{H}_{8} ;[91-20-3]$ & ${ }^{39}$ A. C. Mengarelli, S. B. Bottini, \\
(2) Biphenyl; $\mathrm{C}_{12} \mathrm{H}_{10} ;[92-52-4]$ & and E. A. Brignole, J. Chem. Eng. \\
& Data 40, 746 (1995). \\
\hline Variables: & Prepared by: \\
Temperature & W. E. Acree, Jr. \\
\hline
\end{tabular}

Experimental Values

\begin{tabular}{lcc}
\hline \hline$T / \mathrm{K}$ & $x_{1}{ }^{\mathrm{a}}$ & $x_{2}{ }^{\mathrm{b}}$ \\
\hline 351.88 & 1.00 & 0.00 \\
347.49 & 0.92 & 0.08 \\
342.42 & 0.83 & 0.17 \\
336.26 & 0.74 & 0.26 \\
329.50 & 0.64 & 0.36 \\
321.50 & 0.55 & 0.45 \\
317.69 & 0.50 & 0.50 \\
312.20 & 0.44 & 0.56 \\
316.15 & 0.39 & 0.61 \\
320.99 & 0.34 & 0.66 \\
328.76 & 0.23 & 0.77 \\
335.20 & 0.12 & 0.88 \\
341.62 & 0.00 & 1.00 \\
\hline
\end{tabular}

${ }^{\mathrm{a}} x_{1}$ : mole fraction of the polycyclic aromatic hydrocarbon (component 1).

${ }^{b} x_{2}$ : mole fraction of component 2 in the saturated solution.

\section{Auxiliary Information}

\section{Method/Apparatus/Procedure:}

Thermostatted liquid bath, platinum resistance thermometer, and a coiled wire driven by a small electrical motor to provide stirring.

A liquid mixture of known composition was prepared by mass and placed in a glass tube. The solution was slowly cooled until a solid phase appeared. The temperature of the solution was recorded continuously with a platinum resistance thermometer. A break point in the temperature versus time curve indicated formation of a solid phase. The temperature at break point was taken to be the solid-liquid equilibrium temperature, and the composition of the solution gives the solubility of the crystalline solute in the liquid solvent.

\section{Source and Purity of Chemicals:}

(1) Chemical purity and source not given in the paper.

(2) Chemical purity and source not given in the paper.

Estimated Error:

Temperature: $\pm 0.5 \mathrm{~K}$.

$x_{1}: \pm 0.01$ (estimated by compiler).

\begin{tabular}{ll}
\hline \hline Components: & Original Measurements: \\
(1) Naphthalene; $\mathrm{C}_{10} \mathrm{H}_{8} ;[91-20-3]$ & ${ }^{56} \mathrm{~B}$. L. Sharma, R. Kant, R. \\
(2) Phenanthrene; $\mathrm{C}_{14} \mathrm{H}_{10} ;[85-01-8]$ & Sharma, and S. Tandon, Mater. \\
& Chem. Phys. 82, 216 (2003). \\
\hline Variables: & Prepared by: \\
Temperature & W. E. Acree, Jr. \\
\hline
\end{tabular}


Experimental Values

\begin{tabular}{lll}
\hline \hline$T / \mathrm{K}$ & $x_{2}{ }^{\mathrm{a}}$ & $x_{1}{ }^{\mathrm{b}}$ \\
\hline 373.0 & 1.0 & 0.0 \\
364.0 & 0.9 & 0.1 \\
356.0 & 0.8 & 0.2 \\
348.0 & 0.7 & 0.3 \\
339.0 & 0.6 & 0.4 \\
329.0 & 0.5 & 0.5 \\
326.0 & 0.4 & 0.6 \\
334.0 & 0.3 & 0.7 \\
340.0 & 0.2 & 0.8 \\
346.5 & 0.1 & 0.9 \\
353.0 & 0.0 & 1.0
\end{tabular}

${ }^{a} x_{2}$ : mole fraction of component 2 in the saturated solution.

${ }^{b} x_{1}$ : mole fraction of the polycyclic aromatic hydrocarbon (component 1 ).

\section{Auxiliary Information}

\section{Method/Apparatus/Procedure:}

The only experimental details provided in the paper was a statement by the authors that the solidus-liquid equilibrium data were obtained by the thawpoint melting technique.

Source and Purity of Chemicals:

(1) Laboratory Reagent Grade, Riedel Chemicals, was purified by sublimation. (2) Analytical Reagent Grade, Fluka Chemicals, was purified by repeated recrystallizations from ethyl ethanoate.

Estimated Error:

Temperature: $\pm 0.5 \mathrm{~K}$ (estimated by compiler). $x_{1}: \pm 0.05$ (estimated by compiler).

\subsection{Naphthalene solubility data in esters}

\begin{tabular}{ll}
\hline \hline Components: & Original Measurements: \\
(1) Naphthalene; $\mathrm{C}_{10} \mathrm{H}_{8} ;[91-20-3]$ & ${ }^{53}$ Q.-S. Li, Z.-M. Yi, M.-G. \\
(2) Ethyl ethanoate; $\mathrm{C}_{4} \mathrm{H}_{8} \mathrm{O}_{2} ;[141-78-6]$ & Su, and X.-F. Sun, J. Chem. \\
& Eng. Data 53, 2701 (2008). \\
\hline Variables: & Prepared by: \\
Temperature & W. E. Acree, Jr. \\
\hline
\end{tabular}

Experimental Values

\begin{tabular}{lcc}
\hline \hline$T / \mathrm{K}$ & $x_{2}{ }^{\mathrm{a}}$ & $x_{1}{ }^{\mathrm{b}}$ \\
\hline 278.20 & 0.8256 & 0.1744 \\
283.17 & 0.8090 & 0.1910 \\
288.21 & 0.7826 & 0.2174 \\
293.19 & 0.7670 & 0.2330 \\
298.25 & 0.7236 & 0.2764 \\
303.20 & 0.6861 & 0.3139 \\
308.21 & 0.6429 & 0.3571 \\
313.23 & 0.5940 & 0.4060 \\
318.17 & 0.5349 & 0.4651 \\
323.21 & 0.4743 & 0.5257
\end{tabular}

${ }^{\mathrm{a}} x_{2}$ : mole fraction of component 2 in the saturated solution.

${ }^{b} x_{1}$ : mole fraction of the polycyclic aromatic hydrocarbon (component 1).

\section{Auxiliary Information}

\section{Method/Apparatus/Procedure:}

Double-jacketed glass vessel, laser generator, water bath, photoelectric transformer, and digital display.

Solubilities were determined by a synthetic-dynamic method. Known masses of solute and solvent were placed in the inner chamber of a double-jacketed glass vessel. The vessel was maintained at a constant temperature by circulating water from a water bath equipped with a thermoelectric controller. The solute and solvent mixture was stirred with a magnetic stirrer. 1-3 mg of solute were added at predetermined intervals until no more solid would dissolve. Disappearance of the added solute was observed visually and with laser monitoring.

Source and Purity of Chemicals:

(1) $99 \%$, Tianjin Kermel Chemical Reagent, China, was used as received.

(2) Research Grade, Shanghai Chemical Reagent, China, was used as received.

Estimated Error:

Temperature: $\pm 0.1 \mathrm{~K}$.

$x_{1}: 1.0 \%$ (relative error).

\begin{tabular}{ll}
\hline \hline Components: & Original Measurements: \\
(1) Naphthalene; $\mathrm{C}_{10} \mathrm{H}_{8} ;[91-20-3]$ & ${ }^{53}$ Q.-S. Li, Z.-M. Yi, M.-G. \\
(2) 2-Methylpropyl ethanoate; $\mathrm{C}_{6} \mathrm{H}_{12} \mathrm{O}_{2} ;$ & Su, and X.-F. Sun, J. Chem. \\
[110-19-0] & Eng. Data 53, 2701 (2008). \\
\hline Variables: & Prepared by: \\
Temperature & W. E. Acree, Jr. \\
\hline
\end{tabular}

Experimental Values

\begin{tabular}{lcc}
\hline \hline$T / \mathrm{K}$ & $x_{2}{ }^{\mathrm{a}}$ & $x_{1}{ }^{\mathrm{b}}$ \\
\hline 278.18 & 0.8169 & 0.1831 \\
283.20 & 0.4953 & 0.2047 \\
288.22 & 0.4681 & 0.2319 \\
293.23 & 0.7407 & 0.2593 \\
298.19 & 0.7011 & 0.2989 \\
303.23 & 0.6655 & 0.3345 \\
308.45 & 0.6135 & 0.3865 \\
313.20 & 0.5673 & 0.4327 \\
318.17 & 0.5166 & 0.4834 \\
323.21 & 0.4607 & 0.5393 \\
\hline
\end{tabular}

${ }^{\mathrm{a}} x_{2}$ : mole fraction of component 2 in the saturated solution.

${ }^{\mathrm{b}} x_{1}$ : mole fraction of the polycyclic aromatic hydrocarbon (component 1).

\section{Auxiliary Information}

Method/Apparatus/Procedure:

Double-jacketed glass vessel, laser generator, water bath, photoelectric transformer, and digital display.

Solubilities were determined by a synthetic-dynamic method. Known masses of solute and solvent were placed in the inner chamber of a double-jacketed glass vessel. The vessel was maintained at a constant temperature by circulating water from a water bath equipped with a thermoelectric controller. The solute and solvent mixture was stirred with a magnetic stirrer. 1-3 mg of solute were added at predetermined intervals until no more solid would dissolve. Disappearance of the added solute was observed visually and with laser monitoring.

Source and Purity of Chemicals:

(1) $99 \%$, Tianjin Kermel Chemical Reagent, China, was used as received.

(2) Research Grade, Shanghai Chemical Reagent, China, was used as received. 
Estimated Error:

Temperature: $\pm 0.1 \mathrm{~K}$.

$x_{1}: 1.0 \%$ (relative error).
Variables:

Temperature
Prepared by:

W. E. Acree, Jr.

Experimental Values

\subsection{Naphthalene solubility data in ethers}

\begin{tabular}{ll}
\hline \hline Components: & Original Measurements: \\
(1) Naphthalene; $\mathrm{C}_{10} \mathrm{H}_{8} ;[91-20-3]$ & ${ }^{53}$ Q.-S. Li, Z.-M. Yi, M.-G. \\
(2) Tetrahydrofuran; $\mathrm{C}_{4} \mathrm{H}_{8} \mathrm{O} ;$ & Su, and X.-F. Sun, J. Chem. \\
[109-99-9] & Eng. Data 53, 2701 (2008). \\
\hline Variables: & Prepared by: \\
Temperature & W. E. Acree, Jr. \\
\hline
\end{tabular}

Experimental Values

\begin{tabular}{lcc}
\hline \hline$T / \mathrm{K}$ & $x_{2}{ }^{\mathrm{a}}$ & $x_{1}{ }^{\mathrm{b}}$ \\
\hline 278.60 & 0.7588 & 0.2412 \\
284.35 & 0.7274 & 0.2726 \\
288.25 & 0.7082 & 0.2918 \\
293.25 & 0.6758 & 0.3242 \\
298.00 & 0.6484 & 0.3516 \\
303.20 & 0.6151 & 0.3849 \\
308.19 & 0.5716 & 0.4284 \\
313.25 & 0.5315 & 0.4685 \\
318.35 & 0.4898 & 0.5102 \\
323.20 & 0.4343 & 0.5657 \\
328.21 & 0.4001 & 0.5999 \\
333.22 & 0.3708 & 0.6292
\end{tabular}

${ }^{\mathrm{a}} x_{2}$ : mole fraction of component 2 in the saturated solution.

${ }^{\mathrm{b}} \mathrm{x}_{1}$ : mole fraction of the polycyclic aromatic hydrocarbon (component 1 ).

\section{Auxiliary Information}

\section{Method/Apparatus/Procedure:}

Double-jacketed glass vessel, laser generator, water bath, photoelectric transformer, and digital display.

Solubilities were determined by a synthetic-dynamic method. Known masses of solute and solvent were placed in the inner chamber of a double-jacketed glass vessel. The vessel was maintained at a constant temperature by circulating water from a water bath equipped with a thermoelectric controller. The solute and solvent mixture was stirred with a magnetic stirrer. 1-3 mg of solute were added at predetermined intervals until no more solid would dissolve. Disappearance of the added solute was observed visually and with laser monitoring.

Source and Purity of Chemicals:

(1) $99 \%$, Tianjin Kermel Chemical Reagent, China, was used as received.

(2) Research Grade, Shanghai Chemical Reagent, China, was used as received.

Estimated Error:

Temperature: $\pm 0.1 \mathrm{~K}$.

$x_{1}: 1.0 \%$ (relative error)

\section{Components:}

(1) Naphthalene; $\mathrm{C}_{10} \mathrm{H}_{8}$; [91-20-3]

(2) Methyl nonafluorobutyl ether;

$\mathrm{C}_{5} \mathrm{H}_{3} \mathrm{~F}_{9} \mathrm{O} ;[163702-07-6]$

\section{Original Measurements:}

${ }^{60}$ A. F. Lagalante, A.

Abdulagatov, and T. J. Bruno, J.

Chem. Eng. Data 47, 47 (2002).
Auxiliary Information

Method/Apparatus/Procedure:

UV/visible spectrophotometer.

Solubilities were determined by supersaturating the solvent with naphthalene and measuring the amount of dissolved solute using a spectroscopic method. Aliquots of the saturated solution were pipette into a volumetric flask, and diluted with acetonitrile. If necessary, the sample was further diluted to bring the measured absorbance to within the linear range of the absorbance versus calibration curve obtained from absorbance measurements on solutions of known naphthalene concentration. Absorbance measurements recorded at $275 \mathrm{~nm}$.

Source and Purity of Chemicals:

(1) Purity and Chemical Source were not given, was used as received.

(2) $99 \%$, Name of commercial supplier was not given, used as received.

Estimated Error:

Temperature: $\pm 0.1 \mathrm{~K}$.

$x_{1}: 2.0 \%$ (relative error, estimated by compiler).

\begin{tabular}{ll}
\hline \hline Components: & Original Measurements: \\
(1) Naphthalene; $\mathrm{C}_{10} \mathrm{H}_{8} ;[91-20-3]$ & ${ }^{60}$ A. F. Lagalante, A. \\
(2) Ethyl nonafluorobutyl ether; & Abdulagatov, and T. J. Bruno, J. \\
$\mathrm{C}_{6} \mathrm{H}_{5} \mathrm{~F}_{9} \mathrm{O} ;[163702-05-4]$ & Chem. Eng. Data 47, 47 (2002). \\
\hline Variables: & Prepared by: \\
Temperature & W. E. Acree, Jr. \\
\hline
\end{tabular}

Experimental Values

\begin{tabular}{lll}
\hline \hline$T / \mathrm{K}$ & \multicolumn{1}{c}{$x_{2}{ }^{\mathrm{a}}$} & \multicolumn{1}{c}{$x_{1}{ }^{\mathrm{b}}$} \\
\hline 259.7 & 0.9963 & 0.00374 \\
268.2 & 0.9950 & 0.00500 \\
283.2 & 0.9924 & 0.00763 \\
298.2 & 0.9863 & 0.0137 \\
313.3 & 0.9784 & 0.0216 \\
328.2 & 0.7930 & 0.207 \\
343.2 & 0.7390 & 0.261 \\
\hline
\end{tabular}

${ }^{a_{2}} x_{2}$ mole fraction of component 2 in the saturated solution.

${ }^{\mathrm{b}} x_{1}$ : mole fraction of the polycyclic aromatic hydrocarbon (component 1 ). 


\section{Auxiliary Information}

\section{Method/Apparatus/Procedure:}

UV/visible spectrophotometer.

Solubilities were determined by supersaturating the solvent with naphthalene and measuring the amount of dissolved solute using a spectroscopic method. Aliquots of the saturated solution were pipette into a volumetric flask, and diluted with acetonitrile. If necessary, the sample was further diluted to bring the measured absorbance to within the linear range of the absorbance versus calibration curve obtained from absorbance measurements on solutions of known naphthalene concentration. Absorbance measurements recorded at $275 \mathrm{~nm}$.

Source and Purity of Chemicals:

(2) $99 \%$, Name of commercial supplier was not given, used as received.

Estimated Error:

Temperature: $\pm 0.1 \mathrm{~K}$

$x_{1}: 1.0 \%$ (relative error)
(1) Purity and Chemical Source were not given, was used as received.

Source and Purity of Chemicals:

(1) Analytically pure, Polish Chemical Reagents, Gliwice, Poland, recrystallized twice from distilled benzene and then from anhydrous ethanol. (2) $99.5+\%$, purchased from either Fluka or Aldrich Chemical Company, was used as received.

\section{Estimated Error:}

Temperature: $\pm 0.3 \mathrm{~K}$.

$x_{1}:<1 \%$ (relative error).

\section{Components:}

(1) Naphthalene; $\mathrm{C}_{10} \mathrm{H}_{8}$; [91-20-3]

(2) Tetrachloromethane; $\mathrm{CCl}_{4} ;[56-23-5]$

Original Measurements:

${ }^{13}$ I. Kotula and B. Marciniak, J. Chem. Eng. Data 46, 783 (2001).

\begin{tabular}{ll}
\hline Variables: & Prepared by: \\
Temperature & W. E. Acree, Jr. \\
\hline
\end{tabular}

Experimental Values

\subsection{Naphthalene solubility data in haloalkanes, haloalkenes, and haloaromatic hydrocarbons}

\begin{tabular}{|c|c|}
\hline $\begin{array}{l}\text { Components: } \\
\text { (1) Naphthalene; } \mathrm{C}_{10} \mathrm{H}_{8} ;[91-20-3] \\
\text { (2) Trichloromethane; } \mathrm{CHCl}_{3} ;[67-66-3]\end{array}$ & $\begin{array}{l}\text { Original Measurements: } \\
{ }^{13} \text { I. Kotula and B. Marciniak, J. } \\
\text { Chem. Eng. Data 46, } 783 \text { (2001). }\end{array}$ \\
\hline $\begin{array}{l}\text { Variables: } \\
\text { Temperature }\end{array}$ & W. E. Acree, Jr. \\
\hline
\end{tabular}

Experimental Values

\begin{tabular}{lcc}
\hline \hline$T / \mathrm{K}$ & $x_{2}{ }^{\mathrm{a}}$ & $x_{1}{ }^{\mathrm{b}}$ \\
\hline 288.85 & 0.7501 & 0.2499 \\
290.15 & 0.7415 & 0.2585 \\
293.05 & 0.7214 & 0.2786 \\
296.55 & 0.6956 & 0.3044 \\
300.65 & 0.6633 & 0.3367 \\
305.55 & 0.6215 & 0.3785 \\
310.05 & 0.5799 & 0.4201 \\
312.55 & 0.5554 & 0.4446 \\
315.65 & 0.5237 & 0.4763 \\
318.25 & 0.4959 & 0.5041 \\
320.05 & 0.4759 & 0.5241 \\
322.95 & 0.4426 & 0.5574
\end{tabular}

${ }^{\mathrm{a}} x_{2}$ : mole fraction of component 2 in the saturated solution.

${ }^{b} x_{1}$ : mole fraction of the polycyclic aromatic hydrocarbon (component 1 ).

\section{Auxiliary Information}

\footnotetext{
Method/Apparatus/Procedure:

Constant-temperature bath, electric glass furnace, heater, mercury thermometer.

Solubilities were determined by a synthetic-dynamic method. Known masses of solute and solvent were placed in a Pyrex test tube and sealed by a flexible rubber cap mounted on a calibrated mercury thermometer. The solute-solvent were heated using an electrical glass furnace equipped with a platinum resistor placed near the heater. Samples were agitated and the temperature at which the solid solute disappeared was observed against a dark screen by means of stereoscopic microscope.
}

\begin{tabular}{lcc}
\hline \hline$T / \mathrm{K}$ & $x_{2}{ }^{\mathrm{a}}$ & $x_{1}{ }^{\mathrm{b}}$ \\
\hline 288.45 & 0.8070 & 0.1930 \\
291.35 & 0.7892 & 0.2108 \\
295.35 & 0.7623 & 0.2377 \\
297.05 & 0.7498 & 0.2502 \\
298.65 & 0.7375 & 0.2625 \\
305.15 & 0.6848 & 0.3152 \\
311.05 & 0.6301 & 0.3699 \\
313.55 & 0.6048 & 0.3952 \\
317.95 & 0.5566 & 0.4434 \\
318.75 & 0.5480 & 0.4520 \\
320.15 & 0.5319 & 0.4681 \\
321.85 & 0.5107 & 0.4893
\end{tabular}

${ }^{\mathrm{a}} \mathrm{x}_{2}$ : mole fraction of component 2 in the saturated solution.

${ }^{\mathrm{b}} x_{1}$ : mole fraction of the polycyclic aromatic hydrocarbon (component 1 ).

Auxiliary Information

Method/Apparatus/Procedure:

Constant-temperature bath, electric glass furnace, heater, mercury thermometer.

Solubilities were determined by a synthetic-dynamic method. Known masses of solute and solvent were placed in a Pyrex test tube and sealed by a flexible rubber cap mounted on a calibrated mercury thermometer. The solute-solvent were heated using an electrical glass furnace equipped with a platinum resistor placed near the heater. Samples were agitated and the temperature at which the solid solute disappeared was observed against a dark screen by means of stereoscopic microscope.

Source and Purity of Chemicals:

(1) Analytically pure, Polish Chemical Reagents, Gliwice, Poland, recrystallized twice from distilled benzene and then from anhydrous ethanol. (2) Purity not given, Polish Chemical Reagents, Gliwice, Poland, was dried over anhydrous sodium sulfate and then fractionally distilled.

\section{Estimated Error:}

Temperature: $\pm 0.3 \mathrm{~K}$.

$x_{1}:<1 \%$ (relative error). 


\section{Components:}

(1) Naphthalene; $\mathrm{C}_{10} \mathrm{H}_{8} ;$ [91-20-3]

(2) 1,1-Dichloroethane; $\mathrm{C}_{2} \mathrm{H}_{4} \mathrm{Cl}_{2} ;$ [75-34-3]

\begin{tabular}{ll} 
& $(2001)$. \\
\hline Variables: & Prepared by: \\
Temperature & W. E. Acree, Jr. \\
\hline
\end{tabular}

Experimental Values

\begin{tabular}{lcc}
\hline \hline$T / \mathrm{K}$ & $x_{2}{ }^{\mathrm{a}}$ & $x_{1}{ }^{\mathrm{b}}$ \\
\hline 288.35 & 0.7663 & 0.2337 \\
289.85 & 0.7566 & 0.2434 \\
296.35 & 0.7105 & 0.2895 \\
300.65 & 0.6773 & 0.3227 \\
305.95 & 0.6313 & 0.3687 \\
313.45 & 0.5601 & 0.4399 \\
318.25 & 0.5086 & 0.4914 \\
323.75 & 0.4457 & 0.5543 \\
\hline
\end{tabular}

${ }^{\mathrm{a}} x_{2}$ : mole fraction of component 2 in the saturated solution.

${ }^{\mathrm{b}} x_{1}$ : mole fraction of the polycyclic aromatic hydrocarbon (component 1 ).

\section{Auxiliary Information}

\section{Method/Apparatus/Procedure:}

Constant-temperature bath, electric glass furnace, heater, mercury thermometer.

Solubilities were determined by a synthetic-dynamic method. Known masses rubber cap mounted on a calibrated mercury thermometer. The solute-solvent were heated using an electrical glass furnace equipped with a platinum resistor placed near the heater. Samples were agitated and the temperature at which the solid solute disappeared was observed against a dark screen by means of stereoscopic microscope.

\section{Source and Purity of Chemicals:}

(1) Analytically pure, Polish Chemical Reagents, Gliwice, Poland, recrystallized twice from distilled benzene and then from anhydrous ethanol. (2) Purity not given, Polish Chemical Reagents, Glowice, Poland, was dried over anhydrous sodium sulfate and then fractionally distilled.

Estimated Error:

Temperature: $\pm 0.3 \mathrm{~K}$.

$x_{1}:<1 \%$ (relative error). of solute and solvent were placed in a Pyrex test tube and sealed by a flexible

\begin{tabular}{lcc}
\hline \hline$T / \mathrm{K}$ & $x_{2}{ }^{\mathrm{a}}$ & $x_{1}{ }^{\mathrm{b}}$ \\
\hline 298.55 & 0.6801 & 0.3199 \\
302.55 & 0.6475 & 0.3525 \\
306.05 & 0.6170 & 0.3830 \\
308.55 & 0.5941 & 0.4059 \\
310.05 & 0.5799 & 0.4201 \\
313.05 & 0.5504 & 0.4496 \\
315.05 & 0.5299 & 0.4701 \\
316.85 & 0.5110 & 0.4890 \\
318.55 & 0.4926 & 0.5074 \\
320.05 & 0.4759 & 0.5241 \\
321.85 & 0.4555 & 0.5445
\end{tabular}

${ }^{\mathrm{a}} x_{2}$ : mole fraction of component 2 in the saturated solution.

${ }^{\mathrm{b}} x_{1}$ : mole fraction of the polycyclic aromatic hydrocarbon (component 1 ).

\section{Auxiliary Information}

Method/Apparatus/Procedure:

Constant-temperature bath, electric glass furnace, heater, mercury thermometer.

Solubilities were determined by a synthetic-dynamic method. Known masses of solute and solvent were placed in a Pyrex test tube and sealed by a flexible rubber cap mounted on a calibrated mercury thermometer. The solute-solvent were heated using an electrical glass furnace equipped with a platinum resistor placed near the heater. Samples were agitated and the temperature at which the solid solute disappeared was observed against a dark screen by means of stereoscopic microscope.

\section{Source and Purity of Chemicals:}

(1) Analytically pure, Polish Chemical Reagents, Gliwice, Poland, recrystallized twice from distilled benzene and then from anhydrous ethanol. (2) $99.5+\%$, purchased from either Fluka or Aldrich Chemical Company, was used as received.

Estimated Error:

Temperature: $\pm 0.3 \mathrm{~K}$.

$x_{1}:<1 \%$ (relative error).

\begin{tabular}{|c|c|}
\hline $\begin{array}{l}\text { Components: } \\
\text { (1) Naphthalene; } \mathrm{C}_{10} \mathrm{H}_{8} ;[91-20-3] \\
\text { (2) Trichloroethene; } \mathrm{C}_{2} \mathrm{HCl}_{3} ;[79-01-6]\end{array}$ & $\begin{array}{l}\text { Original Measurements: } \\
{ }^{13} \text { I. Kotula and B. Marciniak, J. } \\
\text { Chem. Eng. Data 46, } 783 \text { (2001) }\end{array}$ \\
\hline $\begin{array}{l}\text { Variables: } \\
\text { Temperature }\end{array}$ & $\begin{array}{l}\text { Prepared by: } \\
\text { W. E. Acree, Jr. }\end{array}$ \\
\hline
\end{tabular}

Experimental Values

\begin{tabular}{ll}
\hline \hline Components: & Original Measurements: \\
(1) Naphthalene; $\mathrm{C}_{10} \mathrm{H}_{8} ;[91-20-3]$ & ${ }^{13}$ I. Kotula and B. Marciniak, \\
(2) 1,2-Dichloroethane; $\mathrm{C}_{2} \mathrm{H}_{4} \mathrm{Cl}_{2} ;$ & J. Chem. Eng. Data 46, 783 \\
[107-06-2] & $(2001)$. \\
\hline Variables: & Prepared by: \\
Temperature & W. E. Acree, Jr. \\
\hline
\end{tabular}

Experimental Values

\begin{tabular}{lcc}
\hline \hline$T / \mathrm{K}$ & $x_{2}{ }^{\mathrm{a}}$ & $x_{1}{ }^{\mathrm{b}}$ \\
\hline 288.35 & 0.7534 & 0.2466 \\
291.65 & 0.7312 & 0.2688 \\
294.15 & 0.7134 & 0.2866 \\
296.55 & 0.6956 & 0.3044
\end{tabular}

\begin{tabular}{lcc}
\hline \hline$T / \mathrm{K}$ & $x_{2}{ }^{\mathrm{a}}$ & $x_{1}{ }^{\mathrm{b}}$ \\
\hline 288.35 & 0.7839 & 0.2161 \\
290.85 & 0.7679 & 0.2321 \\
298.55 & 0.7126 & 0.2874 \\
300.85 & 0.6944 & 0.3056 \\
303.35 & 0.6735 & 0.3265 \\
310.15 & 0.6115 & 0.3885 \\
315.85 & 0.5531 & 0.4469 \\
318.45 & 0.5244 & 0.4756 \\
321.75 & 0.4861 & 0.5139 \\
323.25 & 0.4679 & 0.5321 \\
\hline
\end{tabular}

${ }^{\mathrm{a}} x_{2}$ : mole fraction of component 2 in the saturated solution.

${ }^{\mathrm{b}} x_{1}$ : mole fraction of the polycyclic aromatic hydrocarbon (component 1 ). 


\section{Auxiliary Information}

\section{Method/Apparatus/Procedure:}

Constant-temperature bath, electric glass furnace, heater, mercury thermometer.

Solubilities were determined by a synthetic-dynamic method. Known masses of solute and solvent were placed in a Pyrex test tube and sealed by a flexible rubber cap mounted on a calibrated mercury thermometer. The solute-solvent were heated using an electrical glass furnace equipped with a platinum resistor placed near the heater. Samples were agitated and the temperature at which the solid solute disappeared was observed against a dark screen by means of stereoscopic microscope.

Source and Purity of Chemicals:

(1) Analytically pure, Polish Chemical Reagents, Gliwice, Poland, recrystallized twice from distilled benzene and then from anhydrous ethanol. (2) $99.5+\%$, purchased from either Fluka or Aldrich Chemical Company, was used as received.

Estimated Error:

Temperature: $\pm 0.3 \mathrm{~K}$

$x_{1}:<1 \%$ (relative error).

\begin{tabular}{ll}
\hline \hline Components: & Original Measurements: \\
(1) Naphthalene; $\mathrm{C}_{10} \mathrm{H}_{8} ;[91-20-3]$ & ${ }^{13}$ I. Kotula and B. Marciniak, \\
(2) Tetrachloroethene; $\mathrm{C}_{2} \mathrm{Cl}_{4} ;[127-18-4]$ & J. Chem. Eng. Data 46, 783 \\
& $(2001)$. \\
\hline Variables: & Prepared by: \\
Temperature & W. E. Acree, Jr. \\
\hline
\end{tabular}

Experimental Values

\begin{tabular}{lcc}
\hline \hline$T / \mathrm{K}$ & $x_{2}{ }^{\mathrm{a}}$ & $x_{1}{ }^{\mathrm{b}}$ \\
\hline 288.45 & 0.8324 & 0.1676 \\
290.65 & 0.8196 & 0.1804 \\
292.65 & 0.8071 & 0.1929 \\
294.45 & 0.7957 & 0.2043 \\
294.85 & 0.7932 & 0.2068 \\
296.25 & 0.7840 & 0.2160 \\
297.95 & 0.7712 & 0.2288 \\
302.65 & 0.7350 & 0.2650 \\
306.15 & 0.7055 & 0.2945 \\
309.65 & 0.6734 & 0.3266 \\
312.45 & 0.6458 & 0.3542 \\
318.35 & 0.5819 & 0.4181 \\
323.95 & 0.5139 & 0.4861
\end{tabular}

${ }^{\mathrm{a}} x_{2}$ : mole fraction of component 2 in the saturated solution.

${ }^{b} x_{1}$ : mole fraction of the polycyclic aromatic hydrocarbon (component 1 ).

\section{Auxiliary Information}

\section{Method/Apparatus/Procedure:}

Constant-temperature bath, electric glass furnace, heater, mercury thermometer.

Solubilities were determined by a synthetic-dynamic method. Known masses of solute and solvent were placed in a Pyrex test tube and sealed by a flexible rubber cap mounted on a calibrated mercury thermometer. The solute-solvent were heated using an electrical glass furnace equipped with a platinum resistor placed near the heater. Samples were agitated and the temperature at which the solid solute disappeared was observed against a dark screen by means of stereoscopic microscope.
Source and Purity of Chemicals:

(1) Analytically pure, Polish Chemical Reagents, Gliwice, Poland, recrystallized twice from distilled benzene and then from anhydrous ethanol. (2) $99.5+\%$, purchased from either Fluka or Aldrich Chemical Company, was used as received.

\section{Estimated Error:}

Temperature: $\pm 0.3 \mathrm{~K}$.

$x_{1}:<1 \%$ (relative error).

\begin{tabular}{ll}
\hline \hline Components: & Original Measurements: \\
(1) Naphthalene; $\mathrm{C}_{10} \mathrm{H}_{8} ;[91-20-3]$ & ${ }^{57} \mathrm{D}$. Wei and K. Jin, J. \\
(2) 1,2-Dichlorobenzene; $\mathrm{C}_{6} \mathrm{H}_{4} \mathrm{Cl}_{2} ;[95-50-1]$ & Chem. Thermodyn. 41, \\
& $145(2009)$. \\
\hline Variables: & Prepared by: \\
Temperature & W. E. Acree, Jr. \\
\hline
\end{tabular}

Experimental Values

\begin{tabular}{lcc}
\hline \hline$T / \mathrm{K}$ & $x_{2}{ }^{\mathrm{a}}$ & $x_{1}{ }^{\mathrm{b}}$ \\
\hline 255.85 & 1.0000 & 0.0000 \\
244.25 & 0.9711 & 0.0289 \\
240.65 & 0.9251 & 0.0749 \\
252.15 & 0.8579 & 0.1421 \\
270.75 & 0.7910 & 0.2090 \\
278.25 & 0.7568 & 0.2432 \\
285.35 & 0.7199 & 0.2801 \\
289.25 & 0.7018 & 0.2982 \\
295.65 & 0.6585 & 0.3415 \\
304.95 & 0.6036 & 0.3964 \\
309.15 & 0.5700 & 0.4300 \\
313.55 & 0.5331 & 0.4669 \\
319.45 & 0.4710 & 0.5290 \\
323.35 & 0.4388 & 0.5612 \\
329.45 & 0.3770 & 0.6230 \\
330.65 & 0.3510 & 0.6490 \\
336.65 & 0.2787 & 0.7213 \\
339.25 & 0.2308 & 0.7692 \\
344.45 & 0.1529 & 0.8471 \\
347.65 & 0.1098 & 0.8902 \\
349.15 & 0.0779 & 0.9221 \\
353.25 & 0.0000 & 1.0000 \\
\hline
\end{tabular}

${ }^{a} x_{2}$ : mole fraction of component 2 in the saturated solution.

${ }^{\mathrm{b}} x_{1}$ : mole fraction of the polycyclic aromatic hydrocarbon (component 1 ).

The authors report that the system is of an incongruent eutectic type. The eutectic point occurs at $T / \mathrm{K}=232.55$ and $x_{1}=0.130$, and the peritectic point occurs at $T / \mathrm{K}=250.15$ and $x_{1}=0.077$. The composition of the incongruent compound is a molar ratio of 1:1 between naphthalene and 1,2dichlorobenzene. 


\section{Auxiliary Information}

\section{Method/Apparatus/Procedure:}

Differential scanning calorimeter.

Phase diagram was determined using a differential scanning calorimeter.

Samples of naphthalene and dichlorobenzene were heated very slowly inside a glass tube near the melting point of the major component. The fluid solution was then allowed to solidify with continuous stirring. A small quantity of the solid material was then sealed into a dsc sampling crucible for analysis.

Measurements were performed at a constant fixed scanning rate of $1 \mathrm{~K} / \mathrm{min}$

Source and Purity of Chemicals:

(1) $99.5+\%$, Analytical Research Grade, Tianjin Kewei Chemical Reagent Company, China, was used as received.

(2) $99.5+\%$, Analytical Research Grade, Tianjin Kewei Chemical Reagent Company, China, was used as received.

Estimated Error:

Temperature: $\pm 0.2 \mathrm{~K}$.

$x_{1}: \pm 0.002$ (estimated by compiler).

\begin{tabular}{|c|c|}
\hline $\begin{array}{l}\text { Components: } \\
\text { (1) Naphthalene; } \mathrm{C}_{10} \mathrm{H}_{8} ;[91-20-3] \\
\text { (2) 1,3-Dichlorobenzene; } \mathrm{C}_{6} \mathrm{H}_{4} \mathrm{Cl}_{2} \text {; } \\
\text { [541-73-1] }\end{array}$ & $\begin{array}{l}\text { Original Measurements: } \\
{ }^{57} \text { D. Wei and K. Jin, J. Chem. } \\
\text { Thermodyn. 41, } 145 \text { (2009). }\end{array}$ \\
\hline $\begin{array}{l}\text { Variables: } \\
\text { Temperature }\end{array}$ & $\begin{array}{l}\text { Prepared by: } \\
\text { W. E. Acree, Jr. }\end{array}$ \\
\hline
\end{tabular}

Experimental Values

\begin{tabular}{lcc}
\hline \hline$T / \mathrm{K}$ & $x_{2}{ }^{\mathrm{a}}$ & $x_{1}{ }^{\mathrm{b}}$ \\
\hline 248.25 & 1.0000 & 0.0000 \\
261.15 & 0.8886 & 0.1114 \\
272.35 & 0.8420 & 0.1580 \\
283.45 & 0.7822 & 0.2178 \\
293.05 & 0.7189 & 0.2811 \\
299.25 & 0.6750 & 0.3250 \\
304.15 & 0.6329 & 0.3671 \\
311.65 & 0.5640 & 0.4360 \\
317.55 & 0.5038 & 0.4962 \\
321.85 & 0.4542 & 0.5458 \\
324.15 & 0.4299 & 0.5701 \\
326.55 & 0.4030 & 0.5970 \\
331.05 & 0.3420 & 0.6580 \\
334.45 & 0.2949 & 0.7051 \\
336.35 & 0.2679 & 0.7321 \\
338.15 & 0.2458 & 0.7542 \\
340.95 & 0.2038 & 0.7962 \\
343.65 & 0.1681 & 0.8319 \\
346.15 & 0.1252 & 0.8748 \\
347.95 & 0.0918 & 0.9082 \\
349.15 & 0.0711 & 0.9289 \\
350.95 & 0.0429 & 0.9571 \\
353.25 & 0.0000 & 1.0000 \\
\hline
\end{tabular}

${ }^{a} x_{2}$ : mole fraction of component 2 in the saturated solution.

${ }^{b} x_{1}$ : mole fraction of the polycyclic aromatic hydrocarbon (component 1).

The authors report that the system is of an incongruent eutectic type. The eutectic point occurs at $T / \mathrm{K}=244.85$ and $x_{1}$ $=0.058$.

\section{Auxiliary Information}

Method/Apparatus/Procedure:

Phase diagram was determined using a differential scanning calorimeter.

Samples of naphthalene and dichlorobenzene were heated very slowly inside a glass tube near the melting point of the major component. The fluid solution was then allowed to solidify with continuous stirring. A small quantity of the solid material was then sealed into a dsc sampling crucible for analysis.

Measurements were performed at a constant fixed scanning rate of $1 \mathrm{~K} / \mathrm{min}$.

Source and Purity of Chemicals:

(1) $99.5+\%$, Analytical Research Grade, Tianjin Kewei Chemical Reagent Company, China, was used as received.

(2) $99.5+\%$, Analytical Research Grade, Tianjin Kewei Chemical Reagent Company, China, was used as received.

Estimated Error:

Temperature: $\pm 0.2 \mathrm{~K}$.

$x_{1}: \pm 0.002$ (estimated by compiler).

\begin{tabular}{|c|c|}
\hline $\begin{array}{l}\text { Components: } \\
\text { (1) Naphthalene; } \mathrm{C}_{10} \mathrm{H}_{8} ;[91-20-3] \\
\text { (2) } 1,4-\text { Dichlorobenzene; } \mathrm{C}_{6} \mathrm{H}_{4} \mathrm{Cl}_{2} \text {; } \\
\text { [106-46-7] }\end{array}$ & $\begin{array}{l}\text { Original Measurements: } \\
{ }^{57} \text { D. Wei and K. Jin, J. Chem. } \\
\text { Thermodyn. 41, } 145 \text { (2009). }\end{array}$ \\
\hline $\begin{array}{l}\text { Variables: } \\
\text { Temperature }\end{array}$ & $\begin{array}{l}\text { Prepared by: } \\
\text { W. E. Acree, Jr. }\end{array}$ \\
\hline
\end{tabular}

Experimental Values

\begin{tabular}{lcc}
\hline \hline$T / \mathrm{K}$ & $x_{2}{ }^{\mathrm{a}}$ & $x_{1}{ }^{\mathrm{b}}$ \\
\hline 326.15 & 1.0000 & 0.0000 \\
321.95 & 0.9263 & 0.0737 \\
317.05 & 0.8500 & 0.1500 \\
314.15 & 0.8078 & 0.1922 \\
311.65 & 0.7630 & 0.2370 \\
310.65 & 0.7437 & 0.2563 \\
307.15 & 0.6938 & 0.3062 \\
305.15 & 0.6562 & 0.3438 \\
306.25 & 0.5772 & 0.4228 \\
312.35 & 0.5169 & 0.4831 \\
320.15 & 0.4410 & 0.5590 \\
326.65 & 0.3740 & 0.6260 \\
331.35 & 0.3157 & 0.6843 \\
335.05 & 0.2689 & 0.7311 \\
339.65 & 0.2042 & 0.7958 \\
343.55 & 0.1568 & 0.8432 \\
347.95 & 0.0791 & 0.9209 \\
350.75 & 0.0470 & 0.9530 \\
353.25 & 0.0000 & 1.0000 \\
\hline${ }^{\mathrm{a}} x_{2}:$ mole fraction of component 2 in the saturated solution. \\
${ }^{\mathrm{b}} x_{1}:$ mole fraction of the polycyclic aromatic hydrocarbon (component 1$)$.
\end{tabular}

The authors report that the system is of a simple eutectic type. The eutectic point occurs at $T / \mathrm{K}=302.85$ and $x_{1}=0.390$. 


\section{Auxiliary Information}

\section{Method/Apparatus/Procedure:}

Differential scanning calorimeter.

The phase diagram was determined using a differential scanning calorimeter Samples of naphthalene and dichlorobenzene were heated very slowly inside a glass tube near the melting point of the major component. The fluid solution was then allowed to solidify with continuous stirring. A small quantity of the solid material was then sealed into a dsc sampling crucible for analysis.

Measurements were performed at a constant fixed scanning rate of $1 \mathrm{~K} / \mathrm{min}$.

Source and Purity of Chemicals:

(1) $99.5+\%$, Analytical Research Grade, Tianjin Kewei Chemical Reagent Company, China, was used as received.

(2) $99.5+\%$, Analytical Research Grade, Tianjin Kewei Chemical Reagent Company, China, was used as received.

Estimated Error:

Temperature: $\pm 0.2 \mathrm{~K}$

$x_{1}: \pm 0.002$ (estimated by compiler).

\subsection{Naphthalene solubility data in alcohols}

Components:
(1) Naphthalene; $\mathrm{C}_{10} \mathrm{H}_{8} ;[91-20-3]$
(2) Methanol; $\left.\mathrm{CH}_{4} \mathrm{O} ; 67-56-1\right]$

(2) Methanol; $\left.\mathrm{CH}_{4} \mathrm{O} ; 67-56-1\right]$

\begin{tabular}{ll}
\hline Variables: & Prepared by: \\
$T / \mathrm{K}=298.15$ & W. E. Acree, Jr. \\
\hline
\end{tabular}

Experimental Values

\begin{tabular}{|c|c|c|}
\hline$c_{1}^{a}$ & $x_{2}{ }^{\mathrm{b}}$ & $x_{1}{ }^{c}$ \\
\hline 0.540 & 0.9771 & 0.0229 \\
\hline
\end{tabular}

The mole fraction solubility of the solute was calculated by the compiler using the experimental density data given in the paper.

\section{Auxiliary Information}

\section{Method/Apparatus/Procedure:}

Incubator, shaker, and an ultraviolet/visible spectrophotometer.

Solubility was determined by equilibrating an excess amount of solid naphthalene with the organic solvent using a shaker placed in an incubator equipped with a temperature controlling system. After an equilibration period of at least $48 \mathrm{~h}$, the samples were withdrawn and filtered through hydrophobic Durapore filters $(0.45 \mu \mathrm{m})$. The filtered sample was diluted quantitatively with methanol. Concentrations were determined by spectrophotmetric analysis at $274 \mathrm{~nm}$.

\section{Source and Purity of Chemicals:}

(1) $99 \%$, Fluka Chemical Company, was used as received.

(2) $99.5 \%$, Merck Chemical Company, Germany. No purification details were provided in the paper.
Estimated Error:

Temperature: $\pm 0.2 \mathrm{~K}$.

$x_{1}: \pm 2 \%$ (relative error).

\begin{tabular}{ll}
\hline \hline Components: & Original Measurements: \\
(1) Naphthalene; $\mathrm{C}_{10} \mathrm{H}_{8} ;[91-20-3]$ & ${ }^{59}$ A. Shayanfar, S. Soltani, F. \\
(2) Ethanol; $\mathrm{C}_{2} \mathrm{H}_{6} \mathrm{O} ;[64-17-5]$ & Jabbaribar, A. A. Hamidi, W. E. \\
& Acree, Jr., and A. Jouyban, J. \\
& Chem. Eng. Data 53, 574 (2008). \\
\hline Variables: & Prepared by: \\
$T / \mathrm{K}=298.15$ & W. E. Acree, Jr. \\
\hline
\end{tabular}

Experimental Values

\begin{tabular}{lcc}
\hline \hline$c_{1}^{\mathrm{a}}$ & $x_{2}{ }^{\mathrm{b}}$ & $x_{1}{ }^{\mathrm{c}}$ \\
\hline 0.558 & 0.9664 & 0.0336 \\
\hline${ }^{\mathrm{a}} c_{1}:$ molar solubility of the solute. & \\
${ }^{\mathrm{b}} x_{2}:$ mole fraction of component 2 in the saturated solution. \\
${ }^{\mathrm{c}} x_{1}:$ mole fraction solubility of the solute.
\end{tabular}

The mole fraction solubility of the solute was calculated by the compiler using the experimental density data given in the paper.

\section{Auxiliary Information}

\section{Method/Apparatus/Procedure:}

Incubator, shaker, and an ultraviolet/visible spectrophotometer.

Solubility was determined by equilibrating an excess amount of solid naphthalene with the organic solvent using a shaker placed in an incubator equipped with a temperature controlling system. After an equilibration period of at least $48 \mathrm{~h}$ the samples were withdrawn and filtered through hydrophobic Durapore filters $(0.45 \mu \mathrm{m})$. The filtered sample was diluted quantitatively with methanol. Concentrations were determined by spectrophotmetric analysis at $274 \mathrm{~nm}$.

Source and Purity of Chemicals:

(1) $99 \%$, Fluka Chemical Company, was used as received.

(2) $99.5 \%$, Merck Chemical Company, Germany. No purification details were provided in the paper.

Estimated Error:

Temperature: $\pm 0.2 \mathrm{~K}$.

$x_{1}: \pm 2 \%$ (relative error).

\begin{tabular}{ll}
\hline \hline Components: & Original Measurements: \\
(1) Naphthalene; $\mathrm{C}_{10} \mathrm{H}_{8} ;[91-20-3]$ & ${ }^{52}$ T.-B. Cui, T.-L. Luo, C. Zhang, \\
(2) Ethanol; $\mathrm{C}_{2} \mathrm{H}_{6} \mathrm{O} ;[64-17-5]$ & Z.-B. Mao, and G.-J. Liu, J. Chem. \\
& Eng. Data 54, 1065 (2009). \\
\hline Variables: & Prepared by: \\
Temperature & W. E. Acree, Jr. \\
\hline
\end{tabular}


Experimental Values

\begin{tabular}{lcc}
\hline \hline$T / \mathrm{K}$ & $x_{2}{ }^{\mathrm{a}}$ & $x_{1}{ }^{\mathrm{b}}$ \\
\hline 304.26 & 0.9536 & 0.04640 \\
307.44 & 0.9469 & 0.05311 \\
309.53 & 0.9434 & 0.05656 \\
311.93 & 0.9386 & 0.06141 \\
314.52 & 0.3642 & 0.06584 \\
315.93 & 0.9297 & 0.07025 \\
318.07 & 0.9240 & 0.07601 \\
319.80 & 0.9184 & 0.08161 \\
321.46 & 0.9124 & 0.08761 \\
323.45 & 0.8965 & 0.1035 \\
325.68 & 0.8838 & 0.1162 \\
327.32 & 0.8715 & 0.1285 \\
329.34 & 0.8605 & 0.1395 \\
330.43 & 0.8461 & 0.1539 \\
332.00 & 0.8258 & 0.1742 \\
333.90 & 0.7964 & 0.2036 \\
335.90 & 0.7618 & 0.2382 \\
337.76 & 0.7065 & 0.2935 \\
339.76 & 0.6185 & 0.3815 \\
\hline
\end{tabular}

${ }^{\mathrm{a}} x_{2}$ : mole fraction of component 2 in the saturated solution.

${ }^{b} x_{1}$ : mole fraction of the polycyclic aromatic hydrocarbon (component 1 ).

\section{Auxiliary Information}

\section{Method/Apparatus/Procedure:}

Double-jacketed glass vessel, laser generator, water bath, photoelectric transformer, and digital display.

Solubilities were determined by a synthetic-dynamic method. Known masses of solute and solvent were placed in a dissolving flask. The solid in the solution dissolved as the temperature was increased. The temperature at which the last amount of solid dissolved was recorded as the solid-liquid equilibrium temperature. The disappearance of the solid solute was determined by laser monitoring.

\section{Source and Purity of Chemicals:}

(1) Initial purity not given, Tianjin Kermel Chemical Reagent Purchase and Supply Station, China, was dissolved in acetone, filtered and the acetone evaporated. The sample was then recrystallized several times from methanol. The purity of the recrystallized sample was $99.8 \%$ as determined by UV spectrometry.

(2) 99.7\%, Tianjin Kermel Chemical Reagent Company, Ltd, China, no information given regarding any further purification.

Estimated Error:

Temperature: $\pm 0.2 \mathrm{~K}$.

$x_{1}: 3 \%$ (relative error).

\section{Components:}

(1) Naphthalene; $\mathrm{C}_{10} \mathrm{H}_{8} ;[91-20-3]$

(2) 1-Propanol; $\mathrm{C}_{3} \mathrm{H}_{8} \mathrm{O}$; [71-23-8]

\section{Original Measurements:}

${ }^{59}$ A. Shayanfar, S. Soltani, F. Jabbaribar, A. A. Hamidi, W. E. Acree, Jr., and A. Jouyban, J. Chem. Eng. Data 53, 574 (2008).

\section{Variables:}

$T / \mathrm{K}=298.15$

\section{Prepared by:}

W. E. Acree, Jr

Experimental Values

\begin{tabular}{|c|c|c|}
\hline$c_{1}{ }^{\mathrm{a}}$ & $x_{2}{ }^{\mathrm{b}}$ & $x_{1}{ }^{\mathrm{c}}$ \\
\hline 0.601 & 0.9539 & 0.0461 \\
\hline
\end{tabular}

The mole fraction solubility of the solute was calculated by the compiler using the experimental density data given in the paper.

\section{Auxiliary Information}

Method/Apparatus/Procedure:

Incubator, shaker, and an ultraviolet/visible spectrophotometer.

Solubility was determined by equilibrating an excess amount of solid naphthalene with the organic solvent using a shaker placed in an incubator equipped with a temperature controlling system. After an equilibration period of at least $48 \mathrm{~h}$, the samples were withdrawn and filtered through hydrophobic Durapore filters $(0.45 \mu \mathrm{m})$. The filtered sample was diluted quantitatively with methanol. Concentrations were determined by spectrophotmetric analysis at $274 \mathrm{~nm}$.

Source and Purity of Chemicals:

(1) $99 \%$, Fluka Chemical Company, was used as received.

(2) $99.5 \%$, Merck Chemical Company, Germany. No purification details were provided in the paper.

Estimated Error:

Temperature: $\pm 0.2 \mathrm{~K}$.

$x_{1}: \pm 2 \%$ (relative error).

\section{Components:}

(1) Naphthalene; $\mathrm{C}_{10} \mathrm{H}_{8} ;$ [91-20-3]

(2) 2-Propanol: $\mathrm{C}_{3} \mathrm{H}_{8} \mathrm{O}$; [67-63-0]

\begin{tabular}{ll}
\hline Variables: & Prepared by: \\
$T / \mathrm{K}=298.15$ & W. E. Acree, Jr. \\
\hline
\end{tabular}

Original Measurements:

${ }^{59}$ A. Shayanfar, S. Soltani, F. Jabbaribar, A. A. Hamidi, W. E. Acree, Jr., and A. Jouyban, J. Chem. Eng. Data 53, 574 (2008). Prepared by:
Experimental Values

\begin{tabular}{lcc}
\hline \hline$c_{1}{ }^{\mathrm{a}}$ & $x_{2}{ }^{\mathrm{b}}$ & $x_{1}{ }^{\mathrm{c}}$ \\
\hline 0.571 & 0.9549 & 0.0451 \\
\hline
\end{tabular}

${ }^{\mathrm{a}} c_{1}$ : molar solubility of the solute.

${ }^{\mathrm{b}} x_{2}$ : mole fraction of component 2 in the saturated solution.

${ }^{c} x_{1}$ : mole fraction solubility of the solute.

The mole fraction solubility of the solute was calculated by the compiler using the experimental density data given in the paper. 


\section{Auxiliary Information}

\section{Method/Apparatus/Procedure:}

Incubator, shaker, and an ultraviolet/visible spectrophotometer.

Solubility was determined by equilibrating an excess amount of solid naphthalene with the organic solvent using a shaker placed in an incubator equipped with a temperature controlling system. After an equilibration period of at least $48 \mathrm{~h}$, the samples were withdrawn and filtered through hydrophobic Durapore filters $(0.45 \mu \mathrm{m})$. The filtered sample was diluted quantitatively with methanol. Concentrations were determined by spectrophotmetric analysis at $274 \mathrm{~nm}$.

\section{Source and Purity of Chemicals:}

(1) $99 \%$, Fluka Chemical Company, was used as received.

(2) $99.5 \%$, Merck Chemical Company, Germany. No purification details were provided in the paper.

Estimated Error:

Temperature: $\pm 0.2 \mathrm{~K}$.

$x_{1}: \pm 2 \%$ (relative error).

Components:

Original Measurements:

${ }^{59}$ A. Shayanfar, S. Soltani, F. Jabbaribar, A. A. Hamidi, W. E. Acree, Jr., and A. Jouyban, J. Chem. Eng. Data 53, 574 (2008).

Variables:

$T / \mathrm{K}=298.15$

\section{Prepared by:}

W. E. Acree, Jr.

Experimental Values

\begin{tabular}{lcc}
\hline \hline$c_{1}{ }^{\mathrm{a}}$ & $x_{2}{ }^{\mathrm{b}}$ & $x_{1}{ }^{\mathrm{c}}$ \\
\hline 0.705 & 0.9339 & 0.0661 \\
${ }^{\mathrm{a}} c_{1}:$ molar solubility of the solute. & \\
${ }^{\mathrm{b}} x_{2}:$ mole fraction of component 2 in the saturated solution. \\
${ }^{\mathrm{c}} x_{1}$ : mole fraction solubility of the solute.
\end{tabular}

The mole fraction solubility of the solute was calculated by the compiler using the experimental density data given in the paper.

\section{Auxiliary Information}

\footnotetext{
Method/Apparatus/Procedure:

Incubator, shaker, and an ultraviolet/visible spectrophotometer.

Solubility was determined by equilibrating an excess amount of solid naphthalene with the organic solvent using a shaker placed in an incubator equipped with a temperature controlling system. After an equilibration period of at least $48 \mathrm{~h}$, the samples were withdrawn and filtered through hydrophobic Durapore filters $(0.45 \mu \mathrm{m})$. The filtered sample was diluted quantitatively with methanol. Concentrations were determined by spectrophotmetric analysis at $274 \mathrm{~nm}$.
}

Source and Purity of Chemicals:

(1) $99 \%$, Fluka Chemical Company, was used as received.

(2) $99.5 \%$, Merck Chemical Company, Germany. No purification details were provided in the paper.

\section{Estimated Error:}

Temperature: $\pm 0.2 \mathrm{~K}$.

$x_{1}: \pm 2 \%$ (relative error).

\begin{tabular}{ll}
\hline \hline Components: & Original Measurements: \\
(1) Naphthalene; $\mathrm{C}_{10} \mathrm{H}_{8} ;[91-20-3]$ & ${ }^{52}$ T.-B. Cui, T.-L. Luo, C. Zhang, \\
(2) 1-Butanol; $\mathrm{C}_{4} \mathrm{H}_{10} \mathrm{O} ;[71-36-3]$ & Z.-B. Mao, and G.-J. Liu, J. Chem. \\
& Eng. Data 54, 1065 (2009). \\
\hline Variables: & Prepared by: \\
Temperature & W. E. Acree, Jr. \\
\hline
\end{tabular}

Experimental Values

\begin{tabular}{lcc}
\hline \hline$T / \mathrm{K}$ & $x_{2}{ }^{\mathrm{a}}$ & $x_{1}{ }^{\mathrm{b}}$ \\
\hline 313.77 & 0.8873 & 0.1127 \\
315.17 & 0.8805 & 0.1195 \\
317.98 & 0.8612 & 0.1388 \\
320.74 & 0.8410 & 0.1590 \\
323.67 & 0.8172 & 0.1828 \\
325.60 & 0.7954 & 0.2046 \\
327.76 & 0.7721 & 0.2279 \\
329.03 & 0.7476 & 0.2524 \\
331.80 & 0.7063 & 0.2937 \\
333.65 & 0.6674 & 0.3326 \\
335.10 & 0.6251 & 0.3749 \\
337.00 & 0.5637 & 0.4363 \\
338.15 & 0.5234 & 0.4766 \\
339.63 & 0.4735 & 0.5265 \\
340.65 & 0.4326 & 0.5674 \\
342.04 & 0.3803 & 0.6197 \\
342.80 & 0.3395 & 0.6605 \\
\hline
\end{tabular}

${ }^{a} x_{2}$ : mole fraction of component 2 in the saturated solution.

${ }^{\mathrm{b}} x_{1}$ : mole fraction of the polycyclic aromatic hydrocarbon (component 1 ).

\section{Auxiliary Information}

\section{Method/Apparatus/Procedure:}

Double-jacketed glass vessel, laser generator, water bath, photoelectric transformer, and digital display.

Solubilities were determined by a synthetic-dynamic method. Known masses of solute and solvent were placed in a dissolving flask. The solid in the solution dissolved as the temperature was increased. The temperature at which the last amount of solid dissolved was recorded as the solid-liquid equilibrium temperature. The disappearance of the solid solute was determined by laser monitoring.

\section{Source and Purity of Chemicals:}

(1) Initial purity not given, Tianjin Kermel Chemical Reagent Purchase and Supply Station, China, was dissolved in acetone, filtered and the acetone evaporated. The sample was then recrystallized several times from methanol. The purity of the recrystallized sample was $99.8 \%$ as determined by UV spectrometry.

(2) $99.0 \%$, Tianjin Kermel Chemical Reagent Company, Ltd, China, no information given regarding any further purification.

\section{Estimated Error:}

Temperature: $\pm 0.15 \mathrm{~K}$.

$x_{1}: 2 \%$ (relative error). 


\subsection{Naphthalene solubility data in ketones}

\begin{tabular}{|c|c|}
\hline $\begin{array}{l}\text { Components: } \\
\text { (1) Naphthalene; } \mathrm{C}_{10} \mathrm{H}_{8} ;[91-20-3] \\
\text { (2) Propanone; } \mathrm{C}_{3} \mathrm{H}_{6} \mathrm{O} ;[67-64-1]\end{array}$ & $\begin{array}{l}\text { Original Measurements: } \\
{ }^{53} \text { Q.-S. Li, Z.-M. Yi, M.-G. Su, } \\
\text { and X.-F. Sun, J. Chem. Eng. Data } \\
\text { 53, } 2701 \text { (2008). }\end{array}$ \\
\hline Variables: & Prepared by: \\
\hline Temperature & W. E. Acree, Jr. \\
\hline
\end{tabular}

Experimental Values

\begin{tabular}{lcc}
\hline \hline$T / \mathrm{K}$ & $x_{2}{ }^{\mathrm{a}}$ & $x_{1}{ }^{\mathrm{b}}$ \\
\hline 278.15 & 0.8763 & 0.1237 \\
283.15 & 0.8567 & 0.1433 \\
288.05 & 0.8293 & 0.1707 \\
293.25 & 0.8059 & 0.1941 \\
298.15 & 0.7740 & 0.2260 \\
303.00 & 0.7319 & 0.2681 \\
308.10 & 0.6927 & 0.3073 \\
313.05 & 0.6394 & 0.3606 \\
318.15 & 0.5825 & 0.4175 \\
323.05 & 0.5229 & 0.4771
\end{tabular}

${ }^{a} x_{2}$ : mole fraction of component 2 in the saturated solution.

${ }^{\mathrm{b}} \mathrm{x}_{1}$ : mole fraction of the polycyclic aromatic hydrocarbon (component 1 ).

\section{Auxiliary Information}

\section{Method/Apparatus/Procedure:}

Double-jacketed glass vessel, laser generator, water bath, photoelectric transformer, and digital display.

Solubilities were determined by a synthetic-dynamic method. Known masses of solute and solvent were placed in the inner chamber of a double-jacketed glass vessel. The vessel was maintained at a constant temperature by circulating water from a water bath equipped with a thermoelectric controller. The solute and solvent mixture was stirred with a magnetic stirrer. 1-3 mg of solute were added at predetermined intervals until no more solid would dissolve. Disappearance of the added solute was observed visually and with laser monitoring.

Source and Purity of Chemicals:

(1) $99 \%$, Tianjin Kermel Chemical Reagent, China, was used as received.

(2) Research Grade, Shanghai Chemical Reagent, China, was used as received.

Estimated Error:

Temperature: $\pm 0.1 \mathrm{~K}$.

$x_{1}: 1.0 \%$ (relative error).

\begin{tabular}{ll}
\hline \hline Components: & Original Measurements: \\
(1) Naphthalene; $\mathrm{C}_{10} \mathrm{H}_{8} ;[91-20-3]$ & ${ }^{52}$ T.-B. Cui, T.-L. Luo, C. Zhang, \\
(2) Propanone; $\mathrm{C}_{3} \mathrm{H}_{6} \mathrm{O} ;[67-64-1]$ & Z.-B. Mao, and G.-J. Liu, J. Chem. \\
& Eng. Data 54, 1065 (2009). \\
\hline Variables: & Prepared by: \\
Temperature & W. E. Acree, Jr. \\
\hline
\end{tabular}

Experimental Values

\begin{tabular}{lcc}
\hline \hline$T / \mathrm{K}$ & $x_{2}{ }^{\mathrm{a}}$ & $x_{1}{ }^{\mathrm{b}}$ \\
\hline 297.17 & 0.7894 & 0.2106 \\
298.11 & 0.7815 & 0.2185 \\
300.23 & 0.7671 & 0.2329 \\
301.55 & 0.7540 & 0.2460 \\
303.47 & 0.7411 & 0.2589 \\
305.14 & 0.7276 & 0.2724 \\
306.63 & 0.7154 & 0.2846 \\
307.76 & 0.7028 & 0.2972 \\
309.37 & 0.6899 & 0.3101 \\
311.03 & 0.6770 & 0.3230 \\
312.03 & 0.6654 & 0.3346 \\
312.97 & 0.6532 & 0.3468 \\
314.37 & 0.6392 & 0.3608 \\
315.74 & 0.6238 & 0.3762 \\
317.26 & 0.6058 & 0.3942 \\
319.06 & 0.5846 & 0.4154 \\
320.87 & 0.5603 & 0.4397 \\
322.70 & 0.5414 & 0.4586 \\
\hline${ }^{\mathrm{a}} x_{2}:$ mole fraction of component 2 in the saturated solution. \\
${ }^{{ }^{2}} x_{1}:$ mole fraction of the polycyclic aromatic hydrocarbon (component 1$)$.
\end{tabular}

Auxiliary Information

Method/Apparatus/Procedure:

Double-jacketed glass vessel, laser generator, water bath, photoelectric transformer, and digital display.

Solubilities were determined by a synthetic-dynamic method. Known masses of solute and solvent were placed in a dissolving flask. The solid in the solution dissolved as the temperature was increased. The temperature at which the last amount of solid dissolved was recorded as the solid-liquid equilibrium temperature. The disappearance of the solid solute was determined by laser monitoring.

Source and Purity of Chemicals:

(1) Initial purity not given, Tianjin Kermel Chemical Reagent Purchase and Supply Station, China, was dissolved in acetone, filtered and the acetone evaporated. The sample was then recrystallized several times from methanol. The purity of the recrystallized sample was $99.8 \%$ as determined by UV spectrometry.

(2) $99.5 \%$, Tianjin Kermel Chemical Reagent Company, Ltd, China, no information given regarding any further purification.

Estimated Error:

Temperature: $\pm 0.2 \mathrm{~K}$.

$x_{1}: 0.7 \%$ (relative error).

\begin{tabular}{|c|c|}
\hline $\begin{array}{l}\text { Components: } \\
\text { (1) Naphthalene; } \mathrm{C}_{10} \mathrm{H}_{8} ; \text { [91-20-3] } \\
\text { (2) 1,2-Diphenyl-1,2-ethanedione; } \\
\mathrm{C}_{14} \mathrm{H}_{10} \mathrm{O}_{2} ;[134-81-6]\end{array}$ & $\begin{array}{l}\text { Original Measurements: } \\
{ }^{58} \text { U. S. Rai and R. N. Rai, Asian J. } \\
\text { Chem. 10, } 421 \text { (1998). }\end{array}$ \\
\hline $\begin{array}{l}\text { Variables: } \\
\text { Temperature }\end{array}$ & $\begin{array}{l}\text { Prepared by: } \\
\text { W. E. Acree, Jr. }\end{array}$ \\
\hline
\end{tabular}




\section{Experimental Values}

The binary phase diagram was graphically depicted in the paper. The phase diagram showed formation of a simple eutectic at $T / \mathrm{K}=372$ and a mole fraction composition of naphthalene of $x_{1}=0.592$.

\section{Auxiliary Information}

\section{Method/Apparatus/Procedure:}

Melting-point apparatus equipped with a precision thermometer.

The phase diagram was determined by the thaw-melt method. Binary mixtures of the two components were prepared by mass, placed in a test tube, and homogenized by repeated melting and thawing. The melting point and thawing temperature for the phase diagram was determined using a melting point apparatus equipped with a precision thermometer.

Source and Purity of Chemicals:

(1) Purity not given, Fluka Chemicals, purified by sublimation followed by recrystallization from cyclohexane.

(2) Purity not given, $\mathrm{CDH}$, China, was purified by repeated recrystallization from ethyl ethanoate.

Estimated Error:

Temperature: $\pm 0.5 \mathrm{~K}$.

$x_{1}: 0.005$ (estimated by compiler).

\subsection{Naphthalene solubility data in miscellaneous organic solvents}

\begin{tabular}{ll}
\hline \hline Components: & Original Measurements: \\
(1) Naphthalene; $\mathrm{C}_{10} \mathrm{H}_{8} ;[91-20-3]$ & ${ }^{53}$ Q.-S. Li, Z.-M. Yi, M.-G. Su, \\
$(2) N, N$-Dimethylformamide; $\mathrm{C}_{3} \mathrm{H}_{7} \mathrm{NO} ;$ & and X.-F. Sun, J. Chem. Eng. Data \\
{$[111-84-2]$} & $\mathbf{5 3}, 2701(2008)$. \\
\hline Variables: & Prepared by: \\
Temperature & W. E. Acree, Jr. \\
\hline
\end{tabular}

Experimental Values

\begin{tabular}{lcc}
\hline \hline$T / \mathrm{K}$ & $x_{2}{ }^{\mathrm{a}}$ & $x_{1}{ }^{\mathrm{b}}$ \\
\hline 278.05 & 0.8385 & 0.1615 \\
283.05 & 0.8148 & 0.1852 \\
288.15 & 0.7850 & 0.2150 \\
292.75 & 0.7556 & 0.2444 \\
298.05 & 0.7186 & 0.2814 \\
303.25 & 0.6929 & 0.3071 \\
307.95 & 0.6461 & 0.3539 \\
313.15 & 0.5941 & 0.4059 \\
318.15 & 0.5351 & 0.4649 \\
\hline
\end{tabular}

${ }^{a} x_{2}$ : mole fraction of component 2 in the saturated solution.

${ }^{\mathrm{b}} x_{1}$ : mole fraction of the polycyclic aromatic hydrocarbon (component 1 ).

\section{Auxiliary Information}

\section{Method/Apparatus/Procedure:}

Double-jacketed glass vessel, laser generator, water bath, photoelectric transformer, and digital display.

Solubilities were determined by a synthetic-dynamic method. Known masses of solute and solvent were placed in the inner chamber of a double-jacketed glass vessel. The vessel was maintained at a constant temperature by circulating water from a water bath equipped with a thermoelectric controller. The solute and solvent mixture was stirred with a magnetic stirrer. 1-3 mg of solute were added at predetermined intervals until no more solid would dissolve. Disappearance of the added solute was observed visually and with laser monitoring.

Source and Purity of Chemicals:

(1) $99 \%$, Tianjin Kermel Chemical Reagent, China, was used as received.

(2) Research Grade, Shanghai Chemical Reagent, China, was used as received.

Estimated Error:

Temperature: $\pm 0.1 \mathrm{~K}$.

$x_{1}: 1.0 \%$ (relative error).

\begin{tabular}{ll}
\hline \hline Components: & Original Measurements: \\
(1) Naphthalene; $\mathrm{C}_{10} \mathrm{H}_{8} ;[91-20-3]$ & ${ }^{53} \mathrm{Q}$.-S. Li, Z.-M. Yi, M.-G. Su, \\
(2) 1-Methyl-2-pyrrolidone; $\mathrm{C}_{5} \mathrm{H}_{9} \mathrm{NO} ;$ & and X.-F. Sun, J. Chem. Eng. Data \\
[872-50-4] & $\mathbf{5 3}, 2701(2008)$. \\
\hline Variables: & Prepared by: \\
Temperature & W. E. Acree, Jr. \\
\hline
\end{tabular}

Experimental Values

\begin{tabular}{lcc}
\hline \hline$T / \mathrm{K}$ & $x_{2}{ }^{\mathrm{a}}$ & $x_{1}{ }^{\mathrm{b}}$ \\
\hline 278.18 & 0.7315 & 0.2685 \\
283.25 & 0.7107 & 0.2893 \\
288.17 & 0.6927 & 0.3073 \\
293.20 & 0.6642 & 0.3358 \\
298.16 & 0.6215 & 0.3785 \\
303.21 & 0.5899 & 0.4101 \\
308.17 & 0.5617 & 0.4383 \\
313.16 & 0.5136 & 0.4864 \\
318.21 & 0.4654 & 0.5346 \\
323.27 & 0.4233 & 0.5767 \\
\hline
\end{tabular}

${ }^{\mathrm{a}} x_{2}$ : mole fraction of component 2 in the saturated solution.

${ }^{b} x_{1}$ : mole fraction of the polycyclic aromatic hydrocarbon (component 1).

\section{Auxiliary Information}

\section{Method/Apparatus/Procedure:}

Double-jacketed glass vessel, laser generator, water bath, photoelectric transformer, and digital display.

Solubilities were determined by a synthetic-dynamic method. Known masses of solute and solvent were placed in the inner chamber of a double-jacketed glass vessel. The vessel was maintained at a constant temperature by circulating water from a water bath equipped with a thermoelectric controller. The solute and solvent mixture was stirred with a magnetic stirrer. 1-3 mg of solute were added at predetermined intervals until no more solid would dissolve. Disappearance of the added solute was observed visually and with laser monitoring. 
Source and Purity of Chemicals:

(1) $99 \%$, Tianjin Kermel Chemical Reagent, China, was used as received.

(2) Research Grade, Shanghai Chemical Reagent, China, was used as received.

Estimated Error:

Temperature: $\pm 0.1 \mathrm{~K}$.

$x_{1}: 1.0 \%$ (relative error).

\section{Solubility of Phenanthrene in Organic Solvents}

\subsection{Critical evaluation of experimental solubility data}

Volume 59 in the IUPAC Solubility Data Series ${ }^{3}$ contained experimental solubility data for phenanthrene dissolved in four linear alkanes (hexane, heptane, octane, and octadecane) and two cycloalkanes (cyclohexane and decahydronaphthalene), in three aromatic hydrocarbons (benzene, methylbenzene, and 1,2,3,4-tetrahydronaphthalene), in three halogenated alkanes (tetrachloromethane, 1-hydroperfluoroheptane, and 1,8-dihydroperfluorooctane), in two alkanols (methanol and 1-octanol), and in five miscellaneous organic solvents (pyridine, thiophene, perfluorotributylamine, nitrobenzene, and carbon disulfide). The volume also included phase diagram information for binary phenanthrene + biphenyl, phenanthrene + fluoranthene, phenanthrene + acenaphthene, phenanthrene $+1,4$-dibromobenzene, phenanthrene +1 ,4-dibromobenzene, phenanthrene + octafluoronaphthalene, phenanthrene + 1,2-dinitrobenzene, phenanthrene $+1,3$-dinitrobenzene, phenanthrene +1 ,4-dinitrobenzene, phenanthrene + 1,3,5-trinitrobenzene, phenanthrene + 1,2,3,5-tetranitrobenzene, phenanthrene $+2,4-$ dinitromethylbenzene, phenanthrene $+2,6$-dinitromethylbenzene, phenanthrene + 3,4-dinitromethylbenzene, phenanthrene $+2,4,6$-trinitromethylbenzene, phenanthrene $+2,4-$ dinitrophenol, phenanthrene + 1-chloro-4-nitrobenzene, phenanthrene + dibenzothiophene, phenanthrene + thianthrene, phenanthrene + benzoic acid, phenanthrene + transcinnamic acid, phenanthrene +2 -hydroxybenzoic acid, phenanthrene +3 -hydroxybenzoic acid, and phenanthrene + 6methyl-2,3,4-trinitrophenol mixtures. Solubility data contained in Vol. 59 will not be republished here. The listing above is provided so that readers will know what solubility data are available in the earlier volume for phenanthrene.

After Vol. 59 appeared in print, there have been several experimental solubility studies involving phenanthrene in organic solvents. Acree and co-workers ${ }^{17,62}$ measured the solubility of anthracene in eight linear alkanes (hexane, heptane, octane, nonane, decane, undecane, dodecane, and hexadecane), four cycloalkanes (cyclohexane, methylcyclohexane, cyclooctane, and tert-butylcyclohexane) and one branched alkane (2,2,4-trimethylpentane), in two alkyl alkanoates (ethyl ethanoate and butyl ethanoate), in one dialkyl ether $\left(1,1^{\prime}\right.$-oxybisbutane) and two cyclic ethers (tetrahydrofuran and 1,4-dioxane), in one chloroalkane (tetrachloromethane), in 14 primary alkanols (methanol, ethanol, 1propanol, 1-butanol, 2-methyl-1-propanol, 1-pentanol, 3- methyl-1-butanol, 1-hexanol, 2-methyl-1-pentanol, 1-heptanol, 1-octanol, 2-ethyl-1-hexanol, 1,2-ethanediol, and 2,2,2trifluoroethanol), in five secondary alkanols (2-propanol, 2butanol, 2-pentanol, 4-methyl-2-pentanol, and cyclopentanol) and one tertiary alkanol (2-methyl-2-butanol), in two alkanones (butanone, cyclohexanone), and in three miscellaneous organic solvents (ethanenitrile, propanenitrile, and butanenitrile). For the majority of the solvents studied by Acree and coworkers there is only the single experimental value, and it is not possible to perform a critical evaluation on most of the published data.

There do exist independent solubility measurements for phenanthrene dissolved in 2,2,4-trimethylpentane and in several of the smaller alkanols. Shayanfar et al. ${ }^{34}$ measured the solubility of phenanthrene in binary 2,2,4-trimethylpentane and ethanol mixtures at 298.2,308.2, and $318.2 \mathrm{~K}$. The authors measured mole fraction solubilities at $298.2 \mathrm{~K}$ in 2,2,4-trimethylpentane $\left(x_{1}=0.0246\right)$ and ethanol $\left(x_{1}=0.0119\right)$ were in reasonably good agreement with the earlier published values of $x_{1}=0.002486$ for 2,2,4-trimethylpentane and $x_{1}$ $=0.001114$ for ethanol determined by Hernández et al. ${ }^{61}$ Since only three temperatures were studied by Shayanfar et al.,${ }^{34}$ it is not feasible to use the three-parameter Apelblat equation to assess the internal consistency of the authors' experimental data since the equation would yield essentially a perfect fit. There is one additional independent experimental measurement for the solubility of phenanthrene in ethanol. Fakhree et al. ${ }^{62,63}$ measured the solubilities of phenanthrene in binary and ternary mixtures of $\mathrm{C}_{1}-\mathrm{C}_{4}$ alcohols. The measured mole fraction solubilities compare favorably with the values determined by Acree and co-workers, ${ }^{17,62}$ namely, $x_{1}$ $=0.00549$ versus $x_{1}=0.00589$ for methanol, $x_{1}=0.0108$ versus $x_{1}=0.01114$ for ethanol, $x_{1}=0.0132$ versus $x_{1}$ $=0.01355$ for 1 -propanol, and $x_{1}=0.0178$ versus $x_{1}$ $=0.01771$ for 1-butanol.

Lisicki and Jamróz ${ }^{35}$ reported solid-liquid equilibria data for binary mixtures containing phenanthrene with $N, N$ dimethylacetamide, 1-methyl-2-pyrrolidone, and hexahydro1-methyl-2 $\mathrm{H}$-azepin-2-one (also called $\mathrm{N}$-methyl-e-caprolactam). The phenanthrene $+N, N$-dimethylformamide and phenanthrene +1 -methyl-2-pyrrolidone systems both exhibited simple eutectic behavior. The published experimental liquidus curves for the latter three phenanthrene systems did not indicate any outlier data points.

The experimental solubility data for phenanthrene dissolved in the different organic solvents are given in Secs. 11.2-11.9.

\subsection{Phenanthrene solubility data in saturated hydrocarbons (including cycloalkanes)}

\begin{tabular}{|c|c|}
\hline $\begin{array}{l}\text { Components: } \\
\text { (1) Phenanthrene; } \mathrm{C}_{14} \mathrm{H}_{10} ;[85-01-8] \\
\text { (2) Hexane; } \mathrm{C}_{6} \mathrm{H}_{14} ;[110-54-3]\end{array}$ & $\begin{array}{l}\text { Original Measurements: } \\
{ }^{61} \text { C. E. Hernández, K. M. De Fina, L. } \\
\text { E. Roy, T. L. Sharp, and W. E. Acree, } \\
\text { Jr., Can. J. Chem. 77, } 1465 \text { (1999). }\end{array}$ \\
\hline $\begin{array}{l}\text { Variables: } \\
T / \mathrm{K}=298.15\end{array}$ & $\begin{array}{l}\text { Prepared by: } \\
\text { W. E. Acree, Jr. }\end{array}$ \\
\hline
\end{tabular}


Experimental Values

\begin{tabular}{lcc}
\hline \hline$x_{2}{ }^{(\mathrm{s}) \mathrm{a}}$ & $x_{2}{ }^{\mathrm{b}}$ & $x_{1}{ }^{\mathrm{c}}$ \\
\hline 1.0000 & 0.9681 & 0.03189 \\
$\frac{{ }^{a} x_{2}{ }^{(\mathrm{s})}: \text { initial mole fraction of component } 2 \text { in the solution. }}{{ }^{\mathrm{b}} x_{2}: \text { mole fraction of component } 2 \text { in the saturated solution. }}$ \\
${ }^{\mathrm{c}} x_{1}:$ mole fraction solubility of the solute.
\end{tabular}

\section{Auxiliary Information}

\section{Method/Apparatus/Procedure:}

Constant-temperature bath, calorimetric thermometer, and an ultraviolet/ visible spectrophotometer.

Excess solute and solvent were placed in amber glass bottles and allowed to equilibrate for several days at constant temperature. Attainment of equilibrium was verified by several repetitive measurements and by approaching equilibrium from supersaturation. Aliquots of saturated solutions were transferred through a coarse filter into tared volumetric flasks, weighed and diluted with methanol. Concentrations were determined by spectrophotometric measurements at $347 \mathrm{~nm}$

\section{Source and Purity of Chemicals:}

(1) $98 \%$, Aldrich Chemical Company, Milwaukee, WI, USA, was recrystallized several times from methanol.

(2) $99 \%$, Aldrich Chemical Company, stored over molecular sieves before use.

\section{Estimated Error:}

Temperature: $\pm 0.1 \mathrm{~K}$.

$x_{1}: \pm 1.5 \%$ (relative error)

\begin{tabular}{|c|c|}
\hline $\begin{array}{l}\text { Components: } \\
\text { (1) Phenanthrene; } \mathrm{C}_{14} \mathrm{H}_{10} ;[85-01-8] \\
\text { (2) Heptane; } \mathrm{C}_{7} \mathrm{H}_{16} ;[142-82-5]\end{array}$ & $\begin{array}{l}\text { Original Measurements: } \\
{ }^{61} \text { C. E. Hernández, K. M. De Fina, L. } \\
\text { E. Roy, T. L. Sharp, and W. E. Acree, } \\
\text { Jr., Can. J. Chem. 77, } 1465 \text { (1999). }\end{array}$ \\
\hline $\begin{array}{l}\text { Variables: } \\
T / \mathrm{K}=298.15\end{array}$ & $\begin{array}{l}\text { Prepared by: } \\
\text { W. E. Acree, Jr. }\end{array}$ \\
\hline
\end{tabular}

Experimental Values

\begin{tabular}{lcc}
\hline \hline$x_{2}{ }^{(\mathrm{s}) \mathrm{a}}$ & $x_{2}{ }^{\mathrm{b}}$ & $x_{1}{ }^{\mathrm{c}}$ \\
\hline 1.0000 & 0.9611 & 0.03888 \\
\hline
\end{tabular}

${ }^{\mathrm{a}} x_{2}{ }^{(\mathrm{s})}$ : initial mole fraction of component 2 in the solution.

${ }^{b} x_{2}$ : mole fraction of component 2 in the saturated solution.

${ }^{c} x_{1}$ : mole fraction solubility of the solute.

\section{Auxiliary Information}

\footnotetext{
Method/Apparatus/Procedure:

Constant-temperature bath, calorimetric thermometer, and an ultraviolet/ visible spectrophotometer.

Excess solute and solvent were placed in amber glass bottles and allowed to equilibrate for several days at constant temperature. Attainment of equilibrium was verified by several repetitive measurements and by approaching equilibrium from supersaturation. Aliquots of saturated solutions were transferred through a coarse filter into tared volumetric flasks, weighed and diluted with methanol. Concentrations were determined by spectrophotometric measurements at $347 \mathrm{~nm}$.
}

Source and Purity of Chemicals:

(1) $98 \%$, Aldrich Chemical Company, Milwaukee, WI, USA, was recrystallized several times from methanol.

(2) HPLC Grade, Aldrich Chemical Company, stored over molecular sieves before use.

\section{Estimated Error:}

Temperature: $\pm 0.1 \mathrm{~K}$.

$x_{1}: \pm 1.5 \%$ (relative error)

\begin{tabular}{ll}
\hline \hline Components: & $\begin{array}{l}\text { Original Measurements: } \\
\text { (1) Phenanthrene; } \mathrm{C}_{14} \mathrm{H}_{10} ;[85-01-8]\end{array}$ \\
$\begin{array}{l}{ }^{6} \text { C. E. Hernández, K. M. De Fina, L. } \\
\text { (2) Octane; } \mathrm{C}_{8} \mathrm{H}_{18} ;[111-65-9]\end{array}$ & E. Roy, T. L. Sharp, and W. E. Acree, \\
& Jr., Can. J. Chem. 77, 1465 (1999). \\
\hline Variables: & Prepared by: \\
$T / \mathrm{K}=298.15$ & W. E. Acree, Jr. \\
\hline
\end{tabular}

\section{Experimental Values}

\begin{tabular}{lcc}
\hline \hline$x_{2}{ }^{(\mathrm{s}) \mathrm{a}}$ & $x_{2}{ }^{\mathrm{b}}$ & $x_{1}{ }^{\mathrm{c}}$ \\
\hline 1.0000 & 0.9556 & 0.04443 \\
${ }^{\mathrm{a}} x_{2}{ }^{(\mathrm{s})}:$ initial mole fraction of component 2 in the solution. \\
${ }^{\mathrm{b}} x_{2}:$ mole fraction of component 2 in the saturated solution. \\
${ }^{\mathrm{c}} x_{1}:$ mole fraction solubility of the solute.
\end{tabular}

\section{Auxiliary Information}

\section{Method/Apparatus/Procedure:}

Constant-temperature bath, calorimetric thermometer, and an ultraviolet/ visible spectrophotometer.

Excess solute and solvent were placed in amber glass bottles and allowed to equilibrate for several days at constant temperature. Attainment of equilibrium was verified by several repetitive measurements and by approaching equilibrium from supersaturation. Aliquots of saturated solutions were transferred through a coarse filter into tared volumetric flasks, weighed and diluted with methanol. Concentrations were determined by spectrophotometric measurements at $347 \mathrm{~nm}$.

\section{Source and Purity of Chemicals:}

(1) $98 \%$, Aldrich Chemical Company, Milwaukee, WI, USA, was recrystallized several times from methanol.

(2) $99+\%$, anhydrous, Aldrich Chemical Company, stored over molecular sieves before use.

\section{Estimated Error:}

Temperature: $\pm 0.1 \mathrm{~K}$.

$x_{1}: \pm 1.5 \%$ (relative error)

\begin{tabular}{|c|c|}
\hline $\begin{array}{l}\text { Components: } \\
\text { (1) Phenanthrene; } \mathrm{C}_{14} \mathrm{H}_{10} ;[85-01-8] \\
\text { (2) Nonane; } \mathrm{C}_{9} \mathrm{H}_{20} ;[111-84-2]\end{array}$ & $\begin{array}{l}\text { Original Measurements: } \\
{ }^{61} \text { C. E. Hernández, K. M. De Fina, L. } \\
\text { E. Roy, T. L. Sharp, and W. E. Acree, } \\
\text { Jr., Can. J. Chem. 77, } 1465 \text { (1999). }\end{array}$ \\
\hline $\begin{array}{l}\text { Variables: } \\
T / \mathrm{K}=298.15\end{array}$ & $\begin{array}{l}\text { Prepared by: } \\
\text { W. E. Acree, Jr. }\end{array}$ \\
\hline
\end{tabular}


Experimental Values

\begin{tabular}{lcc}
\hline \hline$x_{2}{ }^{(\mathrm{s}) \mathrm{a}}$ & $x_{2}{ }^{\mathrm{b}}$ & $x_{1}{ }^{\mathrm{c}}$ \\
\hline 1.0000 & 0.9522 & 0.04785 \\
$\frac{{ }^{\mathrm{a}} x_{2}{ }^{(\mathrm{s})}: \text { initial mole fraction of component } 2 \text { in the solution. }}{{ }^{\mathrm{b}}{ }_{x_{2}}: \text { mole fraction of component } 2 \text { in the saturated solution. }}$ \\
${ }^{\mathrm{c}}{ }_{x_{1}}:$ mole fraction solubility of the solute.
\end{tabular}

\section{Auxiliary Information}

\section{Method/Apparatus/Procedure:}

Constant-temperature bath, calorimetric thermometer, and an ultraviolet/ visible spectrophotometer.

Excess solute and solvent were placed in amber glass bottles and allowed to equilibrate for several days at constant temperature. Attainment of equilibrium was verified by several repetitive measurements and by approaching equilibrium from supersaturation. Aliquots of saturated solutions were transferred through a coarse filter into tared volumetric flasks, weighed and diluted with methanol. Concentrations were determined by spectrophotometric measurements at $347 \mathrm{~nm}$

Source and Purity of Chemicals:

(1) $98 \%$, Aldrich Chemical Company, Milwaukee, WI, USA, was recrystallized several times from methanol.

(2) $99+\%$, TCI America, Portland, OR, USA, stored over molecular sieves before use.

Estimated Error:

Temperature: $\pm 0.1 \mathrm{~K}$

$x_{1}: \pm 1.5 \%$ (relative error).

\begin{tabular}{ll}
\hline \hline Components: & Original Measurements: \\
$\begin{array}{l}\text { (1) Phenanthrene; } \mathrm{C}_{14} \mathrm{H}_{10} ;[85-01-8] \\
\text { (2) Decane; } \mathrm{C}_{10} \mathrm{H}_{22} ;[124-18-5]\end{array}$ & $\begin{array}{l}\text { E. Roy, Ternández, K. M. Sharp, and W. E. Acree, } \\
\end{array}$ \\
\hline Jr., Can. J. Chem. 77, 1465 (1999). \\
\hline Variables: & Prepared by: \\
$T / \mathrm{K}=298.15$ & W. E. Acree, Jr. \\
\hline
\end{tabular}

Experimental Values

\begin{tabular}{|c|c|c|}
\hline$x_{2}{ }^{(\mathrm{s}) \mathrm{a}}$ & $x_{2}{ }^{b}$ & $x_{1}{ }^{c}$ \\
\hline 1.0000 & 0.9447 & 0.05531 \\
\hline
\end{tabular}

\section{Auxiliary Information}

\section{Method/Apparatus/Procedure:}

Constant-temperature bath, calorimetric thermometer, and an ultraviolet/ visible spectrophotometer.

Excess solute and solvent were placed in amber glass bottles and allowed to equilibrate for several days at constant temperature. Attainment of equilibrium was verified by several repetitive measurements and by approaching equilibrium from supersaturation. Aliquots of saturated solutions were transferred through a coarse filter into tared volumetric flasks, weighed and diluted with methanol. Concentrations were determined by spectrophotometric measurements at $347 \mathrm{~nm}$.
Source and Purity of Chemicals:

(1) $98 \%$, Aldrich Chemical Company, Milwaukee, WI, USA, was recrystallized several times from methanol.

(2) $99+\%$, TCI America, Portland, OR, USA, stored over molecular sieves before use.

\section{Estimated Error:}

Temperature: $\pm 0.1 \mathrm{~K}$.

$x_{1}: \pm 1.5 \%$ (relative error).

\begin{tabular}{ll}
\hline \hline Components: & Original Measurements: \\
(1) Phenanthrene; $\mathrm{C}_{14} \mathrm{H}_{10} ;[85-01-8]$ & ${ }^{17}$ M. H. Abraham and W. E. \\
(2) Undecane; $\mathrm{C}_{11} \mathrm{H}_{24} ;[1120-21-4]$ & $\begin{array}{l}\text { Acree, Jr., New J. Chem. 28, 1538 } \\
(2004) .\end{array}$ \\
\hline Variables: & Prepared by: \\
$T / \mathrm{K}=298.15$ & W. E. Acree, Jr. \\
\hline
\end{tabular}

Experimental Values

\begin{tabular}{lcc}
\hline \hline$x_{2}{ }^{(\mathrm{s}) \mathrm{a}}$ & $x_{2}{ }^{\mathrm{b}}$ & $x_{1}{ }^{\mathrm{c}}$ \\
\hline 1.0000 & 0.9402 & 0.05980 \\
${ }^{\mathrm{a}} x_{2}{ }^{(\mathrm{s})}$ : initial mole fraction of component 2 in the solution. \\
${ }^{\mathrm{b}} x_{2}$ : mole fraction of component 2 in the saturated solution. \\
${ }^{\mathrm{c}} x_{1}$ : mole fraction solubility of the solute.
\end{tabular}

Experimental values were reported as the logarithm of the solute's molar solubility in undecane divided by the molar solubility in water. Mole fraction solubilities were provided by the authors of the paper.

\section{Auxiliary Information}

\section{Method/Apparatus/Procedure:}

Constant-temperature bath, calorimetric thermometer, and an ultraviolet/ visible spectrophotometer.

Excess solute and solvent were placed in amber glass bottles and allowed to equilibrate for several days at constant temperature. Attainment of equilibrium was verified by several repetitive measurements and by approaching equilibrium from supersaturation. Aliquots of saturated solutions were transferred through a coarse filter into tared volumetric flasks, weighed and diluted with methanol. Concentrations were determined by spectrophotometric measurements at $347 \mathrm{~nm}$.

\section{Source and Purity of Chemicals:}

(1) $98 \%$, Aldrich Chemical Company, Milwaukee, WI, USA, was recrystallized several times from methanol.

(2) $99 \%$, Aldrich Chemical Company, stored over molecular sieves before use.

\section{Estimated Error:}

Temperature: $\pm 0.1 \mathrm{~K}$.

$x_{1}: \pm 1.5 \%$ (relative error). 


\section{Components:}

(1) Phenanthrene; $\mathrm{C}_{14} \mathrm{H}_{10}$; [85-01-8]

(2) Dodecane; $\mathrm{C}_{12} \mathrm{H}_{26} ;[112-40-3]$

\section{Variables:}

$T / \mathrm{K}=298.15$

Original Measurements:

${ }^{17} \mathrm{M}$. H. Abraham and W. E.

Acree, Jr., New J. Chem. 28, 1538 (2004).

Prepared by:

W. E. Acree, Jr.

Experimental Values

\begin{tabular}{lcc}
\hline \hline$x_{2}{ }^{(\mathrm{s}) \mathrm{a}}$ & $x_{2}{ }^{\mathrm{b}}$ & $x_{1}{ }^{\mathrm{c}}$ \\
\hline 1.0000 & 0.9365 & 0.06348
\end{tabular}

${ }^{\mathrm{a}} x_{2}{ }^{(\mathrm{s})}$ : initial mole fraction of component 2 in the solution.

${ }^{b} x_{2}$ : mole fraction of component 2 in the saturated solution.

${ }^{c} x_{1}$ : mole fraction solubility of the solute.

Experimental values were reported as the logarithm of the solute's molar solubility in dodecane divided by the molar solubility in water. Mole fraction solubilities were provided by the authors of the paper.

\section{Auxiliary Information}

\section{Method/Apparatus/Procedure:}

Constant-temperature bath, calorimetric thermometer, and an ultraviolet/ visible spectrophotometer.

Excess solute and solvent were placed in amber glass bottles and allowed to equilibrate for several days at constant temperature. Attainment of equilibrium was verified by several repetitive measurements and by approaching equilibrium from supersaturation. Aliquots of saturated solutions were transferred through a coarse filter into tared volumetric flasks, weighed and diluted with methanol. Concentrations were determined by

spectrophotometric measurements at $347 \mathrm{~nm}$.

Source and Purity of Chemicals:

(1) 98\%, Aldrich Chemical Company, Milwaukee, WI, USA, was recrystallized several times from methanol.

(2) $99 \%$, Aldrich Chemical Company, stored over molecular sieves before use.

Estimated Error:

Temperature: $\pm 0.1 \mathrm{~K}$.

$x_{1}: \pm 1.5 \%$ (relative error).

\section{Components:}

(1) Phenanthrene; $\mathrm{C}_{14} \mathrm{H}_{10} ;[85-01-8]$

(2) Hexadecane; $\mathrm{C}_{16} \mathrm{H}_{34} ;[$ [544-76-3]

Original Measurements:

${ }^{61}$ C. E. Hernández, K. M. De Fina,

L. E. Roy, T. L. Sharp, and W. E. Acree, Jr., Can. J. Chem. 77, 1465 (1999).

\section{Variables:}

$T / \mathrm{K}=298.15$

\section{Prepared by:}

W. E. Acree, Jr.

\section{Experimental Values}

\begin{tabular}{lcc}
\hline \hline$x_{2}{ }^{(\mathrm{s}) \mathrm{a}}$ & $x_{2}{ }^{\mathrm{b}}$ & $x_{1}{ }^{\mathrm{c}}$ \\
\hline 1.0000 & 0.9203 & 0.07972 \\
$\frac{{ }^{a} x_{2}(\mathrm{~s})}{\text { initial mole fraction of component } 2 \text { in the solution. }}$ \\
${ }^{\mathrm{b}} x_{2}:$ mole fraction of component 2 in the saturated solution. \\
${ }^{\mathrm{c}} x_{1}:$ mole fraction solubility of the solute.
\end{tabular}

\section{Auxiliary Information}

Method/Apparatus/Procedure:

Constant-temperature bath, calorimetric thermometer, and an ultraviolet/ visible spectrophotometer.

Excess solute and solvent were placed in amber glass bottles and allowed to equilibrate for several days at constant temperature. Attainment of equilibrium was verified by several repetitive measurements and by approaching equilibrium from supersaturation. Aliquots of saturated solutions were transferred through a coarse filter into tared volumetric flasks, weighed and diluted with methanol. Concentrations were determined by spectrophotometric measurements at $347 \mathrm{~nm}$.

\section{Source and Purity of Chemicals:}

(1) $98 \%$, Aldrich Chemical Company, Milwaukee, WI, USA, was recrystallized several times from methanol.

(2) $99 \%$, Aldrich Chemical Company, stored over molecular sieves before use.

\section{Estimated Error:}

Temperature: $\pm 0.1 \mathrm{~K}$.

$x_{1}: \pm 1.5 \%$ (relative error).

\begin{tabular}{ll}
\hline \hline Components: & Original Measurements: \\
(1) Phenanthrene; $\mathrm{C}_{14} \mathrm{H}_{10} ;[85-01-8]$ & ${ }^{15}$ A. Aoulmi, M. Bouroukba, R. \\
(2) Octacosane; $\mathrm{C}_{28} \mathrm{H}_{58} ;[630-02-4]$ & Solimando, and M. Rogalski, \\
& Fluid Phase Equilib. 110, 283 \\
& $(1995)$. \\
\hline Variables: & Prepared by: \\
Temperature & W. E. Acree, Jr. \\
\hline
\end{tabular}

Experimental Values

\begin{tabular}{lcc}
\hline \hline$T / \mathrm{K}$ & $x_{2}{ }^{\mathrm{a}}$ & $x_{1}{ }^{\mathrm{b}}$ \\
\hline 333.91 & 1.0000 & 0.0000 \\
333.81 & 0.8969 & 0.1031 \\
333.22 & 0.8004 & 0.1996 \\
331.26 & 0.6915 & 0.3085 \\
341.01 & 0.5001 & 0.4999 \\
344.75 & 0.4485 & 0.5515 \\
350.95 & 0.4004 & 0.5996 \\
360.10 & 0.2801 & 0.7199 \\
364.14 & 0.2000 & 0.8000 \\
367.60 & 0.1002 & 0.8998 \\
371.35 & 0.0000 & 1.0000 \\
\hline
\end{tabular}

${ }^{\mathrm{a}} x_{2}$ : mole fraction of component 2 in the saturated solution.

${ }^{\mathrm{b}} x_{1}$ : mole fraction of the polycyclic aromatic hydrocarbon (component 1 ).

The authors employed a SSF to describe the activity coefficients of phenanthrene and octacosane calculated from the solid-liquid equilibrium data. The SSF model gave a calculated eutectic temperature of $T / \mathrm{K}=329.0$ and eutectic mole fraction of phenanthrene of $x_{1}=0.420$. 


\section{Auxiliary Information}

\section{Method/Apparatus/Procedure:}

Differential scanning calorimeter

The phase diagram was determined using a differential scanning calorimeter. Measurements were performed at a constant fixed scanning rate of $0.5 \mathrm{~K} / \mathrm{min}$.

Source and Purity of Chemicals:

(1) $98 \%$, Aldrich Chemical Company, Milwaukee, WI, USA, no purification details were given in the paper.

(2) $99 \%$, Aldrich Chemical Company, purification details were not given in the paper.

\section{Estimated Error:}

Temperature: Authors state a global accuracy of $\pm 1 \%$ regarding their measurements.

$x_{1}: \pm 0.0002$ (estimated by compiler).

\begin{tabular}{|c|c|}
\hline $\begin{array}{l}\text { Components: } \\
\text { (1) Phenanthrene; } \mathrm{C}_{14} \mathrm{H}_{10} ;[85-01-8] \\
\text { (2) Cyclohexane; } \mathrm{C}_{6} \mathrm{H}_{12} ;[110-82-7]\end{array}$ & $\begin{array}{l}\text { Original Measurements: } \\
{ }^{61} \text { C. E. Hernández, K. M. De Fina, } \\
\text { L. E. Roy, T. L. Sharp, and W. E. } \\
\text { Acree, Jr., Can. J. Chem. 77, } 1465 \\
\text { (1999). }\end{array}$ \\
\hline $\begin{array}{l}\text { Variables: } \\
T / \mathrm{K}=298.15\end{array}$ & $\begin{array}{l}\text { Prepared by: } \\
\text { W. E. Acree, Jr. }\end{array}$ \\
\hline
\end{tabular}

Experimental Values

\begin{tabular}{lcc}
\hline \hline$x_{2}{ }^{(\mathrm{s}) \mathrm{a}}$ & $x_{2}{ }^{\mathrm{b}}$ & $x_{1}{ }^{\mathrm{c}}$ \\
\hline 1.0000 & 0.9635 & 0.03648 \\
\hline
\end{tabular}

${ }^{\mathrm{a}} x_{2}{ }^{(\mathrm{s})}$ : initial mole fraction of component 2 in the solution.

${ }^{\mathrm{b}} x_{2}$ : mole fraction of component 2 in the saturated solution.

${ }^{c} x_{1}$ : mole fraction solubility of the solute.

\section{Auxiliary Information}

Method/Apparatus/Procedure:
Constant-temperature bath, calorimetric thermometer, and an ultraviolet/
visible spectrophotometer.
Excess solute and solvent were placed in amber glass bottles and allowed to
equilibrate for several days at constant temperature. Attainment of equilibrium
was verified by several repetitive measurements and by approaching
equilibrium from supersaturation. Aliquots of saturated solutions were
transferred through a coarse filter into tared volumetric flasks, weighed and
diluted with methanol. Concentrations were determined by
spectrophotometric measurements at 347 nm.
Source and Purity of Chemicals:
(1) $98 \%$, Aldrich Chemical Company, Milwaukee, WI, USA, was
recrystallized several times from methanol.
(2) HPLC Grade, Aldrich Chemical Company, stored over molecular sieves
before use.
Estimated Error:
Temperature: $\pm 0.1 \mathrm{~K}$.
$x_{1}: \pm 1.5 \%$ (relative error).

\begin{tabular}{ll}
\hline \hline Components: & Original Measurements: \\
(1) Phenanthrene; $\mathrm{C}_{14} \mathrm{H}_{10} ;[85-01-8]$ & ${ }^{61} \mathrm{C}$. E. Hernández, K. M. De Fina, \\
(2) Methylcyclohexane; $\mathrm{C}_{7} \mathrm{H}_{14} ;$ & L. E. Roy, T. L. Sharp, and W. E. \\
[108-87-2] & Acree, Jr., Can. J. Chem. 77, 1465 \\
& $(1999)$. \\
\hline Variables: & Prepared by: \\
$T / \mathrm{K}=298.15$ & W. E. Acree, Jr. \\
\hline
\end{tabular}

Experimental Values

\begin{tabular}{lcc}
\hline \hline$x_{2}{ }^{(\mathrm{s}) \mathrm{a}}$ & $x_{2}{ }^{\mathrm{b}}$ & $x_{1}{ }^{\mathrm{c}}$ \\
\hline 1.0000 & 0.9543 & 0.04572 \\
\hline
\end{tabular}

${ }^{\mathrm{a}} x_{2}{ }^{(\mathrm{s})}$ : initial mole fraction of component 2 in the solution.

${ }^{\mathrm{b}} \mathrm{x}_{2}$ : mole fraction of component 2 in the saturated solution.

${ }^{c} x_{1}$ : mole fraction solubility of the solute.

\section{Auxiliary Information}

\section{Method/Apparatus/Procedure:}

Constant-temperature bath, calorimetric thermometer, and an ultraviolet/ visible spectrophotometer.

Excess solute and solvent were placed in amber glass bottles and allowed to equilibrate for several days at constant temperature. Attainment of equilibrium was verified by several repetitive measurements and by approaching equilibrium from supersaturation. Aliquots of saturated solutions were transferred through a coarse filter into tared volumetric flasks, weighed and diluted with methanol. Concentrations were determined by spectrophotometric measurements at $347 \mathrm{~nm}$.

\section{Source and Purity of Chemicals:}

(1) $98 \%$, Aldrich Chemical Company, Milwaukee, WI, USA, was recrystallized several times from methanol.

(2) $99+\%$, anhydrous, Aldrich Chemical Company, stored over molecular sieves before use.

Estimated Error:

Temperature: $\pm 0.1 \mathrm{~K}$.

$x_{1}: \pm 1.5 \%$ (relative error)

\begin{tabular}{|c|c|}
\hline $\begin{array}{l}\text { Components: } \\
\text { (1) Phenanthrene; } \mathrm{C}_{14} \mathrm{H}_{10} ;[85-01-8] \\
\text { (2) Cyclooctane; } \mathrm{C}_{8} \mathrm{H}_{16} ;[292-64-8]\end{array}$ & $\begin{array}{l}\text { Original Measurements: } \\
{ }^{61} \text { C. E. Hernández, K. M. De Fina, } \\
\text { L. E. Roy, T. L. Sharp, and W. E. } \\
\text { Acree, Jr., Can. J. Chem. 77, } 1465 \\
\text { (1999). }\end{array}$ \\
\hline $\begin{array}{l}\text { Variables: } \\
T / \mathrm{K}=298.15\end{array}$ & $\begin{array}{l}\text { Prepared by: } \\
\text { W. E. Acree, Jr. }\end{array}$ \\
\hline
\end{tabular}

Experimental Values

\begin{tabular}{lcc}
\hline \hline$x_{2}{ }^{(\mathrm{s}) \mathrm{a}}$ & $x_{2}{ }^{\mathrm{b}}$ & $x_{1}{ }^{\mathrm{c}}$ \\
\hline 1.0000 & 0.9400 & 0.06002 \\
$\frac{{ }^{\mathrm{a}} x_{2}{ }^{(\mathrm{s})}: \text { initial mole fraction of component } 2 \text { in the solution. }}{{ }^{\mathrm{b}} x_{2}: \text { mole fraction of component } 2 \text { in the saturated solution. }}$ \\
${ }^{\mathrm{c}}{ }_{x_{1}}$ : mole fraction solubility of the solute.
\end{tabular}




\section{Auxiliary Information}

\section{Method/Apparatus/Procedure:}

Constant-temperature bath, calorimetric thermometer, and an ultraviolet/ visible spectrophotometer.

Excess solute and solvent were placed in amber glass bottles and allowed to equilibrate for several days at constant temperature. Attainment of equilibrium was verified by several repetitive measurements and by approaching equilibrium from supersaturation. Aliquots of saturated solutions were transferred through a coarse filter into tared volumetric flasks, weighed and diluted with methanol. Concentrations were determined by spectrophotometric measurements at $347 \mathrm{~nm}$.

Source and Purity of Chemicals:

(1) $98 \%$, Aldrich Chemical Company, Milwaukee, WI, USA, was recrystallized several times from methanol.

(2) $99+\%$, Aldrich Chemical Company, stored over molecular sieves before use.

Estimated Error:

Temperature: $\pm 0.1 \mathrm{~K}$

$x_{1}: \pm 1.5 \%$ (relative error).

\begin{tabular}{|c|c|}
\hline $\begin{array}{l}\text { Components: } \\
\text { (1) Phenanthrene; } \mathrm{C}_{14} \mathrm{H}_{10} ;[85-01-8] \\
\text { (2) 2,2,4-Trimethylpentane; } \mathrm{C}_{8} \mathrm{H}_{18} \text {; } \\
\text { [540-84-1] }\end{array}$ & $\begin{array}{l}\text { Original Measurements: } \\
{ }^{61} \text { C. E. Hernández, K. M. De Fina, } \\
\text { L. E. Roy, T. L. Sharp, and W. E. } \\
\text { Acree, Jr., Can. J. Chem. 77, } 1465 \\
(1999) \text {. }\end{array}$ \\
\hline $\begin{array}{l}\text { Variables: } \\
T / \mathrm{K}=298.15\end{array}$ & $\begin{array}{l}\text { Prepared by: } \\
\text { W. E. Acree, Jr. }\end{array}$ \\
\hline
\end{tabular}

Experimental Values

\begin{tabular}{lcc}
\hline \hline$x_{2}{ }^{(\mathrm{s}) \mathrm{a}}$ & $x_{2}{ }^{\mathrm{b}}$ & $x_{1}{ }^{\mathrm{c}}$ \\
\hline 1.0000 & 0.9751 & 0.02486
\end{tabular}

${ }^{\mathrm{a}} x_{2}{ }^{(\mathrm{s})}$ : initial mole fraction of component 2 in the solution.

${ }^{b} x_{2}$ : mole fraction of component 2 in the saturated solution.

${ }^{c} x_{1}$ : mole fraction solubility of the solute.

\section{Auxiliary Information}

\section{Method/Apparatus/Procedure:}

Constant-temperature bath, calorimetric thermometer, and an ultraviolet/ visible spectrophotometer.

Excess solute and solvent were placed in amber glass bottles and allowed to equilibrate for several days at constant temperature. Attainment of equilibrium was verified by several repetitive measurements and by approaching equilibrium from supersaturation. Aliquots of saturated solutions were transferred through a coarse filter into tared volumetric flasks, weighed and diluted with methanol. Concentrations were determined by

spectrophotometric measurements at $347 \mathrm{~nm}$.

Source and Purity of Chemicals:

(1) $98 \%$, Aldrich Chemical Company, Milwaukee, WI, USA, was recrystallized several times from methanol.

(2) HPLC Grade, Aldrich Chemical Company, stored over molecular sieves before use.

Estimated Error:

Temperature: $\pm 0.1 \mathrm{~K}$.

$x_{1}: \pm 1.5 \%$ (relative error).

\begin{tabular}{ll}
\hline \hline Components: & Original Measurements: \\
(1) Phenanthrene; $\mathrm{C}_{14} \mathrm{H}_{10} ;[85-01-8]$ & ${ }^{34}$ A. Shayanfar, S. H. Eghrary, F. \\
(2) 2,2,4-Trimethylpentane; $\mathrm{C}_{8} \mathrm{H}_{18} ;$ & Sardari, W. E. Acree, Jr., and A. \\
[540-84-1] & Jouyban, J. Chem. Eng. Data 56, \\
& $2290(2011)$. \\
\hline Variables: & Prepared by: \\
Temperature & W. E. Acree, Jr. \\
\hline
\end{tabular}

Experimental Values

\begin{tabular}{lcc}
\hline \hline$T / \mathrm{K}$ & $x_{2}{ }^{\mathrm{a}}$ & $x_{1}{ }^{\mathrm{b}}$ \\
\hline 298.2 & 0.9754 & 0.0246 \\
308.2 & 0.9622 & 0.0378 \\
318.2 & 0.9516 & 0.0484 \\
\hline
\end{tabular}

${ }^{\mathrm{a}} x_{2}$ : mole fraction of component 2 in the saturated solution.

${ }^{b} x_{1}$ : mole fraction of the polycyclic aromatic hydrocarbon (component 1 ).

\section{Auxiliary Information}

\section{Method/Apparatus/Procedure:}

Incubator, shaker, and an ultraviolet/visible spectrophotometer.

Solubility was determined by equilibrating an excess amount of solid naphthalene with the organic solvent using a shaker placed in an incubator equipped with a temperature controlling system. After an equilibration period of at least $48 \mathrm{~h}$, the samples were withdrawn and filtered through hydrophobic Durapore filters $(0.45 \mu \mathrm{m})$. The filtered sample was diluted quantitatively with methanol. Concentrations were determined by spectrophotmetric analysis at $356 \mathrm{~nm}$.

\section{Source and Purity of Chemicals:}

(1) $98 \%$, Merck Chemicals, Germany, was recrystallized several times from propanone before use.

(2) $99+\%$, Merck Chemicals, no information given regarding any further purification.

\section{Estimated Error:}

Temperature: $\pm 0.2 \mathrm{~K}$.

$x_{1}: \pm 2.5 \%$ (relative uncertainty).

\begin{tabular}{ll}
\hline \hline Components: & Original Measurements: \\
$\begin{array}{l}\text { (1) Phenanthrene; } \mathrm{C}_{14} \mathrm{H}_{10} ;[85-01-8] \\
\text { (2) tert-Butylcyclohexane; } \mathrm{C}_{10} \mathrm{H}_{20} ;\end{array}$ & L. E. Roy, T. L. Sharp, and W. E. \\
[3178-22-1] & Acree, Jr., Can. J. Chem. 77, 1465 \\
& $(1999)$. \\
\hline Variables: & Prepared by: \\
$T / \mathrm{K}=298.15$ & W. E. Acree, Jr. \\
\hline
\end{tabular}

Experimental Values

\begin{tabular}{lcc}
\hline \hline$x_{2}{ }^{(\mathrm{s}) \mathrm{a}}$ & $x_{2}{ }^{\mathrm{b}}$ & $x_{1}{ }^{\mathrm{c}}$ \\
\hline 1.0000 & 0.9488 & 0.05124 \\
$\frac{{ }^{a} x_{2}{ }^{(\mathrm{s})}: \text { initial mole fraction of component } 2 \text { in the solution. }}{{ }^{\mathrm{b}} x_{2}: \text { mole fraction of component } 2 \text { in the saturated solution. }}$ \\
${ }^{c} x_{1}:$ mole fraction solubility of the solute.
\end{tabular}




\section{Auxiliary Information}

\section{Method/Apparatus/Procedure:}

Constant-temperature bath, calorimetric thermometer, and an ultraviolet/ visible spectrophotometer.

Excess solute and solvent were placed in amber glass bottles and allowed to equilibrate for several days at constant temperature. Attainment of equilibrium was verified by several repetitive measurements and by approaching equilibrium from supersaturation. Aliquots of saturated solutions were diluted with methanol. Concentrations were determined by spectrophotometric measurements at $347 \mathrm{~nm}$.

Source and Purity of Chemicals:

(1) $98 \%$, Aldrich Chemical Company, Milwaukee, WI, USA, was recrystallized several times from methanol.

(2) $99+\%$, Aldrich Chemical Company, stored over molecular sieves before use.

Temperature: $\pm 0.1 \mathrm{~K}$.

$x_{1}: \pm 1.5 \%$ (relative error). transferred through a coarse filter into tared volumetric flasks, weighed and

Estimated Error:

\section{Auxiliary Information}

Method/Apparatus/Procedure:

Simple thermal analysis device constructed in the authors' laboratory, and a high precision platinum-resistance thermometer.

Phase diagram was determined using a simple thermal device. A sample of known composition was placed in a thermostatted glass tube, melted and then slowly cooled at a rate of about $1 \mathrm{~K} / \mathrm{min}$. The temperature of the sample was measured with a high precision platinum-resistance thermometer. The crystallization temperature was obtained from a plot of the cooling curve versus time. Each measurement was repeated three times.

Source and Purity of Chemicals:

(1) $98+\%$, Aldrich Chemical Company, Milwaukee, WI, USA, no purification details were given in the paper.

(2) $99 \%$, Aldrich Chemical Company, purification details were not given in the paper.

\section{Estimated Error:}

Temperature: Authors state a global accuracy of $\pm 1 \%$ regarding their measurements.

$x_{1}: \pm 0.0002$ (estimated by compiler).

\subsection{Phenanthrene solubility data in aromatic hydrocarbons}

\begin{tabular}{ll} 
Components: & Original Measurements: \\
(1) Phenanthrene; $\mathrm{C}_{14} \mathrm{H}_{10} ;[85-01-8]$ & ${ }^{15}$ A. Aoulmi, M. Bouroukba, R. \\
(2) Squalane; $\mathrm{C}_{30} \mathrm{H}_{62} ;[111-01-3]$ & Solimando, and M. Rogalski, Fluid \\
& Phase Equilib. 110, 283 (1995). \\
\hline Variables: & Prepared by: \\
Temperature & W. E. Acree, Jr. \\
\hline
\end{tabular}

Experimental Values

\begin{tabular}{lcc}
\hline \hline$T / \mathrm{K}$ & $x_{2}{ }^{\mathrm{a}}$ & $x_{1}{ }^{\mathrm{b}}$ \\
\hline 331.65 & 0.6984 & 0.3016 \\
343.15 & 0.5953 & 0.4047 \\
353.15 & 0.4803 & 0.5197 \\
356.55 & 0.4463 & 0.5537 \\
359.25 & 0.3967 & 0.6033 \\
360.75 & 0.3501 & 0.6499 \\
362.55 & 0.2939 & 0.7061 \\
363.95 & 0.2497 & 0.7503 \\
365.15 & 0.2021 & 0.7979 \\
366.35 & 0.1540 & 0.8460 \\
367.65 & 0.0988 & 0.9012 \\
369.15 & 0.0507 & 0.9493 \\
\hline
\end{tabular}

${ }^{\mathrm{a}} x_{2}$ : mole fraction of component 2 in the saturated solution.

${ }^{\mathrm{b}} x_{1}$ : mole fraction of the polycyclic aromatic hydrocarbon (component 1).

The authors employed a SSF to describe the activity coefficients of phenanthrene and squalane calculated from the solidliquid equilibrium data. The SSF model gave a calculated eutectic temperature of $T / \mathrm{K}=233.8$ and eutectic mole fraction of phenanthrene of $x_{1}=0.03$.
Solubilities were reported in the paper as the grams of phenanthrene dissolved per $100 \mathrm{~g}$ of solvent. Mole fraction solubilities were calculated by the compiler. 
Experimental Values

\begin{tabular}{lcc}
\hline \hline$T / \mathrm{K}$ & $x_{2}^{\mathrm{a}, \mathrm{b}}$ & $x_{1}^{\mathrm{b}, \mathrm{c}}$ \\
\hline 298.15 & 0.8029 & 0.1971 \\
303.15 & 0.7953 & 0.2047 \\
308.15 & 0.7627 & 0.2373 \\
313.15 & 0.7293 & 0.2707 \\
318.15 & 0.6846 & 0.3154 \\
323.15 & 0.6499 & 0.3501 \\
328.15 & 0.5988 & 0.4012 \\
333.15 & 0.5587 & 0.4413 \\
338.15 & 0.5033 & 0.4967 \\
\hline
\end{tabular}

${ }^{a_{x_{2}}}$ : mole fraction of component 2 in the saturated solution.

${ }^{\mathrm{b}}$ Experimental values based on gravimetric method.

${ }^{c} x_{1}$ : mole fraction of the polycyclic aromatic hydrocarbon (component 1 ).

Solubilities were reported in the paper as the grams of phenanthrene dissolved per $100 \mathrm{~g}$ of solvent. Mole fraction solubilities were calculated by the compiler.

\section{Auxiliary Information}

\section{Method/Apparatus/Procedure:}

Three-neck glass vessel, attenuated total reflectance FTIR spectrophotometer; regular diamond immersion probe, temperature controller.

Solubilities were determined by a synthetic-undersaturation method. Known masses of solute and solvent were placed in a three-neck glass vessel. Once equilibrium is achieved a steady-stated ATR-FTIR reading is recorded. An additional known mass of phenanthrene is added to the solution. Once equilibrium is achieved, a new steady-state ATR-FTIR reading is recorded and compared to the previous reading. As long as there is no change in FTIR reading, the measurement is taken as the solubility of phenanthrene at the solution temperature. If the reading has changed, additional phenanthrene is added until a saturated solution is obtained. The authors checked the method by performing phenanthrene solubility measurements using a gravimetric method. The solubilities determined using the gravimetric and ATR-FTIR methods were in good agreement.

Source and Purity of Chemicals:

(1) $98 \%$, Sigma-Aldrich Chemical Company, USA, no further purification details were provided in the paper.

(2) Analytical Grade, Sigma-Aldrich Chemical Company, USA, no further purification details were provided in the paper.

\section{Estimated Error:}

Temperature: $\pm 0.1 \mathrm{~K}$ (estimated by compiler). $x_{1}: 2 \%$ (relative error, estimated by compiler).

\begin{tabular}{ll}
\hline \hline Components: & Original Measurements: \\
(1) Phenanthrene; $\mathrm{C}_{14} \mathrm{H}_{10} ;[85-01-8]$ & ${ }^{56}$ B. L. Sharma, R. Kant, R. \\
(2) Naphthalene; $\mathrm{C}_{10} \mathrm{H}_{8} ;[91-20-3]$ & Sharma, and S. Tandon, Mater. \\
& Chem. Phys. 82, 216 (2003). \\
\hline Variables: & Prepared by: \\
Temperature & W. E. Acree, Jr. \\
\hline
\end{tabular}

Experimental Values

\begin{tabular}{lll}
\hline \hline$T / \mathrm{K}$ & $x_{1}{ }^{\mathrm{a}}$ & $x_{2}{ }^{\mathrm{b}}$ \\
\hline 373.0 & 1.0 & 0.0 \\
364.0 & 0.9 & 0.1 \\
356.0 & 0.8 & 0.2 \\
348.0 & 0.7 & 0.3 \\
339.0 & 0.6 & 0.4 \\
329.0 & 0.5 & 0.5 \\
326.0 & 0.4 & 0.6 \\
334.0 & 0.3 & 0.7 \\
340.0 & 0.2 & 0.8 \\
346.5 & 0.1 & 0.9 \\
353.0 & 0.0 & 1.0 \\
\hline
\end{tabular}

${ }^{a} x_{1}$ : mole fraction of the component 1 in the saturated solution.

${ }^{b} x_{2}$ : mole fraction of component 2 in the saturated solution.

\section{Auxiliary Information}

Method/Apparatus/Procedure:

The only experimental details provided in the paper was a statement by the authors that the solidus-liquid equilibrium data were obtained by the thawpoint melting technique.

Source and Purity of Chemicals:

(1) Analytical Reagent Grade, Fluka Chemicals, was purified by repeated recrystallizations from ethyl ethanoate.

(2) Laboratory Reagent Grade, Riedel Chemicals, was purified by sublimation.

Estimated Error:

Temperature: $\pm 0.5 \mathrm{~K}$ (estimated by compiler).

$x_{1}: \pm 0.05$ (estimated by compiler).

\subsection{Phenanthrene solubility data in esters}

\begin{tabular}{ll}
\hline \hline Components: & Original Measurements: \\
(1) Phenanthrene; $\mathrm{C}_{14} \mathrm{H}_{10} ;[85-01-8]$ & ${ }^{61}$ C. E. Hernández, K. M. De \\
(2) Ethyl ethanoate; $\mathrm{C}_{4} \mathrm{H}_{8} \mathrm{O}_{2} ;[141-78-6]$ & Fina, L. E. Roy, T. L. Sharp, \\
& and W. E. Acree, Jr., Can. J. \\
& Chem. 77, 1465 (1999). \\
\hline Variables: & Prepared by: \\
$T / \mathrm{K}=298.15$ & W. E. Acree, Jr. \\
\hline
\end{tabular}

Experimental Values

\begin{tabular}{lcc}
\hline \hline$x_{2}{ }^{(\mathrm{s}) \mathrm{a}}$ & $x_{2}{ }^{\mathrm{b}}$ & $x_{1}{ }^{\mathrm{c}}$ \\
\hline 1.0000 & 0.8501 & 0.1499 \\
$\frac{{ }^{\mathrm{a}} x_{2}{ }^{(\mathrm{s})}: \text { initial mole fraction of component } 2 \text { in the solution. }}{{ }^{\mathrm{b}} x_{2}: \text { mole fraction of component } 2 \text { in the saturated solution. }}$ \\
${ }^{c_{x_{1}}: \text { mole fraction solubility of the solute. }}$
\end{tabular}




\section{Auxiliary Information}

Method/Apparatus/Procedure:

Constant-temperature bath, calorimetric thermometer, and an ultraviolet/ visible spectrophotometer.

Excess solute and solvent were placed in amber glass bottles and allowed to equilibrate for several days at constant temperature. Attainment of equilibrium was verified by several repetitive measurements and by approaching equilibrium from supersaturation. Aliquots of saturated solutions were transferred through a coarse filter into tared volumetric flasks, weighed and diluted with methanol. Concentrations were determined by spectrophotometric measurements at $347 \mathrm{~nm}$.

Source and Purity of Chemicals:

(1) $98 \%$, Aldrich Chemical Company, Milwaukee, WI, USA, was recrystallized several times from methanol.

(2) $99.9 \%$, HPLC Grade, Aldrich Chemical Company, stored over molecular sieves before use.

Estimated Error:

Temperature: $\pm 0.1 \mathrm{~K}$.

$x_{1}: \pm 1.5 \%$ (relative error).

\begin{tabular}{ll}
\hline \hline Components: & Original Measurements: \\
(1) Phenanthrene; $\mathrm{C}_{14} \mathrm{H}_{10} ;[85-01-8]$ & ${ }^{61}$ C. E. Hernández, K. M. De \\
(2) Butyl ethanoate; $\mathrm{C}_{6} \mathrm{H}_{12} \mathrm{O}_{2} ;[123-86-4]$ & Fina, L. E. Roy, T. L. Sharp, \\
& and W. E. Acree, Jr., Can. J. \\
& Chem. 77, 1465 (1999). \\
\hline Variables: & Prepared by: \\
$T / \mathrm{K}=298.15$ & W. E. Acree, Jr. \\
\hline
\end{tabular}

Experimental Values

\begin{tabular}{lcc}
\hline \hline$x_{2}{ }^{(\mathrm{s}) \mathrm{a}}$ & $x_{2}{ }^{\mathrm{b}}$ & $x_{1}{ }^{\mathrm{c}}$ \\
\hline 1.0000 & 0.8188 & 0.1812 \\
$\frac{{ }^{\mathrm{a}} x_{2}{ }^{(\mathrm{s})}: \text { initial mole fraction of component } 2 \text { in the solution. }}{{ }^{\mathrm{b}} x_{2}: \text { mole fraction of component } 2 \text { in the saturated solution. }}$ \\
${ }^{\mathrm{c}}{ }_{x_{1}: \text { mole fraction solubility of the solute. }}$
\end{tabular}

\section{Auxiliary Information}

\section{Method/Apparatus/Procedure:}

Constant-temperature bath, calorimetric thermometer, and an ultraviolet/ visible spectrophotometer.

Excess solute and solvent were placed in amber glass bottles and allowed to equilibrate for several days at constant temperature. Attainment of equilibrium was verified by several repetitive measurements and by approaching equilibrium from supersaturation. Aliquots of saturated solutions were transferred through a coarse filter into tared volumetric flasks, weighed and diluted with methanol. Concentrations were determined by

spectrophotometric measurements at $347 \mathrm{~nm}$.

Source and Purity of Chemicals:

(1) $98 \%$, Aldrich Chemical Company, Milwaukee, WI, USA, was recrystallized several times from methanol.

(2) $99.8 \%$, HPLC Grade, Aldrich Chemical Company, stored over molecular sieves before use.

\section{Estimated Error:}

Temperature: $\pm 0.1 \mathrm{~K}$.

$x_{1}: \pm 1.5 \%$ (relative error).

\subsection{Phenanthrene solubility data in ethers}

\begin{tabular}{|c|c|}
\hline $\begin{array}{l}\text { Components: } \\
\text { (1) Phenanthrene; } \mathrm{C}_{14} \mathrm{H}_{10} ;[85-01-8] \\
\text { (2); 1,1'-Oxybisbutane; } \mathrm{C}_{8} \mathrm{H}_{18} \mathrm{O} \text {; } \\
{[142-96-1]}\end{array}$ & $\begin{array}{l}\text { Original Measurements: } \\
{ }^{61} \text { C. E. Hernández, K. M. De Fina, } \\
\text { L. E. Roy, T. L. Sharp, and W. E. } \\
\text { Acree, Jr., Can. J. Chem. 77, } 1465 \\
\text { (1999). }\end{array}$ \\
\hline $\begin{array}{l}\text { Variables: } \\
T / \mathrm{K}=298.15\end{array}$ & $\begin{array}{l}\text { Prepared by: } \\
\text { W. E. Acree, Jr. }\end{array}$ \\
\hline
\end{tabular}

Experimental Values

\begin{tabular}{lcc}
\hline \hline$x_{2}{ }^{(\mathrm{s}) \mathrm{a}}$ & $x_{2}{ }^{\mathrm{b}}$ & $x_{1}{ }^{\mathrm{c}}$ \\
\hline 1.0000 & 0.9055 & 0.09454 \\
${ }^{\mathrm{a}}{ }{ }^{(\mathrm{s})}:$ initial mole fraction of component 2 in the solution. \\
${ }^{\mathrm{b}} x_{2}:$ mole fraction of component 2 in the saturated solution. \\
${ }^{\mathrm{c}}{ }_{x_{1}}$ : mole fraction solubility of the solute.
\end{tabular}

\section{Auxiliary Information}

Method/Apparatus/Procedure:

Constant-temperature bath, calorimetric thermometer, and an ultraviolet/ visible spectrophotometer.

Excess solute and solvent were placed in amber glass bottles and allowed to equilibrate for several days at constant temperature. Attainment of equilibrium was verified by several repetitive measurements and by approaching equilibrium from supersaturation. Aliquots of saturated solutions were transferred through a coarse filter into tared volumetric flasks, weighed and diluted with methanol. Concentrations were determined by spectrophotometric measurements at $347 \mathrm{~nm}$.

Source and Purity of Chemicals:

(1) $98 \%$, Aldrich Chemical Company, Milwaukee, WI, USA, was recrystallized several times from methanol.

(2) $99 \%$, Aldrich Chemical Company, stored over molecular sieves before use.

Estimated Error:

Temperature: $\pm 0.1 \mathrm{~K}$.

$x_{1}: \pm 1.5 \%$ (relative error).

\begin{tabular}{|c|c|}
\hline $\begin{array}{l}\text { Components: } \\
\text { (1) Phenanthrene; } \mathrm{C}_{14} \mathrm{H}_{10} ;[85-01-8] \\
\text { (2) Tetrahydrofuran; } \mathrm{C}_{4} \mathrm{H}_{8} \mathrm{O} \text {; } \\
\text { [109-99-9] }\end{array}$ & $\begin{array}{l}\text { Original Measurements: } \\
{ }^{61} \text { C. E. Hernández, K. M. De Fina, L. } \\
\text { E. Roy, T. L. Sharp, and W. E. Acree, } \\
\text { Jr., Can. J. Chem. 77, } 1465 \text { (1999). }\end{array}$ \\
\hline $\begin{array}{l}\text { Variables: } \\
T / \mathrm{K}=298.15\end{array}$ & $\begin{array}{l}\text { Prepared by: } \\
\text { W. E. Acree, Jr. }\end{array}$ \\
\hline
\end{tabular}

Experimental Values

\begin{tabular}{lcc}
\hline \hline$x_{2}{ }^{(\mathrm{s}) \mathrm{a}}$ & $x_{2}{ }^{\mathrm{b}}$ & $x_{1}{ }^{\mathrm{c}}$ \\
\hline 1.0000 & 0.7116 & 0.2884 \\
$\frac{{ }^{\mathrm{a}} x_{2}{ }^{(\mathrm{s})}: \text { initial mole fraction of component } 2 \text { in the solution. }}{}$ \\
${ }^{\mathrm{b}} x_{2}:$ mole fraction of component 2 in the saturated solution. \\
${ }^{\mathrm{c}}{ }_{x_{1}: \text { mole fraction solubility of the solute. }}$
\end{tabular}




\section{Auxiliary Information}

\section{Method/Apparatus/Procedure:}

Constant-temperature bath, calorimetric thermometer, and an ultraviolet/ visible spectrophotometer.

Excess solute and solvent were placed in amber glass bottles and allowed to equilibrate for several days at constant temperature. Attainment of equilibrium was verified by several repetitive measurements and by approaching equilibrium from supersaturation. Aliquots of saturated solutions were transferred through a coarse filter into tared volumetric flasks, weighed and diluted with methanol. Concentrations were determined by spectrophotometric measurements at $347 \mathrm{~nm}$.

Source and Purity of Chemicals:

(1) $98 \%$, Aldrich Chemical Company, Milwaukee, WI, USA, was recrystallized several times from methanol.

(2) $99.9 \%$, Aldrich Chemical Company, stored over molecular sieves before use.

Estimated Error:

Temperature: $\pm 0.1 \mathrm{~K}$

$x_{1}: \pm 1.5 \%$ (relative error).

\subsection{Phenanthrene solubility data in haloalkanes, haloalkenes, and haloaromatic hydrocarbons}

\begin{tabular}{ll}
\hline \hline Components: & Original Measurements: \\
(1) Phenanthrene; $\mathrm{C}_{14} \mathrm{H}_{10} ;[85-01-8]$ & ${ }^{61}$ C. E. Hernández, K. M. De Fina, L. \\
(2) Tetrachloromethane; $\mathrm{CCl}_{4} ;$ & E. Roy, T. L. Sharp, and W. E. Acree, \\
[56-23-5] & Jr., Can. J. Chem. 77, 1465 (1999). \\
\hline Variables: & Prepared by: \\
$T / \mathrm{K}=298.15$ & W. E. Acree, Jr. \\
\hline
\end{tabular}

Experimental Values

\begin{tabular}{lcc}
\hline \hline$x_{2}{ }^{(\mathrm{s}) \mathrm{a}}$ & $x_{2}{ }^{\mathrm{b}}$ & $x_{1}{ }^{\mathrm{c}}$ \\
\hline 1.0000 & 0.8738 & 0.1262 \\
$\frac{{ }^{a} x_{2}{ }^{(\mathrm{s})}: \text { initial mole fraction of component } 2 \text { in the solution. }}{{ }^{\mathrm{b}} x_{2}: \text { mole fraction of component } 2 \text { in the saturated solution. }}$ \\
${ }^{\mathrm{c}} x_{1}:$ mole fraction solubility of the solute.
\end{tabular}

\section{Auxiliary Information}

\section{(1) Phenanthrene; $\mathrm{C}_{14} \mathrm{H}_{10} ;$ [85-01-8] \\ (2) 1,4-Dioxane; $\mathrm{C}_{4} \mathrm{H}_{8} \mathrm{O}_{2} ;[123-91-1]$}

Components:

\section{Variables:}

$T / \mathrm{K}=298.15$
Original Measurements:

${ }^{61}$ C. E. Hernández, K. M. De Fina, L. E. Roy, T. L. Sharp, and W. E. Acree, Jr., Can. J. Chem. 77, 1465 (1999).

\section{Prepared by:}

W. E. Acree, Jr

\section{Experimental Values}

\begin{tabular}{lcc}
\hline \hline$x_{2}{ }^{(\mathrm{s}) \mathrm{a}}$ & $x_{2}{ }^{\mathrm{b}}$ & $x_{1}{ }^{\mathrm{c}}$ \\
\hline 1.0000 & 0.7835 & 0.2165
\end{tabular}

${ }^{\mathrm{a}} x_{2}{ }^{(\mathrm{s})}$ : initial mole fraction of component 2 in the solution.

${ }^{b} x_{2}$ : mole fraction of component 2 in the saturated solution.

${ }^{c} x_{1}$ : mole fraction solubility of the solute.

\section{Auxiliary Information}

\section{Method/Apparatus/Procedure:}

Constant-temperature bath, calorimetric thermometer, and an ultraviolet/ visible spectrophotometer.

Excess solute and solvent were placed in amber glass bottles and allowed to equilibrate for several days at constant temperature. Attainment of equilibrium was verified by several repetitive measurements and by approaching equilibrium from supersaturation. Aliquots of saturated solutions were transferred through a coarse filter into tared volumetric flasks, weighed and diluted with methanol. Concentrations were determined by spectrophotometric measurements at $347 \mathrm{~nm}$.

Source and Purity of Chemicals:

(1) $98 \%$, Aldrich Chemical Company, Milwaukee, WI, USA, was recrystallized several times from methanol.

(2) $99.8 \%$, Aldrich Chemical Company, stored over molecular sieves before use.

Estimated Error:

Temperature: $\pm 0.1 \mathrm{~K}$.

$x_{1}: \pm 1.5 \%$ (relative error).

\subsection{Phenanthrene solubility data in alcohols}

\begin{tabular}{ll}
\hline \hline Components: & Original Measurements: \\
(1) Phenanthrene; $\mathrm{C}_{14} \mathrm{H}_{10} ;[85-01-8]$ & ${ }^{61}$ C. E. Hernández, K. M. De Fina, L. \\
(2) Methanol; $\mathrm{CH}_{4} \mathrm{O} ;[67-56-1]$ & E. Roy, T. L. Sharp, and W. E. Acree, \\
& Jr., Can. J. Chem. 77, 1465 (1999). \\
\hline Variables: & Prepared by: \\
$T / \mathrm{K}=298.15$ & W. E. Acree, Jr. \\
\hline
\end{tabular}

$T / \mathrm{K}=298.15$

\section{Method/Apparatus/Procedure:}

Constant-temperature bath, calorimetric thermometer, and an ultraviolet/ visible spectrophotometer.

Excess solute and solvent were placed in amber glass bottles and allowed to equilibrate for several days at constant temperature. Attainment of equilibrium was verified by several repetitive measurements and by approaching equilibrium from supersaturation. Aliquots of saturated solutions were transferred through a coarse filter into tared volumetric flasks, weighed and diluted with methanol. Concentrations were determined by spectrophotometric measurements at $347 \mathrm{~nm}$.

Source and Purity of Chemicals:

(1) $98 \%$, Aldrich Chemical Company, Milwaukee, WI, USA, was recrystallized several times from methanol.

(2) $99.9+\%$, HPLC Grade, Aldrich Chemical Company, stored over molecular sieves before use.

\section{Estimated Error:}

Temperature: $\pm 0.1 \mathrm{~K}$.

$x_{1}: \pm 1.5 \%$ (relative error). 
Experimental Values

\begin{tabular}{lcc}
\hline \hline$x_{2}{ }^{(\mathrm{s}) \mathrm{a}}$ & $x_{2}{ }^{\mathrm{b}}$ & $x_{1}{ }^{\mathrm{c}}$ \\
\hline 1.0000 & 0.9941 & 0.00589 \\
$\frac{{ }^{\mathrm{a}} x_{2}{ }^{(\mathrm{s})}: \text { initial mole fraction of component } 2 \text { in the solution. }}{{ }^{\mathrm{b}}{ }_{x_{2}}: \text { mole fraction of component } 2 \text { in the saturated solution. }}$ \\
${ }^{\mathrm{c}}{ }_{x_{1}}:$ mole fraction solubility of the solute.
\end{tabular}

\section{Auxiliary Information}

\section{Method/Apparatus/Procedure:}

Constant-temperature bath, calorimetric thermometer, and an ultraviolet/ visible spectrophotometer.

Excess solute and solvent were placed in amber glass bottles and allowed to equilibrate for several days at constant temperature. Attainment of equilibrium was verified by several repetitive measurements and by approaching equilibrium from supersaturation. Aliquots of saturated solutions were transferred through a coarse filter into tared volumetric flasks, weighed and diluted with methanol. Concentrations were determined by spectrophotometric measurements at $347 \mathrm{~nm}$.

\section{Source and Purity of Chemicals:}

(1) $98 \%$, Aldrich Chemical Company, Milwaukee, WI, USA, was recrystallized several times from methanol.

(2) $99.9 \%$, Aldrich Chemical Company, stored over molecular sieves before use.

\section{Estimated Error:}

Temperature: $\pm 0.1 \mathrm{~K}$.

$x_{1}: \pm 1.5 \%$ (relative error).

\begin{tabular}{|c|c|}
\hline $\begin{array}{l}\text { Components: } \\
\text { (1) Phenanthrene; } \mathrm{C}_{14} \mathrm{H}_{10} ;[85-01-8] \\
\text { (2) Methanol; } \mathrm{CH}_{4} \mathrm{O} ;[67-56-1]\end{array}$ & $\begin{array}{l}\text { Original Measurements: } \\
{ }^{62} \text { M. A. A. Fakhree, W. E. Acree, } \\
\text { Jr., and A. Jouyban, J. Chem. Eng. } \\
\text { Data 55, } 531 \text { (2010). }\end{array}$ \\
\hline $\begin{array}{l}\text { Variables: } \\
T / \mathrm{K}=298.15\end{array}$ & $\begin{array}{l}\text { Prepared by: } \\
\text { W. E. Acree, Jr. }\end{array}$ \\
\hline
\end{tabular}

Experimental Values

\begin{tabular}{lcc}
\hline \hline$c_{1}^{\mathrm{a}}$ & $x_{2}{ }^{\mathrm{b}}$ & $x_{1}{ }^{\mathrm{c}}$ \\
\hline 0.133 & 0.9945 & 0.00549 \\
\hline
\end{tabular}

${ }^{\mathrm{a}} c_{1}$ : molar solubility of the solute.

${ }^{\mathrm{b}} x_{2}$ : mole fraction of component 2 in the saturated solution.

${ }^{c} x_{1}$ : mole fraction solubility of the solute.

The mole fraction solubility of the solute was calculated by the compiler using the experimental density data given in the paper.

\section{Auxiliary Information}

Method/Apparatus/Procedure:

Incubator, shaker, and an ultraviolet/visible spectrophotometer.

Solubility was determined by equilibrating an excess amount of solid naphthalene with the organic solvent using a shaker placed in an incubator equipped with a temperature controlling system. After an equilibration period of at least $48 \mathrm{~h}$, the samples were withdrawn and filtered through hydrophobic Durapore filters $(0.45 \mu \mathrm{m})$. The filtered sample was diluted quantitatively with methanol. Concentrations were determined by spectrophotmetric analysis at $345 \mathrm{~nm}$.

Source and Purity of Chemicals:

(1) $99 \%$, Merck Chemical Company, Germany, was recrystallized several times from propanone.

(2) $99.5 \%$, Merck Chemical Company. No purification details were provided in the paper. Water content was checked by Karl Fischer titration and found to be $0.04 \%$ by mass.

Estimated Error:

Temperature: $\pm 0.2 \mathrm{~K}$.

$x_{1}: \pm 4.2 \%$ (relative error).

\begin{tabular}{|c|c|}
\hline $\begin{array}{l}\text { Components: } \\
\text { (1) Phenanthrene; } \mathrm{C}_{14} \mathrm{H}_{10} ;[85-01-8] \\
\text { (2) Ethanol; } \mathrm{C}_{2} \mathrm{H}_{6} \mathrm{O} ;[64-17-5]\end{array}$ & $\begin{array}{l}\text { Original Measurements: } \\
{ }^{61} \text { C. E. Hernández, K. M. De Fina, L. } \\
\text { E. Roy, T. L. Sharp, and W. E. Acree, } \\
\text { Jr., Can. J. Chem. 77, } 1465 \text { (1999). }\end{array}$ \\
\hline $\begin{array}{l}\text { Variables: } \\
T / \mathrm{K}=298.15\end{array}$ & $\begin{array}{l}\text { Prepared by: } \\
\text { W. E. Acree, Jr. }\end{array}$ \\
\hline
\end{tabular}

Experimental Values

\begin{tabular}{|c|c|c|}
\hline$x_{2}{ }^{(\mathrm{s}) \mathrm{a}}$ & $x_{2}{ }^{b}$ & $x_{1}{ }^{\mathrm{c}}$ \\
\hline 1.0000 & 0.9889 & 0.01114 \\
\hline
\end{tabular}

\section{Auxiliary Information}

\section{Method/Apparatus/Procedure:}

Constant-temperature bath, calorimetric thermometer, and an ultraviolet/ visible spectrophotometer.

Excess solute and solvent were placed in amber glass bottles and allowed to equilibrate for several days at constant temperature. Attainment of equilibrium was verified by several repetitive measurements and by approaching equilibrium from supersaturation. Aliquots of saturated solutions were transferred through a coarse filter into tared volumetric flasks, weighed and diluted with methanol. Concentrations were determined by

spectrophotometric measurements at $347 \mathrm{~nm}$.

\section{Source and Purity of Chemicals:}

(1) $98 \%$, Aldrich Chemical Company, Milwaukee, WI, USA, was recrystallized several times from methanol.

(2) Absolute, Aaper Alcohol and Chemical Company, USA, stored over molecular sieves before use.

Estimated Error:

Temperature: $\pm 0.1 \mathrm{~K}$.

$x_{1}: \pm 1.5 \%$ (relative error). 


\section{Components:}

(1) Phenanthrene; $\mathrm{C}_{14} \mathrm{H}_{10}$; [85-01-8]

(2) Ethanol; $\mathrm{C}_{2} \mathrm{H}_{6} \mathrm{O}$; [64-17-5]
Original Measurements:

${ }^{34}$ A. Shayanfar, S. H. Eghrary, F. Sardari, W. E. Acree, Jr., and A. Jouyban, J. Chem. Eng. Data 56, 2290 (2011).

\begin{tabular}{ll}
\hline Variables: & Prepared by: \\
Temperature & W. E. Acree, Jr. \\
\hline
\end{tabular}

Experimental Values

\begin{tabular}{lcc}
\hline \hline$T / K$ & $x_{2}{ }^{\mathrm{a}}$ & $x_{1}{ }^{\mathrm{b}}$ \\
\hline 298.2 & 0.9881 & 0.0119 \\
308.2 & 0.9841 & 0.0159 \\
318.2 & 0.9810 & 0.0190 \\
\hline
\end{tabular}

${ }^{a} x_{2}$ : mole fraction of the component 2 in the saturated solution.

${ }^{b} x_{1}$ : mole fraction of component 1 in the saturated solution.

\section{Auxiliary Information}

\section{Method/Apparatus/Procedure:}

Incubator, shaker, and an ultraviolet/visible spectrophotometer.

Solubility was determined by equilibrating an excess amount of solid naphthalene with the organic solvent using a shaker placed in an incubator equipped with a temperature controlling system. After an equilibration period of at least $48 \mathrm{~h}$, the samples were withdrawn and filtered through hydrophobic Durapore filters $(0.45 \mu \mathrm{m})$. The filtered sample was diluted quantitatively with methanol. Concentrations were determined by spectrophotmetric analysis at $356 \mathrm{~nm}$.

\section{Source and Purity of Chemicals:}

(1) $98 \%$, Merck Chemicals, Germany, was recrystallized several times from propanone before use.

(2) $99+\%$, Absolute, Merck Chemicals, no information given regarding any further purification.

Estimated Error:

Temperature: $\pm 0.2 \mathrm{~K}$.

$x_{1}: \pm 2.5 \%$ (relative uncertainty).

\begin{tabular}{|c|c|}
\hline $\begin{array}{l}\text { Components: } \\
\text { (1) Phenanthrene; } \mathrm{C}_{14} \mathrm{H}_{10} ;[85-01-8] \\
\text { (2) Ethanol; } \mathrm{C}_{2} \mathrm{H}_{6} \mathrm{O} ;[64-17-5]\end{array}$ & $\begin{array}{l}\text { Original Measurements: } \\
{ }^{62} \text { M. A. A. Fakhree, W. E. Acree, } \\
\text { Jr., and A. Jouyban, J. Chem. Eng. } \\
\text { Data 55, } 531 \text { (2010). }\end{array}$ \\
\hline $\begin{array}{l}\text { Variables: } \\
T / \mathrm{K}=298.15\end{array}$ & $\begin{array}{l}\text { Prepared by: } \\
\text { W. E. Acree, Jr. }\end{array}$ \\
\hline
\end{tabular}

Experimental Values

\begin{tabular}{lcc}
\hline \hline$c_{1}{ }^{\mathrm{a}}$ & $x_{2}{ }^{\mathrm{b}}$ & $x_{1}{ }^{\mathrm{c}}$ \\
\hline 0.182 & 0.9892 & 0.0108 \\
\hline
\end{tabular}

${ }^{\mathrm{a}} c_{1}$ : molar solubility of the solute.

${ }^{\mathrm{b}} x_{2}$ : mole fraction of component 2 in the saturated solution.

${ }^{c} x_{1}$ : mole fraction solubility of the solute.
The mole fraction solubility of the solute was calculated by the compiler using the experimental density data given in the paper.

\section{Auxiliary Information}

\section{Method/Apparatus/Procedure:}

Incubator, shaker, and an ultraviolet/visible spectrophotometer.

Solubility was determined by equilibrating an excess amount of solid naphthalene with the organic solvent using a shaker placed in an incubator equipped with a temperature controlling system. After an equilibration period of at least $48 \mathrm{~h}$, the samples were withdrawn and filtered through hydrophobic Durapore filters $(0.45 \mu \mathrm{m})$. The filtered sample was diluted quantitatively with methanol. Concentrations were determined by spectrophotmetric analysis at $345 \mathrm{~nm}$.

Source and Purity of Chemicals:

(1) $99 \%$, Merck Chemical Company, Germany, was recrystallized several times from propanone.

(2) $99.9 \%$, Merck Chemical Company. No purification details were provided in the paper. Water content was checked by Karl Fischer titration and found to be $0.13 \%$ by mass.

\section{Estimated Error:}

Temperature: $\pm 0.2 \mathrm{~K}$.

$x_{1}: \pm 4.2 \%$ (relative error).

\begin{tabular}{ll}
\hline \hline $\begin{array}{l}\text { Components: } \\
\text { (1) Phenanthrene; } \mathrm{C}_{14} \mathrm{H}_{10} ;[85-01-8]\end{array}$ & $\begin{array}{l}\text { Original Measurements: } \\
{ }_{61} \text { C. E. Hernández, K. M. De Fina, } \\
\text { (2) 1-Propanol; } \mathrm{C}_{3} \mathrm{H}_{8} \mathrm{O} ;[71-23-8]\end{array}$ \\
& L. E. Roy, T. L. Sharp, and W. E. \\
& Acree, Jr., Can. J. Chem. 77, 1465 \\
& $(1999)$. \\
\hline Variables: & Prepared by: \\
$T / \mathrm{K}=298.15$ & W. E. Acree, Jr. \\
\hline
\end{tabular}

Experimental Values

\begin{tabular}{lcc}
\hline \hline$x_{2}{ }^{(\mathrm{s}) \mathrm{a}}$ & $x_{2}{ }^{\mathrm{b}}$ & $x_{1}{ }^{\mathrm{c}}$ \\
\hline 1.0000 & 0.9865 & 0.01355 \\
$\frac{{ }^{\mathrm{a}} x_{2}{ }^{(\mathrm{s})}: \text { initial mole fraction of component } 2 \text { in the solution. }}{}$ \\
${ }^{\mathrm{b}} x_{x_{2}}$ : mole fraction of component 2 in the saturated solution. \\
${ }^{\mathrm{c}} x_{1}$ : mole fraction solubility of the solute.
\end{tabular}

\section{Auxiliary Information}

\section{Method/Apparatus/Procedure:}

Constant-temperature bath, calorimetric thermometer, and an ultraviolet/ visible spectrophotometer.

Excess solute and solvent were placed in amber glass bottles and allowed to equilibrate for several days at constant temperature. Attainment of equilibrium was verified by several repetitive measurements and by approaching equilibrium from supersaturation. Aliquots of saturated solutions were transferred through a coarse filter into tared volumetric flasks, weighed and diluted with methanol. Concentrations were determined by spectrophotometric measurements at $347 \mathrm{~nm}$.

\section{Source and Purity of Chemicals:}

(1) $98 \%$, Aldrich Chemical Company, Milwaukee, WI, USA, was recrystallized several times from methanol.

(2) $99+\%$, anhydrous, Aldrich Chemical Company, stored over molecular sieves before use. 
Estimated Error:

Temperature: $\pm 0.1 \mathrm{~K}$.

$x_{1}: \pm 1.5 \%$ (relative error).

Original Measurements:

${ }^{62}$ M. A. A. Fakhree, W. E. Acree, Jr., and A. Jouyban, J. Chem. Eng. Data 55, 531 (2010).

Prepared by: W. E. Acree, Jr.

Experimental Values

\begin{tabular}{lcc}
\hline \hline$c_{1}^{\mathrm{a}}$ & $x_{2}{ }^{\mathrm{b}}$ & $x_{1}{ }^{\mathrm{c}}$ \\
\hline 0.174 & 0.9868 & 0.0132 \\
\hline
\end{tabular}

${ }^{\mathrm{a}} c_{1}$ : molar solubility of the solute.

${ }^{\mathrm{b}} x_{2}$ : mole fraction of component 2 in the saturated solution.

${ }^{c} x_{1}$ : mole fraction solubility of the solute.

The mole fraction solubility of the solute was calculated by the compiler using the experimental density data given in the paper.

\section{Auxiliary Information}

\section{Method/Apparatus/Procedure:}

Incubator, shaker, and an ultraviolet/visible spectrophotometer.

Solubility was determined by equilibrating an excess amount of solid naphthalene with the organic solvent using a shaker placed in an incubator equipped with a temperature controlling system. After an equilibration period of at least $48 \mathrm{~h}$, the samples were withdrawn and filtered through hydrophobic Durapore filters $(0.45 \mu \mathrm{m})$. The filtered sample was diluted quantitatively with methanol. Concentrations were determined by spectrophotmetric analysis at $345 \mathrm{~nm}$.

\section{Source and Purity of Chemicals:}

(1) $99 \%$, Merck Chemical Company, Germany, was recrystallized several times from propanone.

(2) $99.5 \%$, Merck Chemical Company. No purification details were provided in the paper. Water content was checked by Karl Fischer titration and found to be $0.07 \%$ by mass.

\section{Estimated Error:}

Temperature: $\pm 0.2 \mathrm{~K}$

$x_{1}: \pm 4.2 \%$ (relative error).

\section{Components:}

(1) Phenanthrene; $\mathrm{C}_{14} \mathrm{H}_{10}$; [85-01-8]

(2) 2-Propanol; $\mathrm{C}_{3} \mathrm{H}_{8} \mathrm{O}$; [67-63-0]
Original Measurements:

${ }^{61}$ C. E. Hernández, K. M. De Fina, L. E. Roy, T. L. Sharp, and W. E. Acree, Jr., Can. J. Chem. 77, 1465 (1999).

\begin{tabular}{ll}
\hline Variables: & Prepared by: \\
$T / \mathrm{K}=298.15$ & W. E. Acree, Jr. \\
\hline
\end{tabular}

Experimental Values

\begin{tabular}{lcc}
\hline \hline$x_{2}{ }^{(\mathrm{s}) \mathrm{a}}$ & $x_{2}{ }^{\mathrm{b}}$ & $x_{1}{ }^{\mathrm{c}}$ \\
\hline 1.0000 & 0.9902 & 0.00977 \\
$\frac{{ }^{\mathrm{a}} x_{2}{ }^{(\mathrm{s})}: \text { initial mole fraction of component } 2 \text { in the solution. }}{{ }^{\mathrm{b}} x_{x_{2}}: \text { mole fraction of component } 2 \text { in the saturated solution. }}$ \\
${ }^{\mathrm{c}} x_{1}:$ mole fraction solubility of the solute.
\end{tabular}

\section{Auxiliary Information}

\section{Method/Apparatus/Procedure:}

Constant-temperature bath, calorimetric thermometer, and an ultraviolet/ visible spectrophotometer.

Excess solute and solvent were placed in amber glass bottles and allowed to equilibrate for several days at constant temperature. Attainment of equilibrium was verified by several repetitive measurements and by approaching equilibrium from supersaturation. Aliquots of saturated solutions were transferred through a coarse filter into tared volumetric flasks, weighed and diluted with methanol. Concentrations were determined by spectrophotometric measurements at $347 \mathrm{~nm}$.

Source and Purity of Chemicals:

(1) $98 \%$, Aldrich Chemical Company, Milwaukee, WI, USA, was recrystallized several times from methanol.

(2) $99+\%$, anhydrous, Aldrich Chemical Company, stored over molecular sieves before use.

Estimated Error:

Temperature: $\pm 0.1 \mathrm{~K}$.

$x_{1}: \pm 1.5 \%$ (relative error).

\begin{tabular}{ll}
\hline \hline Components: & Original Measurements: \\
(1) Phenanthrene; $\mathrm{C}_{14} \mathrm{H}_{10} ;[85-01-8]$ & ${ }^{61} \mathrm{C}$. E. Hernández, K. M. De Fina, \\
(2) 1-Butanol; $\mathrm{C}_{4} \mathrm{H}_{10} \mathrm{O} ;[71-36-3]$ & L. E. Roy, T. L. Sharp, and W. E. \\
& Acree, Jr., Can. J. Chem. 77, 1465 \\
& $(1999)$. \\
\hline Variables: & Prepared by: \\
$T / \mathrm{K}=298.15$ & W. E. Acree, Jr. \\
\hline
\end{tabular}

Experimental Values

\begin{tabular}{lcc}
\hline \hline$x_{2}{ }^{(\mathrm{s}) \mathrm{a}}$ & $x_{2}{ }^{\mathrm{b}}$ & $x_{1}{ }^{\mathrm{c}}$ \\
\hline 1.0000 & 0.9823 & 0.01771
\end{tabular}



$\mathrm{b}_{x_{2}}$ : mole fraction of component 2 in the saturated solution.

${ }^{c} x_{1}$ : mole fraction solubility of the solute.

\section{Auxiliary Information}

\section{Method/Apparatus/Procedure:}

Constant-temperature bath, calorimetric thermometer, and an ultraviolet/ visible spectrophotometer.

Excess solute and solvent were placed in amber glass bottles and allowed to equilibrate for several days at constant temperature. Attainment of equilibrium was verified by several repetitive measurements and by approaching equilibrium from supersaturation. Aliquots of saturated solutions were transferred through a coarse filter into tared volumetric flasks, weighed and diluted with methanol. Concentrations were determined by spectrophotometric measurements at $347 \mathrm{~nm}$. 
Source and Purity of Chemicals:

(1) $98 \%$, Aldrich Chemical Company, Milwaukee, WI, USA, was recrystallized several times from methanol.

(2) 99.8\%, HPLC Grade, Aldrich Chemical Company, stored over molecular sieves before use.

\section{Estimated Error:}

Temperature: $\pm 0.1 \mathrm{~K}$.

$x_{1}: \pm 1.5 \%$ (relative error).

\begin{tabular}{ll}
\hline \hline Components: & Original Measurements: \\
(1) Phenanthrene; $\mathrm{C}_{14} \mathrm{H}_{10} ;[85-01-8]$ & ${ }^{62} \mathrm{M}$. A. A. Fakhree, W. E. Acree, \\
(2) 1-Butanol; $\mathrm{C}_{4} \mathrm{H}_{10} \mathrm{O} ;[71-36-3]$ & Jr., and A. Jouyban, J. Chem. Eng. \\
& Data 55, 531 (2010). \\
\hline Variables: & Prepared by: \\
$T / \mathrm{K}=298.15$ & W. E. Acree, Jr. \\
\hline
\end{tabular}

Experimental Values

\begin{tabular}{lcc}
\hline \hline$c_{1}{ }^{\mathrm{a}}$ & $x_{2}{ }^{\mathrm{b}}$ & $x_{1}{ }^{\mathrm{c}}$ \\
\hline 0.194 & 0.9822 & 0.0178
\end{tabular}

${ }^{\mathrm{a}} c_{1}$ : molar solubility of the solute.

${ }^{b} x_{2}$ : mole fraction of component 2 in the saturated solution.

${ }^{\mathrm{c}} x_{1}$ : mole fraction solubility of the solute.

The mole fraction solubility of the solute was calculated by the compiler using the experimental density data given in the paper.

\section{Auxiliary Information}

\section{Method/Apparatus/Procedure:}

Incubator, shaker, and an ultraviolet/visible spectrophotometer.

Solubility was determined by equilibrating an excess amount of solid naphthalene with the organic solvent using a shaker placed in an incubator equipped with a temperature controlling system. After an equilibration period of at least $48 \mathrm{~h}$, the samples were withdrawn and filtered through hydrophobic Durapore filters $(0.45 \mu \mathrm{m})$. The filtered sample was diluted quantitatively with methanol. Concentrations were determined by spectrophotmetric analysis at $345 \mathrm{~nm}$.

\section{Source and Purity of Chemicals:}

(1) $99 \%$, Merck Chemical Company, Germany, was recrystallized several times from propanone.

(2) $99.5 \%$, Merck Chemical Company. No purification details were provided in the paper. Water content was checked by Karl Fischer titration and found to be $0.40 \%$ by mass.

\section{Estimated Error:}

Temperature: $\pm 0.2 \mathrm{~K}$

$x_{1}: \pm 4.2 \%$ (relative error).

\begin{tabular}{ll}
\hline \hline Components: & Original Measurements: \\
(1) Phenanthrene; $\mathrm{C}_{14} \mathrm{H}_{10} ;[85-01-8]$ & ${ }^{61}$ C. E. Hernández, K. M. De Fina, \\
(2) 2-Butanol; $\mathrm{C}_{4} \mathrm{H}_{10} \mathrm{O} ;[78-92-2]$ & L. E. Roy, T. L. Sharp, and W. E. \\
& Acree, Jr., Can. J. Chem. 77, 1465 \\
& $(1999)$. \\
\hline Variables: & Prepared by: \\
$T / \mathrm{K}=298.15$ & W. E. Acree, Jr. \\
\hline
\end{tabular}

Experimental Values

\begin{tabular}{lcc}
\hline \hline$x_{2}{ }^{(\mathrm{s}) \mathrm{a}}$ & $x_{2}{ }^{\mathrm{b}}$ & $x_{1}{ }^{\mathrm{c}}$ \\
\hline 1.0000 & 0.9882 & 0.01178 \\
\hline
\end{tabular}

${ }^{\mathrm{a}} x_{2}{ }^{(\mathrm{s})}$ : initial mole fraction of component 2 in the solution.

${ }^{\mathrm{b}} x_{2}$ : mole fraction of component 2 in the saturated solution.

${ }^{c} x_{1}$ : mole fraction solubility of the solute.

\section{Auxiliary Information}

\section{Method/Apparatus/Procedure:}

Constant-temperature bath, calorimetric thermometer, and an ultraviolet/ visible spectrophotometer.

Excess solute and solvent were placed in amber glass bottles and allowed to equilibrate for several days at constant temperature. Attainment of equilibrium was verified by several repetitive measurements and by approaching equilibrium from supersaturation. Aliquots of saturated solutions were transferred through a coarse filter into tared volumetric flasks, weighed and diluted with methanol. Concentrations were determined by spectrophotometric measurements at $347 \mathrm{~nm}$.

\section{Source and Purity of Chemicals:}

(1) $98 \%$, Aldrich Chemical Company, Milwaukee, WI, USA, was recrystallized several times from methanol.

(2) $99+\%$, Aldrich Chemical Company, stored over molecular sieves before use.

Estimated Error:

Temperature: $\pm 0.1 \mathrm{~K}$.

$x_{1}: \pm 1.5 \%$ (relative error)

\begin{tabular}{ll}
\hline \hline Components: & Original Measurements: \\
$\begin{array}{l}\text { (1) Phenanthrene; } \mathrm{C}_{14} \mathrm{H}_{10} ;[85-01-8] \\
\text { (2) 2-Methyl-1-propanol; } \mathrm{C}_{4} \mathrm{H}_{10} \mathrm{O} ;\end{array}$ & $\begin{array}{l}{ }^{61} \text { C. E. Hernández, K. M. De Fina, } \\
\text { [78-83-1] }\end{array}$ \\
& $\begin{array}{l}\text { Acree, Jr., Can. J. Chem. 77, 1465 } \\
(1999) .\end{array}$ \\
\hline Variables: & Prepared by: \\
$T / \mathrm{K}=298.15$ & W. E. Acree, Jr. \\
\hline
\end{tabular}

Experimental Values

\begin{tabular}{lcc}
\hline \hline$x_{2}{ }^{(\mathrm{s}) \mathrm{a}}$ & $x_{2}{ }^{\mathrm{b}}$ & $x_{1}{ }^{\mathrm{c}}$ \\
\hline 1.0000 & 0.9898 & 0.01020 \\
$\frac{{ }^{a} x_{2}{ }^{(\mathrm{s})}: \text { initial mole fraction of component } 2 \text { in the solution. }}{{ }^{\mathrm{b}} x_{2}: \text { mole fraction of component } 2 \text { in the saturated solution. }}$ \\
${ }^{{ }^{c} x_{1}: \text { mole fraction solubility of the solute. }}$
\end{tabular}




\section{Auxiliary Information}

\section{Method/Apparatus/Procedure:}

Constant-temperature bath, calorimetric thermometer, and an ultraviolet/ visible spectrophotometer.

Excess solute and solvent were placed in amber glass bottles and allowed to equilibrate for several days at constant temperature. Attainment of equilibrium was verified by several repetitive measurements and by approaching equilibrium from supersaturation. Aliquots of saturated solutions were transferred through a coarse filter into tared volumetric flasks, weighed and diluted with methanol. Concentrations were determined by spectrophotometric measurements at $347 \mathrm{~nm}$.

Source and Purity of Chemicals:

(1) $98 \%$, Aldrich Chemical Company, Milwaukee, WI, USA, was recrystallized several times from methanol.

(2) $99+\%$, Aldrich Chemical Company, stored over molecular sieves before use.

Estimated Error:

Temperature: $\pm 0.1 \mathrm{~K}$.

$x_{1}: \pm 1.5 \%$ (relative error).

\section{Components:}

(1) Phenanthrene; $\mathrm{C}_{14} \mathrm{H}_{10}$; [85-01-8]

(2) 1-Pentanol; $\mathrm{C}_{5} \mathrm{H}_{12} \mathrm{O} ;$ [71-41-0]

\begin{tabular}{ll}
\hline Variables: & Prepared by: \\
$T / \mathrm{K}=298.15$ & W. E. Acree, Jr. \\
\hline
\end{tabular}

Experimental Values

\begin{tabular}{lcc}
\hline \hline$x_{2}{ }^{(\mathrm{s}) \mathrm{a}}$ & $x_{2}^{\mathrm{b}}$ & $x_{1}{ }^{\mathrm{c}}$ \\
\hline 1.0000 & 0.9751 & 0.02491 \\
$\frac{{ }^{\mathrm{a}} x_{2}{ }^{(\mathrm{s})}: \text { initial mole fraction of component } 2 \text { in the solution. }}{{ }^{\mathrm{b}} x_{2}: \text { mole fraction of component } 2 \text { in the saturated solution. }}$ \\
${ }^{c} x_{1}:$ mole fraction solubility of the solute.
\end{tabular}

\section{Auxiliary Information}

\section{Method/Apparatus/Procedure:}

Constant-temperature bath, calorimetric thermometer, and an ultraviolet/ visible spectrophotometer.

Excess solute and solvent were placed in amber glass bottles and allowed to equilibrate for several days at constant temperature. Attainment of equilibrium was verified by several repetitive measurements and by approaching equilibrium from supersaturation. Aliquots of saturated solutions were transferred through a coarse filter into tared volumetric flasks, weighed and diluted with methanol. Concentrations were determined by

spectrophotometric measurements at $347 \mathrm{~nm}$.

\section{Source and Purity of Chemicals:}

(1) $98 \%$, Aldrich Chemical Company, Milwaukee, WI, USA, was recrystallized several times from methanol.

(2) $99+\%$, Aldrich Chemical Company, stored over molecular sieves before use.

\section{Estimated Error:}

Temperature: $\pm 0.1 \mathrm{~K}$.

$x_{1}: \pm 1.5 \%$ (relative error).

\begin{tabular}{ll}
\hline \hline Components: & Original Measurements: \\
(1) Phenanthrene; $\mathrm{C}_{14} \mathrm{H}_{10} ;[85-01-8]$ & ${ }^{61}$ C. E. Hernández, K. M. De Fina, \\
(2) 2-Pentanol; $\mathrm{C}_{5} \mathrm{H}_{12} \mathrm{O} ;[6032-29-7]$ & L. E. Roy, T. L. Sharp, and W. E. \\
& Acree, Jr., Can. J. Chem. 77, 1465 \\
& $(1999)$. \\
\hline Variables: & Prepared by: \\
$T / \mathrm{K}=298.15$ & W. E. Acree, Jr. \\
\hline
\end{tabular}

Experimental Values

\begin{tabular}{lcc}
\hline \hline$x_{2}{ }^{(\mathrm{s}) \mathrm{a}}$ & $x_{2}{ }^{\mathrm{b}}$ & $x_{1}{ }^{\mathrm{c}}$ \\
\hline 1.0000 & 0.9824 & 0.01764 \\
\hline
\end{tabular}

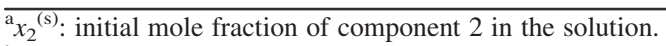

${ }^{\mathrm{b}} x_{2}$ : mole fraction of component 2 in the saturated solution.

${ }^{c} x_{1}$ : mole fraction solubility of the solute.

\section{Auxiliary Information}

\section{Method/Apparatus/Procedure:}

Constant-temperature bath, calorimetric thermometer, and an ultraviolet/ visible spectrophotometer.

Excess solute and solvent were placed in amber glass bottles and allowed to equilibrate for several days at constant temperature. Attainment of equilibrium was verified by several repetitive measurements and by approaching equilibrium from supersaturation. Aliquots of saturated solutions were transferred through a coarse filter into tared volumetric flasks, weighed and diluted with methanol. Concentrations were determined by spectrophotometric measurements at $347 \mathrm{~nm}$.

\section{Source and Purity of Chemicals:}

(1) $98 \%$, Aldrich Chemical Company, Milwaukee, WI, USA, was recrystallized several times from methanol.

(2) $99+\%$, Acros Organics, USA, stored over molecular sieves before use.

\section{Estimated Error:}

Temperature: $\pm 0.1 \mathrm{~K}$.

$x_{1}: \pm 1.5 \%$ (relative error).

\begin{tabular}{|c|c|}
\hline $\begin{array}{l}\text { Components: } \\
\text { (1) Phenanthrene; } \mathrm{C}_{14} \mathrm{H}_{10} ;[85-01-8] \\
\text { (2) 3-Methyl-1-butanol; } \mathrm{C}_{5} \mathrm{H}_{12} \mathrm{O} \text {; } \\
\text { [123-51-3] }\end{array}$ & $\begin{array}{l}\text { Original Measurements: } \\
{ }^{61} \text { C. E. Hernández, K. M. De Fina, } \\
\text { L. E. Roy, T. L. Sharp, and W. E. } \\
\text { Acree, Jr., Can. J. Chem. 77, } 1465 \\
\text { (1999). }\end{array}$ \\
\hline $\begin{array}{l}\text { Variables: } \\
T / \mathrm{K}=298.15\end{array}$ & $\begin{array}{l}\text { Prepared by: } \\
\text { W. E. Acree, Jr. }\end{array}$ \\
\hline
\end{tabular}

Experimental Values

\begin{tabular}{lcc}
\hline \hline$x_{2}{ }^{(\mathrm{s}) \mathrm{a}}$ & $x_{2}{ }^{\mathrm{b}}$ & $x_{1}{ }^{\mathrm{c}}$ \\
\hline 1.0000 & 0.9839 & 0.01606 \\
\hline
\end{tabular}

${ }^{\mathrm{a}} x_{2}{ }^{(\mathrm{s})}$ : initial mole fraction of component 2 in the solution.

${ }^{\mathrm{b}} x_{2}$ : mole fraction of component 2 in the saturated solution.

${ }^{c} x_{1}$ : mole fraction solubility of the solute. 


\section{Auxiliary Information}

\section{Method/Apparatus/Procedure:}

Constant-temperature bath, calorimetric thermometer, and an ultraviolet/ visible spectrophotometer.

Excess solute and solvent were placed in amber glass bottles and allowed to equilibrate for several days at constant temperature. Attainment of equilibrium was verified by several repetitive measurements and by approaching equilibrium from supersaturation. Aliquots of saturated solutions were transferred through a coarse filter into tared volumetric flasks, weighed and diluted with methanol. Concentrations were determined by spectrophotometric measurements at $347 \mathrm{~nm}$.

Source and Purity of Chemicals:

(1) $98 \%$, Aldrich Chemical Company, Milwaukee, WI, USA, was recrystallized several times from methanol.

(2) $99+\%$, anhydrous, Aldrich Chemical Company, stored over molecular sieves before use.

Estimated Error:

Temperature: $\pm 0.1 \mathrm{~K}$.

$x_{1}: \pm 1.5 \%$ (relative error)

\begin{tabular}{ll}
\hline \hline Components: & Original Measurements: \\
$\begin{array}{l}\text { (1) Phenanthrene; } \mathrm{C}_{14} \mathrm{H}_{10} ;[85-01-8] \\
\text { (2) 2-Methyl-2-butanol; } \mathrm{C}_{5} \mathrm{H}_{12} \mathrm{O} ;\end{array}$ & $\begin{array}{l}{ }^{1} \mathrm{C} . \text { E. Hernández, K. M. De Fina, } \\
\text { [75-85-4] }\end{array}$ \\
& L. E. Roy, T. L. Sharp, and W. E. \\
& Acree, Jr., Can. J. Chem. 77, 1465 \\
& (1999). \\
\hline Variables: & Prepared by: \\
$T / \mathrm{K}=298.15$ & W. E. Acree, Jr. \\
\hline
\end{tabular}

Experimental Values

\begin{tabular}{lcc}
\hline \hline$x_{2}{ }^{(\mathrm{s}) \mathrm{a}}$ & $x_{2}{ }^{\mathrm{b}}$ & $x_{1}{ }^{\mathrm{c}}$ \\
\hline 1.0000 & 0.9807 & 0.01926 \\
\hline${ }^{\mathrm{a}}{ }_{x_{2}}{ }^{(\mathrm{s})}:$ initial mole fraction of component 2 in the solution. \\
${ }^{\mathrm{b}} x_{2}:$ mole fraction of component 2 in the saturated solution. \\
${ }^{{ }^{c} x_{1}: \text { mole fraction solubility of the solute. }}$
\end{tabular}

\section{Auxiliary Information}

\section{Method/Apparatus/Procedure:}

Constant-temperature bath, calorimetric thermometer, and an ultraviolet/ visible spectrophotometer.

Excess solute and solvent were placed in amber glass bottles and allowed to equilibrate for several days at constant temperature. Attainment of equilibrium was verified by several repetitive measurements and by approaching equilibrium from supersaturation. Aliquots of saturated solutions were transferred through a coarse filter into tared volumetric flasks, weighed and diluted with methanol. Concentrations were determined by spectrophotometric measurements at $347 \mathrm{~nm}$.

Source and Purity of Chemicals:

(1) $98 \%$, Aldrich Chemical Company, Milwaukee, WI, USA, was recrystallized several times from methanol.

(2) $99+\%$, Acros Organics, USA, stored over molecular sieves before use.

Estimated Error:

Temperature: $\pm 0.1 \mathrm{~K}$.

$x_{1}: \pm 1.5 \%$ (relative error).

\begin{tabular}{ll}
\hline \hline Components: & Original Measurements: \\
(1) Phenanthrene; $\mathrm{C}_{14} \mathrm{H}_{10} ;[85-01-8]$ & ${ }^{61}$ C. E. Hernández, K. M. De Fina, \\
(2) 1-Hexanol; $\mathrm{C}_{6} \mathrm{H}_{14} \mathrm{O} ;[111-27-3]$ & L. E. Roy, T. L. Sharp, and W. E. \\
& Acree, Jr., Can. J. Chem. 77, 1465 \\
& $(1999)$. \\
\hline Variables: & Prepared by: \\
$T / \mathrm{K}=298.15$ & W. E. Acree, Jr. \\
\hline
\end{tabular}

Experimental Values

\begin{tabular}{lcc}
\hline \hline$x_{2}{ }^{(\mathrm{s}) \mathrm{a}}$ & $x_{2}{ }^{\mathrm{b}}$ & $x_{1}{ }^{\mathrm{c}}$ \\
\hline 1.0000 & 0.9697 & 0.03028 \\
\hline
\end{tabular}

${ }^{\mathrm{a}} x_{2}{ }^{(\mathrm{s})}$ : initial mole fraction of component 2 in the solution.

${ }^{b} x_{2}$ : mole fraction of component 2 in the saturated solution.

${ }^{c} x_{1}$ : mole fraction solubility of the solute.

\section{Auxiliary Information}

\section{Method/Apparatus/Procedure:}

Constant-temperature bath, calorimetric thermometer, and an ultraviolet/ visible spectrophotometer.

Excess solute and solvent were placed in amber glass bottles and allowed to equilibrate for several days at constant temperature. Attainment of equilibrium was verified by several repetitive measurements and by approaching equilibrium from supersaturation. Aliquots of saturated solutions were transferred through a coarse filter into tared volumetric flasks, weighed and diluted with methanol. Concentrations were determined by spectrophotometric measurements at $347 \mathrm{~nm}$.

\section{Source and Purity of Chemicals:}

(1) $98 \%$, Aldrich Chemical Company, Milwaukee, WI, USA, was recrystallized several times from methanol.

(2) $99+\%$, Alfa Aesar, USA, stored over molecular sieves before use.

\section{Estimated Error:}

Temperature: $\pm 0.1 \mathrm{~K}$.

$x_{1}: \pm 1.5 \%$ (relative error).

\begin{tabular}{|c|c|}
\hline $\begin{array}{l}\text { Components: } \\
\text { (1) Phenanthrene; } \mathrm{C}_{14} \mathrm{H}_{10} ;[85-01-8] \\
\text { (2) 2-Methyl-1-pentanol; } \mathrm{C}_{6} \mathrm{H}_{14} \mathrm{O} \text {; } \\
\text { [105-30-6] }\end{array}$ & $\begin{array}{l}\text { Original Measurements: } \\
{ }^{61} \text { C. E. Hernández, K. M. De Fina, } \\
\text { L. E. Roy, T. L. Sharp, and W. E. } \\
\text { Acree, Jr., Can. J. Chem. 77, } 1465 \\
\text { (1999). }\end{array}$ \\
\hline $\begin{array}{l}\text { Variables: } \\
T / \mathrm{K}=298.15\end{array}$ & $\begin{array}{l}\text { Prepared by: } \\
\text { W. E. Acree, Jr. }\end{array}$ \\
\hline
\end{tabular}

Experimental Values

\begin{tabular}{lcc}
\hline \hline$x_{2}{ }^{(\mathrm{s}) \mathrm{a}}$ & $x_{2}{ }^{\mathrm{b}}$ & $x_{1}{ }^{\mathrm{c}}$ \\
\hline 1.0000 & 0.9820 & 0.01801 \\
$\frac{{ }^{a} x_{2}{ }^{(\mathrm{s})}: \text { initial mole fraction of component } 2 \text { in the solution. }}{{ }^{\mathrm{b}} x_{2}: \text { mole fraction of component } 2 \text { in the saturated solution. }}$ \\
${ }^{\mathrm{c}} x_{1}:$ mole fraction solubility of the solute.
\end{tabular}




\section{Auxiliary Information}

\section{Method/Apparatus/Procedure:}

Constant-temperature bath, calorimetric thermometer, and an ultraviolet/ visible spectrophotometer.

Excess solute and solvent were placed in amber glass bottles and allowed to equilibrate for several days at constant temperature. Attainment of equilibrium was verified by several repetitive measurements and by approaching equilibrium from supersaturation. Aliquots of saturated solutions were transferred through a coarse filter into tared volumetric flasks, weighed and diluted with methanol. Concentrations were determined by spectrophotometric measurements at $347 \mathrm{~nm}$.

Source and Purity of Chemicals:

(1) $98 \%$, Aldrich Chemical Company, Milwaukee, WI, USA, was recrystallized several times from methanol.

(2) $99 \%$, Aldrich Chemical Company, stored over molecular sieves before use.

\section{Estimated Error:}

Temperature: $\pm 0.1 \mathrm{~K}$.

$x_{1}: \pm 1.5 \%$ (relative error)

\begin{tabular}{ll}
\hline \hline Components: & Original Measurements: \\
(1) Phenanthrene; $\mathrm{C}_{14} \mathrm{H}_{10} ;[85-01-8]$ & ${ }^{61} \mathrm{C}$. E. Hernández, K. M. De Fina, \\
(2) 1-Heptanol; $\mathrm{C}_{7} \mathrm{H}_{16} \mathrm{O} ;[111-70-6]$ & L. E. Roy, T. L. Sharp, and W. E. \\
& Acree, Jr., Can. J. Chem. 77, 1465 \\
& $(1999)$. \\
\hline Variables: & Prepared by: \\
$T / \mathrm{K}=298.15$ & W. E. Acree, Jr. \\
\hline
\end{tabular}

Experimental Values

\begin{tabular}{lcc}
\hline \hline$x_{2}{ }^{(\mathrm{s}) \mathrm{a}}$ & $x_{2}{ }^{\mathrm{b}}$ & $x_{1}{ }^{\mathrm{c}}$ \\
\hline 1.0000 & 0.9606 & 0.03937 \\
\hline
\end{tabular}

${ }^{\mathrm{a}} x_{2}{ }^{(\mathrm{s})}$ : initial mole fraction of component 2 in the solution.

${ }^{b} x_{2}$ : mole fraction of component 2 in the saturated solution.

${ }^{c} x_{1}$ : mole fraction solubility of the solute.

\section{Auxiliary Information}

\section{Components: \\ (1) Phenanthrene; $\mathrm{C}_{14} \mathrm{H}_{10} ;$ [85-01-8] \\ (2) 4-Methyl-2-pentanol; $\mathrm{C}_{6} \mathrm{H}_{14} \mathrm{O}$;}

[108-11-2]

\section{Variables:}

$T / \mathrm{K}=298.15$

\section{Original Measurements:}

${ }^{61}$ C. E. Hernández, K. M. De Fina, L. E. Roy, T. L. Sharp, and W. E. Acree, Jr., Can. J. Chem. 77, 1465 (1999).

\section{Prepared by:}

W. E. Acree, Jr.

Experimental Values

\begin{tabular}{lcc}
\hline \hline$x_{2}{ }^{(\mathrm{s}) \mathrm{a}}$ & $x_{2}{ }^{\mathrm{b}}$ & $x_{1}{ }^{\mathrm{c}}$ \\
\hline 1.0000 & 0.9825 & 0.01754 \\
\hline
\end{tabular}

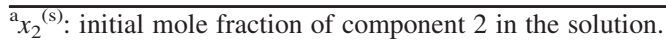

${ }^{b} x_{2}$ : mole fraction of component 2 in the saturated solution.

${ }^{c} x_{1}$ : mole fraction solubility of the solute.

\section{Auxiliary Information}

\footnotetext{
Method/Apparatus/Procedure:

Constant-temperature bath, calorimetric thermometer, and an ultraviolet/ visible spectrophotometer.

Excess solute and solvent were placed in amber glass bottles and allowed to equilibrate for several days at constant temperature. Attainment of equilibrium was verified by several repetitive measurements and by approaching equilibrium from supersaturation. Aliquots of saturated solutions were transferred through a coarse filter into tared volumetric flasks, weighed and diluted with methanol. Concentrations were determined by

spectrophotometric measurements at $347 \mathrm{~nm}$.
}

\section{Source and Purity of Chemicals:}

(1) $98 \%$, Aldrich Chemical Company, Milwaukee, WI, USA, was recrystallized several times from methanol.

(2) $99 \%$, Acros Organics, USA, stored over molecular sieves before use.

\section{Estimated Error:}

Temperature: $\pm 0.1 \mathrm{~K}$

$x_{1}: \pm 1.5 \%$ (relative error).

\begin{abstract}
Method/Apparatus/Procedure:
Constant-temperature bath, calorimetric thermometer, and an ultraviolet/ visible spectrophotometer.

Excess solute and solvent were placed in amber glass bottles and allowed to equilibrate for several days at constant temperature. Attainment of equilibrium was verified by several repetitive measurements and by approaching equilibrium from supersaturation. Aliquots of saturated solutions were transferred through a coarse filter into tared volumetric flasks, weighed and diluted with methanol. Concentrations were determined by spectrophotometric measurements at $347 \mathrm{~nm}$.
\end{abstract}

\section{Source and Purity of Chemicals:}

(1) $98 \%$, Aldrich Chemical Company, Milwaukee, WI, USA, was recrystallized several times from methanol.

(2) $99+\%$, Alfa Aesar, USA, stored over molecular sieves before use.

\section{Estimated Error:}

Temperature: $\pm 0.1 \mathrm{~K}$.

$x_{1}: \pm 1.5 \%$ (relative error).

\begin{tabular}{|c|c|}
\hline $\begin{array}{l}\text { Components: } \\
\text { (1) Phenanthrene; } \mathrm{C}_{14} \mathrm{H}_{10} ;[85-01-8] \\
\text { (2) 1-Octanol; } \mathrm{C}_{8} \mathrm{H}_{18} \mathrm{O} ;[111-87-5]\end{array}$ & $\begin{array}{l}\text { Original Measurements: } \\
{ }^{61} \text { C. E. Hernández, K. M. De Fina, } \\
\text { L. E. Roy, T. L. Sharp, and W. E. } \\
\text { Acree, Jr., Can. J. Chem. 77, } 1465 \\
\text { (1999). }\end{array}$ \\
\hline $\begin{array}{l}\text { Variables: } \\
T / \mathrm{K}=298.15\end{array}$ & $\begin{array}{l}\text { Prepared by: } \\
\text { W. E. Acree, Jr. }\end{array}$ \\
\hline
\end{tabular}

Experimental Values

\begin{tabular}{|c|c|c|}
\hline$x_{2}{ }^{(\mathrm{s}) \mathrm{a}}$ & $x_{2}{ }^{\mathrm{b}}$ & $x_{1}{ }^{\mathrm{c}}$ \\
\hline 1.0000 & 0.9458 & 0.05418 \\
\hline
\end{tabular}




\section{Auxiliary Information}

\section{Method/Apparatus/Procedure:}

Constant-temperature bath, calorimetric thermometer, and an ultraviolet/ visible spectrophotometer.

Excess solute and solvent were placed in amber glass bottles and allowed to equilibrate for several days at constant temperature. Attainment of equilibrium was verified by several repetitive measurements and by approaching equilibrium from supersaturation. Aliquots of saturated solutions were transferred through a coarse filter into tared volumetric flasks, weighed and diluted with methanol. Concentrations were determined by spectrophotometric measurements at $347 \mathrm{~nm}$.

Source and Purity of Chemicals:

(1) $98 \%$, Aldrich Chemical Company, Milwaukee, WI, USA, was recrystallized several times from methanol.

(2) $99+\%$, anhydrous, Aldrich Chemical Company, stored over molecular sieves before use.

Estimated Error:

Temperature: $\pm 0.1 \mathrm{~K}$.

$x_{1}: \pm 1.5 \%$ (relative error)

\begin{tabular}{|c|c|}
\hline $\begin{array}{l}\text { Components: } \\
\text { (1) Phenanthrene; } \mathrm{C}_{14} \mathrm{H}_{10} ;[85-01-8] \\
\text { (2) 2-Ethyl-1-hexanol; } \mathrm{C}_{8} \mathrm{H}_{18} \mathrm{O} \text {; } \\
\text { [104-76-7] }\end{array}$ & $\begin{array}{l}\text { Original Measurements: } \\
{ }^{61} \text { C. E. Hernández, K. M. De Fina, } \\
\text { L. E. Roy, T. L. Sharp, and W. E. } \\
\text { Acree, Jr., Can. J. Chem. 77, } 1465 \\
(1999) \text {. }\end{array}$ \\
\hline $\begin{array}{l}\text { Variables: } \\
T / \mathrm{K}=298.15\end{array}$ & $\begin{array}{l}\text { Prepared by: } \\
\text { W. E. Acree, Jr. }\end{array}$ \\
\hline
\end{tabular}

Experimental Values

\begin{tabular}{lcc}
\hline \hline$x_{2}{ }^{(\mathrm{s}) \mathrm{a}}$ & $x_{2}{ }^{\mathrm{b}}$ & $x_{1}{ }^{\mathrm{c}}$ \\
\hline 1.0000 & 0.9712 & 0.02876
\end{tabular}

${ }^{\mathrm{a}} x_{2}{ }^{(\mathrm{s})}$ : initial mole fraction of component 2 in the solution.

${ }^{b_{x_{2}}}$ : mole fraction of component 2 in the saturated solution.

${ }^{c} x_{1}$ : mole fraction solubility of the solute.

\section{Auxiliary Information}

\section{Method/Apparatus/Procedure:}

Constant-temperature bath, calorimetric thermometer, and an ultraviolet/ visible spectrophotometer.

Excess solute and solvent were placed in amber glass bottles and allowed to equilibrate for several days at constant temperature. Attainment of equilibrium was verified by several repetitive measurements and by approaching equilibrium from supersaturation. Aliquots of saturated solutions were transferred through a coarse filter into tared volumetric flasks, weighed and diluted with methanol. Concentrations were determined by spectrophotometric measurements at $347 \mathrm{~nm}$.

Source and Purity of Chemicals:

(1) $98 \%$, Aldrich Chemical Company, Milwaukee, WI, USA, was recrystallized several times from methanol.

(2) $99+\%$, Aldrich Chemical Company, stored over molecular sieves before use.

Estimated Error:

Temperature: $\pm 0.1 \mathrm{~K}$.

$x_{1}: \pm 1.5 \%$ (relative error).

\begin{tabular}{ll}
\hline \hline Components: & Original Measurements: \\
(1) Phenanthrene; $\mathrm{C}_{14} \mathrm{H}_{10} ;[85-01-8]$ & ${ }^{61}$ C. E. Hernández, K. M. De Fina, \\
(2) Cyclopentanol; $\mathrm{C}_{5} \mathrm{H}_{10} \mathrm{O} ;[96-41-3]$ & L. E. Roy, T. L. Sharp, and W. E. \\
& Acree, Jr., Can. J. Chem. 77, 1465 \\
& $(1999)$. \\
\hline Variables: & Prepared by: \\
$T / \mathrm{K}=298.15$ & W. E. Acree, Jr. \\
\hline
\end{tabular}

Experimental Values

\begin{tabular}{lcc}
\hline \hline$x_{2}{ }^{(\mathrm{s}) \mathrm{a}}$ & $x_{2}{ }^{\mathrm{b}}$ & $x_{1}{ }^{\mathrm{c}}$ \\
\hline 1.0000 & 0.9693 & 0.03070 \\
\hline
\end{tabular}

${ }^{\mathrm{a}} x_{2}{ }^{(\mathrm{s})}$ : initial mole fraction of component 2 in the solution.

${ }^{b} x_{2}$ : mole fraction of component 2 in the saturated solution.

${ }^{c} x_{1}$ : mole fraction solubility of the solute.

\section{Auxiliary Information}

\section{Method/Apparatus/Procedure:}

Constant-temperature bath, calorimetric thermometer, and an ultraviolet/ visible spectrophotometer.

Excess solute and solvent were placed in amber glass bottles and allowed to equilibrate for several days at constant temperature. Attainment of equilibrium was verified by several repetitive measurements and by approaching equilibrium from supersaturation. Aliquots of saturated solutions were transferred through a coarse filter into tared volumetric flasks, weighed and diluted with methanol. Concentrations were determined by spectrophotometric measurements at $347 \mathrm{~nm}$.

\section{Source and Purity of Chemicals:}

(1) $98 \%$, Aldrich Chemical Company, Milwaukee, WI, USA, was recrystallized several times from methanol.

(2) $99 \%$, Aldrich Chemical Company, stored over molecular sieves before use.

\section{Estimated Error:}

Temperature: $\pm 0.1 \mathrm{~K}$.

$x_{1}: \pm 1.5 \%$ (relative error).

\begin{tabular}{|c|c|}
\hline $\begin{array}{l}\text { Components: } \\
\text { (1) Phenanthrene; } \mathrm{C}_{14} \mathrm{H}_{10} ;[85-01-8] \\
\text { (2) } 1,2 \text {-Ethanediol; } \mathrm{C}_{2} \mathrm{H}_{6} \mathrm{O}_{2} ;[107-21-1]\end{array}$ & $\begin{array}{l}\text { Original Measurements: } \\
{ }^{6}{ }^{1} \text { C. E. Hernández, K. M. De Fina, } \\
\text { L. E. Roy, T. L. Sharp, and W. E. } \\
\text { Acree, Jr., Can. J. Chem. 77, } 1465 \\
\text { (1999). }\end{array}$ \\
\hline $\begin{array}{l}\text { Variables: } \\
T / \mathrm{K}=298.15\end{array}$ & $\begin{array}{l}\text { Prepared by: } \\
\text { W. E. Acree, Jr. }\end{array}$ \\
\hline
\end{tabular}

Experimental Values

\begin{tabular}{|c|c|c|}
\hline$x_{2}{ }^{(\mathrm{s}) \mathrm{a}}$ & $x_{2}{ }^{\mathrm{b}}$ & $x_{1}{ }^{\mathrm{c}}$ \\
\hline 1.0000 & 0.9989 & 0.001134 \\
\hline
\end{tabular}




\section{Auxiliary Information}

Method/Apparatus/Procedure:

Constant-temperature bath, calorimetric thermometer, and an ultraviolet/ visible spectrophotometer.

Excess solute and solvent were placed in amber glass bottles and allowed to equilibrate for several days at constant temperature. Attainment of equilibrium was verified by several repetitive measurements and by approaching equilibrium from supersaturation. Aliquots of saturated solutions were transferred through a coarse filter into tared volumetric flasks, weighed and diluted with methanol. Concentrations were determined by spectrophotometric measurements at $347 \mathrm{~nm}$.

Source and Purity of Chemicals:

(1) $98 \%$, Aldrich Chemical Company, Milwaukee, WI, USA, was recrystallized several times from methanol.

(2) $99.8 \%$, anhydrous, Aldrich Chemical Company, stored over molecular sieves before use.

Estimated Error:

Temperature: $\pm 0.1 \mathrm{~K}$.

$x_{1}: \pm 1.5 \%$ (relative error).

\subsection{Phenanthrene solubility data in ketones}

\begin{tabular}{ll}
\hline \hline Components: & Original Measurements: \\
(1) Phenanthrene; $\mathrm{C}_{14} \mathrm{H}_{10} ;[85-01-8]$ & ${ }^{61}$ C. E. Hernández, K. M. De Fina, \\
(2) 2-Butanone; $\mathrm{C}_{4} \mathrm{H}_{8} \mathrm{O} ;[78-93-3]$ & L. E. Roy, T. L. Sharp, and W. E. \\
& Acree, Jr., Can. J. Chem. 77, 1465 \\
& (1999). \\
\hline Variables: & Prepared by: \\
$T / \mathrm{K}=298.15$ & W. E. Acree, Jr. \\
\hline
\end{tabular}

Experimental Values

\begin{tabular}{lcc}
\hline \hline$x_{2}{ }^{(\mathrm{s}) \mathrm{a}}$ & $x_{2}{ }^{\mathrm{b}}$ & $x_{1}{ }^{\mathrm{c}}$ \\
\hline 1.0000 & 0.7910 & 0.2090 \\
${ }^{\mathrm{a}} x_{2}{ }^{(\mathrm{s})}:$ initial mole fraction of component 2 in the solution. \\
${ }^{\mathrm{b}} x_{2}:$ mole fraction of component 2 in the saturated solution. \\
${ }^{\mathrm{c}}{ }_{x_{1}: \text { mole fraction solubility of the solute. }}$
\end{tabular}

\section{Auxiliary Information}

\section{Components:}

(1) Phenanthrene; $\mathrm{C}_{14} \mathrm{H}_{10} ;$ [85-01-8]

(2) 2,2,2-Trifluoroethanol; $\mathrm{C}_{2} \mathrm{H}_{3} \mathrm{~F}_{3} \mathrm{O}$;

[75-89-8]

\section{Variables:}

$T / \mathrm{K}=298.15$
Original Measurements:

${ }^{61}$ C. E. Hernández, K. M. De Fina, L. E. Roy, T. L. Sharp, and W. E. Acree, Jr., Can. J. Chem. 77, 1465 (1999).

\section{Prepared by:}

W. E. Acree, Jr

\section{Experimental Values}

\begin{tabular}{|c|c|c|}
\hline$x_{2}^{(\mathrm{s}) \mathrm{a}}$ & $x_{2}{ }^{\mathrm{b}}$ & $x_{1}{ }^{\mathrm{c}}$ \\
\hline 1.0000 & 0.9982 & 0.001826 \\
\hline
\end{tabular}

\section{Auxiliary Information}

\footnotetext{
Method/Apparatus/Procedure:

Constant-temperature bath, calorimetric thermometer, and an ultraviolet/ visible spectrophotometer.

Excess solute and solvent were placed in amber glass bottles and allowed to equilibrate for several days at constant temperature. Attainment of equilibrium was verified by several repetitive measurements and by approaching equilibrium from supersaturation. Aliquots of saturated solutions were transferred through a coarse filter into tared volumetric flasks, weighed and diluted with methanol. Concentrations were determined by spectrophotometric measurements at $347 \mathrm{~nm}$.
}

Source and Purity of Chemicals:

(1) $98 \%$, Aldrich Chemical Company, Milwaukee, WI, USA, was recrystallized several times from methanol.

(2) $99+\%$, Aldrich Chemical Company, stored over molecular sieves before use.

Estimated Error:

Temperature: $\pm 0.1 \mathrm{~K}$.

$x_{1}: \pm 1.5 \%$ (relative error).

\begin{abstract}
Method/Apparatus/Procedure:
Constant-temperature bath, calorimetric thermometer, and an ultraviolet/ visible spectrophotometer.

Excess solute and solvent were placed in amber glass bottles and allowed to equilibrate for several days at constant temperature. Attainment of equilibrium was verified by several repetitive measurements and by approaching equilibrium from supersaturation. Aliquots of saturated solutions were transferred through a coarse filter into tared volumetric flasks, weighed and diluted with methanol. Concentrations were determined by spectrophotometric measurements at $347 \mathrm{~nm}$.
\end{abstract}

\section{Source and Purity of Chemicals:}

(1) $98 \%$, Aldrich Chemical Company, Milwaukee, WI, USA, was recrystallized several times from methanol.

(2) $99.5 \%$, HPLC Grade, Aldrich Chemical Company, stored over molecular sieves before use.

Estimated Error:

Temperature: $\pm 0.1 \mathrm{~K}$.

$x_{1}: \pm 1.5 \%$ (relative error).

\begin{tabular}{|c|c|}
\hline $\begin{array}{l}\text { Components: } \\
\text { (1) Phenanthrene; } \mathrm{C}_{14} \mathrm{H}_{10} ;[85-01-8] \\
\text { (2) Cyclohexanone; } \mathrm{C}_{6} \mathrm{H}_{10} \mathrm{O} ;[108-94-1]\end{array}$ & $\begin{array}{l}\text { Original Measurements: } \\
{ }^{61} \text { C. E. Hernández, K. M. De Fina, } \\
\text { L. E. Roy, T. L. Sharp, and W. E. } \\
\text { Acree, Jr., Can. J. Chem. 77, } 1465 \\
\text { (1999). }\end{array}$ \\
\hline $\begin{array}{l}\text { Variables: } \\
T / \mathrm{K}=298.15\end{array}$ & $\begin{array}{l}\text { Prepared by: } \\
\text { W. E. Acree, Jr. }\end{array}$ \\
\hline
\end{tabular}

Experimental Values

\begin{tabular}{lcc}
\hline \hline$x_{2}{ }^{(\mathrm{s}) \mathrm{a}}$ & $x_{2}{ }^{\mathrm{b}}$ & $x_{1}{ }^{\mathrm{c}}$ \\
\hline 1.0000 & 0.7284 & 0.2716 \\
$\frac{{ }^{a} x_{2}{ }^{(\mathrm{s})}: \text { initial mole fraction of component } 2 \text { in the solution. }}{{ }^{\mathrm{b}} x_{2}: \text { mole fraction of component } 2 \text { in the saturated solution. }}$ \\
${ }^{\mathrm{c}} x_{1}:$ mole fraction solubility of the solute.
\end{tabular}




\section{Auxiliary Information}

\section{Method/Apparatus/Procedure:}

Constant-temperature bath, calorimetric thermometer, and an ultraviolet/ visible spectrophotometer.

Excess solute and solvent were placed in amber glass bottles and allowed to equilibrate for several days at constant temperature. Attainment of equilibrium was verified by several repetitive measurements and by approaching equilibrium from supersaturation. Aliquots of saturated solutions were transferred through a coarse filter into tared volumetric flasks, weighed and diluted with methanol. Concentrations were determined by spectrophotometric measurements at $347 \mathrm{~nm}$.

Source and Purity of Chemicals:

(1) $98 \%$, Aldrich Chemical Company, Milwaukee, WI, USA, was recrystallized several times from methanol.

(2) $99.8 \%$, Aldrich Chemical Company, stored over molecular sieves before use.

Estimated Error:

Temperature: $\pm 0.1 \mathrm{~K}$.

$x_{1}: \pm 1.5 \%$ (relative error).

Estimated Error:

Temperature: $\pm 0.1 \mathrm{~K}$.

$x_{1}: \pm 1.5 \%$ (relative error)

\begin{tabular}{|c|c|}
\hline $\begin{array}{l}\text { Components: } \\
\text { (1) Phenanthrene; } \mathrm{C}_{14} \mathrm{H}_{10} ;[85-01-8] \\
\text { (2) Propanenitrile; } \mathrm{C}_{3} \mathrm{H}_{5} \mathrm{~N} ;[107-12-0]\end{array}$ & $\begin{array}{l}\text { Original Measurements: } \\
\text { W. E. Acree, Jr., unpublished data. }\end{array}$ \\
\hline $\begin{array}{l}\text { Variables: } \\
T / \mathrm{K}=298.15\end{array}$ & $\begin{array}{l}\text { Prepared by: } \\
\text { W. E. Acree, Jr. }\end{array}$ \\
\hline
\end{tabular}

Experimental Values

\begin{tabular}{lcc}
\hline \hline$x_{2}{ }^{(\mathrm{s}) \mathrm{a}}$ & $x_{2}{ }^{\mathrm{b}}$ & $x_{1}{ }^{\mathrm{c}}$ \\
\hline 1.0000 & 0.8794 & 0.1206 \\
\hline${ }^{{ }^{a} x_{2}{ }^{(\mathrm{s})}: \text { initial mole fraction of component } 2 \text { in the solution. }}$ \\
${ }^{\mathrm{b}} x_{2}:$ mole fraction of component 2 in the saturated solution. \\
${ }^{{ }^{c} x_{1}: \text { mole fraction solubility of the solute. }}$
\end{tabular}

\section{Auxiliary Information}

\subsection{Phenanthrene solubility data in miscellaneous organic solvents}

\section{Components:}

(1) Phenanthrene; $\mathrm{C}_{14} \mathrm{H}_{10} ;[85-01-8]$

(2) Ethanenitrile; $\mathrm{C}_{2} \mathrm{H}_{3} \mathrm{~N}$; [75-05-8]

Original Measurements:

${ }^{61}$ C. E. Hernández, K. M. De Fina, L. E. Roy, T. L. Sharp, and W. E. Acree, Jr., Can. J. Chem. 77, 1465 (1999).

\begin{tabular}{ll}
\hline Variables: & Prepared by: \\
$T / \mathrm{K}=298.15$ & W. E. Acree, Jr. \\
\hline
\end{tabular}

Experimental Values

\begin{tabular}{lcc}
\hline \hline$x_{2}{ }^{(\mathrm{s}) \mathrm{a}}$ & $x_{2}{ }^{\mathrm{b}}$ & $x_{1}{ }^{\mathrm{c}}$ \\
\hline 1.0000 & 0.9673 & 0.03267 \\
\hline
\end{tabular}

${ }^{\mathrm{a}} x_{2}{ }^{(\mathrm{s})}$ : initial mole fraction of component 2 in the solution.

${ }^{\mathrm{b}} x_{2}$ : mole fraction of component 2 in the saturated solution.

${ }^{\mathrm{c}} x_{1}$ : mole fraction solubility of the solute.

\section{Auxiliary Information}

\section{Method/Apparatus/Procedure:}

Constant-temperature bath, calorimetric thermometer, and an ultraviolet/ visible spectrophotometer.

Excess solute and solvent were placed in amber glass bottles and allowed to equilibrate for several days at constant temperature. Attainment of equilibrium was verified by several repetitive measurements and by approaching equilibrium from supersaturation. Aliquots of saturated solutions were transferred through a coarse filter into tared volumetric flasks, weighed and diluted with methanol. Concentrations were determined by spectrophotometric measurements at $347 \mathrm{~nm}$.

Source and Purity of Chemicals:

(1) $98 \%$, Aldrich Chemical Company, Milwaukee, WI, USA, was recrystallized several times from methanol.

(2) $99.9+\%$, HPLC Grade, Aldrich Chemical Company, stored over molecular sieves before use.

\section{Method/Apparatus/Procedure:}

Constant-temperature bath, calorimetric thermometer, and an ultraviolet/ visible spectrophotometer.

Excess solute and solvent were placed in amber glass bottles and allowed to equilibrate for several days at constant temperature. Attainment of equilibrium was verified by several repetitive measurements and by approaching equilibrium from supersaturation. Aliquots of saturated solutions were transferred through a coarse filter into tared volumetric flasks, weighed and diluted with 2-propanol. Concentrations were determined by spectrophotometric measurements at $347 \mathrm{~nm}$.

Source and Purity of Chemicals:

(1) $98 \%$, Aldrich Chemical Company, Milwaukee, WI, USA, was recrystallized several times from methanol.

(2) $99 \%$, Aldrich Chemical Company, stored over molecular sieves before use.

\section{Estimated Error:}

Temperature: $\pm 0.1 \mathrm{~K}$.

$x_{1}: \pm 1.5 \%$ (relative error).

\begin{tabular}{ll}
\hline \hline $\begin{array}{l}\text { Components: } \\
\text { (1) Phenanthrene; } \mathrm{C}_{14} \mathrm{H}_{10} ;[85-01-8]\end{array}$ & Original Measurements: \\
(2) Butanenitrile; $\mathrm{C}_{4} \mathrm{H}_{7} \mathrm{~N} ;[109-74-0]$ & \\
\hline Variables: & Prepared by: \\
$T / \mathrm{K}=298.15$ & W. E. Acree, Jr.
\end{tabular}

Experimental Values

\begin{tabular}{lcc}
\hline \hline$x_{2}{ }^{(\mathrm{s}) \mathrm{a}}$ & $x_{2}{ }^{\mathrm{b}}$ & $x_{1}{ }^{\mathrm{c}}$ \\
\hline 1.0000 & 0.8376 & 0.1624 \\
${ }^{\mathrm{a}} x_{2}{ }^{(\mathrm{s})}:$ initial mole fraction of component 2 in the solution. \\
${ }^{\mathrm{b}} x_{2}:$ mole fraction of component 2 in the saturated solution. \\
${ }^{\mathrm{c}} x_{1}:$ mole fraction solubility of the solute.
\end{tabular}




\section{Auxiliary Information}

\section{Method/Apparatus/Procedure:}

Constant-temperature bath, calorimetric thermometer, and an ultraviolet/ visible spectrophotometer.

Excess solute and solvent were placed in amber glass bottles and allowed to equilibrate for several days at constant temperature. Attainment of equilibrium was verified by several repetitive measurements and by approaching equilibrium from supersaturation. Aliquots of saturated solutions were transferred through a coarse filter into tared volumetric flasks, weighed and diluted with 2-propanol. Concentrations were determined by spectrophotometric measurements at $347 \mathrm{~nm}$.

Source and Purity of Chemicals:

(1) $98 \%$, Aldrich Chemical Company, Milwaukee, WI, USA, was recrystallized several times from methanol.

(2) $99 \%$, Aldrich Chemical Company, stored over molecular sieves before use.

Estimated Error:

Temperature: $\pm 0.1 \mathrm{~K}$.

$x_{1}: \pm 1.5 \%$ (relative error).

\begin{tabular}{ll}
\hline \hline Components: & Original Measurements: \\
(1) Phenanthrene; $\mathrm{C}_{14} \mathrm{H}_{10} ;[85-01-8]$ & ${ }^{35} \mathrm{Z}$. Lisicki and M. E. Jamróz, J. \\
$(2) N, N$-Dimethylacetamide; $\mathrm{C}_{4} \mathrm{H}_{9} \mathrm{NO} ;$ & Chem. Thermodyn. 32, 1335 \\
{$[127-19-5]$} & $(2000)$. \\
\hline Variables: & Prepared by: \\
Temperature & W. E. Acree, Jr. \\
\hline
\end{tabular}

Experimental Values

\begin{tabular}{lcc}
\hline \hline$T / \mathrm{K}$ & $x_{2}{ }^{\mathrm{a}}$ & $x_{1}{ }^{\mathrm{b}}$ \\
\hline 253.2 & 1.0000 & 0.0000 \\
252.7 & 0.9900 & 0.0100 \\
251.3 & 0.9712 & 0.0288 \\
250.2 & 0.9497 & 0.0503 \\
249.2 & 0.9395 & 0.0605 \\
248.1 & 0.9249 & 0.0751 \\
245.9 & 0.9006 & 0.0994 \\
250.7 & 0.8797 & 0.1203 \\
260.2 & 0.8508 & 0.1492 \\
274.8 & 0.8009 & 0.1991 \\
287.8 & 0.7509 & 0.2491 \\
298.4 & 0.8968 & 0.3032 \\
308.3 & 0.6385 & 0.3615 \\
314.3 & 0.5997 & 0.4003 \\
318.4 & 0.5713 & 0.4287 \\
328.0 & 0.4974 & 0.5026 \\
339.6 & 0.3915 & 0.6085 \\
349.1 & 0.2942 & 0.7058 \\
359.4 & 0.1812 & 0.8188 \\
366.4 & 0.0959 & 0.9041 \\
372.9 & 0.0000 & 1.0000 \\
\hline${ }^{\mathrm{a}} x_{2}:$ mole fraction of component 2 in the saturated solution. \\
${ }_{x_{1}: \text { mole fraction of the polycyclic aromatic hydrocarbon }}($ component 1$)$.
\end{tabular}

The authors reported that the binary system exhibits simple eutectic behavior, with a eutectic temperature of $T / \mathrm{K}=245.5$ and eutectic composition of $x_{1}=0.105$.

\section{Auxiliary Information}

\section{Method/Apparatus/Procedure:}

Temperature-controlled heating/cooling system and a shaking-mixing system. Synthetic mixtures of known compositions were weighed in small glass ampoules. The mixture composition was known to within \pm 0.0002 mole fraction. Ampoules were cooled to very low temperatures of about $220 \mathrm{~K}$, pumped down and sealed. The sealed mixtures were then melted, cooled rapidly, and reheated. The rate of reheating was at an approximate rate of $8 \times$ $10^{-5} \mathrm{~K} / \mathrm{s}$ just before complete dissolution. The temperature at which the solid completely dissolved was recorded. The average of two or three independent measurements was taken as the solid-liquid equilibrium.

\section{Source and Purity of Chemicals:}

(1) 98\%, Koch-Light Lab. Ltd., United Kingdom, was purified with concentrated sulfuric acid, followed by maleic anhydride, and then recrystallized to yield a sample of $99.86 \%$ purity (mass percent).

(2) Purest grade, Merck-Schuchardt, Federal Republic of Germany, was distilled under reduced pressure and dried over molecular sieves to produce a sample of $99.9 \%$ purity (mass percent).

\section{Estimated Error:}

Temperature: $\pm 0.2 \mathrm{~K}$ (estimated by compiler). $x_{1}: \pm 0.0002$.

\begin{tabular}{ll}
\hline \hline Components: & Original Measurements: \\
(1) Phenanthrene; $\mathrm{C}_{14} \mathrm{H}_{10} ;[85-01-8]$ & ${ }^{35} \mathrm{Z}$. Lisicki and M. E. Jamróz, J. \\
(2) 1-Methyl-2-pyrrolidone; $\mathrm{C}_{5} \mathrm{H}_{9} \mathrm{NO} ;$ & Chem. Thermodyn. 32, 1335 \\
[872-50-4] & $(2000)$. \\
\hline Variables: & Prepared by: \\
Temperature & W. E. Acree, Jr. \\
\hline
\end{tabular}

Experimental Values

\begin{tabular}{lcc}
\hline \hline$T / \mathrm{K}$ & $x_{2}{ }^{\mathrm{a}}$ & $x_{1}{ }^{\mathrm{b}}$ \\
\hline 248.5 & 1.0000 & 0.0000 \\
248.1 & 0.9898 & 0.0102 \\
246.5 & 0.9507 & 0.0493 \\
245.3 & 0.9249 & 0.0751 \\
243.8 & 0.8993 & 0.1007 \\
246.7 & 0.8570 & 0.1430 \\
262.9 & 0.8009 & 0.1991 \\
275.0 & 0.7520 & 0.2480 \\
278.3 & 0.7340 & 0.2660 \\
280.4 & 0.7274 & 0.2726 \\
282.8 & 0.7139 & 0.2861 \\
288.4 & 0.6920 & 0.3080 \\
293.4 & 0.6663 & 0.3337 \\
305.4 & 0.5964 & 0.4036 \\
319.4 & 0.4988 & 0.5012 \\
330.0 & 0.4197 & 0.5803 \\
335.1 & 0.3800 & 0.6200 \\
340.7 & 0.3328 & 0.6672 \\
356.8 & 0.2020 & 0.7980 \\
365.3 & 0.0996 & 0.9004 \\
372.9 & 0.0000 & 1.0000
\end{tabular}

${ }^{a} x_{2}$ : mole fraction of component 2 in the saturated solution.

${ }^{\mathrm{b}} x_{1}$ : mole fraction of the polycyclic aromatic hydrocarbon (component 1 ).

The authors reported that the binary system exhibits simple eutectic behavior, with a eutectic temperature of $T / \mathrm{K}=242.4$ and eutectic composition of $x_{1}=0.162$. 


\section{Auxiliary Information}

\section{Method/Apparatus/Procedure:}

Temperature controlled heating/cooling system and a shaking-mixing system. Synthetic mixtures of known compositions were weighed in small glass ampoules. The mixture composition was known to within \pm 0.0002 mole fraction. Ampoules were cooled to very low temperatures of about $220 \mathrm{~K}$, pumped down and sealed. The sealed mixtures were then melted, cooled rapidly, and reheated. The rate of reheating was at an approximate rate of $8 \times 10^{-5} \mathrm{~K} / \mathrm{s}$ just before complete dissolution. The temperature at which the solid completely dissolved was recorded. The average of two or three independent measurements was taken as the solid-liquid equilibrium.

Source and Purity of Chemicals:

(1) 98\%, Koch-Light Lab. Ltd., United Kingdom, was purified with concentrated sulfuric acid, followed by maleic anhydride, and then recrystallized to yield a sample of $99.86 \%$ purity (mass percent).

(2) Technical Grade, BASF, Federal Republic of Germany, was purified by treatment with a $2 \%$ solution of potassium permanganate, and then distilled under reduced pressure to collect the distillate having $99.8 \%$ purity (mass percent). The purified sample was dried over molecular sieves.

\section{Estimated Error:}

Temperature: $\pm 0.2 \mathrm{~K}$ (estimated by compiler). $x_{1}: \pm 0.0002$.

\begin{tabular}{|c|c|}
\hline $\begin{array}{l}\text { Components: } \\
\text { (1) Phenanthrene; } \mathrm{C}_{14} \mathrm{H}_{10} ;[85-01-8] \\
\text { (2) Hexahydro-1-methyl- } 2 \mathrm{H} \text {-azepin-2-one }(\mathrm{N}- \\
\text { Methyl- } \varepsilon \text {-caprolactam); } \mathrm{C}_{7} \mathrm{H}_{13} \mathrm{NO} ;[2556-73-2]\end{array}$ & $\begin{array}{l}\text { Original Measurements: } \\
{ }^{35} \text { Z. Lisicki and M. E. } \\
\text { Jamróz, J. Chem. } \\
\text { Thermodyn. 32, } 1335 \\
\text { (2000). }\end{array}$ \\
\hline $\begin{array}{l}\text { Variables: } \\
\text { Temperature }\end{array}$ & $\begin{array}{l}\text { Prepared by: } \\
\text { W. E. Acree, Jr. }\end{array}$ \\
\hline
\end{tabular}

Experimental Values

\begin{tabular}{lcc}
\hline \hline$T / \mathrm{K}$ & $x_{2}{ }^{\mathrm{a}}$ & $x_{1}{ }^{\mathrm{b}}$ \\
\hline 279.6 & 1.0000 & 0.0000 \\
281.3 & 0.7225 & 0.2775 \\
294.1 & 0.6749 & 0.3251 \\
300.2 & 0.6363 & 0.3637 \\
308.4 & 0.5992 & 0.4008 \\
316.3 & 0.5479 & 0.4521 \\
323.6 & 0.5044 & 0.4956 \\
325.2 & 0.4988 & 0.5012 \\
334.9 & 0.4207 & 0.5793 \\
343.5 & 0.3464 & 0.6536 \\
353.5 & 0.2480 & 0.7520 \\
362.1 & 0.1497 & 0.8503 \\
369.7 & 0.0486 & 0.9514 \\
372.9 & 0.0000 & 1.0000 \\
\hline
\end{tabular}

${ }^{\mathrm{a}} x_{2}$ : mole fraction of component 2 in the saturated solution.

${ }^{\mathrm{b}} \mathrm{x}_{1}$ : mole fraction of the polycyclic aromatic hydrocarbon (component 1 ).

\section{Auxiliary Information}

Method/Apparatus/Procedure:

Temperature controlled heating/cooling system and a shaking-mixing system. Synthetic mixtures of known compositions were weighed in small glass ampoules. The mixture composition was known to within \pm 0.0002 mole fraction. Ampoules were cooled to very low temperatures of about $220 \mathrm{~K}$, pumped down and sealed. The sealed mixtures were then melted, cooled rapidly, and reheated. The rate of reheating was at an approximate rate of $8 \times 10^{-5} \mathrm{~K} / \mathrm{s}$ just before complete dissolution. The temperature at which the solid completely dissolved was recorded. The average of two or three independent measurements was taken as the solid-liquid equilibrium.

Source and Purity of Chemicals:

(1) 98\%, Koch-Light Lab. Ltd., United Kingdom, was purified with concentrated sulfuric acid, followed by maleic anhydride, and then recrystallized to yield a sample of $99.86 \%$ purity (mass percent).

(2) Technical Grade, Leuna Werke, Federal Republic of Germany, was purified by super-rectification and then dried over molecular sieves to give a sample having a purity of $99.9 \%$ by mass.

\section{Estimated Error:}

Temperature: $\pm 0.2 \mathrm{~K}$ (estimated by compiler).

$x_{1}: \pm 0.0002$.

\section{Solubility of Phenothiazine in Organic Solvents \\ 12.1. Critical evaluation of experimental solubility data}

The three earlier volumes in the IUPAC Solubility Data Series on solubilities of polycyclic aromatic hydrocarbons ${ }^{1-3}$ contained very little solubility data for phenothiazine. Volume 59 (Ref. 3) contained phase diagram information for four binary systems, namely phenothiazine + phenoxazine, phenothiazine + thianthrene, phenothiazine + phenoxanthiin, and phenothiazine + dibenzo[b,e][1,4]dioxine. The experimental data will not be repeated here. The solubility information contained in Vol. 59 is listed so that readers will know what phenothiazine data are available in earlier volumes in the series.

There have been three studies that reported solubility data for phenothiazine in organic solvents after Vol. 59 was published in 1995. Hoover $\mathrm{et} \mathrm{al.} .^{65}$ determined the solubility of phenothiazine in nine alkanes, 20 alkanols, one alkanediol, four dialkyl ethers, one cyclic ether, and three alkanenitriles at $298.15 \mathrm{~K}$. They used the experimental solubility data to determine the Abraham model solute descriptors for phenothiazine. The calculated solute descriptors described observed solubility data to within $\pm 0.094 \log$ units. Saifullah et al. ${ }^{66}$ reported the solubility of phenothiazine in tetrahydrofuran at $298.15 \mathrm{~K}$ as part of a computational study aimed at updating the Abraham model equation coefficients of tetrahydrofuran and 1,4-dioxane and extending the basic model to include ionic solute species. Finally, Ahmadian et al. ${ }^{67}$ measured the solubility of phenothiazine in ethanol and 1,2-propanediol at 298.2, 308.2, 318.2, and 328.2 K. 
There is only one solvent common to the three solubility studies. The mole fraction solubility of phenothiazine in ethanol of $x_{1}$ $=0.00890$ reported by Hoover et al ${ }^{65}$ differs from the experimental value of $x_{1}=0.00876$ from the Ahmadian et al. ${ }^{67}$ study by less than $2 \%$.

The experimental solubility data for phenothiazine dissolved in the different organic solvents are given in Secs. 12.2-12.6.

\subsection{Phenothiazine solubility data in saturated hydrocarbons (including cycloalkanes)}

\begin{tabular}{|c|c|}
\hline $\begin{array}{l}\text { Components: } \\
\text { (1) Phenothiazine; } \mathrm{C}_{12} \mathrm{H}_{9} \mathrm{NS} \text {; [92-84-2] } \\
\text { (2) Hexane; } \mathrm{C}_{6} \mathrm{H}_{14} ;[110-54-3]\end{array}$ & $\begin{array}{l}\text { Original Measurements: } \\
{ }^{65} \text { K. R. Hoover, W. E. Acree, Jr., } \\
\text { and M. H. Abraham, Phys. Chem. } \\
\text { Liq. 44, } 367 \text { (2006). }\end{array}$ \\
\hline Variables: & Prepared by: \\
\hline$T / \mathrm{K}=298.15$ & W. E. Acree, Jr. \\
\hline
\end{tabular}

Experimental Values

\begin{tabular}{lcc}
\hline \hline$x_{2}{ }^{(\mathrm{s}) \mathrm{a}}$ & $x_{2}{ }^{\mathrm{b}}$ & $x_{1}{ }^{\mathrm{c}}$ \\
\hline 1.0000 & 0.9994 & 0.000585 \\
${ }^{\mathrm{a}} x_{2}{ }^{(\mathrm{s})}:$ initial mole fraction of component 2 in the solution. \\
${ }^{\mathrm{b}} x_{2}:$ mole fraction of component 2 in the saturated solution. \\
${ }^{\mathrm{c}}{ }_{x_{1}}$ : mole fraction solubility of the solute.
\end{tabular}

\section{Auxiliary Information}

\section{Method/Apparatus/Procedure:}

Constant-temperature bath, calorimetric thermometer, and an ultraviolet/ visible spectrophotometer.

Excess solute and solvent were placed in amber glass bottles and allowed to equilibrate for several days at constant temperature. Attainment of equilibrium was verified by several repetitive measurements and by approaching equilibrium from supersaturation. Aliquots of saturated solutions were transferred through a coarse filter into tared volumetric flasks, weighed and diluted with methanol. Concentrations were determined by spectrophotometric measurements at $281 \mathrm{~nm}$.

Source and Purity of Chemicals:

(1) $99 \%$, Acros Organics, USA, was used as received.

(2) $99 \%$, Aldrich Chemical Company, Milwaukee, WI, USA, stored over molecular sieves before use.

Estimated Error:

Temperature: $\pm 0.1 \mathrm{~K}$.

$x_{1}: \pm 1.5 \%$ (relative error).

\begin{tabular}{|c|c|}
\hline $\begin{array}{l}\text { Components: } \\
\text { (1) Phenothiazine; } \mathrm{C}_{12} \mathrm{H}_{9} \mathrm{NS} \text {; [92-84-2] } \\
\text { (2) Heptane; } \mathrm{C}_{7} \mathrm{H}_{16} ;[142-82-5]\end{array}$ & $\begin{array}{l}\text { Original Measurements: } \\
{ }^{65} \text { K. R. Hoover, W. E. Acree, Jr., } \\
\text { and M. H. Abraham, Phys. Chem. } \\
\text { Liq. 44, } 367 \text { (2006). }\end{array}$ \\
\hline $\begin{array}{l}\text { Variables: } \\
T / \mathrm{K}=298.15\end{array}$ & $\begin{array}{l}\text { Prepared by: } \\
\text { W. E. Acree, Jr. }\end{array}$ \\
\hline
\end{tabular}

Experimental Values

\begin{tabular}{lcc}
\hline \hline$x_{2}{ }^{(\mathrm{s}) \mathrm{a}}$ & $x_{2}{ }^{\mathrm{b}}$ & $x_{1}{ }^{\mathrm{c}}$ \\
\hline 1.0000 & 0.9993 & 0.000696 \\
$\frac{{ }^{\mathrm{a}} x_{2}{ }^{(\mathrm{s})}: \text { initial mole fraction of component } 2 \text { in the solution. }}{{ }^{\mathrm{b}} x_{2}: \text { mole fraction of component } 2 \text { in the saturated solution. }}$ \\
${ }^{\mathrm{c}} x_{1}:$ mole fraction solubility of the solute.
\end{tabular}

\section{Auxiliary Information}

\section{Method/Apparatus/Procedure:}

Constant-temperature bath, calorimetric thermometer, and an ultraviolet/ visible spectrophotometer.

Excess solute and solvent were placed in amber glass bottles and allowed to equilibrate for several days at constant temperature. Attainment of equilibrium was verified by several repetitive measurements and by approaching equilibrium from supersaturation. Aliquots of saturated solutions were transferred through a coarse filter into tared volumetric flasks, weighed and diluted with methanol. Concentrations were determined by spectrophotometric measurements at $281 \mathrm{~nm}$.

Source and Purity of Chemicals:

(1) $99 \%$, Acros Organics, USA, was used as received.

(2) $99 \%$, anhydrous, Aldrich Chemical Company, Milwaukee, WI, USA, stored over molecular sieves before use.

Estimated Error:

Temperature: $\pm 0.1 \mathrm{~K}$.

$x_{1}: \pm 1.5 \%$ (relative error).

\begin{tabular}{|c|c|}
\hline $\begin{array}{l}\text { Components: } \\
\text { (1) Phenothiazine; } \mathrm{C}_{12} \mathrm{H}_{9} \mathrm{NS} \text {; [92-84-2] } \\
\text { (2) Octane; } \mathrm{C}_{8} \mathrm{H}_{18} ;[111-65-9]\end{array}$ & $\begin{array}{l}\text { Original Measurements: } \\
{ }^{65} \text { K. R. Hoover, W. E. Acree, Jr., } \\
\text { and M. H. Abraham, Phys. Chem. } \\
\text { Liq. 44, } 367 \text { (2006). }\end{array}$ \\
\hline Variables: & Prepared by: \\
\hline$T / \mathrm{K}=298.15$ & W. E. Acree, Jr. \\
\hline
\end{tabular}

Experimental Values

\begin{tabular}{|c|c|c|}
\hline$x_{2}{ }^{(\mathrm{s}) \mathrm{a}}$ & $x_{2}{ }^{\mathrm{b}}$ & $x_{1}{ }^{\mathrm{c}}$ \\
\hline 1.0000 & 0.9991 & 0.000858 \\
\hline
\end{tabular}

Auxiliary Information

Method/Apparatus/Procedure:

Constant-temperature bath, calorimetric thermometer, and an ultraviolet/ visible spectrophotometer.

Excess solute and solvent were placed in amber glass bottles and allowed to equilibrate for several days at constant temperature. Attainment of equilibrium was verified by several repetitive measurements and by approaching equilibrium from supersaturation. Aliquots of saturated solutions were transferred through a coarse filter into tared volumetric flasks, weighed and diluted with methanol. Concentrations were determined by spectrophotometric measurements at $281 \mathrm{~nm}$. 
Source and Purity of Chemicals:

(1) $99 \%$, Acros Organics, USA, was used as received.

(2) $99+\%$, anhydrous, Aldrich Chemical Company, Milwaukee, WI, USA, stored over molecular sieves before use.

Estimated Error:

Temperature: $\pm 0.1 \mathrm{~K}$.

$x_{1}: \pm 1.5 \%$ (relative error).

\begin{tabular}{|c|c|}
\hline $\begin{array}{l}\text { Components: } \\
\text { (1) Phenothiazine; } \mathrm{C}_{12} \mathrm{H}_{9} \mathrm{NS} ;[\text { [92-84-2] } \\
\text { (2) Decane; } \mathrm{C}_{10} \mathrm{H}_{22} ;[124-18-5]\end{array}$ & $\begin{array}{l}\text { Original Measurements: } \\
{ }^{65} \text { K. R. Hoover, W. E. Acree, Jr., } \\
\text { and M. H. Abraham, Phys. Chem. } \\
\text { Liq. 44, } 367 \text { (2006). }\end{array}$ \\
\hline $\begin{array}{l}\text { Variables: } \\
T / \mathrm{K}=298.15\end{array}$ & $\begin{array}{l}\text { Prepared by: } \\
\text { W. E. Acree, Jr. }\end{array}$ \\
\hline
\end{tabular}

Experimental Values

\begin{tabular}{lcc}
\hline \hline$x_{2}{ }^{(\mathrm{s}) \mathrm{a}}$ & $x_{2}{ }^{\mathrm{b}}$ & $x_{1}{ }^{\mathrm{c}}$ \\
\hline 1.0000 & 0.9989 & 0.001056 \\
\hline
\end{tabular}

${ }^{\mathrm{a}} x_{2}{ }^{(\mathrm{s})}$ : initial mole fraction of component 2 in the solution.

${ }^{b} x_{2}$ : mole fraction of component 2 in the saturated solution.

${ }^{\mathrm{c}} x_{1}$ : mole fraction solubility of the solute.

\section{Auxiliary Information}

\section{Method/Apparatus/Procedure:}

Constant-temperature bath, calorimetric thermometer, and an ultraviolet/ visible spectrophotometer.

Excess solute and solvent were placed in amber glass bottles and allowed to equilibrate for several days at constant temperature. Attainment of equilibrium was verified by several repetitive measurements and by approaching equilibrium from supersaturation. Aliquots of saturated solutions were transferred through a coarse filter into tared volumetric flasks, weighed and diluted with methanol. Concentrations were determined by

spectrophotometric measurements at $281 \mathrm{~nm}$.

Source and Purity of Chemicals:

(1) $99 \%$, Acros Organics, USA, was used as received.

(2) $99+\%$, TCI America, Portland, OR, USA, stored over molecular sieves before use.

\section{Estimated Error:}

Temperature: $\pm 0.1 \mathrm{~K}$.

$x_{1}: \pm 1.5 \%$ (relative error).

\begin{tabular}{|c|c|}
\hline $\begin{array}{l}\text { Components: } \\
\text { (1) Phenothiazine; } \mathrm{C}_{12} \mathrm{H}_{9} \mathrm{NS} \text {; [92-84-2] } \\
\text { (2) Hexadecane; } \mathrm{C}_{16} \mathrm{H}_{34} ;[544-76-3]\end{array}$ & $\begin{array}{l}\text { Original Measurements: } \\
{ }^{65} \text { K. R. Hoover, W. E. Acree, Jr., } \\
\text { and M. H. Abraham, Phys. Chem. } \\
\text { Liq. 44, } 367 \text { (2006). }\end{array}$ \\
\hline $\begin{array}{l}\text { Variables: } \\
T / \mathrm{K}=298.15\end{array}$ & $\begin{array}{l}\text { Prepared by: } \\
\text { W. E. Acree, Jr. }\end{array}$ \\
\hline
\end{tabular}

Experimental Values

\begin{tabular}{|c|c|c|}
\hline$x_{2}{ }^{(\mathrm{s}) \mathrm{a}}$ & $x_{2}{ }^{b}$ & $x_{1}{ }^{\mathrm{c}}$ \\
\hline 1.0000 & 0.9983 & 0.001661 \\
\hline
\end{tabular}

\section{Auxiliary Information}

\section{Method/Apparatus/Procedure:}

Constant-temperature bath, calorimetric thermometer, and an ultraviolet/ visible spectrophotometer.

Excess solute and solvent were placed in amber glass bottles and allowed to equilibrate for several days at constant temperature. Attainment of equilibrium was verified by several repetitive measurements and by approaching equilibrium from supersaturation. Aliquots of saturated solutions were transferred through a coarse filter into tared volumetric flasks, weighed and diluted with methanol. Concentrations were determined by spectrophotometric measurements at $281 \mathrm{~nm}$.

\section{Source and Purity of Chemicals:}

(1) $99 \%$, Acros Organics, USA, was used as received.

(2) $99 \%$, Aldrich Chemical Company, Milwaukee, WI, USA, stored over molecular sieves before use.

\section{Estimated Error:}

Temperature: $\pm 0.1 \mathrm{~K}$.

$x_{1}: \pm 1.5 \%$ (relative error)

\begin{tabular}{ll}
\hline \hline $\begin{array}{l}\text { Components: } \\
\text { (1) Phenothiazine; } \mathrm{C}_{12} \mathrm{H}_{9} \mathrm{NS} ;[\text { [92-84-2] }\end{array}$ & $\begin{array}{l}\text { Original Measurements: } \\
{ }^{65} \mathrm{~K} . \mathrm{R} . \text { Hoover, W. E. Acree, Jr., } \\
\text { (2) Cyclohexane; } \mathrm{C}_{6} \mathrm{H}_{12} ;[110-82-7]\end{array}$ \\
& $\begin{array}{l}\text { and M. H. Abraham, Phys. Chem. } \\
\text { Liq. 44, 367 (2006). }\end{array}$ \\
\hline Variables: & Prepared by: \\
$T / \mathrm{K}=298.15$ & W. E. Acree, Jr. \\
\hline
\end{tabular}

Experimental Values

\begin{tabular}{lcc}
\hline \hline$x_{2}{ }^{(\mathrm{s}) \mathrm{a}}$ & $x_{2}{ }^{\mathrm{b}}$ & $x_{1}{ }^{\mathrm{c}}$ \\
\hline 1.0000 & 0.9990 & 0.000979
\end{tabular}

${ }^{\mathrm{a}} x_{2}{ }^{(\mathrm{s})}$ : initial mole fraction of component 2 in the solution.

${ }^{\mathrm{b}} x_{2}$ : mole fraction of component 2 in the saturated solution.

${ }^{c} x_{1}$ : mole fraction solubility of the solute.

\section{Auxiliary Information}

\section{Method/Apparatus/Procedure:}

Constant-temperature bath, calorimetric thermometer, and an ultraviolet/ visible spectrophotometer.

Excess solute and solvent were placed in amber glass bottles and allowed to equilibrate for several days at constant temperature. Attainment of equilibrium was verified by several repetitive measurements and by approaching equilibrium from supersaturation. Aliquots of saturated solutions were transferred through a coarse filter into tared volumetric flasks, weighed and diluted with methanol. Concentrations were determined by spectrophotometric measurements at $281 \mathrm{~nm}$. 
Source and Purity of Chemicals:

(1) $99 \%$, Acros Organics, USA, was used as received.

(2) $99.9+\%$, HPLC Grade, Aldrich Chemical Company, Milwaukee, WI, USA, stored over molecular sieves before use.

Estimated Error:

Temperature: $\pm 0.1 \mathrm{~K}$.

$x_{1}: \pm 1.5 \%$ (relative error).

\begin{tabular}{ll}
\hline \hline Components: & Original Measurements: \\
$\begin{array}{l}\text { (1) Phenothiazine; } \mathrm{C}_{12} \mathrm{H}_{9} \mathrm{NS} ;[\text { [92-84-2] } \\
\text { (2) Methylcyclohexane; } \mathrm{C}_{7} \mathrm{H}_{14} ;\end{array}$ & $\begin{array}{l}\text { K5. R. Hoover, W. E. Acree, Jr., } \\
\text { and M. H. Abraham, Phys. Chem. }\end{array}$ \\
\hline $108-87-2]$ & Liq. 44, 367 (2006). \\
\hline Variables: & Prepared by: \\
$T / \mathrm{K}=298.15$ & W. E. Acree, Jr. \\
\hline
\end{tabular}

Experimental Values

\begin{tabular}{lcc}
\hline \hline$x_{2}{ }^{(\mathrm{s}) \mathrm{a}}$ & $x_{2}{ }^{\mathrm{b}}$ & $x_{1}{ }^{\mathrm{c}}$ \\
\hline 1.0000 & 0.9990 & 0.001027 \\
\hline
\end{tabular}

${ }^{\mathrm{a}} x_{2}{ }^{(\mathrm{s})}$ : initial mole fraction of component 2 in the solution.

${ }^{\mathrm{b}} x_{2}$ : mole fraction of component 2 in the saturated solution.

${ }^{\mathrm{c}} x_{1}$ : mole fraction solubility of the solute.

\section{Auxiliary Information}

\section{Method/Apparatus/Procedure:}

Constant-temperature bath, calorimetric thermometer, and an ultraviolet/ visible spectrophotometer.

Excess solute and solvent were placed in amber glass bottles and allowed to equilibrate for several days at constant temperature. Attainment of equilibrium was verified by several repetitive measurements and by approaching equilibrium from supersaturation. Aliquots of saturated solutions were transferred through a coarse filter into tared volumetric flasks, weighed and diluted with methanol. Concentrations were determined by

spectrophotometric measurements at $281 \mathrm{~nm}$.

Source and Purity of Chemicals:

(1) $99 \%$, Acros Organics, USA, was used as received.

(2) $99+\%$, anhydrous, Aldrich Chemical Company, Milwaukee, WI, USA, stored over molecular sieves before use.

\section{Estimated Error:}

Temperature: $\pm 0.1 \mathrm{~K}$.

$x_{1}: \pm 1.5 \%$ (relative error).

\begin{tabular}{ll}
\hline \hline Components: & Original Measurements: \\
(1) Phenothiazine; $\mathrm{C}_{12} \mathrm{H}_{9} \mathrm{NS} ;[$ [92-84-2] & ${ }^{65} \mathrm{~K}$. R. Hoover, W. E. Acree, Jr., \\
(2) Cyclooctane; $\mathrm{C}_{8} \mathrm{H}_{16} ;$ [292-64-8] & and M. H. Abraham, Phys. Chem. \\
& Liq. 44, 367 (2006). \\
\hline Variables: & Prepared by: \\
$T / \mathrm{K}=298.15$ & W. E. Acree, Jr. \\
\hline
\end{tabular}

Experimental Values

\begin{tabular}{lcc}
\hline \hline$\frac{x_{2}{ }^{(\mathrm{s}) \mathrm{a}}}{x_{2}{ }^{\mathrm{b}}}$ & \multicolumn{1}{c}{$x_{1}{ }^{\mathrm{c}}$} \\
\hline 1.0000 & 0.9984 & 0.001577 \\
${ }^{{ }^{\mathrm{a}} x_{2}{ }^{(\mathrm{s})}: \text { initial mole fraction of component } 2 \text { in the solution. }}$ \\
${ }^{\mathrm{b}} x_{2}:$ mole fraction of component 2 in the saturated solution. \\
${ }^{\mathrm{c}}{ }_{x_{1}: \text { mole fraction solubility of the solute. }}$
\end{tabular}

\section{Auxiliary Information}

\section{Method/Apparatus/Procedure:}

Constant-temperature bath, calorimetric thermometer, and an ultraviolet/ visible spectrophotometer.

Excess solute and solvent were placed in amber glass bottles and allowed to equilibrate for several days at constant temperature. Attainment of equilibrium was verified by several repetitive measurements and by approaching equilibrium from supersaturation. Aliquots of saturated solutions were transferred through a coarse filter into tared volumetric flasks, weighed and diluted with methanol. Concentrations were determined by spectrophotometric measurements at $281 \mathrm{~nm}$.

\section{Source and Purity of Chemicals:}

(1) $99 \%$, Acros Organics, USA, was used as received.

(2) $99 \%$, Lancaster Synthesis, Ward Hill, Massachusetts, USA, stored over molecular sieves before use.

\section{Estimated Error:}

Temperature: $\pm 0.1 \mathrm{~K}$.

$x_{1}: \pm 1.5 \%$ (relative error).

\begin{tabular}{|c|c|}
\hline $\begin{array}{l}\text { Components: } \\
\text { (1) Phenothiazine; } \mathrm{C}_{12} \mathrm{H}_{9} \mathrm{NS} ;[92-84-2] \\
\text { (2) } 2,2,4-\text { Trimethylpentane; } \mathrm{C}_{8} \mathrm{H}_{18} ; \\
\text { [540-84-1] }\end{array}$ & $\begin{array}{l}\text { Original Measurements: } \\
{ }^{65} \text { K. R. Hoover, W. E. Acree, Jr., } \\
\text { and M. H. Abraham, Phys. Chem. } \\
\text { Liq. 44, } 367 \text { (2006). }\end{array}$ \\
\hline $\begin{array}{l}\text { Variables: } \\
T / \mathrm{K}=298.15\end{array}$ & $\begin{array}{l}\text { Prepared by: } \\
\text { W. E. Acree, Jr. }\end{array}$ \\
\hline
\end{tabular}

Experimental Values

\begin{tabular}{lcc}
\hline \hline$x_{2}{ }^{(\mathrm{s}) \mathrm{a}}$ & $x_{2}{ }^{\mathrm{b}}$ & $x_{1}{ }^{\mathrm{c}}$ \\
\hline 1.0000 & 0.9995 & 0.000532 \\
\hline
\end{tabular}

${ }^{\mathrm{a}} x_{2}{ }^{(\mathrm{s})}$ : initial mole fraction of component 2 in the solution.

${ }^{\mathrm{b}} x_{2}$ : mole fraction of component 2 in the saturated solution.

${ }^{c} x_{1}$ : mole fraction solubility of the solute.

\section{Auxiliary Information}

\section{Method/Apparatus/Procedure:}

Constant-temperature bath, calorimetric thermometer, and an ultraviolet/ visible spectrophotometer.

Excess solute and solvent were placed in amber glass bottles and allowed to equilibrate for several days at constant temperature. Attainment of equilibrium was verified by several repetitive measurements and by approaching equilibrium from supersaturation. Aliquots of saturated solutions were transferred through a coarse filter into tared volumetric flasks, weighed and diluted with methanol. Concentrations were determined by spectrophotometric measurements at $281 \mathrm{~nm}$. 
Source and Purity of Chemicals:

(1) $99 \%$, Acros Organics, USA, was used as received.

(2) $99 \%$, Aldrich Chemical Company, Milwaukee, WI, USA, stored over molecular sieves before use.

Estimated Error:

Temperature: $\pm 0.1 \mathrm{~K}$.

$x_{1}: \pm 1.5 \%$ (relative error).

\subsection{Phenothiazine solubility data in ethers}

\begin{tabular}{ll}
\hline \hline Components: & Original Measurements: \\
(1) Phenothiazine; $\mathrm{C}_{12} \mathrm{H}_{9} \mathrm{NS} ;$ [92-84-2] & ${ }^{65} \mathrm{~K}$. R. Hoover, W. E. Acree, Jr., \\
$(2) 1,1^{\prime}-$ Oxybisethane; $\mathrm{C}_{4} \mathrm{H}_{10} \mathrm{O} ;$ & and M. H. Abraham, Phys. Chem. \\
{$[60-29-7]$} & Liq. 44, 367 (2006). \\
\hline Variables: & Prepared by: \\
$T / \mathrm{K}=298.15$ & W. E. Acree, Jr. \\
\hline
\end{tabular}

Experimental Values

\begin{tabular}{lcc}
\hline \hline$x_{2}{ }^{(\mathrm{s}) \mathrm{a}}$ & $x_{2}{ }^{\mathrm{b}}$ & $x_{1}{ }^{\mathrm{c}}$ \\
\hline 1.0000 & 0.9742 & 0.02581 \\
\hline
\end{tabular}

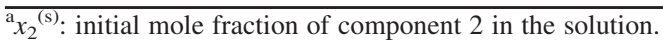

${ }^{b} x_{2}$ : mole fraction of component 2 in the saturated solution.

${ }^{c} x_{1}$ : mole fraction solubility of the solute.

\section{Auxiliary Information}

\section{Method/Apparatus/Procedure:}

Constant-temperature bath, calorimetric thermometer, and an ultraviolet/ visible spectrophotometer.

Excess solute and solvent were placed in amber glass bottles and allowed to equilibrate for several days at constant temperature. Attainment of equilibrium was verified by several repetitive measurements and by approaching equilibrium from supersaturation. Aliquots of saturated solutions were transferred through a coarse filter into tared volumetric flasks, weighed and diluted with methanol. Concentrations were determined by spectrophotometric measurements at $281 \mathrm{~nm}$.

\section{Source and Purity of Chemicals:}

(1) $99 \%$, Acros Organics, USA, was used as received.

(2) 99+\%, anhydrous, Aldrich Chemical Company, Milwaukee, WI, USA, stored over molecular sieves before use.

\section{Estimated Error:}

Temperature: $\pm 0.1 \mathrm{~K}$

$x_{1}: \pm 1.5 \%$ (relative error).

\begin{tabular}{ll}
\hline \hline Components: & $\begin{array}{l}\text { Original Measurements: } \\
\text { (1) Phenothiazine; } \mathrm{C}_{12} \mathrm{H}_{9} \mathrm{NS} ;[\text { [92-84-2] }\end{array}$ \\
$\begin{array}{l}{ }^{65} \mathrm{~K} . \mathrm{R} . \text { Hoover, W. E. Acree, Jr., } \\
\text { (2) } 2,2^{\prime}-\text {-Oxybispropane; } \mathrm{C}_{6} \mathrm{H}_{14} \mathrm{O} ;\end{array}$ & and M. H. Abraham, Phys. Chem. \\
{$[108-20-3]$} & Liq. 44, 367 (2006). \\
\hline Variables: & Prepared by: \\
$T / \mathrm{K}=298.15$ & W. E. Acree, Jr. \\
\hline
\end{tabular}

Experimental Values

\begin{tabular}{lcc}
\hline \hline$x_{2}{ }^{(\mathrm{s}) \mathrm{a}}$ & $x_{2}{ }^{\mathrm{b}}$ & $x_{1}{ }^{\mathrm{c}}$ \\
\hline 1.0000 & 0.9881 & 0.01185 \\
$\frac{{ }^{\mathrm{a}} x_{2}{ }^{(\mathrm{s})}: \text { initial mole fraction of component } 2 \text { in the solution. }}{{ }^{\mathrm{b}} x_{2}: \text { mole fraction of component } 2 \text { in the saturated solution. }}$ \\
${ }^{\mathrm{c}} x_{1}$ : mole fraction solubility of the solute.
\end{tabular}

\section{Auxiliary Information}

\section{Method/Apparatus/Procedure:}

Constant-temperature bath, calorimetric thermometer, and an ultraviolet/ visible spectrophotometer.

Excess solute and solvent were placed in amber glass bottles and allowed to equilibrate for several days at constant temperature. Attainment of equilibrium was verified by several repetitive measurements and by approaching equilibrium from supersaturation. Aliquots of saturated solutions were transferred through a coarse filter into tared volumetric flasks, weighed and diluted with methanol. Concentrations were determined by spectrophotometric measurements at $281 \mathrm{~nm}$.

Source and Purity of Chemicals:

(1) $99 \%$, Acros Organics, USA, was used as received.

(2) 99\%, anhydrous, Aldrich Chemical Company, Milwaukee, WI, USA, stored over molecular sieves before use.

\section{Estimated Error:}

Temperature: $\pm 0.1 \mathrm{~K}$.

$x_{1}: \pm 1.5 \%$ (relative error)

\begin{tabular}{ll}
\hline \hline Components: & $\begin{array}{l}\text { Original Measurements: } \\
\text { (1) Phenothiazine; } \mathrm{C}_{12} \mathrm{H}_{9} \mathrm{NS} ;[\text { [92-84-2] }\end{array}$ \\
$\begin{array}{l}{ }^{65} \mathrm{~K} . \text { R. Hoover, W. E. Acree, Jr., } \\
\text { (2) } 1,1^{\prime}-\text {-Oxybisbutane; } \mathrm{C}_{8} \mathrm{H}_{18} \mathrm{O} ;\end{array}$ & and M. H. Abraham, Phys. Chem. \\
{$[142-96-1]$} & Liq. 44, 367 (2006). \\
\hline Variables: & Prepared by: \\
$T / \mathrm{K}=298.15$ & W. E. Acree, Jr. \\
\hline
\end{tabular}

\section{Experimental Values}

\begin{tabular}{lcc}
\hline \hline$x_{2}{ }^{(\mathrm{s}) \mathrm{a}}$ & $x_{2}^{\mathrm{b}}$ & $x_{1}{ }^{\mathrm{c}}$ \\
\hline 1.0000 & 0.9886 & 0.01144
\end{tabular}

${ }^{\mathrm{a}} x_{2}{ }^{(\mathrm{s})}$ : initial mole fraction of component 2 in the solution.

${ }^{\mathrm{b}} x_{2}$ : mole fraction of component 2 in the saturated solution.

${ }^{c} x_{1}$ : mole fraction solubility of the solute.

\section{Auxiliary Information}

\section{Method/Apparatus/Procedure:}

Constant-temperature bath, calorimetric thermometer, and an ultraviolet/ visible spectrophotometer.

Excess solute and solvent were placed in amber glass bottles and allowed to equilibrate for several days at constant temperature. Attainment of equilibrium was verified by several repetitive measurements and by approaching equilibrium from supersaturation. Aliquots of saturated solutions were transferred through a coarse filter into tared volumetric flasks, weighed and diluted with methanol. Concentrations were determined by spectrophotometric measurements at $281 \mathrm{~nm}$. 
Source and Purity of Chemicals:

(1) $99 \%$, Acros Organics, USA, was used as received.

(2) $99.3 \%$, anhydrous, Aldrich Chemical Company, Milwaukee, WI, USA, stored over molecular sieves before use.

Estimated Error:

Temperature: $\pm 0.1 \mathrm{~K}$.

$x_{1}: \pm 1.5 \%$ (relative error).

\begin{tabular}{|c|c|}
\hline $\begin{array}{l}\text { Components: } \\
\text { (1) Phenothiazine; } \mathrm{C}_{12} \mathrm{H}_{9} \mathrm{NS} \text {; [92-84-2] } \\
\text { (2) 2-Methoxy-2-methylpropane; } \\
\mathrm{C}_{5} \mathrm{H}_{12} \mathrm{O} \text {; [1634-04-4] }\end{array}$ & $\begin{array}{l}\text { Original Measurements: } \\
{ }^{65} \text { K. R. Hoover, W. E. Acree, Jr., } \\
\text { and M. H. Abraham, Phys. Chem. } \\
\text { Liq. 44, } 367 \text { (2006). }\end{array}$ \\
\hline $\begin{array}{l}\text { Variables: } \\
T / \mathrm{K}=298.15\end{array}$ & $\begin{array}{l}\text { Prepared by: } \\
\text { W. E. Acree, Jr. }\end{array}$ \\
\hline
\end{tabular}

Experimental Values

\begin{tabular}{lcc}
\hline \hline$x_{2}{ }^{(\mathrm{s}) \mathrm{a}}$ & $x_{2}{ }^{\mathrm{b}}$ & $x_{1}{ }^{\mathrm{c}}$ \\
\hline 1.0000 & 0.9766 & 0.02339 \\
\hline
\end{tabular}

${ }^{\mathrm{a}} x_{2}{ }^{(\mathrm{s})}$ : initial mole fraction of component 2 in the solution.

${ }^{\mathrm{b}} x_{2}$ : mole fraction of component 2 in the saturated solution.

${ }^{\mathrm{c}} x_{1}$ : mole fraction solubility of the solute.

\section{Auxiliary Information}

\section{Method/Apparatus/Procedure:}

Constant-temperature bath, calorimetric thermometer, and an ultraviolet/ visible spectrophotometer.

Excess solute and solvent were placed in amber glass bottles and allowed to equilibrate for several days at constant temperature. Attainment of equilibrium was verified by several repetitive measurements and by approaching equilibrium from supersaturation. Aliquots of saturated solutions were transferred through a coarse filter into tared volumetric flasks, weighed and diluted with methanol. Concentrations were determined by

spectrophotometric measurements at $281 \mathrm{~nm}$.

Source and Purity of Chemicals:

(1) $99 \%$, Acros Organics, USA, was used as received.

(2) $99.9+\%$, Arco Chemical Company, USA, stored over molecular sieves

before use.

Estimated Error:

Temperature: $\pm 0.1 \mathrm{~K}$.

$x_{1}: \pm 1.5 \%$ (relative error).

\begin{tabular}{ll}
\hline \hline Components: & Original Measurements: \\
(1) Phenothiazine; $\mathrm{C}_{12} \mathrm{H}_{9} \mathrm{NS} ;$ [92-84-2] & ${ }^{66}$ M. Saifullah, S. Ye, L. M. \\
(2) Tetrahydrofuran; $\mathrm{C}_{4} \mathrm{H}_{8} \mathrm{O}$; [109-99-9] & Grubbs, N. E. De La Rosa, W. E. \\
& Acree, Jr., and M. H. Abraham, J. \\
& Solution Chem. 40, 2082 (2011). \\
\hline Variables: & Prepared by: \\
$T / \mathrm{K}=298.15$ & W. E. Acree, Jr. \\
\hline
\end{tabular}

Experimental Values

\begin{tabular}{lcc}
\hline \hline$x_{2}{ }^{(\mathrm{s}) \mathrm{a}}$ & $x_{2}{ }^{\mathrm{b}}$ & $x_{1}{ }^{\mathrm{c}}$ \\
\hline 1.0000 & 0.8388 & 0.1612 \\
\hline${ }^{\mathrm{a}} x_{2}{ }^{(\mathrm{s})}:$ initial mole fraction of component 2 in the solution. \\
${ }^{\mathrm{b}} x_{2}:$ mole fraction of component 2 in the saturated solution. \\
${ }^{\mathrm{c}} x_{1}:$ mole fraction solubility of the solute.
\end{tabular}

\section{Auxiliary Information}

\section{Method/Apparatus/Procedure:}

Constant-temperature bath, calorimetric thermometer, and an ultraviolet/ visible spectrophotometer.

Excess solute and solvent were placed in amber glass bottles and allowed to equilibrate for several days at constant temperature. Attainment of equilibrium was verified by several repetitive measurements and by approaching equilibrium from supersaturation. Aliquots of saturated solutions were transferred through a coarse filter into tared volumetric flasks, weighed and diluted with methanol. Concentrations were determined by spectrophotometric measurements at $281 \mathrm{~nm}$.

\section{Source and Purity of Chemicals:}

(1) $99 \%$, Acros Organics, USA, was used as received.

(2) $99.9 \%$, anhydrous, Aldrich Chemical Company, Milwaukee, WI, USA, stored over molecular sieves before use.

\section{Estimated Error:}

Temperature: $\pm 0.1 \mathrm{~K}$.

$x_{1}: \pm 1.5 \%$ (relative error).

\begin{tabular}{ll}
\hline \hline Components: & Original Measurements: \\
(1) Phenothiazine; $\mathrm{C}_{12} \mathrm{H}_{9} \mathrm{NS} ;[$ [92-84-2] & $\begin{array}{l}{ }^{65} \mathrm{~K} \text {. R. Hoover, W. E. Acree, Jr., } \\
\text { (2) 1,4-Dioxane; } \mathrm{C}_{4} \mathrm{H}_{8} \mathrm{O}_{2} ;[123-91-1]\end{array}$ \\
& $\begin{array}{l}\text { and M. H. Abraham, Phys. Chem. } \\
\text { Liq. 44, 367 (2006). }\end{array}$ \\
\hline Variables: & Prepared by: \\
$T / \mathrm{K}=298.15$ & W. E. Acree, Jr. \\
\hline
\end{tabular}

Experimental Values

\begin{tabular}{lcc}
\hline \hline$x_{2}{ }^{(\mathrm{s}) \mathrm{a}}$ & $x_{2}{ }^{\mathrm{b}}$ & $x_{1}{ }^{\mathrm{c}}$ \\
\hline 1.0000 & 0.8974 & 0.1026
\end{tabular}

${ }^{\mathrm{a}} x_{2}{ }^{(\mathrm{s})}$ : initial mole fraction of component 2 in the solution.

${ }^{\mathrm{b}} x_{2}$ : mole fraction of component 2 in the saturated solution.

${ }^{\mathrm{c}} x_{1}$ : mole fraction solubility of the solute.

\section{Auxiliary Information}

\section{Method/Apparatus/Procedure:}

Constant-temperature bath, calorimetric thermometer, and an ultraviolet/ visible spectrophotometer.

Excess solute and solvent were placed in amber glass bottles and allowed to equilibrate for several days at constant temperature. Attainment of equilibrium was verified by several repetitive measurements and by approaching equilibrium from supersaturation. Aliquots of saturated solutions were transferred through a coarse filter into tared volumetric flasks, weighed and diluted with methanol. Concentrations were determined by spectrophotometric measurements at $281 \mathrm{~nm}$. 
Source and Purity of Chemicals:

(1) $99 \%$, Acros Organics, USA, was used as received.

(2) $99.8 \%$, anhydrous, Aldrich Chemical Company, Milwaukee, WI, USA, stored over molecular sieves before use.

Estimated Error:

Temperature: $\pm 0.1 \mathrm{~K}$.

$x_{1}: \pm 1.5 \%$ (relative error).

\subsection{Phenothiazine solubility data in alcohols}

\begin{tabular}{ll}
\hline \hline Components: & Original Measurements: \\
(1) Phenothiazine; $\mathrm{C}_{12} \mathrm{H}_{9} \mathrm{NS} ;[$ [92-84-2] & ${ }^{65} \mathrm{~K}$. R. Hoover, W. E. Acree, Jr., \\
(2) Methanol; $\mathrm{CH}_{4} \mathrm{O} ;[67-56-1]$ & and M. H. Abraham, Phys. Chem. \\
& Liq. 44, 367 (2006). \\
\hline Variables: & Prepared by: \\
$T / \mathrm{K}=298.15$ & W. E. Acree, Jr. \\
\hline
\end{tabular}

Experimental Values

\begin{tabular}{lcc}
\hline \hline$x_{2}{ }^{(\mathrm{s}) \mathrm{a}}$ & $x_{2}{ }^{\mathrm{b}}$ & $x_{1}{ }^{\mathrm{c}}$ \\
\hline 1.0000 & 0.9949 & 0.00512 \\
\hline
\end{tabular}

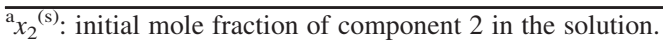

${ }^{b_{x_{2}}}$ : mole fraction of component 2 in the saturated solution.

${ }^{c} x_{1}$ : mole fraction solubility of the solute.

\section{Auxiliary Information}

\section{Method/Apparatus/Procedure:}

Constant-temperature bath, calorimetric thermometer, and an ultraviolet/ visible spectrophotometer.

Excess solute and solvent were placed in amber glass bottles and allowed to equilibrate for several days at constant temperature. Attainment of equilibrium was verified by several repetitive measurements and by approaching equilibrium from supersaturation. Aliquots of saturated solutions were transferred through a coarse filter into tared volumetric flasks, weighed and diluted with methanol. Concentrations were determined by spectrophotometric measurements at $281 \mathrm{~nm}$.

\section{Source and Purity of Chemicals:}

(1) $99 \%$, Acros Organics, USA, was used as received.

(2) $99.8 \%$, anhydrous, Aldrich Chemical Company, Milwaukee, WI, USA, stored over molecular sieves before use.

\section{Estimated Error:}

Temperature: $\pm 0.1 \mathrm{~K}$

$x_{1}: \pm 1.5 \%$ (relative error)

\begin{tabular}{ll}
\hline \hline Components: & Original Measurements: \\
(1) Phenothiazine; $\mathrm{C}_{12} \mathrm{H}_{9} \mathrm{NS} ;[92-84-2]$ & ${ }^{65} \mathrm{~K}$. R. Hoover, W. E. Acree, Jr., \\
(2) Ethanol; $\mathrm{C}_{2} \mathrm{H}_{6} \mathrm{O} ;[64-17-5]$ & and M. H. Abraham, Phys. Chem. \\
& Liq. 44, 367 (2006). \\
\hline Variables: & Prepared by: \\
$T / \mathrm{K}=298.15$ & W. E. Acree, Jr. \\
\hline
\end{tabular}

Experimental Values

\begin{tabular}{|c|c|c|}
\hline$x_{2}{ }^{(\mathrm{s}) \mathrm{a}}$ & $x_{2}{ }^{b}$ & $x_{1}{ }^{\mathrm{c}}$ \\
\hline 1.0000 & 0.9911 & 0.00890 \\
\hline
\end{tabular}

\section{Auxiliary Information}

\section{Method/Apparatus/Procedure:}

Constant-temperature bath, calorimetric thermometer, and an ultraviolet/ visible spectrophotometer.

Excess solute and solvent were placed in amber glass bottles and allowed to equilibrate for several days at constant temperature. Attainment of equilibrium was verified by several repetitive measurements and by approaching equilibrium from supersaturation. Aliquots of saturated solutions were transferred through a coarse filter into tared volumetric flasks, weighed and diluted with methanol. Concentrations were determined by spectrophotometric measurements at $281 \mathrm{~nm}$.

Source and Purity of Chemicals:

(1) $99 \%$, Acros Organics, USA, was used as received.

(2) Absolute, Aapar Alcohol and Chemical Company, USA, stored over molecular sieves before use.

\section{Estimated Error:}

Temperature: $\pm 0.1 \mathrm{~K}$.

$x_{1}: \pm 1.5 \%$ (relative error)

\begin{tabular}{ll}
\hline \hline Components: & $\begin{array}{l}\text { Original Measurements: } \\
\text { (1) Phenothiazine; } \mathrm{C}_{12} \mathrm{H}_{9} \mathrm{NS} ;[\text { [92-84-2] }\end{array}$ \\
$\begin{array}{l}{ }^{67} \text { S. Ahmadian, V. Panahi-Azar, } \\
\text { (2) Ethanol; } \mathrm{C}_{2} \mathrm{H}_{6} \mathrm{O} ;[64-17-5]\end{array}$ & $\begin{array}{l}\text { M. A. A. Fakhree, W. E. Acree, Jr., } \\
\text { and A. Jouyban, J. Chem. Eng. }\end{array}$ \\
& Data 56, 4352 (2011). \\
\hline Variables: & Prepared by: \\
Temperature & W. E. Acree, Jr. \\
\hline
\end{tabular}

Experimental Values

\begin{tabular}{lcc}
\hline \hline$T / \mathrm{K}$ & $x_{2}{ }^{\mathrm{a}}$ & $x_{1}{ }^{\mathrm{b}}$ \\
\hline 298.2 & 0.9912 & 0.00876 \\
308.2 & 0.9900 & 0.0100 \\
318.2 & 0.9878 & 0.0122 \\
328.2 & 0.9855 & 0.0145 \\
\hline${ }^{a} x_{2}:$ mole fraction of component 2 in the saturated solution. \\
${ }^{\mathrm{b}} x_{1}:$ mole fraction of the polycyclic aromatic hydrocarbon (component 1$)$.
\end{tabular}

\section{Auxiliary Information}

\section{Method/Apparatus/Procedure:}

Incubator, shaker, and an ultraviolet/visible spectrophotometer.

Solubility was determined by equilibrating an excess amount of solid naphthalene with the organic solvent using a shaker placed in an incubator equipped with a temperature controlling system. After an equilibration period of at least $48 \mathrm{~h}$, the samples were withdrawn and filtered through hydrophobic Durapore filters $(0.45 \mu \mathrm{m})$. The filtered sample was diluted quantitatively with methanol. Concentrations were determined by spectrophotmetric analysis at $356 \mathrm{~nm}$. 
Source and Purity of Chemicals:

(1) $98 \%$, Merck Chemicals, Germany, was recrystallized from propanone before use.

(2) $99.9 \%$, Scharlau Chemie, Spain, no information given regarding any further purification.

\section{Estimated Error:}

Temperature: $\pm 0.2 \mathrm{~K}$ to $\pm 0.6 \mathrm{~K}$, the higher temperatures have greater uncertainty.

$x_{1}: \pm 2.3 \%$ (relative uncertainty).

\begin{tabular}{|c|c|}
\hline $\begin{array}{l}\text { Components: } \\
\text { (1) Phenothiazine; } \mathrm{C}_{12} \mathrm{H}_{9} \mathrm{NS} \text {; [92-84-2] } \\
\text { (2) 1-Propanol; } \mathrm{C}_{3} \mathrm{H}_{8} \mathrm{O} ;[71-23-8]\end{array}$ & $\begin{array}{l}\text { Original Measurements: } \\
{ }^{65} \text { K. R. Hoover, W. E. Acree, Jr., } \\
\text { and M. H. Abraham, Phys. Chem. } \\
\text { Liq. 44, } 367 \text { (2006). }\end{array}$ \\
\hline $\begin{array}{l}\text { Variables: } \\
T / \mathrm{K}=298.15\end{array}$ & $\begin{array}{l}\text { Prepared by: } \\
\text { W. E. Acree, Jr. }\end{array}$ \\
\hline
\end{tabular}

Experimental Values

\begin{tabular}{|c|c|c|}
\hline$x_{2}{ }^{(\mathrm{s}) \mathrm{a}}$ & $x_{2}{ }^{\mathrm{b}}$ & $x_{1}{ }^{\mathrm{c}}$ \\
\hline 1.0000 & 0.9911 & 0.00885 \\
\hline
\end{tabular}

\section{Auxiliary Information}

\section{Method/Apparatus/Procedure:}

Constant-temperature bath, calorimetric thermometer, and an ultraviolet/ visible spectrophotometer.

Excess solute and solvent were placed in amber glass bottles and allowed to equilibrate for several days at constant temperature. Attainment of equilibrium was verified by several repetitive measurements and by approaching equilibrium from supersaturation. Aliquots of saturated solutions were transferred through a coarse filter into tared volumetric flasks, weighed and diluted with methanol. Concentrations were determined by spectrophotometric measurements at $281 \mathrm{~nm}$.

\section{Source and Purity of Chemicals:}

(1) $99 \%$, Acros Organics, USA, was used as received.

(2) 99+\%, anhydrous, Aldrich Chemical Company, Milwaukee, WI, USA, stored over molecular sieves before use.

\section{Estimated Error:}

Temperature: $\pm 0.1 \mathrm{~K}$

$x_{1}: \pm 1.5 \%$ (relative error).

\begin{tabular}{|c|c|}
\hline $\begin{array}{l}\text { Components: } \\
\text { (1) Phenothiazine; } \mathrm{C}_{12} \mathrm{H}_{9} \mathrm{NS} \text {; [92-84-2] } \\
\text { (2) 2-Propanol; } \mathrm{C}_{3} \mathrm{H}_{8} \mathrm{O} ;[67-63-0]\end{array}$ & $\begin{array}{l}\text { Original Measurements: } \\
{ }^{65} \text { K. R. Hoover, W. E. Acree, Jr., } \\
\text { and M. H. Abraham, Phys. Chem. } \\
\text { Liq. 44, } 367 \text { (2006). }\end{array}$ \\
\hline $\begin{array}{l}\text { Variables: } \\
T / \mathrm{K}=298 .\end{array}$ & $\begin{array}{l}\text { Prepared by: } \\
\text { W E Acree }\end{array}$ \\
\hline
\end{tabular}

Experimental Values

\begin{tabular}{|c|c|c|}
\hline$x_{2}{ }^{(\mathrm{s}) \mathrm{a}}$ & $x_{2}{ }^{b}$ & $x_{1}{ }^{\mathrm{c}}$ \\
\hline 1.0000 & 0.9940 & 0.00600 \\
\hline
\end{tabular}

\section{Auxiliary Information}

\section{Method/Apparatus/Procedure:}

Constant-temperature bath, calorimetric thermometer, and an ultraviolet/ visible spectrophotometer.

Excess solute and solvent were placed in amber glass bottles and allowed to equilibrate for several days at constant temperature. Attainment of equilibrium was verified by several repetitive measurements and by approaching equilibrium from supersaturation. Aliquots of saturated solutions were transferred through a coarse filter into tared volumetric flasks, weighed and diluted with methanol. Concentrations were determined by spectrophotometric measurements at $281 \mathrm{~nm}$.

Source and Purity of Chemicals:

(1) $99 \%$, Acros Organics, USA, was used as received.

(2) $99+\%$, anhydrous, Aldrich Chemical Company, Milwaukee, WI, USA, stored over molecular sieves before use.

\section{Estimated Error:}

Temperature: $\pm 0.1 \mathrm{~K}$.

$x_{1}: \pm 1.5 \%$ (relative error).

\begin{tabular}{|c|c|}
\hline $\begin{array}{l}\text { Components: } \\
\text { (1) Phenothiazine; } \mathrm{C}_{12} \mathrm{H}_{9} \mathrm{NS} \text {; [92-84-2] } \\
\text { (2) 1-Butanol; } \mathrm{C}_{4} \mathrm{H}_{10} \mathrm{O} ;[71-36-3]\end{array}$ & $\begin{array}{l}\text { Original Measurements: } \\
{ }^{65} \text { K. R. Hoover, W. E. Acree, Jr., } \\
\text { and M. H. Abraham, Phys. Chem. } \\
\text { Liq. 44, } 367 \text { (2006). }\end{array}$ \\
\hline Variables: & Prepared by: \\
\hline$T / \mathrm{K}=298.15$ & W. E. Acree, Jr. \\
\hline
\end{tabular}

Experimental Values

\begin{tabular}{lcc}
\hline \hline$x_{2}{ }^{(\mathrm{s}) \mathrm{a}}$ & $x_{2}{ }^{\mathrm{b}}$ & $x_{1}{ }^{\mathrm{c}}$ \\
\hline 1.0000 & 0.9890 & 0.01099
\end{tabular}

${ }^{\mathrm{a}} x_{2}{ }^{(\mathrm{s})}$ : initial mole fraction of component 2 in the solution.

${ }^{\mathrm{b}} x_{2}$ : mole fraction of component 2 in the saturated solution.

${ }^{c} x_{1}$ : mole fraction solubility of the solute.

\section{Auxiliary Information}

\section{Method/Apparatus/Procedure:}

Constant-temperature bath, calorimetric thermometer, and an ultraviolet/ visible spectrophotometer.

Excess solute and solvent were placed in amber glass bottles and allowed to equilibrate for several days at constant temperature. Attainment of equilibrium was verified by several repetitive measurements and by approaching equilibrium from supersaturation. Aliquots of saturated solutions were transferred through a coarse filter into tared volumetric flasks, weighed and diluted with methanol. Concentrations were determined by spectrophotometric measurements at $281 \mathrm{~nm}$. 
Source and Purity of Chemicals:

(1) $99 \%$, Acros Organics, USA, was used as received.

(2) $99.8+\%$, HPLC Grade, Aldrich Chemical Company, Milwaukee, WI, USA, stored over molecular sieves before use.

Estimated Error:

Temperature: $\pm 0.1 \mathrm{~K}$.

$x_{1}: \pm 1.5 \%$ (relative error)

\begin{tabular}{|c|c|}
\hline $\begin{array}{l}\text { Components: } \\
\text { (1) Phenothiazine; } \mathrm{C}_{12} \mathrm{H}_{9} \mathrm{NS} ;[92-84-2] \\
\text { (2) 2-Butanol; } \mathrm{C}_{4} \mathrm{H}_{10} \mathrm{O} ;[78-92-2]\end{array}$ & $\begin{array}{l}\text { Original Measurements: } \\
{ }^{65} \text { K. R. Hoover, W. E. Acree, Jr., } \\
\text { and M. H. Abraham, Phys. Chem. } \\
\text { Liq. 44, } 367 \text { (2006). }\end{array}$ \\
\hline $\begin{array}{l}\text { Variables: } \\
T / \mathrm{K}=298.15\end{array}$ & $\begin{array}{l}\text { Prepared by: } \\
\text { W. E. Acree, Jr. }\end{array}$ \\
\hline
\end{tabular}

Experimental Values

\begin{tabular}{lcc}
\hline \hline$x_{2}{ }^{(\mathrm{s}) \mathrm{a}}$ & $x_{2}{ }^{\mathrm{b}}$ & $x_{1}{ }^{\mathrm{c}}$ \\
\hline 1.0000 & 0.9927 & 0.00732 \\
\hline
\end{tabular}

${ }^{\mathrm{a}} x_{2}{ }^{(\mathrm{s})}$ : initial mole fraction of component 2 in the solution.

${ }^{\mathrm{b}} x_{2}$ : mole fraction of component 2 in the saturated solution.

${ }^{\mathrm{c}} x_{1}$ : mole fraction solubility of the solute.

\section{Auxiliary Information}

\section{Method/Apparatus/Procedure:}

Constant-temperature bath, calorimetric thermometer, and an ultraviolet/ visible spectrophotometer.

Excess solute and solvent were placed in amber glass bottles and allowed to equilibrate for several days at constant temperature. Attainment of equilibrium was verified by several repetitive measurements and by approaching equilibrium from supersaturation. Aliquots of saturated solutions were transferred through a coarse filter into tared volumetric flasks, weighed and diluted with methanol. Concentrations were determined by

spectrophotometric measurements at $281 \mathrm{~nm}$.

Source and Purity of Chemicals:

(1) $99 \%$, Acros Organics, USA, was used as received.

(2) $99+\%$, anhydrous, Aldrich Chemical Company, Milwaukee, WI, USA, stored over molecular sieves before use.

\section{Estimated Error:}

Temperature: $\pm 0.1 \mathrm{~K}$.

$x_{1}: \pm 1.5 \%$ (relative error).

Experimental Values

\begin{tabular}{|c|c|c|}
\hline$x_{2}{ }^{(\mathrm{s}) \mathrm{a}}$ & $x_{2}{ }^{b}$ & $x_{1}{ }^{\mathrm{c}}$ \\
\hline 1.0000 & 0.9947 & 0.00534 \\
\hline
\end{tabular}

\section{Auxiliary Information}

\section{Method/Apparatus/Procedure:}

Constant-temperature bath, calorimetric thermometer, and an ultraviolet/ visible spectrophotometer.

Excess solute and solvent were placed in amber glass bottles and allowed to equilibrate for several days at constant temperature. Attainment of equilibrium was verified by several repetitive measurements and by approaching equilibrium from supersaturation. Aliquots of saturated solutions were transferred through a coarse filter into tared volumetric flasks, weighed and diluted with methanol. Concentrations were determined by spectrophotometric measurements at $281 \mathrm{~nm}$.

\section{Source and Purity of Chemicals:}

(1) $99 \%$, Acros Organics, USA, was used as received.

(2) $99+\%$, anhydrous, Aldrich Chemical Company, Milwaukee, WI, USA, stored over molecular sieves before use.

\section{Estimated Error:}

Temperature: $\pm 0.1 \mathrm{~K}$.

$x_{1}: \pm 1.5 \%$ (relative error)

\begin{tabular}{|c|c|}
\hline $\begin{array}{l}\text { Components: } \\
\text { (1) Phenothiazine; } \mathrm{C}_{12} \mathrm{H}_{9} \mathrm{NS} \text {; [92-84-2] } \\
\text { (2) 2-Methyl-2-propanol; } \mathrm{C}_{4} \mathrm{H}_{10} \mathrm{O} \text {; } \\
\text { [75-65-0] }\end{array}$ & $\begin{array}{l}\text { Original Measurements: } \\
{ }^{65} \text { K. R. Hoover, W. E. Acree, Jr., } \\
\text { and M. H. Abraham, Phys. Chem. } \\
\text { Liq. 44, } 367 \text { (2006). }\end{array}$ \\
\hline $\begin{array}{l}\text { Variables: } \\
T / \mathrm{K}=298.15\end{array}$ & $\begin{array}{l}\text { Prepared by: } \\
\text { W. E. Acree, Jr. }\end{array}$ \\
\hline
\end{tabular}

Experimental Values

\begin{tabular}{lcc}
\hline \hline$x_{2}{ }^{(\mathrm{s}) \mathrm{a}}$ & $x_{2}{ }^{\mathrm{b}}$ & $x_{1}{ }^{\mathrm{c}}$ \\
\hline 1.0000 & 0.9942 & 0.00583
\end{tabular}

${ }^{\mathrm{a}} x_{2}{ }^{(\mathrm{s})}$ : initial mole fraction of component 2 in the solution.

${ }^{\mathrm{b}} x_{2}$ : mole fraction of component 2 in the saturated solution.

${ }^{c} x_{1}$ : mole fraction solubility of the solute.

\section{Auxiliary Information}

\section{Components:}

(1) Phenothiazine; $\mathrm{C}_{12} \mathrm{H}_{9} \mathrm{NS}$; [92-84-2]

(2) 2-Methyl-1-propanol; $\mathrm{C}_{4} \mathrm{H}_{10} \mathrm{O}$;

[78-83-1]

\begin{tabular}{ll}
\hline Variables: & Prepared by: \\
$T / \mathrm{K}=298.15$ & W. E. Acree, Jr. \\
\hline
\end{tabular}

Original Measurements:

${ }^{65}$ K. R. Hoover, W. E. Acree, Jr., and M. H. Abraham, Phys. Chem. Liq. 44, 367 (2006).

Prepared by:

\section{Method/Apparatus/Procedure:}

Constant-temperature bath, calorimetric thermometer, and an ultraviolet/ visible spectrophotometer.

Excess solute and solvent were placed in amber glass bottles and allowed to equilibrate for several days at constant temperature. Attainment of equilibrium was verified by several repetitive measurements and by approaching equilibrium from supersaturation. Aliquots of saturated solutions were transferred through a coarse filter into tared volumetric flasks, weighed and diluted with methanol. Concentrations were determined by spectrophotometric measurements at $281 \mathrm{~nm}$. 
Source and Purity of Chemicals:

(1) $99 \%$, Acros Organics, USA, was used as received.

(2) $99 \%$, Arco Chemical Company, USA, stored over molecular sieves before use.

Estimated Error:

Temperature: $\pm 0.1 \mathrm{~K}$.

$x_{1}: \pm 1.5 \%$ (relative error).

\begin{tabular}{ll}
\hline \hline Components: & Original Measurements: \\
(1) Phenothiazine; $\mathrm{C}_{12} \mathrm{H}_{9} \mathrm{NS} ;$ [92-84-2] & ${ }^{65} \mathrm{~K}$. R. Hoover, W. E. Acree, Jr., \\
(2) 1-Pentanol; $\mathrm{C}_{5} \mathrm{H}_{12} \mathrm{O} ;$ [71-41-0] & and M. H. Abraham, Phys. Chem. \\
& Liq. 44, 367 (2006). \\
\hline Variables: & Prepared by: \\
$T / \mathrm{K}=298.15$ & W. E. Acree, Jr. \\
\hline
\end{tabular}

Experimental Values

\begin{tabular}{lcc}
\hline \hline$x_{2}{ }^{(\mathrm{s}) \mathrm{a}}$ & $x_{2}{ }^{\mathrm{b}}$ & $x_{1}{ }^{\mathrm{c}}$ \\
\hline 1.0000 & 0.9866 & 0.01339 \\
\hline
\end{tabular}

${ }^{\mathrm{a}} x_{2}{ }^{(\mathrm{s})}$ : initial mole fraction of component 2 in the solution.

${ }^{\mathrm{b}} x_{2}$ : mole fraction of component 2 in the saturated solution.

${ }^{\mathrm{c}} x_{1}$ : mole fraction solubility of the solute.

\section{Auxiliary Information}

\section{Method/Apparatus/Procedure:}

Constant-temperature bath, calorimetric thermometer, and an ultraviolet/ visible spectrophotometer.

Excess solute and solvent were placed in amber glass bottles and allowed to equilibrate for several days at constant temperature. Attainment of equilibrium was verified by several repetitive measurements and by approaching equilibrium from supersaturation. Aliquots of saturated solutions were transferred through a coarse filter into tared volumetric flasks, weighed and diluted with methanol. Concentrations were determined by

spectrophotometric measurements at $281 \mathrm{~nm}$.

Source and Purity of Chemicals:

(1) $99 \%$, Acros Organics, USA, was used as received.

(2) $99+\%$, Aldrich Chemical Company, Milwaukee, WI, USA, stored over molecular sieves before use.

\section{Estimated Error:}

Temperature: $\pm 0.1 \mathrm{~K}$.

$x_{1}: \pm 1.5 \%$ (relative error).

Experimental Values

\begin{tabular}{lcc}
\hline \hline$x_{2}{ }^{(\mathrm{s}) \mathrm{a}}$ & $x_{2}{ }^{\mathrm{b}}$ & $x_{1}{ }^{\mathrm{c}}$ \\
\hline 1.0000 & 0.9927 & 0.00726 \\
$\frac{{ }^{a} x_{2}{ }^{(\mathrm{s})}: \text { initial mole fraction of component } 2 \text { in the solution. }}{{ }^{\mathrm{b}} x_{2}: \text { mole fraction of component } 2 \text { in the saturated solution. }}$ \\
${ }^{c} x_{1}:$ mole fraction solubility of the solute.
\end{tabular}

\section{Auxiliary Information}

\section{Method/Apparatus/Procedure:}

Constant-temperature bath, calorimetric thermometer, and an ultraviolet/ visible spectrophotometer.

Excess solute and solvent were placed in amber glass bottles and allowed to equilibrate for several days at constant temperature. Attainment of equilibrium was verified by several repetitive measurements and by approaching equilibrium from supersaturation. Aliquots of saturated solutions were transferred through a coarse filter into tared volumetric flasks, weighed and diluted with methanol. Concentrations were determined by spectrophotometric measurements at $281 \mathrm{~nm}$.

\section{Source and Purity of Chemicals:}

(1) $99 \%$, Acros Organics, USA, was used as received.

(2) $99 \%$, Aldrich Chemical Company, Milwaukee, WI, USA, stored over molecular sieves before use.

\section{Estimated Error:}

Temperature: $\pm 0.1 \mathrm{~K}$.

$x_{1}: \pm 1.5 \%$ (relative error).

\begin{tabular}{|c|c|}
\hline $\begin{array}{l}\text { Components: } \\
\text { (1) Phenothiazine; } \mathrm{C}_{12} \mathrm{H}_{9} \mathrm{NS} \text {; [92-84-2] } \\
\text { (2) 3-Methyl-1-butanol; } \mathrm{C}_{5} \mathrm{H}_{12} \mathrm{O} \text {; } \\
\text { [123-51-3] }\end{array}$ & $\begin{array}{l}\text { Original Measurements: } \\
{ }^{65} \text { K. R. Hoover, W. E. Acree, Jr., } \\
\text { and M. H. Abraham, Phys. Chem. } \\
\text { Liq. 44, } 367 \text { (2006). }\end{array}$ \\
\hline $\begin{array}{l}\text { Variables: } \\
T / \mathrm{K}=298.15\end{array}$ & $\begin{array}{l}\text { Prepared by: } \\
\text { W. E. Acree, Jr. }\end{array}$ \\
\hline
\end{tabular}

Experimental Values

\begin{tabular}{lcc}
\hline \hline$x_{2}{ }^{(\mathrm{s}) \mathrm{a}}$ & $x_{2}{ }^{\mathrm{b}}$ & $x_{1}{ }^{\mathrm{c}}$ \\
\hline 1.0000 & 0.9910 & 0.00896
\end{tabular}

${ }^{\mathrm{a}} x_{2}{ }^{(\mathrm{s})}$ : initial mole fraction of component 2 in the solution.

${ }^{\mathrm{b}} x_{2}$ : mole fraction of component 2 in the saturated solution.

${ }^{\mathrm{c}} x_{1}$ : mole fraction solubility of the solute.

\section{Auxiliary Information}

\section{Components:}

(1) Phenothiazine; $\mathrm{C}_{12} \mathrm{H}_{9} \mathrm{NS}$; [92-84-2]

(2) 2-Methyl-1-butanol; $\mathrm{C}_{5} \mathrm{H}_{12} \mathrm{O}$;

[132-32-6]

\begin{tabular}{ll}
\hline Variables: & Prepared by: \\
$T / \mathrm{K}=298.15$ & W. E. Acree, Jr. \\
\hline
\end{tabular}

\section{Original Measurements:}

${ }^{65}$ K. R. Hoover, W. E. Acree, Jr., and M. H. Abraham, Phys. Chem. Liq. 44, 367 (2006).

Prepared by:
W. E. Acree, Jr

\section{Method/Apparatus/Procedure:}

Constant-temperature bath, calorimetric thermometer, and an ultraviolet/ visible spectrophotometer.

Excess solute and solvent were placed in amber glass bottles and allowed to equilibrate for several days at constant temperature. Attainment of equilibrium was verified by several repetitive measurements and by approaching equilibrium from supersaturation. Aliquots of saturated solutions were transferred through a coarse filter into tared volumetric flasks, weighed and diluted with methanol. Concentrations were determined by spectrophotometric measurements at $281 \mathrm{~nm}$. 
Source and Purity of Chemicals:

(1) $99 \%$, Acros Organics, USA, was used as received.

(2) $99 \%$, anhydrous, Aldrich Chemical Company, Milwaukee, WI, USA, stored over molecular sieves before use.

Estimated Error:

Temperature: $\pm 0.1 \mathrm{~K}$.

$x_{1}: \pm 1.5 \%$ (relative error).

\begin{tabular}{ll}
\hline \hline Components: & Original Measurements: \\
(1) Phenothiazine; $\mathrm{C}_{12} \mathrm{H}_{9} \mathrm{NS} ;[$ [92-84-2] & ${ }^{65} \mathrm{~K}$. R. Hoover, W. E. Acree, Jr., \\
(2) 2-Pentanol; $\mathrm{C}_{5} \mathrm{H}_{12} \mathrm{O} ;[6032-29-7]$ & and M. H. Abraham, Phys. Chem. \\
& Liq. 44, 367 (2006). \\
\hline Variables: & Prepared by: \\
$T / \mathrm{K}=298.15$ & W. E. Acree, Jr. \\
\hline
\end{tabular}

Experimental Values

\begin{tabular}{|c|c|c|}
\hline$x_{2}^{(\mathrm{s}) \mathrm{a}}$ & $x_{2}{ }^{\mathrm{b}}$ & $x_{1}^{\mathrm{c}}$ \\
\hline 1.0000 & 0.9913 & 0.00871 \\
\hline
\end{tabular}

\section{Auxiliary Information}

\section{Method/Apparatus/Procedure:}

Constant-temperature bath, calorimetric thermometer, and an ultraviolet/ visible spectrophotometer.

Excess solute and solvent were placed in amber glass bottles and allowed to equilibrate for several days at constant temperature. Attainment of equilibrium was verified by several repetitive measurements and by approaching equilibrium from supersaturation. Aliquots of saturated solutions were transferred through a coarse filter into tared volumetric flasks, weighed and diluted with methanol. Concentrations were determined by

spectrophotometric measurements at $281 \mathrm{~nm}$.

Source and Purity of Chemicals:

(1) $99 \%$, Acros Organics, USA, was used as received.

(2) $99+\%$, Acros Organics, USA, stored over molecular sieves before use.

\section{Estimated Error:}

Temperature: $\pm 0.1 \mathrm{~K}$

$x_{1}: \pm 1.5 \%$ (relative error).

\section{Components:}

(2) 1-Hexanol; $\mathrm{C}_{6} \mathrm{H}_{14} \mathrm{O} ;[111-27-3]$
(1) Phenothiazine; $\mathrm{C}_{12} \mathrm{H}_{9} \mathrm{NS}$; [92-84-2]

Original Measurements:

${ }^{65}$ K. R. Hoover, W. E. Acree, Jr., and M. H. Abraham, Phys. Chem. Liq. 44, 367 (2006).

\section{Variables:}

$T / \mathrm{K}=298.15$

\section{Prepared by:}

W. E. Acree, Jr.
Experimental Values

\begin{tabular}{|c|c|c|}
\hline$x_{2}^{(\mathrm{s}) \mathrm{a}}$ & $x_{2}{ }^{\mathrm{b}}$ & $x_{1}{ }^{\mathrm{c}}$ \\
\hline 1.0000 & 0.9844 & 0.01562 \\
\hline
\end{tabular}

\section{Auxiliary Information}

\section{Method/Apparatus/Procedure:}

Constant-temperature bath, calorimetric thermometer, and an ultraviolet/ visible spectrophotometer.

Excess solute and solvent were placed in amber glass bottles and allowed to equilibrate for several days at constant temperature. Attainment of equilibrium was verified by several repetitive measurements and by approaching equilibrium from supersaturation. Aliquots of saturated solutions were transferred through a coarse filter into tared volumetric flasks, weighed and diluted with methanol. Concentrations were determined by spectrophotometric measurements at $281 \mathrm{~nm}$.

\section{Source and Purity of Chemicals:}

(1) $99 \%$, Acros Organics, USA, was used as received.

(2) $99+\%$, Alfa Aesar, USA, stored over molecular sieves before use.

Estimated Error:

Temperature: $\pm 0.1 \mathrm{~K}$.

$x_{1}: \pm 1.5 \%$ (relative error).

\begin{tabular}{|c|c|}
\hline $\begin{array}{l}\text { Components: } \\
\text { (1) Phenothiazine; } \mathrm{C}_{12} \mathrm{H}_{9} \mathrm{NS} ;[92-84-2] \\
\text { (2) 2-Methyl-1-pentanol; } \mathrm{C}_{6} \mathrm{H}_{14} \mathrm{O} \text {; } \\
\text { [105-30-6] }\end{array}$ & $\begin{array}{l}\text { Original Measurements: } \\
{ }^{65} \text { K. R. Hoover, W. E. Acree, Jr., } \\
\text { and M. H. Abraham, Phys. Chem. } \\
\text { Liq. 44, } 367 \text { (2006). }\end{array}$ \\
\hline $\begin{array}{l}\text { Variables: } \\
T / \mathrm{K}=298.15\end{array}$ & $\begin{array}{l}\text { Prepared by: } \\
\text { W. E. Acree, Jr. }\end{array}$ \\
\hline
\end{tabular}

Experimental Values

\begin{tabular}{lcc}
\hline \hline$x_{2}{ }^{(\mathrm{s}) \mathrm{a}}$ & $x_{2}{ }^{\mathrm{b}}$ & $x_{1}{ }^{\mathrm{c}}$ \\
\hline 1.0000 & 0.9913 & 0.00866 \\
${ }^{\mathrm{a}} x_{2}{ }^{(\mathrm{s})}:$ initial mole fraction of component 2 in the solution. \\
${ }^{\mathrm{b}} x_{2}:$ mole fraction of component 2 in the saturated solution. \\
${ }^{\mathrm{c}} x_{1}:$ mole fraction solubility of the solute.
\end{tabular}

\section{Auxiliary Information}

\section{Method/Apparatus/Procedure:}

Constant-temperature bath, calorimetric thermometer, and an ultraviolet/ visible spectrophotometer.

Excess solute and solvent were placed in amber glass bottles and allowed to equilibrate for several days at constant temperature. Attainment of equilibrium was verified by several repetitive measurements and by approaching equilibrium from supersaturation. Aliquots of saturated solutions were transferred through a coarse filter into tared volumetric flasks, weighed and diluted with methanol. Concentrations were determined by spectrophotometric measurements at $281 \mathrm{~nm}$. 
Source and Purity of Chemicals:

(1) $99 \%$, Acros Organics, USA, was used as received.

(2) $99 \%$, Aldrich Chemical Company, Milwaukee, WI, USA, stored over molecular sieves before use.

Estimated Error:

Temperature: $\pm 0.1 \mathrm{~K}$.

$x_{1}: \pm 1.5 \%$ (relative error)

\begin{tabular}{|c|c|}
\hline $\begin{array}{l}\text { Components: } \\
\text { (1) Phenothiazine; } \mathrm{C}_{12} \mathrm{H}_{9} \mathrm{NS} \text {; [92-84-2] } \\
\text { (2) 4-Methyl-2-pentanol; } \mathrm{C}_{6} \mathrm{H}_{14} \mathrm{O} \text {; } \\
\text { [108-11-2] }\end{array}$ & $\begin{array}{l}\text { Original Measurements: } \\
{ }^{65} \text { K. R. Hoover, W. E. Acree, Jr., } \\
\text { and M. H. Abraham, Phys. Chem. } \\
\text { Liq. 44, } 367 \text { (2006). }\end{array}$ \\
\hline $\begin{array}{l}\text { Variables: } \\
T / \mathrm{K}=298.15\end{array}$ & $\begin{array}{l}\text { Prepared by: } \\
\text { W. E. Acree, Jr. }\end{array}$ \\
\hline
\end{tabular}

Experimental Values

\begin{tabular}{|c|c|c|}
\hline$x_{2}^{(\mathrm{s}) \mathrm{a}}$ & $x_{2}{ }^{\mathrm{b}}$ & $x_{1}^{\mathrm{c}}$ \\
\hline 1.0000 & 0.9927 & 0.00728 \\
\hline
\end{tabular}

\section{Auxiliary Information}

\section{Method/Apparatus/Procedure:}

Constant-temperature bath, calorimetric thermometer, and an ultraviolet/ visible spectrophotometer.

Excess solute and solvent were placed in amber glass bottles and allowed to equilibrate for several days at constant temperature. Attainment of equilibrium was verified by several repetitive measurements and by approaching equilibrium from supersaturation. Aliquots of saturated solutions were transferred through a coarse filter into tared volumetric flasks, weighed and diluted with methanol. Concentrations were determined by

spectrophotometric measurements at $281 \mathrm{~nm}$.

Source and Purity of Chemicals:

(1) $99 \%$, Acros Organics, USA, was used as received.

(2) $99+\%$, Acros Organics, USA, stored over molecular sieves before use.

\section{Estimated Error:}

Temperature: $\pm 0.1 \mathrm{~K}$.

$x_{1}: \pm 1.5 \%$ (relative error).

\section{Components:}

(2) Heptanol; $\mathrm{C}_{7} \mathrm{H}_{16} \mathrm{O}$; [111-70-6]
(1) Phenothiazine; $\mathrm{C}_{12} \mathrm{H}_{9} \mathrm{NS}$; [92-84-2]

Original Measurements:

${ }^{65}$ K. R. Hoover, W. E. Acree, Jr., and M. H. Abraham, Phys. Chem. Liq. 44, 367 (2006).

\section{Variables:}

$T / \mathrm{K}=298.15$

\section{Prepared by:}

W. E. Acree, Jr.
Experimental Values

\begin{tabular}{|c|c|c|}
\hline$x_{2}{ }^{(\mathrm{s}) \mathrm{a}}$ & $x_{2}{ }^{b}$ & $x_{1}{ }^{\mathrm{c}}$ \\
\hline 1.0000 & 0.9825 & 0.01754 \\
\hline
\end{tabular}

\section{Auxiliary Information}

\section{Method/Apparatus/Procedure:}

Constant-temperature bath, calorimetric thermometer, and an ultraviolet/ visible spectrophotometer.

Excess solute and solvent were placed in amber glass bottles and allowed to equilibrate for several days at constant temperature. Attainment of equilibrium was verified by several repetitive measurements and by approaching equilibrium from supersaturation. Aliquots of saturated solutions were transferred through a coarse filter into tared volumetric flasks, weighed and diluted with methanol. Concentrations were determined by spectrophotometric measurements at $281 \mathrm{~nm}$.

\section{Source and Purity of Chemicals:}

(1) $99 \%$, Acros Organics, USA, was used as received.

(2) $99+\%$, Alfa Aesar, USA, stored over molecular sieves before use.

Estimated Error:

Temperature: $\pm 0.1 \mathrm{~K}$.

$x_{1}: \pm 1.5 \%$ (relative error)

\begin{tabular}{ll}
\hline \hline Components: & $\begin{array}{l}\text { Original Measurements: } \\
\text { (1) Phenothiazine; } \mathrm{C}_{12} \mathrm{H}_{9} \mathrm{NS} ;[\text { [92-84-2] }\end{array}$ \\
$\begin{array}{ll}{ }^{65} \mathrm{~K} . \mathrm{R} . \text { Hoover, W. E. Acree, Jr., } \\
\text { (2) 1-Octanol; } \mathrm{C}_{8} \mathrm{H}_{18} \mathrm{O} ;[111-87-5]\end{array}$ & $\begin{array}{l}\text { and M. H. Abraham, Phys. Chem. } \\
\text { Liq. 44, 367 (2006). }\end{array}$ \\
\hline Variables: & Prepared by: \\
$T / \mathrm{K}=298.15$ & W. E. Acree, Jr. \\
\hline
\end{tabular}

Experimental Values

\begin{tabular}{lcc}
\hline \hline$x_{2}{ }^{(\mathrm{s}) \mathrm{a}}$ & $x_{2}{ }^{\mathrm{b}}$ & $x_{1}{ }^{\mathrm{c}}$ \\
\hline 1.0000 & 0.9814 & 0.01855 \\
${ }^{\mathrm{a}} x_{2}{ }^{(\mathrm{s})}:$ initial mole fraction of component 2 in the solution. \\
${ }^{\mathrm{b}} x_{2}:$ mole fraction of component 2 in the saturated solution. \\
${ }^{\mathrm{c}} x_{1}:$ mole fraction solubility of the solute.
\end{tabular}

\section{Auxiliary Information}

\section{Method/Apparatus/Procedure:}

Constant-temperature bath, calorimetric thermometer, and an ultraviolet/ visible spectrophotometer.

Excess solute and solvent were placed in amber glass bottles and allowed to equilibrate for several days at constant temperature. Attainment of equilibrium was verified by several repetitive measurements and by approaching equilibrium from supersaturation. Aliquots of saturated solutions were transferred through a coarse filter into tared volumetric flasks, weighed and diluted with methanol. Concentrations were determined by spectrophotometric measurements at $281 \mathrm{~nm}$. 
Source and Purity of Chemicals:

(1) $99 \%$, Acros Organics, USA, was used as received.

(2) $99+\%$, anhydrous, Aldrich Chemical Company, Milwaukee, WI, USA, stored over molecular sieves before use.

Estimated Error:

Temperature: $\pm 0.1 \mathrm{~K}$.

$x_{1}: \pm 1.5 \%$ (relative error).

\begin{tabular}{|c|c|}
\hline $\begin{array}{l}\text { Components: } \\
\text { (1) Phenothiazine; } \mathrm{C}_{12} \mathrm{H}_{9} \mathrm{NS} \text {; [92-84-2] } \\
\text { (2) 2-Ethyl-1-hexanol; } \mathrm{C}_{8} \mathrm{H}_{18} \mathrm{O} \text {; } \\
\text { [104-76-7] }\end{array}$ & $\begin{array}{l}\text { Original Measurements: } \\
{ }^{65} \text { K. R. Hoover, W. E. Acree, Jr., } \\
\text { and M. H. Abraham, Phys. Chem. } \\
\text { Liq. 44, } 367 \text { (2006). }\end{array}$ \\
\hline $\begin{array}{l}\text { Variables: } \\
T / \mathrm{K}=298.15\end{array}$ & $\begin{array}{l}\text { Prepared by: } \\
\text { W. E. Acree, Jr. }\end{array}$ \\
\hline
\end{tabular}

Experimental Values

\begin{tabular}{lcc}
\hline \hline$x_{2}{ }^{(\mathrm{s}) \mathrm{a}}$ & $x_{2}{ }^{\mathrm{b}}$ & $x_{1}{ }^{\mathrm{c}}$ \\
\hline 1.0000 & 0.9899 & 0.01009 \\
\hline
\end{tabular}

${ }^{\mathrm{a}} x_{2}{ }^{(\mathrm{s})}$ : initial mole fraction of component 2 in the solution.

${ }^{\mathrm{b}} x_{2}$ : mole fraction of component 2 in the saturated solution.

${ }^{\mathrm{c}} x_{1}$ : mole fraction solubility of the solute.

\section{Auxiliary Information}

\section{Method/Apparatus/Procedure:}

Constant-temperature bath, calorimetric thermometer, and an ultraviolet/ visible spectrophotometer.

Excess solute and solvent were placed in amber glass bottles and allowed to equilibrate for several days at constant temperature. Attainment of equilibrium was verified by several repetitive measurements and by approaching equilibrium from supersaturation. Aliquots of saturated solutions were transferred through a coarse filter into tared volumetric flasks, weighed and diluted with methanol. Concentrations were determined by

spectrophotometric measurements at $281 \mathrm{~nm}$.

Source and Purity of Chemicals:

(1) $99 \%$, Acros Organics, USA, was used as received.

(2) $99 \%$, Aldrich Chemical Company, Milwaukee, WI, USA, stored over molecular sieves before use.

\section{Estimated Error:}

Temperature: $\pm 0.1 \mathrm{~K}$.

$x_{1}: \pm 1.5 \%$ (relative error).
Experimental Values

\begin{tabular}{|c|c|c|}
\hline$x_{2}{ }^{(\mathrm{s}) \mathrm{a}}$ & $x_{2}{ }^{b}$ & $x_{1}{ }^{\mathrm{c}}$ \\
\hline 1.0000 & 0.9802 & 0.01984 \\
\hline
\end{tabular}

\section{Auxiliary Information}

\section{Method/Apparatus/Procedure:}

Constant-temperature bath, calorimetric thermometer, and an ultraviolet/ visible spectrophotometer.

Excess solute and solvent were placed in amber glass bottles and allowed to equilibrate for several days at constant temperature. Attainment of equilibrium was verified by several repetitive measurements and by approaching equilibrium from supersaturation. Aliquots of saturated solutions were transferred through a coarse filter into tared volumetric flasks, weighed and diluted with methanol. Concentrations were determined by spectrophotometric measurements at $281 \mathrm{~nm}$.

\section{Source and Purity of Chemicals:}

(1) $99 \%$, Acros Organics, USA, was used as received.

(2) $99+\%$, Alfa Aesar, USA, stored over molecular sieves before use.

Estimated Error:

Temperature: $\pm 0.1 \mathrm{~K}$.

$x_{1}: \pm 1.5 \%$ (relative error).

\begin{tabular}{ll}
\hline \hline Components: & $\begin{array}{l}\text { Original Measurements: } \\
\text { (1) Phenothiazine; } \mathrm{C}_{12} \mathrm{H}_{9} \mathrm{NS} ;[92-84-2]\end{array}$ \\
$\begin{array}{ll}{ }^{65} \mathrm{~K} . \mathrm{R} . \text { Hoover, W. E. Acree, Jr., } \\
\text { (2) Cyclopentanol; } \mathrm{C}_{5} \mathrm{H}_{10} \mathrm{O} ;[96-41-3]\end{array}$ & $\begin{array}{l}\text { and M. H. Abraham, Phys. Chem. } \\
\text { Liq. 44, 367 (2006). }\end{array}$ \\
\hline Variables: & Prepared by: \\
$T / \mathrm{K}=298.15$ & W. E. Acree, Jr. \\
\hline
\end{tabular}

Experimental Values

\begin{tabular}{lcc}
\hline \hline$x_{2}{ }^{(\mathrm{s}) \mathrm{a}}$ & $x_{2}{ }^{\mathrm{b}}$ & $x_{1}{ }^{\mathrm{c}}$ \\
\hline 1.0000 & 0.9788 & 0.02119 \\
$\frac{{ }^{\mathrm{a}} x_{2}{ }^{(\mathrm{s})}: \text { initial mole fraction of component } 2 \text { in the solution. }}{{ }^{\mathrm{b}} x_{2}: \text { mole fraction of component } 2 \text { in the saturated solution. }}$ \\
${ }^{\mathrm{c}} x_{1}:$ mole fraction solubility of the solute.
\end{tabular}

\section{Auxiliary Information}

\section{Components:}

(2) 1-Decanol; $\mathrm{C}_{10} \mathrm{H}_{22} \mathrm{O}$; [112-30-1]

\section{Variables:}

$T / \mathrm{K}=298.15$
(1) Phenothiazine; $\mathrm{C}_{12} \mathrm{H}_{9} \mathrm{NS}$; [92-84-2]
Original Measurements:

${ }^{65}$ K. R. Hoover, W. E. Acree, Jr., and M. H. Abraham, Phys. Chem. Liq. 44, 367 (2006).

Prepared by:

W. E. Acree, Jr

\section{Method/Apparatus/Procedure:}

Constant-temperature bath, calorimetric thermometer, and an ultraviolet/ visible spectrophotometer.

Excess solute and solvent were placed in amber glass bottles and allowed to equilibrate for several days at constant temperature. Attainment of equilibrium was verified by several repetitive measurements and by approaching equilibrium from supersaturation. Aliquots of saturated solutions were transferred through a coarse filter into tared volumetric flasks, weighed and diluted with methanol. Concentrations were determined by spectrophotometric measurements at $281 \mathrm{~nm}$. 
Source and Purity of Chemicals:

(1) $99 \%$, Acros Organics, USA, was used as received.

(2) $99 \%$, Aldrich Chemical Company, Milwaukee, WI, USA, stored over molecular sieves before use.

Estimated Error:

Temperature: $\pm 0.1 \mathrm{~K}$.

$x_{1}: \pm 1.5 \%$ (relative error)

\begin{tabular}{ll}
\hline \hline Components: & Original Measurements: \\
(1) Phenothiazine; $\mathrm{C}_{12} \mathrm{H}_{9} \mathrm{NS} ;[92-84-2]$ & ${ }^{65} \mathrm{~K}$. R. Hoover, W. E. Acree, Jr., \\
(2) 1,2-Ethanediol; $\mathrm{C}_{2} \mathrm{H}_{6} \mathrm{O}_{2} ;[107-21-1]$ & and M. H. Abraham, Phys. Chem. \\
& Liq. 44, 367 (2006). \\
\hline Variables: & Prepared by: \\
$T / \mathrm{K}=298.15$ & W. E. Acree, Jr. \\
\hline
\end{tabular}

Experimental Values

\begin{tabular}{lcc}
\hline \hline$x_{2}{ }^{(\mathrm{s}) \mathrm{a}}$ & $x_{2}{ }^{\mathrm{b}}$ & $x_{1}{ }^{\mathrm{c}}$ \\
\hline 1.0000 & 0.9981 & 0.00191 \\
\hline
\end{tabular}

${ }^{\mathrm{a}} x_{2}{ }^{(\mathrm{s})}$ : initial mole fraction of component 2 in the solution.

${ }^{\mathrm{b}} x_{2}$ : mole fraction of component 2 in the saturated solution.

${ }^{\mathrm{c}} x_{1}$ : mole fraction solubility of the solute.

\section{Auxiliary Information}

\section{Method/Apparatus/Procedure:}

Constant-temperature bath, calorimetric thermometer, and an ultraviolet/ visible spectrophotometer.

Excess solute and solvent were placed in amber glass bottles and allowed to equilibrate for several days at constant temperature. Attainment of equilibrium was verified by several repetitive measurements and by approaching equilibrium from supersaturation. Aliquots of saturated solutions were transferred through a coarse filter into tared volumetric flasks, weighed and diluted with methanol. Concentrations were determined by spectrophotometric measurements at $281 \mathrm{~nm}$.

Source and Purity of Chemicals:

(1) $99 \%$, Acros Organics, USA, was used as received.

(2) $99.8 \%$, anhydrous, Aldrich Chemical Company, Milwaukee, WI, USA, stored over molecular sieves before use.

Estimated Error:

Temperature: $\pm 0.1 \mathrm{~K}$.

$x_{1}: \pm 1.5 \%$ (relative error).

\begin{tabular}{|c|c|}
\hline $\begin{array}{l}\text { Components: } \\
\text { (1) Phenothiazine; } \mathrm{C}_{12} \mathrm{H}_{9} \mathrm{NS} \text {; [92-84-2] } \\
\text { (2) 1,2-Propanediol; } \mathrm{C}_{3} \mathrm{H}_{8} \mathrm{O}_{2} ;[57-55-6]\end{array}$ & $\begin{array}{l}\text { Original Measurements: } \\
{ }^{67} \text { S. Ahmadian, V. Panahi-Azar, } \\
\text { M. A. A. Fakhree, W. E. Acree, Jr., } \\
\text { and A. Jouyban, J. Chem. Eng. } \\
\text { Data 56, } 4352 \text { (2011). }\end{array}$ \\
\hline Variables: & Prepared by: \\
\hline Temperature & W. E. Acree, Jr. \\
\hline
\end{tabular}

Experimental Values

\begin{tabular}{lcc}
\hline \hline$T / \mathrm{K}$ & $x_{2}{ }^{\mathrm{a}}$ & $x_{1}{ }^{\mathrm{b}}$ \\
\hline 298.2 & 0.9958 & 0.00416 \\
308.2 & 0.9946 & 0.00540 \\
318.2 & 0.9934 & 0.00656 \\
328.2 & 0.9923 & 0.00763 \\
338.2 & 0.9915 & 0.00852 \\
\hline
\end{tabular}

${ }^{\mathrm{a}} x_{2}$ : mole fraction of component 2 in the saturated solution.

${ }^{b} x_{1}$ : mole fraction of the polycyclic aromatic hydrocarbon (component 1).

\section{Auxiliary Information}

\section{Method/Apparatus/Procedure:}

Incubator, shaker, and an ultraviolet/visible spectrophotometer.

Solubility was determined by equilibrating an excess amount of solid naphthalene with the organic solvent using a shaker placed in an incubator equipped with a temperature controlling system. After an equilibration period of at least $48 \mathrm{~h}$, the samples were withdrawn and filtered through hydrophobic Durapore filters $(0.45 \mu \mathrm{m})$. The filtered sample was diluted quantitatively with methanol. Concentrations were determined by spectrophotmetric analysis at $356 \mathrm{~nm}$.

Source and Purity of Chemicals:

(1) $98 \%$, Merck Chemicals, Germany, was recrystallized from propanone before use.

(2) $99.9 \%$, Scharlau Chemie, Spain, no information given regarding any further purification.

\section{Estimated Error:}

Temperature: \pm 0.2 to $\pm 0.6 \mathrm{~K}$, the higher temperatures have greater uncertainty.

$x_{1}: \pm 2.3 \%$ (relative uncertainty).

\subsection{Phenothiazine solubility data in alkoxyalcohols}

\begin{tabular}{ll}
\hline \hline Components: & Original Measurements: \\
(1) Phenothiazine; $\mathrm{C}_{12} \mathrm{H}_{9} \mathrm{NS} ;[$ [92-84-2] & W. E. Acree, unpublished \\
(2) 2-Ethoxyethanol; $\mathrm{C}_{4} \mathrm{H}_{10} \mathrm{O}_{2} ;[110-80-5]$ & data. \\
\hline Variables: & Prepared by: \\
$T / \mathrm{K}=298.15$ & W. E. Acree, Jr. \\
\hline
\end{tabular}

\section{Experimental Values}

\begin{tabular}{lcc}
\hline \hline$x_{2}{ }^{(\mathrm{s}) \mathrm{a}}$ & $x_{2}{ }^{\mathrm{b}}$ & $x_{1}{ }^{\mathrm{c}}$ \\
\hline 1.0000 & 0.9195 & 0.08051 \\
\hline
\end{tabular}

${ }^{\mathrm{a}} x_{2}{ }^{(\mathrm{s})}$ : initial mole fraction of component 2 in the solution.

${ }^{\mathrm{b}} x_{2}$ : mole fraction of component 2 in the saturated solution.

${ }^{c} x_{1}$ : mole fraction solubility of the solute. 


\section{Auxiliary Information}

\section{Method/Apparatus/Procedure:}

Constant-temperature bath, calorimetric thermometer, and an ultraviolet/ visible spectrophotometer.

Excess solute and solvent were placed in amber glass bottles and allowed to equilibrate for several days at constant temperature. Attainment of equilibrium was verified by several repetitive measurements and by approaching equilibrium from supersaturation. Aliquots of saturated solutions were transferred through a coarse filter into tared volumetric flasks, weighed and diluted with methanol. Concentrations were determined by spectrophotometric measurements at $281 \mathrm{~nm}$.

Source and Purity of Chemicals:

(1) $99 \%$, Acros Organics, USA, used as received.

(2) $99 \%$, Aldrich Chemical Company, Milwaukee, WI, USA, stored over molecular sieves before use.

Estimated Error:

Temperature: $\pm 0.1 \mathrm{~K}$.

$x_{1}: \pm 1.5 \%$ (relative error).

\begin{tabular}{|c|c|}
\hline $\begin{array}{l}\text { Components: } \\
\text { (1) Phenothiazine; } \mathrm{C}_{12} \mathrm{H}_{9} \mathrm{NS} \text {; [92-84-2] } \\
\text { (2) 2-Propoxyethanol; } \mathrm{C}_{5} \mathrm{H}_{12} \mathrm{O}_{2} \text {; } \\
\text { [2807-30-9] }\end{array}$ & $\begin{array}{l}\text { Original Measurements: } \\
\text { W. E. Acree, Jr., } \\
\text { unpublished data. }\end{array}$ \\
\hline $\begin{array}{l}\text { Variables: } \\
T / \mathrm{K}=298.15\end{array}$ & $\begin{array}{l}\text { Prepared by: } \\
\text { W. E. Acree, Jr. }\end{array}$ \\
\hline
\end{tabular}

Experimental Values

\begin{tabular}{lcc}
\hline$\frac{x_{2}{ }^{(\mathrm{s}) \mathrm{a}}}{x_{2}{ }^{\mathrm{b}}}$ & $x_{1}{ }^{\mathrm{c}}$ \\
\hline 1.0000 & 0.9276 & 0.07241 \\
$\frac{{ }^{\mathrm{a}} x_{2}{ }^{(\mathrm{s})}: \text { initial mole fraction of component } 2 \text { in the solution. }}{{ }^{\mathrm{b}}{ }_{x_{2}}: \text { mole fraction of component } 2 \text { in the saturated solution. }}$ \\
${ }^{\mathrm{c}} x_{1}:$ mole fraction solubility of the solute.
\end{tabular}

\section{Auxiliary Information}

\section{Method/Apparatus/Procedure:}

Constant-temperature bath, calorimetric thermometer, and an ultraviolet/ visible spectrophotometer.

Excess solute and solvent were placed in amber glass bottles and allowed to equilibrate for several days at constant temperature. Attainment of equilibrium was verified by several repetitive measurements and by approaching equilibrium from supersaturation. Aliquots of saturated solutions were transferred through a coarse filter into tared volumetric flasks, weighed and diluted with methanol. Concentrations were determined by spectrophotometric measurements at $281 \mathrm{~nm}$.

\section{Source and Purity of Chemicals:}

(1) $99 \%$, Acros Organics, USA, used as received.

(2) $99 \%$, Aldrich Chemical Company, Milwaukee, WI, USA, stored over molecular sieves before use.

\section{Estimated Error:}

Temperature: $\pm 0.1 \mathrm{~K}$

$x_{1}: \pm 1.5 \%$ (relative error).

\section{Components:}

(1) Phenothiazine; $\mathrm{C}_{12} \mathrm{H}_{9} \mathrm{NS}$; [92-84-2]

(2) 2-Isopropoxyethanol; $\mathrm{C}_{5} \mathrm{H}_{12} \mathrm{O}_{2}$; [109-59-1]

Variables:

$T / \mathrm{K}=298.15$

Prepared by:

W. E. Acree, Jr.

Experimental Values

\begin{tabular}{lcc}
\hline \hline$x_{2}{ }^{(\mathrm{s}) \mathrm{a}}$ & $x_{2}{ }^{\mathrm{b}}$ & $x_{1}{ }^{\mathrm{c}}$ \\
\hline 1.0000 & 0.9288 & 0.07122 \\
$\frac{{ }^{a} x_{2}{ }^{(\mathrm{s})}: \text { initial mole fraction of component } 2 \text { in the solution. }}{{ }^{\mathrm{b}} x_{2}: \text { mole fraction of component } 2 \text { in the saturated solution. }}$ \\
${ }^{c} x_{1}:$ mole fraction solubility of the solute.
\end{tabular}

Original Measurements:

W. E. Acree, Jr.,

unpublished data.

\section{Auxiliary Information}

\section{Method/Apparatus/Procedure:}

Constant-temperature bath, calorimetric thermometer, and an ultraviolet/ visible spectrophotometer.

Excess solute and solvent were placed in amber glass bottles and allowed to equilibrate for several days at constant temperature. Attainment of equilibrium was verified by several repetitive measurements and by approaching equilibrium from supersaturation. Aliquots of saturated solutions were transferred through a coarse filter into tared volumetric flasks, weighed and diluted with methanol. Concentrations were determined by spectrophotometric measurements at $281 \mathrm{~nm}$.

Source and Purity of Chemicals:

(1) $99 \%$, Acros Organics, USA, used as received.

(2) $99 \%$, Aldrich Chemical Company, Milwaukee, WI, USA, stored over molecular sieves before use.

\section{Estimated Error:}

Temperature: $\pm 0.1 \mathrm{~K}$.

$x_{1}: \pm 1.5 \%$ (relative error)

\section{Components:}

(1) Phenothiazine; $\mathrm{C}_{12} \mathrm{H}_{9} \mathrm{NS}$; [92-84-2]

(2) 2-Butoxyethanol; $\mathrm{C}_{6} \mathrm{H}_{14} \mathrm{O}_{2}$; [111-76-2]

\begin{tabular}{ll}
\hline Variables: & Prepared by: \\
$T / \mathrm{K}=298.15$ & W. E. Acree, Jr. \\
\hline
\end{tabular}

Experimental Values

\begin{tabular}{lcc}
\hline \hline$x_{2}{ }^{(\mathrm{s}) \mathrm{a}}$ & $x_{2}{ }^{\mathrm{b}}$ & $x_{1}{ }^{\mathrm{c}}$ \\
\hline 1.0000 & 0.9437 & 0.05625 \\
\hline
\end{tabular}

${ }^{\mathrm{a}} x_{2}{ }^{(\mathrm{s})}$ : initial mole fraction of component 2 in the solution.

${ }^{\mathrm{b}} x_{2}$ : mole fraction of component 2 in the saturated solution.

${ }^{c} x_{1}$ : mole fraction solubility of the solute. 


\section{Auxiliary Information}

\section{Method/Apparatus/Procedure:}

Constant-temperature bath, calorimetric thermometer, and an ultraviolet/ visible spectrophotometer.

Excess solute and solvent were placed in amber glass bottles and allowed to equilibrate for several days at constant temperature. Attainment of equilibrium was verified by several repetitive measurements and by approaching equilibrium from supersaturation. Aliquots of saturated solutions were transferred through a coarse filter into tared volumetric flasks, weighed and diluted with methanol. Concentrations were determined by spectrophotometric measurements at $281 \mathrm{~nm}$.

Source and Purity of Chemicals:

(1) $99 \%$, Acros Organics, USA, used as received.

(2) $99+\%$, Acros Organics, USA, stored over anhydrous sodium sulfate and molecular sieves before use.

Estimated Error:

Temperature: $\pm 0.1 \mathrm{~K}$.

$x_{1}: \pm 1.5 \%$ (relative error).

\subsection{Phenothiazine solubility data in miscellaneous organic solvents}

\section{Components:}

(1) Phenothiazine; $\mathrm{C}_{12} \mathrm{H}_{9} \mathrm{NS}$; [92-84-2]

(2) Ethanenitrile; $\mathrm{C}_{2} \mathrm{H}_{3} \mathrm{~N} ;[$ [75-05-8]

\section{Variables:}

$T / \mathrm{K}=298.15$

Experimental Values

\begin{tabular}{lcc}
\hline \hline$x_{2}{ }^{(\mathrm{s}) \mathrm{a}}$ & $x_{2}{ }^{\mathrm{b}}$ & $x_{1}{ }^{\mathrm{c}}$ \\
\hline 1.0000 & 0.9883 & 0.01169 \\
\hline
\end{tabular}

${ }^{\mathrm{a}} x_{2}{ }^{(\mathrm{s})}$ : initial mole fraction of component 2 in the solution.

${ }^{\mathrm{b}} \mathrm{x}_{2}$ : mole fraction of component 2 in the saturated solution.

${ }^{c} x_{1}$ : mole fraction solubility of the solute.

\section{Auxiliary Information}

\section{Method/Apparatus/Procedure:}

Constant-temperature bath, calorimetric thermometer, and an ultraviolet/ visible spectrophotometer.

Excess solute and solvent were placed in amber glass bottles and allowed to equilibrate for several days at constant temperature. Attainment of equilibrium was verified by several repetitive measurements and by approaching equilibrium from supersaturation. Aliquots of saturated solutions were transferred through a coarse filter into tared volumetric flasks, weighed and diluted with methanol. Concentrations were determined by

spectrophotometric measurements at $281 \mathrm{~nm}$.

Source and Purity of Chemicals:

(1) $99 \%$, Acros Organics, USA, was used as received.

(2) $99.8 \%$, anhydrous, Aldrich Chemical Company, Milwaukee, WI, USA, stored over molecular sieves before use.
Estimated Error:

Temperature: $\pm 0.1 \mathrm{~K}$.

$x_{1}: \pm 1.5 \%$ (relative error).

\begin{tabular}{ll}
\hline \hline Components: & Original Measurements: \\
(1) Phenothiazine; $\mathrm{C}_{12} \mathrm{H}_{9} \mathrm{NS} ;[$ [92-84-2] & ${ }^{65} \mathrm{~K}$. R. Hoover, W. E. Acree, Jr., \\
(2) Propanenitrile; $\mathrm{C}_{3} \mathrm{H}_{5} \mathrm{~N} ;[107-12-0]$ & and M. H. Abraham, Phys. Chem. \\
& Liq. 44, 367 (2006). \\
\hline Variables: & Prepared by: \\
$T / \mathrm{K}=298.15$ & W. E. Acree, Jr. \\
\hline
\end{tabular}

Experimental Values

\begin{tabular}{|c|c|c|}
\hline$x_{2}{ }^{(\mathrm{s}) \mathrm{a}}$ & $x_{2}{ }^{b}$ & $x_{1}{ }^{\mathrm{c}}$ \\
\hline 1.0000 & 0.9613 & 0.03872 \\
\hline
\end{tabular}

\section{Auxiliary Information}

\section{Method/Apparatus/Procedure:}

Constant-temperature bath, calorimetric thermometer, and an ultraviolet/ visible spectrophotometer.

Excess solute and solvent were placed in amber glass bottles and allowed to equilibrate for several days at constant temperature. Attainment of equilibrium was verified by several repetitive measurements and by approaching equilibrium from supersaturation. Aliquots of saturated solutions were transferred through a coarse filter into tared volumetric flasks, weighed and diluted with methanol. Concentrations were determined by spectrophotometric measurements at $281 \mathrm{~nm}$.

Source and Purity of Chemicals:

(1) $99 \%$, Acros Organics, USA, was used as received.

(2) $99 \%$, Aldrich Chemical Company, Milwaukee, WI, USA, stored over molecular sieves before use.

\section{Estimated Error:}

Temperature: $\pm 0.1 \mathrm{~K}$.

$x_{1}: \pm 1.5 \%$ (relative error).

\begin{tabular}{|c|c|}
\hline $\begin{array}{l}\text { Components: } \\
\text { (1) Phenothiazine; } \mathrm{C}_{12} \mathrm{H}_{9} \mathrm{NS} \text {; [92-84-2] } \\
\text { (2) Butanenitrile; } \mathrm{C}_{4} \mathrm{H}_{7} \mathrm{~N} ;[109-74-0]\end{array}$ & $\begin{array}{l}\text { Original Measurements: } \\
{ }^{65} \text { K. R. Hoover, W. E. Acree, Jr., } \\
\text { and M. H. Abraham, Phys. Chem. } \\
\text { Liq. 44, } 367 \text { (2006). }\end{array}$ \\
\hline $\begin{array}{l}\text { Variables: } \\
T / \mathrm{K}=298.15\end{array}$ & $\begin{array}{l}\text { Prepared by: } \\
\text { W. E. Acree, Jr. }\end{array}$ \\
\hline
\end{tabular}

Experimental Values

\begin{tabular}{lcc}
\hline \hline$x_{2}{ }^{(\mathrm{s}) \mathrm{a}}$ & $x_{2}{ }^{\mathrm{b}}$ & $x_{1}{ }^{\mathrm{c}}$ \\
\hline 1.0000 & 0.9426 & 0.05741 \\
\hline
\end{tabular}

${ }^{\mathrm{a}} x_{2}{ }^{(\mathrm{s})}$ : initial mole fraction of component 2 in the solution.

${ }^{\mathrm{b}} x_{2}$ : mole fraction of component 2 in the saturated solution.

${ }^{c} x_{1}$ : mole fraction solubility of the solute. 


\section{Auxiliary Information}

\begin{abstract}
Method/Apparatus/Procedure:
Constant-temperature bath, calorimetric thermometer, and an ultraviolet/ visible spectrophotometer.

Excess solute and solvent were placed in amber glass bottles and allowed to equilibrate for several days at constant temperature. Attainment of equilibrium was verified by several repetitive measurements and by approaching equilibrium from supersaturation. Aliquots of saturated solutions were transferred through a coarse filter into tared volumetric flasks, weighed and diluted with methanol. Concentrations were determined by spectrophotometric measurements at $281 \mathrm{~nm}$.
\end{abstract}

Source and Purity of Chemicals:

(1) $99 \%$, Acros Organics, USA, was used as received.

(2) $99.7 \%$, anhydrous, Aldrich Chemical Company, Milwaukee, WI, USA, stored over molecular sieves before use.

Estimated Error:

Temperature: $\pm 0.1 \mathrm{~K}$.

$x_{1}: \pm 1.5 \%$ (relative error).

\section{Solubility of Pyrene in Organic Solvents}

\subsection{Critical evaluation of experimental solubility data}

Volume 59 in the IUPAC Solubility Data Series ${ }^{3}$ contained experimental solubility data for pyrene dissolved in nine saturated hydrocarbons (hexane, heptanes, octane, octadecane, cyclohexane, methylcyclohexane, cyclooctane, 2,2,4trimethylpentane, and tert-butylcyclohexane), in five aromatic hydrocarbons (benzene, methylbenzene, 1,2-dimethylbenzene, 1,3-dimethylbenzene, and 1,4-dimethylbenzene), in three alkyl alkanoates (ethyl ethanoate, butyl ethanoate, and ethyl butanoate), in one dialkyl ether (1,1'-oxybisbutane) and one cyclic ether (1,4-dioxane), in four haloalkanes (tetrachloromethane, 1,2-dichloroethane, 1-chlorobutane, and 1,4dichlorobutane), in 11 alkanols (methanol, ethanol, 1-propanol, 2-propanol, 1-butanol, 2-butanol, 1-pentanol, 2-methyl-1propanol, 2-methyl-1-propanol, 3-methyl-1-butanol, 1-octanol, and cyclopentanol) and one phenol (1-hydroxy-2-methylbenzene), and in three miscellaneous organic solvents (pyridine, dimethyl sulfoxide, and acetonitrile). Most of the experimental measurements were performed between 293 and $303 \mathrm{~K}$. The compiled solubility data also included phase diagrams for binary pyrene + chrysene, pyrene + naphthalene, pyrene + octafluoronaphthalene, pyrene $+1,3$-dinitrobenzene, pyrene +1 ,4-dinitrobenzene, pyrene + 1,3,5-trinitrobenzene, pyrene $+1,2,3,5$-tetranitrobenzene, pyrene $+2,4$-dinitromethylbenzene, pyrene +2 ,4-dinitrophenol, pyrene $+2,4,6$ trinitromethylbenzene, pyrene + 2,4,6-trinitromethoxybenzene, pyrene $+2,4,6$-trinitoraniline, pyrene +2 -chloro1,3,5-trinitrobenzene, and pyrene +3 -methyl-2,4,6-trinitrophenol mixtures. Solubility data contained in Vol. 59 will not be republished here. The listing above is provided so that readers will know what solubility data are available in the earlier volume for pyrene.
There have been several studies that reported solubility data for pyrene in organic solvents after Vol. 59 was published in 1995. Acree and co-workers ${ }^{10,17,22,45,61,68-70}$ determined the solubility of pyrene in six alkanes (nonane, decane, undecane, dodecane, hexadecane, and squalane), in one aromatic hydrocarbon (ethylbenzene), in four alkyl alkanoates (methyl ethanoate, pentyl ethanoate, methyl butanoate, and dibutyl oxalate), in three dialkyl ethers $\left(1,1^{\prime}\right.$-oxybisethane, $2,2^{\prime}$-oxybispropane, and 2-methoxy-2-methylpropane) and two cyclic ethers (tetrahydrofuran and 1,4-dioxane), in three halogenated alkanes (1-chlorohexane, 1-chlorooctane, and chlorocyclohexane) and two halogenated benzenes (fluorobenzene and (trifluoromethyl)benzene), ten alkanols (2-methyl-2-propanol, 1-hexanol, 2-methyl-1-pentanol, 4-methyl-2-pentanol, 1-heptanol, 1-decanol, 3,7-dimethyl-1-octanol, cyclohexanol, 1,2-ethanediol, and 2,2,2-trifluoroethanol), in five alkoxyalkanols (2-methoxyethanol, 2-methoxyethanol, 2-propoxyethanol, 2-butoxyethanol, and 3-methoxy-1-butanol), in two alkanones (butanone, cyclohexanone) and acetophenone, and in 15 different miscellaneous organic solvents (ethanenitrile, propanenitrile, butanenitrile, hexanedinitrile, benzonitrile, methyl acetoacetate, $N$-methylformamide, $N, N$-dimethylformamide, $N, N$-dimethylacetamide, benzeneamine, dimethyl sulfoxide, propylene carbonate, tributyl phosphate, morpholine, and ethanolamine). The experimental solubilities were measured at a single temperature of $299.15 \mathrm{~K}$. Critical evaluations are not possible as there are no independent measurements for the phenanthrene-organic solvent systems studied by Acree and co-workers. ${ }^{10,17,22,45,61,68-70}$

Two research groups have studied the solubility behavior of pyrene as a function of temperature. Yu et al. ${ }^{71}$ examined the solubility of pyrene in methylbenzene, ethyl ethanoate, tetrahydrofuran, methanol, ethanol, 1-propanol, 2-propanol and propanone at several temperatures between 293 and $333 \mathrm{~K}$. In addition to reporting experimental solubilities, the authors provided a mathematical representation describing how the solubility varied with temperature based on the Apelblat model (see Eq. (3)) and calculated both the dissolution enthalpy and entropy of pyrene in the eight different solvents from plots of the natural logarithm of mole fraction solubility versus reciprocal absolute temperature. The authors' calculated curve-fit coefficients are given in Table 6, along with the root-mean-square deviations (RMSDs) for the respective mathematical representations. The root-mean-square deviation is defined by Eq. (9)

$$
\operatorname{RMSD}=\frac{1}{N} \sum_{i=1}^{N}\left|\left(x_{1}{ }^{\text {calc }}-x_{1}{ }^{\exp }\right)^{2}\right|^{1 / 2},
$$

where $N$ is the number of experimental solubility measurements in an individual solute-solvent data set. The Apelblat model provided a very good mathematical description of the observed solubility behavior of pyrene as evidenced by the small RMSD values. It was also noted in the manuscript that the authors' experimental data at $298 \mathrm{~K}$ were in good 
TABLE 6. Parameters of the Apelblat equation for describing the solubility of pyrene in various organic solvents

\begin{tabular}{|c|c|c|c|c|}
\hline Solvent & $A$ & $B$ & $C$ & RMSD \\
\hline Methylbenzene $^{\mathrm{a}}$ & -231.518 & 8511.8 & 35.145 & 0.001396 \\
\hline Ethyl ethanoate $^{\mathrm{a}}$ & -295.480 & 11362.5 & 44.605 & 0.000970 \\
\hline Methanol $^{\mathrm{a}}$ & -21.919 & -1578.6 & 3.630 & 0.000041 \\
\hline Ethanol $^{\mathrm{a}}$ & -147.196 & 4300.3 & 22.292 & 0.000042 \\
\hline 1-Propanol ${ }^{\mathrm{a}}$ & -180.557 & 5549.2 & 27.463 & 0.000067 \\
\hline 2-Propanol ${ }^{\mathrm{a}}$ & -190.169 & 5835.4 & 28.929 & 0.000049 \\
\hline Tetrahydrofuran $^{\mathrm{a}}$ & -84.110 & 2415.7 & 12.995 & 0.001421 \\
\hline Propanone $^{\mathrm{a}}$ & -184.688 & 6062.2 & 28.246 & 0.000367 \\
\hline Hexane $^{\mathrm{b}}$ & 44.139 & -4126.7 & -6.112 & 0.000231 \\
\hline Methylcyclohexane $^{\mathrm{b}}$ & 34.845 & -4126.4 & -4.404 & 0.000440 \\
\hline Benzene $^{\mathrm{b}}$ & 17.063 & -4126.8 & -1.029 & 0.000753 \\
\hline Ethylbenzene $^{\mathrm{b}}$ & 15.133 & -4126.8 & -0.670 & 0.003241 \\
\hline 1-Hexanol ${ }^{\mathrm{b}}$ & 46.292 & -4126.1 & -6.436 & 0.000224 \\
\hline Octane $e^{c}$ & 42.018 & -4126.2 & -5.657 & 0.000188 \\
\hline 2,2,4-Trimethylpentane $\mathrm{e}^{\mathrm{c}}$ & 36.658 & -4126.3 & -4.837 & 0.000133 \\
\hline 1,4-Dimethylbenzene ${ }^{c}$ & 48.383 & -4126.0 & -6.491 & 0.000298 \\
\hline $1-$ Octanol $^{\mathrm{c}}$ & 41.770 & -4126.2 & -5.550 & 0.000207 \\
\hline
\end{tabular}

${ }^{a}$ Numerical values of the coefficients and the percent mean relative deviations were taken from Yu et al. $^{71}$

${ }^{b}$ Numerical values of the coefficients and the percent mean relative deviations were taken from Ali and Al-Rashed. ${ }^{73}$

${ }^{\mathrm{c}}$ Numerical values of the coefficients and the percent mean relative deviations were taken from Ali. ${ }^{74}$

agreement with previously published by Powell et al. ${ }^{72}$ (which is given in Vol. 59 of the IUPAC Solubility Data Series ${ }^{3}$ ). It is further noted that the measured solubility of pyrene in tetrahydrofuran at $298.75 \mathrm{~K}, x_{1}=0.1427$, is in good agreement with the value of $x_{1}=0.1402$ determined by Acree and Abraham ${ }^{10}$ for $299.15 \mathrm{~K}$.

Ali and Al-Rashed ${ }^{73}$ studied the solubility of pyrene in both single solvents and in multi-component solvent mixtures containing hexane, methylcyclohexane, benzene, methylbenzene, and 1-hexanol over the temperature range from 293 to $318 \mathrm{~K}$. The measured mole fraction solubilities were used to test the descriptive and predictive abilities of the Wilson, Combined NIBS/Redlich-Kister, UNIQAC, Non-Random Two Liquid solution models. The models were found to describe the observed solubility behavior of pyrene in quaternary benzene + hexane +1 -hexanol + methylcyclohexane, quaternary ethylbenzene + hexane +1 -hexanol + methylcyclohexane and pentinary benzene + ethylbenzene + hexane + 1-hexanol + methylcyclohexane to within a deviation of $15 \%$ or less using interaction parameters calculated from the measured pyrene solubilities in the contributing sub-binary solvent systems. The authors further noted that plots of the natural logarithm of the mole fraction solubility versus reciprocal temperature (e.g., $\ln x_{1}$ versus $1 / T$ ) resulted in linear plots having squared correlation coefficients of $\mathrm{R}^{2}=0.96$ to $\mathrm{R}^{2}=$ 0.99 as would be expected for internally consistent experimental solubility data. $\mathrm{Ali}^{74}$ had previously measured the solubility of pyrene in octane, 2,2,4-trimethylpentane, 1,4dimethylbenzene and 1-octanol. Also tabulated in Table 6 are the equation coefficients for the Apelblat equation and the corresponding RMSDs for these latter nine pyrene-organic solvent systems.

The experimental solubility data for pyrene in the different organic solvents are given in Secs. 13.2-13.10.

\subsection{Pyrene solubility data in saturated hydrocarbons (including cycloalkanes)}

\begin{tabular}{ll}
\hline \hline Components: & Original Measurements: \\
(1) Pyrene; $\mathrm{C}_{16} \mathrm{H}_{10} ;[129-00-0]$ & ${ }^{73}$ S. H. Ali and O. A. Al-Rashed, \\
(2) Hexane; $\mathrm{C}_{6} \mathrm{H}_{14} ;[110-54-3]$ & $\begin{array}{l}\text { Fluid Phase Equilib. 281, 133 } \\
(2009) .\end{array}$ \\
\hline Variables: & Prepared by: \\
Temperature & W. E. Acree, Jr. \\
\hline
\end{tabular}

Experimental Values

\begin{tabular}{lcc}
\hline \hline$T / \mathrm{K}$ & $x_{2}{ }^{\mathrm{a}}$ & $x_{1}{ }^{\mathrm{b}}$ \\
\hline 293 & 0.9900 & 0.0100 \\
298 & 0.9896 & 0.0104 \\
303 & 0.9877 & 0.0123 \\
308 & 0.9871 & 0.0129 \\
313 & 0.9843 & 0.0157 \\
318 & 0.9819 & 0.0181 \\
\hline
\end{tabular}

${ }^{\mathrm{a}} x_{2}$ : mole fraction of component 2 in the saturated solution.

${ }^{b} x_{1}$ : mole fraction of the polycyclic aromatic hydrocarbon (component 1 ).

Auxiliary Information

\section{Method/Apparatus/Procedure:}

Constant-temperature bath, gas chromatograph equipped with a flame ionization detector and an uv/visible spectrophotometer.

Saturated solutions were prepared by placing excess solute in glass bottles containing the solvent. Samples were equilibrated in a constant temperature with shaking for at least three days, followed by a three-day period in which the solid was allowed to settle to the bottom of the container. Aliquots were transferred to tared volumetric flasks, weighed and then diluted with the solvent for spectrophotometric analysis. Concentrations of the dilute solutions were determined from absorbance versus concentration curves based on absorbance measurements for standard solutions of known concentration. The spectrophotometric results were verified by gas chromatographic analyses. 
Source and Purity of Chemicals:

(1) $97 \%$, Fluka, recrystallized several times from methanol to yield a sample of $99 \%$ purity.

(2) $99.9+\%$, chemical source not given, no purification details were provided in the paper.

\section{Estimated Error:}

Temperature: $\pm 0.1 \mathrm{~K}$.

$x_{1}:<3.0 \%$ (relative error).

\begin{tabular}{ll}
\hline \hline Components: & Original Measurements: \\
(1) Pyrene; $\mathrm{C}_{16} \mathrm{H}_{10} ;[129-00-0]$ & ${ }^{74} \mathrm{~S} . \mathrm{H}$. Ali, Fluid Phase Equilib. \\
(2) Octane; $\mathrm{C}_{8} \mathrm{H}_{18} ;[111-65-9]$ & $\mathbf{2 6 4}, 29(2008)$. \\
\hline Variables: & Prepared by: \\
Temperature & W. E. Acree, Jr. \\
\hline
\end{tabular}

Experimental Values

\begin{tabular}{lcc}
\hline \hline$T / \mathrm{K}$ & $x_{2}{ }^{\mathrm{a}}$ & $x_{1}{ }^{\mathrm{b}}$ \\
\hline 293 & 0.9844 & 0.0156 \\
298 & 0.9831 & 0.0169 \\
303 & 0.9805 & 0.0195 \\
308 & 0.9781 & 0.0219 \\
313 & 0.9741 & 0.0259 \\
318 & 0.9710 & 0.0290 \\
\hline
\end{tabular}

${ }^{a} x_{2}$ : mole fraction of component 2 in the saturated solution.

${ }^{\mathrm{b}} x_{1}$ : mole fraction of the polycyclic aromatic hydrocarbon (component 1).

\section{Auxiliary Information}

\section{Method/Apparatus/Procedure:}

Constant-temperature bath, gas chromatograph equipped with a flame ionization detector and an uv/visible spectrophotometer.

Saturated solutions were prepared by placing excess solute in glass bottles containing the solvent. Samples were equilibrated in a constant temperature with shaking for at least three days, followed by a three-day period in which the solid was allowed to settle to the bottom of the container. Aliquots were transferred to tared volumetric flasks, weighed and then diluted with the solvent for spectrophotometric analysis. Concentrations of the dilute solutions were determined from absorbance versus concentration curves based on absorbance measurements for standard solutions of known concentration. The spectrophotometric results were verified by gas chromatographic analyses.

Source and Purity of Chemicals:

(1) $97 \%$, Fluka, recrystallized several times from methanol to yield a sample of $99 \%$ purity.

(2) $99.9+\%$, Fluka, no purification details were provided in the paper.

Estimated Error:

Temperature: $\pm 0.1 \mathrm{~K}$.

$x_{1}:<3.0 \%$ (relative error).

\begin{tabular}{ll}
\hline \hline Components: & Original Measurements: \\
(1) Pyrene; $\mathrm{C}_{16} \mathrm{H}_{10} ;[129-00-0]$ & ${ }^{45}$ L. E. Roy, C. E. Hernández, and \\
(2) Nonane; $\mathrm{C}_{9} \mathrm{H}_{20} ;[111-84-2]$ & W. E. Acree, Jr., Polycyclic \\
& Aromat. Compds. 13, 205 (1999). \\
\hline Variables: & Prepared by: \\
$T / \mathrm{K}=299.15$ & W. E. Acree, Jr. \\
\hline
\end{tabular}

Experimental Values

\begin{tabular}{|c|c|c|}
\hline$x_{2}{ }^{(\mathrm{s}) \mathrm{a}}$ & $x_{2}{ }^{b}$ & $x_{1}{ }^{\mathrm{c}}$ \\
\hline 1.0000 & 0.9832 & 0.01678 \\
\hline
\end{tabular}

\section{Auxiliary Information}

\section{Method/Apparatus/Procedure:}

Constant-temperature bath, calorimetric thermometer, and an ultraviolet/ visible spectrophotometer.

Excess solute and solvent were placed in amber glass bottles and allowed to equilibrate for several days at constant temperature. Attainment of equilibrium was verified by several repetitive measurements and by approaching equilibrium from supersaturation. Aliquots of saturated solutions were transferred through a coarse filter into tared volumetric flasks, weighed and diluted with methanol. Concentrations were determined by spectrophotometric measurements at $372 \mathrm{~nm}$.

\section{Source and Purity of Chemicals:}

(1) $99+\%$, Aldrich Chemical Company, Milwaukee, WI, USA, was recrystallized several times from methanol.

(2) $99+\%$, TCI America, Portland, OR, USA, stored over molecular sieves and distilled shortly before use.

Estimated Error:

Temperature: $\pm 0.1 \mathrm{~K}$.

$x_{1}: \pm 1.5 \%$ (relative error).

\begin{tabular}{ll}
\hline \hline Components: & Original Measurements: \\
(1) Pyrene; $\mathrm{C}_{16} \mathrm{H}_{10} ;[129-00-0]$ & ${ }^{45}$ L. E. Roy, C. E. Hernández and \\
(2) Decane; $\mathrm{C}_{10} \mathrm{H}_{22} ;[124-18-5]$ & W. E. Acree, Jr., Polycyclic \\
& Aromat. Compd. 13, 205 (1999). \\
\hline Variables: & Prepared by: \\
$T / \mathrm{K}=299.15$ & W. E. Acree, Jr. \\
\hline
\end{tabular}

\section{Experimental Values}

\begin{tabular}{lcc}
\hline \hline$x_{2}{ }^{(\mathrm{s}) \mathrm{a}}$ & $x_{2}{ }^{\mathrm{b}}$ & $x_{1}{ }^{\mathrm{c}}$ \\
\hline 1.0000 & 0.9806 & 0.01936 \\
\hline
\end{tabular}

${ }^{\mathrm{a}} x_{2}{ }^{(\mathrm{s})}$ : initial mole fraction of component 2 in the solution.

${ }^{\mathrm{b}} x_{2}$ : mole fraction of component 2 in the saturated solution.

${ }^{c} x_{1}$ : mole fraction solubility of the solute.

\section{Auxiliary Information}

\section{Method/Apparatus/Procedure:}

Constant-temperature bath, calorimetric thermometer, and an ultraviolet/ visible spectrophotometer.

Excess solute and solvent were placed in amber glass bottles and allowed to equilibrate for several days at constant temperature. Attainment of equilibrium was verified by several repetitive measurements and by approaching equilibrium from supersaturation. Aliquots of saturated solutions were transferred through a coarse filter into tared volumetric flasks, weighed and diluted with ethanol. Concentrations were determined by spectrophotometric measurements at $372 \mathrm{~nm}$. 
Source and Purity of Chemicals:

(1) $99+\%$, Aldrich Chemical Company, Milwaukee, WI, USA, was recrystallized several times from methanol.

(2) $99+\%$, TCI America, Portland, OR, USA, stored over molecular sieves and distilled shortly before use.

Estimated Error:

Temperature: $\pm 0.1 \mathrm{~K}$.

$x_{1}: \pm 1.5 \%$ (relative error)

\begin{tabular}{|c|c|}
\hline $\begin{array}{l}\text { Components: } \\
\text { (1) Pyrene; } \mathrm{C}_{16} \mathrm{H}_{10} ;[129-00-0] \\
\text { (2) Undecane; } \mathrm{C}_{11} \mathrm{H}_{24} ;[1120-21-4]\end{array}$ & $\begin{array}{l}\text { Original Measurements: } \\
{ }^{17} \text { M. H. Abraham and W. E. } \\
\text { Acree, Jr., New J. Chem. 28, } 1538 \\
(2004) .\end{array}$ \\
\hline $\begin{array}{l}\text { Variables: } \\
T / \mathrm{K}=299.15\end{array}$ & $\begin{array}{l}\text { Prepared by: } \\
\text { W. E. Acree, Jr. }\end{array}$ \\
\hline
\end{tabular}

Experimental Values

\begin{tabular}{lcc}
\hline \hline$x_{2}{ }^{(\mathrm{s}) \mathrm{a}}$ & $x_{2}{ }^{\mathrm{b}}$ & $x_{1}{ }^{\mathrm{c}}$ \\
\hline 1.0000 & 0.9779 & 0.02213 \\
$\frac{{ }^{\mathrm{a}} x_{2}{ }^{(\mathrm{s})}: \text { initial mole fraction of component } 2 \text { in the solution. }}{}$ \\
${ }^{\mathrm{b}} x_{2}:$ mole fraction of component 2 in the saturated solution. \\
${ }^{\mathrm{c}} x_{1}:$ mole fraction solubility of the solute.
\end{tabular}

Experimental values were reported as the logarithm of the solute's molar solubility in undecane divided by the molar solubility in water. Mole fraction solubilities were provided by the authors of the paper.

\section{Auxiliary Information}

\section{Method/Apparatus/Procedure:}

Constant-temperature bath, calorimetric thermometer, and an ultraviolet/ visible spectrophotometer.

Excess solute and solvent were placed in amber glass bottles and allowed to equilibrate for several days at constant temperature. Attainment of equilibrium was verified by several repetitive measurements and by approaching equilibrium from supersaturation. Aliquots of saturated solutions were transferred through a coarse filter into tared volumetric flasks, weighed and diluted with ethanol. Concentrations were determined by spectrophotometric measurements at $372 \mathrm{~nm}$.

\section{Source and Purity of Chemicals:}

(1) $99+\%$, Aldrich Chemical Company, Milwaukee, WI, USA, was recrystallized several times from methanol.

(2) $99 \%$, Aldrich Chemical Company, stored over molecular sieves before use.

\section{Estimated Error:}

Temperature: $\pm 0.1 \mathrm{~K}$.

$x_{1}: \pm 1.5 \%$ (relative error).

\begin{tabular}{ll}
\hline \hline Components: & Original Measurements: \\
(1) Pyrene; $\mathrm{C}_{16} \mathrm{H}_{10} ;[129-00-0]$ & ${ }^{17} \mathrm{M} . \mathrm{H}$. Abraham and W. E. \\
(2) Dodecane; $\mathrm{C}_{12} \mathrm{H}_{26} ;[112-40-3]$ & Acree, Jr., New J. Chem. 28, 1538 \\
& $(2004)$. \\
\hline Variables: & Prepared by: \\
$T / \mathrm{K}=299.15$ & W. E. Acree, Jr. \\
\hline
\end{tabular}

Experimental Values

\begin{tabular}{lcc}
\hline \hline$x_{2}{ }^{(\mathrm{s}) \mathrm{a}}$ & $x_{2}{ }^{\mathrm{b}}$ & $x_{1}{ }^{\mathrm{c}}$ \\
\hline 1.0000 & 0.9756 & 0.02439 \\
$\frac{{ }^{\mathrm{a}} x_{2}{ }^{(\mathrm{s})}: \text { initial mole fraction of component } 2 \text { in the solution. }}{{ }^{\mathrm{b}} x_{2}: \text { mole fraction of component } 2 \text { in the saturated solution. }}$ \\
${ }^{\mathrm{c}} x_{1}:$ mole fraction solubility of the solute.
\end{tabular}

Experimental values were reported as the logarithm of the solute's molar solubility in dodecane divided by the molar solubility in water. Mole fraction solubilities were provided by the authors of the paper.

\section{Auxiliary Information}

Method/Apparatus/Procedure:

Constant-temperature bath, calorimetric thermometer, and an ultraviolet/ visible spectrophotometer.

Excess solute and solvent were placed in amber glass bottles and allowed to equilibrate for several days at constant temperature. Attainment of equilibrium was verified by several repetitive measurements and by approaching equilibrium from supersaturation. Aliquots of saturated solutions were transferred through a coarse filter into tared volumetric flasks, weighed and diluted with ethanol. Concentrations were determined by spectrophotometric measurements at $372 \mathrm{~nm}$.

\section{Source and Purity of Chemicals:}

(1) $99+\%$, Aldrich Chemical Company, Milwaukee, WI, USA, was recrystallized several times from methanol.

(2) $99 \%$, Aldrich Chemical Company, stored over molecular sieves before use.

\section{Estimated Error:}

Temperature: $\pm 0.1 \mathrm{~K}$.

$x_{1}: \pm 1.5 \%$ (relative error).

\begin{tabular}{ll}
\hline \hline Components: & Original Measurements: \\
(1) Pyrene; $\mathrm{C}_{16} \mathrm{H}_{10} ;[129-00-0]$ & ${ }^{45}$ L. E. Roy, C. E. Hernández, and \\
(2) Hexadecane; $\mathrm{C}_{16} \mathrm{H}_{34} ;[544-76-3]$ & W. E. Acree, Jr., Polycyclic \\
& Aromat. Compd. 13, 205 (1999). \\
\hline Variables: & Prepared by: \\
$T / \mathrm{K}=299.15$ & W. E. Acree, Jr. \\
\hline
\end{tabular}

Experimental Values

\begin{tabular}{lcc}
\hline \hline$x_{2}{ }^{(\mathrm{s}) \mathrm{a}}$ & $x_{2}{ }^{\mathrm{b}}$ & $x_{1}{ }^{\mathrm{c}}$ \\
\hline 1.0000 & 0.9657 & 0.03431 \\
${ }^{\mathrm{a}} x_{2}{ }^{(\mathrm{s})}$ : initial mole fraction of component 2 in the solution. \\
${ }^{\mathrm{b}} x_{2}:$ mole fraction of component 2 in the saturated solution. \\
${ }^{\mathrm{c}}{ }_{x_{1}}$ : mole fraction solubility of the solute.
\end{tabular}




\section{Auxiliary Information}

\section{Method/Apparatus/Procedure:}

Constant-temperature bath, calorimetric thermometer, and an ultraviolet/ visible spectrophotometer.

Excess solute and solvent were placed in amber glass bottles and allowed to equilibrate for several days at constant temperature. Attainment of equilibrium was verified by several repetitive measurements and by approaching equilibrium from supersaturation. Aliquots of saturated solutions were transferred through a coarse filter into tared volumetric flasks, weighed and diluted with ethanol. Concentrations were determined by spectrophotometric measurements at $372 \mathrm{~nm}$.

Source and Purity of Chemicals:

(1) $99+\%$, Aldrich Chemical Company, Milwaukee, WI, USA, was recrystallized several times from methanol.

(2) 99\%, Aldrich Chemical Company, stored over molecular sieves and distilled shortly before use.

Estimated Error:

Temperature: $\pm 0.1 \mathrm{~K}$.

$x_{1}: \pm 1.5 \%$ (relative error).

\begin{tabular}{ll}
\hline \hline Components: & Original Measurements: \\
(1) Pyrene; $\mathrm{C}_{16} \mathrm{H}_{10} ;[129-00-0]$ & ${ }^{15}$ A. Aoulmi, M. Bouroukba, R. \\
(2) Octacosane; $\mathrm{C}_{28} \mathrm{H}_{58} ;[630-02-4]$ & Solimando, and M. Rogalski, Fluid \\
& Phase Equilib. 110, 283 (1995). \\
\hline Variables: & Prepared by: \\
Temperature & W. E. Acree, Jr. \\
\hline
\end{tabular}

Experimental Values

\begin{tabular}{lcc}
\hline \hline$T / \mathrm{K}$ & $x_{2}{ }^{\mathrm{a}}$ & $x_{1}{ }^{\mathrm{b}}$ \\
\hline 333.91 & 1.0000 & 0.0000 \\
333.64 & 0.8810 & 0.1190 \\
333.54 & 0.7577 & 0.2423 \\
344.00 & 0.6993 & 0.3007 \\
370.57 & 0.5965 & 0.4035 \\
387.15 & 0.4994 & 0.5006 \\
398.87 & 0.4001 & 0.5999 \\
407.36 & 0.2998 & 0.7002 \\
414.52 & 0.2000 & 0.8000 \\
418.36 & 0.1001 & 0.8999 \\
422.65 & 0.0000 & 1.0000 \\
\hline
\end{tabular}

${ }^{\mathrm{a}} x_{2}$ : mole fraction of component 2 in the saturated solution.

${ }^{\mathrm{b}} x_{1}$ : mole fraction of the polycyclic aromatic hydrocarbon (component 1 ).

The authors employed a SSF to describe the activity coefficients of pyrene and octacosane calculated from the solidliquid equilibrium data. The SSF model gave a calculated eutectic temperature of $T / \mathrm{K}=330.6$ and eutectic mole fraction of pyrene of $x_{1}=0.218$.

\section{Auxiliary Information}

\section{Method/Apparatus/Procedure:}

Differential scanning calorimeter.

The phase diagram was determined using a differential scanning calorimeter Measurements were performed at a constant fixed scanning rate of $0.5 \mathrm{~K} / \mathrm{min}$
Source and Purity of Chemicals:

(1) $99+\%$, Aldrich Chemical Company, Milwaukee, WI, USA, no purification details were given in the paper.

(2) $99 \%$, Aldrich Chemical Company, purification details were not given in the paper.

\section{Estimated Error:}

Temperature: Authors state a global accuracy of $\pm 1 \%$ regarding their measurements.

$x_{1}: \pm 0.0002$ (estimated by compiler).

\begin{tabular}{ll}
\hline \hline Components: & Original Measurements: \\
(1) Pyrene; $\mathrm{C}_{16} \mathrm{H}_{10} ;[129-00-0]$ & ${ }^{73} \mathrm{~S}$. H. Ali and O. A. Al-Rashed, \\
(2) Methylcyclohexane; $\mathrm{C}_{7} \mathrm{H}_{14} ;$ & Fluid Phase Equilib. 281, 133 \\
{$[108-87-2]$} & $(2009)$. \\
\hline Variables: & Prepared by: \\
Temperature & W. E. Acree, Jr. \\
\hline
\end{tabular}

Experimental Values

\begin{tabular}{lcc}
\hline \hline$T / \mathrm{K}$ & $x_{2}{ }^{\mathrm{a}}$ & $x_{1}{ }^{\mathrm{b}}$ \\
\hline 293 & 0.9850 & 0.0150 \\
298 & 0.9842 & 0.0158 \\
303 & 0.9814 & 0.0186 \\
308 & 0.9763 & 0.0237 \\
313 & 0.9723 & 0.0277 \\
318 & 0.9711 & 0.0289 \\
\hline
\end{tabular}

${ }^{a} x_{2}$ : mole fraction of component 2 in the saturated solution.

${ }^{\mathrm{b}} x_{1}$ : mole fraction of the polycyclic aromatic hydrocarbon (component 1).

\section{Auxiliary Information}

\section{Method/Apparatus/Procedure:}

Constant-temperature bath, gas chromatograph equipped with a flame ionization detector and an uv/visible spectrophotometer.

Saturated solutions were prepared by placing excess solute in glass bottles containing the solvent. Samples were equilibrated in a constant temperature with shaking for at least three days, followed by a three-day period in which the solid was allowed to settle to the bottom of the container. Aliquots were transferred to tared volumetric flasks, weighed and then diluted with the solvent for spectrophotometric analysis. Concentrations of the dilute solutions were determined from absorbance versus concentration curves based on absorbance measurements for standard solutions of known concentration. The spectrophotometric results were verified by gas chromatographic analyses.

\section{Source and Purity of Chemicals:}

(1) $97 \%$, Fluka, recrystallized several times from methanol to yield a sample of $99 \%$ purity.

(2) $99.9+\%$, chemical source not given, no purification details were provided in the paper.

\section{Estimated Error:}

Temperature: $\pm 0.1 \mathrm{~K}$.

$x_{1}:<3.0 \%$ (relative error). 


\section{Components:}

(1) Pyrene; $\mathrm{C}_{16} \mathrm{H}_{10} ;[129-00-0]$

(2) 2,2,4-Trimethylpentane; $\mathrm{C}_{8} \mathrm{H}_{18}$; [540-84-1]

\section{Variables:}

Temperature
Original Measurements:

${ }^{74}$ S. H. Ali, Fluid Phase Equilib. 264, 29 (2008).

\section{Auxiliary Information}

\section{Method/Apparatus/Procedure:}

Constant-temperature bath, calorimetric thermometer, and an ultraviolet/ visible spectrophotometer.

Excess solute and solvent were placed in amber glass bottles and allowed to equilibrate for several days at constant temperature. Attainment of equilibrium was verified by several repetitive measurements and by approaching equilibrium from supersaturation. Aliquots of saturated solutions were transferred through a coarse filter into tared volumetric flasks, weighed and diluted with 2-propanol. Concentrations were determined by spectrophotometric measurements at $372 \mathrm{~nm}$.

\begin{tabular}{lcc}
\hline \hline$T / \mathrm{K}$ & $x_{2}{ }^{\mathrm{a}}$ & $x_{1}{ }^{\mathrm{b}}$ \\
\hline 293 & 0.9927 & 0.0073 \\
298 & 0.9914 & 0.0086 \\
303 & 0.9896 & 0.0104 \\
308 & 0.9880 & 0.0120 \\
313 & 0.9865 & 0.0135 \\
318 & 0.9854 & 0.0146 \\
\hline
\end{tabular}

${ }^{\mathrm{a}} x_{2}$ : mole fraction of component 2 in the saturated solution.

${ }^{b} x_{1}$ : mole fraction of the polycyclic aromatic hydrocarbon (component 1).

\section{Auxiliary Information}

\section{Method/Apparatus/Procedure:}

Constant-temperature bath, gas chromatograph equipped with a flame ionization detector and an uv/visible spectrophotometer.

Saturated solutions were prepared by placing excess solute in glass bottles containing the solvent. Samples were equilibrated in a constant temperature with shaking for at least three days, followed by a three-day period in which the solid was allowed to settle to the bottom of the container. Aliquots were transferred to tared volumetric flasks, weighed and then diluted with the solvent for spectrophotometric analysis. Concentrations of the dilute solutions were determined from absorbance versus concentration curves based on absorbance measurements for standard solutions of known concentration. The spectrophotometric results were verified by gas chromatographic analyses.

\section{Source and Purity of Chemicals:}

(1) $97 \%$, Fluka, recrystallized several times from methanol to yield a sample of $99 \%$ purity.

(2) $99.9+\%$, Fluka, no purification details were provided in the paper.

Estimated Error:

Temperature: $\pm 0.1 \mathrm{~K}$.

$x_{1}:<3.0 \%$ (relative error).

\begin{tabular}{ll}
\hline \hline Components: & Original Measurements: \\
(1) Pyrene; $\mathrm{C}_{16} \mathrm{H}_{10} ;[129-00-0]$ & ${ }^{10}$ W. E. Acree, Jr. and M. H. \\
(2) Squalane; $\mathrm{C}_{30} \mathrm{H}_{62} ;[111-01-3]$ & Abraham, Fluid Phase Equilib. \\
& $\mathbf{2 0 1 , 2 4 5 ( 2 0 0 2 ) .}$ \\
\hline Variables: & Prepared by: \\
$T / \mathrm{K}=299.15$ & W. E. Acree, Jr. \\
\hline
\end{tabular}

Experimental Values

\begin{tabular}{lcc}
\hline \hline$x_{2}{ }^{(\mathrm{s})}$ & $x_{2}$ & $x_{1}$ \\
\hline 1.0000 & 0.9622 & 0.03776 \\
$\frac{{ }^{a} x_{2}{ }^{(\mathrm{s})}: \text { initial mole fraction of component } 2 \text { in the solution. }}{{ }^{\mathrm{b}} x_{2}: \text { mole fraction of component } 2 \text { in the saturated solution. }}$ \\
${ }^{c} x_{1}:$ mole fraction solubility of the solute.
\end{tabular}

\section{Source and Purity of Chemicals:}

(1) $98 \%$, Aldrich Chemical Company, Milwaukee, WI, USA, was recrystallized several times from methanol.

(2) $99 \%$, Aldrich Chemical Company, stored over molecular sieves before use.

\section{Estimated Error:}

Temperature: $\pm 0.1 \mathrm{~K}$.

$x_{1}: \pm 1.5 \%$ (relative error).

\begin{tabular}{ll}
\hline \hline Components: & Original Measurements: \\
(1) Pyrene; $\mathrm{C}_{16} \mathrm{H}_{10} ;[129-00-0]$ & ${ }^{15}$ A. Aoulmi, M. Bouroukba, R. \\
(2) Squalane; $\mathrm{C}_{30} \mathrm{H}_{62} ;[111-01-3]$ & Solimando, and M. Rogalski, Fluid \\
& Phase Equilib. 110, 283 (1995). \\
\hline Variables: & Prepared by: \\
Temperature & W. E. Acree, Jr. \\
\hline
\end{tabular}

Experimental Values

\begin{tabular}{lcc}
\hline \hline$T / \mathrm{K}$ & $x_{2}{ }^{\mathrm{a}}$ & $x_{1}{ }^{\mathrm{b}}$ \\
\hline 345.75 & 0.8480 & 0.1520 \\
361.35 & 0.7971 & 0.2029 \\
380.15 & 0.6984 & 0.3016 \\
392.65 & 0.5943 & 0.4057 \\
400.15 & 0.4996 & 0.5004 \\
405.35 & 0.4005 & 0.5995 \\
410.45 & 0.2999 & 0.7001 \\
413.85 & 0.2004 & 0.7996 \\
415.35 & 0.1491 & 0.8509 \\
416.95 & 0.1070 & 0.8930 \\
419.45 & 0.0505 & 0.9495 \\
\hline
\end{tabular}

${ }^{\mathrm{a}} x_{2}$ : mole fraction of component 2 in the saturated solution.

${ }^{b} x_{1}$ : mole fraction of the polycyclic aromatic hydrocarbon (component 1 ).

The authors employed a SSF to describe the activity coefficients of pyrene and squalane calculated from the solid-liquid equilibrium data. The SSF model gave a calculated eutectic temperature of $T / \mathrm{K}=243.1$ and eutectic mole fraction of pyrene of $x_{1}=0.006$. 


\section{Auxiliary Information}

\section{Method/Apparatus/Procedure:}

Simple thermal analysis device constructed in the authors' laboratory, and a high precision platinum-resistance thermometer.

The phase diagram was determined using a simple thermal device. A sample of known composition was placed in a thermostatted glass tube, melted and then slowly cooled at a rate of about $1 \mathrm{~K} / \mathrm{min}$. The temperature of the sample was measured with a high precision platinum-resistance thermometer. The crystallization temperature was obtained from a plot of the cooling curve versus time. Each measurement was repeated three times.

\section{Source and Purity of Chemicals:}

(1) $99+\%$, Aldrich Chemical Company, Milwaukee, WI, USA, no purification details were given in the paper.

(2) $99 \%$, Aldrich Chemical Company, purification details were not given in the paper.

\section{Estimated Error:}

Temperature: Authors state a global accuracy of $\pm 1 \%$ regarding their measurements.

$x_{1}: \pm 0.0002$ (estimated by compiler).

\subsection{Pyrene solubility data in aromatic hydrocarbons}

\section{Components: \\ (1) Pyrene; $\mathrm{C}_{16} \mathrm{H}_{10} ;[129-00-0]$ \\ (2) Benzene; $\mathrm{C}_{6} \mathrm{H}_{6} ;$ [71-43-2]}

Variables:

Temperature
Original Measurements:

${ }^{73}$ S. H. Ali and O. A. Al-Rashed, Fluid Phase Equilib. 281, 133 (2009).

Prepared by:

W. E. Acree, Jr.
Experimental Values

\begin{tabular}{lcc}
\hline \hline$T / \mathrm{K}$ & $x_{2}{ }^{\mathrm{a}}$ & $x_{1}{ }^{\mathrm{b}}$ \\
\hline 293 & 0.9416 & 0.0584 \\
298 & 0.9290 & 0.0710 \\
303 & 0.9151 & 0.0849 \\
308 & 0.8936 & 0.1064 \\
313 & 0.8687 & 0.1313 \\
318 & 0.8383 & 0.1617 \\
\hline
\end{tabular}

${ }^{\mathrm{a}} x_{2}$ : mole fraction of component 2 in the saturated solution.

${ }^{b} x_{1}$ : mole fraction of the polycyclic aromatic hydrocarbon (component 1).

\section{Auxiliary Information}

\footnotetext{
Method/Apparatus/Procedure:

Constant-temperature bath, gas chromatograph equipped with a flame ionization detector and an uv/visible spectrophotometer.

Saturated solutions were prepared by placing excess solute in glass bottles containing the solvent. Samples were equilibrated in a constant temperature with shaking for at least three days, followed by a three-day period in which the solid was allowed to settle to the bottom of the container. Aliquots were transferred to tared volumetric flasks, weighed and then diluted with the solvent for spectrophotometric analysis. Concentrations of the dilute solutions were determined from absorbance versus concentration curves based on absorbance measurements for standard solutions of known concentration. The spectrophotometric results were verified by gas chromatographic analyses.
}

Source and Purity of Chemicals:

(1) $97 \%$, Fluka, recrystallized several times from methanol to yield a sample of $99 \%$ purity.

(2) $99.9+\%$, chemical source not given, no purification details were provided in the paper.

\section{Estimated Error:}

Temperature: $\pm 0.1 \mathrm{~K}$.

$x_{1}:<3.0 \%$ (relative error).

\begin{tabular}{ll}
\hline \hline Components: & Original Measurements: \\
(1) Pyrene; $\mathrm{C}_{16} \mathrm{H}_{10} ;[129-00-0]$ & ${ }^{71} \mathrm{Q}$. Yu, X. Ma, and L. Xu, Fluid \\
(2) Methylbenzene; $\mathrm{C}_{7} \mathrm{H}_{8} ;[108-88-3]$ & Phase Equilib. 319, 5 (2012). \\
\hline Variables: & Prepared by: \\
Temperature & W. E. Acree, Jr. \\
\hline
\end{tabular}

Experimental Values

\begin{tabular}{lcc}
\hline \hline$T / \mathrm{K}$ & $x_{2}{ }^{\mathrm{a}}$ & $x_{1}{ }^{\mathrm{b}}$ \\
\hline 295.35 & 0.9389 & 0.0611 \\
303.75 & 0.9243 & 0.0757 \\
308.65 & 0.9134 & 0.0866 \\
313.55 & 0.9050 & 0.0950 \\
318.45 & 0.8900 & 0.1100 \\
323.35 & 0.8780 & 0.1220 \\
328.15 & 0.8600 & 0.1400 \\
333.15 & 0.8355 & 0.1645 \\
\hline
\end{tabular}

${ }^{\mathrm{a}} x_{2}$ : mole fraction of component 2 in the saturated solution.

${ }^{\mathrm{b}} x_{1}$ : mole fraction of the polycyclic aromatic hydrocarbon (component 1).

\section{Auxiliary Information}

\begin{abstract}
Method/Apparatus/Procedure:
Constant-temperature bath, oven and electronic balance.

Saturated solutions were prepared by placing excess solute in a cylindrical double-jacketed glass vial. The solution was equilibrated at constant temperature with continuous stirring for at least $4 \mathrm{~h}$. The stirring was discontinued and the solution was allowed to stand for $3 \mathrm{~h}$. An aliquot of the upper clear solution was removed, filtered through a $0.45 \mu \mathrm{m}$ membrane, and transferred to a tared double dish. The double dish and contents were quickly weighed. The solvent in the double dish was evaporated, dried at $325 \mathrm{~K}$ and then reweighed. The solubility was determined from the mass of the solid residue and the mass of the sample analyzed. Measurements were performed in triplicate.
\end{abstract}

Source and Purity of Chemicals:

(1) $99 \%$, Alfa Aesar Reagent Company, was recrystallized twice from propanone.

(2) $99.9+\%$, Tianjin Kemel Chemical, China, was used as received.

\section{Estimated Error:}

Temperature: $\pm 0.1 \mathrm{~K}$.

$x_{1}:<1.0 \%$ (relative error). 


\section{Components:}

(1) Pyrene; $\mathrm{C}_{16} \mathrm{H}_{10} ;[129-00-0]$

(2) Ethylbenzene; $\mathrm{C}_{8} \mathrm{H}_{10} ;[100-41-4]$

\section{Original Measurements:}

${ }^{61}$ C. E. Hernández, K. M. De Fina,

L. E. Roy, T. L. Sharp, and W. E.

Acree, Jr., Can. J. Chem. 77, 1465 (1999).

\begin{tabular}{ll}
\hline Variables: & Prepared by: \\
$T / \mathrm{K}=299.15$ & W. E. Acree, Jr. \\
\hline
\end{tabular}

Experimental Values

\begin{tabular}{lcc}
\hline \hline$x_{2}{ }^{(\mathrm{s}) \mathrm{a}}$ & $x_{2}{ }^{\mathrm{b}}$ & $x_{1}{ }^{\mathrm{c}}$ \\
\hline 1.0000 & 0.9358 & 0.06424 \\
${ }^{\mathrm{a}} x_{2}{ }^{\left({ }^{()}\right.}:$initial mole fraction of component 2 in the solution. \\
${ }^{\mathrm{b}} x_{2}:$ mole fraction of component 2 in the saturated solution. \\
${ }^{\mathrm{c}} x_{1}:$ mole fraction solubility of the solute.
\end{tabular}

\section{Auxiliary Information}

\section{Method/Apparatus/Procedure:}

Constant-temperature bath, calorimetric thermometer, and an ultraviolet/ visible spectrophotometer.

Excess solute and solvent were placed in amber glass bottles and allowed to equilibrate for several days at constant temperature. Attainment of equilibrium was verified by several repetitive measurements and by approaching equilibrium from supersaturation. Aliquots of saturated solutions were transferred through a coarse filter into tared volumetric flasks, weighed and diluted with methanol. Concentrations were determined by spectrophotometric measurements at $372 \mathrm{~nm}$.

\section{Source and Purity of Chemicals:}

(1) $99+\%$, Aldrich Chemical Company, Milwaukee, WI, USA, was recrystallized several times from methanol.

(2) $99.8 \%$, anhydrous, Aldrich Chemical Company, stored over molecular sieves before use.

\section{Estimated Error:}

Temperature: $\pm 0.1 \mathrm{~K}$

$x_{1}: \pm 1.5 \%$ (relative error).

\section{Components:}

(1) Pyrene; $\mathrm{C}_{16} \mathrm{H}_{10} ;[129-00-0]$

(2) Ethylbenzene; $\mathrm{C}_{8} \mathrm{H}_{10} ;[100-41-4]$

\section{Original Measurements:}

${ }^{73}$ S. H. Ali and O. A. Al-Rashed, Fluid Phase Equilib. 281, 133 (2009).

\section{Variables:}

Temperature

Prepared by:

W. E. Acree, Jr.

\section{Experimental Values}

\begin{tabular}{lcc}
\hline \hline$T / \mathrm{K}$ & $x_{2}{ }^{\mathrm{a}}$ & $x_{1}{ }^{\mathrm{b}}$ \\
\hline 293 & 0.9349 & 0.0651 \\
298 & 0.9224 & 0.0776 \\
303 & 0.9056 & 0.0944 \\
308 & 0.8776 & 0.1224 \\
313 & 0.8353 & 0.1647 \\
318 & 0.8290 & 0.1710 \\
\hline
\end{tabular}

${ }^{\mathrm{a}} x_{2}$ : mole fraction of component 2 in the saturated solution.

${ }^{b} x_{1}$ : mole fraction of the polycyclic aromatic hydrocarbon (component 1 ).

\section{Auxiliary Information}

\section{Method/Apparatus/Procedure:}

Constant-temperature bath, gas chromatograph equipped with a flame ionization detector and an uv/visible spectrophotometer.

Saturated solutions were prepared by placing excess solute in glass bottles containing the solvent. Samples were equilibrated in a constant temperature with shaking for at least three days, followed by a three-day period in which the solid was allowed to settle to the bottom of the container. Aliquots were transferred to tared volumetric flasks, weighed and then diluted with the solvent for spectrophotometric analysis. Concentrations of the dilute solutions were determined from absorbance versus concentration curves based on absorbance measurements for standard solutions of known concentration. The spectrophotometric results were verified by gas chromatographic analyses.

\section{Source and Purity of Chemicals:}

(1) $97 \%$, Fluka, recrystallized several times from methanol to yield a sample of $99 \%$ purity.

(2) $99.9+\%$, chemical source not given, no purification details were provided in the paper.

\section{Estimated Error:}

Temperature: $\pm 0.1 \mathrm{~K}$.

$x_{1}:<3.0 \%$ (relative error).

\begin{tabular}{|c|c|}
\hline $\begin{array}{l}\text { Components: } \\
\text { (1) Pyrene; } \mathrm{C}_{16} \mathrm{H}_{10} ;[129-00-0] \\
\text { (2) 1,4-Dimethylbenzene; } \mathrm{C}_{8} \mathrm{H}_{10} \text {; } \\
{[106-42-3]}\end{array}$ & $\begin{array}{l}\text { Original Measurements: } \\
{ }^{74} \text { S. H. Ali, Fluid Phase Equilib. } \\
\text { 264, } 29 \text { (2008). }\end{array}$ \\
\hline $\begin{array}{l}\text { Variables: } \\
\text { Temperature }\end{array}$ & $\begin{array}{l}\text { Prepared by: } \\
\text { W. E. Acree, Jr. }\end{array}$ \\
\hline
\end{tabular}

Experimental Values

\begin{tabular}{lcc}
\hline \hline$T / \mathrm{K}$ & $x_{2}{ }^{\mathrm{a}}$ & $x_{1}{ }^{\mathrm{b}}$ \\
\hline 293 & 0.9223 & 0.0777 \\
298 & 0.9137 & 0.0863 \\
303 & 0.9024 & 0.0976 \\
308 & 0.8907 & 0.1093 \\
313 & 0.8764 & 0.1236 \\
318 & 0.8633 & 0.1367
\end{tabular}

${ }^{\mathrm{a}} x_{2}$ : mole fraction of component 2 in the saturated solution.

${ }^{\mathrm{b}} x_{1}$ : mole fraction of the polycyclic aromatic hydrocarbon (component 1 ).

\section{Auxiliary Information}

\section{Method/Apparatus/Procedure:}

Constant-temperature bath, gas chromatograph equipped with a flame ionization detector and an uv/visible spectrophotometer.

Saturated solutions were prepared by placing excess solute in glass bottles containing the solvent. Samples were equilibrated in a constant temperature with shaking for at least three days, followed by a three-day period in which the solid was allowed to settle to the bottom of the container. Aliquots were transferred to tared volumetric flasks, weighed and then diluted with the solvent for spectrophotometric analysis. Concentrations of the dilute solutions were determined from absorbance versus concentration curves based on absorbance measurements for standard solutions of known concentration. The spectrophotometric results were verified by gas chromatographic analyses. 
Source and Purity of Chemicals:

(1) $97 \%$, Fluka, recrystallized several times from methanol to yield a sample of $99 \%$ purity.

(2) $99.9+\%$, Fluka, no purification details were provided in the paper.

Estimated Error:

Temperature: $\pm 0.1 \mathrm{~K}$

$x_{1}:<3.0 \%$ (relative error).

\subsection{Pyrene solubility data in esters}

\begin{tabular}{|c|c|}
\hline $\begin{array}{l}\text { Components: } \\
\text { (1) Pyrene; } \mathrm{C}_{16} \mathrm{H}_{10} ;[129-00-0] \\
\text { (2) Methyl ethanoate; } \mathrm{C}_{3} \mathrm{H}_{6} \mathrm{O}_{2} \text {; } \\
\text { [79-20-9] }\end{array}$ & $\begin{array}{l}\text { Original Measurements: } \\
{ }^{10} \text { W. E. Acree, Jr. and M. H. } \\
\text { Abraham, Fluid Phase Equilib. } \\
\text { 201, } 245 \text { (2002). }\end{array}$ \\
\hline $\begin{array}{l}\text { Variables: } \\
T / \mathrm{K}=299.15\end{array}$ & $\begin{array}{l}\text { Prepared by: } \\
\text { W. E. Acree, Jr. }\end{array}$ \\
\hline
\end{tabular}

\section{Experimental Values}

\begin{tabular}{lcc}
\hline \hline$x_{2}{ }^{(\mathrm{s}) \mathrm{a}}$ & $x_{2}{ }^{\mathrm{b}}$ & $x_{1}{ }^{\mathrm{c}}$ \\
\hline 1.0000 & 0.9670 & 0.03296 \\
\hline
\end{tabular}

${ }^{\mathrm{a}} x_{2}{ }^{(\mathrm{s})}$ : initial mole fraction of component 2 in the solution.

${ }^{b} x_{2}$ : mole fraction of component 2 in the saturated solution.

${ }^{c} x_{1}$ : mole fraction solubility of the solute.

\section{Auxiliary Information}

\section{Method/Apparatus/Procedure:}

Constant-temperature bath, calorimetric thermometer, and an ultraviolet/ visible spectrophotometer.

Excess solute and solvent were placed in amber glass bottles and allowed to equilibrate for several days at constant temperature. Attainment of equilibrium was verified by several repetitive measurements and by approaching equilibrium from supersaturation. Aliquots of saturated solutions were transferred through a coarse filter into tared volumetric flasks, weighed and diluted with 2-propanol. Concentrations were determined by spectrophotometric measurements at $372 \mathrm{~nm}$.

\section{Source and Purity of Chemicals:}

(1) $98 \%$, Aldrich Chemical Company, Milwaukee, WI, USA, was recrystallized several times from methanol.

(2) $99.5 \%$, anhydrous, Aldrich Chemical Company, stored over molecular sieves before use.

\section{Estimated Error:}

Temperature: $\pm 0.1 \mathrm{~K}$.

$x_{1}: \pm 1.5 \%$ (relative error).

\section{Components:}

(1) Pyrene; $\mathrm{C}_{16} \mathrm{H}_{10} ;[129-00-0]$

(2) Ethyl ethanoate; $\mathrm{C}_{4} \mathrm{H}_{8} \mathrm{O}_{2} ;$ [141-78-6]
Original Measurements:

${ }^{71}$ Q. Yu, X. Ma, and L. Xu, Fluid Phase Equilib. 319, 5 (2012).

Prepared by:

Variables:

Temperature
Experimental Values

\begin{tabular}{lcc}
\hline \hline$T / \mathrm{K}$ & $x_{2}{ }^{\mathrm{a}}$ & $x_{1}{ }^{\mathrm{b}}$ \\
\hline 295.35 & 0.9634 & 0.0366 \\
298.75 & 0.9602 & 0.0398 \\
303.75 & 0.9544 & 0.0456 \\
308.65 & 0.9486 & 0.0514 \\
313.45 & 0.9418 & 0.0582 \\
318.45 & 0.9349 & 0.0651 \\
323.35 & 0.9264 & 0.0736 \\
328.15 & 0.9114 & 0.0886 \\
333.15 & 0.8981 & 0.1019 \\
\hline
\end{tabular}

${ }^{a_{2}}$ : mole fraction of component 2 in the saturated solution.

${ }^{\mathrm{b}} x_{1}$ : mole fraction of the polycyclic aromatic hydrocarbon (component 1).

\section{Auxiliary Information}

\section{Method/Apparatus/Procedure:}

Constant-temperature bath, oven and electronic balance.

Saturated solutions were prepared by placing excess solute in a cylindrical double-jacketed glass vial. The solution was equilibrated at constant temperature with continuous stirring for at least $4 \mathrm{~h}$. The stirring was discontinued and the solution was allowed to stand for $3 \mathrm{~h}$. An aliquot of the upper clear solution was removed, filtered through a $0.45 \mu \mathrm{m}$ membrane, and transferred to a tared double dish. The double dish and contents were quickly weighed. The solvent in the double dish was evaporated, dried at $325 \mathrm{~K}$ and then reweighed. The solubility was determined from the mass of the solid residue and the mass of the sample analyzed. Measurements were performed in triplicate.

Source and Purity of Chemicals:

(1) $99 \%$, Alfa Aesar Reagent Company, was recrystallized twice from propanone.

(2) $99.9+\%$, Tianjin Kemel Chemical, China, was used as received.

\section{Estimated Error:}

Temperature: $\pm 0.1 \mathrm{~K}$.

$x_{1}:<1.3 \%$ (relative error).

\section{Components:}

(1) Pyrene; $\mathrm{C}_{16} \mathrm{H}_{10} ;[129-00-0]$

(2) Pentyl ethanoate; $\mathrm{C}_{7} \mathrm{H}_{14} \mathrm{O}_{2} ;[628-63-7]$

Original Measurements:

W. E. Acree, Jr.,

\begin{tabular}{ll}
\hline Variables: & Prepared by: \\
$T / \mathrm{K}=299.15$ & W. E. Acree, Jr. \\
\hline
\end{tabular}

Experimental Values

\begin{tabular}{lcc}
\hline \hline$\frac{x_{2}{ }^{(\mathrm{s}) \mathrm{a}}}{x_{2}{ }^{\mathrm{b}}}$ & $x_{1}{ }^{\mathrm{c}}$ \\
\hline 1.0000 & 0.9292 & 0.07077 \\
${ }^{{ }^{\mathrm{a}} x_{2}{ }^{(\mathrm{s})}: \text { initial mole fraction of component } 2 \text { in the solution. }}$ \\
${ }^{\mathrm{b}} x_{2}:$ mole fraction of component 2 in the saturated solution. \\
${ }^{\mathrm{c}} x_{1}$ : mole fraction solubility of the solute.
\end{tabular}




\section{Auxiliary Information}

\section{Method/Apparatus/Procedure:}

Constant-temperature bath, calorimetric thermometer, and an ultraviolet/ visible spectrophotometer.

Excess solute and solvent were placed in amber glass bottles and allowed to equilibrate for several days at constant temperature. Attainment of equilibrium was verified by several repetitive measurements and by approaching equilibrium from supersaturation. Aliquots of saturated solutions were transferred through a coarse filter into tared volumetric flasks, weighed and diluted with 2-propanol. Concentrations were determined by spectrophotometric measurements at $372 \mathrm{~nm}$.

Source and Purity of Chemicals:

(1) $98 \%$, Aldrich Chemical Company, Milwaukee, WI, USA, was recrystallized several times from methanol.

(2) $99 \%$, Aldrich Chemical Company, stored over molecular sieves before use

Estimated Error:

Temperature: $\pm 0.1 \mathrm{~K}$.

$x_{1}: \pm 1.5 \%$ (relative error).

\begin{tabular}{ll}
\hline \hline Components: & Original Measurements: \\
(1) Pyrene; $\mathrm{C}_{16} \mathrm{H}_{10} ;[129-00-0]$ & W. E. Acree, Jr., \\
(2) Methyl butanoate; $\mathrm{C}_{5} \mathrm{H}_{10} \mathrm{O}_{2} ;[623-42-7]$ & unpublished data. \\
\hline Variables: & Prepared by: \\
$T / \mathrm{K}=299.15$ & W. E. Acree, Jr. \\
\hline
\end{tabular}

Experimental Values

\begin{tabular}{lcc}
\hline \hline$x_{2}{ }^{(\mathrm{s}) \mathrm{a}}$ & $x_{2}{ }^{\mathrm{b}}$ & $x_{1}{ }^{\mathrm{c}}$ \\
\hline 1.0000 & 0.9463 & 0.05367
\end{tabular}

${ }^{\mathrm{a}} x_{2}{ }^{(\mathrm{s})}$ : initial mole fraction of component 2 in the solution.

${ }^{\mathrm{b}} x_{2}$ : mole fraction of component 2 in the saturated solution.

${ }^{c} x_{1}$ : mole fraction solubility of the solute.

\section{Auxiliary Information}

\section{Method/Apparatus/Procedure:}

Constant-temperature bath, calorimetric thermometer, and an ultraviolet/ visible spectrophotometer.

Excess solute and solvent were placed in amber glass bottles and allowed to equilibrate for several days at constant temperature. Attainment of equilibrium was verified by several repetitive measurements and by approaching equilibrium from supersaturation. Aliquots of saturated solutions were transferred through a coarse filter into tared volumetric flasks, weighed and diluted with 2-propanol. Concentrations were determined by spectrophotometric measurements at $372 \mathrm{~nm}$.

Source and Purity of Chemicals:

(1) $98 \%$, Aldrich Chemical Company, Milwaukee, WI, USA, was recrystallized several times from methanol.

(2) $99 \%$, Aldrich Chemical Company, stored over molecular sieves before use.

Estimated Error:

Temperature: $\pm 0.1 \mathrm{~K}$.

$x_{1}: \pm 1.5 \%$ (relative error).

\begin{tabular}{ll}
\hline \hline Components: & Original Measurements: \\
(1) Pyrene; $\mathrm{C}_{16} \mathrm{H}_{10} ;[129-00-0]$ & W. E. Acree, Jr., \\
(2) Dibutyl oxalate; $\mathrm{C}_{10} \mathrm{H}_{18} \mathrm{O}_{4} ;[2050-60-4]$ & unpublished data. \\
\hline Variables: & Prepared by: \\
$T / \mathrm{K}=299.15$ & W. E. Acree, Jr. \\
\hline
\end{tabular}

Experimental Values

\begin{tabular}{lcc}
\hline \hline$x_{2}{ }^{(\mathrm{s}) \mathrm{a}}$ & $x_{2}{ }^{\mathrm{b}}$ & $x_{1}{ }^{\mathrm{c}}$ \\
\hline 1.0000 & 0.8891 & 0.1109 \\
\hline${ }^{\mathrm{a}} x_{2}{ }^{(\mathrm{s})}:$ initial mole fraction of component 2 in the solution. \\
${ }^{\mathrm{b}} x_{2}:$ mole fraction of component 2 in the saturated solution. \\
${ }^{\mathrm{c}} x_{1}:$ mole fraction solubility of the solute.
\end{tabular}

\section{Auxiliary Information}

\section{Method/Apparatus/Procedure:}

Constant-temperature bath, calorimetric thermometer, and an ultraviolet/ visible spectrophotometer.

Excess solute and solvent were placed in amber glass bottles and allowed to equilibrate for several days at constant temperature. Attainment of equilibrium was verified by several repetitive measurements and by approaching equilibrium from supersaturation. Aliquots of saturated solutions were transferred through a coarse filter into tared volumetric flasks, weighed and diluted with 2-propanol. Concentrations were determined by spectrophotometric measurements at $372 \mathrm{~nm}$.

\section{Source and Purity of Chemicals:}

(1) $98 \%$, Aldrich Chemical Company, Milwaukee, WI, USA, was recrystallized several times from methanol.

(2) $99 \%$, Aldrich Chemical Company, stored over molecular sieves before use.

\section{Estimated Error:}

Temperature: $\pm 0.1 \mathrm{~K}$.

$x_{1}: \pm 1.5 \%$ (relative error).

\subsection{Pyrene solubility data in ethers}

\begin{tabular}{ll}
\hline \hline Components: & Original Measurements: \\
(1) Pyrene; $\mathrm{C}_{16} \mathrm{H}_{10} ;[129-00-0]$ & ${ }^{10}$ W. E. Acree, Jr. and M. H. \\
$(2) 1,1^{\prime}-$ Oxybisethane; $\mathrm{C}_{4} \mathrm{H}_{10} \mathrm{O} ;[60-29-7]$ & Abraham, Fluid Phase \\
& Equilib. 201, 245 (2002). \\
\hline Variables: & Prepared by: \\
$T / \mathrm{K}=299.15$ & W. E. Acree, Jr. \\
\hline
\end{tabular}

Experimental Values

\begin{tabular}{|c|c|c|}
\hline$x_{2}{ }^{(\mathrm{s}) \mathrm{a}}$ & $x_{2}{ }^{b}$ & $x_{1}{ }^{\mathrm{c}}$ \\
\hline 1.0000 & 0.9713 & 0.02865 \\
\hline
\end{tabular}




\section{Auxiliary Information}

\section{Method/Apparatus/Procedure:}

Constant-temperature bath, calorimetric thermometer, and an ultraviolet/ visible spectrophotometer.

Excess solute and solvent were placed in amber glass bottles and allowed to equilibrate for several days at constant temperature. Attainment of equilibrium was verified by several repetitive measurements and by approaching equilibrium from supersaturation. Aliquots of saturated solutions were transferred through a coarse filter into tared volumetric flasks, weighed and diluted with 2-propanol. Concentrations were determined by spectrophotometric measurements at $372 \mathrm{~nm}$.

Source and Purity of Chemicals:

(1) $98 \%$, Aldrich Chemical Company, Milwaukee, WI, USA, was recrystallized several times from methanol.

(2) $99+\%$, anhydrous, Aldrich Chemical Company, stored over molecular sieves before use.

Estimated Error:

Temperature: $\pm 0.1 \mathrm{~K}$.

$x_{1}: \pm 1.5 \%$ (relative error).

\begin{tabular}{ll}
\hline \hline Components: & Original Measurements: \\
(1) Pyrene; $\mathrm{C}_{16} \mathrm{H}_{10} ;[129-00-0]$ & ${ }^{10}$ W. E. Acree, Jr. and M. H. \\
(2) 2,2'-Oxybispropane; $\mathrm{C}_{6} \mathrm{H}_{14} \mathrm{O} ;$ & Abraham, Fluid Phase Equilib. \\
{$[108-20-3]$} & $\mathbf{2 0 1 ,} 245(2002)$. \\
\hline Variables: & Prepared by: \\
$T / \mathrm{K}=299.15$ & W. E. Acree, Jr. \\
\hline
\end{tabular}

Experimental Values

\begin{tabular}{lcc}
\hline \hline$x_{2}{ }^{(\mathrm{s}) \mathrm{a}}$ & $x_{2}{ }^{\mathrm{b}}$ & $x_{1}{ }^{\mathrm{c}}$ \\
\hline 1.0000 & 0.9798 & 0.02015 \\
\hline
\end{tabular}

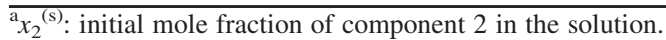

${ }^{b} x_{2}$ : mole fraction of component 2 in the saturated solution.

${ }^{c} x_{1}$ : mole fraction solubility of the solute.

\section{Auxiliary Information}

\section{Method/Apparatus/Procedure:}

Constant-temperature bath, calorimetric thermometer, and an ultraviolet/ visible spectrophotometer.

Excess solute and solvent were placed in amber glass bottles and allowed to equilibrate for several days at constant temperature. Attainment of equilibrium was verified by several repetitive measurements and by approaching equilibrium from supersaturation. Aliquots of saturated solutions were transferred through a coarse filter into tared volumetric flasks, weighed and diluted with 2-propanol. Concentrations were determined by

spectrophotometric measurements at $372 \mathrm{~nm}$.

\section{Source and Purity of Chemicals:}

(1) $98 \%$, Aldrich Chemical Company, Milwaukee, WI, USA, was recrystallized several times from methanol.

(2) $99 \%$, anhydrous, Aldrich Chemical Company, stored over molecular sieves before use.

Estimated Error:

Temperature: $\pm 0.1 \mathrm{~K}$.

$x_{1}: \pm 1.5 \%$ (relative error)

\begin{tabular}{ll}
\hline \hline Components: & Original Measurements: \\
(1) Pyrene; $\mathrm{C}_{16} \mathrm{H}_{10} ;[129-00-0]$ & ${ }^{10}$ W. E. Acree, Jr. and M. H. \\
(2) 2-Methoxy-2-methylpropane; & Abraham, Fluid Phase Equilib. \\
$\mathrm{C}_{5} \mathrm{H}_{12} \mathrm{O} ;[1634-04-4]$ & $\mathbf{2 0 1}, 245(2002)$. \\
\hline Variables: & Prepared by: \\
$T / \mathrm{K}=299.15$ & W. E. Acree, Jr. \\
\hline
\end{tabular}

Experimental Values

\begin{tabular}{lcc}
\hline \hline$x_{2}{ }^{(\mathrm{s}) \mathrm{a}}$ & $x_{2}{ }^{\mathrm{b}}$ & $x_{1}{ }^{\mathrm{c}}$ \\
\hline 1.0000 & 0.9757 & 0.02432 \\
$\frac{{ }^{\mathrm{a}} x_{2}{ }^{(\mathrm{s})}: \text { initial mole fraction of component } 2 \text { in the solution. }}{{ }^{\mathrm{b}} x_{2}: \text { mole fraction of component } 2 \text { in the saturated solution. }}$ \\
${ }^{\mathrm{c}} x_{1}:$ mole fraction solubility of the solute.
\end{tabular}

The published value was given as molar solubility of pyrene in 2-methoxy-2-methylpropane (also called methyl tert-butyl ether). The mole fraction solubility was provided by the authors of the paper.

\section{Auxiliary Information}

\section{Method/Apparatus/Procedure:}

Constant-temperature bath, calorimetric thermometer, and an ultraviolet/ visible spectrophotometer.

Excess solute and solvent were placed in amber glass bottles and allowed to equilibrate for several days at constant temperature. Attainment of equilibrium was verified by several repetitive measurements and by approaching equilibrium from supersaturation. Aliquots of saturated solutions were transferred through a coarse filter into tared volumetric flasks, weighed and diluted with methanol. Concentrations were determined by spectrophotometric measurements at $372 \mathrm{~nm}$.

\section{Source and Purity of Chemicals:}

(1) $99+\%$, Aldrich Chemical Company, Milwaukee, WI, USA, was recrystallized several times from methanol.

(2) $99.9+\%$, Arco Chemical Company, USA, stored over molecular sieves before use.

Estimated Error:

Temperature: $\pm 0.1 \mathrm{~K}$.

$x_{1}: \pm 1.5 \%$ (relative error).

\begin{tabular}{ll}
\hline \hline Components: & Original Measurements: \\
(1) Pyrene; $\mathrm{C}_{16} \mathrm{H}_{10} ;[129-00-0]$ & ${ }^{10}$ W. E. Acree, Jr. and M. H. \\
(2) Tetrahydrofuran; $\mathrm{C}_{4} \mathrm{H}_{8} \mathrm{O} ;[109-99-9]$ & Abraham, Fluid Phase \\
& Equilib. 201, 245 (2002). \\
\hline Variables: & Prepared by: \\
$T / \mathrm{K}=299.15$ & W. E. Acree, Jr. \\
\hline
\end{tabular}

Experimental Values

\begin{tabular}{lcc}
\hline \hline$x_{2}{ }^{(\mathrm{s}) \mathrm{a}}$ & $x_{2}{ }^{\mathrm{b}}$ & $x_{1}{ }^{\mathrm{c}}$ \\
\hline 1.0000 & 0.8598 & 0.1402 \\
$\frac{{ }^{\mathrm{a}} x_{2}{ }^{(\mathrm{s})}: \text { initial mole fraction of component } 2 \text { in the solution. }}{}$ \\
${ }^{\mathrm{b}} x_{2}$ : mole fraction of component 2 in the saturated solution. \\
${ }^{\mathrm{c}}{ }_{x_{1}}$ : mole fraction solubility of the solute.
\end{tabular}




\section{Auxiliary Information}

\section{Method/Apparatus/Procedure:}

Constant-temperature bath, calorimetric thermometer, and an ultraviolet/ visible spectrophotometer.

Excess solute and solvent were placed in amber glass bottles and allowed to equilibrate for several days at constant temperature. Attainment of equilibrium was verified by several repetitive measurements and by approaching equilibrium from supersaturation. Aliquots of saturated solutions were transferred through a coarse filter into tared volumetric flasks, weighed and diluted with 2-propanol. Concentrations were determined by spectrophotometric measurements at $372 \mathrm{~nm}$.

Source and Purity of Chemicals:

(1) $98 \%$, Aldrich Chemical Company, Milwaukee, WI, USA, was recrystallized several times from methanol.

(2) $99.9 \%$, anhydrous, Aldrich Chemical Company, stored over molecular sieves before use.

Estimated Error:

Temperature: $\pm 0.1 \mathrm{~K}$.

$x_{1}: \pm 1.5 \%$ (relative error).

\begin{tabular}{ll}
\hline \hline Components: & Original Measurements: \\
(1) Pyrene; $\mathrm{C}_{16} \mathrm{H}_{10} ;[129-00-0]$ & ${ }^{71} \mathrm{Q}$. Yu, X. Ma, and L. Xu, Fluid \\
(2) Tetrahydrofuran; $\mathrm{C}_{4} \mathrm{H}_{8} \mathrm{O} ;[109-99-9]$ & Phase Equilib. 319, 5 (2012). \\
\hline Variables: & Prepared by: \\
Temperature & W. E. Acree, Jr. \\
\hline
\end{tabular}

Experimental Values

\begin{tabular}{lcc}
\hline \hline$T / \mathrm{K}$ & $x_{2}{ }^{\mathrm{a}}$ & $x_{1}{ }^{\mathrm{b}}$ \\
\hline 295.35 & 0.8653 & 0.1347 \\
298.75 & 0.8573 & 0.1427 \\
303.75 & 0.8484 & 0.1516 \\
308.65 & 0.8345 & 0.1655 \\
313.45 & 0.8199 & 0.1801 \\
318.45 & 0.8005 & 0.1995 \\
323.35 & 0.7864 & 0.2136 \\
328.15 & 0.7668 & 0.2332 \\
333.15 & 0.7477 & 0.2523 \\
\hline
\end{tabular}

${ }^{a} x_{2}$ : mole fraction of component 2 in the saturated solution.

${ }^{\mathrm{b}} \mathrm{x}_{1}$ : mole fraction of the polycyclic aromatic hydrocarbon (component 1 ).

\section{Auxiliary Information}

\section{Method/Apparatus/Procedure:}

Constant-temperature bath, oven and electronic balance.

Saturated solutions were prepared by placing excess solute in a cylindrical double-jacketed glass vial. The solution was equilibrated at constant temperature with continuous stirring for at least $4 \mathrm{~h}$. The stirring was discontinued and the solution was allowed to stand for $3 \mathrm{~h}$. An aliquot of the upper clear solution was removed, filtered through a $0.45 \mu \mathrm{m}$ membrane, and transferred to a tared double dish. The double dish and contents were quickly weighed. The solvent in the double dish was evaporated, dried at $325 \mathrm{~K}$ and then reweighed. The solubility was determined from the mass of the solid residue and the mass of the sample analyzed. Measurements were performed in triplicate.
Source and Purity of Chemicals:

(1) $99 \%$, Alfa Aesar Reagent Company, was recrystallized twice from propanone.

(2) $99.9+\%$, Tianjin Kemel Chemical, China, was used as received.

Estimated Error:

Temperature: $\pm 0.1 \mathrm{~K}$.

$x_{1}:<5 \%$ (relative error).

\begin{tabular}{ll}
\hline \hline Components: & Original Measurements: \\
(1) Pyrene; $\mathrm{C}_{16} \mathrm{H}_{10} ;[129-00-0]$ & ${ }^{10}$ W. E. Acree, Jr. and M. H. \\
(2) 1,4-Dioxane; $\mathrm{C}_{4} \mathrm{H}_{8} \mathrm{O}_{2} ;[123-91-1]$ & Abraham, Fluid Phase \\
& Equilib. 201, 245 (2002). \\
\hline Variables: & Prepared by: \\
$T / \mathrm{K}=299.15$ & W. E. Acree, Jr. \\
\hline
\end{tabular}

Experimental Values

\begin{tabular}{lcc}
\hline \hline$x_{2}{ }^{(\mathrm{s}) \mathrm{a}}$ & $x_{2}{ }^{\mathrm{b}}$ & $x_{1}{ }^{\mathrm{c}}$ \\
\hline 1.0000 & 0.9232 & 0.07676 \\
$\frac{{ }^{\mathrm{a}} x_{2}{ }^{(\mathrm{s})}: \text { initial mole fraction of component } 2 \text { in the solution. }}{{ }^{\mathrm{b}} x_{2}: \text { mole fraction of component } 2 \text { in the saturated solution. }}$ \\
${ }^{\mathrm{c}} x_{1}:$ mole fraction solubility of the solute.
\end{tabular}

The solubility was re-measured because an earlier published value $^{72}$ was out of line with observed pyrene solubility data for other ethers. The authors suspected that a twofold dilution had been missed when converting the earlier set of measured absorbances to mole fraction solubilities.

\section{Auxiliary Information}

Method/Apparatus/Procedure:

Constant-temperature bath, calorimetric thermometer, and an ultraviolet/ visible spectrophotometer.

Excess solute and solvent were placed in amber glass bottles and allowed to equilibrate for several days at constant temperature. Attainment of equilibrium was verified by several repetitive measurements and by approaching equilibrium from supersaturation. Aliquots of saturated solutions were transferred through a coarse filter into tared volumetric flasks, weighed and diluted with 2-propanol. Concentrations were determined by

spectrophotometric measurements at $372 \mathrm{~nm}$.

Source and Purity of Chemicals:

(1) $98 \%$, Aldrich Chemical Company, Milwaukee, WI, USA, was recrystallized several times from methanol.

(2) $99.8 \%$, anhydrous, Aldrich Chemical Company, stored over molecular sieves before use.

Estimated Error:

Temperature: $\pm 0.1 \mathrm{~K}$.

$x_{1}: \pm 1.5 \%$ (relative error) 


\subsection{Pyrene solubility data in haloalkanes, haloalkenes, and haloaromatic hydrocarbons}

\begin{tabular}{|c|c|}
\hline $\begin{array}{l}\text { Components: } \\
\text { (1) Pyrene; } \mathrm{C}_{16} \mathrm{H}_{10} ;[129-00-0] \\
\text { (2) 1-Chlorohexane; } \mathrm{C}_{6} \mathrm{H}_{13} \mathrm{Cl} \text {; } \\
{[544-10-5]}\end{array}$ & $\begin{array}{l}\text { Original Measurements: } \\
{ }^{45} \text { L. E. Roy, C. E. Hernández, and } \\
\text { W. E. Acree, Jr., Polycyclic } \\
\text { Aromat. Compd. 13, } 205 \text { (1999). }\end{array}$ \\
\hline $\begin{array}{l}\text { Variables: } \\
T / \mathrm{K}=299.15\end{array}$ & $\begin{array}{l}\text { Prepared by: } \\
\text { W. E. Acree, Jr. }\end{array}$ \\
\hline
\end{tabular}

Experimental Values

\begin{tabular}{lcc}
\hline \hline$x_{2}{ }^{(\mathrm{s}) \mathrm{a}}$ & $x_{2}{ }^{\mathrm{b}}$ & $x_{1}{ }^{\mathrm{c}}$ \\
\hline 1.0000 & 0.9244 & 0.07557 \\
$\frac{{ }^{\mathrm{a}} x_{2}{ }^{(\mathrm{s})}: \text { initial mole fraction of component 2 in the solution. }}{{ }^{\mathrm{b}} x_{2}: \text { mole fraction of component } 2 \text { in the saturated solution. }}$ \\
${ }^{\mathrm{c}} x_{1}:$ mole fraction solubility of the solute.
\end{tabular}

\section{Auxiliary Information}

\section{Method/Apparatus/Procedure:}

Constant-temperature bath, calorimetric thermometer, and an ultraviolet/ visible spectrophotometer.

Excess solute and solvent were placed in amber glass bottles and allowed to equilibrate for several days at constant temperature. Attainment of equilibrium was verified by several repetitive measurements and by approaching equilibrium from supersaturation. Aliquots of saturated solutions were transferred through a coarse filter into tared volumetric flasks, weighed and diluted with methanol. Concentrations were determined by

spectrophotometric measurements at $372 \mathrm{~nm}$.

\section{Source and Purity of Chemicals:}

(1) $99+\%$, Aldrich Chemical Company, Milwaukee, WI, USA, was recrystallized several times from methanol.

(2) $99 \%$, Aldrich Chemical Company, stored over molecular sieves and distilled shortly before use.

\section{Estimated Error:}

Temperature: $\pm 0.1 \mathrm{~K}$.

$x_{1}: \pm 1.5 \%$ (relative error).

\begin{tabular}{|c|c|}
\hline $\begin{array}{l}\text { Components: } \\
\text { (1) Pyrene; } \mathrm{C}_{16} \mathrm{H}_{10} ;[129-00-0] \\
\text { (2) } 1-\text {-Chlorooctane; } \mathrm{C}_{8} \mathrm{H}_{17} \mathrm{Cl} ; \\
{[111-85-3]}\end{array}$ & $\begin{array}{l}\text { Original Measurements: } \\
{ }^{45} \text { L. E. Roy, C. E. Hernández, and } \\
\text { W. E. Acree, Jr., Polycyclic } \\
\text { Aromat. Compd. 13, } 205 \text { (1999). }\end{array}$ \\
\hline $\begin{array}{l}\text { Variables: } \\
T / \mathrm{K}=299.15\end{array}$ & $\begin{array}{l}\text { Prepared by: } \\
\text { W. E. Acree, Jr. }\end{array}$ \\
\hline
\end{tabular}

Experimental Values

\begin{tabular}{|c|c|c|}
\hline$x_{2}^{(\mathrm{s}) \mathrm{a}}$ & $x_{2}{ }^{\mathrm{b}}$ & $x_{1}{ }^{\mathrm{c}}$ \\
\hline 1.0000 & 0.9140 & 0.08601 \\
\hline
\end{tabular}

\section{Auxiliary Information}

Method/Apparatus/Procedure:

Constant-temperature bath, calorimetric thermometer, and an ultraviolet/ visible spectrophotometer.

Excess solute and solvent were placed in amber glass bottles and allowed to equilibrate for several days at constant temperature. Attainment of equilibrium was verified by several repetitive measurements and by approaching equilibrium from supersaturation. Aliquots of saturated solutions were transferred through a coarse filter into tared volumetric flasks, weighed and diluted with methanol. Concentrations were determined by spectrophotometric measurements at $372 \mathrm{~nm}$.

Source and Purity of Chemicals:

(1) $99+\%$, Aldrich Chemical Company, Milwaukee, WI, USA, was recrystallized several times from methanol.

(2) $99 \%$, Aldrich Chemical Company, stored over molecular sieves and distilled shortly before use.

Estimated Error:

Temperature: $\pm 0.1 \mathrm{~K}$.

$x_{1}: \pm 1.5 \%$ (relative error).

\begin{tabular}{ll}
\hline \hline Components: & Original Measurements: \\
(1) Pyrene; $\mathrm{C}_{16} \mathrm{H}_{10} ;[129-00-0]$ & ${ }^{45}$ L. E. Roy, C. E. Hernández, and \\
(2) Chlorocyclohexane; $\mathrm{C}_{6} \mathrm{H}_{11} \mathrm{Cl} ;$ & W. E. Acree, Jr., Polycyclic \\
[542-18-7] & Aromat. Compd. 13, 205 (1999). \\
\hline Variables: & Prepared by: \\
$T / \mathrm{K}=299.15$ & W. E. Acree, Jr. \\
\hline
\end{tabular}

Experimental Values

\begin{tabular}{lcc}
\hline \hline$x_{2}{ }^{(\mathrm{s}) \mathrm{a}}$ & $x_{2}{ }^{\mathrm{b}}$ & $x_{1}{ }^{\mathrm{c}}$ \\
\hline 1.0000 & 0.9337 & 0.06625 \\
$\frac{{ }^{\mathrm{a}} x_{2}{ }^{(\mathrm{s})}: \text { initial mole fraction of component } 2 \text { in the solution. }}{{ }^{\mathrm{b}} x_{2}: \text { mole fraction of component } 2 \text { in the saturated solution. }}$ \\
${ }^{\mathrm{c}} x_{1}:$ mole fraction solubility of the solute.
\end{tabular}

\section{Auxiliary Information}

\section{Method/Apparatus/Procedure:}

Constant-temperature bath, calorimetric thermometer, and an ultraviolet/ visible spectrophotometer.

Excess solute and solvent were placed in amber glass bottles and allowed to equilibrate for several days at constant temperature. Attainment of equilibrium was verified by several repetitive measurements and by approaching equilibrium from supersaturation. Aliquots of saturated solutions were transferred through a coarse filter into tared volumetric flasks, weighed and diluted with methanol. Concentrations were determined by spectrophotometric measurements at $372 \mathrm{~nm}$.

\section{Source and Purity of Chemicals:}

(1) $99+\%$, Aldrich Chemical Company, Milwaukee, WI, USA, was recrystallized several times from methanol.

(2) $99 \%$, Aldrich Chemical Company, stored over molecular sieves and distilled shortly before use.

Estimated Error:

Temperature: $\pm 0.1 \mathrm{~K}$.

$x_{1}: \pm 1.5 \%$ (relative error). 


\section{Components:}

(1) Pyrene; $\mathrm{C}_{16} \mathrm{H}_{10} ;[129-00-0]$

(2) Fluorobenzene; $\mathrm{C}_{6} \mathrm{H}_{5} \mathrm{~F}$; [462-06-6]

Variables:
$T / \mathrm{K}=299.15$

Original Measurements:

${ }^{22}$ M. H. Abraham, W. E. Acree,

Jr., A. J. Leo, and D. Hoekman, New J. Chem. 33, 1685 (2009).

Prepared by:

W. E. Acree, Jr.

Experimental Values

\begin{tabular}{lcc}
\hline \hline$x_{2}{ }^{(\mathrm{s}) \mathrm{a}}$ & $x_{2}{ }^{\mathrm{b}}$ & $x_{1}{ }^{\mathrm{c}}$ \\
\hline 1.0000 & 0.9383 & 0.06168 \\
\hline
\end{tabular}

${ }^{\mathrm{a}} x_{2}{ }^{(\mathrm{s})}$ : initial mole fraction of component 2 in the solution.

${ }^{b} x_{2}$ : mole fraction of component 2 in the saturated solution.

${ }^{c} x_{1}$ : mole fraction solubility of the solute.

The experimental value was reported as the logarithm of pyrene's molar solubility in fluorobenzene dissolved by the solute's molar solubility in water. Experimental mole fraction solubility was obtained from the authors.

\section{Auxiliary Information}

\section{Method/Apparatus/Procedure:}

Constant-temperature bath, calorimetric thermometer, and an ultraviolet/ visible spectrophotometer.

Excess solute and solvent were placed in amber glass bottles and allowed to equilibrate for several days at constant temperature. Attainment of equilibrium was verified by several repetitive measurements and by approaching equilibrium from supersaturation. Aliquots of saturated solutions were transferred through a coarse filter into tared volumetric flasks, weighed and diluted with methanol. Concentrations were determined by spectrophotometric measurements at $372 \mathrm{~nm}$.

Source and Purity of Chemicals:

(1) $99+\%$, Aldrich Chemical Company, Milwaukee, WI, USA, was recrystallized several times from methanol.

(2) $99 \%$, Aldrich Chemical Company, stored over molecular sieves before use.

\section{Estimated Error:}

Temperature: $\pm 0.1 \mathrm{~K}$.

$x_{1}: \pm 1.5 \%$ (relative error).

\begin{tabular}{|c|c|}
\hline $\begin{array}{l}\text { Components: } \\
\text { (1) Pyrene; } \mathrm{C}_{16} \mathrm{H}_{10} ;[129-00-0] \\
\text { (2) (Trifluoromethyl)benzene; } \mathrm{C}_{7} \mathrm{H}_{5} \mathrm{~F}_{3} \text {; } \\
\text { [98-08-8] }\end{array}$ & $\begin{array}{l}\text { Original Measurements: } \\
\text { W. E. Acree, Jr., } \\
\text { unpublished data. }\end{array}$ \\
\hline $\begin{array}{l}\text { Variables: } \\
T / \mathrm{K}=299.15\end{array}$ & $\begin{array}{l}\text { Prepared by: } \\
\text { W. E. Acree, Jr. }\end{array}$ \\
\hline
\end{tabular}

Experimental Values

\begin{tabular}{lcc}
\hline \hline$x_{2}{ }^{(\mathrm{s}) \mathrm{a}}$ & $x_{2}{ }^{\mathrm{b}}$ & $x_{1}{ }^{\mathrm{c}}$ \\
\hline 1.0000 & 0.9624 & 0.03756 \\
$\frac{{ }^{\mathrm{a}} x_{2}{ }^{(\mathrm{s})}: \text { initial mole fraction of component } 2 \text { in the solution. }}{{ }^{\mathrm{b}} x_{2}: \text { mole fraction of component } 2 \text { in the saturated solution. }}$ \\
${ }^{\mathrm{c}}{ }_{x_{1}: \text { mole fraction solubility of the solute. }}$
\end{tabular}

Auxiliary Information

\section{Method/Apparatus/Procedure:}

Constant-temperature bath, calorimetric thermometer, and an ultraviolet/ visible spectrophotometer.

Excess solute and solvent were placed in amber glass bottles and allowed to equilibrate for several days at constant temperature. Attainment of equilibrium was verified by several repetitive measurements and by approaching equilibrium from supersaturation. Aliquots of saturated solutions were transferred through a coarse filter into tared volumetric flasks, weighed and diluted with 2-propanol. Concentrations were determined by

spectrophotometric measurements at $372 \mathrm{~nm}$.

Source and Purity of Chemicals:

(1) $98 \%$, Aldrich Chemical Company, Milwaukee, WI, USA, was recrystallized several times from methanol.

(2) $99 \%$, Aldrich Chemical Company, stored over molecular sieves before use.

\section{Estimated Error:}

Temperature: $\pm 0.1 \mathrm{~K}$.

$x_{1}: \pm 1.5 \%$ (relative error).

\subsection{Pyrene solubility data in alcohols}

\begin{tabular}{ll}
\hline \hline Components: & Original Measurements: \\
(1) Pyrene; $\mathrm{C}_{16} \mathrm{H}_{10} ;[129-00-0]$ & ${ }^{71} \mathrm{Q}$. Yu, X. Ma, and L. Xu, Fluid \\
(2) Methanol; $\mathrm{CH}_{4} \mathrm{O} ;[67-56-1]$ & Phase Equilib. 319, 5 (2012). \\
\hline Variables: & Prepared by: \\
Temperature & W. E. Acree, Jr. \\
\hline
\end{tabular}

Experimental Values

\begin{tabular}{lcc}
\hline \hline$T / \mathrm{K}$ & $x_{2}{ }^{\mathrm{a}}$ & $x_{1}{ }^{\mathrm{b}}$ \\
\hline 293.95 & 0.9987 & 0.00127 \\
298.35 & 0.9985 & 0.00149 \\
303.85 & 0.9983 & 0.00170 \\
308.65 & 0.9980 & 0.00202 \\
313.35 & 0.9978 & 0.00223 \\
318.35 & 0.9974 & 0.00262 \\
323.35 & 0.9971 & 0.00288 \\
328.15 & 0.9966 & 0.00341 \\
333.15 & 0.9962 & 0.00379 \\
${ }^{a}{ }^{a} x_{2}:$ mole fraction of component 2 in the saturated solution. \\
${ }^{\mathrm{b}}{ }_{x_{1}}$ : mole fraction of the polycyclic aromatic hydrocarbon (component 1).
\end{tabular}




\section{Auxiliary Information}

\section{Method/Apparatus/Procedure:}

Constant-temperature bath, oven and electronic balance.

Saturated solutions were prepared by placing excess solute in a cylindrical double-jacketed glass vial. The solution was equilibrated at constant temperature with continuous stirring for at least $4 \mathrm{~h}$. The stirring was discontinued and the solution was allowed to stand for $3 \mathrm{~h}$. An aliquot of the upper clear solution was removed, filtered through a $0.45 \mu \mathrm{m}$ membrane, and transferred to a tared double dish. The double dish and contents were quickly weighed. The solvent in the double dish was evaporated, dried at $325 \mathrm{~K}$, and then reweighed. The solubility was determined from the mass of the solid residue and the mass of the sample analyzed. Measurements were performed in triplicate.

\section{Source and Purity of Chemicals:}

(1) $99 \%$, Alfa Aesar Reagent Company, was recrystallized twice from propanone.

(2) $99.9+\%$, Tianjin Kemel Chemical, China, was used as received.

\section{Estimated Error:}

Temperature: $\pm 0.1 \mathrm{~K}$.

$x_{1}:<5 \%$ (relative error).

\begin{tabular}{ll}
\hline \hline Components: & Original Measurements: \\
(1) Pyrene; $\mathrm{C}_{16} \mathrm{H}_{10} ;[129-00-0]$ & ${ }^{71} \mathrm{Q}$. Yu, X. Ma, and L. Xu, Fluid \\
(2) Ethanol; $\mathrm{C}_{2} \mathrm{H}_{6} \mathrm{O} ;[64-17-5]$ & Phase Equilib. 319, 5 (2012). \\
\hline Variables: & Prepared by: \\
Temperature & W. E. Acree, Jr. \\
\hline
\end{tabular}

Experimental Values

\begin{tabular}{lcc}
\hline \hline$T / \mathrm{K}$ & $x_{2}{ }^{\mathrm{a}}$ & $x_{1}{ }^{\mathrm{b}}$ \\
\hline 299.55 & 0.9971 & 0.00294 \\
298.85 & 0.9968 & 0.00321 \\
303.75 & 0.9964 & 0.00363 \\
308.75 & 0.9958 & 0.00424 \\
313.55 & 0.9952 & 0.00479 \\
318.45 & 0.9946 & 0.00536 \\
323.45 & 0.9937 & 0.00628 \\
328.15 & 0.9928 & 0.00720 \\
333.15 & 0.9918 & 0.00820 \\
\hline
\end{tabular}

${ }^{a} x_{2}$ : mole fraction of component 2 in the saturated solution.

${ }^{b} x_{1}$ : mole fraction of the polycyclic aromatic hydrocarbon (component 1 ).

\section{Auxiliary Information}

\footnotetext{
Method/Apparatus/Procedure:

Constant-temperature bath, oven and electronic balance.

Saturated solutions were prepared by placing excess solute in a cylindrical double-jacketed glass vial. The solution was equilibrated at constant temperature with continuous stirring for at least $4 \mathrm{~h}$. The stirring was discontinued and the solution was allowed to stand for $3 \mathrm{~h}$. An aliquot of the upper clear solution was removed, filtered through a $0.45 \mu \mathrm{m}$ membrane, and transferred to a tared double dish. The double dish and contents were quickly weighed. The solvent in the double dish was evaporated, dried at $325 \mathrm{~K}$, and then reweighed. The solubility was determined from the mass of the solid residue and the mass of the sample analyzed. Measurements were performed in triplicate.
}

Source and Purity of Chemicals:

(1) $99 \%$, Alfa Aesar Reagent Company, was recrystallized twice from propanone.

(2) $99.9+\%$, Tianjin Kemel Chemical, China, was used as received.

Estimated Error:

Temperature: $\pm 0.1 \mathrm{~K}$.

$x_{1}:<1.3 \%$ (relative error).

\begin{tabular}{ll}
\hline \hline Components: & Original Measurements: \\
(1) Pyrene; $\mathrm{C}_{16} \mathrm{H}_{10} ;[129-00-0]$ & ${ }^{71} \mathrm{Q}$. Yu, X. Ma, and L. Xu, Fluid \\
(2) 1-Propanol; $\mathrm{C}_{3} \mathrm{H}_{8} \mathrm{O} ;[71-23-8]$ & Phase Equilib. 319, 5 (2012). \\
\hline Variables: & Prepared by: \\
Temperature & W. E. Acree, Jr. \\
\hline
\end{tabular}

Experimental Values

\begin{tabular}{lcc}
\hline \hline$T / \mathrm{K}$ & $x_{2}{ }^{\mathrm{a}}$ & $x_{1}{ }^{\mathrm{b}}$ \\
\hline 283.25 & 0.9973 & 0.00268 \\
290.05 & 0.9966 & 0.00342 \\
296.95 & 0.9960 & 0.00404 \\
303.75 & 0.9950 & 0.00499 \\
310.85 & 0.9939 & 0.00607 \\
317.55 & 0.9924 & 0.00761 \\
323.55 & 0.9907 & 0.00930 \\
331.35 & 0.9882 & 0.01179 \\
\hline
\end{tabular}

${ }^{\mathrm{a}} x_{2}$ : mole fraction of component 2 in the saturated solution.

${ }^{b} x_{1}$ : mole fraction of the polycyclic aromatic hydrocarbon (component 1).

\section{Auxiliary Information}

\section{Method/Apparatus/Procedure:}

Constant-temperature bath, oven and electronic balance.

Saturated solutions were prepared by placing excess solute in a cylindrical double-jacketed glass vial. The solution was equilibrated at constant temperature with continuous stirring for at least $4 \mathrm{~h}$. The stirring was discontinued and the solution was allowed to stand for $3 \mathrm{~h}$. An aliquot of the upper clear solution was removed, filtered through a $0.45 \mu \mathrm{m}$ membrane, and transferred to a tared double dish. The double dish and contents were quickly weighed. The solvent in the double dish was evaporated, dried at $325 \mathrm{~K}$ and then reweighed. The solubility was determined from the mass of the solid residue and the mass of the sample analyzed. Measurements were performed in triplicate.

Source and Purity of Chemicals:

(1) $99 \%$, Alfa Aesar Reagent Company, was recrystallized twice from propanone.

(2) $99.9+\%$, Tianjin Kemel Chemical, China, was used as received.

\section{Estimated Error:}

Temperature: $\pm 0.1 \mathrm{~K}$.

$x_{1}:<4 \%$ (relative error).

\begin{tabular}{|c|c|}
\hline $\begin{array}{l}\text { Components: } \\
\text { (1) Pyrene; } \mathrm{C}_{16} \mathrm{H}_{10} ;[129-00-0] \\
\text { (2) 2-Propanol; } \mathrm{C}_{3} \mathrm{H}_{8} \mathrm{O} ;[67-63-0]\end{array}$ & $\begin{array}{l}\text { Original Measurements: } \\
{ }^{71} \text { Q. Yu, X. Ma, and L. Xu, Fluid } \\
\text { Phase Equilib. 319, } 5 \text { (2012). }\end{array}$ \\
\hline $\begin{array}{l}\text { Variables: } \\
\text { Temperature }\end{array}$ & $\begin{array}{l}\text { Prepared by: } \\
\text { W. E. Acree, Jr. }\end{array}$ \\
\hline
\end{tabular}


Experimental Values

\begin{tabular}{lcc}
\hline \hline$T / \mathrm{K}$ & $x_{2}{ }^{\mathrm{a}}$ & $x_{1}{ }^{\mathrm{b}}$ \\
\hline 283.35 & 0.9980 & 0.00201 \\
290.05 & 0.9976 & 0.00242 \\
296.95 & 0.9971 & 0.00292 \\
300.85 & 0.9965 & 0.00350 \\
307.85 & 0.9958 & 0.00420 \\
314.55 & 0.9947 & 0.00530 \\
321.45 & 0.9933 & 0.00667 \\
328.15 & 0.9916 & 0.00835 \\
333.15 & 0.9901 & 0.00989 \\
${ }^{\mathrm{a}}{ }_{x_{2}: \text { mole fraction of component } 2 \text { in the saturated solution. }}$ \\
${ }^{\mathrm{b}}{ }_{\left.x_{1}: \text { mole fraction of the polycyclic aromatic hydrocarbon (component } 1\right) .}$
\end{tabular}

\section{Auxiliary Information}

\section{Method/Apparatus/Procedure:}

Constant-temperature bath, oven and electronic balance.

Saturated solutions were prepared by placing excess solute in a cylindrical double-jacketed glass vial. The solution was equilibrated at constant temperature with continuous stirring for at least $4 \mathrm{~h}$. The stirring was discontinued and the solution was allowed to stand for $3 \mathrm{~h}$. An aliquot of the upper clear solution was removed, filtered through a $0.45 \mu \mathrm{m}$ membrane, and transferred to a tared double dish. The double dish and contents were quickly weighed. The solvent in the double dish was evaporated, dried at $325 \mathrm{~K}$ and then reweighed. The solubility was determined from the mass of the solid residue and the mass of the sample analyzed. Measurements were performed in triplicate.

Source and Purity of Chemicals:

(1) $99 \%$, Alfa Aesar Reagent Company, was recrystallized twice from propanone.

(2) $99.9+\%$, Tianjin Kemel Chemical, China, was used as received.

Estimated Error:

Temperature: $\pm 0.1 \mathrm{~K}$.

$x_{1}:<5 \%$ (relative error).

\section{Auxiliary Information}

Method/Apparatus/Procedure:

Constant-temperature bath, calorimetric thermometer, and an ultraviolet/ visible spectrophotometer.

Excess solute and solvent were placed in amber glass bottles and allowed to equilibrate for several days at constant temperature. Attainment of equilibrium was verified by several repetitive measurements and by approaching equilibrium from supersaturation. Aliquots of saturated solutions were transferred through a coarse filter into tared volumetric flasks, weighed and diluted with 2-propanol. Concentrations were determined by spectrophotometric measurements at $372 \mathrm{~nm}$.

Source and Purity of Chemicals:

(1) $98 \%$, Aldrich Chemical Company, Milwaukee, WI, USA, was recrystallized several times from methanol.

(2) $99 \%$, Aldrich Chemical Company, stored over molecular sieves before use.

Estimated Error:

Temperature: $\pm 0.1 \mathrm{~K}$.

$x_{1}: \pm 1.5 \%$ (relative error).

\begin{tabular}{ll}
\hline \hline Components: & Original Measurements: \\
(1) Pyrene; $\mathrm{C}_{16} \mathrm{H}_{10} ;[129-00-0]$ & ${ }^{68}$ M. E. R. McHale, A.-S. M. Horton, S. A. \\
(2) 2-Pentanol; $\mathrm{C}_{5} \mathrm{H}_{12} \mathrm{O} ;$ & Padilla, A. L. Trufant, N. U. De La \\
[6032-29-7] & Sancha, E. Vela, and W. E. Acree, Jr., J. \\
& Chem. Eng. Data 41, 1522 (1996). \\
\hline Variables: & Prepared by: \\
$T / \mathrm{K}=299.15$ & W. E. Acree, Jr. \\
\hline
\end{tabular}

Experimental Values

\begin{tabular}{lcc}
\hline \hline$x_{2}{ }^{(\mathrm{s}) \mathrm{a}}$ & $x_{2}{ }^{\mathrm{b}}$ & $x_{1}{ }^{\mathrm{c}}$ \\
\hline 1.0000 & 0.9936 & 0.00640 \\
\hline${ }^{\mathrm{a}} x_{2}{ }^{(\mathrm{s})}:$ initial mole fraction of component 2 in the solution. \\
${ }^{\mathrm{b}} x_{2}:$ mole fraction of component 2 in the saturated solution. \\
${ }^{\mathrm{c}}{ }_{x_{1}: \text { mole fraction solubility of the solute. }}$
\end{tabular}

\section{Auxiliary Information}

\section{Components:}

(1) Pyrene; $\mathrm{C}_{16} \mathrm{H}_{10} ;[129-00-0]$

(2) 2-Methyl-2-propanol; $\mathrm{C}_{4} \mathrm{H}_{10} \mathrm{O}$;

[75-65-0]

\begin{tabular}{ll}
\hline Variables: & Prepared by: \\
$T / \mathrm{K}=299.15$ & W. E. Acree, Jr. \\
\hline
\end{tabular}

Experimental Values

\begin{tabular}{lcc}
\hline \hline$x_{2}{ }^{(\mathrm{s}) \mathrm{a}}$ & $x_{2}{ }^{\mathrm{b}}$ & $x_{1}{ }^{\mathrm{c}}$ \\
\hline 1.0000 & 0.9969 & 0.003134 \\
\hline
\end{tabular}

${ }^{\mathrm{a}} x_{2}{ }^{(\mathrm{s})}$ : initial mole fraction of component 2 in the solution.

${ }^{\mathrm{b}} x_{2}$ : mole fraction of component 2 in the saturated solution.

${ }^{c} x_{1}$ : mole fraction solubility of the solute.
Original Measurements:

${ }^{10}$ W. E. Acree, Jr. and M. H. Abraham, Fluid Phase Equilib. 201, 245 (2002)

Prepared by:

.

\footnotetext{
$x_{1}$ : mole fraction solubility of the solute.
}

r




\begin{tabular}{ll}
\hline \hline Components: & Original Measurements: \\
(1) Pyrene; $\mathrm{C}_{16} \mathrm{H}_{10} ;[129-00-0]$ & ${ }^{69}$ M. E. R. McHale, K. S. Coym, K. A. \\
(2) 2-Methyl-2-butanol; $\mathrm{C}_{5} \mathrm{H}_{12} \mathrm{O} ;$ & Fletcher, and W. E. Acree, Jr., J. \\
[75-85-4] & Chem. Eng. Data 42, 511 (1997). \\
\hline Variables: & Prepared by: \\
$T / \mathrm{K}=299.15$ & W. E. Acree, Jr. \\
\hline
\end{tabular}

Experimental Values

\begin{tabular}{lcc}
\hline \hline$x_{2}{ }^{(\mathrm{s}) \mathrm{a}}$ & $x_{2}{ }^{\mathrm{b}}$ & $x_{1}{ }^{\mathrm{c}}$ \\
\hline 1.0000 & 0.9938 & 0.00617 \\
$\frac{{ }^{a} x_{2}{ }^{(\mathrm{s})}: \text { initial mole fraction of component } 2 \text { in the solution. }}{{ }^{\mathrm{b}} x_{2}: \text { mole fraction of component } 2 \text { in the saturated solution. }}$ \\
${ }^{\mathrm{c}} x_{1}:$ mole fraction solubility of the solute.
\end{tabular}

${ }^{c} x_{1}$ : mole fraction solubility of the solute.

\section{Auxiliary Information}

Method/Apparatus/Procedure:
Constant-temperature bath, calorimetric thermometer, and an ultraviolet/
visible spectrophotometer.
Excess solute and solvent were placed in amber glass bottles and allowed to
equilibrate for several days at constant temperature. Attainment of equilibrium
was verified by several repetitive measurements and by approaching
equilibrium from supersaturation. Aliquots of saturated solutions were
transferred through a coarse filter into tared volumetric flasks, weighed and
diluted with methanol. Concentrations were determined by
spectrophotometric measurements at 372 nm.
Source and Purity of Chemicals:
(1) $99 \%$, Aldrich Chemical Company, Milwaukee, WI, USA, was
recrystallized several times from methanol.
(2) $99 \%$, Acros Organics, USA, stored over anhydrous sodium sulfate and
molecular sieves before use.
Estimated Error:
Temperature: $\pm 0.1 \mathrm{~K}$.
$x_{1}: \pm 1.3 \%$ (relative error).

\begin{tabular}{|c|c|}
\hline $\begin{array}{l}\text { Components: } \\
\text { (1) Pyrene; } \mathrm{C}_{16} \mathrm{H}_{10} ;[129-00-0] \\
\text { (2) 1-Hexanol; } \mathrm{C}_{6} \mathrm{H}_{14} \mathrm{O} ;[111-27-3]\end{array}$ & $\begin{array}{l}\text { Original Measurements: } \\
{ }^{61} \text { C. E. Hernández, K. M. De Fina, L. } \\
\text { E. Roy, T. L. Sharp, and W. E. Acree, } \\
\text { Jr., Can. J. Chem. 77, } 1465 \text { (1999). }\end{array}$ \\
\hline $\begin{array}{l}\text { Variables: } \\
T / \mathrm{K}=299.15\end{array}$ & $\begin{array}{l}\text { Prepared by: } \\
\text { W. E. Acree, Jr. }\end{array}$ \\
\hline
\end{tabular}

Experimental Values

\begin{tabular}{lcc}
\hline \hline$x_{2}{ }^{(\mathrm{s}) \mathrm{a}}$ & $x_{2}{ }^{\mathrm{b}}$ & $x_{1}{ }^{\mathrm{c}}$ \\
\hline 1.0000 & 0.9873 & 0.01273 \\
${ }^{\mathrm{a}} x_{2}{ }^{(\mathrm{s})}:$ initial mole fraction of component 2 in the solution. \\
${ }^{\mathrm{b}} x_{2}:$ mole fraction of component 2 in the saturated solution. \\
${ }^{\mathrm{c}} x_{1}:$ mole fraction solubility of the solute.
\end{tabular}

\section{Auxiliary Information}

Method/Apparatus/Procedure:

Constant-temperature bath, calorimetric thermometer, and an ultraviolet/ visible spectrophotometer.

Excess solute and solvent were placed in amber glass bottles and allowed to equilibrate for several days at constant temperature. Attainment of equilibrium was verified by several repetitive measurements and by approaching equilibrium from supersaturation. Aliquots of saturated solutions were transferred through a coarse filter into tared volumetric flasks, weighed and diluted with methanol. Concentrations were determined by spectrophotometric measurements at $372 \mathrm{~nm}$.

\section{Source and Purity of Chemicals:}

(1) $99+\%$, Aldrich Chemical Company, Milwaukee, WI, USA, was recrystallized several times from methanol.

(2) $99+\%$, Alfa Aesar, USA, stored over molecular sieves before use.

\section{Estimated Error:}

Temperature: $\pm 0.1 \mathrm{~K}$.

$x_{1}: \pm 1.5 \%$ (relative error).

\begin{tabular}{ll}
\hline \hline Components: & Original Measurements: \\
(1) Pyrene; $\mathrm{C}_{16} \mathrm{H}_{10} ;[129-00-0]$ & ${ }^{73}$ S. H. Ali and O. A. Al-Rashed, Fluid \\
(2) 1-Hexanol; $\mathrm{C}_{6} \mathrm{H}_{14} \mathrm{O} ;[111-27-3]$ & Phase Equilib. 281, 133 (2009). \\
\hline Variables: & Prepared by: \\
Temperature & W. E. Acree, Jr. \\
\hline
\end{tabular}

Experimental Values

\begin{tabular}{lcc}
\hline \hline$T / \mathrm{K}$ & $x_{2}{ }^{\mathrm{a}}$ & $x_{1}{ }^{\mathrm{b}}$ \\
\hline 293 & 0.9863 & 0.0137 \\
298 & 0.9857 & 0.0143 \\
303 & 0.9841 & 0.0159 \\
308 & 0.9817 & 0.0183 \\
313 & 0.9788 & 0.0212 \\
318 & 0.9763 & 0.0237 \\
\hline
\end{tabular}

${ }^{a} x_{2}$ : mole fraction of component 2 in the saturated solution.

${ }^{\mathrm{b}} x_{1}$ : mole fraction of the polycyclic aromatic hydrocarbon (component 1 ).

\section{Auxiliary Information}

\section{Method/Apparatus/Procedure:}

Constant-temperature bath, gas chromatograph equipped with a flame ionization detector and an uv/visible spectrophotometer

Saturated solutions were prepared by placing excess solute in glass bottles containing the solvent. Samples were equilibrated in a constant temperature with shaking for at least three days, followed by a three-day period in which the solid was allowed to settle to the bottom of the container. Aliquots were transferred to tared volumetric flasks, weighed and then diluted with the solvent for spectrophotometric analysis. Concentrations of the dilute solutions were determined from absorbance versus concentration curves based on absorbance measurements for standard solutions of known concentration. The spectrophotometric results were verified by gas chromatographic analyses.

\section{Source and Purity of Chemicals:}

(1) $97 \%$, Fluka, recrystallized several times from methanol to yield a sample of $99 \%$ purity.

(2) $99.9+\%$, chemical source not given, no purification details were provided in the paper. 
Estimated Error:

Temperature: $\pm 0.1 \mathrm{~K}$.

$x_{1}:<3.0 \%$ (relative error).

\begin{tabular}{ll}
\hline \hline Components: & Original Measurements: \\
(1) Pyrene; $\mathrm{C}_{16} \mathrm{H}_{10} ;[129-00-0]$ & ${ }^{70}$ M. E. R. McHale, K. A. Fletcher, \\
(2) 2-Methyl-1-pentanol; $\mathrm{C}_{6} \mathrm{H}_{14} \mathrm{O} ;$ & K. S. Coym, W. E. Acree, Jr., V. G. \\
[105-30-6] & Varanasi, and S. W. Campbell, \\
& Phys. Chem. Liq. 34, 103 (1997). \\
\hline Variables: & Prepared by: \\
$T / \mathrm{K}=299.15$ & W. E. Acree, Jr. \\
\hline
\end{tabular}

\section{Experimental Values}

\begin{tabular}{lcc}
\hline \hline$x_{2}{ }^{(\mathrm{s}) \mathrm{a}}$ & $x_{2}{ }^{\mathrm{b}}$ & $x_{1}{ }^{\mathrm{c}}$ \\
\hline 1.0000 & 0.9921 & 0.00789
\end{tabular}

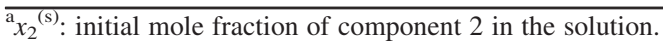

${ }^{b} x_{2}$ : mole fraction of component 2 in the saturated solution.

${ }^{c} x_{1}$ : mole fraction solubility of the solute.

\section{Auxiliary Information}

\section{Method/Apparatus/Procedure:}

Constant-temperature bath, calorimetric thermometer, and an ultraviolet/ visible spectrophotometer.

Excess solute and solvent were placed in amber glass bottles and allowed to equilibrate for several days at constant temperature. Attainment of equilibrium was verified by several repetitive measurements and by approaching equilibrium from supersaturation. Aliquots of saturated solutions were transferred through a coarse filter into tared volumetric flasks, weighed and diluted with methanol. Concentrations were determined by spectrophotometric measurements at $372 \mathrm{~nm}$.

\section{Source and Purity of Chemicals:}

(1) $99 \%$, Aldrich Chemical Company, Milwaukee, WI, USA, was recrystallized several times from anhydrous methanol.

(2) $99 \%$, Aldrich Chemical Company, stored over anhydrous sodium sulfate and molecular sieves before use.

\section{Estimated Error:}

Temperature: $\pm 0.1 \mathrm{~K}$.

$x_{1}: \pm 1.5 \%$ (relative error).

\begin{tabular}{|c|c|}
\hline $\begin{array}{l}\text { Components: } \\
\text { (1) Pyrene; } \mathrm{C}_{16} \mathrm{H}_{10} ;[129-00-0] \\
\text { (2) 4-Methyl-2-pentanol; } \mathrm{C}_{6} \mathrm{H}_{14} \mathrm{O} \text {; } \\
\text { [108-11-2] }\end{array}$ & $\begin{array}{l}\text { Original Measurements: } \\
{ }^{69} \text { M. E. R. McHale, K. S. Coym, K. A. } \\
\text { Fletcher, and W. E. Acree, Jr., J. } \\
\text { Chem. Eng. Data 42, } 511 \text { (1997). }\end{array}$ \\
\hline $\begin{array}{l}\text { Variables: } \\
T / \mathrm{K}=299.15\end{array}$ & $\begin{array}{l}\text { Prepared by: } \\
\text { W. E. Acree, Jr. }\end{array}$ \\
\hline
\end{tabular}

Experimental Values

\begin{tabular}{lcc}
\hline \hline$x_{2}{ }^{(\mathrm{s}) \mathrm{a}}$ & $x_{2}{ }^{\mathrm{b}}$ & $x_{1}{ }^{\mathrm{c}}$ \\
\hline 1.0000 & 0.9938 & 0.00621 \\
$\frac{{ }^{\mathrm{a}} x_{2}{ }^{(\mathrm{s})}: \text { initial mole fraction of component } 2 \text { in the solution. }}{{ }^{\mathrm{b}} x_{2}: \text { mole fraction of component } 2 \text { in the saturated solution. }}$ \\
${ }^{\mathrm{c}} x_{1}:$ mole fraction solubility of the solute.
\end{tabular}

\section{Auxiliary Information}

\section{Method/Apparatus/Procedure:}

Constant-temperature bath, calorimetric thermometer, and an ultraviolet/ visible spectrophotometer.

Excess solute and solvent were placed in amber glass bottles and allowed to equilibrate for several days at constant temperature. Attainment of equilibrium was verified by several repetitive measurements and by approaching equilibrium from supersaturation. Aliquots of saturated solutions were transferred through a coarse filter into tared volumetric flasks, weighed and diluted with methanol. Concentrations were determined by spectrophotometric measurements at $372 \mathrm{~nm}$.

Source and Purity of Chemicals:

(1) $99 \%$, Aldrich Chemical Company, Milwaukee, WI, USA, was recrystallized several times from methanol.

(2) $99+\%$, Acros Organics, USA, stored over anhydrous sodium sulfate and molecular sieves before use.

\section{Estimated Error:}

Temperature: $\pm 0.1 \mathrm{~K}$.

$x_{1}: \pm 1.3 \%$ (relative error).

\begin{tabular}{|c|c|}
\hline $\begin{array}{l}\text { Components: } \\
\text { (1) Pyrene; } \mathrm{C}_{16} \mathrm{H}_{10} ;[129-00-0] \\
\text { (2) } 1-H e p t a n o l ; \mathrm{C}_{7} \mathrm{H}_{16} \mathrm{O} ;[111-70-6]\end{array}$ & $\begin{array}{l}\text { Original Measurements: } \\
{ }^{61} \text { C. E. Hernández, K. M. De Fina, L. } \\
\text { E. Roy, T. L. Sharp, and W. E. Acree, } \\
\text { Jr., Can. J. Chem. 77, } 1465 \text { (1999). }\end{array}$ \\
\hline $\begin{array}{l}\text { Variables: } \\
T / \mathrm{K}=299.15\end{array}$ & $\begin{array}{l}\text { Prepared by: } \\
\text { W. E. Acree, Jr. }\end{array}$ \\
\hline
\end{tabular}

\section{Experimental Values}

\begin{tabular}{|c|c|c|}
\hline$x_{2}{ }^{(\mathrm{s}) \mathrm{a}}$ & $x_{2}{ }^{b}$ & $x_{1}{ }^{\mathrm{c}}$ \\
\hline 1.0000 & 0.9831 & 0.01694 \\
\hline
\end{tabular}

\section{Auxiliary Information}

\section{Method/Apparatus/Procedure:}

Constant-temperature bath, calorimetric thermometer, and an ultraviolet/ visible spectrophotometer.

Excess solute and solvent were placed in amber glass bottles and allowed to equilibrate for several days at constant temperature. Attainment of equilibrium was verified by several repetitive measurements and by approaching equilibrium from supersaturation. Aliquots of saturated solutions were transferred through a coarse filter into tared volumetric flasks, weighed and diluted with methanol. Concentrations were determined by spectrophotometric measurements at $372 \mathrm{~nm}$. 
Source and Purity of Chemicals:

(1) $99+\%$, Aldrich Chemical Company, Milwaukee, WI, USA, was recrystallized several times from methanol.

(2) $99+\%$, Alfa Aesar, USA, stored over molecular sieves before use.

Estimated Error:

Temperature: $\pm 0.1 \mathrm{~K}$

$x_{1}: \pm 1.5 \%$ (relative error).

\begin{tabular}{ll}
\hline \hline Components: & Original Measurements: \\
(1) Pyrene; $\mathrm{C}_{16} \mathrm{H}_{10} ;[129-00-0]$ & ${ }^{74} \mathrm{~S} . \mathrm{H}$. Ali, Fluid Phase Equilib. \\
(2) 1-Octanol; $\mathrm{C}_{8} \mathrm{H}_{18} \mathrm{O} ;[111-87-5]$ & $\mathbf{2 6 4}, 29(2008)$. \\
\hline Variables: & Prepared by: \\
Temperature & W. E. Acree, Jr. \\
\hline
\end{tabular}

Experimental Values

\begin{tabular}{lcc}
\hline \hline$T / \mathrm{K}$ & $x_{2}{ }^{\mathrm{a}}$ & $x_{1}{ }^{\mathrm{b}}$ \\
\hline 293 & 0.9781 & 0.0219 \\
298 & 0.9759 & 0.0241 \\
303 & 0.9715 & 0.0285 \\
308 & 0.9667 & 0.0333 \\
313 & 0.9634 & 0.0366 \\
318 & 0.9589 & 0.0411 \\
\hline
\end{tabular}

${ }^{\mathrm{a}} x_{2}$ : mole fraction of component 2 in the saturated solution.

${ }^{b} x_{1}$ : mole fraction of the polycyclic aromatic hydrocarbon (component 1 ).

\section{Auxiliary Information}

\section{Method/Apparatus/Procedure:}

Constant-temperature bath, gas chromatograph equipped with a flame ionization detector and an uv/visible spectrophotometer.

Saturated solutions were prepared by placing excess solute in glass bottles containing the solvent. Samples were equilibrated in a constant temperature with shaking for at least three days, followed by a three-day period in which the solid was allowed to settle to the bottom of the container. Aliquots were transferred to tared volumetric flasks, weighed and then diluted with the solvent for spectrophotometric analysis. Concentrations of the dilute solutions were determined from absorbance versus concentration curves based on absorbance measurements for standard solutions of known concentration. The spectrophotometric results were verified by gas chromatographic analyses.

Source and Purity of Chemicals:

(1) $97 \%$, Fluka, recrystallized several times from methanol to yield a sample of $99 \%$ purity.

(2) $99.9+\%$, Baker Chemical Company, USA, no purification details were provided in the paper.

Estimated Error:

Temperature: $\pm 0.1 \mathrm{~K}$.

$x_{1}:<3.0 \%$ (relative error).
Experimental Values

\begin{tabular}{|c|c|c|}
\hline$x_{2}{ }^{(\mathrm{s}) \mathrm{a}}$ & $x_{2}{ }^{b}$ & $x_{1}{ }^{\mathrm{c}}$ \\
\hline 1.0000 & 0.9714 & 0.02859 \\
\hline
\end{tabular}

The published value was given as molar solubility of pyrene in 1-decanol. The mole fraction solubility was provided by the authors of the paper.

\section{Auxiliary Information}

\section{Method/Apparatus/Procedure:}

Constant-temperature bath, calorimetric thermometer, and an ultraviolet/ visible spectrophotometer.

Excess solute and solvent were placed in amber glass bottles and allowed to equilibrate for several days at constant temperature. Attainment of equilibrium was verified by several repetitive measurements and by approaching equilibrium from supersaturation. Aliquots of saturated solutions were transferred through a coarse filter into tared volumetric flasks, weighed and diluted with methanol. Concentrations were determined by spectrophotometric measurements at $372 \mathrm{~nm}$.

Source and Purity of Chemicals:

(1) $99+\%$, Aldrich Chemical Company, Milwaukee, WI, USA, was recrystallized several times from methanol.

(2) $99+\%$, Alfa Aesar, USA, stored over molecular sieves before use.

Estimated Error:

Temperature: $\pm 0.1 \mathrm{~K}$.

$x_{1}: \pm 1.5 \%$ (relative error)

\begin{tabular}{ll}
\hline \hline Components: & Original Measurements: \\
(1) Pyrene; $\mathrm{C}_{16} \mathrm{H}_{10} ;[129-00-0]$ & ${ }^{10}$ W. E. Acree, Jr. and M. H. \\
(2) 3,7-Dimethyl-1-octanol; $\mathrm{C}_{10} \mathrm{H}_{22} \mathrm{O} ;$ & Abraham, Fluid Phase Equilib. \\
{$[106-21-8]$} & $\mathbf{2 0 1 ,} 245$ (2002). \\
\hline Variables: & Prepared by: \\
$T / \mathrm{K}=299.15$ & W. E. Acree, Jr. \\
\hline
\end{tabular}

Experimental Values

\begin{tabular}{lcc}
\hline \hline$\frac{x_{2}{ }^{(\mathrm{s}) \mathrm{a}}}{x_{2}{ }^{\mathrm{b}}}$ & $x_{1}{ }^{\mathrm{c}}$ \\
\hline 1.0000 & 0.9831 & 0.01694 \\
$\frac{{ }^{\mathrm{a}} x_{2}{ }^{(\mathrm{s})}: \text { initial mole fraction of component } 2 \text { in the solution. }}{{ }^{\mathrm{b}}{ }_{x_{2}}: \text { mole fraction of component } 2 \text { in the saturated solution. }}$ \\
${ }^{\mathrm{c}}{ }_{x_{1}}$ : mole fraction solubility of the solute.
\end{tabular}

\begin{tabular}{ll}
\hline \hline Components: & Original Measurements: \\
(1) Pyrene; $\mathrm{C}_{16} \mathrm{H}_{10} ;[129-00-0]$ & ${ }^{10}$ W. E. Acree, Jr. and M. H. \\
(2) 1-Decanol; $\mathrm{C}_{10} \mathrm{H}_{22} \mathrm{O} ;[112-30-1]$ & Abraham, Fluid Phase Equilib. \\
& $\mathbf{2 0 1 , 2 4 5 ( 2 0 0 2 ) .}$ \\
\hline Variables: & Prepared by: \\
$T / \mathrm{K}=299.15$ & W. E. Acree, Jr. \\
\hline
\end{tabular}




\section{Auxiliary Information}

Method/Apparatus/Procedure:

Constant-temperature bath, calorimetric thermometer, and an ultraviolet/ visible spectrophotometer.

Excess solute and solvent were placed in amber glass bottles and allowed to equilibrate for several days at constant temperature. Attainment of equilibrium was verified by several repetitive measurements and by approaching equilibrium from supersaturation. Aliquots of saturated solutions were transferred through a coarse filter into tared volumetric flasks, weighed and diluted with 2-propanol. Concentrations were determined by spectrophotometric measurements at $372 \mathrm{~nm}$.

Source and Purity of Chemicals:

(1) $98 \%$, Aldrich Chemical Company, Milwaukee, WI, USA, was recrystallized several times from methanol.

(2) $99 \%$, Aldrich Chemical Company, stored over molecular sieves before use.

\section{Estimated Error:}

Temperature: $\pm 0.1 \mathrm{~K}$.

$x_{1}: \pm 1.5 \%$ (relative error).

\begin{tabular}{|c|c|}
\hline $\begin{array}{l}\text { Components: } \\
\text { (1) Pyrene; } \mathrm{C}_{16} \mathrm{H}_{10} ;[129-00-0] \\
\text { (2) Cyclohexanol; } \mathrm{C}_{6} \mathrm{H}_{12} \mathrm{O} \text {; } \\
{[108-93-0]}\end{array}$ & $\begin{array}{l}\text { Original Measurements: } \\
{ }^{68} \text { M. E. R. McHale, A.-S. M. Horton, S. A. } \\
\text { Padilla, A. L. Trufant, N. U. De La } \\
\text { Sancha, E. Vela, and W. E. Acree, Jr., } \\
\text { J. Chem. Eng. Data 41, } 1522 \text { (1996). }\end{array}$ \\
\hline $\begin{array}{l}\text { Variables: } \\
T / \mathrm{K}=299.15\end{array}$ & $\begin{array}{l}\text { Prepared by: } \\
\text { W. E. Acree, Jr. }\end{array}$ \\
\hline
\end{tabular}

Experimental Values

\begin{tabular}{lcc}
\hline \hline$x_{2}{ }^{(\mathrm{s}) \mathrm{a}}$ & $x_{2}{ }^{\mathrm{b}}$ & $x_{1}{ }^{\mathrm{c}}$ \\
\hline 1.0000 & 0.9903 & 0.00965 \\
\hline
\end{tabular}

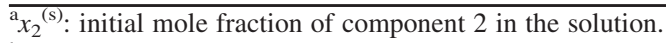

${ }^{\mathrm{b}} x_{2}$ : mole fraction of component 2 in the saturated solution.

${ }^{c} x_{1}$ : mole fraction solubility of the solute.

\section{Auxiliary Information}

Method/Apparatus/Procedure:
Constant-temperature bath, calorimetric thermometer, and an ultraviolet/
visible spectrophotometer.
Excess solute and solvent were placed in amber glass bottles and allowed to
equilibrate for several days at constant temperature. Attainment of equilibrium
was verified by several repetitive measurements and by approaching
equilibrium from supersaturation. Aliquots of saturated solutions were
transferred through a coarse filter into tared volumetric flasks, weighed and
diluted with methanol. Concentrations were determined by
spectrophotometric measurements at 372 nm.
Source and Purity of Chemicals:
(1) $99 \%$, Aldrich Chemical Company, Milwaukee, WI, USA, was
recrystallized several times from methanol.
(2) $99+\%$, Aldrich Chemical Company, stored over molecular sieves before
use.
Estimated Error:
Temperature: $\pm 0.1 \mathrm{~K}$.
$x_{1}: \pm 1.5 \%$ (relative error).

\begin{tabular}{ll}
\hline \hline Components: & Original Measurements: \\
(1) Pyrene; $\mathrm{C}_{16} \mathrm{H}_{10} ;[129-00-0]$ & ${ }^{45}$ L. E. Roy, C. E. Hernández, and \\
(2) 1,2 -Ethanediol; $\mathrm{C}_{2} \mathrm{H}_{6} \mathrm{O}_{2} ;[107-21-1]$ & W. E. Acree, Jr., Polycyclic \\
& Aromat. Compd. 13, 205 (1999). \\
\hline Variables: & Prepared by: \\
$T / \mathrm{K}=299.15$ & W. E. Acree, Jr. \\
\hline
\end{tabular}

Experimental Values

\begin{tabular}{|c|c|c|}
\hline$x_{2}{ }^{(\mathrm{s}) \mathrm{a}}$ & $x_{2}{ }^{\mathrm{b}}$ & $x_{1}{ }^{\mathrm{c}}$ \\
\hline 1.0000 & 0.9995 & 0.000519 \\
\hline
\end{tabular}

Auxiliary Information

\begin{abstract}
Method/Apparatus/Procedure:
Constant-temperature bath, calorimetric thermometer, and an ultraviolet/ visible spectrophotometer.

Excess solute and solvent were placed in amber glass bottles and allowed to equilibrate for several days at constant temperature. Attainment of equilibrium was verified by several repetitive measurements and by approaching equilibrium from supersaturation. Aliquots of saturated solutions were transferred through a coarse filter into tared volumetric flasks, weighed and diluted with methanol. Concentrations were determined by spectrophotometric measurements at $372 \mathrm{~nm}$.
\end{abstract}

Source and Purity of Chemicals:

(1) $99+\%$, Aldrich Chemical Company, Milwaukee, WI, USA, was recrystallized several times from methanol.

(2) $99+\%$, Aldrich Chemical Company, stored over molecular sieves and distilled shortly before use.

\section{Estimated Error:}

Temperature: $\pm 0.1 \mathrm{~K}$.

$x_{1}: \pm 1.5 \%$ (relative error).

\begin{tabular}{|c|c|}
\hline $\begin{array}{l}\text { Components: } \\
\text { (1) Pyrene; } \mathrm{C}_{16} \mathrm{H}_{10} ;[129-00-0] \\
\text { (2) 2,2,2-Trifluoroethanol; } \mathrm{C}_{2} \mathrm{H}_{3} \mathrm{~F}_{3} \mathrm{O} \text {; } \\
\text { [75-89-8] }\end{array}$ & $\begin{array}{l}\text { Original Measurements: } \\
{ }^{45} \text { L. E. Roy, C. E. Hernández, and } \\
\text { W. E. Acree, Jr., Polycyclic } \\
\text { Aromat. Compd. 13, } 205 \text { (1999). }\end{array}$ \\
\hline $\begin{array}{l}\text { Variables: } \\
T / \mathrm{K}=299.15\end{array}$ & $\begin{array}{l}\text { Prepared by: } \\
\text { W. E. Acree, Jr. }\end{array}$ \\
\hline
\end{tabular}

Experimental Values

\begin{tabular}{|c|c|c|}
\hline$x_{2}^{(\mathrm{s}) \mathrm{a}}$ & $x_{2}{ }^{b}$ & $x_{1}^{\mathrm{c}}$ \\
\hline 1.0000 & 0.9995 & 0.000467 \\
\hline
\end{tabular}




\section{Auxiliary Information}

\section{Method/Apparatus/Procedure:}

Constant-temperature bath, calorimetric thermometer, and an ultraviolet/ visible spectrophotometer.

Excess solute and solvent were placed in amber glass bottles and allowed to equilibrate for several days at constant temperature. Attainment of equilibrium was verified by several repetitive measurements and by approaching equilibrium from supersaturation. Aliquots of saturated solutions were transferred through a coarse filter into tared volumetric flasks, weighed and diluted with methanol. Concentrations were determined by spectrophotometric measurements at $372 \mathrm{~nm}$.

Source and Purity of Chemicals:

(1) $99+\%$, Aldrich Chemical Company, Milwaukee, WI, USA, was recrystallized several times from methanol.

(2) $99 \%$, Aldrich Chemical Company, stored over molecular sieves and distilled shortly before use.

Estimated Error:

Temperature: $\pm 0.1 \mathrm{~K}$.

$x_{1}: \pm 1.5 \%$ (relative error).

\subsection{Pyrene solubility data in alkoxyalcohols}

\begin{tabular}{ll}
\hline \hline Components: & $\begin{array}{l}\text { Original Measurements: } \\
\text { (1) Pyrene; } \mathrm{C}_{16} \mathrm{H}_{10} ;[129-00-0]\end{array}$ \\
$\begin{array}{l}{ }^{69} \text { M. E. R. McHale, K. S. Coym, K. A. } \\
\text { (2) 2-Methoxyethanol; } \mathrm{C}_{3} \mathrm{H}_{8} \mathrm{O}_{2} ;\end{array}$ & Fletcher, and W. E. Acree, Jr., J. \\
[109-86-4] & Chem. Eng. Data 42, 511 (1997). \\
\hline Variables: & Prepared by: \\
$T / \mathrm{K}=299.15$ & W. E. Acree, Jr. \\
\hline
\end{tabular}

Experimental Values

\begin{tabular}{lcc}
\hline \hline$x_{2}{ }^{(\mathrm{s}) \mathrm{a}}$ & $x_{2}{ }^{\mathrm{b}}$ & $x_{1}{ }^{\mathrm{c}}$ \\
\hline 1.0000 & 0.9828 & 0.01717
\end{tabular}

${ }^{\mathrm{a}} x_{2}{ }^{(\mathrm{s})}$ : initial mole fraction of component 2 in the solution.

${ }^{b} x_{2}$ : mole fraction of component 2 in the saturated solution.

${ }^{c} x_{1}$ : mole fraction solubility of the solute.

\section{Auxiliary Information}

\section{Method/Apparatus/Procedure:}

Constant-temperature bath, calorimetric thermometer, and an ultraviolet/ visible spectrophotometer.

Excess solute and solvent were placed in amber glass bottles and allowed to equilibrate for several days at constant temperature. Attainment of equilibrium was verified by several repetitive measurements and by approaching equilibrium from supersaturation. Aliquots of saturated solutions were transferred through a coarse filter into tared volumetric flasks, weighed and diluted with methanol. Concentrations were determined by spectrophotometric measurements at $372 \mathrm{~nm}$.

\section{Source and Purity of Chemicals:}

(1) $99 \%$, Aldrich Chemical Company, Milwaukee, WI, USA, was recrystallized several times from methanol.

(2) $99.5+\%$, anhydrous, Aldrich Chemical Company, stored over anhydrous sodium sulfate and molecular sieves before use.
Estimated Error:

Temperature: $\pm 0.1 \mathrm{~K}$.

$x_{1}: \pm 1.3 \%$ (relative error).

\begin{tabular}{ll}
\hline \hline Components: & Original Measurements: \\
(1) Pyrene; $\mathrm{C}_{16} \mathrm{H}_{10} ;[129-00-0]$ & ${ }^{69}$ M. E. R. McHale, K. S. Coym, K. A. \\
(2) 2-Ethoxyethanol; $\mathrm{C}_{4} \mathrm{H}_{10} \mathrm{O}_{2} ;$ & Fletcher, and W. E. Acree, Jr., J. \\
{$[110-80-5]$} & Chem. Eng. Data 42, 511 (1997). \\
\hline Variables: & Prepared by: \\
$T / \mathrm{K}=299.15$ & W. E. Acree, Jr. \\
\hline
\end{tabular}

Experimental Values

\begin{tabular}{lcc}
\hline \hline$x_{2}{ }^{(\mathrm{s}) \mathrm{a}}$ & $x_{2}^{\mathrm{b}}$ & $x_{1}{ }^{\mathrm{c}}$ \\
\hline 1.0000 & 0.9695 & 0.03046 \\
$\frac{{ }^{a} x_{2}{ }^{(\mathrm{s})}: \text { initial mole fraction of component } 2 \text { in the solution. }}{{ }^{\mathrm{b}} x_{2}: \text { mole fraction of component } 2 \text { in the saturated solution. }}$ \\
${ }^{c} x_{1}:$ mole fraction solubility of the solute.
\end{tabular}

\section{Auxiliary Information}

Method/Apparatus/Procedure:

Constant-temperature bath, calorimetric thermometer, and an ultraviolet/ visible spectrophotometer.

Excess solute and solvent were placed in amber glass bottles and allowed to equilibrate for several days at constant temperature. Attainment of equilibrium was verified by several repetitive measurements and by approaching equilibrium from supersaturation. Aliquots of saturated solutions were transferred through a coarse filter into tared volumetric flasks, weighed and diluted with methanol. Concentrations were determined by spectrophotometric measurements at $372 \mathrm{~nm}$.

\section{Source and Purity of Chemicals:}

(1) $99 \%$, Aldrich Chemical Company, Milwaukee, WI, USA, was recrystallized several times from methanol.

(2) $99 \%$, Acros Organics, USA, stored over anhydrous sodium sulfate molecular sieves before use.

\section{Estimated Error:}

Temperature: $\pm 0.1 \mathrm{~K}$.

$x_{1}: \pm 1.3 \%$ (relative error)

\begin{tabular}{ll}
\hline \hline Components: & Original Measurements: \\
(1) Pyrene; $\mathrm{C}_{16} \mathrm{H}_{10} ;[129-00-0]$ & ${ }^{69}$ M. E. R. McHale, K. S. Coym, K. A. \\
(2) 2-Propoxyethanol; $\mathrm{C}_{5} \mathrm{H}_{12} \mathrm{O}_{2} ;$ & Fletcher, and W. E. Acree, Jr., J. \\
{$[2807-30-9]$} & Chem. Eng. Data 42, 511 (1997). \\
\hline Variables: & Prepared by: \\
$T / \mathrm{K}=299.15$ & W. E. Acree, Jr. \\
\hline
\end{tabular}


Experimental Values

\begin{tabular}{lcc}
\hline \hline$x_{2}{ }^{(\mathrm{s}) \mathrm{a}}$ & $x_{2}{ }^{\mathrm{b}}$ & $x_{1}{ }^{\mathrm{c}}$ \\
\hline 1.0000 & 0.9660 & 0.03400 \\
$\frac{{ }^{\mathrm{a}} x_{2}{ }^{(\mathrm{s})}: \text { initial mole fraction of component } 2 \text { in the solution. }}{{ }^{\mathrm{b}}{ }_{x_{2}}: \text { mole fraction of component } 2 \text { in the saturated solution. }}$ \\
${ }^{\mathrm{c}}{ }_{x_{1}}:$ mole fraction solubility of the solute.
\end{tabular}

\section{Auxiliary Information}

\section{Method/Apparatus/Procedure:}

Constant-temperature bath, calorimetric thermometer, and an ultraviolet/ visible spectrophotometer.

Excess solute and solvent were placed in amber glass bottles and allowed to equilibrate for several days at constant temperature. Attainment of equilibrium was verified by several repetitive measurements and by approaching equilibrium from supersaturation. Aliquots of saturated solutions were transferred through a coarse filter into tared volumetric flasks, weighed and diluted with methanol. Concentrations were determined by spectrophotometric measurements at $372 \mathrm{~nm}$.

Source and Purity of Chemicals:

(1) $99 \%$, Aldrich Chemical Company, Milwaukee, WI, USA, was recrystallized several times from methanol.

(2) $99+\%$, Aldrich Chemical Company, stored over anhydrous sodium sulfate and molecular sieves before use.

Estimated Error:

Temperature: $\pm 0.1 \mathrm{~K}$.

$x_{1}: \pm 1.3 \%$ (relative error).

\begin{tabular}{ll}
\hline \hline Components: & Original Measurements: \\
(1) Pyrene; $\mathrm{C}_{16} \mathrm{H}_{10} ;[129-00-0]$ & ${ }^{69}$ M. E. R. McHale, K. S. Coym, K. A. \\
(2) 2-Butoxyethanol; $\mathrm{C}_{6} \mathrm{H}_{14} \mathrm{O}_{2} ;$ & Fletcher, and W. E. Acree, Jr., J. \\
[111-76-2] & Chem. Eng. Data 42, 511 (1997). \\
\hline Variables: & Prepared by: \\
$T / \mathrm{K}=299.15$ & W. E. Acree, Jr. \\
\hline
\end{tabular}

Experimental Values

\begin{tabular}{|c|c|c|}
\hline$x_{2}{ }^{(\mathrm{s}) \mathrm{a}}$ & $x_{2}{ }^{b}$ & $x_{1}{ }^{c}$ \\
\hline 1.0000 & 0.9621 & 0.03790 \\
\hline
\end{tabular}

\section{Auxiliary Information}

\footnotetext{
Method/Apparatus/Procedure:

Constant-temperature bath, calorimetric thermometer, and an ultraviolet/ visible spectrophotometer.

Excess solute and solvent were placed in amber glass bottles and allowed to equilibrate for several days at constant temperature. Attainment of equilibrium was verified by several repetitive measurements and by approaching equilibrium from supersaturation. Aliquots of saturated solutions were transferred through a coarse filter into tared volumetric flasks, weighed and diluted with methanol. Concentrations were determined by spectrophotometric measurements at $372 \mathrm{~nm}$.
}

Source and Purity of Chemicals:

(1) $99 \%$, Aldrich Chemical Company, Milwaukee, WI, USA, was recrystallized several times from methanol.

(2) $99 \%$, Acros Organics, USA, stored over anhydrous sodium sulfate and molecular sieves before use.

\section{Estimated Error:}

Temperature: $\pm 0.1 \mathrm{~K}$.

$x_{1}: \pm 1.3 \%$ (relative error).

\begin{tabular}{|c|c|}
\hline $\begin{array}{l}\text { Components: } \\
\text { (1) Pyrene; } \mathrm{C}_{16} \mathrm{H}_{10} ;[129-00-0] \\
\text { (2) 3-Methoxy-1-butanol; } \mathrm{C}_{5} \mathrm{H}_{12} \mathrm{O}_{2} \text {; } \\
\text { [2517-43-3] }\end{array}$ & $\begin{array}{l}\text { Original Measurements: } \\
{ }^{69} \text { M. E. R. McHale, K. S. Coym, K. A. } \\
\text { Fletcher, and W. E. Acree, Jr., J. } \\
\text { Chem. Eng. Data 42, } 511 \text { (1997). }\end{array}$ \\
\hline $\begin{array}{l}\text { Variables: } \\
T / \mathrm{K}=299.15\end{array}$ & $\begin{array}{l}\text { Prepared by: } \\
\text { W. E. Acree, Jr. }\end{array}$ \\
\hline
\end{tabular}

Experimental Values

\begin{tabular}{lcc}
\hline \hline$x_{2}{ }^{(\mathrm{s}) \mathrm{a}}$ & $x_{2}{ }^{\mathrm{b}}$ & $x_{1}{ }^{\mathrm{c}}$ \\
\hline 1.0000 & 0.9746 & 0.02541 \\
${ }^{\mathrm{a}} x_{2}{ }^{(\mathrm{s})}$ : initial mole fraction of component 2 in the solution. \\
${ }^{\mathrm{b}} x_{2}:$ mole fraction of component 2 in the saturated solution. \\
${ }^{\mathrm{c}}{ }_{x_{1}: \text { mole fraction solubility of the solute. }}$
\end{tabular}

\section{Auxiliary Information}

Method/Apparatus/Procedure:

Constant-temperature bath, calorimetric thermometer, and an ultraviolet/ visible spectrophotometer.

Excess solute and solvent were placed in amber glass bottles and allowed to equilibrate for several days at constant temperature. Attainment of equilibrium was verified by several repetitive measurements and by approaching equilibrium from supersaturation. Aliquots of saturated solutions were transferred through a coarse filter into tared volumetric flasks, weighed and diluted with methanol. Concentrations were determined by spectrophotometric measurements at $372 \mathrm{~nm}$.

Source and Purity of Chemicals:

(1) $99 \%$, Aldrich Chemical Company, Milwaukee, WI, USA, was recrystallized several times from methanol.

(2) $99+\%$, Aldrich Chemical Company, stored over anhydrous sodium sulfate and molecular sieves before use.

Estimated Error:

Temperature: $\pm 0.1 \mathrm{~K}$.

$x_{1}: \pm 1.3 \%$ (relative error).

\subsection{Pyrene solubility data in ketones}

\begin{tabular}{ll}
\hline \hline Components: & Original Measurements: \\
(1) Pyrene; $\mathrm{C}_{16} \mathrm{H}_{10} ;[129-00-0]$ & ${ }^{71} \mathrm{Q}$. Yu, X. Ma, and L. Xu, Fluid \\
(2) Propanone; $\mathrm{C}_{3} \mathrm{H}_{6} \mathrm{O} ;[67-64-1]$ & Phase Equilib. 319, 5 (2012). \\
\hline Variables: & Prepared by: \\
Temperature & W. E. Acree, Jr. \\
\hline
\end{tabular}


Experimental Values

\begin{tabular}{lcc}
\hline \hline$T / \mathrm{K}$ & $x_{2}{ }^{\mathrm{a}}$ & $x_{1}{ }^{\mathrm{b}}$ \\
\hline 295.35 & 0.9697 & 0.0303 \\
299.05 & 0.9668 & 0.0332 \\
303.75 & 0.9618 & 0.0382 \\
308.65 & 0.9564 & 0.0436 \\
313.55 & 0.9498 & 0.0502 \\
318.45 & 0.9433 & 0.0567 \\
323.35 & 0.9334 & 0.0666 \\
\hline
\end{tabular}

${ }^{\mathrm{a}} x_{2}$ : mole fraction of component 2 in the saturated solution.

${ }^{\mathrm{b}} x_{1}$ : mole fraction of the polycyclic aromatic hydrocarbon (component 1).

\section{Auxiliary Information}

\section{Method/Apparatus/Procedure:}

Constant-temperature bath, oven and electronic balance.

Saturated solutions were prepared by placing excess solute in a cylindrical double-jacketed glass vial. The solution was equilibrated at constant temperature with continuous stirring for at least $4 \mathrm{~h}$. The stirring was discontinued and the solution was allowed to stand for $3 \mathrm{~h}$. An aliquot of the upper clear solution was removed, filtered through a $0.45 \mu \mathrm{m}$ membrane, and transferred to a tared double dish. The double dish and contents were quickly weighed. The solvent in the double dish was evaporated, dried at $325 \mathrm{~K}$ and then reweighed. The solubility was determined from the mass of the solid residue and the mass of the sample analyzed. Measurements were performed in triplicate.

Source and Purity of Chemicals:

(1) $99 \%$, Alfa Aesar Reagent Company, was recrystallized twice from propanone.

(2) $99.9+\%$, Tianjin Kemel Chemical, China, was used as received.

\section{Estimated Error:}

Temperature: $\pm 0.1 \mathrm{~K}$.

$x_{1}: \pm 1 \%$ (relative error).

\begin{tabular}{ll}
\hline \hline Components: & Original Measurements: \\
(1) Pyrene; $\mathrm{C}_{16} \mathrm{H}_{10} ;[129-00-0]$ & ${ }^{61}$ C. E. Hernández, K. M. De Fina, L. \\
(2) 2-Butanone; $\mathrm{C}_{4} \mathrm{H}_{8} \mathrm{O} ;[78-93-3]$ & E. Roy, T. L. Sharp, and W. E. Acree, \\
& Jr., Can. J. Chem. 77, 1465 (1999). \\
\hline Variables: & Prepared by: \\
$T / \mathrm{K}=299.15$ & W. E. Acree, Jr. \\
\hline
\end{tabular}

Experimental Values

\begin{tabular}{lcc}
\hline \hline$x_{2}{ }^{(\mathrm{s}) \mathrm{a}}$ & $x_{2}{ }^{\mathrm{b}}$ & $x_{1}{ }^{\mathrm{c}}$ \\
\hline 1.0000 & 0.9325 & 0.06752 \\
$\frac{{ }^{\mathrm{a}} x_{2}{ }^{(\mathrm{s})}: \text { initial mole fraction of component 2 in the solution. }}{{ }^{\mathrm{b}} x_{2}: \text { mole fraction of component } 2 \text { in the saturated solution. }}$ \\
${ }^{\mathrm{c}}{ }_{x_{1}}:$ mole fraction solubility of the solute.
\end{tabular}

\section{Auxiliary Information}

\section{Method/Apparatus/Procedure:}

Constant-temperature bath, calorimetric thermometer, and an ultraviolet/ visible spectrophotometer.

Excess solute and solvent were placed in amber glass bottles and allowed to equilibrate for several days at constant temperature. Attainment of equilibrium was verified by several repetitive measurements and by approaching equilibrium from supersaturation. Aliquots of saturated solutions were transferred through a coarse filter into tared volumetric flasks, weighed and diluted with methanol. Concentrations were determined by spectrophotometric measurements at $372 \mathrm{~nm}$.

\section{Source and Purity of Chemicals:}

(1) $99+\%$, Aldrich Chemical Company, Milwaukee, WI, USA, was recrystallized several times from methanol.

(2) $99.5+\%$, HPLC Grade, Aldrich Chemical Company, stored over molecular sieves before use.

\section{Estimated Error:}

Temperature: $\pm 0.1 \mathrm{~K}$.

$x_{1}: \pm 1.5 \%$ (relative error).

\begin{tabular}{ll}
\hline \hline Components: & Original Measurements: \\
(1) Pyrene; $\mathrm{C}_{16} \mathrm{H}_{10} ;[129-00-0]$ & ${ }^{10}$ W. E. Acree, Jr. and M. H. \\
(2) Cyclohexanone; $\mathrm{C}_{6} \mathrm{H}_{10} \mathrm{O} ;[108-94-1]$ & Abraham, Fluid Phase \\
& Equilib. 201, 245 (2002). \\
\hline Variables: & Prepared by: \\
$T / \mathrm{K}=299.15$ & W. E. Acree, Jr. \\
\hline
\end{tabular}

Experimental Values

\begin{tabular}{lcc}
\hline \hline$x_{2}{ }^{(\mathrm{s}) \mathrm{a}}$ & $x_{2}{ }^{\mathrm{b}}$ & $x_{1}{ }^{\mathrm{c}}$ \\
\hline 1.0000 & 0.8900 & 0.1100 \\
\hline${ }^{\mathrm{a}} x_{2}{ }^{(\mathrm{s})}:$ initial mole fraction of component 2 in the solution. \\
${ }^{\mathrm{b}} x_{2}:$ mole fraction of component 2 in the saturated solution. \\
${ }^{\mathrm{c}} x_{1}:$ mole fraction solubility of the solute.
\end{tabular}

\section{Auxiliary Information}

\section{Method/Apparatus/Procedure:}

Constant-temperature bath, calorimetric thermometer, and an ultraviolet/ visible spectrophotometer.

Excess solute and solvent were placed in amber glass bottles and allowed to equilibrate for several days at constant temperature. Attainment of equilibrium was verified by several repetitive measurements and by approaching equilibrium from supersaturation. Aliquots of saturated solutions were transferred through a coarse filter into tared volumetric flasks, weighed and diluted with 2-propanol. Concentrations were determined by spectrophotometric measurements at $372 \mathrm{~nm}$.

\section{Source and Purity of Chemicals:}

(1) $98 \%$, Aldrich Chemical Company, Milwaukee, WI, USA, was recrystallized several times from methanol.

(2) $99.8 \%$, Aldrich Chemical Company, stored over molecular sieves before use.

\section{Estimated Error:}

Temperature: $\pm 0.1 \mathrm{~K}$.

$x_{1}: \pm 1.5 \%$ (relative error). 


\section{Components:}

(1) Pyrene; $\mathrm{C}_{16} \mathrm{H}_{10} ;[129-00-0]$

(2) Acetophenone; $\mathrm{C}_{8} \mathrm{H}_{8} \mathrm{O}$; [98-86-2]

Original Measurements:

${ }^{10}$ W. E. Acree, Jr. and M. H.

Abraham, Fluid Phase Equilib. 201, 245 (2002).

\section{Variables:}

$T / \mathrm{K}=299.15$

Prepared by:

W. E. Acree, Jr.

\section{Experimental Values}

\begin{tabular}{lcc}
\hline \hline$x_{2}{ }^{(\mathrm{s}) \mathrm{a}}$ & $x_{2}{ }^{\mathrm{b}}$ & $x_{1}{ }^{\mathrm{c}}$ \\
\hline 1.0000 & 0.8848 & 0.1152 \\
\hline
\end{tabular}

${ }^{\mathrm{a}} x_{2}{ }^{(\mathrm{s})}$ : initial mole fraction of component 2 in the solution.

${ }^{b} x_{2}$ : mole fraction of component 2 in the saturated solution.

${ }^{c} x_{1}$ : mole fraction solubility of the solute.

\section{Auxiliary Information}

Method/Apparatus/Procedure:
Constant-temperature bath, calorimetric thermometer, and an ultraviolet/
visible spectrophotometer.
Excess solute and solvent were placed in amber glass bottles and allowed to
equilibrate for several days at constant temperature. Attainment of equilibrium
was verified by several repetitive measurements and by approaching
equilibrium from supersaturation. Aliquots of saturated solutions were
transferred through a coarse filter into tared volumetric flasks, weighed and
diluted with 2-propanol. Concentrations were determined by
spectrophotometric measurements at $372 \mathrm{~nm}$.
Source and Purity of Chemicals:
(1) $98 \%$, Aldrich Chemical Company, Milwaukee, WI, USA, was
recrystallized several times from methanol.
(2) $99 \%$, Aldrich Chemical Company, stored over molecular sieves before use.
Estimated Error:
Temperature: $\pm 0.1 \mathrm{~K}$.
$x_{1}: \pm 1.5 \%$ (relative error).

\section{Auxiliary Information}

\section{Method/Apparatus/Procedure:}

Constant-temperature bath, calorimetric thermometer, and an ultraviolet/ visible spectrophotometer.

Excess solute and solvent were placed in amber glass bottles and allowed to equilibrate for several days at constant temperature. Attainment of equilibrium was verified by several repetitive measurements and by approaching equilibrium from supersaturation. Aliquots of saturated solutions were transferred through a coarse filter into tared volumetric flasks, weighed and diluted with methanol. Concentrations were determined by spectrophotometric measurements at $372 \mathrm{~nm}$.

\section{Source and Purity of Chemicals:}

(1) $99+\%$, Aldrich Chemical Company, Milwaukee, WI, USA, was recrystallized several times from methanol.

(2) $99.9+\%$, HPLC Grade, Aldrich Chemical Company, stored over molecular sieves and distilled shortly before use.

\section{Estimated Error:}

Temperature: $\pm 0.1 \mathrm{~K}$.

$x_{1}: \pm 1.5 \%$ (relative error).

\begin{tabular}{ll}
\hline \hline Components: & Original Measurements: \\
(1) Pyrene; $\mathrm{C}_{16} \mathrm{H}_{10} ;[129-00-0]$ & ${ }^{10}$ W. E. Acree, Jr. and M. H. \\
(2) Propanenitrile; $\mathrm{C}_{3} \mathrm{H}_{5} \mathrm{~N} ;[107-12-0]$ & Abraham, Fluid Phase Equilib. \\
& 201, 245 (2002). \\
\hline Variables: & Prepared by: \\
$T / \mathrm{K}=299.15$ & W. E. Acree, Jr. \\
\hline
\end{tabular}

Experimental Values

\begin{tabular}{lcc}
\hline \hline$x_{2}{ }^{(\mathrm{s}) \mathrm{a}}$ & $x_{2}{ }^{\mathrm{b}}$ & $x_{1}{ }^{\mathrm{c}}$ \\
\hline 1.0000 & 0.9744 & 0.02562 \\
$\frac{{ }^{\mathrm{a}} x_{2}{ }^{(\mathrm{s})}: \text { initial mole fraction of component } 2 \text { in the solution. }}{{ }^{\mathrm{b}} x_{2}: \text { mole fraction of component } 2 \text { in the saturated solution. }}$ \\
${ }^{\mathrm{c}} x_{1}:$ mole fraction solubility of the solute.
\end{tabular}

\section{Auxiliary Information}

\section{Method/Apparatus/Procedure:}

Constant-temperature bath, calorimetric thermometer, and an ultraviolet/ visible spectrophotometer.

Excess solute and solvent were placed in amber glass bottles and allowed to equilibrate for several days at constant temperature. Attainment of equilibrium was verified by several repetitive measurements and by approaching equilibrium from supersaturation. Aliquots of saturated solutions were transferred through a coarse filter into tared volumetric flasks, weighed and diluted with 2-propanol. Concentrations were determined by spectrophotometric measurements at $372 \mathrm{~nm}$.

\section{Source and Purity of Chemicals:}

(1) $98 \%$, Aldrich Chemical Company, Milwaukee, WI, USA, was recrystallized several times from methanol.

(2) $99 \%$, Aldrich Chemical Company, stored over molecular sieves before use.

\section{Estimated Error:}

Temperature: $\pm 0.1 \mathrm{~K}$.

$x_{1}: \pm 1.5 \%$ (relative error). 


\section{Components:}

(1) Pyrene; $\mathrm{C}_{16} \mathrm{H}_{10} ;[129-00-0]$

(2) Butanenitrile; $\mathrm{C}_{4} \mathrm{H}_{7} \mathrm{~N}$; [109-74-0]

Variables:

$T / \mathrm{K}=299.15$

Original Measurements:

${ }^{10}$ W. E. Acree, Jr. and M. H.

Abraham, Fluid Phase Equilib. 201, 245 (2002).

Prepared by:

W. E. Acree, Jr.

Experimental Values

\begin{tabular}{lcc}
\hline \hline$x_{2}{ }^{(\mathrm{s}) \mathrm{a}}$ & $x_{2}{ }^{\mathrm{b}}$ & $x_{1}{ }^{\mathrm{c}}$ \\
\hline 1.0000 & 0.9558 & 0.04417 \\
\hline
\end{tabular}

${ }^{\mathrm{a}} x_{2}{ }^{(\mathrm{s})}$ : initial mole fraction of component 2 in the solution.

${ }^{b} x_{2}$ : mole fraction of component 2 in the saturated solution.

${ }^{c} x_{1}$ : mole fraction solubility of the solute.

\section{Auxiliary Information}

Method/Apparatus/Procedure:
Constant-temperature bath, calorimetric thermometer, and an ultraviolet/
visible spectrophotometer.
Excess solute and solvent were placed in amber glass bottles and allowed to
equilibrate for several days at constant temperature. Attainment of equilibrium
was verified by several repetitive measurements and by approaching
equilibrium from supersaturation. Aliquots of saturated solutions were
transferred through a coarse filter into tared volumetric flasks, weighed and
diluted with 2-propanol. Concentrations were determined by
spectrophotometric measurements at 372 nm.
Source and Purity of Chemicals:
(1) $98 \%$, Aldrich Chemical Company, Milwaukee, WI, USA, was
recrystallized several times from methanol.
(2) $99 \%$, Aldrich Chemical Company, stored over molecular sieves before use.
Estimated Error:
Temperature: $\pm 0.1 \mathrm{~K}$.
$x_{1}: \pm 1.5 \%$ (relative error).

\begin{tabular}{ll}
\hline \hline Components: & Original Measurements: \\
(1) Pyrene; $\mathrm{C}_{16} \mathrm{H}_{10} ;[129-00-0]$ & ${ }^{10}$ W. E. Acree, Jr. and M. H. \\
(2) Hexanedinitrile; $\mathrm{C}_{6} \mathrm{H}_{8} \mathrm{~N}_{2} ;[111-69-3]$ & Abraham, Fluid Phase \\
& Equilib. 201, 245 (2002). \\
\hline Variables: & Prepared by: \\
$T / \mathrm{K}=299.15$ & W. E. Acree, Jr. \\
\hline
\end{tabular}

Experimental Values

\begin{tabular}{lcc}
\hline \hline$x_{2}{ }^{(\mathrm{s}) \mathrm{a}}$ & $x_{2}{ }^{\mathrm{b}}$ & $x_{1}{ }^{\mathrm{c}}$ \\
\hline 1.0000 & 0.9747 & 0.02528 \\
${ }^{\mathrm{a}}{ }_{x_{2}}{ }^{(\mathrm{s})}:$ initial mole fraction of component 2 in the solution. \\
${ }^{\mathrm{b}} x_{2}:$ mole fraction of component 2 in the saturated solution. \\
${ }^{\mathrm{c}} x_{1}:$ mole fraction solubility of the solute.
\end{tabular}

\section{Auxiliary Information}

Method/Apparatus/Procedure:

Constant-temperature bath, calorimetric thermometer, and an ultraviolet/ visible spectrophotometer.

Excess solute and solvent were placed in amber glass bottles and allowed to equilibrate for several days at constant temperature. Attainment of equilibrium was verified by several repetitive measurements and by approaching equilibrium from supersaturation. Aliquots of saturated solutions were transferred through a coarse filter into tared volumetric flasks, weighed and diluted with 2-propanol. Concentrations were determined by spectrophotometric measurements at $372 \mathrm{~nm}$.

\section{Source and Purity of Chemicals:}

(1) $98 \%$, Aldrich Chemical Company, Milwaukee, WI, USA, was recrystallized several times from methanol.

(2) $99 \%$, Aldrich Chemical Company, stored over molecular sieves before use.

\section{Estimated Error:}

Temperature: $\pm 0.1 \mathrm{~K}$.

$x_{1}: \pm 1.5 \%$ (relative error).

\begin{tabular}{ll}
\hline \hline Components: & Original Measurements: \\
(1) Pyrene; $\mathrm{C}_{16} \mathrm{H}_{10} ;[129-00-0]$ & ${ }^{45}$ L. E. Roy, C. E. Hernández, and \\
(2) Benzonitrile; $\mathrm{C}_{7} \mathrm{H}_{5} \mathrm{~N} ;[100-47-0]$ & W. E. Acree, Jr., Polycyclic \\
& Aromat. Compd. 13, 205 (1999). \\
\hline Variables: & Prepared by: \\
$T / \mathrm{K}=299.15$ & W. E. Acree, Jr. \\
\hline
\end{tabular}

Experimental Values

\begin{tabular}{|c|c|c|}
\hline$\underline{x}_{2}{ }^{(\mathrm{s}) \mathrm{a}}$ & $x_{2}{ }^{\mathrm{b}}$ & $x_{1}{ }^{\mathrm{c}}$ \\
\hline 1.0000 & 0.8985 & 0.1015 \\
\hline
\end{tabular}

\section{Auxiliary Information}

\section{Method/Apparatus/Procedure:}

Constant-temperature bath, calorimetric thermometer, and an ultraviolet/ visible spectrophotometer.

Excess solute and solvent were placed in amber glass bottles and allowed to equilibrate for several days at constant temperature. Attainment of equilibrium was verified by several repetitive measurements and by approaching equilibrium from supersaturation. Aliquots of saturated solutions were transferred through a coarse filter into tared volumetric flasks, weighed and diluted with methanol. Concentrations were determined by spectrophotometric measurements at $372 \mathrm{~nm}$.

\section{Source and Purity of Chemicals:}

(1) $99+\%$, Aldrich Chemical Company, Milwaukee, WI, USA, was recrystallized several times from methanol.

(2) $99 \%$, Aldrich Chemical Company, stored over molecular sieves and distilled shortly before use.

Estimated Error:

Temperature: $\pm 0.1 \mathrm{~K}$.

$x_{1}: \pm 1.5 \%$ (relative error). 


\section{Components:}

(1) Pyrene; $\mathrm{C}_{16} \mathrm{H}_{10} ;$ [129-00-0]

(2) Methyl acetoacetate; $\mathrm{C}_{5} \mathrm{H}_{8} \mathrm{O}_{3}$; [105-45-3]

\section{Variables:}

$T / \mathrm{K}=299.15$

Original Measurements:

${ }^{45}$ L. E. Roy, C. E. Hernández, and

W. E. Acree, Jr., Polycyclic Aromat. Compd. 13, 205 (1999).

Prepared by:

W. E. Acree, Jr.

Experimental Values

\begin{tabular}{lcc}
\hline \hline$x_{2}{ }^{(\mathrm{s}) \mathrm{a}}$ & $x_{2}{ }^{\mathrm{b}}$ & $x_{1}{ }^{\mathrm{c}}$ \\
\hline 1.0000 & 0.9689 & 0.03106 \\
\hline
\end{tabular}

${ }^{\mathrm{a}} x_{2}{ }^{(\mathrm{s})}$ : initial mole fraction of component 2 in the solution.

${ }^{b} x_{2}$ : mole fraction of component 2 in the saturated solution.

${ }^{c} x_{1}$ : mole fraction solubility of the solute.

\section{Auxiliary Information}

Method/Apparatus/Procedure:
Constant-temperature bath, calorimetric thermometer, and an ultraviolet/
visible spectrophotometer.
Excess solute and solvent were placed in amber glass bottles and allowed to
equilibrate for several days at constant temperature. Attainment of equilibrium
was verified by several repetitive measurements and by approaching
equilibrium from supersaturation. Aliquots of saturated solutions were
transferred through a coarse filter into tared volumetric flasks, weighed and
diluted with methanol. Concentrations were determined by
spectrophotometric measurements at 372 nm.
Source and Purity of Chemicals:
(1) $99+\%$, Aldrich Chemical Company, Milwaukee, WI, USA, was
recrystallized several times from methanol.
(2) $99 \%$, Aldrich Chemical Company, stored over molecular sieves and
distilled shortly before use.
Estimated Error:
Temperature: $\pm 0.1 \mathrm{~K}$.
$x_{1}: \pm 1.5 \%$ (relative error).

\begin{tabular}{ll}
\hline \hline Components: & Original Measurements: \\
(1) Pyrene; $\mathrm{C}_{16} \mathrm{H}_{10} ;[129-00-0]$ & ${ }^{10}$ W. E. Acree, Jr. and M. H. \\
$(2) \mathrm{N}$-Methylformamide; $\mathrm{C}_{2} \mathrm{H}_{5} \mathrm{NO} ;$ & Abraham, Fluid Phase \\
{$[123-39-7]$} & Equilib. 201, 245 (2002). \\
\hline Variables: & Prepared by: \\
$T / \mathrm{K}=299.15$ & W. E. Acree, Jr. \\
\hline
\end{tabular}

Experimental Values

\begin{tabular}{l}
\hline \hline$\frac{x_{2}{ }^{(\mathrm{s}) \mathrm{a}}}{{ }^{\mathrm{b}}}$ \\
$\begin{array}{l}1.0000 \\
{ }^{\mathrm{a}} x_{2}{ }^{(\mathrm{s})}: \text { initial mole fraction of component } 2 \text { in the solution. }\end{array}$ \\
${ }^{\mathrm{b}} x_{2}:$ mole fraction of component 2 in the saturated solution. \\
${ }^{c} x_{1}:$ mole fraction solubility of the solute.
\end{tabular}

\section{Auxiliary Information}

\section{Method/Apparatus/Procedure:}

Constant-temperature bath, calorimetric thermometer, and an ultraviolet/ visible spectrophotometer.

Excess solute and solvent were placed in amber glass bottles and allowed to equilibrate for several days at constant temperature. Attainment of equilibrium was verified by several repetitive measurements and by approaching equilibrium from supersaturation. Aliquots of saturated solutions were transferred through a coarse filter into tared volumetric flasks, weighed and diluted with 2-propanol. Concentrations were determined by spectrophotometric measurements at $372 \mathrm{~nm}$.

\section{Source and Purity of Chemicals:}

(1) $98 \%$, Aldrich Chemical Company, Milwaukee, WI, USA, was recrystallized several times from methanol.

(2) $99 \%$, Aldrich Chemical Company, stored over molecular sieves before use.

\section{Estimated Error:}

Temperature: $\pm 0.1 \mathrm{~K}$.

$x_{1}: \pm 1.5 \%$ (relative error).

\begin{tabular}{|c|c|}
\hline $\begin{array}{l}\text { Components: } \\
\text { (1) Pyrene; } \mathrm{C}_{16} \mathrm{H}_{10} ;[129-00-0] \\
\text { (2) } \mathrm{N}, \mathrm{N} \text {-Dimethylformamide; } \mathrm{C}_{3} \mathrm{H}_{7} \mathrm{NO} \text {; } \\
{[68-12-2]}\end{array}$ & $\begin{array}{l}\text { Original Measurements: } \\
{ }^{45} \text { L. E. Roy, C. E. Hernández, and } \\
\text { W. E. Acree, Jr., Polycyclic } \\
\text { Aromat. Compd. 13, } 205 \text { (1999). }\end{array}$ \\
\hline $\begin{array}{l}\text { Variables: } \\
T / \mathrm{K}=299.15\end{array}$ & $\begin{array}{l}\text { Prepared by: } \\
\text { W. E. Acree, Jr. }\end{array}$ \\
\hline
\end{tabular}

Experimental Values

\begin{tabular}{lcc}
\hline \hline$x_{2}{ }^{(\mathrm{s}) \mathrm{a}}$ & $x_{2}{ }^{\mathrm{b}}$ & $x_{1}{ }^{\mathrm{c}}$ \\
\hline 1.0000 & 0.9116 & 0.08844 \\
$\frac{1}{{ }^{a} x_{2}{ }^{(\mathrm{s})}: \text { initial mole fraction of component } 2 \text { in the solution. }}$ \\
${ }^{\mathrm{b}} x_{x_{2}}$ : mole fraction of component 2 in the saturated solution. \\
${ }^{c} x_{1}:$ mole fraction solubility of the solute.
\end{tabular}

\section{Auxiliary Information}

\section{Method/Apparatus/Procedure:}

Constant-temperature bath, calorimetric thermometer, and an ultraviolet/ visible spectrophotometer.

Excess solute and solvent were placed in amber glass bottles and allowed to equilibrate for several days at constant temperature. Attainment of equilibrium was verified by several repetitive measurements and by approaching equilibrium from supersaturation. Aliquots of saturated solutions were transferred through a coarse filter into tared volumetric flasks, weighed and diluted with methanol. Concentrations were determined by spectrophotometric measurements at $372 \mathrm{~nm}$.

\section{Source and Purity of Chemicals:}

(1) $99+\%$, Aldrich Chemical Company, Milwaukee, WI, USA, was recrystallized several times from methanol.

(2) $99.8 \%$, anhydrous, Aldrich Chemical Company, stored over molecular sieves and distilled shortly before use.

Estimated Error:

Temperature: $\pm 0.1 \mathrm{~K}$.

$x_{1}: \pm 1.5 \%$ (relative error). 


\section{Components:}

(1) Pyrene; $\mathrm{C}_{16} \mathrm{H}_{10} ;[129-00-0]$

(2) $N, N$-Dimethylacetamide; $\mathrm{C}_{4} \mathrm{H}_{9} \mathrm{NO}$;

[127-19-5]

\section{Variables:}

$T / \mathrm{K}=299.15$

Original Measurements:

${ }^{45}$ L. E. Roy, C. E. Hernández, and

W. E. Acree, Jr., Polycyclic

Aromat. Compd. 13, 205 (1999).

Prepared by:

W. E. Acree, Jr.

\section{Experimental Values}

\begin{tabular}{lcc}
\hline \hline$x_{2}{ }^{(\mathrm{s}) \mathrm{a}}$ & $x_{2}{ }^{\mathrm{b}}$ & $x_{1}{ }^{\mathrm{c}}$ \\
\hline 1.0000 & 0.8625 & 0.1375 \\
\hline
\end{tabular}

${ }^{\mathrm{a}} x_{2}{ }^{(\mathrm{s})}$ : initial mole fraction of component 2 in the solution.

${ }^{b} x_{2}$ : mole fraction of component 2 in the saturated solution.

${ }^{c} x_{1}$ : mole fraction solubility of the solute.

\section{Auxiliary Information}

\begin{abstract}
Method/Apparatus/Procedure:
Constant-temperature bath, calorimetric thermometer, and an ultraviolet/ visible spectrophotometer.

Excess solute and solvent were placed in amber glass bottles and allowed to equilibrate for several days at constant temperature. Attainment of equilibrium was verified by several repetitive measurements and by approaching equilibrium from supersaturation. Aliquots of saturated solutions were transferred through a coarse filter into tared volumetric flasks, weighed and diluted with methanol. Concentrations were determined by

spectrophotometric measurements at $372 \mathrm{~nm}$.
\end{abstract}

Source and Purity of Chemicals:

(1) $99+\%$, Aldrich Chemical Company, Milwaukee, WI, USA, was recrystallized several times from methanol.

(2) $99.8 \%$, anhydrous, Aldrich Chemical Company, stored over molecular sieves and distilled shortly before use.

\section{Estimated Error:}

Temperature: $\pm 0.1 \mathrm{~K}$.

$x_{1}: \pm 1.5 \%$ (relative error).

\begin{tabular}{|c|c|}
\hline $\begin{array}{l}\text { Components: } \\
\text { (1) Pyrene; } \mathrm{C}_{16} \mathrm{H}_{10} ;[129-00-0] \\
\text { (2) Benzenamine; } \mathrm{C}_{6} \mathrm{H}_{7} \mathrm{~N} ;[62-53-3]\end{array}$ & $\begin{array}{l}\text { Original Measurements: } \\
{ }^{61} \text { C. E. Hernández, K. M. De Fina, } \\
\text { L. E. Roy, T. L. Sharp, and W. E. } \\
\text { Acree, Jr., Can. J. Chem. 77, } 1465 \\
\text { (1999). }\end{array}$ \\
\hline $\begin{array}{l}\text { Variables: } \\
T / \mathrm{K}=299.15\end{array}$ & $\begin{array}{l}\text { Prepared by: } \\
\text { W. E. Acree, Jr. }\end{array}$ \\
\hline
\end{tabular}

Experimental Values

\begin{tabular}{|c|c|c|}
\hline$x_{2}{ }^{(s) a}$ & $x_{2}{ }^{\mathrm{b}}$ & $x_{1}{ }^{\mathrm{c}}$ \\
\hline 1.0000 & 0.9660 & 0.03398 \\
\hline
\end{tabular}

\section{Auxiliary Information}

\section{Method/Apparatus/Procedure:}

Constant-temperature bath, calorimetric thermometer, and an ultraviolet/ visible spectrophotometer.

Excess solute and solvent were placed in amber glass bottles and allowed to equilibrate for several days at constant temperature. Attainment of equilibrium was verified by several repetitive measurements and by approaching equilibrium from supersaturation. Aliquots of saturated solutions were transferred through a coarse filter into tared volumetric flasks, weighed and diluted with methanol. Concentrations were determined by spectrophotometric measurements at $372 \mathrm{~nm}$.

\section{Source and Purity of Chemicals:}

(1) $99+\%$, Aldrich Chemical Company, Milwaukee, WI, USA, was recrystallized several times from methanol.

(2) ACS Reagent Grade, Aldrich Chemical Company, stored over molecular sieves before use.

Estimated Error:

Temperature: $\pm 0.1 \mathrm{~K}$.

$x_{1}: \pm 1.5 \%$ (relative error).

\begin{tabular}{ll}
\hline \hline Components: & Original Measurements: \\
(1) Pyrene; $\mathrm{C}_{16} \mathrm{H}_{10} ;[129-00-0]$ & ${ }^{10}$ W. E. Acree, Jr. and M. H. \\
(2) Dimethyl sulfoxide; $\mathrm{C}_{2} \mathrm{H}_{6} \mathrm{OS} ;$ & Abraham, Fluid Phase Equilib. \\
[67-68-5] & 201, 245 (2002). \\
\hline Variables: & Prepared by: \\
$T / \mathrm{K}=299.15$ & W. E. Acree, Jr. \\
\hline
\end{tabular}

Experimental Values

\begin{tabular}{|c|c|c|}
\hline$x_{2}{ }^{(\mathrm{s}) \mathrm{a}}$ & $x_{2}{ }^{b}$ & $x_{1}{ }^{\mathrm{c}}$ \\
\hline 1.0000 & 0.9665 & 0.03354 \\
\hline
\end{tabular}

\section{Auxiliary Information}

\section{Method/Apparatus/Procedure:}

Constant-temperature bath, calorimetric thermometer, and an ultraviolet/ visible spectrophotometer.

Excess solute and solvent were placed in amber glass bottles and allowed to equilibrate for several days at constant temperature. Attainment of equilibrium was verified by several repetitive measurements and by approaching equilibrium from supersaturation. Aliquots of saturated solutions were transferred through a coarse filter into tared volumetric flasks, weighed and diluted with 2-propanol. Concentrations were determined by spectrophotometric measurements at $372 \mathrm{~nm}$.

\section{Source and Purity of Chemicals:}

(1) $98 \%$, Aldrich Chemical Company, Milwaukee, WI, USA, was recrystallized several times from methanol.

(2) $99.9 \%$, HPLC grade, Aldrich Chemical Company, stored over molecular sieves before use.

\section{Estimated Error:}

Temperature: $\pm 0.1 \mathrm{~K}$.

$x_{1}: \pm 1.5 \%$ (relative error) 


\section{Components:}

(1) Pyrene; $\mathrm{C}_{16} \mathrm{H}_{10} ;[129-00-0]$

(2) Propylene carbonate; $\mathrm{C}_{4} \mathrm{H}_{6} \mathrm{O}_{3}$; [108-32-7]

\section{Variables:}

$T / \mathrm{K}=299.15$

Original Measurements:

${ }^{10}$ W. E. Acree, Jr. and M. H.

Abraham, Fluid Phase

Equilib. 201, 245 (2002).

Prepared by:

W. E. Acree, Jr.

Experimental Values

\begin{tabular}{lcc}
\hline \hline$x_{2}{ }^{(\mathrm{s}) \mathrm{a}}$ & $x_{2}{ }^{\mathrm{b}}$ & $x_{1}{ }^{\mathrm{c}}$ \\
\hline 1.0000 & 0.9815 & 0.01847 \\
\hline
\end{tabular}

${ }^{\mathrm{a}} x_{2}{ }^{(\mathrm{s})}$ : initial mole fraction of component 2 in the solution.

${ }^{b} x_{2}$ : mole fraction of component 2 in the saturated solution.

${ }^{c} x_{1}$ : mole fraction solubility of the solute.

\section{Auxiliary Information}

Method/Apparatus/Procedure:
Constant-temperature bath, calorimetric thermometer, and an ultraviolet/
visible spectrophotometer.
Excess solute and solvent were placed in amber glass bottles and allowed to
equilibrate for several days at constant temperature. Attainment of equilibrium
was verified by several repetitive measurements and by approaching
equilibrium from supersaturation. Aliquots of saturated solutions were
transferred through a coarse filter into tared volumetric flasks, weighed and
diluted with 2-propanol. Concentrations were determined by
spectrophotometric measurements at $372 \mathrm{~nm}$.
Source and Purity of Chemicals:
(1) $98 \%$, Aldrich Chemical Company, Milwaukee, WI, USA, was
recrystallized several times from methanol.
(2) $99.7 \%$, anhydrous, Aldrich Chemical Company, stored over molecular
sieves before use.
Estimated Error:
Temperature: $\pm 0.1 \mathrm{~K}$.
$x_{1}: \pm 1.5 \%$ (relative error).

\begin{tabular}{ll}
\hline \hline Components: & Original Measurements: \\
(1) Pyrene; $\mathrm{C}_{16} \mathrm{H}_{10} ;[129-00-0]$ & ${ }^{10}$ W. E. Acree, Jr. and M. H. \\
(2) Tributyl phosphate; $\mathrm{C}_{12} \mathrm{H}_{27} \mathrm{O}_{4} \mathrm{P} ;$ & Abraham, Fluid Phase \\
{$[126-73-8]$} & Equilib. 201, 245 (2002). \\
\hline Variables: & Prepared by: \\
$T / \mathrm{K}=299.15$ & W. E. Acree, Jr. \\
\hline
\end{tabular}

Experimental Values

\begin{tabular}{|c|c|c|}
\hline$x_{2}^{(\mathrm{s}) \mathrm{a}}$ & $x_{2}{ }^{b}$ & $x_{1}^{\mathrm{c}}$ \\
\hline 1.0000 & 0.8707 & 0.1293 \\
\hline
\end{tabular}

\section{Auxiliary Information}

\section{Method/Apparatus/Procedure:}

Constant-temperature bath, calorimetric thermometer, and an ultraviolet/ visible spectrophotometer.

Excess solute and solvent were placed in amber glass bottles and allowed to equilibrate for several days at constant temperature. Attainment of equilibrium was verified by several repetitive measurements and by approaching equilibrium from supersaturation. Aliquots of saturated solutions were transferred through a coarse filter into tared volumetric flasks, weighed and diluted with 2-propanol. Concentrations were determined by spectrophotometric measurements at $372 \mathrm{~nm}$.

\section{Source and Purity of Chemicals:}

(1) $98 \%$, Aldrich Chemical Company, Milwaukee, WI, USA, was recrystallized several times from methanol.

(2) $99+\%$, Aldrich Chemical Company, stored over molecular sieves before use.

Estimated Error:

Temperature: $\pm 0.1 \mathrm{~K}$.

$x_{1}: \pm 1.5 \%$ (relative error)

\begin{tabular}{ll}
\hline \hline Components: & Original Measurements: \\
$\begin{array}{ll}\text { (1) Pyrene; } \mathrm{C}_{16} \mathrm{H}_{10} ;[129-00-0] \\
\text { (2) Morpholine; } \mathrm{C}_{4} \mathrm{H}_{9} \mathrm{NO} ;[110-91-8]\end{array}$ & W. E. Acree, Jr., unpublished data. \\
\hline Variables: & Prepared by: \\
$T / \mathrm{K}=299.15$ & W. E. Acree, Jr. \\
\hline
\end{tabular}

Experimental Values

\begin{tabular}{|c|c|c|}
\hline$x_{2}{ }^{(\mathrm{s}) \mathrm{a}}$ & $x_{2}{ }^{b}$ & $x_{1}{ }^{\mathrm{c}}$ \\
\hline 1.0000 & 0.9178 & 0.08218 \\
\hline
\end{tabular}

\section{Auxiliary Information}

\section{Method/Apparatus/Procedure:}

Constant-temperature bath, calorimetric thermometer, and an ultraviolet/ visible spectrophotometer.

Excess solute and solvent were placed in amber glass bottles and allowed to equilibrate for several days at constant temperature. Attainment of equilibrium was verified by several repetitive measurements and by approaching equilibrium from supersaturation. Aliquots of saturated solutions were transferred through a coarse filter into tared volumetric flasks, weighed and diluted with 2-propanol. Concentrations were determined by spectrophotometric measurements at $372 \mathrm{~nm}$.

\section{Source and Purity of Chemicals:}

(1) $98 \%$, Aldrich Chemical Company, Milwaukee, WI, USA, was recrystallized several times from methanol.

(2) $99 \%$, Aldrich Chemical Company, stored over molecular sieves before use.

\section{Estimated Error:}

Temperature: $\pm 0.1 \mathrm{~K}$.

$x_{1}: \pm 1.5 \%$ (relative error) 


\section{Components:}

(1) Pyrene; $\mathrm{C}_{16} \mathrm{H}_{10} ;[129-00-0]$

(2) Ethanolamine; $\mathrm{C}_{2} \mathrm{H}_{7} \mathrm{NO}$; [141-43-5]

\begin{tabular}{ll}
\hline Variables: & Prepared by: \\
$T / \mathrm{K}=299.15$ & W. E. Acree, Jr. \\
\hline
\end{tabular}

Experimental Values

\begin{tabular}{lcc}
\hline \hline$x_{2}{ }^{(\mathrm{s}) \mathrm{a}}$ & $x_{2}^{\mathrm{b}}$ & $x_{1}{ }^{\mathrm{c}}$ \\
\hline 1.0000 & 0.9980 & 0.001953 \\
${ }^{{ }^{\mathrm{a}} x_{2}{ }^{(\mathrm{s})}: \text { initial mole fraction of component } 2 \text { in the solution. }}$ \\
${ }^{\mathrm{b}} x_{2}:$ mole fraction of component 2 in the saturated solution. \\
${ }^{\mathrm{c}} x_{1}:$ mole fraction solubility of the solute.
\end{tabular}

\section{Auxiliary Information}

Method/Apparatus/Procedure:

Constant-temperature bath, calorimetric thermometer, and an ultraviolet/ visible spectrophotometer.

Excess solute and solvent were placed in amber glass bottles and allowed to equilibrate for several days at constant temperature. Attainment of equilibrium was verified by several repetitive measurements and by approaching equilibrium from supersaturation. Aliquots of saturated solutions were transferred through a coarse filter into tared volumetric flasks, weighed and diluted with 2-propanol. Concentrations were determined by

spectrophotometric measurements at $372 \mathrm{~nm}$.

Source and Purity of Chemicals:

(1) $98 \%$, Aldrich Chemical Company, Milwaukee, WI, USA, was recrystallized several times from methanol.

(2) $99 \%$, Aldrich Chemical Company, stored over molecular sieves before use.

Estimated Error:

Temperature: $\pm 0.1 \mathrm{~K}$.

$x_{1}: \pm 1.5 \%$ (relative error).

\section{Solubility of Thianthrene in Organic Solvents}

\subsection{Critical evaluation of experimental solubility data}

Volume 59 in the IUPAC Solubility Data Series ${ }^{3}$ contained only experimental solubility data for thianthrene dissolved in eight saturated hydrocarbon solutes, namely hexane, heptanes, octane, cyclohexane, methylcyclohexane, cyclooctane, and 2,2,4-trimethylpentane. Experimental measurements were performed at a single temperature of $298.15 \mathrm{~K}$. The compiled solubility data also included phase diagram information for binary thianthrene + phenanthrene, thianthrene + phenothiazine, thianthrene + phenoxanthiin, and thianthrene + phenoxazine mixtures. Solubility data contained in Vol. 59 will not be republished here. The listing above is provided so that readers will know what solubility data are available in the earlier volume for thianthrene.

There have been two studies that reported solubility data for thianthrene in organic solvents after Vol. 59 was published in 1995. Fletcher et al. $^{75}$ determined the solubility of thianthrene in 21 different organic solvents containing hydroxyl-, ether- or tert-butyl functional groups. The measured solubility data were used to test the applications and limitations of predictive expressions derived from Mobile Order theory. Stovall et al. ${ }^{76}$ subsequently determined solubilities in nonane, decane, undecane, hexadecane, 1,1'-oxybisethane, 2,2'-oxybispropane, 1,4dioxane, 1-decanol, ethylene glycol, and ethanenitrile to increase the experimental data available for determining the numerical values of the Abraham model solute descriptors of thianthrene. The calculated solute descriptors derived from the experimental solubility data provided a very good mathematical description of the solubility behavior of thianthrene in organic solvents, with an average absolute deviation between calculated and experimental values on the order of $\pm 0.12 \mathrm{log}$ units. Both research groups performed the solubility measurements at the single temperature of $298.15 \mathrm{~K}$. Critical evaluations are not possible as there are no independent measurements for the thianthrene-organic solvent systems studied by Fletcher et $\mathrm{al}^{75}$ and Stovall et al. ${ }^{76}$

The solubility data for thianthrene are given in Secs. 14.214.5.

\subsection{Thianthrene solubility data in saturated hydrocarbons (including cycloalkanes)}

\begin{tabular}{ll}
\hline \hline Components: & Original Measurements: \\
(1) Thianthrene; $\mathrm{C}_{12} \mathrm{H}_{8} \mathrm{~S}_{2} ;[92-85-3]$ & ${ }^{76} \mathrm{D}$. M. Stovall, W. E. Acree, Jr., \\
(2) Nonane; $\mathrm{C}_{9} \mathrm{H}_{20} ;[111-84-2]$ & and M. H. Abraham, Fluid Phase \\
& Equilib. 232, 113 (2005). \\
\hline Variables: & Prepared by: \\
$T / \mathrm{K}=298.15$ & W. E. Acree, Jr. \\
\hline
\end{tabular}

Experimental Values

\begin{tabular}{lcc}
\hline \hline$\frac{x_{2}{ }^{(\mathrm{s}) \mathrm{a}}}{x_{2}{ }^{\mathrm{b}}}$ & $x_{1}{ }^{\mathrm{c}}$ \\
\hline 1.0000 & 0.9955 & 0.004465 \\
${ }^{\mathrm{a}}{ }_{x_{2}{ }^{(\mathrm{s})}: \text { initial mole fraction of component } 2 \text { in the solution. }}$ \\
${ }_{\mathrm{b}} x_{2}:$ mole fraction of component 2 in the saturated solution. \\
${ }^{\mathrm{c}}{ }_{x_{1}: \text { mole fraction solubility of the solute. }}$
\end{tabular}

\section{Auxiliary Information}

\section{Method/Apparatus/Procedure:}

Constant-temperature bath, calorimetric thermometer, and an ultraviolet/ visible spectrophotometer.

Excess solute and solvent were placed in amber glass bottles and allowed to equilibrate for several days at constant temperature. Attainment of equilibrium was verified by several repetitive measurements and by approaching equilibrium from supersaturation. Aliquots of saturated solutions were transferred through a coarse filter into tared volumetric flasks, weighed and diluted with methanol. Concentrations were determined by spectrophotometric measurements at $255 \mathrm{~nm}$.

Source and Purity of Chemicals:

(1) $99+\%$, Aldrich Chemical Company, Milwaukee, WI, USA, was recrystallized several times from methanol.

(2) $99+\%$, TCI America, Portland, OR, USA, stored over molecular sieves before use. 
Estimated Error:

Temperature: $\pm 0.1 \mathrm{~K}$

$x_{1}: \pm 2.0 \%$ (relative error).

Original Measurements:

${ }^{76}$ D. M. Stovall, W. E. Acree, Jr., and M. H. Abraham, Fluid Phase Equilib. 232, 113 (2005).

\section{Variables:}

$T / \mathrm{K}=298.15$

\section{Prepared by:}

W. E. Acree, Jr.

Experimental Values

\begin{tabular}{lcc}
\hline \hline$x_{2}{ }^{(\mathrm{s}) \mathrm{a}}$ & $x_{2}{ }^{\mathrm{b}}$ & $x_{1}{ }^{\mathrm{c}}$ \\
\hline 1.0000 & 0.9950 & 0.004980 \\
\hline
\end{tabular}



${ }^{b} x_{2}$ : mole fraction of component 2 in the saturated solution.

${ }^{c} x_{1}$ : mole fraction solubility of the solute.

\section{Auxiliary Information}

\section{Method/Apparatus/Procedure:}

Constant-temperature bath, calorimetric thermometer, and an ultraviolet/ visible spectrophotometer.

Excess solute and solvent were placed in amber glass bottles and allowed to equilibrate for several days at constant temperature. Attainment of equilibrium was verified by several repetitive measurements and by approaching equilibrium from supersaturation. Aliquots of saturated solutions were transferred through a coarse filter into tared volumetric flasks, weighed and diluted with methanol. Concentrations were determined by

spectrophotometric measurements at $255 \mathrm{~nm}$.

\section{Source and Purity of Chemicals:}

(1) $99+\%$, Aldrich Chemical Company, Milwaukee, WI, USA, was recrystallized several times from methanol.

(2) $99+\%$, TCI America, Portland, OR, USA, stored over molecular sieves before use.

\section{Estimated Error:}

Temperature: $\pm 0.1 \mathrm{~K}$

$x_{1}: \pm 2.0 \%$ (relative error).

\section{Components:}

(1) Thianthrene; $\mathrm{C}_{12} \mathrm{H}_{8} \mathrm{~S}_{2} ;$ [92-85-3]

(2) Undecane; $\mathrm{C}_{11} \mathrm{H}_{24} ;[1120-21-4]$
Original Measurements:

${ }^{76}$ D. M. Stovall, W. E. Acree, Jr., and M. H. Abraham, Fluid Phase Equilib. 232, 113 (2005).

Prepared by:

\section{Variables:}

$T / \mathrm{K}=298.15$
Experimental Values

\begin{tabular}{|c|c|c|}
\hline$x_{2}{ }^{(\mathrm{s}) \mathrm{a}}$ & $x_{2}{ }^{b}$ & $x_{1}{ }^{\mathrm{c}}$ \\
\hline 1.0000 & 0.9944 & 0.005636 \\
\hline
\end{tabular}

\section{Auxiliary Information}

\section{Method/Apparatus/Procedure:}

Constant-temperature bath, calorimetric thermometer, and an ultraviolet/ visible spectrophotometer.

Excess solute and solvent were placed in amber glass bottles and allowed to equilibrate for several days at constant temperature. Attainment of equilibrium was verified by several repetitive measurements and by approaching equilibrium from supersaturation. Aliquots of saturated solutions were transferred through a coarse filter into tared volumetric flasks, weighed and diluted with methanol. Concentrations were determined by spectrophotometric measurements at $255 \mathrm{~nm}$.

\section{Source and Purity of Chemicals:}

(1) $99+\%$, Aldrich Chemical Company, Milwaukee, WI, USA, was recrystallized several times from methanol.

(2) $99 \%$, Acros Organics, USA, stored over molecular sieves before use.

\section{Estimated Error:}

Temperature: $\pm 0.1 \mathrm{~K}$.

$x_{1}: \pm 2.0 \%$ (relative error).

\begin{tabular}{ll}
\hline \hline Components: & Original Measurements: \\
(1) Thianthrene; $\mathrm{C}_{12} \mathrm{H}_{8} \mathrm{~S}_{2} ;[92-85-3]$ & ${ }^{76} \mathrm{D}$. M. Stovall, W. E. Acree, Jr., \\
(2) Hexadecane; $\mathrm{C}_{16} \mathrm{H}_{34} ;[544-76-3]$ & and M. H. Abraham, Fluid Phase \\
& Equilib. 232, 113 (2005). \\
\hline Variables: & Prepared by: \\
$T / \mathrm{K}=298.15$ & W. E. Acree, Jr. \\
\hline
\end{tabular}

Experimental Values

\begin{tabular}{lcc}
\hline \hline$x_{2}{ }^{(\mathrm{s}) \mathrm{a}}$ & $x_{2}^{\mathrm{b}}$ & $x_{1}{ }^{\mathrm{c}}$ \\
\hline 1.0000 & 0.9918 & 0.008166
\end{tabular}

${ }^{\mathrm{a}} x_{2}{ }^{(\mathrm{s})}$ : initial mole fraction of component 2 in the solution.

${ }^{\mathrm{b}} x_{2}$ : mole fraction of component 2 in the saturated solution.

${ }^{c} x_{1}$ : mole fraction solubility of the solute.

\section{Auxiliary Information}

\section{Method/Apparatus/Procedure:}

Constant-temperature bath, calorimetric thermometer, and an ultraviolet/ visible spectrophotometer.

Excess solute and solvent were placed in amber glass bottles and allowed to equilibrate for several days at constant temperature. Attainment of equilibrium was verified by several repetitive measurements and by approaching equilibrium from supersaturation. Aliquots of saturated solutions were transferred through a coarse filter into tared volumetric flasks, weighed and diluted with methanol. Concentrations were determined by spectrophotometric measurements at $255 \mathrm{~nm}$. 
Source and Purity of Chemicals:

(1) $99+\%$, Aldrich Chemical Company, Milwaukee, WI, USA, was

recrystallized several times from methanol.

(2) $99 \%$, Aldrich Chemical Company, stored over molecular sieves before use.

Estimated Error:

Temperature: $\pm 0.1 \mathrm{~K}$.

$x_{1}: \pm 2.0 \%$ (relative error).

\begin{tabular}{ll}
\hline \hline Components: & Original Measurements: \\
(1) Thianthrene; $\mathrm{C}_{12} \mathrm{H}_{8} \mathrm{~S}_{2} ;[92-85-3]$ & ${ }^{75}$ K. A. Fletcher, M. E. R. McHale, \\
(2) tert-Butylcyclohexane; $\mathrm{C}_{10} \mathrm{H}_{20} ;$ & J. R. Powell, K. S. Coym, and W. \\
[3178-22-1] & E. Acree, Jr., Phys. Chem. Liq. 34, \\
& 41 (1997). \\
\hline Variables: & Prepared by: \\
$T / \mathrm{K}=298.15$ & W. E. Acree, Jr. \\
\hline
\end{tabular}

Experimental Values

\begin{tabular}{lcc}
\hline \hline$x_{2}{ }^{(\mathrm{s}) \mathrm{a}}$ & $x_{2}{ }^{\mathrm{b}}$ & $x_{1}{ }^{\mathrm{c}}$ \\
\hline 1.0000 & 0.9934 & 0.00658
\end{tabular}

${ }^{a} x_{2}{ }^{(\mathrm{s})}$ : initial mole fraction of component 2 in the solution.

${ }^{b} x_{2}$ : mole fraction of component 2 in the saturated solution.

${ }^{c} x_{1}$ : mole fraction solubility of the solute.

\section{Auxiliary Information}

Method/Apparatus/Procedure:
Constant-temperature bath, calorimetric thermometer, and an ultraviolet/
visible spectrophotometer.
Excess solute and solvent were placed in amber glass bottles and allowed to
equilibrate for several days at constant temperature. Attainment of equilibrium
was verified by several repetitive measurements and by approaching
equilibrium from supersaturation. Aliquots of saturated solutions were
transferred through a coarse filter into tared volumetric flasks, weighed and
diluted with methanol. Concentrations were determined by
spectrophotometric measurements at $255 \mathrm{~nm}$.
Source and Purity of Chemicals:
(1) $99+\%$, Aldrich Chemical Company, Milwaukee, WI, USA, was
recrystallized several times from methanol.
(2) $99+\%$, anhydrous, Aldrich Chemical Company, stored over molecular
sieves before use.
Estimated Error:
Temperature: $\pm 0.1 \mathrm{~K}$.
$x_{1}: \pm 2.0 \%$ (relative error).

\subsection{Thianthrene solubility data in ethers}

\begin{tabular}{|c|c|}
\hline $\begin{array}{l}\text { Components: } \\
\text { (1) Thianthrene; } \mathrm{C}_{12} \mathrm{H}_{8} \mathrm{~S}_{2} ;[92-85-3] \\
\text { (2) } 1,1^{\prime}-\text { Oxybisethane; } \mathrm{C}_{4} \mathrm{H}_{10} \mathrm{O} \text {; } \\
{[60-29-7]}\end{array}$ & $\begin{array}{l}\text { Original Measurements: } \\
{ }^{76} \text { D. M. Stovall, W. E. Acree, Jr., } \\
\text { and M. H. Abraham, Fluid Phase } \\
\text { Equilib. 232, } 113 \text { (2005). }\end{array}$ \\
\hline $\begin{array}{l}\text { Variables: } \\
T / \mathrm{K}=298.15\end{array}$ & $\begin{array}{l}\text { Prepared by: } \\
\text { W. E. Acree, Jr. }\end{array}$ \\
\hline
\end{tabular}

Experimental Values

\begin{tabular}{|c|c|c|}
\hline$\underline{x_{2}{ }^{(\mathrm{s}) \mathrm{a}}}$ & $x_{2}{ }^{b}$ & $x_{1}{ }^{\mathrm{c}}$ \\
\hline 1.0000 & 0.9916 & 0.008363 \\
\hline
\end{tabular}

\section{Auxiliary Information}

\section{Method/Apparatus/Procedure:}

Constant-temperature bath, calorimetric thermometer, and an ultraviolet/ visible spectrophotometer.

Excess solute and solvent were placed in amber glass bottles and allowed to equilibrate for several days at constant temperature. Attainment of equilibrium was verified by several repetitive measurements and by approaching equilibrium from supersaturation. Aliquots of saturated solutions were transferred through a coarse filter into tared volumetric flasks, weighed and diluted with methanol. Concentrations were determined by spectrophotometric measurements at $255 \mathrm{~nm}$.

\section{Source and Purity of Chemicals:}

(1) $99+\%$, Aldrich Chemical Company, Milwaukee, WI, USA, was recrystallized several times from methanol.

(2) 99+\%, anhydrous, Aldrich Chemical Company, stored over molecular sieves before use.

Estimated Error:

Temperature: $\pm 0.1 \mathrm{~K}$.

$x_{1}: \pm 2.0 \%$ (relative error).

\begin{tabular}{ll}
\hline \hline Components: & Original Measurements: \\
(1) Thianthrene; $\mathrm{C}_{12} \mathrm{H}_{8} \mathrm{~S}_{2} ;[92-85-3]$ & ${ }^{76} \mathrm{D}$. M. Stovall, W. E. Acree, Jr., \\
(2) $2,2^{\prime}-$ Oxybispropane; $\mathrm{C}_{6} \mathrm{H}_{14} \mathrm{O} ;$ & and M. H. Abraham, Fluid Phase \\
{$[108-20-3]$} & Equilib. 232, 113 (2005). \\
\hline Variables: & Prepared by: \\
$T / \mathrm{K}=298.15$ & W. E. Acree, Jr. \\
\hline
\end{tabular}

\section{Experimental Values}

\begin{tabular}{|c|c|c|}
\hline$x_{2}{ }^{(\mathrm{s}) \mathrm{a}}$ & $x_{2}{ }^{b}$ & $x_{1}{ }^{c}$ \\
\hline 1.0000 & 0.9936 & 0.006376 \\
\hline
\end{tabular}

\section{Auxiliary Information}

\section{Method/Apparatus/Procedure:}

Constant-temperature bath, calorimetric thermometer, and an ultraviolet/ visible spectrophotometer.

Excess solute and solvent were placed in amber glass bottles and allowed to equilibrate for several days at constant temperature. Attainment of equilibrium was verified by several repetitive measurements and by approaching equilibrium from supersaturation. Aliquots of saturated solutions were transferred through a coarse filter into tared volumetric flasks, weighed and diluted with methanol. Concentrations were determined by spectrophotometric measurements at $255 \mathrm{~nm}$. 
Source and Purity of Chemicals:

(1) $99+\%$, Aldrich Chemical Company, Milwaukee, WI, USA, was recrystallized several times from methanol.

(2) $99 \%$, anhydrous, Aldrich Chemical Company, stored over molecular sieves before use.

\section{Estimated Error:}

Temperature: $\pm 0.1 \mathrm{~K}$

$x_{1}: \pm 2.0 \%$ (relative error).

\begin{tabular}{|c|c|}
\hline $\begin{array}{l}\text { Components: } \\
\text { (1) Thianthrene; } \mathrm{C}_{12} \mathrm{H}_{8} \mathrm{~S}_{2} ;[92-85-3] \\
\text { (2) 1,1'-Oxybisbutane; } \mathrm{C}_{8} \mathrm{H}_{18} \mathrm{O} \text {; } \\
\text { [142-96-1] }\end{array}$ & $\begin{array}{l}\text { Original Measurements: } \\
{ }^{75} \text { K. A. Fletcher, M. E. R. McHale, } \\
\text { J. R. Powell, K. S. Coym, and W. } \\
\text { E. Acree, Jr., Phys. Chem. Liq. 34, } \\
41 \text { (1997). }\end{array}$ \\
\hline $\begin{array}{l}\text { Variables: } \\
T / \mathrm{K}=298.15\end{array}$ & $\begin{array}{l}\text { Prepared by: } \\
\text { W. E. Acree, Jr. }\end{array}$ \\
\hline
\end{tabular}

Experimental Values

\begin{tabular}{lcc}
\hline \hline$x_{2}{ }^{(\mathrm{s}) \mathrm{a}}$ & $x_{2}{ }^{\mathrm{b}}$ & $x_{1}{ }^{\mathrm{c}}$ \\
\hline 1.0000 & 0.9903 & 0.00970 \\
\hline
\end{tabular}

${ }^{\mathrm{a}} x_{2}{ }^{(\mathrm{s})}$ : initial mole fraction of component 2 in the solution.

${ }^{\mathrm{b}} x_{2}$ : mole fraction of component 2 in the saturated solution.

${ }^{\mathrm{c}} x_{1}$ : mole fraction solubility of the solute.

\section{Auxiliary Information}

\section{Method/Apparatus/Procedure:}

Constant-temperature bath, calorimetric thermometer, and an ultraviolet/ visible spectrophotometer.

Excess solute and solvent were placed in amber glass bottles and allowed to equilibrate for several days at constant temperature. Attainment of equilibrium was verified by several repetitive measurements and by approaching equilibrium from supersaturation. Aliquots of saturated solutions were transferred through a coarse filter into tared volumetric flasks, weighed and diluted with methanol. Concentrations were determined by

spectrophotometric measurements at $255 \mathrm{~nm}$.

Source and Purity of Chemicals:

(1) $99+\%$, Aldrich Chemical Company, Milwaukee, WI, USA, was recrystallized several times from methanol.

(2) $99 \%$, anhydrous, Aldrich Chemical Company, stored over molecular sieves before use.

\section{Estimated Error:}

Temperature: $\pm 0.1 \mathrm{~K}$.

$x_{1}: \pm 2.0 \%$ (relative error).

\section{Components:}

(1) Thianthrene; $\mathrm{C}_{12} \mathrm{H}_{8} \mathrm{~S}_{2} ;$ [92-85-3]

(2) 2-Methoxy-2-methylpropane;

$\mathrm{C}_{5} \mathrm{H}_{12} \mathrm{O}$; [1634-04-4]

\section{Original Measurements:}

${ }^{75}$ K. A. Fletcher, M.E. R. McHale,

J. R. Powell, K. S. Coym, and W.

E. Acree, Jr., Phys. Chem. Liq. 34, 41 (1997).

\begin{tabular}{ll}
\hline Variables: & Prepared by: \\
$T / \mathrm{K}=298.15$ & W. E. Acree, Jr. \\
\hline
\end{tabular}

Experimental Values

\begin{tabular}{lcc}
\hline \hline$x_{2}{ }^{(\mathrm{s}) \mathrm{a}}$ & $x_{2}{ }^{\mathrm{b}}$ & $x_{1}{ }^{\mathrm{c}}$ \\
\hline 1.0000 & 0.9906 & 0.00939 \\
$\frac{{ }^{\mathrm{a}} x_{2}{ }^{(\mathrm{s})}: \text { initial mole fraction of component } 2 \text { in the solution. }}{{ }^{\mathrm{b}} x_{x_{2}}: \text { mole fraction of component } 2 \text { in the saturated solution. }}$ \\
${ }^{\mathrm{c}} x_{1}:$ mole fraction solubility of the solute.
\end{tabular}

\section{Auxiliary Information}

\section{Method/Apparatus/Procedure:}

Constant-temperature bath, calorimetric thermometer, and an ultraviolet/ visible spectrophotometer.

Excess solute and solvent were placed in amber glass bottles and allowed to equilibrate for several days at constant temperature. Attainment of equilibrium was verified by several repetitive measurements and by approaching equilibrium from supersaturation. Aliquots of saturated solutions were transferred through a coarse filter into tared volumetric flasks, weighed and diluted with methanol. Concentrations were determined by spectrophotometric measurements at $255 \mathrm{~nm}$.

Source and Purity of Chemicals:

(1) $99+\%$, Aldrich Chemical Company, Milwaukee, WI, USA, was recrystallized several times from methanol.

(2) $99.9+\%$, Arco Chemical Company, USA, stored over molecular sieves before use.

Estimated Error:

Temperature: $\pm 0.1 \mathrm{~K}$.

$x_{1}: \pm 2.0 \%$ (relative error).

\section{Components:}

(1) Thianthrene; $\mathrm{C}_{12} \mathrm{H}_{8} \mathrm{~S}_{2}$; [92-85-3]

Variables:

$T / \mathrm{K}=298.15$
(2) 1,4-Dioxane; $\mathrm{C}_{4} \mathrm{H}_{8} \mathrm{O}_{2}$; [123-91-1]
Original Measurements:

${ }^{76}$ D. M. Stovall, W. E. Acree, Jr., and M. H. Abraham, Fluid Phase Equilib. 232, 113 (2005).

Prepared by: W. E. Acree, Jr.

\section{Experimental Values}

\begin{tabular}{lcc}
\hline \hline$x_{2}{ }^{(\mathrm{s}) \mathrm{a}}$ & $x_{2}{ }^{\mathrm{b}}$ & $x_{1}{ }^{\mathrm{c}}$ \\
\hline 1.0000 & 0.9757 & 0.02431 \\
$\frac{{ }^{\mathrm{a}} x_{2}{ }^{(\mathrm{s})}: \text { initial mole fraction of component } 2 \text { in the solution. }}{{ }^{\mathrm{b}} x_{2}: \text { mole fraction of component } 2 \text { in the saturated solution. }}$ \\
${ }^{\mathrm{c}} x_{1}:$ mole fraction solubility of the solute.
\end{tabular}

\section{Auxiliary Information}

\section{Method/Apparatus/Procedure:}

Constant-temperature bath, calorimetric thermometer, and an ultraviolet/ visible spectrophotometer.

Excess solute and solvent were placed in amber glass bottles and allowed to equilibrate for several days at constant temperature. Attainment of equilibrium was verified by several repetitive measurements and by approaching equilibrium from supersaturation. Aliquots of saturated solutions were transferred through a coarse filter into tared volumetric flasks, weighed and diluted with methanol. Concentrations were determined by spectrophotometric measurements at $255 \mathrm{~nm}$. 
Source and Purity of Chemicals:

(1) $99+\%$, Aldrich Chemical Company, Milwaukee, WI, USA, was recrystallized several times from methanol.

(2) $99.8 \%$, anhydrous, Aldrich Chemical Company, stored over molecular sieves before use.

Estimated Error:

Temperature: $\pm 0.1 \mathrm{~K}$.

$x_{1}: \pm 2.0 \%$ (relative error)

\subsection{Thianthrene solubility data in alcohols}

\begin{tabular}{ll}
\hline \hline Components: & Original Measurements: \\
(1) Thianthrene; $\mathrm{C}_{12} \mathrm{H}_{8} \mathrm{~S}_{2} ;$ [92-85-3] & ${ }^{75}$ K. A. Fletcher, M. E. R. McHale, \\
(2) Methanol; $\mathrm{CH}_{4} \mathrm{O} ;[67-56-1]$ & J. R. Powell, K. S. Coym, and W. \\
& E. Acree, Jr., Phys. Chem. Liq. 34, \\
& 41 (1997). \\
\hline Variables: & Prepared by: \\
$T / \mathrm{K}=298.15$ & W. E. Acree, Jr. \\
\hline
\end{tabular}

Experimental Values

\begin{tabular}{|c|c|c|}
\hline$x_{2}^{(\mathrm{s}) \mathrm{a}}$ & $x_{2}{ }^{\mathrm{b}}$ & $x_{1}^{\mathrm{c}}$ \\
\hline 1.0000 & 0.9995 & 0.000472 \\
\hline
\end{tabular}

\section{Auxiliary Information}

\section{Method/Apparatus/Procedure:}

Constant-temperature bath, calorimetric thermometer, and an ultraviolet/ visible spectrophotometer.

Excess solute and solvent were placed in amber glass bottles and allowed to equilibrate for several days at constant temperature. Attainment of equilibrium was verified by several repetitive measurements and by approaching equilibrium from supersaturation. Aliquots of saturated solutions were transferred through a coarse filter into tared volumetric flasks, weighed and diluted with methanol. Concentrations were determined by

spectrophotometric measurements at $255 \mathrm{~nm}$.

\section{Source and Purity of Chemicals:}

(1) $99+\%$, Aldrich Chemical Company, Milwaukee, WI, USA, was recrystallized several times from methanol.

(2) $99.8 \%$, anhydrous, Aldrich Chemical Company, stored over molecular sieves before use.

Estimated Error:

Temperature: $\pm 0.1 \mathrm{~K}$

$x_{1}: \pm 2.0 \%$ (relative error).
Experimental Values

\begin{tabular}{|c|c|c|}
\hline$x_{2}{ }^{(\mathrm{s}) \mathrm{a}}$ & $x_{2}{ }^{b}$ & $x_{1}{ }^{\mathrm{c}}$ \\
\hline 1.0000 & 0.9990 & 0.001038 \\
\hline
\end{tabular}

\section{Auxiliary Information}

\section{Method/Apparatus/Procedure:}

Constant-temperature bath, calorimetric thermometer, and an ultraviolet/ visible spectrophotometer.

Excess solute and solvent were placed in amber glass bottles and allowed to equilibrate for several days at constant temperature. Attainment of equilibrium was verified by several repetitive measurements and by approaching equilibrium from supersaturation. Aliquots of saturated solutions were transferred through a coarse filter into tared volumetric flasks, weighed and diluted with methanol. Concentrations were determined by spectrophotometric measurements at $255 \mathrm{~nm}$.

\section{Source and Purity of Chemicals:}

(1) $99+\%$, Aldrich Chemical Company, Milwaukee, WI, USA, was recrystallized several times from methanol.

(2) Absolute, Aaper Alcohol and Chemical Company, USA, stored over molecular sieves before use.

\section{Estimated Error:}

Temperature: $\pm 0.1 \mathrm{~K}$.

$x_{1}: \pm 2.0 \%$ (relative error)

\begin{tabular}{ll}
\hline \hline Components: & Original Measurements: \\
(1) Thianthrene; $\mathrm{C}_{12} \mathrm{H}_{8} \mathrm{~S}_{2} ;[92-85-3]$ & ${ }^{75}$ K. A. Fletcher, M. E. R. McHale, \\
(2) 1-Propanol; $\mathrm{C}_{3} \mathrm{H}_{6} \mathrm{O} ;[71-23-8]$ & J. R. Powell, K. S. Coym, and W. \\
& E. Acree, Jr., Phys. Chem. Liq. 34, \\
& 41 (1997). \\
\hline Variables: & Prepared by: \\
$T / \mathrm{K}=298.15$ & W. E. Acree, Jr. \\
\hline
\end{tabular}

\section{Experimental Values}

\begin{tabular}{lcc}
\hline \hline$x_{2}{ }^{(\mathrm{s}) \mathrm{a}}$ & $x_{2}{ }^{\mathrm{b}}$ & $x_{1}{ }^{\mathrm{c}}$ \\
\hline 1.0000 & 0.9984 & 0.00162 \\
$\frac{{ }^{\mathrm{a}} x_{2}{ }^{(\mathrm{s})}: \text { initial mole fraction of component } 2 \text { in the solution. }}{{ }^{\mathrm{b}} x_{x_{2}} \text { : mole fraction of component } 2 \text { in the saturated solution. }}$ \\
${ }^{\mathrm{c}} x_{1}:$ mole fraction solubility of the solute.
\end{tabular}

\begin{tabular}{ll}
\hline \hline Components: & Original Measurements: \\
(1) Thianthrene; $\mathrm{C}_{12} \mathrm{H}_{8} \mathrm{~S}_{2} ;[92-85-3]$ & ${ }^{75}$ K. A. Fletcher, M. E. R. McHale, \\
(2) Ethanol; $\mathrm{C}_{2} \mathrm{H}_{6} \mathrm{O} ;[64-17-5]$ & J. R. Powell, K. S. Coym, and W. \\
& E. Acree, Jr., Phys. Chem. Liq. 34, \\
& 41 (1997). \\
\hline Variables: & Prepared by: \\
$T / \mathrm{K}=298.15$ & W. E. Acree, Jr. \\
\hline
\end{tabular}




\section{Auxiliary Information}

\section{Method/Apparatus/Procedure:}

Constant-temperature bath, calorimetric thermometer, and an ultraviolet/ visible spectrophotometer.

Excess solute and solvent were placed in amber glass bottles and allowed to equilibrate for several days at constant temperature. Attainment of equilibrium was verified by several repetitive measurements and by approaching equilibrium from supersaturation. Aliquots of saturated solutions were transferred through a coarse filter into tared volumetric flasks, weighed and diluted with methanol. Concentrations were determined by spectrophotometric measurements at $255 \mathrm{~nm}$.

Source and Purity of Chemicals:

(1) $99+\%$, Aldrich Chemical Company, Milwaukee, WI, USA, was recrystallized several times from methanol.

(2) $99+\%$, anhydrous, Aldrich Chemical Company, stored over molecular sieves before use.

Estimated Error:

Temperature: $\pm 0.1 \mathrm{~K}$.

$x_{1}: \pm 2.0 \%$ (relative error).

\section{Components:}

(1) Thianthrene; $\mathrm{C}_{12} \mathrm{H}_{8} \mathrm{~S}_{2} ;[92-85-3]$

(2) 2-Propanol; $\mathrm{C}_{3} \mathrm{H}_{6} \mathrm{O}$; [67-63-0]

\section{Variables:}

$T / \mathrm{K}=298.15$
Original Measurements:

${ }^{75}$ K. A. Fletcher, M. E. R. McHale, J. R. Powell, K. S. Coym, and W. E. Acree, Jr., Phys. Chem. Liq. 34 41 (1997).

Prepared by:

W. E. Acree, Jr

\section{Experimental Values}

\begin{tabular}{lcc}
\hline \hline$x_{2}{ }^{(\mathrm{s}) \mathrm{a}}$ & $x_{2}^{\mathrm{b}}$ & $x_{1}{ }^{\mathrm{c}}$ \\
\hline 1.0000 & 0.9990 & 0.001007
\end{tabular}

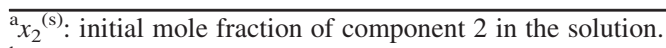

${ }^{\mathrm{b}} x_{2}$ : mole fraction of component 2 in the saturated solution.

${ }^{c} x_{1}$ : mole fraction solubility of the solute.

\section{Auxiliary Information}

\section{Method/Apparatus/Procedure:}

Constant-temperature bath, calorimetric thermometer, and an ultraviolet/ visible spectrophotometer.

Excess solute and solvent were placed in amber glass bottles and allowed to equilibrate for several days at constant temperature. Attainment of equilibrium was verified by several repetitive measurements and by approaching equilibrium from supersaturation. Aliquots of saturated solutions were transferred through a coarse filter into tared volumetric flasks, weighed and diluted with methanol. Concentrations were determined by

spectrophotometric measurements at $255 \mathrm{~nm}$.

Source and Purity of Chemicals:

(1) $99+\%$, Aldrich Chemical Company, Milwaukee, WI, USA, was recrystallized several times from methanol.

(2) $99+\%$, anhydrous, Aldrich Chemical Company, stored over molecular sieves before use.

\section{Estimated Error:}

Temperature: $\pm 0.1 \mathrm{~K}$.

$x_{1}: \pm 2.0 \%$ (relative error).

\begin{tabular}{ll}
\hline \hline Components: & Original Measurements: \\
(1) Thianthrene; $\mathrm{C}_{12} \mathrm{H}_{8} \mathrm{~S}_{2} ;[92-85-3]$ & ${ }^{75}$ K. A. Fletcher, M. E. R. McHale, \\
(2) 1-Butanol; $\mathrm{C}_{4} \mathrm{H}_{10} \mathrm{O} ;[71-36-3]$ & J. R. Powell, K. S. Coym, and W. \\
& E. Acree, Jr., Phys. Chem. Liq. 34, \\
& 41 (1997). \\
\hline Variables: & Prepared by: \\
$T / \mathrm{K}=298.15$ & W. E. Acree, Jr. \\
\hline
\end{tabular}

Experimental Values

\begin{tabular}{lcc}
\hline \hline$x_{2}{ }^{(\mathrm{s}) \mathrm{a}}$ & $x_{2}{ }^{\mathrm{b}}$ & $x_{1}{ }^{\mathrm{c}}$ \\
\hline 1.0000 & 0.9977 & 0.00227
\end{tabular}

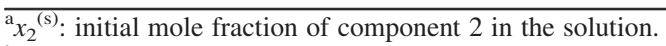

${ }^{\mathrm{b}} x_{2}$ : mole fraction of component 2 in the saturated solution.

${ }^{c} x_{1}$ : mole fraction solubility of the solute.

\section{Auxiliary Information}

\section{Method/Apparatus/Procedure:}

Constant-temperature bath, calorimetric thermometer, and an ultraviolet/ visible spectrophotometer.

Excess solute and solvent were placed in amber glass bottles and allowed to equilibrate for several days at constant temperature. Attainment of equilibrium was verified by several repetitive measurements and by approaching equilibrium from supersaturation. Aliquots of saturated solutions were transferred through a coarse filter into tared volumetric flasks, weighed and diluted with methanol. Concentrations were determined by spectrophotometric measurements at $255 \mathrm{~nm}$.

\section{Source and Purity of Chemicals:}

(1) $99+\%$, Aldrich Chemical Company, Milwaukee, WI, USA, was recrystallized several times from methanol.

(2) $99.8+\%$, HPLC Grade, Aldrich Chemical Company, stored over molecular sieves before use.

Estimated Error:

Temperature: $\pm 0.1 \mathrm{~K}$.

$x_{1}: \pm 2.0 \%$ (relative error).

\begin{tabular}{ll}
\hline \hline Components: & Original Measurements: \\
(1) Thianthrene; $\mathrm{C}_{12} \mathrm{H}_{8} \mathrm{~S}_{2} ;[92-85-3]$ & ${ }^{75}$ K. A. Fletcher, M. E. R. McHale, \\
(2) 2-Butanol; $\mathrm{C}_{4} \mathrm{H}_{10} \mathrm{O} ;[78-92-2]$ & J. R. Powell, K. S. Coym, and W. \\
& E. Acree, Jr., Phys. Chem. Liq. 34, \\
& 41 (1997). \\
\hline Variables: & Prepared by: \\
$T / \mathrm{K}=298.15$ & W. E. Acree, Jr. \\
\hline
\end{tabular}

Experimental Values

\begin{tabular}{lcc}
\hline \hline$x_{2}{ }^{(\mathrm{s}) \mathrm{a}}$ & $x_{2}{ }^{\mathrm{b}}$ & $x_{1}{ }^{\mathrm{c}}$ \\
\hline 1.0000 & 0.9983 & 0.00166 \\
$\frac{{ }^{\mathrm{a}} x_{2}{ }^{(\mathrm{s})}: \text { initial mole fraction of component } 2 \text { in the solution. }}{{ }^{\mathrm{b}} x_{2}: \text { mole fraction of component } 2 \text { in the saturated solution. }}$ \\
${ }^{\mathrm{c}} x_{1}:$ mole fraction solubility of the solute.
\end{tabular}




\section{Auxiliary Information}

\section{Method/Apparatus/Procedure:}

Constant-temperature bath, calorimetric thermometer, and an ultraviolet/ visible spectrophotometer.

Excess solute and solvent were placed in amber glass bottles and allowed to equilibrate for several days at constant temperature. Attainment of equilibrium was verified by several repetitive measurements and by approaching equilibrium from supersaturation. Aliquots of saturated solutions were transferred through a coarse filter into tared volumetric flasks, weighed and diluted with methanol. Concentrations were determined by spectrophotometric measurements at $255 \mathrm{~nm}$.

Source and Purity of Chemicals:

(1) $99+\%$, Aldrich Chemical Company, Milwaukee, WI, USA, was recrystallized several times from methanol.

(2) $99+\%$, anhydrous, Aldrich Chemical Company, stored over molecular sieves before use.

Estimated Error:

Temperature: $\pm 0.1 \mathrm{~K}$

$x_{1}: \pm 2.0 \%$ (relative error).

\begin{tabular}{ll}
\hline \hline Components: & Original Measurements: \\
(1) Thianthrene; $\mathrm{C}_{12} \mathrm{H}_{8} \mathrm{~S}_{2} ;[$ [92-85-3] & ${ }^{75}$ K. A. Fletcher, M. E. R. McHale, \\
(2) 2-Methyl-1-propanol; $\mathrm{C}_{4} \mathrm{H}_{10} \mathrm{O} ;$ & J. R. Powell, K. S. Coym, and W. \\
[78-83-1] & E. Acree, Jr., Phys. Chem. Liq. 34, \\
& 41 (1997). \\
\hline Variables: & Prepared by: \\
$T / \mathrm{K}=298.15$ & W. E. Acree, Jr. \\
\hline
\end{tabular}

\section{Experimental Values}

\begin{tabular}{lcc}
\hline \hline$x_{2}{ }^{(\mathrm{s}) \mathrm{a}}$ & $x_{2}{ }^{\mathrm{b}}$ & $x_{1}{ }^{\mathrm{c}}$ \\
\hline 1.0000 & 0.9985 & 0.00149
\end{tabular}

${ }^{\mathrm{a}} x_{2}{ }^{(\mathrm{s})}$ : initial mole fraction of component 2 in the solution.

${ }^{\mathrm{b}} x_{2}$ : mole fraction of component 2 in the saturated solution.

${ }^{c} x_{1}$ : mole fraction solubility of the solute.

\section{Auxiliary Information}

\section{Method/Apparatus/Procedure:}

Constant-temperature bath, calorimetric thermometer, and an ultraviolet/ visible spectrophotometer.

Excess solute and solvent were placed in amber glass bottles and allowed to equilibrate for several days at constant temperature. Attainment of equilibrium was verified by several repetitive measurements and by approaching equilibrium from supersaturation. Aliquots of saturated solutions were transferred through a coarse filter into tared volumetric flasks, weighed and diluted with methanol. Concentrations were determined by

spectrophotometric measurements at $255 \mathrm{~nm}$.

Source and Purity of Chemicals:

(1) $99+\%$, Aldrich Chemical Company, Milwaukee, WI, USA, was recrystallized several times from methanol.

(2) $99+\%$, anhydrous, Aldrich Chemical Company, stored over molecular sieves before use.

Estimated Error:

Temperature: $\pm 0.1 \mathrm{~K}$

$x_{1}: \pm 2.0 \%$ (relative error).

\begin{tabular}{ll}
\hline \hline Components: & Original Measurements: \\
(1) Thianthrene; $\mathrm{C}_{12} \mathrm{H}_{8} \mathrm{~S}_{2} ;[92-85-3]$ & ${ }^{75}$ K. A. Fletcher, M. E. R. McHale, \\
(2) 1-Pentanol; $\mathrm{C}_{5} \mathrm{H}_{12} \mathrm{O} ;[71-41-0]$ & J. R. Powell, K. S. Coym, and W. \\
& E. Acree, Jr., Phys. Chem. Liq. 34, \\
& 41 (1997). \\
\hline Variables: & Prepared by: \\
$T / \mathrm{K}=298.15$ & W. E. Acree, Jr. \\
\hline
\end{tabular}

Experimental Values

\begin{tabular}{lcc}
\hline \hline$x_{2}{ }^{(\mathrm{s}) \mathrm{a}}$ & $x_{2}{ }^{\mathrm{b}}$ & $x_{1}{ }^{\mathrm{c}}$ \\
\hline 1.0000 & 0.9969 & 0.00308
\end{tabular}

${ }^{\mathrm{a}} x_{2}{ }^{(\mathrm{s})}$ : initial mole fraction of component 2 in the solution.

${ }^{b} x_{2}$ : mole fraction of component 2 in the saturated solution.

${ }^{c} x_{1}$ : mole fraction solubility of the solute.

\section{Auxiliary Information}

\section{Method/Apparatus/Procedure:}

Constant-temperature bath, calorimetric thermometer, and an ultraviolet/ visible spectrophotometer.

Excess solute and solvent were placed in amber glass bottles and allowed to equilibrate for several days at constant temperature. Attainment of equilibrium was verified by several repetitive measurements and by approaching equilibrium from supersaturation. Aliquots of saturated solutions were transferred through a coarse filter into tared volumetric flasks, weighed and diluted with methanol. Concentrations were determined by spectrophotometric measurements at $255 \mathrm{~nm}$.

\section{Source and Purity of Chemicals:}

(1) $99+\%$, Aldrich Chemical Company, Milwaukee, WI, USA, was recrystallized several times from methanol.

(2) $99+\%$, Aldrich Chemical Company, stored over molecular sieves before use.

\section{Estimated Error:}

Temperature: $\pm 0.1 \mathrm{~K}$.

$x_{1}: \pm 2.0 \%$ (relative error)

\begin{tabular}{ll}
\hline \hline Components: & Original Measurements: \\
(1) Thianthrene; $\mathrm{C}_{12} \mathrm{H}_{8} \mathrm{~S}_{2} ;[92-85-3]$ & ${ }^{75}$ K. A. Fletcher, M. E. R. McHale, \\
(2) 2-Pentanol; $\mathrm{C}_{5} \mathrm{H}_{12} \mathrm{O} ;[6032-29-7]$ & J. R. Powell, K. S. Coym, and W. \\
& E. Acree, Jr., Phys. Chem. Liq. 34, \\
& 41 (1997). \\
\hline Variables: & Prepared by: \\
$T / \mathrm{K}=298.15$ & W. E. Acree, Jr. \\
\hline
\end{tabular}

Experimental Values

\begin{tabular}{lcc}
\hline \hline$x_{2}{ }^{(\mathrm{s}) \mathrm{a}}$ & $x_{2}{ }^{\mathrm{b}}$ & $x_{1}{ }^{\mathrm{c}}$ \\
\hline 1.0000 & 0.9979 & 0.00208 \\
$\frac{{ }^{\mathrm{a}} x_{2}{ }^{(\mathrm{s})}: \text { initial mole fraction of component } 2 \text { in the solution. }}{{ }^{\mathrm{b}}{ }_{x_{2}}: \text { mole fraction of component } 2 \text { in the saturated solution. }}$ \\
${ }^{\mathrm{c}} x_{1}:$ mole fraction solubility of the solute.
\end{tabular}




\section{Auxiliary Information}

\section{Method/Apparatus/Procedure:}

Constant-temperature bath, calorimetric thermometer, and an ultraviolet/ visible spectrophotometer.

Excess solute and solvent were placed in amber glass bottles and allowed to equilibrate for several days at constant temperature. Attainment of equilibrium was verified by several repetitive measurements and by approaching equilibrium from supersaturation. Aliquots of saturated solutions were transferred through a coarse filter into tared volumetric flasks, weighed and diluted with methanol. Concentrations were determined by spectrophotometric measurements at $255 \mathrm{~nm}$.

Source and Purity of Chemicals:

(1) $99+\%$, Aldrich Chemical Company, Milwaukee, WI, USA, was recrystallized several times from methanol.

(2) $99+\%$, Acros Organics, USA, stored over molecular sieves before use.

\section{Estimated Error:}

Temperature: $\pm 0.1 \mathrm{~K}$.

$x_{1}: \pm 2.0 \%$ (relative error).

\begin{tabular}{ll}
\hline \hline Components: & Original Measurements: \\
(1) Thianthrene; $\mathrm{C}_{12} \mathrm{H}_{8} \mathrm{~S}_{2} ;$ [92-85-3] & ${ }^{75}$ K. A. Fletcher, M. E. R. McHale, \\
(2) 2-Methyl-2-butanol; $\mathrm{C}_{5} \mathrm{H}_{12} \mathrm{O} ;$ & J. R. Powell, K. S. Coym, and W. \\
[75-85-4] & E. Acree, Jr., Phys. Chem. Liq. 34, \\
& 41 (1997). \\
\hline Variables: & Prepared by: \\
$T / \mathrm{K}=298.15$ & W. E. Acree, Jr. \\
\hline
\end{tabular}

Experimental Values

\begin{tabular}{lcc}
\hline \hline$x_{2}{ }^{(\mathrm{s}) \mathrm{a}}$ & $x_{2}{ }^{\mathrm{b}}$ & $x_{1}{ }^{\mathrm{c}}$ \\
\hline 1.0000 & 0.9976 & 0.00235 \\
\hline
\end{tabular}

${ }^{\mathrm{a}} x_{2}{ }^{(\mathrm{s})}$ : initial mole fraction of component 2 in the solution.

${ }^{b} x_{2}$ : mole fraction of component 2 in the saturated solution.

${ }^{c} x_{1}$ : mole fraction solubility of the solute.

\section{Auxiliary Information}

\section{Components:}

(1) Thianthrene; $\mathrm{C}_{12} \mathrm{H}_{8} \mathrm{~S}_{2} ;$ [92-85-3]

(2) 3-Methyl-1-butanol; $\mathrm{C}_{5} \mathrm{H}_{12} \mathrm{O}$;

[123-51-3]

\section{Variables:}

$T / \mathrm{K}=298.15$

\section{Original Measurements:}

${ }^{75}$ K. A. Fletcher, M. E. R. McHale, J. R. Powell, K. S. Coym, and W. E. Acree, Jr., Phys. Chem. Liq. 34 41 (1997).

Prepared by:

W. E. Acree, Jr.

Experimental Values

\begin{tabular}{lcc}
\hline \hline$x_{2}{ }^{(\mathrm{s}) \mathrm{a}}$ & $x_{2}{ }^{\mathrm{b}}$ & $x_{1}{ }^{\mathrm{c}}$ \\
\hline 1.0000 & 0.9976 & 0.00243 \\
\hline
\end{tabular}

${ }^{\mathrm{a}} x_{2}{ }^{(\mathrm{s})}$ : initial mole fraction of component 2 in the solution.

${ }^{\mathrm{b}} x_{2}$ : mole fraction of component 2 in the saturated solution.

${ }^{c} x_{1}$ : mole fraction solubility of the solute.

\section{Auxiliary Information}

Method/Apparatus/Procedure:
Constant-temperature bath, calorimetric thermometer, and an ultraviolet/
visible spectrophotometer.
Excess solute and solvent were placed in amber glass bottles and allowed to
equilibrate for several days at constant temperature. Attainment of equilibrium
was verified by several repetitive measurements and by approaching
equilibrium from supersaturation. Aliquots of saturated solutions were
transferred through a coarse filter into tared volumetric flasks, weighed and
diluted with methanol. Concentrations were determined by
spectrophotometric measurements at 255 nm.
Source and Purity of Chemicals:
(1) $99+\%$, Aldrich Chemical Company, Milwaukee, WI, USA, was
recrystallized several times from methanol.
(2) $99+\%$, anhydrous, Aldrich Chemical Company, stored over molecular
sieves before use.
Estimated Error:
Temperature: $\pm 0.1 \mathrm{~K}$.
$x_{1}: \pm 2.0 \%$ (relative error).

\author{
Method/Apparatus/Procedure: \\ Constant-temperature bath, calorimetric thermometer, and an ultraviolet/ \\ visible spectrophotometer. \\ Excess solute and solvent were placed in amber glass bottles and allowed to \\ equilibrate for several days at constant temperature. Attainment of equilibrium \\ was verified by several repetitive measurements and by approaching \\ equilibrium from supersaturation. Aliquots of saturated solutions were \\ transferred through a coarse filter into tared volumetric flasks, weighed and \\ diluted with methanol. Concentrations were determined by \\ spectrophotometric measurements at $255 \mathrm{~nm}$.

\section{Source and Purity of Chemicals:} \\ (1) $99+\%$, Aldrich Chemical Company, Milwaukee, WI, USA, was \\ recrystallized several times from methanol. \\ (2) $99+\%$, Acros Organics, USA, stored over molecular sieves before use.

\section{Estimated Error:} \\ Temperature: $\pm 0.1 \mathrm{~K}$. \\ $x_{1}: \pm 2.0 \%$ (relative error).
}

\begin{tabular}{|c|c|}
\hline $\begin{array}{l}\text { Components: } \\
\text { (1) Thianthrene; } \mathrm{C}_{12} \mathrm{H}_{8} \mathrm{~S}_{2} ;[92-85-3] \\
\text { (2) 1-Hexanol; } \mathrm{C}_{6} \mathrm{H}_{14} \mathrm{O} ;[111-27-3]\end{array}$ & $\begin{array}{l}\text { Original Measurements: } \\
{ }^{75} \text { K. A. Fletcher, M. E. R. McHale, } \\
\text { J. R. Powell, K. S. Coym, and W. } \\
\text { E. Acree, Jr., Phys. Chem. Liq. 34, } \\
41 \text { (1997). }\end{array}$ \\
\hline $\begin{array}{l}\text { Variables: } \\
T / \mathrm{K}=298.15\end{array}$ & $\begin{array}{l}\text { Prepared by: } \\
\text { W. E. Acree, Jr. }\end{array}$ \\
\hline
\end{tabular}

Experimental Values

\begin{tabular}{|c|c|c|}
\hline$x_{2}{ }^{(\mathrm{s}) \mathrm{a}}$ & $x_{2}{ }^{b}$ & $x_{1}{ }^{\mathrm{c}}$ \\
\hline 1.0000 & 0.9961 & 0.00390 \\
\hline
\end{tabular}




\section{Auxiliary Information}

\section{Method/Apparatus/Procedure:}

Constant-temperature bath, calorimetric thermometer, and an ultraviolet/ visible spectrophotometer.

Excess solute and solvent were placed in amber glass bottles and allowed to equilibrate for several days at constant temperature. Attainment of equilibrium was verified by several repetitive measurements and by approaching equilibrium from supersaturation. Aliquots of saturated solutions were transferred through a coarse filter into tared volumetric flasks, weighed and diluted with methanol. Concentrations were determined by spectrophotometric measurements at $255 \mathrm{~nm}$.

Source and Purity of Chemicals:

(1) $99+\%$, Aldrich Chemical Company, Milwaukee, WI, USA, was recrystallized several times from methanol.

(2) $99+\%$, Alfa Aesar, USA, stored over molecular sieves before use.

Estimated Error:

Temperature: $\pm 0.1 \mathrm{~K}$.

$x_{1}: \pm 2.0 \%$ (relative error).

\begin{tabular}{ll}
\hline \hline Components: & Original Measurements: \\
(1) Thianthrene; $\mathrm{C}_{12} \mathrm{H}_{8} \mathrm{~S}_{2} ;[92-85-3]$ & ${ }^{75}$ K. A. Fletcher, M. E. R. McHale, \\
(2) 4-Methyl-2-pentanol; $\mathrm{C}_{6} \mathrm{H}_{14} \mathrm{O} ;$ & J. R. Powell, K. S. Coym, and W. \\
[108-11-2] & E. Acree, Jr., Phys. Chem. Liq. 34, \\
& 41 (1997). \\
\hline Variables: & Prepared by: \\
$T / \mathrm{K}=298.15$ & W. E. Acree, Jr. \\
\hline
\end{tabular}

Experimental Values

\begin{tabular}{lcc}
\hline \hline$x_{2}{ }^{(\mathrm{s}) \mathrm{a}}$ & $x_{2}{ }^{\mathrm{b}}$ & $x_{1}{ }^{\mathrm{c}}$ \\
\hline 1.0000 & 0.9977 & 0.00230 \\
$\frac{{ }^{\mathrm{a}} x_{2}{ }^{(\mathrm{s})}: \text { initial mole fraction of component } 2 \text { in the solution. }}{{ }^{\mathrm{b}} x_{2}: \text { mole fraction of component } 2 \text { in the saturated solution. }}$ \\
${ }^{\mathrm{c}} x_{1}:$ mole fraction solubility of the solute.
\end{tabular}

\section{Auxiliary Information}

\begin{tabular}{ll}
\hline Components: & Original Measurements: \\
(1) Thianthrene; $\mathrm{C}_{12} \mathrm{H}_{8} \mathrm{~S}_{2} ;[92-85-3]$ & ${ }^{75}$ K. A. Fletcher, M. E. R. McHale, \\
(2) 2-Methyl-1-pentanol; $\mathrm{C}_{6} \mathrm{H}_{14} \mathrm{O} ;$ & J. R. Powell, K. S. Coym, and W. \\
[105-30-6] & E. Acree, Jr., Phys. Chem. Liq. 34, \\
& 41 (1997). \\
\hline Variables: & Prepared by: \\
$T / \mathrm{K}=298.15$ & W. E. Acree, Jr. \\
\hline
\end{tabular}

Experimental Values

\begin{tabular}{lcc}
\hline \hline$x_{2}{ }^{(\mathrm{s}) \mathrm{a}}$ & $x_{2}{ }^{\mathrm{b}}$ & $x_{1}{ }^{\mathrm{c}}$ \\
\hline 1.0000 & 0.9971 & 0.00287 \\
\hline
\end{tabular}

${ }^{\mathrm{a}} x_{2}{ }^{(\mathrm{s})}$ : initial mole fraction of component 2 in the solution.

${ }^{b} x_{2}$ : mole fraction of component 2 in the saturated solution.

${ }^{\mathrm{c}} x_{1}$ : mole fraction solubility of the solute.

\section{Auxiliary Information}

Method/Apparatus/Procedure:
Constant-temperature bath, calorimetric thermometer, and an ultraviolet/
visible spectrophotometer.
Excess solute and solvent were placed in amber glass bottles and allowed to
equilibrate for several days at constant temperature. Attainment of equilibrium
was verified by several repetitive measurements and by approaching
equilibrium from supersaturation. Aliquots of saturated solutions were
transferred through a coarse filter into tared volumetric flasks, weighed and
diluted with methanol. Concentrations were determined by
spectrophotometric measurements at $255 \mathrm{~nm}$.
Source and Purity of Chemicals:
(1) $99+\%$, Aldrich Chemical Company, Milwaukee, WI, USA, was
recrystallized several times from methanol.
(2) $99 \%$, Aldrich Chemical Company, stored over molecular sieves before use.
Estimated Error:
Temperature: $\pm 0.1 \mathrm{~K}$.
$x_{1}: \pm 2.0 \%$ (relative error).

\begin{abstract}
Method/Apparatus/Procedure:
Constant-temperature bath, calorimetric thermometer, and an ultraviolet/ visible spectrophotometer.

Excess solute and solvent were placed in amber glass bottles and allowed to equilibrate for several days at constant temperature. Attainment of equilibrium was verified by several repetitive measurements and by approaching equilibrium from supersaturation. Aliquots of saturated solutions were transferred through a coarse filter into tared volumetric flasks, weighed and diluted with methanol. Concentrations were determined by spectrophotometric measurements at $255 \mathrm{~nm}$.
\end{abstract}

\section{Source and Purity of Chemicals:}

(1) $99+\%$, Aldrich Chemical Company, Milwaukee, WI, USA, was recrystallized several times from methanol.

(2) $99+\%$, Acros Organics, USA, stored over molecular sieves before use.

\section{Estimated Error:}

Temperature: $\pm 0.1 \mathrm{~K}$.

$x_{1}: \pm 2.0 \%$ (relative error).

\begin{tabular}{ll}
\hline \hline Components: & Original Measurements: \\
(1) Thianthrene; $\mathrm{C}_{12} \mathrm{H}_{8} \mathrm{~S}_{2} ;$ [92-85-3] & ${ }^{75}$ K. A. Fletcher, M. E. R. McHale, \\
(2) 1-Heptanol; $\mathrm{C}_{7} \mathrm{H}_{16} \mathrm{O} ;[111-70-6]$ & J. R. Powell, K. S. Coym, and W. \\
& E. Acree, Jr., Phys. Chem. Liq. 34, \\
& 41 (1997). \\
\hline Variables: & Prepared by: \\
$T / \mathrm{K}=298.15$ & W. E. Acree, Jr. \\
\hline
\end{tabular}

Experimental Values

\begin{tabular}{lcc}
\hline \hline$x_{2}{ }^{(\mathrm{s}) \mathrm{a}}$ & $x_{2}{ }^{\mathrm{b}}$ & $x_{1}{ }^{\mathrm{c}}$ \\
\hline 1.0000 & 0.9950 & 0.00501 \\
$\frac{{ }^{a} x_{2}{ }^{(\mathrm{s})}: \text { initial mole fraction of component } 2 \text { in the solution. }}{{ }^{\mathrm{b}} x_{2}: \text { mole fraction of component } 2 \text { in the saturated solution. }}$ \\
${ }^{\mathrm{c}} x_{1}:$ mole fraction solubility of the solute.
\end{tabular}




\section{Auxiliary Information}

\section{Method/Apparatus/Procedure:}

Constant-temperature bath, calorimetric thermometer, and an ultraviolet/ visible spectrophotometer.

Excess solute and solvent were placed in amber glass bottles and allowed to equilibrate for several days at constant temperature. Attainment of equilibrium was verified by several repetitive measurements and by approaching equilibrium from supersaturation. Aliquots of saturated solutions were transferred through a coarse filter into tared volumetric flasks, weighed and diluted with methanol. Concentrations were determined by spectrophotometric measurements at $255 \mathrm{~nm}$.

Source and Purity of Chemicals:

(1) $99+\%$, Aldrich Chemical Company, Milwaukee, WI, USA, was recrystallized several times from methanol.

(2) $99+\%$, Alfa Aesar, USA, stored over molecular sieves before use.

\section{Estimated Error:}

Temperature: $\pm 0.1 \mathrm{~K}$.

$x_{1}: \pm 2.0 \%$ (relative error).

\begin{tabular}{|c|c|}
\hline $\begin{array}{l}\text { Components: } \\
\text { (1) Thianthrene; } \mathrm{C}_{12} \mathrm{H}_{8} \mathrm{~S}_{2} ;[92-85-3] \\
\text { (2) 1-Octanol; } \mathrm{C}_{8} \mathrm{H}_{18} \mathrm{O} ;[111-87-5]\end{array}$ & $\begin{array}{l}\text { Original Measurements: } \\
{ }^{75} \text { K. A. Fletcher, M. E. R. McHale, } \\
\text { J. R. Powell, K. S. Coym, and W. } \\
\text { E. Acree, Jr., Phys. Chem. Liq. 34, } \\
41 \text { (1997). }\end{array}$ \\
\hline $\begin{array}{l}\text { Variables: } \\
T / \mathrm{K}=298.15\end{array}$ & $\begin{array}{l}\text { Prepared by: } \\
\text { W. E. Acree, Jr. }\end{array}$ \\
\hline
\end{tabular}

Experimental Values

\begin{tabular}{|c|c|c|}
\hline$x_{2}{ }^{(\mathrm{s}) \mathrm{a}}$ & $x_{2}{ }^{b}$ & $x_{1}{ }^{\mathrm{c}}$ \\
\hline 1.0000 & 0.9945 & 0.00553 \\
\hline
\end{tabular}

\section{Auxiliary Information}

Method/Apparatus/Procedure:
Constant-temperature bath, calorimetric thermometer, and an ultraviolet/
visible spectrophotometer.
Excess solute and solvent were placed in amber glass bottles and allowed to
equilibrate for several days at constant temperature. Attainment of equilibrium
was verified by several repetitive measurements and by approaching
equilibrium from supersaturation. Aliquots of saturated solutions were
transferred through a coarse filter into tared volumetric flasks, weighed and
diluted with methanol. Concentrations were determined by
spectrophotometric measurements at 255 nm.
Source and Purity of Chemicals:
(1) $99+\%$, Aldrich Chemical Company, Milwaukee, WI, USA, was
recrystallized several times from methanol.
(2) $99+\%$, anhydrous, Aldrich Chemical Company, stored over molecular
sieves before use.
Estimated Error:
Temperature: $\pm 0.1 \mathrm{~K}$.
$x_{1}: \pm 2.0 \%$ (relative error).

\begin{tabular}{ll}
\hline \hline Components: & Original Measurements: \\
(1) Thianthrene; $\mathrm{C}_{12} \mathrm{H}_{8} \mathrm{~S}_{2} ;$ [92-85-3] & ${ }^{75}$ K. A. Fletcher, M. E. R. McHale, \\
(2) 2-Ethyl-1-hexanol; $\mathrm{C}_{8} \mathrm{H}_{18} \mathrm{O} ;$ & J. R. Powell, K. S. Coym, and W. \\
[104-76-7] & E. Acree, Jr., Phys. Chem. Liq. 34, \\
& 41 (1997). \\
\hline Variables: & Prepared by: \\
$T / \mathrm{K}=298.15$ & W. E. Acree, Jr. \\
\hline
\end{tabular}

Experimental Values

\begin{tabular}{lcc}
\hline \hline$x_{2}{ }^{(\mathrm{s}) \mathrm{a}}$ & $x_{2}{ }^{\mathrm{b}}$ & $x_{1}{ }^{\mathrm{c}}$ \\
\hline 1.0000 & 0.9958 & 0.00415 \\
${ }^{\mathrm{a}} x_{2}{ }^{(\mathrm{s})}:$ initial mole fraction of component 2 in the solution. \\
${ }^{\mathrm{b}} x_{2}:$ mole fraction of component 2 in the saturated solution. \\
${ }^{\mathrm{c}} x_{1}:$ mole fraction solubility of the solute.
\end{tabular}

\section{Auxiliary Information}

\section{Method/Apparatus/Procedure:}

Constant-temperature bath, calorimetric thermometer, and an ultraviolet/ visible spectrophotometer.

Excess solute and solvent were placed in amber glass bottles and allowed to equilibrate for several days at constant temperature. Attainment of equilibrium was verified by several repetitive measurements and by approaching equilibrium from supersaturation. Aliquots of saturated solutions were transferred through a coarse filter into tared volumetric flasks, weighed and diluted with methanol. Concentrations were determined by spectrophotometric measurements at $255 \mathrm{~nm}$.

\section{Source and Purity of Chemicals:}

(1) $99+\%$, Aldrich Chemical Company, Milwaukee, WI, USA, was recrystallized several times from methanol.

(2) $99+\%$, Aldrich Chemical Company, stored over molecular sieves before use.

Estimated Error:

Temperature: $\pm 0.1 \mathrm{~K}$.

$x_{1}: \pm 2.0 \%$ (relative error).

\begin{tabular}{ll}
\hline \hline Components: & Original Measurements: \\
(1) Thianthrene; $\mathrm{C}_{12} \mathrm{H}_{8} \mathrm{~S}_{2} ;[92-85-3]$ & ${ }^{76} \mathrm{D}$. M. Stovall, W. E. Acree, Jr., \\
(2) 1-Decanol; $\mathrm{C}_{10} \mathrm{H}_{22} \mathrm{O} ;[112-30-4]$ & and M. H. Abraham, Fluid Phase \\
& Equilib. 232, 113 (2005). \\
\hline Variables: & Prepared by: \\
$T / \mathrm{K}=298.15$ & W. E. Acree, Jr. \\
\hline
\end{tabular}

Experimental Values

\begin{tabular}{|c|c|c|}
\hline$x_{2}{ }^{(\mathrm{s}) \mathrm{a}}$ & $x_{2}{ }^{\mathrm{b}}$ & $x_{1}{ }^{\mathrm{c}}$ \\
\hline 1.0000 & 0.9930 & 0.007021 \\
\hline
\end{tabular}




\section{Auxiliary Information}

\section{Method/Apparatus/Procedure:}

Constant-temperature bath, calorimetric thermometer, and an ultraviolet/ visible spectrophotometer.

Excess solute and solvent were placed in amber glass bottles and allowed to equilibrate for several days at constant temperature. Attainment of equilibrium was verified by several repetitive measurements and by approaching equilibrium from supersaturation. Aliquots of saturated solutions were transferred through a coarse filter into tared volumetric flasks, weighed and diluted with methanol. Concentrations were determined by spectrophotometric measurements at $255 \mathrm{~nm}$.

Source and Purity of Chemicals:

(1) $99+\%$, Aldrich Chemical Company, Milwaukee, WI, USA, was recrystallized several times from methanol.

(2) 99+\%, Alfa Aesar, USA, stored over molecular sieves before use.

Estimated Error:

Temperature: $\pm 0.1 \mathrm{~K}$

$x_{1}: \pm 2.0 \%$ (relative error)

\begin{tabular}{ll}
\hline \hline Components: & Original Measurements: \\
(1) Thianthrene; $\mathrm{C}_{12} \mathrm{H}_{8} \mathrm{~S}_{2} ;[92-85-3]$ & ${ }^{75}$ K. A. Fletcher, M. E. R. McHale, \\
(2) Cyclopentanol; $\mathrm{C}_{5} \mathrm{H}_{10} \mathrm{O} ;[96-41-3]$ & J. R. Powell, K. S. Coym, and W. \\
& E. Acree, Jr., Phys. Chem. Liq. 34, \\
& 41 (1997). \\
\hline Variables: & Prepared by: \\
$T / \mathrm{K}=298.15$ & W. E. Acree, Jr. \\
\hline
\end{tabular}

Experimental Values

\begin{tabular}{lcc}
\hline \hline$x_{2}{ }^{(\mathrm{s}) \mathrm{a}}$ & $x_{2}{ }^{\mathrm{b}}$ & $x_{1}{ }^{\mathrm{c}}$ \\
\hline 1.0000 & 0.9959 & 0.00408 \\
\hline
\end{tabular}

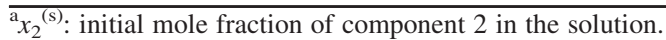

${ }^{\mathrm{b}} x_{2}$ : mole fraction of component 2 in the saturated solution.

${ }^{c} x_{1}$ : mole fraction solubility of the solute.

\section{Auxiliary Information}

\section{Method/Apparatus/Procedure:}

Constant-temperature bath, calorimetric thermometer, and an ultraviolet/ visible spectrophotometer.

Excess solute and solvent were placed in amber glass bottles and allowed to equilibrate for several days at constant temperature. Attainment of equilibrium was verified by several repetitive measurements and by approaching equilibrium from supersaturation. Aliquots of saturated solutions were transferred through a coarse filter into tared volumetric flasks, weighed and diluted with methanol. Concentrations were determined by

spectrophotometric measurements at $255 \mathrm{~nm}$.

\section{Source and Purity of Chemicals:}

(1) $99+\%$, Aldrich Chemical Company, Milwaukee, WI, USA, was recrystallized several times from methanol.

(2) $99 \%$, Aldrich Chemical Company, stored over molecular sieves before use

\section{Estimated Error:}

Temperature: $\pm 0.1 \mathrm{~K}$

$x_{1}: \pm 2.0 \%$ (relative error)

\begin{tabular}{ll}
\hline \hline Components: & Original Measurements: \\
(1) Thianthrene; $\mathrm{C}_{12} \mathrm{H}_{8} \mathrm{~S}_{2} ;[92-85-3]$ & ${ }^{76} \mathrm{D}$. M. Stovall, W. E. Acree, Jr., \\
(2) 1,2-Ethanediol; $\mathrm{C}_{2} \mathrm{H}_{6} \mathrm{O}_{2} ;[107-21-1]$ & and M. H. Abraham, Fluid Phase \\
& Equilib. 232, 113 (2005). \\
\hline Variables: & Prepared by: \\
$T / \mathrm{K}=298.15$ & W. E. Acree, Jr. \\
\hline
\end{tabular}

Experimental Values

\begin{tabular}{|c|c|c|}
\hline$x_{2}{ }^{(\mathrm{s}) a}$ & $x_{2}{ }^{b}$ & $x_{1}{ }^{\mathrm{c}}$ \\
\hline 1.0000 & 0.9987 & 0.001278 \\
\hline
\end{tabular}

Auxiliary Information

\section{Method/Apparatus/Procedure:}

Constant-temperature bath, calorimetric thermometer, and an ultraviolet/ visible spectrophotometer.

Excess solute and solvent were placed in amber glass bottles and allowed to equilibrate for several days at constant temperature. Attainment of equilibrium was verified by several repetitive measurements and by approaching equilibrium from supersaturation. Aliquots of saturated solutions were transferred through a coarse filter into tared volumetric flasks, weighed and diluted with methanol. Concentrations were determined by spectrophotometric measurements at $255 \mathrm{~nm}$.

Source and Purity of Chemicals:

(1) $99+\%$, Aldrich Chemical Company, Milwaukee, WI, USA, was recrystallized several times from methanol.

(2) $99.8 \%$, anhydrous, Aldrich Chemical Company, stored over molecular sieves before use.

Estimated Error:

Temperature: $\pm 0.1 \mathrm{~K}$.

$x_{1}: \pm 2.0 \%$ (relative error)

\subsection{Thianthrene solubility data in miscellaneous organic solvents}

Components:

(1) Thianthrene; $\mathrm{C}_{12} \mathrm{H}_{8} \mathrm{~S}_{2} ;$ [92-85-3]

(2) Ethanenitrile; $\mathrm{C}_{2} \mathrm{H}_{3} \mathrm{~N}$; [75-05-8]

Variables:

$T / \mathrm{K}=298.15$
Original Measurements:

${ }^{76}$ D. M. Stovall, W. E. Acree, Jr., and M. H. Abraham, Fluid Phase Equilib. 232, 113 (2005).

Prepared by: W. E. Acree, Jr.

\section{Experimental Values}

\begin{tabular}{|c|c|c|}
\hline$x_{2}{ }^{(\mathrm{s}) \mathrm{a}}$ & $x_{2}{ }^{\mathrm{b}}$ & $x_{1}{ }^{\mathrm{c}}$ \\
\hline 1.0000 & 0.9986 & 0.001364 \\
\hline
\end{tabular}




\section{Auxiliary Information}

Method/Apparatus/Procedure:

Constant-temperature bath, calorimetric thermometer, and an ultraviolet/ visible spectrophotometer.

Excess solute and solvent were placed in amber glass bottles and allowed to equilibrate for several days at constant temperature. Attainment of equilibrium was verified by several repetitive measurements and by approaching equilibrium from supersaturation. Aliquots of saturated solutions were transferred through a coarse filter into tared volumetric flasks, weighed and diluted with methanol. Concentrations were determined by

spectrophotometric measurements at $255 \mathrm{~nm}$.

Source and Purity of Chemicals:

(1) $99+\%$, Aldrich Chemical Company, Milwaukee, WI, USA, was recrystallized several times from methanol.

(2) $99.8 \%$, anhydrous, Aldrich Chemical Company, stored over molecular sieves before use.

Estimated Error:

Temperature: $\pm 0.1 \mathrm{~K}$.

$x_{1}: \pm 2.0 \%$ (relative error).

\section{Solubility of Xanthene in Organic Solvents}

\subsection{Critical evaluation of experimental solubility data}

Volume 59 in the IUPAC Solubility Data Series ${ }^{3}$ contained experimental solubility data for xanthene in two saturated hydrocarbons (cyclohexane and decahydronaphthalene), in two aromatic hydrocarbons (benzene and 1,2,3,4-tetrahydronaphthalene), and in two miscellaneous organic solvents (pyridine and thiophene). All six systems included measurements at several temperatures covering a 40 to $50 \mathrm{~K}$ range. Solubility data contained in Vol. 59 are not included in the present volume. The above listing is provided so that readers will know what data are available in the earlier volume for xanthene.

There have been three studies that reported solubility data for xanthene in organic solvents after Vol. 59 was published in 1995. Monárrez et al..$^{77}$ determined the solubility of xanthene in 34 different organic solvents containing hydroxyl-, ether-, chloro-, cyano- or tert-butyl functional groups. The measured solubility data were used to test the applications and limitations of predictive expressions derived from Mobile Order theory. Stovall et al. ${ }^{76}$ subsequently determined solubilities in undecane, 1,1'-oxybisethane and 2,2'-oxybispropane to increase the experimental data available for determining the numerical values of the Abraham model solute descriptors of xanthene. The calculated solute descriptors derived from the experimental solubility data provided a very good mathematical description of the solubility behavior of xanthene in organic solvents, with an average absolute deviation between calculated and experimental values being on the order of $\pm 0.09 \log$ units. Saifullah et al. ${ }^{66}$ later reported the solubility of xanthene in both tetrahydrofuran and 1,4-dioxane at $298.15 \mathrm{~K}$ as part of a computational study aimed at updating the Abraham model equation coefficients of tetrahydrofuran and 1,4-dioxane and extending the basic model to include ionic solute species. All three research groups performed the solubility measurements at the single temperature of $298.15 \mathrm{~K}$. Critical evaluations are not possible as there are no independent measurements for the xanthene-organic solvent systems studied by Monárrez et al., ${ }^{77}$ Stovall et al. ${ }^{76}$ and Saifullah et $a{ }^{66}$

The experimental solubility data for xanthene in the different organic solvents are in Secs. 15.2-15.7.

\subsection{Xanthene solubility data in saturated hydrocarbons (including cycloalkanes)}

\begin{tabular}{|c|c|}
\hline $\begin{array}{l}\text { Components: } \\
\text { (1) Xanthene; } \mathrm{C}_{13} \mathrm{H}_{10} \mathrm{O} ;[92-83-1] \\
\text { (2) Hexane; } \mathrm{C}_{6} \mathrm{H}_{14} ;[110-54-3]\end{array}$ & $\begin{array}{l}\text { Original Measurements: } \\
{ }^{77} \text { C. I. Monárrez, D. M. Stovall, J. H. } \\
\text { Woo, P. Taylor, and W. E. Acree, Jr., } \\
\text { Phys. Chem. Liq. 40, } 703 \text { (2002). }\end{array}$ \\
\hline $\begin{array}{l}\text { Variables: } \\
T / \mathrm{K}=298.15\end{array}$ & $\begin{array}{l}\text { Prepared by: } \\
\text { W. E. Acree, Jr. }\end{array}$ \\
\hline
\end{tabular}

Experimental Values

\begin{tabular}{lcc}
\hline \hline$\frac{x_{2}{ }^{(\mathrm{s}) \mathrm{a}}}{x_{2}{ }^{\mathrm{b}}}$ & $x_{1}{ }^{\mathrm{c}}$ \\
\hline 1.0000 & 0.9705 & 0.02949 \\
${ }^{\mathrm{a}} x_{2}{ }^{(\mathrm{s})}:$ initial mole fraction of component 2 in the solution. \\
${ }^{\mathrm{b}} x_{2}:$ mole fraction of component 2 in the saturated solution. \\
${ }^{\mathrm{c}}{ }_{x_{1}}:$ mole fraction solubility of the solute.
\end{tabular}

Auxiliary Information

Method/Apparatus/Procedure:

Constant-temperature bath, calorimetric thermometer, and an ultraviolet/ visible spectrophotometer.

Excess solute and solvent were placed in amber glass bottles and allowed to equilibrate for several days at constant temperature. Attainment of equilibrium was verified by several repetitive measurements and by approaching equilibrium from supersaturation. Aliquots of saturated solutions were transferred through a coarse filter into tared volumetric flasks, weighed and diluted with methanol. Concentrations were determined by spectrophotometric measurements at $280 \mathrm{~nm}$.

Source and Purity of Chemicals:

(1) $98 \%$, Aldrich Chemical Company, Milwaukee, WI, USA, was recrystallized several times from anhydrous methanol.

(2) $99 \%$, Aldrich Chemical Company, stored over molecular sieves and distilled before use.

Estimated Error:

Temperature: $\pm 0.1 \mathrm{~K}$.

$x_{1}: \pm 1.5 \%$ (relative error).

\begin{tabular}{|c|c|}
\hline $\begin{array}{l}\text { Components: } \\
\text { (1) Xanthene; } \mathrm{C}_{13} \mathrm{H}_{10} \mathrm{O} ;[92-83-1] \\
\text { (2) Heptane; } \mathrm{C}_{7} \mathrm{H}_{16} ;[142-82-5]\end{array}$ & $\begin{array}{l}\text { Original Measurements: } \\
{ }^{77} \text { C. I. Monárrez, D. M. Stovall, J. H. } \\
\text { Woo, P. Taylor, and W. E. Acree, Jr., } \\
\text { Phys. Chem. Liq. 40, } 703 \text { (2002). }\end{array}$ \\
\hline $\begin{array}{l}\text { Variables: } \\
T / \mathrm{K}=298.15\end{array}$ & $\begin{array}{l}\text { Prepared by: } \\
\text { W. E. Acree, Jr. }\end{array}$ \\
\hline
\end{tabular}


Experimental Values

\begin{tabular}{lcc}
\hline$\frac{x_{2}{ }^{(\mathrm{s}) \mathrm{a}}}{x_{2}{ }^{\mathrm{b}}}$ & $x_{1}{ }^{\mathrm{c}}$ \\
\hline 1.0000 & 0.9646 & 0.03543 \\
$\frac{{ }^{\mathrm{a}} x_{2}{ }^{(\mathrm{s})}: \text { initial mole fraction of component } 2 \text { in the solution. }}{{ }^{\mathrm{b}}{ }_{x_{2}}: \text { mole fraction of component } 2 \text { in the saturated solution. }}$ \\
${ }^{\mathrm{c}} x_{1}:$ mole fraction solubility of the solute.
\end{tabular}

\section{Auxiliary Information}

\section{Method/Apparatus/Procedure:}

Constant-temperature bath, calorimetric thermometer, and an ultraviolet/ visible spectrophotometer.

Excess solute and solvent were placed in amber glass bottles and allowed to equilibrate for several days at constant temperature. Attainment of equilibrium was verified by several repetitive measurements and by approaching equilibrium from supersaturation. Aliquots of saturated solutions were transferred through a coarse filter into tared volumetric flasks, weighed and diluted with methanol. Concentrations were determined by spectrophotometric measurements at $280 \mathrm{~nm}$.

\section{Source and Purity of Chemicals:}

(1) $98 \%$, Aldrich Chemical Company, Milwaukee, WI, USA, was recrystallized several times from anhydrous methanol.

(2) HPLC Grade, Aldrich Chemical Company, stored over molecular sieves and distilled before use

\section{Estimated Error:}

Temperature: $\pm 0.1 \mathrm{~K}$.

$x_{1}: \pm 1.5 \%$ (relative error).

\begin{tabular}{|c|c|}
\hline $\begin{array}{l}\text { Components: } \\
\text { (1) Xanthene; } \mathrm{C}_{13} \mathrm{H}_{10} \mathrm{O} ;[92-83-1] \\
\text { (2) Octane; } \mathrm{C}_{8} \mathrm{H}_{18} ;[111-65-9]\end{array}$ & $\begin{array}{l}\text { Original Measurements: } \\
{ }^{77} \text { C. I. Monárrez, D. M. Stovall, J. H. } \\
\text { Woo, P. Taylor, and W. E. Acree, Jr., } \\
\text { Phys. Chem. Liq. 40, } 703 \text { (2002). }\end{array}$ \\
\hline $\begin{array}{l}\text { Variables: } \\
T / \mathrm{K}=298.15\end{array}$ & $\begin{array}{l}\text { Prepared by: } \\
\text { W. E. Acree, Jr. }\end{array}$ \\
\hline
\end{tabular}

\section{Experimental Values}

\begin{tabular}{lcc}
\hline \hline$x_{2}{ }^{(\mathrm{s}) \mathrm{a}}$ & $x_{2}^{\mathrm{b}}$ & $x_{1}{ }^{\mathrm{c}}$ \\
\hline 1.0000 & 0.9602 & 0.03976 \\
\hline
\end{tabular}

${ }_{x_{2}}{ }^{(\mathrm{s})}$ : initial mole fraction of component 2 in the solution.

${ }^{b} x_{2}$ : mole fraction of component 2 in the saturated solution.

${ }^{c} x_{1}$ : mole fraction solubility of the solute.

\section{Auxiliary Information}

\section{Method/Apparatus/Procedure:}

Constant-temperature bath, calorimetric thermometer, and an ultraviolet/ visible spectrophotometer.

Excess solute and solvent were placed in amber glass bottles and allowed to equilibrate for several days at constant temperature. Attainment of equilibrium was verified by several repetitive measurements and by approaching equilibrium from supersaturation. Aliquots of saturated solutions were transferred through a coarse filter into tared volumetric flasks, weighed and diluted with methanol. Concentrations were determined by spectrophotometric measurements at $280 \mathrm{~nm}$.

Source and Purity of Chemicals:

(1) $98 \%$, Aldrich Chemical Company, Milwaukee, WI, USA, was recrystallized several times from anhydrous methanol.

(2) $99+\%$, anhydrous, Aldrich Chemical Company, stored over molecular sieves and distilled before use.

\section{Estimated Error:}

Temperature: $\pm 0.1 \mathrm{~K}$.

$x_{1}: \pm 1.5 \%$ (relative error)

\begin{tabular}{|c|c|}
\hline $\begin{array}{l}\text { Components: } \\
\text { (1) Xanthene; } \mathrm{C}_{13} \mathrm{H}_{10} \mathrm{O} ;[92-83-1] \\
\text { (2) Nonane; } \mathrm{C}_{9} \mathrm{H}_{20} ;[111-84-2]\end{array}$ & $\begin{array}{l}\text { Original Measurements: } \\
{ }^{77} \text { C. I. Monárrez, D. M. Stovall, J. H. } \\
\text { Woo, P. Taylor, and W. E. Acree, Jr., } \\
\text { Phys. Chem. Liq. 40, } 703 \text { (2002). }\end{array}$ \\
\hline $\begin{array}{l}\text { Variables: } \\
T / \mathrm{K}=298.15\end{array}$ & $\begin{array}{l}\text { Prepared by: } \\
\text { W. E. Acree, Jr. }\end{array}$ \\
\hline
\end{tabular}

Experimental Values

\begin{tabular}{lcc}
\hline \hline$x_{2}{ }^{(\mathrm{s}) \mathrm{a}}$ & $x_{2}{ }^{\mathrm{b}}$ & $x_{1}{ }^{\mathrm{c}}$ \\
\hline 1.0000 & 0.9569 & 0.04306 \\
$\frac{{ }^{a} x_{2}{ }^{(\mathrm{s})}: \text { initial mole fraction of component } 2 \text { in the solution. }}{{ }^{\mathrm{b}} x_{2}: \text { mole fraction of component } 2 \text { in the saturated solution. }}$ \\
${ }^{\mathrm{c}} x_{1}:$ mole fraction solubility of the solute.
\end{tabular}

\section{Auxiliary Information}

\section{Method/Apparatus/Procedure:}

Constant-temperature bath, calorimetric thermometer, and an ultraviolet/ visible spectrophotometer.

Excess solute and solvent were placed in amber glass bottles and allowed to equilibrate for several days at constant temperature. Attainment of equilibrium was verified by several repetitive measurements and by approaching equilibrium from supersaturation. Aliquots of saturated solutions were transferred through a coarse filter into tared volumetric flasks, weighed and diluted with methanol. Concentrations were determined by spectrophotometric measurements at $280 \mathrm{~nm}$.

\section{Source and Purity of Chemicals:}

(1) $98 \%$, Aldrich Chemical Company, Milwaukee, WI, USA, was recrystallized several times from anhydrous methanol

(2) $99+\%$, TCI America, Portland, OR, USA, stored over molecular sieves and distilled before use.

Estimated Error:

Temperature: $\pm 0.1 \mathrm{~K}$.

$x_{1}: \pm 1.5 \%$ (relative error) 


\section{Components:}

(1) Xanthene; $\mathrm{C}_{13} \mathrm{H}_{10} \mathrm{O}$; [92-83-1]

(2) Decane; $\mathrm{C}_{10} \mathrm{H}_{22} ;$ [124-18-5]
Variables:
$T / \mathrm{K}=298.15$

Original Measurements:

${ }^{77}$ C. I. Monárrez, D. M. Stovall, J. H.

Woo, P. Taylor, and W. E. Acree, Jr., Phys. Chem. Liq. 40, 703 (2002).

Prepared by:

W. E. Acree, Jr.

Experimental Values

\begin{tabular}{lcc}
\hline \hline$x_{2}{ }^{(\mathrm{s}) \mathrm{a}}$ & $x_{2}{ }^{\mathrm{b}}$ & $x_{1}{ }^{\mathrm{c}}$ \\
\hline 1.0000 & 0.9539 & 0.04610 \\
\hline
\end{tabular}

${ }^{\mathrm{a}} x_{2}{ }^{(\mathrm{s})}$ : initial mole fraction of component 2 in the solution.

${ }^{b} x_{2}$ : mole fraction of component 2 in the saturated solution.

${ }^{c} x_{1}$ : mole fraction solubility of the solute.

\section{Auxiliary Information}

Method/Apparatus/Procedure:
Constant-temperature bath, calorimetric thermometer, and an ultraviolet/
visible spectrophotometer.
Excess solute and solvent were placed in amber glass bottles and allowed to
equilibrate for several days at constant temperature. Attainment of equilibrium
was verified by several repetitive measurements and by approaching
equilibrium from supersaturation. Aliquots of saturated solutions were
transferred through a coarse filter into tared volumetric flasks, weighed and
diluted with methanol. Concentrations were determined by
spectrophotometric measurements at $280 \mathrm{~nm}$.
Source and Purity of Chemicals:
(1) $98 \%$, Aldrich Chemical Company, Milwaukee, WI, USA, was
recrystallized several times from anhydrous methanol.
(2) $99+\%$, TCI America, Portland, OR, USA, stored over molecular sieves and
distilled before use.
Estimated Error:
Temperature: $\pm 0.1 \mathrm{~K}$.
$x_{1}: \pm 1.5 \%$ (relative error).

\begin{tabular}{ll}
\hline \hline Components: & Original Measurements: \\
(1) Xanthene; $\mathrm{C}_{13} \mathrm{H}_{10} \mathrm{O} ;[92-83-1]$ & ${ }^{76} \mathrm{D}$. M. Stovall, W. E. Acree, Jr., \\
(2) Undecane; $\mathrm{C}_{11} \mathrm{H}_{24} ;[1120-21-4]$ & and M. H. Abraham, Fluid Phase \\
& Equilib. 232, 111 (2005). \\
\hline Variables: & Prepared by: \\
$T / \mathrm{K}=298.15$ & W. E. Acree, Jr. \\
\hline
\end{tabular}

Experimental Values

\begin{tabular}{lcc}
\hline \hline$x_{2}{ }^{(\mathrm{s}) \mathrm{a}}$ & $x_{2}{ }^{\mathrm{b}}$ & $x_{1}{ }^{\mathrm{c}}$ \\
\hline 1.0000 & 0.9530 & 0.04704 \\
${ }^{\mathrm{a}} x_{2}{ }^{(\mathrm{s})}:$ initial mole fraction of component 2 in the solution. \\
${ }^{\mathrm{b}}{ }_{x_{2}}:$ mole fraction of component 2 in the saturated solution. \\
${ }^{\mathrm{c}} x_{1}:$ mole fraction solubility of the solute.
\end{tabular}

\section{Auxiliary Information}

\section{Method/Apparatus/Procedure:}

Constant-temperature bath, calorimetric thermometer, and an ultraviolet/ visible spectrophotometer.

Excess solute and solvent were placed in amber glass bottles and allowed to equilibrate for several days at constant temperature. Attainment of equilibrium was verified by several repetitive measurements and by approaching equilibrium from supersaturation. Aliquots of saturated solutions were transferred through a coarse filter into tared volumetric flasks, weighed and diluted with methanol. Concentrations were determined by spectrophotometric measurements at $280 \mathrm{~nm}$.

\section{Source and Purity of Chemicals:}

(1) $98 \%$, Aldrich Chemical Company, Milwaukee, WI, USA, was recrystallized several times from anhydrous methanol.

(2) $99 \%$, Acros Organics, USA, stored over molecular sieves and distilled before use.

Estimated Error:

Temperature: $\pm 0.1 \mathrm{~K}$.

$x_{1}: \pm 1.5 \%$ (relative error).

\begin{tabular}{ll}
\hline \hline Components: & Original Measurements: \\
(1) Xanthene; $\mathrm{C}_{13} \mathrm{H}_{10} \mathrm{O} ;[92-83-1]$ & ${ }^{77}$ C. I. Monárrez, D. M. Stovall, J. H. \\
(2) Hexadecane; $\mathrm{C}_{16} \mathrm{H}_{34} ;[544-76-3]$ & Woo, P. Taylor, and W. E. Acree, Jr., \\
& Phys. Chem. Liq. 40, 703 (2002). \\
\hline Variables: & Prepared by: \\
$T / \mathrm{K}=298.15$ & W. E. Acree, Jr. \\
\hline
\end{tabular}

Experimental Values

\begin{tabular}{|c|c|c|}
\hline$x_{2}{ }^{(\mathrm{s}) \mathrm{a}}$ & $x_{2}{ }^{b}$ & $x_{1}{ }^{\mathrm{c}}$ \\
\hline 1.0000 & 0.9316 & 0.06835 \\
\hline
\end{tabular}

\section{Auxiliary Information}

\section{Method/Apparatus/Procedure:}

Constant-temperature bath, calorimetric thermometer, and an ultraviolet/ visible spectrophotometer.

Excess solute and solvent were placed in amber glass bottles and allowed to equilibrate for several days at constant temperature. Attainment of equilibrium was verified by several repetitive measurements and by approaching equilibrium from supersaturation. Aliquots of saturated solutions were transferred through a coarse filter into tared volumetric flasks, weighed and diluted with methanol. Concentrations were determined by spectrophotometric measurements at $280 \mathrm{~nm}$.

\section{Source and Purity of Chemicals:}

(1) $98 \%$, Aldrich Chemical Company, Milwaukee, WI, USA, was recrystallized several times from anhydrous methanol.

(2) $99 \%$, Aldrich Chemical Company, stored over molecular sieves and distilled before use.

\section{Estimated Error:}

Temperature: $\pm 0.1 \mathrm{~K}$.

$x_{1}: \pm 1.5 \%$ (relative error). 


\section{Components:}

(1) Xanthene; $\mathrm{C}_{13} \mathrm{H}_{10} \mathrm{O}$; [92-83-1]

(2) Cyclohexane; $\mathrm{C}_{6} \mathrm{H}_{12} ;[110-82-7]$

\section{Variables:}

$T / \mathrm{K}=298.15$
Original Measurements:

${ }^{77}$ C. I. Monárrez, D. M. Stovall, J. H.

Woo, P. Taylor, and W. E. Acree, Jr., Phys. Chem. Liq. 40, 703 (2002).

Prepared by:

W. E. Acree, Jr

\section{Experimental Values}

\begin{tabular}{lcc}
\hline \hline$x_{2}{ }^{(\mathrm{s}) \mathrm{a}}$ & $x_{2}{ }^{\mathrm{b}}$ & $x_{1}{ }^{\mathrm{c}}$ \\
\hline 1.0000 & 0.9580 & 0.04203 \\
\hline
\end{tabular}

${ }^{\mathrm{a}} x_{2}{ }^{(\mathrm{s})}$ : initial mole fraction of component 2 in the solution.

${ }^{b} x_{2}$ : mole fraction of component 2 in the saturated solution.

${ }^{c} x_{1}$ : mole fraction solubility of the solute.

\section{Auxiliary Information}

\begin{abstract}
Method/Apparatus/Procedure:
Constant-temperature bath, calorimetric thermometer, and an ultraviolet/ visible spectrophotometer.

Excess solute and solvent were placed in amber glass bottles and allowed to equilibrate for several days at constant temperature. Attainment of equilibrium was verified by several repetitive measurements and by approaching equilibrium from supersaturation. Aliquots of saturated solutions were transferred through a coarse filter into tared volumetric flasks, weighed and diluted with methanol. Concentrations were determined by

spectrophotometric measurements at $280 \mathrm{~nm}$.
\end{abstract}

Source and Purity of Chemicals:

(1) $98 \%$, Aldrich Chemical Company, Milwaukee, WI, USA, was recrystallized several times from anhydrous methanol.

(2) HPLC Grade, Aldrich Chemical Company, stored over molecular sieves and distilled before use.

\section{Estimated Error:}

Temperature: $\pm 0.1 \mathrm{~K}$.

$x_{1}: \pm 1.5 \%$ (relative error).

\begin{tabular}{|c|c|}
\hline $\begin{array}{l}\text { Components: } \\
\text { (1) Xanthene; } \mathrm{C}_{13} \mathrm{H}_{10} \mathrm{O} ;[92-83-1] \\
\text { (2) Methylcyclohexane; } \mathrm{C}_{7} \mathrm{H}_{14} ; \\
\text { [108-87-2] }\end{array}$ & $\begin{array}{l}\text { Original Measurements: } \\
{ }^{77} \text { C. I. Monárrez, D. M. Stovall, J. H. } \\
\text { Woo, P. Taylor, and W. E. Acree, Jr., } \\
\text { Phys. Chem. Liq. 40, } 703 \text { (2002). }\end{array}$ \\
\hline $\begin{array}{l}\text { Variables: } \\
T / \mathrm{K}=298.15\end{array}$ & $\begin{array}{l}\text { Prepared by: } \\
\text { W. E. Acree, Jr. }\end{array}$ \\
\hline
\end{tabular}

Experimental Values

\begin{tabular}{|c|c|c|}
\hline$x_{2}^{(\mathrm{s}) \mathrm{a}}$ & $x_{2}{ }^{\mathrm{b}}$ & $x_{1}{ }^{\mathrm{c}}$ \\
\hline 1.0000 & 0.9572 & 0.04275 \\
\hline
\end{tabular}

\section{Auxiliary Information}

Method/Apparatus/Procedure:

Constant-temperature bath, calorimetric thermometer, and an ultraviolet/ visible spectrophotometer.

Excess solute and solvent were placed in amber glass bottles and allowed to equilibrate for several days at constant temperature. Attainment of equilibrium was verified by several repetitive measurements and by approaching equilibrium from supersaturation. Aliquots of saturated solutions were transferred through a coarse filter into tared volumetric flasks, weighed and diluted with methanol. Concentrations were determined by spectrophotometric measurements at $280 \mathrm{~nm}$.

\section{Source and Purity of Chemicals:}

(1) $98 \%$, Aldrich Chemical Company, Milwaukee, WI, USA, was recrystallized several times from anhydrous methanol.

(2) $99+\%$, anhydrous, Aldrich Chemical Company, stored over molecular sieves and distilled before use.

Estimated Error:

Temperature: $\pm 0.1 \mathrm{~K}$.

$x_{1}: \pm 1.5 \%$ (relative error).

\begin{tabular}{|c|c|}
\hline $\begin{array}{l}\text { Components: } \\
\text { (1) Xanthene; } \mathrm{C}_{13} \mathrm{H}_{10} \mathrm{O} ;[92-83-1] \\
\text { (2) Cyclooctane; } \mathrm{C}_{8} \mathrm{H}_{16} ;[292-64-8]\end{array}$ & $\begin{array}{l}\text { Original Measurements: } \\
{ }^{77} \text { C. I. Monárrez, D. M. Stovall, J. H. } \\
\text { Woo, P. Taylor, and W. E. Acree, Jr., } \\
\text { Phys. Chem. Liq. 40, } 703 \text { (2002). }\end{array}$ \\
\hline $\begin{array}{l}\text { Variables: } \\
T / \mathrm{K}=298.15\end{array}$ & $\begin{array}{l}\text { Prepared by: } \\
\text { W. E. Acree, Jr. }\end{array}$ \\
\hline
\end{tabular}

Experimental Values

\begin{tabular}{|c|c|c|}
\hline$x_{2}{ }^{(\mathrm{s}) \mathrm{a}}$ & $x_{2}{ }^{b}$ & $x_{1}{ }^{\mathrm{c}}$ \\
\hline 1.0000 & 0.9459 & 0.05414 \\
\hline
\end{tabular}

\section{Auxiliary Information}

\section{Method/Apparatus/Procedure:}

Constant-temperature bath, calorimetric thermometer, and an ultraviolet/ visible spectrophotometer.

Excess solute and solvent were placed in amber glass bottles and allowed to equilibrate for several days at constant temperature. Attainment of equilibrium was verified by several repetitive measurements and by approaching equilibrium from supersaturation. Aliquots of saturated solutions were transferred through a coarse filter into tared volumetric flasks, weighed and diluted with methanol. Concentrations were determined by spectrophotometric measurements at $280 \mathrm{~nm}$.

\section{Source and Purity of Chemicals:}

(1) $98 \%$, Aldrich Chemical Company, Milwaukee, WI, USA, was recrystallized several times from anhydrous methanol.

(2) $99 \%$, Lancaster Synthesis, USA, stored over molecular sieves and distilled before use.

\section{Estimated Error:}

Temperature: $\pm 0.1 \mathrm{~K}$.

$x_{1}: \pm 1.5 \%$ (relative error). 
(1) Xanthene; $\mathrm{C}_{13} \mathrm{H}_{10} \mathrm{O}$; [92-83-1]

(2) 2,2,4-Trimethylpentane; $\mathrm{C}_{8} \mathrm{H}_{18}$; [540-84-1]

\section{Variables:}

$T / \mathrm{K}=298.15$

\section{Components:}

Original Measurements:

${ }^{77}$ C. I. Monárrez, D. M. Stovall, J. H. Woo, P. Taylor, and W. E. Acree, Jr., Phys. Chem. Liq. 40, 703 (2002).

Prepared by:

W. E. Acree, Jr.

Experimental Values

\begin{tabular}{lcc}
\hline \hline$x_{2}{ }^{(\mathrm{s}) \mathrm{a}}$ & $x_{2}{ }^{\mathrm{b}}$ & $x_{1}{ }^{\mathrm{c}}$ \\
\hline 1.0000 & 0.9755 & 0.02451 \\
\hline
\end{tabular}

${ }^{\mathrm{a}} x_{2}{ }^{(\mathrm{s})}$ : initial mole fraction of component 2 in the solution.

${ }^{b} x_{2}$ : mole fraction of component 2 in the saturated solution.

${ }^{c} x_{1}$ : mole fraction solubility of the solute.

\section{Auxiliary Information}

\begin{abstract}
Method/Apparatus/Procedure:
Constant-temperature bath, calorimetric thermometer, and an ultraviolet/ visible spectrophotometer.

Excess solute and solvent were placed in amber glass bottles and allowed to equilibrate for several days at constant temperature. Attainment of equilibrium was verified by several repetitive measurements and by approaching equilibrium from supersaturation. Aliquots of saturated solutions were transferred through a coarse filter into tared volumetric flasks, weighed and diluted with methanol. Concentrations were determined by spectrophotometric measurements at $280 \mathrm{~nm}$.
\end{abstract}

Source and Purity of Chemicals:

(1) $98 \%$, Aldrich Chemical Company, Milwaukee, WI, USA, was recrystallized several times from anhydrous methanol.

(2) HPLC Grade, Aldrich Chemical Company, stored over molecular sieves and distilled before use.

Estimated Error:

Temperature: $\pm 0.1 \mathrm{~K}$.

$x_{1}: \pm 1.5 \%$ (relative error).

\subsection{Xanthene solubility data in ethers}

\section{Components:}

(1) Xanthene; $\mathrm{C}_{13} \mathrm{H}_{10} \mathrm{O}$; [92-83-1]

(2) $1,1^{\prime}$-Oxybisethane; $\mathrm{C}_{4} \mathrm{H}_{10} \mathrm{O}$;

[60-29-7]

Variables:

$T / \mathrm{K}=298.15$
Original Measurements:

${ }^{76}$ D. M. Stovall, W. E. Acree, Jr., and M. H. Abraham, Fluid Phase Equilib. 232, 111 (2005).

Prepared by:

W. E. Acree, Jr.
Experimental Values

\begin{tabular}{|c|c|c|}
\hline$x_{2}^{(s) a}$ & $x_{2}{ }^{\mathrm{b}}$ & $x_{1}{ }^{\mathrm{c}}$ \\
\hline 1.0000 & 0.9165 & 0.08353 \\
\hline
\end{tabular}

\section{Auxiliary Information}

Method/Apparatus/Procedure:

Constant-temperature bath, calorimetric thermometer, and an ultraviolet/ visible spectrophotometer.

Excess solute and solvent were placed in amber glass bottles and allowed to equilibrate for several days at constant temperature. Attainment of equilibrium was verified by several repetitive measurements and by approaching equilibrium from supersaturation. Aliquots of saturated solutions were transferred through a coarse filter into tared volumetric flasks, weighed and diluted with methanol. Concentrations were determined by spectrophotometric measurements at $280 \mathrm{~nm}$.

\section{Source and Purity of Chemicals:}

(1) $98 \%$, Aldrich Chemical Company, Milwaukee, WI, USA, was recrystallized several times from anhydrous methanol.

(2) $99+\%$, anhydrous, Aldrich Chemical Company, stored over molecular sieves and distilled before use.

Estimated Error:

Temperature: $\pm 0.1 \mathrm{~K}$.

$x_{1}: \pm 1.5 \%$ (relative error).

\begin{tabular}{|c|c|}
\hline $\begin{array}{l}\text { Components: } \\
\text { (1) Xanthene; } \mathrm{C}_{13} \mathrm{H}_{10} \mathrm{O} ;[92-83-1] \\
\text { (2) } 2,2^{\prime} \text {-Oxybispropane; } \mathrm{C}_{6} \mathrm{H}_{14} \mathrm{O} \text {; } \\
{[108-20-3]}\end{array}$ & $\begin{array}{l}\text { Original Measurements: } \\
{ }^{76} \text { D. M. Stovall, W. E. Acree, Jr., } \\
\text { and M. H. Abraham, Fluid Phase } \\
\text { Equilib. 232, } 111 \text { (2005). }\end{array}$ \\
\hline $\begin{array}{l}\text { Variables: } \\
T / \mathrm{K}=298.15\end{array}$ & $\begin{array}{l}\text { Prepared by: } \\
\text { W. E. Acree, Jr. }\end{array}$ \\
\hline
\end{tabular}

Experimental Values

\begin{tabular}{lcc}
\hline \hline$x_{2}{ }^{(\mathrm{s}) \mathrm{a}}$ & $x_{2}{ }^{\mathrm{b}}$ & $x_{1}{ }^{\mathrm{c}}$ \\
\hline 1.0000 & 0.9447 & 0.05531 \\
$\frac{{ }^{\mathrm{a}} x_{2}{ }^{(\mathrm{s})}: \text { initial mole fraction of component } 2 \text { in the solution. }}{{ }^{\mathrm{b}} x_{2}: \text { mole fraction of component } 2 \text { in the saturated solution. }}$ \\
${ }^{\mathrm{c}} x_{1}:$ mole fraction solubility of the solute.
\end{tabular}

\section{Auxiliary Information}

\section{Method/Apparatus/Procedure:}

Constant-temperature bath, calorimetric thermometer, and an ultraviolet/ visible spectrophotometer.

Excess solute and solvent were placed in amber glass bottles and allowed to equilibrate for several days at constant temperature. Attainment of equilibrium was verified by several repetitive measurements and by approaching equilibrium from supersaturation. Aliquots of saturated solutions were transferred through a coarse filter into tared volumetric flasks, weighed and diluted with methanol. Concentrations were determined by

spectrophotometric measurements at $280 \mathrm{~nm}$.

\section{Source and Purity of Chemicals:}

(1) $98 \%$, Aldrich Chemical Company, Milwaukee, WI, USA, was recrystallized several times from anhydrous methanol.

(2) $99 \%$, anhydrous, Aldrich Chemical Company, stored over molecular sieves and distilled before use.

\section{Estimated Error:}

Temperature: $\pm 0.1 \mathrm{~K}$.

$x_{1}: \pm 1.5 \%$ (relative error) 


\section{Components:}

(1) Xanthene; $\mathrm{C}_{13} \mathrm{H}_{10} \mathrm{O}$; [92-83-1]

(2) 1,1'-Oxybisbutane; $\mathrm{C}_{8} \mathrm{H}_{18} \mathrm{O}$;

[142-96-1]

Variables:

$T / \mathrm{K}=298.15$

Original Measurements:

${ }^{77}$ C. I. Monárrez, D. M. Stovall, J. H.

Woo, P. Taylor, and W. E. Acree, Jr., Phys. Chem. Liq. 40, 703 (2002).

Prepared by:

W. E. Acree, Jr.

Experimental Values

\begin{tabular}{lcc}
\hline \hline$x_{2}{ }^{(\mathrm{s}) \mathrm{a}}$ & $x_{2}{ }^{\mathrm{b}}$ & $x_{1}{ }^{\mathrm{c}}$ \\
\hline 1.0000 & 0.9169 & 0.08310 \\
\hline
\end{tabular}

${ }^{\mathrm{a}} x_{2}{ }^{(\mathrm{s})}$ : initial mole fraction of component 2 in the solution.

${ }^{b} x_{2}$ : mole fraction of component 2 in the saturated solution.

${ }^{c} x_{1}$ : mole fraction solubility of the solute.

\section{Auxiliary Information}

\begin{abstract}
Method/Apparatus/Procedure:
Constant-temperature bath, calorimetric thermometer, and an ultraviolet/ visible spectrophotometer.

Excess solute and solvent were placed in amber glass bottles and allowed to equilibrate for several days at constant temperature. Attainment of equilibrium was verified by several repetitive measurements and by approaching equilibrium from supersaturation. Aliquots of saturated solutions were transferred through a coarse filter into tared volumetric flasks, weighed and diluted with methanol. Concentrations were determined by

spectrophotometric measurements at $280 \mathrm{~nm}$.
\end{abstract}

Source and Purity of Chemicals:

(1) $98 \%$, Aldrich Chemical Company, Milwaukee, WI, USA, was

recrystallized several times from anhydrous methanol.

(2) $99 \%$, Aldrich Chemical Company, stored over molecular sieves and distilled before use.

\section{Estimated Error:}

Temperature: $\pm 0.1 \mathrm{~K}$.

$x_{1}: \pm 1.5 \%$ (relative error).

\begin{tabular}{|c|c|}
\hline $\begin{array}{l}\text { Components: } \\
\text { (1) Xanthene; } \mathrm{C}_{13} \mathrm{H}_{10} \mathrm{O} ;[92-83-1] \\
\text { (2) 2-Methoxy-2-methylpropane; } \\
\mathrm{C}_{5} \mathrm{H}_{12} \mathrm{O} ;[1634-04-4]\end{array}$ & $\begin{array}{l}\text { Original Measurements: } \\
{ }^{77} \text { C. I. Monárrez, D. M. Stovall, J. H. } \\
\text { Woo, P. Taylor, and W. E. Acree, Jr., } \\
\text { Phys. Chem. Liq. 40, } 703 \text { (2002). }\end{array}$ \\
\hline $\begin{array}{l}\text { Variables: } \\
T / \mathrm{K}=298.15\end{array}$ & $\begin{array}{l}\text { Prepared by: } \\
\text { W. E. Acree, Jr. }\end{array}$ \\
\hline
\end{tabular}

Experimental Values

\begin{tabular}{|c|c|c|}
\hline$x_{2}^{(\mathrm{s}) \mathrm{a}}$ & $x_{2}{ }^{\mathrm{b}}$ & $x_{1}{ }^{\mathrm{c}}$ \\
\hline 1.0000 & 0.9215 & 0.07846 \\
\hline
\end{tabular}

\section{Auxiliary Information}

\section{Method/Apparatus/Procedure:}

Constant-temperature bath, calorimetric thermometer, and an ultraviolet/ visible spectrophotometer.

Excess solute and solvent were placed in amber glass bottles and allowed to equilibrate for several days at constant temperature. Attainment of equilibrium was verified by several repetitive measurements and by approaching equilibrium from supersaturation. Aliquots of saturated solutions were transferred through a coarse filter into tared volumetric flasks, weighed and diluted with methanol. Concentrations were determined by spectrophotometric measurements at $280 \mathrm{~nm}$.

\section{Source and Purity of Chemicals:}

(1) $98 \%$, Aldrich Chemical Company, Milwaukee, WI, USA, was recrystallized several times from anhydrous methanol.

(2) $99.9+\%$, Arco Chemical Company, USA, stored over molecular sieves and distilled before use.

Estimated Error:

Temperature: $\pm 0.1 \mathrm{~K}$.

$x_{1}: \pm 1.5 \%$ (relative error).

\begin{tabular}{ll}
\hline \hline Components: & Original Measurements: \\
(1) Xanthene; $\mathrm{C}_{13} \mathrm{H}_{10} \mathrm{O} ;$ [92-83-1] & ${ }^{66}$ M. Saifullah, S. Ye, L. M. \\
(2) Tetrahydrofuran; $\mathrm{C}_{4} \mathrm{H}_{8} \mathrm{O} ;$ & Grubbs, N. E. De La Rosa, W. E. \\
{$[109-99-9]$} & Acree, Jr., and M. H. Abraham, J. \\
& Solution Chem. 40, 2082 (2011). \\
\hline Variables: & Prepared by: \\
$T / \mathrm{K}=298.15$ & W. E. Acree, Jr. \\
\hline
\end{tabular}

Experimental Values

\begin{tabular}{lcc}
\hline \hline$x_{2}{ }^{(\mathrm{s}) \mathrm{a}}$ & $x_{2}{ }^{\mathrm{b}}$ & $x_{1}{ }^{\mathrm{c}}$ \\
\hline 1.0000 & 0.8140 & 0.1860 \\
$\frac{{ }^{\mathrm{a}} x_{2}{ }^{(\mathrm{s})}: \text { initial mole fraction of component } 2 \text { in the solution. }}{{ }^{\mathrm{b}}{ }_{x_{2}}: \text { mole fraction of component } 2 \text { in the saturated solution. }}$ \\
${ }^{\mathrm{c}}{ }_{x_{1}}:$ mole fraction solubility of the solute.
\end{tabular}

\section{Auxiliary Information}

\section{Method/Apparatus/Procedure:}

Constant-temperature bath, calorimetric thermometer, and an ultraviolet/ visible spectrophotometer.

Excess solute and solvent were placed in amber glass bottles and allowed to equilibrate for several days at constant temperature. Attainment of equilibrium was verified by several repetitive measurements and by approaching equilibrium from supersaturation. Aliquots of saturated solutions were transferred through a coarse filter into tared volumetric flasks, weighed and diluted with methanol. Concentrations were determined by spectrophotometric measurements at $280 \mathrm{~nm}$.

\section{Source and Purity of Chemicals:}

(1) $98 \%$, Aldrich Chemical Company, Milwaukee, WI, USA, was recrystallized several times from anhydrous methanol.

(2) $99.9 \%$, anhydrous, Aldrich Chemical Company, stored over molecular sieves and distilled before use.

\section{Estimated Error:}

Temperature: $\pm 0.1 \mathrm{~K}$.

$x_{1}: \pm 1.5 \%$ (relative error) 


\section{Components:}

(1) Xanthene; $\mathrm{C}_{13} \mathrm{H}_{10} \mathrm{O}$; [92-83-1]

(2) 1,4-Dioxane; $\mathrm{C}_{4} \mathrm{H}_{8} \mathrm{O}_{2} ;[123-91-1]$

Original Measurements:

${ }^{66}$ M. Saifullah, S. Ye, L. M.

Grubbs, N. E. De La Rosa, W. E. Acree, Jr., and M. H. Abraham, J. Solution Chem. 40, 2082 (2011).

\begin{tabular}{ll}
\hline Variables: & Prepared by: \\
$T / \mathrm{K}=298.15$ & W. E. Acree, Jr. \\
\hline
\end{tabular}

Experimental Values

\begin{tabular}{lcc}
\hline \hline$x_{2}{ }^{(\mathrm{s}) \mathrm{a}}$ & $x_{2}{ }^{\mathrm{b}}$ & $x_{1}{ }^{\mathrm{c}}$ \\
\hline 1.0000 & 0.8498 & 0.1502 \\
${ }^{\mathrm{a}} x_{2}{ }^{\left({ }^{()}\right.}:$initial mole fraction of component 2 in the solution. \\
${ }^{\mathrm{b}} x_{2}:$ mole fraction of component 2 in the saturated solution. \\
${ }^{\mathrm{c}} x_{1}:$ mole fraction solubility of the solute.
\end{tabular}

\section{Auxiliary Information}

\section{Method/Apparatus/Procedure:}

Constant-temperature bath, calorimetric thermometer, and an ultraviolet/ visible spectrophotometer.

Excess solute and solvent were placed in amber glass bottles and allowed to equilibrate for several days at constant temperature. Attainment of equilibrium was verified by several repetitive measurements and by approaching equilibrium from supersaturation. Aliquots of saturated solutions were transferred through a coarse filter into tared volumetric flasks, weighed and diluted with methanol. Concentrations were determined by

spectrophotometric measurements at $280 \mathrm{~nm}$.

Source and Purity of Chemicals:

(1) $98 \%$, Aldrich Chemical Company, Milwaukee, WI, USA, was recrystallized several times from anhydrous methanol.

(2) $99.8 \%$, anhydrous, Aldrich Chemical Company, stored over molecular sieves and distilled before use.

\section{Estimated Error:}

Temperature: $\pm 0.1 \mathrm{~K}$.

$x_{1}: \pm 1.5 \%$ (relative error).

\subsection{Xanthene solubility data in haloalkanes, haloalkenes, and haloaromatic hydrocarbons}

\begin{tabular}{ll}
\hline \hline Components: & Original Measurements: \\
(1) Xanthene; $\mathrm{C}_{13} \mathrm{H}_{10} \mathrm{O} ;[92-83-1]$ & ${ }^{77} \mathrm{C}$. I. Monárrez, D. M. Stovall, \\
(2) Tetrachloromethane; $\mathrm{CCl}_{4} ;$ & J. H. Woo, P. Taylor, and W. E. \\
[56-23-5] & Acree, Jr., Phys. Chem. Liq. 40, \\
& 703 (2002). \\
\hline Variables: & Prepared by: \\
$T / \mathrm{K}=298.15$ & W. E. Acree, Jr. \\
\hline
\end{tabular}

Experimental Values

\begin{tabular}{lcc}
\hline \hline$x_{2}{ }^{(\mathrm{s}) \mathrm{a}}$ & $x_{2}{ }^{\mathrm{b}}$ & $x_{1}{ }^{\mathrm{c}}$ \\
\hline 1.0000 & 0.8763 & 0.1237 \\
\hline${ }^{\mathrm{a}} x_{2}{ }^{(\mathrm{s})}:$ initial mole fraction of component 2 in the solution. \\
${ }^{\mathrm{b}} x_{2}:$ mole fraction of component 2 in the saturated solution. \\
${ }^{\mathrm{c}}{ }_{x_{1}: \text { mole fraction solubility of the solute. }}$
\end{tabular}

\section{Auxiliary Information}

\section{Method/Apparatus/Procedure:}

Constant-temperature bath, calorimetric thermometer, and an ultraviolet/ visible spectrophotometer.

Excess solute and solvent were placed in amber glass bottles and allowed to equilibrate for several days at constant temperature. Attainment of equilibrium was verified by several repetitive measurements and by approaching equilibrium from supersaturation. Aliquots of saturated solutions were transferred through a coarse filter into tared volumetric flasks, weighed and diluted with methanol. Concentrations were determined by spectrophotometric measurements at $280 \mathrm{~nm}$.

Source and Purity of Chemicals:

(1) $98 \%$, Aldrich Chemical Company, Milwaukee, WI, USA, was recrystallized several times from anhydrous methanol.

(2) $99.5+\%$, anhydrous, Aldrich Chemical Company, stored over molecular sieves and distilled before use.

Estimated Error:

Temperature: $\pm 0.1 \mathrm{~K}$.

$x_{1}: \pm 1.5 \%$ (relative error).

\begin{tabular}{|c|c|}
\hline $\begin{array}{l}\text { Components: } \\
\text { (1) Xanthene; } \mathrm{C}_{13} \mathrm{H}_{10} \mathrm{O} ;[92-83-1] \\
\text { (2) } 1,2-\text { Dichloroethane; } \mathrm{C}_{2} \mathrm{H}_{4} \mathrm{Cl}_{2} \text {; } \\
\text { [107-06-2] }\end{array}$ & $\begin{array}{l}\text { Original Measurements: } \\
{ }^{77} \text { C. I. Monárrez, D. M. Stovall, J. H. } \\
\text { Woo, P. Taylor, and W. E. Acree, Jr., } \\
\text { Phys. Chem. Liq. 40, } 703 \text { (2002). }\end{array}$ \\
\hline $\begin{array}{l}\text { Variables: } \\
T / \mathrm{K}=298.15\end{array}$ & $\begin{array}{l}\text { Prepared by: } \\
\text { W. E. Acree, Jr. }\end{array}$ \\
\hline
\end{tabular}

Experimental Values

\begin{tabular}{|c|c|c|}
\hline$x_{2}{ }^{(\mathrm{s}) \mathrm{a}}$ & $x_{2}{ }^{\mathrm{b}}$ & $x_{1}{ }^{\mathrm{c}}$ \\
\hline 1.0000 & 0.8451 & 0.1549 \\
\hline
\end{tabular}

Auxiliary Information

\section{Method/Apparatus/Procedure:}

Constant-temperature bath, calorimetric thermometer, and an ultraviolet/ visible spectrophotometer.

Excess solute and solvent were placed in amber glass bottles and allowed to equilibrate for several days at constant temperature. Attainment of equilibrium was verified by several repetitive measurements and by approaching equilibrium from supersaturation. Aliquots of saturated solutions were transferred through a coarse filter into tared volumetric flasks, weighed and diluted with methanol. Concentrations were determined by spectrophotometric measurements at $280 \mathrm{~nm}$. 
Source and Purity of Chemicals:

(1) $98 \%$, Aldrich Chemical Company, Milwaukee, WI, USA, was recrystallized several times from anhydrous methanol.

(2) $99.8 \%$, anhydrous, Aldrich Chemical Company, stored over molecular sieves and distilled before use.

\section{Estimated Error:}

Temperature: $\pm 0.1 \mathrm{~K}$.

$x_{1}: \pm 1.5 \%$ (relative error).

\subsection{Xanthene solubility data in alcohols}

Components:
(1) Xanthene; $\mathrm{C}_{13} \mathrm{H}_{10} \mathrm{O}$; [92-83-1]
(2) Methanol; $\mathrm{CH}_{4} \mathrm{O} ;[67-56-1]$

Original Measurements:

${ }^{77}$ C. I. Monárrez, D. M. Stovall, J. H. Woo, P. Taylor, and W. E. Acree, Jr., Phys. Chem. Liq. 40, 703 (2002).

\begin{tabular}{ll}
\hline Variables: & Prepared by: \\
$T / \mathrm{K}=298.15$ & W. E. Acree, Jr. \\
\hline
\end{tabular}

Experimental Values

\begin{tabular}{lcc}
\hline \hline$x_{2}{ }^{(\mathrm{s}) \mathrm{a}}$ & $x_{2}{ }^{\mathrm{b}}$ & $x_{1}{ }^{\mathrm{c}}$ \\
\hline 1.0000 & 0.9955 & 0.004455 \\
$\frac{{ }^{a} x_{2}{ }^{(\mathrm{s})}: \text { initial mole fraction of component } 2 \text { in the solution. }}{{ }^{\mathrm{b}} x_{2}: \text { mole fraction of component } 2 \text { in the saturated solution. }}$ \\
${ }^{\mathrm{c}} x_{x_{1}}:$ mole fraction solubility of the solute.
\end{tabular}

\section{Auxiliary Information}

\section{Method/Apparatus/Procedure:}

Constant-temperature bath, calorimetric thermometer, and an ultraviolet/ visible spectrophotometer.

Excess solute and solvent were placed in amber glass bottles and allowed to equilibrate for several days at constant temperature. Attainment of equilibrium was verified by several repetitive measurements and by approaching equilibrium from supersaturation. Aliquots of saturated solutions were transferred through a coarse filter into tared volumetric flasks, weighed and diluted with methanol. Concentrations were determined by

spectrophotometric measurements at $280 \mathrm{~nm}$.

Source and Purity of Chemicals:

(1) $98 \%$, Aldrich Chemical Company, Milwaukee, WI, USA, was recrystallized several times from anhydrous methanol.

(2) $99.9+\%$, Aldrich Chemical Company, stored over molecular sieves and distilled before use.

\section{Estimated Error:}

Temperature: $\pm 0.1 \mathrm{~K}$.

$x_{1}: \pm 1.5 \%$ (relative error).

\section{Components:}

(1) Xanthene; $\mathrm{C}_{13} \mathrm{H}_{10} \mathrm{O}$; [92-83-1]

(2) Ethanol; $\mathrm{C}_{2} \mathrm{H}_{6} \mathrm{O} ;[64-17-5]$

\section{Variables:}

$T / \mathrm{K}=298.15$

\section{Original Measurements:}

${ }^{77}$ C. I. Monárrez, D. M. Stovall, J. H. Woo, P. Taylor, and W. E. Acree, Jr., Phys. Chem. Liq. 40, 703 (2002).

Prepared by:

W. E. Acree, Jr.
Experimental Values

\begin{tabular}{|c|c|c|}
\hline$x_{2}{ }^{(\mathrm{s}) \mathrm{a}}$ & $x_{2}{ }^{b}$ & $x_{1}{ }^{\mathrm{c}}$ \\
\hline 1.0000 & 0.9938 & 0.006231 \\
\hline
\end{tabular}

\section{Auxiliary Information}

Method/Apparatus/Procedure:

Constant-temperature bath, calorimetric thermometer, and an ultraviolet/ visible spectrophotometer.

Excess solute and solvent were placed in amber glass bottles and allowed to equilibrate for several days at constant temperature. Attainment of equilibrium was verified by several repetitive measurements and by approaching equilibrium from supersaturation. Aliquots of saturated solutions were transferred through a coarse filter into tared volumetric flasks, weighed and diluted with methanol. Concentrations were determined by spectrophotometric measurements at $280 \mathrm{~nm}$.

\section{Source and Purity of Chemicals:}

(1) $98 \%$, Aldrich Chemical Company, Milwaukee, WI, USA, was recrystallized several times from anhydrous methanol.

(2) Absolute, Aaper Alcohol and Chemical Company, USA, stored over molecular sieves and distilled before use.

\section{Estimated Error:}

Temperature: $\pm 0.1 \mathrm{~K}$.

$x_{1}: \pm 1.5 \%$ (relative error).

\begin{tabular}{ll}
\hline \hline Components: & Original Measurements: \\
(1) Xanthene; $\mathrm{C}_{13} \mathrm{H}_{10} \mathrm{O} ;[92-83-1]$ & ${ }^{77}$ C. I. Monárrez, D. M. Stovall, J. H. \\
(2) 1-Propanol; $\mathrm{C}_{3} \mathrm{H}_{8} \mathrm{O} ;[71-23-8]$ & Woo, P. Taylor, and W. E. Acree, Jr., \\
& Phys. Chem. Liq. 40, 703 (2002). \\
\hline Variables: & Prepared by: \\
$T / \mathrm{K}=298.15$ & W. E. Acree, Jr.
\end{tabular}

\section{Experimental Values}

\begin{tabular}{lcc}
\hline \hline$x_{2}^{(\mathrm{s}) \mathrm{a}}$ & $x_{2}{ }^{\mathrm{b}}$ & $x_{1}{ }^{\mathrm{c}}$ \\
\hline 1.0000 & 0.9883 & 0.01166 \\
\hline
\end{tabular}

${ }^{\mathrm{a}} x_{2}{ }^{(\mathrm{s})}$ : initial mole fraction of component 2 in the solution.

${ }^{\mathrm{b}} x_{2}$ : mole fraction of component 2 in the saturated solution.

${ }^{c} x_{1}$ : mole fraction solubility of the solute.

\section{Auxiliary Information}

\section{Method/Apparatus/Procedure:}

Constant-temperature bath, calorimetric thermometer, and an ultraviolet/ visible spectrophotometer.

Excess solute and solvent were placed in amber glass bottles and allowed to equilibrate for several days at constant temperature. Attainment of equilibrium was verified by several repetitive measurements and by approaching equilibrium from supersaturation. Aliquots of saturated solutions were transferred through a coarse filter into tared volumetric flasks, weighed and diluted with methanol. Concentrations were determined by spectrophotometric measurements at $280 \mathrm{~nm}$. 
Source and Purity of Chemicals:

(1) $98 \%$, Aldrich Chemical Company, Milwaukee, WI, USA, was recrystallized several times from anhydrous methanol.

(2) $99+\%$, anhydrous, Aldrich Chemical Company, stored over molecular sieves and distilled before use.

\section{Estimated Error:}

Temperature: $\pm 0.1 \mathrm{~K}$.

$x_{1}: \pm 1.5 \%$ (relative error).

\section{Components:}

(1) Xanthene; $\mathrm{C}_{13} \mathrm{H}_{10} \mathrm{O}$; [92-83-1]

(2) 2-Propanol; $\mathrm{C}_{3} \mathrm{H}_{8} \mathrm{O}$; [67-63-0]

\section{Original Measurements:}

${ }^{77}$ C. I. Monárrez, D. M. Stovall, J. H.

Woo, P. Taylor, and W. E. Acree, Jr., Phys. Chem. Liq. 40, 703 (2002).

\begin{tabular}{ll}
\hline Variables: & Prepared by: \\
$T / \mathrm{K}=298.15$ & W. E. Acree, Jr. \\
\hline
\end{tabular}

Experimental Values

\begin{tabular}{lcc}
\hline \hline$x_{2}{ }^{(\mathrm{s}) \mathrm{a}}$ & $x_{2}{ }^{\mathrm{b}}$ & $x_{1}{ }^{\mathrm{c}}$ \\
\hline 1.0000 & 0.9914 & 0.008643
\end{tabular}

${ }^{\mathrm{a}} x_{2}{ }^{(\mathrm{s})}$ : initial mole fraction of component 2 in the solution.

${ }^{\mathrm{b}} x_{2}$ : mole fraction of component 2 in the saturated solution.

${ }^{c} x_{1}$ : mole fraction solubility of the solute.

\section{Auxiliary Information}

\begin{abstract}
Method/Apparatus/Procedure:
Constant-temperature bath, calorimetric thermometer, and an ultraviolet/ visible spectrophotometer.

Excess solute and solvent were placed in amber glass bottles and allowed to equilibrate for several days at constant temperature. Attainment of equilibrium was verified by several repetitive measurements and by approaching equilibrium from supersaturation. Aliquots of saturated solutions were transferred through a coarse filter into tared volumetric flasks, weighed and diluted with methanol. Concentrations were determined by

spectrophotometric measurements at $280 \mathrm{~nm}$.
\end{abstract}

Source and Purity of Chemicals:

(1) $98 \%$, Aldrich Chemical Company, Milwaukee, WI, USA, was recrystallized several times from anhydrous methanol.

(2) $99+\%$, anhydrous, Aldrich Chemical Company, stored over molecular sieves and distilled before use.

\section{Estimated Error:}

Temperature: $\pm 0.1 \mathrm{~K}$.

$x_{1}: \pm 1.5 \%$ (relative error).

\begin{tabular}{|c|c|}
\hline $\begin{array}{l}\text { Components: } \\
\text { (1) Xanthene; } \mathrm{C}_{13} \mathrm{H}_{10} \mathrm{O} ;[92-83-1] \\
\text { (2) 1-Butanol; } \mathrm{C}_{4} \mathrm{H}_{10} \mathrm{O} ;[71-36-3]\end{array}$ & $\begin{array}{l}\text { Original Measurements: } \\
{ }^{77} \text { C. I. Monárrez, D. M. Stovall, J. H. } \\
\text { Woo, P. Taylor, and W. E. Acree, Jr., } \\
\text { Phys. Chem. Liq. 40, } 703 \text { (2002). }\end{array}$ \\
\hline $\begin{array}{l}\text { Variables: } \\
T / \mathrm{K}=298.15\end{array}$ & $\begin{array}{l}\text { Prepared by: } \\
\text { W. E. Acree, Jr. }\end{array}$ \\
\hline
\end{tabular}

Experimental Values

\begin{tabular}{|c|c|c|}
\hline$x_{2}{ }^{(\mathrm{s}) \mathrm{a}}$ & $x_{2}{ }^{b}$ & $x_{1}{ }^{\mathrm{c}}$ \\
\hline 1.0000 & 0.9824 & 0.01756 \\
\hline
\end{tabular}

\section{Auxiliary Information}

\section{Method/Apparatus/Procedure:}

Constant-temperature bath, calorimetric thermometer, and an ultraviolet/ visible spectrophotometer.

Excess solute and solvent were placed in amber glass bottles and allowed to equilibrate for several days at constant temperature. Attainment of equilibrium was verified by several repetitive measurements and by approaching equilibrium from supersaturation. Aliquots of saturated solutions were transferred through a coarse filter into tared volumetric flasks, weighed and diluted with methanol. Concentrations were determined by spectrophotometric measurements at $280 \mathrm{~nm}$.

\section{Source and Purity of Chemicals:}

(1) $98 \%$, Aldrich Chemical Company, Milwaukee, WI, USA, was recrystallized several times from anhydrous methanol.

(2) $99.8+\%$, HPLC Grade, Aldrich Chemical Company, stored over molecular sieves and distilled before use.

Estimated Error:

Temperature: $\pm 0.1 \mathrm{~K}$.

$x_{1}: \pm 1.5 \%$ (relative error).

\begin{tabular}{ll}
\hline \hline Components: & Original Measurements: \\
(1) Xanthene; $\mathrm{C}_{13} \mathrm{H}_{10} \mathrm{O} ;[$ [92-83-1] & ${ }^{77}$ C. I. Monárrez, D. M. Stovall, J. H. \\
(2) 2-Butanol; $\mathrm{C}_{4} \mathrm{H}_{10} \mathrm{O} ;$ [78-92-2] & Woo, P. Taylor, and W. E. Acree, Jr., \\
& Phys. Chem. Liq. 40, 703 (2002). \\
\hline Variables: & Prepared by: \\
$T / \mathrm{K}=298.15$ & W. E. Acree, Jr. \\
\hline
\end{tabular}

\section{Experimental Values}

\begin{tabular}{|c|c|c|}
\hline$x_{2}{ }^{(\mathrm{s}) \mathrm{a}}$ & $x_{2}^{b}$ & $x_{1}{ }^{\mathrm{c}}$ \\
\hline 1.0000 & 0.9875 & 0.01254 \\
\hline
\end{tabular}

\section{Auxiliary Information}

\section{Method/Apparatus/Procedure:}

Constant-temperature bath, calorimetric thermometer, and an ultraviolet/ visible spectrophotometer.

Excess solute and solvent were placed in amber glass bottles and allowed to equilibrate for several days at constant temperature. Attainment of equilibrium was verified by several repetitive measurements and by approaching equilibrium from supersaturation. Aliquots of saturated solutions were transferred through a coarse filter into tared volumetric flasks, weighed and diluted with methanol. Concentrations were determined by spectrophotometric measurements at $280 \mathrm{~nm}$. 
Source and Purity of Chemicals:

(1) $98 \%$, Aldrich Chemical Company, Milwaukee, WI, USA, was recrystallized several times from anhydrous methanol.

(2) $99+\%$, anhydrous, Aldrich Chemical Company, stored over molecular sieves and distilled before use.

\section{Estimated Error:}

Temperature: $\pm 0.1 \mathrm{~K}$.

$x_{1}: \pm 1.5 \%$ (relative error).

\section{Components:}

(1) Xanthene; $\mathrm{C}_{13} \mathrm{H}_{10} \mathrm{O}$; [92-83-1]

(2) 2-Methyl-1-propanol; $\mathrm{C}_{4} \mathrm{H}_{10} \mathrm{O}$;

[78-83-1]

Variables:

$T / \mathrm{K}=298.15$

\section{Original Measurements:}

${ }^{77}$ C. I. Monárrez, D. M. Stovall, J. H.

Woo, P. Taylor, and W. E. Acree, Jr., Phys. Chem. Liq. 40, 703 (2002).

\section{Prepared by:}

W. E. Acree, Jr.

\section{Experimental Values}

\begin{tabular}{lcc}
\hline \hline$x_{2}{ }^{(\mathrm{s}) \mathrm{a}}$ & $x_{2}{ }^{\mathrm{b}}$ & $x_{1}{ }^{\mathrm{c}}$ \\
\hline 1.0000 & 0.9892 & 0.01077
\end{tabular}

${ }^{\mathrm{a}} x_{2}{ }^{(\mathrm{s})}$ : initial mole fraction of component 2 in the solution.

${ }^{\mathrm{b}} x_{2}$ : mole fraction of component 2 in the saturated solution.

${ }^{c} x_{1}$ : mole fraction solubility of the solute.

\section{Auxiliary Information}

\begin{abstract}
Method/Apparatus/Procedure:
Constant-temperature bath, calorimetric thermometer, and an ultraviolet/ visible spectrophotometer.

Excess solute and solvent were placed in amber glass bottles and allowed to equilibrate for several days at constant temperature. Attainment of equilibrium was verified by several repetitive measurements and by approaching equilibrium from supersaturation. Aliquots of saturated solutions were transferred through a coarse filter into tared volumetric flasks, weighed and diluted with methanol. Concentrations were determined by

spectrophotometric measurements at $280 \mathrm{~nm}$.
\end{abstract}

Source and Purity of Chemicals:

(1) $98 \%$, Aldrich Chemical Company, Milwaukee, WI, USA, was recrystallized several times from anhydrous methanol.

(2) $99+\%$, anhydrous, Aldrich Chemical Company, stored over molecular sieves and distilled before use.

\section{Estimated Error:}

Temperature: $\pm 0.1 \mathrm{~K}$.

$x_{1}: \pm 1.5 \%$ (relative error).

\begin{tabular}{ll}
\hline \hline Components: & Original Measurements: \\
(1) Xanthene; $\mathrm{C}_{13} \mathrm{H}_{10} \mathrm{O} ;$ [92-83-1] & ${ }^{77}$ C. I. Monárrez, D. M. Stovall, J. H. \\
(2) 2-Methyl-2-propanol; $\mathrm{C}_{4} \mathrm{H}_{10} \mathrm{O} ;$ & Woo, P. Taylor, and W. E. Acree, Jr., \\
[75-65-0] & Phys. Chem. Liq. 40, 703 (2002). \\
\hline Variables: & Prepared by: \\
$T / \mathrm{K}=298.15$ & W. E. Acree, Jr. \\
\hline
\end{tabular}

Experimental Values

\begin{tabular}{|c|c|c|}
\hline$x_{2}{ }^{(\mathrm{s}) \mathrm{a}}$ & $x_{2}{ }^{b}$ & $x_{1}{ }^{\mathrm{c}}$ \\
\hline 1.0000 & 0.9889 & 0.01112 \\
\hline
\end{tabular}

\section{Auxiliary Information}

\section{Method/Apparatus/Procedure:}

Constant-temperature bath, calorimetric thermometer, and an ultraviolet/ visible spectrophotometer.

Excess solute and solvent were placed in amber glass bottles and allowed to equilibrate for several days at constant temperature. Attainment of equilibrium was verified by several repetitive measurements and by approaching equilibrium from supersaturation. Aliquots of saturated solutions were transferred through a coarse filter into tared volumetric flasks, weighed and diluted with methanol. Concentrations were determined by spectrophotometric measurements at $280 \mathrm{~nm}$.

\section{Source and Purity of Chemicals:}

(1) $98 \%$, Aldrich Chemical Company, Milwaukee, WI, USA, was recrystallized several times from anhydrous methanol.

(2) $99+\%$, Arco Chemical Company, USA, stored over molecular sieves and distilled before use.

\section{Estimated Error:}

Temperature: $\pm 0.1 \mathrm{~K}$.

$x_{1}: \pm 1.5 \%$ (relative error).

\begin{tabular}{ll}
\hline \hline Components: & Original Measurements: \\
(1) Xanthene; $\mathrm{C}_{13} \mathrm{H}_{10} \mathrm{O} ;[92-83-1]$ & ${ }^{77}$ C. I. Monárrez, D. M. Stovall, J. H. \\
(2) 1-Pentanol; $\mathrm{C}_{5} \mathrm{H}_{12} \mathrm{O} ;[71-41-0]$ & Woo, P. Taylor, and W. E. Acree, Jr., \\
& Phys. Chem. Liq. 40, 703 (2002). \\
\hline Variables: & Prepared by: \\
$T / \mathrm{K}=298.15$ & W. E. Acree, Jr. \\
\hline
\end{tabular}

\section{Experimental Values}

\begin{tabular}{|c|c|c|}
\hline$x_{2}{ }^{(\mathrm{s}) \mathrm{a}}$ & $x_{2}{ }^{b}$ & $x_{1}{ }^{\mathrm{c}}$ \\
\hline 1.0000 & 0.9779 & 0.02212 \\
\hline
\end{tabular}

\section{Auxiliary Information}

\section{Method/Apparatus/Procedure:}

Constant-temperature bath, calorimetric thermometer, and an ultraviolet/ visible spectrophotometer.

Excess solute and solvent were placed in amber glass bottles and allowed to equilibrate for several days at constant temperature. Attainment of equilibrium was verified by several repetitive measurements and by approaching equilibrium from supersaturation. Aliquots of saturated solutions were transferred through a coarse filter into tared volumetric flasks, weighed and diluted with methanol. Concentrations were determined by spectrophotometric measurements at $280 \mathrm{~nm}$. 
Source and Purity of Chemicals:

(1) $98 \%$, Aldrich Chemical Company, Milwaukee, WI, USA, was

recrystallized several times from anhydrous methanol.

(2) $99+\%$, Aldrich Chemical Company, stored over molecular sieves and distilled before use.

\section{Estimated Error:}

Temperature: $\pm 0.1 \mathrm{~K}$.

$x_{1}: \pm 1.5 \%$ (relative error).

\section{Components:}

(1) Xanthene; $\mathrm{C}_{13} \mathrm{H}_{10} \mathrm{O}$; [92-83-1]

(2) 2-Pentanol; $\mathrm{C}_{5} \mathrm{H}_{12} \mathrm{O}$; [6032-29-7]

\section{Original Measurements:}

${ }^{77}$ C. I. Monárrez, D. M. Stovall, J. H.

\section{Variables:}

$T / \mathrm{K}=298.15$

Prepared by:

W. E. Acree, Jr.

\section{Experimental Values}

\begin{tabular}{lcc}
\hline \hline$x_{2}{ }^{(\mathrm{s}) \mathrm{a}}$ & $x_{2}{ }^{\mathrm{b}}$ & $x_{1}{ }^{\mathrm{c}}$ \\
\hline 1.0000 & 0.9823 & 0.01766
\end{tabular}

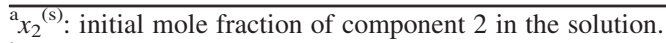

${ }^{\mathrm{b}} x_{2}$ : mole fraction of component 2 in the saturated solution.

${ }^{c} x_{1}$ : mole fraction solubility of the solute.

\section{Auxiliary Information}

\begin{abstract}
Method/Apparatus/Procedure:
Constant-temperature bath, calorimetric thermometer, and an ultraviolet/ visible spectrophotometer.

Excess solute and solvent were placed in amber glass bottles and allowed to equilibrate for several days at constant temperature. Attainment of equilibrium was verified by several repetitive measurements and by approaching equilibrium from supersaturation. Aliquots of saturated solutions were transferred through a coarse filter into tared volumetric flasks, weighed and diluted with methanol. Concentrations were determined by

spectrophotometric measurements at $280 \mathrm{~nm}$.
\end{abstract}

Source and Purity of Chemicals:

(1) $98 \%$, Aldrich Chemical Company, Milwaukee, WI, USA, was recrystallized several times from anhydrous methanol.

(2) $99+\%$, Acros Organics, USA, stored over molecular sieves and distilled before use.

\section{Estimated Error:}

Temperature: $\pm 0.1 \mathrm{~K}$.

$x_{1}: \pm 1.5 \%$ (relative error).

\begin{tabular}{|c|c|}
\hline $\begin{array}{l}\text { Components: } \\
\text { (1) Xanthene; } \mathrm{C}_{13} \mathrm{H}_{10} \mathrm{O} ;[92-83-1] \\
\text { (2) 3-Methyl-1-butanol; } \mathrm{C}_{5} \mathrm{H}_{12} \mathrm{O} \text {; } \\
\text { [123-51-3] }\end{array}$ & $\begin{array}{l}\text { Original Measurements: } \\
{ }^{77} \text { C. I. Monárrez, D. M. Stovall, J. H. } \\
\text { Woo, P. Taylor, and W. E. Acree, Jr., } \\
\text { Phys. Chem. Liq. 40, } 703 \text { (2002). }\end{array}$ \\
\hline $\begin{array}{l}\text { Variables: } \\
T / \mathrm{K}=298.15\end{array}$ & $\begin{array}{l}\text { Prepared by: } \\
\text { W. E. Acree, Jr. }\end{array}$ \\
\hline
\end{tabular}

Experimental Values

\begin{tabular}{|c|c|c|}
\hline$x_{2}{ }^{(\mathrm{s}) \mathrm{a}}$ & $x_{2}{ }^{b}$ & $x_{1}{ }^{\mathrm{c}}$ \\
\hline 1.0000 & 0.9837 & 0.01633 \\
\hline
\end{tabular}

\section{Auxiliary Information}

\section{Method/Apparatus/Procedure:}

Constant-temperature bath, calorimetric thermometer, and an ultraviolet/ visible spectrophotometer.

Excess solute and solvent were placed in amber glass bottles and allowed to equilibrate for several days at constant temperature. Attainment of equilibrium was verified by several repetitive measurements and by approaching equilibrium from supersaturation. Aliquots of saturated solutions were transferred through a coarse filter into tared volumetric flasks, weighed and diluted with methanol. Concentrations were determined by spectrophotometric measurements at $280 \mathrm{~nm}$.

\section{Source and Purity of Chemicals:}

(1) $98 \%$, Aldrich Chemical Company, Milwaukee, WI, USA, was recrystallized several times from anhydrous methanol.

(2) $99+\%$, anhydrous, Aldrich Chemical Company, stored over molecular sieves and distilled before use.

\section{Estimated Error:}

Temperature: $\pm 0.1 \mathrm{~K}$.

$x_{1}: \pm 1.5 \%$ (relative error).

\begin{tabular}{ll}
\hline \hline Components: & Original Measurements: \\
(1) Xanthene; $\mathrm{C}_{13} \mathrm{H}_{10} \mathrm{O} ;[$ [92-83-1] & ${ }^{77}$ C. I. Monárrez, D. M. Stovall, J. H. \\
(2) 2-Methyl-2-butanol; $\mathrm{C}_{5} \mathrm{H}_{12} \mathrm{O} ;$ & Woo, P. Taylor, and W. E. Acree, Jr., \\
[75-85-4] & Phys. Chem. Liq. 40, 703 (2002). \\
\hline Variables: & Prepared by: \\
$T / \mathrm{K}=298.15$ & W. E. Acree, Jr. \\
\hline
\end{tabular}

Experimental Values

\begin{tabular}{|c|c|c|}
\hline$x_{2}{ }^{(\mathrm{s}) \mathrm{a}}$ & $x_{2}^{b}$ & $x_{1}{ }^{c}$ \\
\hline 1.0000 & 0.9805 & 0.01946 \\
\hline
\end{tabular}

\section{Auxiliary Information}

\section{Method/Apparatus/Procedure:}

Constant-temperature bath, calorimetric thermometer, and an ultraviolet/ visible spectrophotometer.

Excess solute and solvent were placed in amber glass bottles and allowed to equilibrate for several days at constant temperature. Attainment of equilibrium was verified by several repetitive measurements and by approaching equilibrium from supersaturation. Aliquots of saturated solutions were transferred through a coarse filter into tared volumetric flasks, weighed and diluted with methanol. Concentrations were determined by spectrophotometric measurements at $280 \mathrm{~nm}$. 
Source and Purity of Chemicals:

(1) $98 \%$, Aldrich Chemical Company, Milwaukee, WI, USA, was

recrystallized several times from anhydrous methanol.

(2) $99+\%$, Acros Organics, USA, stored over molecular sieves and distilled before use.

\section{Estimated Error:}

Temperature: $\pm 0.1 \mathrm{~K}$.

$x_{1}: \pm 1.5 \%$ (relative error).

\section{Components:}

(1) Xanthene; $\mathrm{C}_{13} \mathrm{H}_{10} \mathrm{O}$; [92-83-1]

(2) 1-Hexanol; $\mathrm{C}_{6} \mathrm{H}_{14} \mathrm{O} ;[111-27-3]$

\section{Original Measurements:}

${ }^{77}$ C. I. Monárrez, D. M. Stovall, J. H.

Woo, P. Taylor, and W. E. Acree, Jr., Phys. Chem. Liq. 40, 703 (2002).

\begin{tabular}{ll}
\hline Variables: & Prepared by: \\
$T / \mathrm{K}=298.15$ & W. E. Acree, Jr. \\
\hline
\end{tabular}

Experimental Values

\begin{tabular}{lcc}
\hline \hline$x_{2}{ }^{(\mathrm{s}) \mathrm{a}}$ & $x_{2}{ }^{\mathrm{b}}$ & $x_{1}{ }^{\mathrm{c}}$ \\
\hline 1.0000 & 0.9717 & 0.02831
\end{tabular}

${ }^{\mathrm{a}} x_{2}{ }^{(\mathrm{s})}$ : initial mole fraction of component 2 in the solution.

${ }^{b} x_{2}$ : mole fraction of component 2 in the saturated solution.

${ }^{c} x_{1}$ : mole fraction solubility of the solute.

\section{Auxiliary Information}

\begin{abstract}
Method/Apparatus/Procedure:
Constant-temperature bath, calorimetric thermometer, and an ultraviolet/ visible spectrophotometer.

Excess solute and solvent were placed in amber glass bottles and allowed to equilibrate for several days at constant temperature. Attainment of equilibrium was verified by several repetitive measurements and by approaching equilibrium from supersaturation. Aliquots of saturated solutions were transferred through a coarse filter into tared volumetric flasks, weighed and diluted with methanol. Concentrations were determined by

spectrophotometric measurements at $280 \mathrm{~nm}$.
\end{abstract}

\section{Source and Purity of Chemicals:}

(1) $98 \%$, Aldrich Chemical Company, Milwaukee, WI, USA, was recrystallized several times from anhydrous methanol.

(2) $99+\%$, Alfa Aesar, USA, stored over molecular sieves and distilled before use.

\section{Estimated Error:}

Temperature: $\pm 0.1 \mathrm{~K}$.

$x_{1}: \pm 1.5 \%$ (relative error).

\begin{tabular}{|c|c|}
\hline $\begin{array}{l}\text { Components: } \\
\text { (1) Xanthene; } \mathrm{C}_{13} \mathrm{H}_{10} \mathrm{O} ;[92-83-1] \\
\text { (2) 2-Methyl-1-pentanol; } \mathrm{C}_{6} \mathrm{H}_{14} \mathrm{O} \text {; } \\
{[105-30-6]}\end{array}$ & $\begin{array}{l}\text { Original Measurements: } \\
{ }^{77} \text { C. I. Monárrez, D. M. Stovall, J. H. } \\
\text { Woo, P. Taylor, and W. E. Acree, Jr., } \\
\text { Phys. Chem. Liq. 40, } 703 \text { (2002). }\end{array}$ \\
\hline $\begin{array}{l}\text { Variables: } \\
T / \mathrm{K}=298.15\end{array}$ & $\begin{array}{l}\text { Prepared by: } \\
\text { W. E. Acree, Jr. }\end{array}$ \\
\hline
\end{tabular}

Experimental Values

\begin{tabular}{lcc}
\hline \hline$x_{2}{ }^{(\mathrm{s}) \mathrm{a}}$ & $x_{2}{ }^{\mathrm{b}}$ & $x_{1}{ }^{\mathrm{c}}$ \\
\hline 1.0000 & 0.9803 & 0.01969 \\
$\frac{{ }^{a} x_{2}{ }^{(\mathrm{s})}: \text { initial mole fraction of component } 2 \text { in the solution. }}{{ }^{\mathrm{b}} x_{2}: \text { mole fraction of component } 2 \text { in the saturated solution. }}$ \\
${ }^{c} x_{1}:$ mole fraction solubility of the solute.
\end{tabular}

\section{Auxiliary Information}

\section{Method/Apparatus/Procedure:}

Constant-temperature bath, calorimetric thermometer, and an ultraviolet/ visible spectrophotometer.

Excess solute and solvent were placed in amber glass bottles and allowed to equilibrate for several days at constant temperature. Attainment of equilibrium was verified by several repetitive measurements and by approaching equilibrium from supersaturation. Aliquots of saturated solutions were transferred through a coarse filter into tared volumetric flasks, weighed and diluted with methanol. Concentrations were determined by spectrophotometric measurements at $280 \mathrm{~nm}$.

\section{Source and Purity of Chemicals:}

(1) $98 \%$, Aldrich Chemical Company, Milwaukee, WI, USA, was recrystallized several times from anhydrous methanol.

(2) $99 \%$, Aldrich Chemical Company, stored over molecular sieves and distilled before use.

\section{Estimated Error:}

Temperature: $\pm 0.1 \mathrm{~K}$.

$x_{1}: \pm 1.5 \%$ (relative error).

\begin{tabular}{ll}
\hline \hline Components: & Original Measurements: \\
(1) Xanthene; $\mathrm{C}_{13} \mathrm{H}_{10} \mathrm{O} ;[92-83-1]$ & ${ }^{77}$ C. I. Monárrez, D. M. Stovall, J. H. \\
(2) 4-Methyl-2-pentanol; $\mathrm{C}_{6} \mathrm{H}_{14} \mathrm{O} ;$ & Woo, P. Taylor, and W. E. Acree, Jr., \\
{$[108-11-2]$} & Phys. Chem. Liq. 40, 703 (2002). \\
\hline Variables: & Prepared by: \\
$T / \mathrm{K}=298.15$ & W. E. Acree, Jr. \\
\hline
\end{tabular}

\section{Experimental Values}

\begin{tabular}{|c|c|c|}
\hline$x_{2}{ }^{(\mathrm{s}) \mathrm{a}}$ & $x_{2}{ }^{\mathrm{b}}$ & $x_{1}{ }^{\mathrm{c}}$ \\
\hline 1.0000 & 0.9824 & 0.01762 \\
\hline
\end{tabular}

\section{Auxiliary Information}

\section{Method/Apparatus/Procedure:}

Constant-temperature bath, calorimetric thermometer, and an ultraviolet/ visible spectrophotometer.

Excess solute and solvent were placed in amber glass bottles and allowed to equilibrate for several days at constant temperature. Attainment of equilibrium was verified by several repetitive measurements and by approaching equilibrium from supersaturation. Aliquots of saturated solutions were transferred through a coarse filter into tared volumetric flasks, weighed and diluted with methanol. Concentrations were determined by spectrophotometric measurements at $280 \mathrm{~nm}$. 
Source and Purity of Chemicals:

(1) $98 \%$, Aldrich Chemical Company, Milwaukee, WI, USA, was

recrystallized several times from anhydrous methanol.

(2) $99+\%$, Acros Organics, USA, stored over molecular sieves and distilled before use.

\section{Estimated Error:}

Temperature: $\pm 0.1 \mathrm{~K}$.

$x_{1}: \pm 1.5 \%$ (relative error).

\section{Components:}

(1) Xanthene; $\mathrm{C}_{13} \mathrm{H}_{10} \mathrm{O}$; [92-83-1]

(2) 1-Heptanol; $\mathrm{C}_{7} \mathrm{H}_{16} \mathrm{O} ;[111-70-6]$

\section{Original Measurements:}

${ }^{77}$ C. I. Monárrez, D. M. Stovall, J. H. Woo, P. Taylor, and W. E. Acree, Jr., Phys. Chem. Liq. 40, 703 (2002).

\begin{tabular}{ll}
\hline Variables: & Prepared by: \\
$T / \mathrm{K}=298.15$ & W. E. Acree, Jr.
\end{tabular}

\section{Experimental Values}

\begin{tabular}{lcc}
\hline \hline$x_{2}{ }^{(\mathrm{s}) \mathrm{a}}$ & $x_{2}{ }^{\mathrm{b}}$ & $x_{1}{ }^{\mathrm{c}}$ \\
\hline 1.0000 & 0.9666 & 0.03340
\end{tabular}

${ }^{\mathrm{a}} x_{2}{ }^{(\mathrm{s})}$ : initial mole fraction of component 2 in the solution.

${ }^{\mathrm{b}} x_{2}$ : mole fraction of component 2 in the saturated solution.

${ }^{c} x_{1}$ : mole fraction solubility of the solute.

\section{Auxiliary Information}

\begin{abstract}
Method/Apparatus/Procedure:
Constant-temperature bath, calorimetric thermometer, and an ultraviolet/ visible spectrophotometer.

Excess solute and solvent were placed in amber glass bottles and allowed to equilibrate for several days at constant temperature. Attainment of equilibrium was verified by several repetitive measurements and by approaching equilibrium from supersaturation. Aliquots of saturated solutions were transferred through a coarse filter into tared volumetric flasks, weighed and diluted with methanol. Concentrations were determined by

spectrophotometric measurements at $280 \mathrm{~nm}$.
\end{abstract}

Source and Purity of Chemicals:

(1) $98 \%$, Aldrich Chemical Company, Milwaukee, WI, USA, was recrystallized several times from anhydrous methanol.

(2) $99+\%$, Alfa Aesar, USA, stored over molecular sieves and distilled before use.

\section{Estimated Error:}

Temperature: $\pm 0.1 \mathrm{~K}$.

$x_{1}: \pm 1.5 \%$ (relative error).

\begin{tabular}{|c|c|}
\hline $\begin{array}{l}\text { Components: } \\
\text { (1) Xanthene; } \mathrm{C}_{13} \mathrm{H}_{10} \mathrm{O} ;[92-83-1] \\
\text { (2) 1-Octanol; } \mathrm{C}_{8} \mathrm{H}_{18} \mathrm{O} ;[111-87-5]\end{array}$ & $\begin{array}{l}\text { Original Measurements: } \\
{ }^{77} \text { C. I. Monárrez, D. M. Stovall, J. H. } \\
\text { Woo, P. Taylor, and W. E. Acree, Jr., } \\
\text { Phys. Chem. Liq. 40, } 703 \text { (2002). }\end{array}$ \\
\hline $\begin{array}{l}\text { Variables: } \\
T / \mathrm{K}=298.15\end{array}$ & $\begin{array}{l}\text { Prepared by: } \\
\text { W. E. Acree, Jr. }\end{array}$ \\
\hline
\end{tabular}

Experimental Values

\begin{tabular}{lcc}
\hline \hline$x_{2}{ }^{(\mathrm{s}) \mathrm{a}}$ & $x_{2}{ }^{\mathrm{b}}$ & $x_{1}{ }^{\mathrm{c}}$ \\
\hline 1.0000 & 0.9620 & 0.03800 \\
\hline${ }^{\mathrm{a}} x_{2}{ }^{(\mathrm{s})}:$ initial mole fraction of component 2 in the solution. \\
${ }^{\mathrm{b}} x_{2}:$ mole fraction of component 2 in the saturated solution. \\
${ }^{\mathrm{c}} x_{1}:$ mole fraction solubility of the solute.
\end{tabular}

\section{Auxiliary Information}

\section{Method/Apparatus/Procedure:}

Constant-temperature bath, calorimetric thermometer, and an ultraviolet/ visible spectrophotometer.

Excess solute and solvent were placed in amber glass bottles and allowed to equilibrate for several days at constant temperature. Attainment of equilibrium was verified by several repetitive measurements and by approaching equilibrium from supersaturation. Aliquots of saturated solutions were transferred through a coarse filter into tared volumetric flasks, weighed and diluted with methanol. Concentrations were determined by spectrophotometric measurements at $280 \mathrm{~nm}$.

\section{Source and Purity of Chemicals:}

(1) $98 \%$, Aldrich Chemical Company, Milwaukee, WI, USA, was recrystallized several times from anhydrous methanol.

(2) $99+\%$, anhydrous, Aldrich Chemical Company, stored over molecular sieves and distilled before use.

\section{Estimated Error:}

Temperature: $\pm 0.1 \mathrm{~K}$.

$x_{1}: \pm 1.5 \%$ (relative error).

\begin{tabular}{ll}
\hline \hline Components: & Original Measurements: \\
(1) Xanthene; $\mathrm{C}_{13} \mathrm{H}_{10} \mathrm{O} ;[92-83-1]$ & ${ }^{77} \mathrm{C}$. I. Monárrez, D. M. Stovall, J. H. \\
(2) 1-Decanol; $\mathrm{C}_{10} \mathrm{H}_{22} \mathrm{O} ;[112-30-1]$ & Woo, P. Taylor, and W. E. Acree, Jr., \\
& Phys. Chem. Liq. 40, 703 (2002). \\
\hline Variables: & Prepared by: \\
$T / \mathrm{K}=298.15$ & W. E. Acree, Jr. \\
\hline
\end{tabular}

\section{Experimental Values}

\begin{tabular}{|c|c|c|}
\hline$x_{2}{ }^{(\mathrm{s}) \mathrm{a}}$ & $x_{2}{ }^{b}$ & $x_{1}{ }^{\mathrm{c}}$ \\
\hline 1.0000 & 0.9547 & 0.04528 \\
\hline
\end{tabular}

\section{Auxiliary Information}

\section{Method/Apparatus/Procedure:}

Constant-temperature bath, calorimetric thermometer, and an ultraviolet/ visible spectrophotometer.

Excess solute and solvent were placed in amber glass bottles and allowed to equilibrate for several days at constant temperature. Attainment of equilibrium was verified by several repetitive measurements and by approaching equilibrium from supersaturation. Aliquots of saturated solutions were transferred through a coarse filter into tared volumetric flasks, weighed and diluted with methanol. Concentrations were determined by spectrophotometric measurements at $280 \mathrm{~nm}$. 
Source and Purity of Chemicals:

(1) $98 \%$, Aldrich Chemical Company, Milwaukee, WI, USA, was

recrystallized several times from anhydrous methanol.

(2) $99+\%$, Alfa Aesar, USA, stored over molecular sieves and distilled before use.

\section{Estimated Error:}

Temperature: $\pm 0.1 \mathrm{~K}$.

$x_{1}: \pm 1.5 \%$ (relative error).

Experimental Values

\begin{tabular}{|c|c|c|}
\hline$x_{2}{ }^{(\mathrm{s}) \mathrm{a}}$ & $x_{2}{ }^{b}$ & $x_{1}{ }^{\mathrm{c}}$ \\
\hline 1.0000 & 0.9570 & 0.04299 \\
\hline
\end{tabular}

\section{Auxiliary Information}

\section{Components:}

(1) Xanthene; $\mathrm{C}_{13} \mathrm{H}_{10} \mathrm{O}$; [92-83-1]

(2) Cyclopentanol; $\mathrm{C}_{5} \mathrm{H}_{10} \mathrm{O}$;

[96-41-3]

\section{Variables:}

$T / \mathrm{K}=298.15$

\section{Original Measurements:}

${ }^{77}$ C. I. Monárrez, D. M. Stovall, J. H. Woo, P. Taylor, and W. E. Acree, Jr., Phys. Chem. Liq. 40, 703 (2002).

\section{Prepared by:}

W. E. Acree, Jr.

\section{Experimental Values}

\begin{tabular}{lcc}
\hline \hline$x_{2}{ }^{(\mathrm{s}) \mathrm{a}}$ & $x_{2}{ }^{\mathrm{b}}$ & $x_{1}{ }^{\mathrm{c}}$ \\
\hline 1.0000 & 0.9711 & 0.02886
\end{tabular}

${ }^{a} x_{2}{ }^{(\mathrm{s})}$ : initial mole fraction of component 2 in the solution.

${ }^{\mathrm{b}} x_{2}$ : mole fraction of component 2 in the saturated solution.

${ }^{c} x_{1}$ : mole fraction solubility of the solute.

\section{Auxiliary Information}

\section{Method/Apparatus/Procedure:}

Constant-temperature bath, calorimetric thermometer, and an ultraviolet/ visible spectrophotometer.

Excess solute and solvent were placed in amber glass bottles and allowed to equilibrate for several days at constant temperature. Attainment of equilibrium was verified by several repetitive measurements and by approaching equilibrium from supersaturation. Aliquots of saturated solutions were transferred through a coarse filter into tared volumetric flasks, weighed and diluted with methanol. Concentrations were determined by

spectrophotometric measurements at $280 \mathrm{~nm}$.

Source and Purity of Chemicals:

(1) $98 \%$, Aldrich Chemical Company, Milwaukee, WI, USA, was recrystallized several times from anhydrous methanol.

(2) $99+\%$, Aldrich Chemical Company, stored over molecular sieves and distilled before use.

Estimated Error:

Temperature: $\pm 0.1 \mathrm{~K}$.

$x_{1}: \pm 1.5 \%$ (relative error).

\subsection{Xanthene solubility data in alkoxyalcohols}

\section{Components:}

(1) Xanthene; $\mathrm{C}_{13} \mathrm{H}_{10} \mathrm{O}$; [92-83-1]

(2) 2-Ethoxyethanol; $\mathrm{C}_{4} \mathrm{H}_{10} \mathrm{O}_{2} ;[110-80-5]$

\begin{tabular}{ll}
\hline Variables: & Prepared by: \\
$T / \mathrm{K}=298.15$ & W. E. Acree, Jr. \\
\hline
\end{tabular}

Original Measurements:

W. E. Acree, unpublished W.

Prepared by:
W. E. Acree, Jr.

\section{Auxiliary Information}

\section{Method/Apparatus/Procedure:}

Constant-temperature bath, calorimetric thermometer, and an ultraviolet/ visible spectrophotometer.

Excess solute and solvent were placed in amber glass bottles and allowed to equilibrate for several days at constant temperature. Attainment of equilibrium was verified by several repetitive measurements and by approaching equilibrium from supersaturation. Aliquots of saturated solutions were transferred through a coarse filter into tared volumetric flasks, weighed and diluted with methanol. Concentrations were determined by spectrophotometric measurements at $280 \mathrm{~nm}$. 
Source and Purity of Chemicals:

(1) $98 \%$, Aldrich Chemical Company, Milwaukee, WI, USA, was

recrystallized several times from anhydrous methanol.

(2) $99 \%$, Aldrich Chemical Company, stored over molecular sieves before use.

Estimated Error:

Temperature: $\pm 0.1 \mathrm{~K}$.

$x_{1}: \pm 1.5 \%$ (relative error).

\begin{tabular}{|c|c|}
\hline $\begin{array}{l}\text { Components: } \\
\text { (1) Xanthene; } \mathrm{C}_{13} \mathrm{H}_{10} \mathrm{O} ;[92-83-1] \\
\text { (2) 2-Isopropoxyethanol; } \mathrm{C}_{5} \mathrm{H}_{12} \mathrm{O}_{2} \text {; } \\
\text { [109-59-1] }\end{array}$ & $\begin{array}{l}\text { Original Measurements: } \\
\text { W. E. Acree, Jr., unpublished data }\end{array}$ \\
\hline $\begin{array}{l}\text { Variables: } \\
T / \mathrm{K}=298.15\end{array}$ & $\begin{array}{l}\text { Prepared by: } \\
\text { W. E. Acree, Jr. }\end{array}$ \\
\hline
\end{tabular}

Experimental Values

\begin{tabular}{lcc}
\hline \hline$x_{2}{ }^{(\mathrm{s}) \mathrm{a}}$ & $x_{2}{ }^{\mathrm{b}}$ & $x_{1}{ }^{\mathrm{c}}$ \\
\hline 1.0000 & 0.9471 & 0.05285 \\
\hline $\mathrm{a}^{(\mathrm{s})}:$ initial mat
\end{tabular}

${ }^{\mathrm{a}} x_{2}{ }^{(\mathrm{s})}$ : initial mole fraction of component 2 in the solution.

${ }^{\mathrm{b}} x_{2}$ : mole fraction of component 2 in the saturated solution.

${ }^{\mathrm{c}} x_{1}$ : mole fraction solubility of the solute.

\section{Auxiliary Information}

\section{Method/Apparatus/Procedure:}

Constant-temperature bath, calorimetric thermometer, and an ultraviolet/ visible spectrophotometer.

Excess solute and solvent were placed in amber glass bottles and allowed to equilibrate for several days at constant temperature. Attainment of equilibrium was verified by several repetitive measurements and by approaching equilibrium from supersaturation. Aliquots of saturated solutions were transferred through a coarse filter into tared volumetric flasks, weighed and diluted with methanol. Concentrations were determined by spectrophotometric measurements at $280 \mathrm{~nm}$.

Source and Purity of Chemicals:

(1) $98 \%$, Aldrich Chemical Company, Milwaukee, WI, USA, was recrystallized several times from anhydrous methanol.

(2) $99 \%$, Aldrich Chemical Company, stored over molecular sieves before use.

Estimated Error:

Temperature: $\pm 0.1 \mathrm{~K}$.

$x_{1}: \pm 1.5 \%$ (relative error).

\begin{tabular}{ll}
\hline \hline Components: & Original Measurements: \\
(1) Xanthene; $\mathrm{C}_{13} \mathrm{H}_{10} \mathrm{O} ;[92-83-1]$ & W. E. Acree, Jr., \\
(2) 2-Butoxyethanol; $\mathrm{C}_{6} \mathrm{H}_{14} \mathrm{O}_{2} ;[111-76-2]$ & unpublished data. \\
\hline Variables: & Prepared by: \\
$T / \mathrm{K}=298.15$ & W. E. Acree, Jr. \\
\hline
\end{tabular}

Experimental Values

\begin{tabular}{|c|c|c|}
\hline$x_{2}{ }^{(\mathrm{s}) \mathrm{a}}$ & $x_{2}{ }^{b}$ & $x_{1}{ }^{\mathrm{c}}$ \\
\hline 1.0000 & 0.9471 & 0.05288 \\
\hline
\end{tabular}

Auxiliary Information

\section{Method/Apparatus/Procedure:}

Constant-temperature bath, calorimetric thermometer, and an ultraviolet/ visible spectrophotometer.

Excess solute and solvent were placed in amber glass bottles and allowed to equilibrate for several days at constant temperature. Attainment of equilibrium was verified by several repetitive measurements and by approaching equilibrium from supersaturation. Aliquots of saturated solutions were transferred through a coarse filter into tared volumetric flasks, weighed and diluted with methanol. Concentrations were determined by spectrophotometric measurements at $280 \mathrm{~nm}$.

\section{Source and Purity of Chemicals:}

(1) $98 \%$, Aldrich Chemical Company, Milwaukee, WI, USA, was recrystallized several times from anhydrous methanol.

(2) $99+\%$, Acros Organics, USA, stored over anhydrous sodium sulfate and molecular sieves before use.

\section{Estimated Error:}

Temperature: $\pm 0.1 \mathrm{~K}$.

$x_{1}: \pm 1.5 \%$ (relative error).

\subsection{Xanthene solubility data in miscellaneous organic solvents}

\begin{tabular}{ll}
\hline \hline Components: & Original Measurements: \\
(1) Xanthene; $\mathrm{C}_{13} \mathrm{H}_{10} \mathrm{O} ;[92-83-1]$ & ${ }^{77}$ C. I. Monárrez, D. M. Stovall, J. H. \\
(2) Ethanenitrile; $\mathrm{C}_{2} \mathrm{H}_{3} \mathrm{~N} ;[75-05-8]$ & Woo, P. Taylor, and W. E. Acree, Jr., \\
& Phys. Chem. Liq. 40, 703 (2002). \\
\hline Variables: & Prepared by: \\
$T / \mathrm{K}=298.15$ & W. E. Acree, Jr. \\
\hline
\end{tabular}

Experimental Values

\begin{tabular}{lcc}
\hline \hline$x_{2}{ }^{(\mathrm{s}) \mathrm{a}}$ & $x_{2}{ }^{\mathrm{b}}$ & $x_{1}{ }^{\mathrm{c}}$ \\
\hline 1.0000 & 0.9803 & 0.01970 \\
$\frac{{ }^{\mathrm{a}} x_{2}{ }^{(\mathrm{s})}: \text { initial mole fraction of component } 2 \text { in the solution. }}{{ }^{\mathrm{b}} x_{2}: \text { mole fraction of component } 2 \text { in the saturated solution. }}$ \\
${ }^{\mathrm{c}} x_{1}:$ mole fraction solubility of the solute.
\end{tabular}




\section{Auxiliary Information}

\section{Method/Apparatus/Procedure:}

Constant-temperature bath, calorimetric thermometer, and an ultraviolet/ visible spectrophotometer.

Excess solute and solvent were placed in amber glass bottles and allowed to equilibrate for several days at constant temperature. Attainment of equilibrium was verified by several repetitive measurements and by approaching equilibrium from supersaturation. Aliquots of saturated solutions were transferred through a coarse filter into tared volumetric flasks, weighed and diluted with methanol. Concentrations were determined by spectrophotometric measurements at $280 \mathrm{~nm}$.

\section{Source and Purity of Chemicals:}

(1) $98 \%$, Aldrich Chemical Company, Milwaukee, WI, USA, was recrystallized several times from anhydrous methanol.

(2) $99.8 \%$, anhydrous, Aldrich Chemical Company, stored over molecular sieves and distilled before use.

\section{Estimated Error:}

Temperature: $\pm 0.1 \mathrm{~K}$.

$x_{1}: \pm 1.5 \%$ (relative error).

\section{References}

${ }^{1}$ Polycyclic Aromatic Hydrocarbons in Pure and Binary Solvent Mixtures, edited by W. E. Acree, Jr., IUPAC Solubility Data Series, Vol. 54 (Oxford University Press, London, 1994).

${ }^{2}$ Polycyclic Aromatic Hydrocarbons: Binary Nonaqueous Systems. Part I. Solutes A-E, edited by W. E. Acree, Jr., IUPAC Solubility Data Series, Vol. 58 (Oxford University Press, London, 1995).

${ }^{3}$ W. E. Acree, Jr., Polycyclic Aromatic Hydrocarbons: Binary Nonaqueous Systems. Part 2. Solutes F-Z, IUPAC Solubility Data Series, Vol. 59 (Oxford University Press, London, 1995).

${ }^{4}$ W. E. Acree, Jr., J. Phys. Chem. Ref. Data 42, 013103 (2013).

${ }^{5}$ W. E. Acree, Jr., J. Phys. Chem. Ref. Data 42, 013104 (2013).

${ }^{6} \mathrm{~J}$. M. Prausnitz, Molecular Thermodynamics of Fluid-Phase Equilibria (Prentice-Hall, Englewood Cliffs, NJ, 1969).

${ }^{7}$ H. Buchowski, A. Ksiazczak, and S. J. Pietrzyk, J. Phys. Chem. 84, 975 (1980).

${ }^{8}$ H. Buchowski and A. Khiat, Fluid Phase Equilib. 25, 273 (1986).

${ }^{9}$ K. M. De Fina, T. L. Sharp, and W. E. Acree, Jr., Can. J. Chem. 77, 1537 (1999).

${ }^{10}$ W. E. Acree, Jr. and M. H. Abraham, Fluid Phase Equilib. 201, 245 (2002).

${ }^{11}$ E. McLaughlin and H. A. Zainal, J. Chem. Soc. 3854 (1960).

${ }^{12}$ P. B. Choi, C. P. Williams, K. G. Buehring, and E. McLaughlin, J. Chem. Eng. Data 30, 403 (1985).

${ }^{13}$ I. Kotula and B. Marciniak, J. Chem. Eng. Data 46, 783 (2001).

${ }^{14}$ F. He and P. Liu, J. Chem. Eng. Data 52, 2536 (2007).

${ }^{15}$ A. Aoulmi, M. Bouroukba, R. Solimando, and M. Rogalski, Fluid Phase Equilib. 110, 283 (1995).

${ }^{16}$ L. E. Roy, C. E. Hernández, and W. E. Acree, Jr., Polycyclic Aromat. Compd. 13, 105 (1999).

${ }^{17}$ M. H. Abraham and W. E. Acree, Jr., New J. Chem. 28, 1538 (2004).

${ }^{18}$ L. Alcazar, A. Blanco, R. Cano, L. Fisher, M. Nau, L. Sidransky, and W. E. Acree, Jr., J. Chem. Eng. Data 53, 201 (2008).

${ }^{19}$ M. Carrillo, M. Corella, K. Wolcott, K. R. Bowen, and W. E. Acree, Jr., J. Chem. Eng. Data 52, 270 (2007).

${ }^{20}$ J. R. Powell, M. E. R. McHale, A.-S. M. Kauppila, and W. E. Acree, Jr., J. Chem. Thermodyn. 28, 1215 (1996)

${ }^{21}$ K. S. Coym, L. E. Roy, C. E. Hernández, and W. E. Acree, Jr., Chem. Eng. Commun. 162, 215 (1997).

${ }^{22}$ M. H. Abraham, W. E. Acree, Jr., A. J. Leo, and D. Hoekman, New J. Chem. 33, 1685 (2009).

${ }^{23}$ K. M. De Fina, T. T. Van, A. Ibarra, E. Hamilton, J. Martinez, A. Valdez, and W. E. Acree, Jr., Phys. Chem. Liq. 39, 249 (2001).

${ }^{24}$ J. R. Powell, M. E. R. McHale, A.-S. M. Kauppila, and W. E. Acree, Jr., J. Chem. Eng. Data 41, 728 (1996).
${ }^{25}$ C. I. Monárrez, P. G. Taylor, A. M. Tran, and W. E. Acree, Jr., J. Chem. Eng. Data 48, 1341 (2003).

${ }^{26}$ J. R. Powell, K. A. Fletcher, K. S. Coym, W. E. Acree, Jr., V. G. Varanasi, and S. W. Campbell, Int. J. Thermophys. 18, 1495 (1997).

${ }^{27}$ J. R. Powell, M. E. R. McHale, A.-S. M. Kauppila, P. Otero, M. Jayasekera, and W. E. Acree, Jr., J. Chem. Eng. Data 40, 1270 (1995).

${ }^{28}$ W. E. Acree, Jr. and M. H. Abraham, Can. J. Chem. 79, 1466 (2001).

${ }^{29}$ C. E. Hernández, L. E. Roy, T. Deng, M. B. Tuggle, and W. E. Acree, Jr., Phys. Chem. Liq. 37, 677 (1999).

${ }^{30}$ C. E. Hernández, L. E. Roy, G. D. Reddy, G. L. Martinez, A. Jackson, G. Brown, and W. E. Acree, Jr., Chem. Eng. Commun. 169, 137 (1998).

${ }^{31}$ C. E. Hernández, L. E. Roy, G. D. Reddy, G. L. Martinez, A. Jackson, G. Brown, T. L. Borders, J. T. Sanders, and W. E. Acree, Jr., Phys. Chem. Liq. 36, 257 (1998).

${ }^{32}$ M. E. R. McHale, A.-S. M. Kauppila, J. R. Powell, and W. E. Acree, Jr., J. Chem. Thermodyn. 28, 209 (1996).

${ }^{33}$ C. E. Hernández, L. E. Roy, G. D. Reddy, T. L. Borders, J. T. Sanders, and W. E. Acree, Jr., Phys. Chem. Liq. 37, 31 (1998).

${ }^{34}$ A. Shayanfar, S. H. Eghrary, F. Sardari, W. E. Acree, Jr., and A. Jouyban, J. Chem. Eng. Data 56, 2290 (2011).

${ }^{35}$ Z. Lisicki and M. E. Jamróz, J. Chem. Thermodyn. 32, 1335 (2000).

${ }^{36}$ K. M. De Fina, T. L. Sharp, and W. E. Acree, Jr., Can. J. Chem. 77, 1589 (1999).

${ }^{37}$ L. M. Grubbs, M. Saifullah, N. E. De La Rosa, S. Ye, S. S. Achi, W. E. Acree, Jr., and M. H. Abraham, Fluid Phase Equilib. 298, 48 (2010).

${ }^{38}$ A. C. Mengarelli, S. B. Bottini, and E. A. Brignole, J. Chem. Eng. Data 40, 746 (1995).

${ }^{39}$ J. E. Coon, W. B. Sediawan, J. E. Auwaerter, and E. McLauglin, J. Solution Chem. 17, 519 (1988).

${ }^{40}$ U. Domanska, F. R. Broves, Jr., and E. McLaughlin, J. Chem. Eng. Data 38, 88 (1993).

${ }^{41}$ Y. Wei, L. Dang, Z. Zhang, W. Cui, and H. Wei, J. Chem. Eng. Data 57, 1279 (2012).

${ }^{42}$ S. L. Hafsaoui and R. Mahmoud, J. Therm Anal. Calorim. 88, 565 (2007).

${ }^{43}$ L. F. Ramirez-Verduzco, A. Rojas-Aguilar, J. A. De los Reyes, J. A. MunozArroyo, and F. Murrieta-Guevara, J. Chem. Eng. Data 52, 2212 (2007).

${ }^{44}$ C. E. Hernández and W. E. Acree, Jr., Can. J. Chem. 76, 1312 (1998).

${ }^{45}$ L. E. Roy, C. E. Hernández, and W. E. Acree, Jr., Polycyclic Aromat. Compd. 13, 205 (1999).

${ }^{46}$ R. Pinal, P. S. C. Rao, L. S. Lee, P. V. Cline, and S. H. Yalkowsky, Environ. Sci. Technol. 24, 639 (1990).

${ }^{47}$ M. M. Miller, S. P. Waskik, G.-L. Huang, W.-Y. Shiu, and D. Mackay, Environ. Sci. Technol. 19, 522 (1985).

${ }^{48}$ C. I. Monárrez, W. E. Acree, Jr., and M. H. Abraham, Phys. Chem. Liq. 40, 581 (2002)

${ }^{49}$ D. M. Stovall, K. R. Hoover, W. E. Acree, Jr., and M. H. Abraham, Polycyclic Aromat. Compd. 25, 313 (2005).

${ }^{50}$ Z. Zou, L. Dang, P. Liu, and H. Wei, J. Chem. Eng. Data 52, 1501 (2007).

${ }^{51}$ Y. Han and Z. Wang, J. Chem. Eng. Data 54, 148 (2009).

${ }^{52}$ T.-B. Cui, T.-L. Luo, C. Zhang, Z.-B. Mao, and G.-J. Liu, J. Chem. Eng. Data 54, 1065 (2009).

${ }^{53}$ Q.-S. Li, Z.-M. Yi, M.-G. Su, and X.-F. Sun, J. Chem. Eng. Data 53, 2701 (2008).

${ }^{54}$ P. M. Ghogomu, R. Rakolosaona, M. Bouroukba, D. Petitjean, N. Hubert, and M. Dirand, Mol. Cryst. Liq. Cryst. 408, 103 (2004).

${ }^{55}$ T. Tsuji, K. Sue, T. Hiaki, and N. Itoh, Fluid Phase Equilib. 257, 183 (2007).

${ }^{56}$ B. L. Sharma, R. Kant, R. Sharma, and S. Tandon, Mater. Chem. Phys. 82, 216 (2003)

${ }^{57}$ D. Wei and K. Jin, J. Chem. Thermodyn. 41, 145 (2009).

${ }^{58}$ U. S. Rai and R. N. Rai, Asian J. Chem. 10, 421 (1998).

${ }^{59}$ A. Shayanfar, S. Soltani, F. Jabbaribar, A. A. Hamidi, W. E. Acree, Jr., and A. Jouyban, J. Chem. Eng. Data 53, 574 (2008).

${ }^{60}$ A. F. Lagalante, A. Abdulagatov, and T. J. Bruno, J. Chem. Eng. Data 47, 47 (2002).

${ }^{61}$ C. E. Hernández, K. M. De Fina, L. E. Roy, T. L. Sharp, and W. E. Acree, Jr., Can. J. Chem. 77, 1465 (1999).

${ }^{62}$ M. A. A. Fakhree, W. E. Acree, Jr., and A. Jouyban, J. Chem. Eng. Data 55, 531 (2010)

${ }^{63}$ M. A. A. Fakhree, W. E. Acree, Jr., and A. Jouyban, Ind. Eng. Chem. Res. 49, 6238 (2010).

${ }^{64}$ Y. Bakhbakhi, P. Charpentier, and S. Rohani, Can. J. Chem. Eng. 83, 267 (2005). 
${ }^{65}$ K. R. Hoover, W. E. Acree, Jr., and M. H. Abraham, Phys. Chem. Liq. 44, 367 (2006).

${ }^{66}$ M. Saifullah, S. Ye, L. M. Grubbs, N. E. De La Rosa, W. E. Acree, Jr., and M. H. Abraham, J. Solution Chem. 40, 2082 (2011).

${ }^{67}$ S. Ahmadian, V. Panahi-Azar, M. A. A. Fakhree, W. E. Acree, Jr., and A. Jouyban, J. Chem. Eng. Data 56, 4352 (2011).

${ }^{68}$ M. E. R. McHale, A.-S. M. Horton, S. A. Padilla, A. L. Trufant, N. U. De La Sancha, E. Vela, and W. E. Acree, Jr., J. Chem. Eng. Data 41, 1522 (1996).

${ }^{69}$ M. E. R. McHale, K. S. Coym, K. A. Fletcher, and W. E. Acree, Jr., J. Chem. Eng. Data 42, 511 (1997).

${ }^{70}$ M. E. R. McHale, K. A. Fletcher, K. S. Coym, W. E. Acree, Jr., V. G. Varanasi, and S. W. Campbell, Phys. Chem. Liq. 34, 103 (1997).
${ }^{71}$ Q. Yu, X. Ma, and L. Xu, Fluid Phase Equilib. 319, 5 (2012).

${ }^{72}$ J. R. Powell, D. Voisinet, A. Salazar, and W. E. Acree, Jr., Phys. Chem. Liq. 28, 269 (1994).

${ }^{73}$ S. H. Ali and O. A. Al-Rashed, Fluid Phase Equilib. 281, 133 (2009).

${ }^{74}$ S. H. Ali, Fluid Phase Equilib. 264, 29 (2008).

${ }^{75}$ K. A. Fletcher, M. E. R. McHale, J. R. Powell, K. S. Coym, and W. E. Acree, Jr., Phys. Chem. Liq. 34, 41 (1997).

${ }^{76}$ D. M. Stovall, W. E. Acree, Jr., and M. H. Abraham, Fluid Phase Equilib. 232, 113 (2005).

${ }^{77}$ C. I. Monárrez, D. M. Stovall, J. H. Woo, P. Taylor, and W. E. Acree, Jr., Phys. Chem. Liq. 40, 703 (2002). 